\title{
LEGIBILITY
}

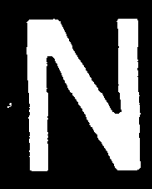

A major purpose of the Technical Information Center is to provide the broadest dissemination possible of information contained in DOE's Research and Development Reports to business, industry, the academic community, and federal, state and local governments.

Although a small portion of this report is not reproducible, it is being made available to expedite the availability of information on the research discussed herein. 
ORNL-6582

Distribution Category UC-413

Nuclear Physics

ORRL- -6582

Er.gineering Physics and Mathematics Divizion

DE90 006440

\section{ENGINEERING PHYSICS AND MATHEMATICS DIVISION PROGRESS REPORT \\ FOR PERIOD ENDING AUGUST 31, 1989}

F. C. Maienschein, Director

Date Published - December 1989

Prepared by the

OAK RIDGE NATIONAL LABORATORY

Oak Ridge, Tennessee 37831 operated by

MARTIN MARIETTA ENERGY SYSTEMS, INC.

for the

U.S. DEPARTMENT OF ENERGY

under contract DE-ACO5-84OR21400 
Reports previously issued in this series are as follows:

ORNL-2081

ORNL-2389

URNL-2609

ORNL-2842

ORNL-3016

ORNL-3193

ORNL-3360

ORNL-3499, Vols. I and II

ORNL-3714, Vols. I and II

ORNL-3858, Vols. I and II

ORNL-3973, Vols. I and II

ORNL-4134

ORNL-4280

ORNL-4433

ORNL-4592

ORNL-4705

ORNL-4800

ORNL-4902

CRNL-4997

ORNL-5101

ORNL-5280

ORNL-5504

ORNL-5725

ORNL-5897, Vols. I and II

ORNL-6030

ORNL-6214

ORNL-6425
Period Ending September 10, 1956

Period Ending September 1, 1957

Period Ending September 1, 1958

Period Ending September 1, 1959

Period Ending September 1, 1960

Period Ending September 1, 1961

Periud Ending September 1, 1962

Period Ending August 1, 1963

Period Ending August 1, 1964

Period Ending August 1, 1965

Period Ending May 31, 1966

Period Ending May 31, 1967

Period Ending May 31, 1968

Period Ending May 31, 1969

Period Ending May 31, 1970

Period Ending May 31, 1971

Periud Ending May 31, 1972

Period Ending May 31, 1973

Period Ending August 31, 1974

Period Ending October 31, 1975

Period Ending February 28, 1977

Period Ending November 30, 1978

Period Ending November 30, 1980

Period Ending May 31, 1982

Period Ending December 31, 1983

Period Ending June 30, 1985

Period Ending September 30, 1987 


\section{PREFACE}

The primary purpose of this report is to provide an archival record of the activities of the Engineering Physics and Matkematics Division during the period October 1. 1987 through August 31, 1989. Earlier reports in this series are identified on the previous page.

Coverage of our research is provided primarily through abstracts of journal articles. reports, and presentations. In addition, this progress report includes appendices which sunumarize publications, presentations, and other professional activities of the division staff members as well as identification of new staff members, guests, ard students. Of necessity, this report tends to look back upon past progress. A look forward toward the slowly changing role and dirertions for the division is provided in the Long-Range Plan for the perind 1987-1991, which was published in Januery 1988.

Unusual challenges strongly impacted the division's work during the period. The continued wait for restart of the Tower Shielding Facility (since March 1987) has delayed the joint U.S.-Japan reactor-shielding program JASPER. The overriding DOE-wide concern with real and possible environmental, safety, and health problems requires increasing resources (of both funds and staff time), which now significantly reduce research productivity. The impact is universal, but is greatly emphasized in experimental programs. The continued difficulties of the Mathematical Sciences Section with a renıote location in an otherwise abandoned building which has peculiar environmental features should be eliminated in 1991 when a building now under contract should be completed.

The report is organized following the division of our research among four sections and an information center. 'The division organization chart, as of October 1, 1989, is included at the back of the report.

Mathematical Sciences. Activities in the mathematical sciences feature increased and broadened interactions with the University of Tennessee. Examples include the Numerical Linear Algebra Year and the establishment of the Advanced Computing Laboratory. The ACL is expected to be equipped with a multi-rigaflop parallel computer in FY90.

In collaboration with Cornell University, a fracture-analysis system, based upon a new boundary-element technique, was developed to make feasible efficient numerical simulation of crack propagation, even for multiple cracks.

Nuclear-Data Measurement and Evaluation. Nuclear-data activities are gradually reduced in the U.S. and in other countries. In this context, about $60 \%$ of the nuclear-data evaluations in the next major release of the U.S. Evaluated Nuclear Data File, Version VI came from our nuclear-data section. ORELA (the Oak Ricige Electron Linear Accelerator) continues as the prime nuclear-data facility in the world and as such it has seen recent collaboration with groups from Harwell (VK), The University of Vienna (Austria), Cadarache (France), and the National Institute of Srience and Terhnology (formerly NBS). Uses other than utuclear lata include ORNL condensed-matter studies and a new intense parasiti: position beam. ORNL support has not developed for basing a free-electron laser at ORELA although this seems feasible for at least much of the spectral range of interest. 
Intelligent Systems. Mobile robots have progressed to HERMIIES-III, with an on-board manipulator and improved on-board sensors and computers. Advances have continued in control algorithms for autonomcus robots. Attention is turning to the use of intelligent robots for environmental remediation and waste management. Close cooperation is in place with four universities (Florida, Michigan, Tennessee, and Texas). Our human-factors specialists have joined this section with the goal of studying human-machine symbiosis in order to optimize the contributions of intelligent machines.

Nuclear Analysis and Shielding. Analysis continued for diverse nuclear systems. New applications emphasize the New Production Reactor, especially in its grafite-moderated version. SDI hypervelocity particle shielding studies have been completed. Deterministic combat models incorporated nonlinear behavior. A major effort is evolving from the application of high-energy physics analysis to detectors planned for the Superconducting Super Collider (SSC). This section will contribute substantially to a new specialized center for detector development which is expected to include participation from many ORNL divisions and from universities, especially in the southeastern U.S.

Engineering Physics Information Center. The work of the Technical Data Management Center was completed, after a decade of operation for the Nuclear Regulatory Commission. For the Radiation Shielding Information Center, international cooperation assumes increasing importance. In the earlier years of operation, much of the technology to be shared came from the U.S. (and especially ORNL). Now the contributions from other countries (especially France and Japan) have assumed a major position. 


\section{TABLE OF CONTENTS}

PREFACE

iii

\section{Section 1. MATHEMATICAL SCIENCES}

1.0. Introduction ................... 3

\section{COMPUTER SCIENCE}

Geraral Parallel Computing

1.1. A Remote Host Facility for Intel Hypercubes

T. H. Dunigen . . . . . . . . . . . . 5

1.2. Performance of a Second Generation Hypercube

T. H. Dunigan . . . . . . . . . . . . 5

1.3. Hypercube Simulation on a Local Area Network

T. H. Dunigan . . . . . . . . . . . 5

1.4. A Parallel Decomposition Algorithm for Staircase Linear Programs

R. Entriken . . . . . . . . . . . . 5

1.5. Hypercube Multiprocessors: What They Are and How to Use Them

G. A. Geist . . . . . . . . . . . 6

1.6. A Machine-Independent Communication Library

G. A. Geist, M. T. Heath, B. W. Peyton, and P. H. Worley . . . . 6

1.7. Parallel Computing

M. T. Heath . . . . . . . . . . . . . 6

1.8. Parallel Computing: Perspectives and Prospects

M. T. Heath . . . . . . . . . . . . . . 7

1.9. Hypercube Applications at Oak Ridge National Laboratory

M. T. Heath . . . . . . . . . . . . . . . . . . 7

1.10. Hypercubes

M. T. Heath . . . . . . . . . . 7 
1.11. Limits to Parallelism

M. T. Heath

1.12. Once Again, Amdahl's Law

M. T. Heath and P. B. Worley . . . . . . . . . 8

1.13. Multiprogramming a Distributed-Memory Multiprocessor

M. R. Leuze, L. W. Dowdy, and K. H. Park . . . . . . . . . 8

1.14. A Bibliography on Parallel and Vector Numerical Algorithms

J. M. Ortega, R. G. Voigt, and C. H. Romine . . . . . . . 8

1.15. A Theoretical Investigation of a Parallel Genetic Algorithm

C. C. Pettey and M. R. Leuze . . . . . . . . . . 8

1.16. Overview of Architectures and Applications in Parallel Prucessing

C. H. Romine . . . . . . . . . . . . . . 9

1.17. The Evolution of Multipmcessing

C. H. Romine

\section{Dense Matrix Computations}

1.18. QR Factorization of a Dense Matrix on a Hypercube Multiprocessor

E. Chu and A. George . . . . . . . . . . . . . . 9

1.19. Reduction of a General Matrix to Tridiagonal Fu'm

G. A. Geist . . . . . . . . . . . . . 10

1.20. Reduction of a General Matrix to Tridiagonal Form Using a

Hypercube Multiprocessor

G. A. Geist . . . . . . . . . . . . 10

1.21. Finding Eigenvalues and Eigenvectors of Unsymmetric Matrices Using

a Distributed-Memory Multiprocessor

G. A. Geist . . . . . . . . . . . . 10

1.22. The Unsymmetric Eigenvalue: Can HQR Be Beat?

G. A. Geist . . . . . . . . . . . . . . . 10

1.23. Finding Eigenvalues and Eigenvectors of Unsymmetric Matrices Using

a Distributed-Memory Multiprocessor

G. A. Geist and G. J. Davis . . . . . . . . . . . . . . . . . . 11 
1.24. Stabilized Gaussian Reduction of an Arbitrary Matrix to Tridiagonal Form G. A. Geist, A. Lu, and E. L. Wachspress . . . . . . . . . . 11

1.25. A Partitioning Strategy for Parallel Sparse Cholesky Factorization

G. A. Geist and E. Ng . . . . . . . . . . . . 11

1.26. LU Factorization on Distributed-Memory Multiprocessors

G. A. Geist and C. E. Romine . . . . . . . . . . 11

1.27. Finding Eigenvalues and Eigenvectors of Unsymmetric Matrices Using a Hypercube Multiprocessor

G. A. Geist, R. C. Ward, G. J. Davis, and R. E. Funderlic . . . . 12

1.28. Regularization, GSVD and Truncated GSVD

P. C. Hansen . . . . . . . . . . . . . . . . 12

1.29. Solution of Ill-Posed Problems by Means of Truncated SVD

P. C. Hanser . . . . . . . . . . . . . . 12

1.30. Parallel Triangular Solutions and Downdating on Distributed-Memory Multiprocessors

M. T. Heath

1.31. Cholesky Downdating on a Hypercube

C. S. Henkel, M. T. Heath, and R. J. Plemmons . . . . . . . 13

1.32. The Influence of Distributed-Memory Architectures on Linear Algebra Algorithms

C. H. Romine

1.33. Orthogonal Factorization on Distributed-Memory Multiprocessors

C. H. Romine and A. Pothen . . . . . . . . . . . . 14

\section{Sparse Matrix Computations}

1.34. Solution of Sparse Positive Definite Systems on a Hypercube A. George, M. T. Heath, J. Liu, and E. Ng . . . . . . . . . 14

1.35. Communication Results for Parallel Sparse Cholesky Factorization on a Hypercube

A. George, J. Liu, and E. Ng . . . . . . . . . . 15 
1.36. Parallel Sparse Gaussian Elimination with Partial Pivoting

A. George and E. Ng . . . . . . . . . . . . . 15

1.37. Some Aspects of the Solution of Sparse Symmetric Positive Definite Systems on Hypercube Multiprocessors

A. George and E. Ng . . . . . . . . . . . . . 15

1.38. Some Shared Memory Is Desirable in Parallel Sparse Matrix Computation

A. George and E. Ng . . . . . . . . . . . 15

1.39. Parallel Solution of Sparse Linear Systems

M. T. Heath . . . . . . . . . . . . . 16

1.40. Parallel Solution of Sparse Linear Systems

M. T. Heath . . . . . . . . . . . . . . 16

1.41. A Fast Algorithm for Reordering Sparse Matrices for Parallel Factorization

J. G. Lewis, B. W. Peyton, ent A. Pothen . . . . . . . . 16

1.42. On the Complexity of Some Sparse Matrix Factorizations

E. Ng . . . . . . . . . . . . . . 17

1.43. Some Ideas in the Solution of Sparse Linear Least Squares Problems

E. Ng . . . . . . . . . . . . . 17

1.44. Sparse Partial Pivoting Using Static Storage Schemes

E. Ng . . . . . . . . . . . . . 17

1.45. Parallel Direct Solution of Sparse Linear Systems

E. Ng . . . . . . . . . . . . . . 17

1.46. A Comparison of Some Methods for Solving Sparse Nonsymmetric Linear Systems E. $\mathrm{Ng} \ldots \ldots \ldots \ldots \ldots$

1.47. A Scheme for Handling Rank Deficiency in the Solution of Sparse Linear Least Squares Problems

E. $\mathrm{Ng} \ldots \ldots \ldots \ldots$ 


\section{Differential Equations}

1.48. A Collection of Test Problems for Ordinary Differential Equation Solvers Which Have Provisions for Rootfinding

S. Thompson ..................... 18

1.49. Explicitly Parallel Algorithms for Hyperbolic PDES - A Proof of Principle P. H. Worley . . . . . . . . . . . . . . . . . 18

1.50. The Effect of Time Constraints on Scaled Speed-Up P. H. Worley . . . . . . . . . . . . . . . . 19

1.51. Limits on Parallelism in the Numerical Solution of Linear PDES P. H. Worley . . . . . . . . . . . . . . 19

1.52. Limits on Parallelism in the Numerical Approximation of Linear PDES P. H. Worley . . . . . . . . . . . . . . . 19

1.53. Information Requirements and the Implications for Parallel Computation P. H. Worley . . . . . . . . . . . . . . . 20

\section{MATHEMATICS}

\section{Phase Change Problems}

1.54. Effect of a Void on Cyclic Melting and Freezing of an Encapsulated PCM V. Alexiades ................. 20

1.55. Shrinkage Effects on Sol:dincation

V. Aleriades

1.56. Equilibrium Size of a Solid Nucleus Forming in Supercooled Liquid

V. Aleriades

1.57. A Weak Formulation for Phase-Change Problems with Bulk Movement Due to Unequal Densities

V. Alexiades and J. B. Drake . . . . . . . . . . . . . 21

1.58. A Convective Flow in the Presence of Melting

J. B. Drake and V. Aleriades . . . . . . . . . . . . 21

1.59. Changing Domains and a Solidification Problem with Void

S. M. Lenhart and D. G. Wilson . . . . . . . . . . . . . . 22 
1.60. Parallel Solutions of a 2-D Phase Change Problem on a Hypercuive H. N. Narang and J. B. Drake . . . . . . . . . . . 22

1.61. Survey of the Literature on Fractals with Possible Applications to Phase Change Processes

L. A. Renker and D. G. Wilson . . . . . . . . . . . 22

1.62. SIMDLA: A FORTRAN Program Simulating Diffusion-Limited Aggregation

L. A. Renker and D. G. Wilson . . . . . . . . . . . 23

1.63. Iterative Solution of a Nonlinear System Arising in Phase Change

Problems

M. A. Williems and D. G. Wilson . . . . . . . . . 23

1.64. Implicit Finite Difference Schemes for Phase Change Problems

D. G. Wilson . . . . . . . . . . . . . 23

1.65. Cyclic Melting and Freezing with Variable Density

D. G. Wilson . . . . . . . . . . . . . . 24

1.66. An Implicit, Finite Difference, Enthalpy Scheme for Phase Change

Problems

D. G. Wilson . . . . . . . . . . . . . . . 24

1.67. Changing Domains and Solidification Problems with Voids

D. G. Wilson . . . . . . . . . . . . . . . . 24

1.68. An Implicit, Finite Difference Enthalpy Scheme Applied to a Multidimensional Cyclic Melting and Freezing Problem with Variable Density

D. G. Wilson, J. B. Drake, and R. E. Flanery . . . . . . . . . 25

1.69. Phase Change Problems with a Void

D. G. Wilson, J. B. Drake, and R. E. Flanery . . . . . . . . . . 25

1.70. Supercomputer Simulation of a 3-D Melting/Freezing Process

D. G. Wilson and R. E. Flanery . . . . . . . . . . . . 25

1.71. Modeling Cyclic Melting and Refreezing in a Hollow Metal Cylinder D. G. Wilson and R. E. Flanery . . . . . . . . . . 26 


\section{Applied Mathematics}

1.72. Parallel Implementation of the Boundary Element Method on the iPSC2 riypercube

J. B. Drake and L. J. Gray . . . . . . . . . . . . . . . . 26

1.73. MHD Calculatioss on a Hypercube Multiprocessor

J. B. Drak';, W. F. Lawkins, B. Carreras, H. R. Hicks, and V. Lynch . . 27

1.74. A Career as a Working Mathematician - What It's Like to Do It for Real

R. E. Flanery . . . . . . . . . . . . . . . 27

1.75. Magnetically Suspended Centrifuge for Stuäies of Nonlinear Phenomena in Stratified Fluids: Developmental Aspects

G. T. Gillies, M. A. Lawoson, R. C. Ritter, W. F. Lawkins, S. Thompson, and W. K. Sartory . . . . . . . . . . . . . . . 27

1.76. Boundary Element Method for Regions with Cracks

L. J. Gray . . . . . . . . . . . . . . . . . . . . . 28

1.77. Evaluation of Hypersingular Integrals in the Boundary Element Method L. J. Gray . . . . . . . . . . . . . . . . . . . . . . 28

1.78. Numerical Experiments with a Boundary Element Technique for Corners L. J. Gray . . . . . . . . . . . . . . . . . . . . . . . 28

1.79. Boundary Element Method for Regions with Thin Internal Cavities L. J. Gray . . . . . . . . . . . . . . . . . . . . . . . . 29

1.80. Boundary Element Method for Contact Heat Transfer L. J. Gray, A. L. Askew, and G. E. Giles . . . . . . . . . . . . . 29

1.81. Application of the Thin Cavity Method to Shield Calculations ir. Electroplating

L. J. Gray and G. E. Giles . . . . . . . . . . . . . . . . . . . . 29

1.82. Electroplating Simulation

L. J. Gray, G. E. Giles, J. Bullock, IV, and P. McKenzie . . . . . . . 29

1.83. Boundary Element Method for Regions with Thin Internal Cavities. II L. J. Gray. G. E. Giles, and M. W. Wendel 
1.84. Hypersingular Integrals in Boundary Element Fracture Analycis L. J. Gray, L. F. Martha, and A. R. Ingraffea . . . . . . . . . 30

1.85. Application of Onsager's Reciprocity Relations to Interface Motion During Phase Transformations T. Kaplan, M. J. Aziz, and L. J. Gray . . . . . . . . . . . 30

1.86. Curvilinear Coordinates Fitted to a Distorted Annular Region W. F. Lawkins

\section{STATISTICS}

\section{Design and Analysis of Computational Experiments}

1.87. Computer Experiments to Optimize a Compression Mold Filling Process

A. Church. Jr. and T. J. Mitchell . . . . . . . . . . . 32

1.88. A Bayesian Approach to the Design and Analysis of Computer Experiments

C. Curtin, T. J. Mitchell, M. D. Morris, and D. Ylvisaker . . . . . 32

1.89. Sensitivity and Uncertainty Analysis in Environmental Models D. J. Downing . I S. Bartell . . . . . . . . . . . . . . . 32

1.90. A Bayesian Approach to the Lesign and Analysis of Computational Experiments

T. J. Mitchell and M. D. Morris . . . . . . . . . . . 33

1.91. Computer Construction of Optimal Designs for Prediction in Computer Ixperiments

T. J. Mitchell and M. D. Morris . . . . . . . . . . . . 33

1.92. Existence of Smoothed Stationary Processes on an Interval T. J. Mitchell, M. D. Morris, and D. Ylvisaker . . . . . . . . . . . 33

1.93. Factorial Sampling Plans for Preliminary Computational Experiments M. D. Morris . . . . . . . . . . . . . . . . . . . . . . . . . . . 33

1.94. Bayesian Design and Analysis of Computer Experiments: Use of Derivatives in Surface Prediction M. D. Morris . . . . . . . . . . . . . . . . . . . . . . 34 
xiii

1.95. Comment On: Design and Analysis of Computer Experiments

M. D. Morris . . . . . . . . . . . . . . . 34

1.96. A Bayesian Approach to the Design and Analysis of Computational Experiments

M. D. Morris and T. J. Mitchell . . . . . . . . . . 34

1.97. Design and Analysis of Computer Experiments

J. Sacks, W. J. Welch, T. J. Mitchell, and H. P. Wynn . . . . . 35

\section{Computational Distribution Theory}

1.98. Moment Methods and the Gram-Charlier Type A

K. O. Bowman and L. R. Shenton . . . . . . . . . . . 35

1.99. Solutions to Johnson's $S_{B}$ and $S_{U}$

K. O. Bowman and L. R. Shenton . . . . . . . . . . 35

1.100. Properties of Estimators for the Gamma Distribution

$K$. O. Bouman and L. R. Shenton . . . . . . . . . 36

1.101. $S_{B}$ and $S_{U}$ Distribution Fitted by Percentiles: A General Criterion

K. O. Bowman and L. R. Shenton . . . . . . . . . . 36

1.102. Continued Fractions and Asymptotic Series in Applications:

With Special Reference to Problems in Statistics

K. O. Bowman and L. R. Shenton . . . . . . . . . . 36

1.103. Some Properties of a Moment Estimator for the Index Parameter of Negative Binomial Distribution

H. K. Lam, L. R. Shenton, and K. O. Bowman . . . . . . . . 37

1.104. A Problem with Transformations of Stieltjes Continued Fractions

L. R. Shenton and K. O. Bowman . . . . . . . . . . . 37

Research in General Statistical Methods

1.105. Generation of an Ordered Simple Random Sample Without Replacement Using One Random Number

Y. H. Etheridge and T. Wright . . . . . . . . . . 38

1.106. Maximum Likelihood Estimation for Cytogenetic Dose-Response Curves

E. L. Frome and R. J DuFrain . . . . . . . . . . . . . . . 38 
xiv

1.107. Survey Data Quality Control Conference: A Report

G. R. Liepins and V. R. R. Uppuluri

1.108. Detection of Associations Between Diseases in Animal Carcinogenicity Experiments

T. J. Mitchell and B. W. Turnbull . . . . . . . . . . 38

1.109. Bayesian Variable Selection in Linear Regression

T. J. Mitchell and J. J. Beüuchamp . . . . . . . . . . . . . 39

1.110. Bayesian Variable Selection in Linear Regression

T. J. Mitchell and J. J. Beauchamp . . . . . . . . . . . 39

1.111. Srnall-Sample Confidence Limits for Parameters Under Inequality

Constraints with Application to Quantal Bioassay

M. D. Morris . . . . . . . . . . . . . . . . . . .40

1.112. Sparse Matrix Computations in Analysis of Variance

G. Ostrouck.ov . . . . . . . . . . . . . 40

1.113. Statistical Computing on a Hypercube

G. Ostrouchov . . . . . . . . . . . . . . 40

1.114. ANOVA Model Fitting via Sparse Matrix Computations: A Fast Direct Method

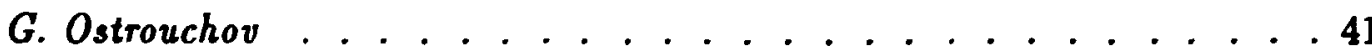

1.115. A Model Search Procedure for Hierarchical Log-Linear Models

G. Ostrouchov and E. L. Frome . . . . . . . . . . . . . . 4 41

1.116. Shrinking Techniques for Robust Regression

R. L. Schmoyer and S. F. Arnold

1.117. Near with Replacement Sampling without Replacement Using Ranks

T. Wright

1.118. On Some Properties of Variable Size Simple Random Sampling and a Limit Theorem

T. Wright

1.119. A Note on Pascal's Triangle and Simple Random Sampling

T. Wright 
1.120. On a New Test of Equality of Two Proportions Based on Directed Divergence

V. R. R. Uppuluri and T. N. Bhargava .......... . 43

\section{Applications of Statistical Methods}

1.121. Equal Opportunities in Science and Engineering

K. O. Bowman . . . . . . . . . . . . . 43

1.122. Evaluation of Special Nuclear Material Monitoring Instruments

K. O. Bowmen and S. A. Wallace . . . . . . . . 43

1.123. Statistical Quality Control Technology in Japan

K. O. Bowman, T. Hopp, R. Kacker, and R. Lundegard . . . . . 43

1.124. Chemical Exposures and Central Nervous System Cancers: A CaseControl Study Among Workers at Two Nuclear Facilities

A. V. Carpenter, W. D. Flanders, E. L. Frome, W. G. Tankersley,

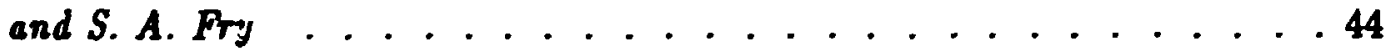

1.125. Characterizing Occupational Dose Distributions Using the Hybrid Lognormal Distribution

B. W. Dolan, P. G. Gröer, and V. R. R. Uppuluri ........ 44

1.126. Statistical Graphics-The Visual Display

D. J. Downing . . . . . . . . . . . . . . . . 44

1.127. Some Outlier Detection Methods in Time Series Analysis

D. J. Downing . . . . . . . . . . . . . . 44

1.128. Evaluating Goodness of Fit of Poisson Regression Models in Cohort Studies

E. L. Frome and M. D. Morris . . . . . . . . . . . . 45

1.129. Is p a Prime Number? Some Probabilistic Tests of Primality

S. R. Jemmalamadake and V.R.R. Uppuluri

1.130. The Mixed Function Oxidase System of Bluegill Sunfish, Lepomis

Macrochirus: Correlation of Activities in Experimental and Wild Fish

B. D. Jimenez, L. S. Burtis, G. H. Ezell, B. Z. Egan, N. E. Lee,

J. J. Beauchamp, and J. F. McCarthy. 
xvi

1.131. Eight Billion Mice Is the Answer. What Is the Question? Commentary for the Citation Classies

M. A. Kastenbaum and K. O. Bowman . . . . . . . . 46

1.132. Mass Loss and Nutrient Changes in Decomposing Upland Oak and Mesic Mixed-Hardwood Leaf Litter

J. M. Kelly and J. J. Beauchamp . . . . . . . . . . . . . 46

1.133. Comparisons of Dose-Response Parameters for Radiation-Induced Acentric Fragments and Micronuclei Observed in Cytokinesis-Arrested Lymphocytes

L. G. Littlefield, A. M. Sayer, and E. L. Frome . . . . . . . . . 47

1.134. Modulation of Radiation-Induced Chromosome Aberrations by DMSO-AN $\mathrm{OH}$ Radical Scavenger. 1. Dose Response Studies in Human Lymphocytes Exposed to $220 \mathrm{kV}$ X-Rays

L. G. Littlefield, E. E. Joiner, S. P. Colyer, A. M. Sayer, and

E. L. Frome . . . . . . . . . . . . . . 47

1.135. Determining Regional Water Quality Patterns and Their Ecological

Relationships

T. W. McDaniel, C. T. Hunsaker, and J. J. Beauchamp . . . . . . 48

1.136. The Role of Assumptions in Statistical Modeling M. D. Morris . . . . . . . . . . . . . . . . . . . 48

1.137. Some Effects of Radiation Dosimetry Errors on an Estimated

Dose-Response Relationship

M. D. Morris and T. D. Jones . . . . . . . . . . . 48

1.138. Hematopoietic Death of Unprotected Man from Photon Irradiations;

Statistical Modeling from Animal Experiments

M. D. Morris and T. D. Jones . . . . . . . . . . . . . 49

1.139. Statistical Approach to Combining the Results of Similar Experiments, with Application to the Hematologic Effects of Extremely-Low-Frequency Electric Field Exposures M. D. Morris, K. T. Kimball, T. E. Aldrich, and C. E. Easterly . . . . 50

1.140. Weighted Voting Models

V. R. R. Uppuluri 
xvii

1.141. An Application of the Maximum Ratio for Comparing Different Petroleum Fstimates

T. Wright . . . . . . . . . . . . . . . . . 50

1.142. When Zero Defectives Appear in a Sample: Upper Confidence Coefficients of Upper Bounds

T. Wright . . . . . . . . . . . . . . . 51

\section{Section 2. NUCI-EAR DATA MEASUREMDNT AND EVALUATION}

2.0. Introduction . . . . . . . . . . . . . . . . . 55

\section{EXPERIMENTAL RESULTS}

2.1. The ${ }^{151} \mathrm{Sm}$ Branching, a Probe for the Irradiation Time Scale of the s-Process

H. Beer and R. L. Macklin . . . . . . . . . . . . . . . 57

2.2. Measurement of the ${ }^{85} \mathrm{Rb}$ and ${ }^{87} \mathrm{Rb}$ Capture Cross Sections for s-Process Studies

H. Beer and R. L. Macklin . . . . . . . . . . . . . . . . . 57

2.3. Tests for "Cold Fusion" in the $P d-D_{2}$ and Ti- $D_{2}$ Systems at 40 to 380 MPA and -196 to $27^{\circ} \mathrm{C}$

J. G. Blencoe, M. T. Naney, D. J. Wesolowski, and F. G. Perey . . . . 57

2.4. Level Densities from Resonance Spectroscopy for $\boldsymbol{n}+{ }^{86} \mathrm{Kr}$

R. F. Carlton, R. R. Winters, C. H. Johnson, N. W. Hill, and

J. A. Harvey . . . . . . . . . . . . . . . . . . . . . . . . . . . 58

2.5. Unified Description of the $n+{ }^{209} \mathrm{Bi}$ Mean Field Between $1 \mathrm{MeV}$ to $40 \mathrm{MeV}$ via Dispersion Relations

R. K. Das, R. W. Finlay, and J. A. Harvey . . . . . . . . . . . . 58

2.6. Use of Monte Carlo Techniques to Derive Yields for $n+{ }^{12} \mathrm{C}$ Multi-Body Breakup Reactions J. K. Dickens . . . . . . . . . . . . . . . . . . . . . 58

2.7. ${ }^{10,11} \mathrm{~B}(n, x \gamma)$ Reactions for Incident Neutron Energies Between 0.1 and $25 \mathrm{MeV}$ J. K. Dickens and D. C. Larson . . . . . . . . . . . . . . . 59 
xviii

2.8. The Ne-110 Scintillator Response to 10 to $100-\mathrm{MeV}$ Carbon Ions

J. K. Dickens and J. W. McConnell

2.9. A Ne 110 Scintillation Detector for keV Energy Neutrons

J. A. Harvey and $\boldsymbol{N}$. W. Hill

2.10. High-Resolution Neutron Transmission Measurements on ${ }^{235} \mathrm{U},{ }^{239} \mathrm{Pu}$, and ${ }^{238} \mathrm{U}$

J. A. Harvey, N. W. Hill, F. G. Perey, G. L. Tweed, and L. Leal . . . . 60

2.11. Cold Fusion Neutron Measurements at ORNL

D. P. Hutchinson, R. K. Richards, C. A. Bennett, C. C. Havener,

C. H. Ma, F. G. Perey, R. R. Spencer, J. K. Dickens, B. D. Rooney,

J. Bullock, IV, and G. L. Powell . . . . . . . . . . . . . .60

2.12. ${ }^{53} \mathrm{Cr}\left(n, n^{\prime} \gamma\right)$ Reactions and the Level Structure of ${ }^{53} \mathrm{Cr}$

D. C. Larson and J. K. Dickens . . . . . . . . . . . . . . 60

2.13. Neutron Capture by ${ }^{79} \mathrm{Br},{ }^{81} \mathrm{Br}$, and ${ }^{75}$ As

R. L. Macklin

2.14. Search for ${ }^{133} \mathrm{Xe}$ Resonance Neutron Capture

R. L. Macklin

2.15. Neutron Absorption Cross Section of Uranium-236

R. L. Macklin and C. W. Alerander . . . . . . . . . . . 61

2.16. High Energy Resolution Measurement of the ${ }^{238} \mathrm{U}$ Neutron Capture

Yield from $1 \mathrm{keV}$ to $100 \mathrm{keV}$

Roger Macklin, R. B. Perez, G. de Saussure, and R. W. Ingle . . . . 61

2.17. Neutron Capture of ${ }^{122} \mathrm{Te},{ }^{123} \mathrm{Te},{ }^{124} \mathrm{Te},{ }^{125} \mathrm{Te}$, and ${ }^{126} \mathrm{Te}$

R. L. Macklin and R. R. Winters

2.18. Resonance Neutron Capture by Argon-40

R. L. Macklin, R. R. Winters, and D. M. Schmidt.

2.19. Neutron Scattering in ${ }^{189}$ Os for Nucleosynthesis Rates of the Odd-A

Os Isotopes and Nucleochronology

M. T. McEllistrem, R. R. Winters, R. L. Hershberger, Z. Cao,

R. L. Macklin, and N.W. Hill 
2.20. ${ }^{58} \mathrm{Ni}+n$ Transmission, Differential Elastic Scattering and Capture Measurements and Analysis from 5 to $813 \mathrm{keV}$

C. M. Perey, F. G. Perey, J. A. Harney, N. W. Hill, N. M. Larson, and $R$. L. Mactlin

2.21. Responses of $\mathrm{C}_{6} \mathrm{D}_{6}$ and $\mathrm{C}_{6} \mathrm{~F}_{6}$ Gamma-Raj Detectors and the Capture in the $1.15-\mathrm{keV}$ Rescnance of ${ }^{56} \mathrm{Fe}$ F. G. Perey, J. O. Johnson, T. A. Gabriel R. L. Macklin, R. R. Winters, J. H. Todd, and N. W. Hill . . . . . . . . 63

2.22. Subthresholds and Near-Subthreshold Fission Physics Measurements at the Oak Ridge Electron Linear Accelerator Facility

R. B. Perez, G. de Seussure, F. C. Dijilippo, L. W. Weston, and

J. A. Hervey . . . . . . . . . . . . . . . 64

2.23. High Resolution Fission Cross Section Measurements at ORELA L. W. Weston ard J. H. Todd . . . . . . . . . . . . . 64

2.24. New Cross Section Evaluations for Reactor Fuel Materials L. W. Weston, P. G. Young, and W. P. Poenitz . . . . . . . . . 64

2.25. $n+{ }^{40} \mathrm{Ar}$ s- and $\mathrm{p}$-Wave Neutron Resonance Spacings and Strength Functions R. R. Winters, R. F. Carlton, and C. H. Johnson . . . . . . . 65

\section{NEUTRON RESONANCE ANALYSES}

2.26. Curnent Plutonium Cross-Section Evaluations in the Resolved Resonance Region

H. Derrien and G. de Saussure . . . . . . . . . . . . . .65

2.27. R-Matrix Analysis of ${ }^{239} \mathrm{Pu}$ Neutron Cross Sections in the Energy Range up to $1000 \mathrm{eV}$

H. Derrien and G. de Saussure . . . . . . . . . . . . 65

2.28. R-Matrix Analysis of the ${ }^{241} \mathrm{Pu}$ Neutron Cross Sections in the Energy Range Thermal to $300 \mathrm{eV}$ H. Derrien and G. de Saussure . . . . . . . . . . . . . . .65

2.29. R-Matrix Analyses of the ${ }^{235} \mathrm{U}$ and ${ }^{239} \mathrm{Pu}$ Neutron Cross Sections H. Derrien, G. de Saussure, N. M. Larson, L. C. Leal, and R. B. Perez 
2.30. Reich-Moore and Adler-Adler Representations of the ${ }^{235} \mathrm{U}$ Cross Sections in the Resolved Resonance Region

G. de Saussurc, L. C. Leal, and R. B. Perez . . . . . . . . . . 66

2.31. A New Resonance-Region Evaluation of Neutron Cross Sections for U-235

G. de Saussure, L. C. Leal, R. B. Perez, N. M. Larson, and

M. S. Moore ...................... 66

2.32. Uncertainties of the ENDF/B-V ${ }^{238}$ U Unresolved Resonance Parameters in the Range $4 \mathrm{keV} \leq \mathrm{E} \leq 45.18 \mathrm{keV}$ (MAT=1398, MF=2, MT=251)

G. de Saussure and J. H. Marable . . . . . . . . . . . . . 67

2.33. Stochastic Nuclear Reaction Theory: Breit-Wigner Nuclear Noise

G. de Saussure and R. B. Perez . . . . . . . . . . . . . 67

2.34. Evaluated Cross Sections for Neutron Scattering from Natural Carbon Below $2 \mathrm{MeV}$ Including R-Matrix Fits to C-13 Resonances

C. Y. Fu

2.35. Use of SAMMY in Resonance Analysis of Neutron Data N. M. Larson . . . . . . . . . . . . . . . . . . . . 68

2.36. Updated Users' Guide for SAMMY: Multilevel R-Matrix Fits to Neutron Data Using Bayes' Equation

N. M. Larson . . . . . . . . . . . . . . . . . . . . . 68

2.37. Resonance Parameter Analysis with SAMMY

N. M. Larson and F. G. Perey . . . . . . . . . . . . 68

2.38. URR Computer Code: A Code to Calculate Resonance Neutron

Cross-Section Probability Tables, Bondarenko Self-Shielding Factors, and Self-Indication Ratios for Fissile and Fertile Nuclides

L. C. Leal, G. de Saussure, and R. B. Perez . . . . . . . . . 69

2.39. Analysis of the U-235 Neutron Cross Sections in the Resolved Resonance Range

L. C. Leal, G. de Saussure, and R. B. Perez . . . . . . . . . 69

2.40. R-Matrix Analysis of the ${ }^{235} \mathrm{U}$ Neutron Cross Sections

L. C. Leal, G. de Saussure, and R. B. Perez . . . . . . . . . . 69

2.41. Statistical Properties of the ${ }^{235} \mathrm{U}$ Resonance Parameters Up to $300 \mathrm{eV}$

L. C. Leal, C. de Saussure, R. R. Winters, and R. B. Perez . . . . 70 
2.42. Resonance Structure in the Fission of $\left({ }^{235} U+n\right)$

M. S. Moore, L. C. Leal, G. de Sausure, R. B. Perez, and

N. M. Lerson . . . . . . . . . . . . . . . . . . . 70

2.43. RFUNC - A Code to Analyze Differential Elastic-Scattering Data

F. G. Perey . . . . . . . . . . . . . . . . 70

2.44. On the Unresolved Resonance Region Representation of Neutron Induced Cross Sections

R. B. Perez, G. de Sarsurur, L. C. Leal, and Roger Macklin . . . . . 71

\section{CROSS SECTION EVALUATION AND MODELING}

2.45. SCINFUL: A Monte Carlo Based Computer Program to Determine a Scintillator Full Energy Response to Neutron Detection for $\boldsymbol{E}_{\boldsymbol{n}}$ Between 0.1 and $80 \mathrm{MeV}$ : User's Manual and FORTRAN Program Listing

J. K. Dickens . . . . . . . . . . . . . . . . 71

2.46. SCINFUL: A Monte Carlo Based Computer Program to Determine a Scintillator Full Energy Response to Neutron Detection for $E_{n}$ Between 0.1 and $80 \mathrm{MeV}$ : Program Development and Comparisons of Program Predictions with Experimental Data J. K. Dickens . . . . . . . . . . . . . . . . . . .71

2.47. A Simplified Unified Hauser-Feshbach/Pre-Equilibrium Model for Calculating Double Differential Cross Sections C. Y. Pu.

2.48. Approximation of Precompound Effects in Hauser-Feshbach Codes for Calculating Double Differential $(n, x n)$ Cross Sections C. Y. Fu.

2.49. Pairing Corrections and Spin Cutoff Fartors in Exciton Level Densities for Two Kinds of Fermions C. Y. Fu . . . . . . . . . . . . . . . . . 72

2.50. Status of Theories Used for Calculations of Long-Lived Activation Cross Sections

C. $\boldsymbol{Y}, \boldsymbol{F u}$. 
2.51. A Review of Activation Cross Sections in the ENDF/B-VI General Purpose Files of $\mathrm{Cr}, \mathrm{Fe}, \mathrm{Ni}, \mathrm{Cu}$, and $\mathrm{Pb}$

C. Y. Fu . 73

2.52. ENDF/B-VI Evaluations for Isotopes of $\mathrm{Cr}, \mathrm{Fe}, \mathrm{Ni}$, and $\mathrm{Cu}$, and for Natural $\mathbf{P b}$

D. M. Hetrick, C. Y. Fu, and D. C. Lerson . . . . . . . 74

2.53. Description of Evaluation of ${ }^{\text {nat }} \mathrm{Pb}$ Performed for ENDF/B-VI D. C. Larson and C. Y. Fu . . . . . . . . . . . . 74

2.54. Some Notes on the Calculation of Energy-Angle Correlated Distributions with TNG and Their Representation in File 6 Formats

D. C. Larson, C. Y. Fu, and D. M. Hetrick . . . . . . . . 74

2.55. Description of Evaluation for ${ }^{54,56,57,58}$ Fe Performed for ENDF/B-VI D. C. Larson, C. Y. Fu, and D. M. Hetrick . . . . . . . 74

2.56. Description of Evaluation for $58,60,61,62,64 \mathrm{Ni}$ Performed for ENDF/B-VI D. C. Larson, D. M. Hetrick, and C. Y. Fu . . . . . . . . 75

2.57. Description of Evaluation for ${ }^{63,65} \mathrm{Cu}$ Performed for ENDF/B-VI D. C. Larson, D. M. Hetrick, and C. Y. Fu . . . . . . . . 75

2.58. Description of Evaluation for ${ }^{50,52.53,54} \mathrm{Cr}$ Performed for ENDF/B-VI D. C. Larson, D. M. Hetrick, and C. Y. Fu . . . . . . . . . 75

2.59. Structural and Shielding Material Evaluations for ENDF/B-VI D. C. Larson and P. G. Young . . . . . . . . . . . . . . . . 76

2.60. Test of the ENDF/B Unresolved Resonance Formalism for U-235 L. C. Leal, G. de Saussure, R. B. Perez, and R. Q. Wright . . . . . 76

2.61. Covariance Data for ENDF/B-VI R. W. Peelle

2.62. Neutron Standard Data

R. W. Peelle and H. Conde

2.63. Extended Covariance Data Formats for the ENDF/B-VI Differential Data Evaluation

R. W. Peelle and D. W. Muir . . . . . . . . . . . .77 
2.64. Covariances of Physical Constants

F. G. Perey . . . . . . . . . . . . 77

2.65. A Stochastic Method for Nuclear Power Plant Diagnosis

R. B. Perez and R. T. Wood . . . . . . . . . . . . . 77

\section{REVIEWS}

2.66. Electron Linear Accelerators for Fast Neutron Data Measurements in Support of Fusion Energy Applications

K. H. Bockhoff, A. D. Cerlson, O. A. Wesson, J. A. Hervey, and

D. C. Lerson . . . . . . . . . . . . . . . . 78

2.67. Decay Heat Measurements and the Loss-of-Coolant Accident

J. K. Dickens . . . . . . . . . . . . . . . . 78

2.68. Reactor Safety and the Status of Commercial Nuclear Power in the United States

J. K. Dickers . . . . . . . . . . . . . 78

2.69. Neutron Research at the Oak Ridge Electron Linear Accelerator

J. K. Dickens

2.70. ENDF/B-VI Nuclear Data Eraluations for Fusion Applications

C. L. Dunford, D. C. Larson, and P. G. Young . . . . . . . . . . . 79

2.71. Reports on Nuclear Data Activities for the DOE Nuclear Data Committee

D. C. Larson

\section{Section 3. INTELLIGENT SYSTEMS}

3.0. Introduction

\section{AUTONOMOUS MOBILE ROBOTS}

3.1. Robot Navigation Research at CESAR

D. L. Barnett, G. de Saussure, S. S. Iyengar, F. G. Pin, A. Sabharwal, and $C$. R. Weisbin . . . . . . . . . . . 85

3.2. The Use of an Expert System for Autonomous Robot Control

D. L. Barnett, G. de Saussure, and P. F. Spelt 
xoiv

3.3. Robot Navigation Research Using the HERMIES Mobile Robot D. L. Barnett and F. G. Pin

3.4. Spatial Reasoning in the Treatment of Systematic Sensor Errors

M. Beckerman, J. P. Jones, R. C. Mann, L. A. Parkas, and

S. E. Johnsion

3.5. Robust Performance of Multiple Tasks by an Autonomous Robot

M. Beckerman, D. L. Barnett, J. R. Einstein, J. P. Jones, P. F. Spelt, and C. R. Weisbin ................... 87

3.6. World Modelling and Multi-Sensur Integration for a Mobile Robot

M. Beckerman, L. A. Farkas, J. P. Jones, R. C. Mann, and

C. W. Glover .

3.7. Treatment of Systematic Errors in the Processing of Wide Angle Sonar Sensor Data for Robotic Navigation

M. Beckerman and E. M. Oblow . . . . . . . . . . . . . . . 87

3.8. An Approach to Elemental Task Learning

P. F. R. Belmans . . . . . . . . . . . . . . . . . 87

3.9. Object Recognition for the HERMIES Robot

K. W. Bowyer . . . . . . . . . . . . . . . . . 88

3.10. A Demonstration of Autonomous Navigation and Machine Vision Using the HERMIES-IIB Robot

B. L. Burks, D. L. Barnett, J. P. Jones, and S. M. Killough . . . . . . 88

3.11. Ongoing Research Using HERMIES - the Hostile Environment Robotic Machine Intelligence Experiment Series

B. L. Burks and P. F. Spelt . . . . . . . . . . . . . . . . 89

3.12. Automatic Learning by Autonomous Mobile Robot

G. de Saussure, P. F. Spelt, S. M. Killough, F. G. Pin, and

C. R. Weisbin . . . . . . . . . . . . . . . . . 89

3.13. Navigation and Learning Experiments by an Autonomous Robot

G. de Saussure, C. R. Weisbin, and P. F. Spelt . . . . . . . . . . . 89

3.14. Non-Linear Surface Fitting of Laser Range Images Using a Hypercube

Concurrent Computer

M. Finley 
3.15. Navigation Planning Using Quadtrees

R. C. Fryxell

3.16. The Man-Robot Symbiosis Project

W. R. Hamel and F. G. Pin . . . . . . . . . . . 90

3.17. Smelting Net,works for Real Time Cooperative Planni $g$ in the Presence of Uncertainties

S. S. Iyengar, S. Gulati, and J. Barhen . . . . . . . . . . 91

3.18. Asynchronous Production Systems for Controlling Autonomous Mobile Robots

S. S. Iyengar, C. R. Weisbin, and F. G. Pin . . . . . . . . 91

3.19. The U.S. DOE/NE University Program in Robotics for Advanced Reactors R. C. Mann . . . . . . . . . . . . . . 91

3.20. The Development of an Intelligent Nuclear Hraintenance Robot R. C. Mann, W. R. Hamel, and C. R. Weisbin

3.21. An Intelligent Integrated Sensor System for the ORNL Mobile Robot R. C. Mann, J. P. Jones, M. Beckerman, C. W. Glover, L. Farkas, J. Han, E. Wacholder, and J. R. Einstein . . . . . . . . . 92

3.22. U.S. Department of Energy Nuciear Energy University Program in Robotics for Advanced Reactors

R. C. Mann, R. C. Gonzalez, D. Tesar, J. S. Tulenko, D. K. Wehe . . 92

3.23. Research. in Mobile Robotics at ORNL/CESAR R. C. Mann, C. R. Weisbin, and F. G. Pin

3.24. Adaptive Optimal Control of Uncertain Nonlinear Systems: On-Line Microprocessor-Based Algorithm to Control Mechanical Manipulators C. March-Leuba and R. B. Perez . . . . . . . . . . . . . . . 93

3.25. Optimal Control of Uncertain Nonlinear Systems: An Application to a Two-Link Robotic Arm

C. March Leuba and R. B. Perez . . . . . . . . . . . . . . .93

3.26. A Probabilistic-Propositional Framework for the O-Theory Intersection Rule

E. M. Oblow . . . . . . . . . . . . . . . . . . . . 
xxvi

3.27. Foundations of O-Theory: Measurements and Relation to Fuzzy Set Theory

E. M. Oblow

3.28. Supertracks, Supertrack Functions and Chaos in the Quadratic Map

E. M. Oblow

3.29. ' 'an-Robot Symbiosis: A Framework for Cooperative Intelligence and Control

L. E. Parker and F. G. Pin . . . . . . . . . . . . . . 94

3.30. 1988 Workshof on Human-Machine Symbiotic Systems

L. E. Parker and C. R. Weisbin, Editors . . . . . . . . . . . . 95

3.31. Review of the 1988 Workshop on Human-Machine Symbiotic Systems

I. E. Parker and C. R. Weisbin . . . . . . . . . . . . . . 95

3.32. Autonomous Mobile Robot Research Using the HERMIES-III Robot

F. G. Pin, M. Beckerman, P. F. Spelt, J. T. Robinson, and

C. R. Weisbin

3.33. Recent CESAR Research Activities in Sensor Based Reasoning for Autonomous Machines

F. G. Pin, G. de Saussure, P. F. Spelt, S. M. Killough, and

C. R. Weisbin . . . . . . . . . . . . . . . . . 96

3.34. Design of an Obstacle Avoidance System for AIMS

D. B. Keister and M. W. Noakes . . . . . . . . . . . . . . . . 96

3.35. Learning by an Autonomous Robot at a Process Control Panel

P. F. Spelt, G. de Saussure, E. Lyness, F. G. Pin, and C. R. Weisbin . 97

3.36. Path-Planning in a Known I'nvirousisent with Unexpected Obstacles:

Potential Application to an Automatic Alarm Testing Robot

N. Sreenath

3.37. Parallelism in Prinduction Systems

A. Sabharwal, S. S. Iyengar, G. de Saussure, and C. R. Weisbin . . . . 97

3.38. Asynchronous Production Systeins

A. Sabharwal, S. S. Iyengar, C. R. Weishin, and F. G. Pin . . . . . 98 
xxvii

3.39. Asynchronous Production Systems for Real-Time Expert Systems A. Sabharwal, S. S. Iyengar, C. R. Weisbin, and F. G. Pin . . . . . 98

3.40. A Two-PC Simulation for Development and Training of a Learning Expert System in an Autonomous Mobile Robot P. F. Spelt, E. Lyness, and G. de Saussurc . . . . . . . . . . . . . . 98

3.41. Dynamics for Space Based Robotic Manipulation $N$. Sreenath and P. S. Krishnaprasad

3.42. Intelligent Robotics and Remote Systems for the Nuclear Industry D. K. Wehe, J. C. Lee, W. R. Martin, R. C. Mann, W. R. Hamel, and $J$. S. Tulenko

3.43. Research in Autonomous Robotics at ORNL Using HERMIES-III

C. R. Weisbin, B. L. Burks, J. R. Einstein, R. R. Feezell,

W. W. Manges, and D. B. Thompson . . . . . . . . . . . 100

3.44. HERMIES-III: A Step Toward Autonomous Mobility, Manipulation and Perception

C. R. Weisbin, B. L. Burks, J. R. Einstein, R. R. Feezell,

W. W. Manges, and D. H. Thompscí . . . . . . . . . . . 100

3.45. Autonomous Mobiie Robot Navigation and Learning

C. R. Weisbin, G. de Saussure, J. R. Einstein, E. Heer, and

F. G. Pin

3.46. The Robotics and Intelligent Systems Program at ORNL

C. R. Weisbin, W. R. Hamel, D. P. Kuban, S. A. Meacham, and F. G. Pin 101

\section{CONCURRENT COMPUTING}

3.47. Computing the Hough Transform on an MIMD Hypercube

K. W. vowyer, J. P. Jones, and C. H. Lake

3.48. A Concurrent Approach to Model-Based Motion Detection

H. I. Christensen

3.49. Concurrent Spatio-Temporal Image Analysis

H. I. Christensen 
3.50. Concurrent Multi-Resolution Image Analysis

H. I. Christersen and J. P. Jones . . . . . . . . . . . 103

3.51. A Tool to Aid in Mapping Computational Tasks to a Hypercube

J. R. Einstein, C. W. Glover, and M. Dickens . . . . . . . 103

3.52. Design and Implementation of a Multi-Sensor Fusion Algorithm on a Hypercube Computer Architecture

C. W. Glover . . . . . . . . . . . . . . 103

3.53. Multi-Sensor Integration on the NCUBE ${ }^{\mathrm{TM}}$ Hypercube Computer

C. W. Glover . . . . . . . . . . . . . . . . . 104

3.54. Concurrency in Mobile Robotics at ORNL

J. P. Jones . . . . . . . . . . . . . . . . . . 104

3.55. Concurrent Computer Vision on a Hypercube Multicomputer

J. P. Jones . . . . . . . . . . . . . . . . . . . 105

3.56. A Concurrent On-Board Vision System for a Mobile Robot

J. P. Jones . . . . . . . . . . . . . . . . 105

3.57. The Butterfy Accumulator and Its Application in Concurrent Computer Vision on Hypercube Multicomputers

J. P. Jones . . . . . . . . . . . . . . . . . . . . . . . 105

3.58. Parallel Relaxation Matching

J. P. Jones and K. M. Clinard . . . . . . . . . . . . . . . . 106

3.59. A Computer Vision System for a Hypercube Concurrent Ensemble

J. P. Jones, R. C. Mann, and E. M. Simpson . . . . . . . . . 106

3.60. A Sensor Integration Using Concurrent Computing On-Board the ORNL Mobile Robot

R. C. Mann, J. P. Jones, M. Beckernian, C. W. Glover, L. Farkas, and J. R. Einstein . . . . . . . . . . . . 106

\section{OPTIMIZATION FOR INTELLIGENT SYSTEMS}

3.1. Yet Another Non-Linear Approach to Linear Programming

J. C. Culioli 
xxix

3.62. Decomposition/Coordination Algorithms in Convex Stochastic Optimization J.-C. Culioli and G. Cohen . . . . . . . . . . . . . . . . 107

3.63. Decomposition/Coordination Algorithms in Stochastic Optimization J.-C. Culioli and G. Cohen . . . . . . . . . . . . . . . . 107

3.64. NCUBE Implementation of Some Heuristics and an Optimal Algorithm for Large-Scale Assignment Problems J.-C. Culioli, C. W. Glover, J. P. Jones, and C. Roe . . . . . . . 107

3.65. A New Algorithm for Linear Programming That Is Easy to Implement:

Application to the Transportation Problem J.-C. Culioli and V. Protopopescu . . . . . . . . . . . . . . . . . 108

3.66. An Algorithm for Linear Programming That Is Easy to Implement. J.-C. Culioli and V. Protopopescu . . . . . . . . . . . . . . 108

3.67. Bifurcating Optimization Algorithms and Their Possible Application J.-C. Culioli and V. Protopopescu . . . . . . . . . . . . . . . . 108

3.68. Sigmoidic Linear and Non-Linear Optimization Algorithms J. C. Culioli and V. Protopopescu . . . . . . . . . . . . . . 109

3.69. A New Class of Optimization Algorithms with Bifurcations and Chaotic Behavior J.-C. Culioli and V. Protopopescu . . . . . . . . . . . . . . 109

3.70. A Linear-Programming Method Inspired by the Neural Networks Framework J.-C. Culioli and V. Protopopescu . . . . . . . . . . . . . . 109

3.71. Stability and Chaos in a Numerical Scheme Inspired by Neural Networks J.-C. Culioli and V. Protopopescu . . . . . . . . . . . . . . . . . 109

3.72. Neural tetworks Models for Linear Programming J.-C. Culioli, V. Protopopescu, C. Britton, and N. Ericson . . . . . . 110

3.73. A Neural Network for Bounded Linear Programming J..C. Culioli, V. Protopopescu, C. Britton, and N. Ericson . . . . . . 110 
$\mathbf{x 0 x}$

3.74. A Comparison Study of Optimization Methods for the Bipartite Matching Problem (BMP)

M. Goldstein, N. Toomarian, and J. Barhen . . . . . . . . 110

3.75. A Concurrent Neural Network Algorithm for the Traveling Salesman

Problem

N. Toomarian . . . . . . . . . . . . . 110

3.76. A Neural Network-Based Optimization Algorithm for the WeaponTarget Assignment Problem

E. Wacholder . . . . . . . . . . . . . . 111

3.77. An Extension of the Hopfield Tank Model for Solution of the Multiple Traveling Salesmen Problem

E. Wacholder, J. Han, and R. C. Mann . . . . . . . . 111

3.78. A Neural Network Algorithm for the Multiple Traveling Salesmen

Problem

E. Wacholder, J. Han, and R. C. Mann . . . . . . . 111

3.79. A Neural Network Algorithm for the Target-Weapon Assignment

Problem

E. Wacholder, J. Han, and R. C. Mann . . . . . . . . . . 112

\section{AUTOMATED METHODS FOR SENSITIVITY}

AND UNCERTAINTY ANALYSIS

3.80. GRESS Adjoint Matrix Reduction Algorithms

J. E. Horwedel . . . . . . . . . . . . . . . . . 112

3.81. EXAP-Precompiler for the GRESS and ADGEN Automated Sensitivity Calculation Systems

J. E. Horwedel, F. G. Pin, and B. A. Worley . . . . . . . . . . 113

3.82. GRESS Version 0.0 User's Manual

J. E. Horwedel, B. A. Worley, E. M. Oblow, F. G. Pin, and

R. Q. Wright.

3.83. Comparison of Results Baseu on a Deterministic Versus a Statistical Uncertainty Analysis

R. E. Maerk:er 
$\mathbf{x x x i}$

3.84. Development and Application of a New Deterministic Method for Calculating Computer Model Result Uncertainties

R. E. Maerker and B. A. Worley . . . . . . . . . . . . . . . 114

3.85. Analytical Methods for Large-Scale Sensitivity Analysis Using GRESS and ADGEN

F. G. Pin

3.86. Sensitivity Analysis of Two-Phase Flow Problems

N. Toomarian, E. Wacholder, and S. Kaizerman . . . . . . . . 114

3.87. A FORTRAN Precompiler and System for Automating the Implementation of the Forward and Adjoint Deterministic Sensitivity Methods into Existing FORTRAN Computer Codes

B. A. Worley . . . . . . . . . . . . . . . . . . 115

3.88. Calculation and Use of Computer Model Derivatives

B. A. Worley . . . . . . . . . . . . . . . . . 115

3.89. ADGEN - Adjoint Generator for Computer Models

B. A. Worley, F. G. Pin, J. E. Horwedel, and E. M. Oblow . . . . 115

3.90. Deterministic Sensitivity and Uncertainty Analysis for Large Scale

Computer Models

B. A. Worley, F. G. Pin, E. M. Oblow, R. E. Maerker, J. E. Horwedel, and $\boldsymbol{R}$. Q. Wright . . . . . . . . . . . . . . . 11

\section{ADVANCED CARBOLS PROGRAM}

3.91. An Intelligent Simulation Environment for Control System Design

J. T. Robinson

3.92. An Intelligent Dynamic Simulation Environment: An Object-

Oriented Approach

J. T. Robinson and R. A. Kisner . . . . . . . . . . . 117

3.93. An Application of Object Oriented Programming to I'rocess Simulatınn J. T. Robinson and P. J. Otaduy . . . . . . . . . . . . . . . . 117

3.94. Void Distributions ir Bubbly Flow Through Yawed Rod Arrays J. T. Robinson, N. E. Todreas, and D. Edeling-Koning 
xxoxii

3.95. Hierarchical Control of a Nuclear Reactor Using Uncertain Dynamics Techniques

L. A. Rovere, P. J. Otaduy, C. Britton, and R. B. Perez . . . . . . 118

3.96. Development of an Uncertain Model Based Nonlinear Optimal Pressure Control System for a Nuclear Reactor

L. A. Rovere, P. J. Otaduy, and C. March-Leube

\section{ENERGY ANALYSIS}

3.97. Estimating Finding Rates for U.S. Crude Oil

D. S. Christiansen arod D. B. Reister ............. 119

3.98. The Aggregate Production Profile for U.S. Crude Oil

D. S. Christiensen and D. B. Reister ............. 119

3.99. Oil Vulnerability and Intermediate Price Fluctuations: A Preliminary Assessment and Proposal

T. R. Curlee and D. B. Reister . . . . . . . . . . . . . 120

3.100. Energy Technology R\&D: What Could Make a Difference?

W. Fulkerson, S. I. Auerbach, A. T. Crane, D. E. Kash, A. M. Perry,

D. B. Reister. and C. W. Hagan, Jr. . . . . . . . . . . 120

3.101. Global Warming - An Energy Technology R\&D Challenge

W. Fulkerson, D. B. Reister, A. M. Perry, A. T. Crane, D. E. Kash, and $S$. I. A uerbach

3.102. Energy Technology R\&D: What Could Make a Difference

D. B. Reister.

3.103. Future Emissions of Carbon Dioxide: Can We Make a Dramatic Reduction?

D. B. Reister . . . . . . . . . . . . . . . . 121

3.104. The Replacement Cost Integration Program User's Guide

D. B. Reister

3.105. Replacement Cost Integration Program Model Description

D. B. Reister .

3.106. Replacement Cost Integration Program Model Overview

D. B. Reister 
xxoxiii

3.107. Validation of the Replacement Cost Integration Progran

D. B. Reister and D. S. Christiansen .................

3.108. The Replacement Cost Integration Program, an Engineering-Economic Model of Oil Supply

D. B. Reister and D. S. Christiensen . . . . . . . . . 123

3.109. The RAMS Coal Model: Volume I - Model Overview, Volume II User's Guide, Volume III - Model Description

D. B. Reister, J. V. Conopask, and P. E. Mihlmester . . . . . . . 123

\section{DOSIMETRY}

3.110. Pressure Vessel Fluence Analysis and Neutron Dosimetry

F. B. K. Kam, R. E. Maerker, M. L. Williams, and F. W. Stallmann . 124

3.111. Analysis of the VENUS-3 Experiments

R. E. Maerker . . . . . . . . . . . . . 125

3.112. The LEPRICON Code System: Consolidation of Transport

Analytical and Unfolding Procedures in LWR Pressure Vessel Dosimetry

R. E. Maerker . . . . . . . . . . . . . 125

3.113. Benchmark Verification of a Method for Calculating Leakage from PLSA Modified Cores

R. E. Maerker, P. D'hondt, L. Leenders, and A. Fabry . . . . . 126

3.114. Analysis of the VENUS-3 Experiments

R. E. Maerker, P. D'hondt, L. Leenders, and A. Faory . . . . . . 126

\section{HUMAN-COMPUTER INTERACTIONS}

3.115. Hurnan Factors in the Naval Air Systems Command: Computer-Based Training

T. L. Seamster, C. E. Snyder, M. Terranova, W. J. Walker, and

D. T. Jones

3.116. Teamwork and Data Deliverables: A Map to Software and Training

Design Integration

C. E. Snyder, N. E. Clapp, Jr., D. E. Smith, M. Terranova, J. Davis,

J. L. Finney, P. G. Guthrie, G. B. Payne, and S. Webster. 
xxxiv

3.117. A Part-Task Trainer in the Naval Air Systems Command C. E. Snyder and M. Terranova . . . . . . . . . . . 128

3.118. Dynamic Allocation of Responsibility Between Operators with Different Models of System Information Using Computer-Mediated Communication M. Terranova

3.119. Cognitive Task Analysis: Techniques Applied to Airborne Weapons Training M. Terrenova, T. L. Seamster, C. E. Snyder, and I. E. Treitler . . . 129

\section{COGNITIVE MODELING/OPERATOR ROLES}

3.120. Feasibility of Quantitative Performance Measures for Evaluating Nuclear Power Plant Operators R. J. Carter, E. M. Connelly, and P. A. Krois . . . . . . . . 129

3.121. Operator Role Definition and Human System Integration

H. E. Knee and J. C. Schryver . . . . . . . . . . . 130

3.122. An Operator Model-Based Filtering Scheme

R. S. Sawhey and J. C. Schryoer

3.123. A Hypothesis Generation Model of Initiating Events for Nuclear Power Plant Operators

R. S. Sawhney, J. C. Schryver, H. E. Knee, and H. L. Dodds . . . . . 131

3.124. Operator Model-Based Design and Evaluation of Advanced Systems:

Computational Models

J. C. Schryver

3.125. Operator Model-Based Design and Evaluation of Advanced Systems: Conceptual Models

J. C. Schryver

\section{USAGE OF ADVANCED INSTRUMENTATION AND CONTROLS - THE NUCLEAR INDUSTRY}

3.126. Digital Instrumentation and Interactive Computer Systems: A Survey of the Use of Advanced Technology in the Nuclear Industry

R. J. Carter 
$\mathbf{x x x y}$

3.127. Human Factors Aspects of Advanced Instrumentation in the Nuclear Industry

R. J. Carter

3.128. Human Factors Survey of Advarced Instrumentation and Controls

R. J. Carter . . . . . . . . . . . . . . . . . . . . . 133

3.129. Human Factors Issues Related tc Expert Systems for Electric Power Plant Control Rooms

R. J. Carter and R. E. Uhrig . . . . . . . . . . . . . . . 133

\section{HAZARDOUS WASTE MANAGEMENT}

3.130. Designing a HAZMAT Incident Management System for Facilities with Widely Varying Emergency Organization Structures

R. J. Carter and C. E. Basterly

3.131. A Generic Hazardous Waste Management Training Program

R. J. Carter and B. Karnofsky

\section{RELIABILITY}

3.132. Assessment of Critical Component Unavailability in Liquid Metal Reactors

K. H. Koger, J. Haire, B. L. Humphrys, J. F. Manneschmidt,

K. Setoguchi, and R. Nakai . . . . . . . . . . . . 135

3.133. Development of FREEDOM/CREDO Data Base for LMFBR PSA

R. Nakai, K. Setoguchi, H. Yasuda, and H. E. Knee . . . . . . . 135

3.134. Interaction of CREDO with the EBR-II PRA Development

M. S. Smith and W. A. Ragland . . . . . . . . . . . . . . 136

\section{Section 4. NUCLEAR ANALYSIS AND SHIELDING}

4.0. Introduction . . . . . . . . . . . . . . . 139

\section{RADIATION TRANSPORT AND PHYSICS FOR FISSION REACTORS}

4.1. Artificial Intelligence Search Techniques for Optimization of the Cold Source Geometry 
4.2. A State Space Search Technique for Optimizing the Shape of a Cold Neutron Source

Y. Y. Azmy

4.3. Geometrical Shape Optimization of a Cold Neutron Source Using Artificial Intelligence Strategies

Y. Y. Azmy

4.4. A Parallel Algorithm for Solving the Multidimensional Neutron Transport Equation Y. Y. Azmy

4.5. Multidimensional Nodal Transport Methods for Multiple-Instruction Multiple-Data, Distributed Memory Machines

Y. Y. Azmy

4.6. Accuracy of Transport Calcuiations for the Advanced Neutron Source Y. Y. Azmy

4.7. Comparison of the Diffusion and Transport Calculations for the Advanced Neutron Source Reactor Y. Y. Azmy and F. C. Difilippo

4.8. Convergence Properties of Iterative Algorithms for Solving the Nodal Diffusion Equations

Y. Y. Azmy and B. L. Kirk

4.9. Three-Dimensional Calculations of Neutron Streaming in the Beam Tubes of the ORNL HFIR Reactor

R. L. Childs, W. A. Rhoades, and L. R. Williams . . . . . . . . . 144

4.10. Determination of Kinetics Parameters Using Stochastic Methods in a ${ }^{252}$ Cf Driven System

F. C. Difilippo

4.11. Theoretical Analysis of Reactivity Measurement with the ${ }^{252} \mathrm{Cf}$ Source Ratio Method

F. C. Difilippo

4.12. Harmonic Analysis of Stochastic Descriptors and the Interpretation of ${ }^{252} \mathrm{Cf}$ Neutron Source Experiments

F. C. Diflippo 
xocrii

4.13. Neutron Kinetics of High-Intense Fission Neutron Sources

F. C. Difilippo . . . . . . . . . . . . . 145

4.14. Flattening the Power Distribution of the Advanced Neutron Source

Reactor

F. C. Difilippo . . . . . . . . . . . 146

4.15. Reactor Physics Caiculations for the Control of the Advanced Neutron Source Reactor

F. C. Difilippo, M. Abu-Shehadeh, and R. B. Perez . . . . . . . 146

4.16. Two-Point and Two-Energy Group Kinetics Model of the ANS Reactor F. C. Difilippo, M. Abu-Shehadeh, and R. B. Perez . . . . . . . 147

4.17. Radiation Transport Calculations for the ANS Beam Tubes

W. W. Engle, Jr., R. A. Lialien, und C. O. Slater . . . . . . . . 147

4.18. Advanced Neutron Source Design: Burnout Heat Flux Correlation

Development

W. R. Gambill and T. Mochizuki . . . . . . . . . 148

4.19. Preliminary ANS Reactor Cold Source Gain Factor Calculations

for Liquid Deuterium and Liquid Nitrogen-15

D. L. Henderson . . . . . . . . . . . . . . . . . 148

4.20. Some Preliminary Design Considerations for the ANS Reacto: Cold Source

D. L. Eenderson . . . . . . . . . . . . . 148

4.21. Preliminary One-Dimensional Neutronics Scoping Study for a

Californium-252 Cold Neutron Source Moderating Device

D. L. Henderson and R. G. Alsmiller, Jr.

4.22. Time-Dependent Single-Collision Kernels for Integral Transport Theory D. L. Henderson and C. W. Maynard . . . . . . . . . . 149

4.23. Generation of Broad-Group Neutron/Photon Cross-Section Libraries for Shielding Applications

D. T. Ingersoll, R. W. Roussin, C. Y. Fu, and J. E. White . . . . 149

4.24. A User's Guide to MICAP: A Monte Carlo Ionization Chamber Analysis Package.

J. O. Johnson and T. A. Gabriel 
4.25. Design Calculations for the ANS Cold Source

R. A. Lillie and R. G. Alsmiller, Jr.

4.26. Cross-Section Processing Methodology Used to Design the Advanced Neutron Source Reactor

R. T. Primm, III . . . . . . . . . . . 150

4.27. Generation of Lumped Fission Froduct Cross Sections for High Burnup, Highly Enriched Uranium Fuel

R. T. Primm, III and N. M. Greene . . . . . . . . . . 151

4.28. Reactor Physics Studies of Various Advanced Neutron Source Reactor Core Configurations

R. T. Primm, III . . . . . . . . . . . . . 151

4.29. The DORT Two-Dimensional Discrete Ordinates Transport Code

W. A. Rhoades and R. L. Childs . . . . . . . . . . 151

4.30. 3-D Discrete Orclinates Calculations with Parallel-Vector Processors

W. A. Rhoades and R. E. Flanery . . . . . . . . . 151

4.31. User's Manual for FERD-PC

B. D. Rooney . . . . . . . . . . . . . . 152

4.32. Reactor Physics Methods for the Preconceptual Core Design of the Advanced Neutron Source

J. M. Ryskamp, F. C. Difilippo, and R. T. Primm, III . . . . . . 152

4.33. DOS-HEATING6: A General Conduction Code with Nuclear Heat Generation Derived from DOT-IV Transport Calculations

M. L. Williams, A. Yücel, and S. Nadkarny . . . . . . . . . 153

4.34. Impact of ${ }^{235} \mathrm{U}$ (MAT 9235) Evaluation on $k_{\text {eff }}$ of Thermal Systems R. Q. Wright, M. L. Williams, and C. O. Slater . . . . . 153

\section{HIGH ENERGY RADIATION AND PARTICLE TRANSPORT}

4.35. Inclusion of Correlations in the Empirical Selection of Intranuclear Cascade Nucleons from High-Energy Hadron-Nucleus Collisions F. S. Alsmiller and R. G. Alsmiller, Jr. 
xocix

4.36. The High-Energy Transport Code HETC88

R. G. Alsmiller, Jr., F. S. Alsmiller, T. A. Gabriel, O. W. Hermann, and J. M. Barnes . . . . . . . . . . . . . . . . . 154

4.37. The Modified High-Energy Transport Code HETC and Design Calculations for the SSC

R. G. Alsmiller, Jr., F. S. Alsmiller, T. A. Gabriel, O. W. Hermann, and B. L. Bishop

4.38. Calculated Inclusive Neutron Production from $400 \mathrm{GeV}$ Proton-Nucleus Collisions

R. G. Alsmiller, Jr., F. S. Alsmiller, and O. W. Hermann . . . . . 155

4.39. Theoretical Studies of Hadronic Calorimetry for High Luminosity, High Energy Colliders

J E. Brau and T. A. Gabriel

4.40. Prospects for and Tests of Hadron Calorimetry with Silicon

J. E. Brau, T. A. Gabriel, and P. G. Rancoita

4.41. The Calculation of Cosmogenic Nuclide Abundance in Stony Meteorites M. Divadeenam, T. A. Gabriel, O. Lazereth, M. S. Spergel, and

T. E. Ward

4.42. Production Rates of Cusmogenic Nuclides in Stony Meteorites

M. Divadeenam, T. A. Gabriel, O. Lazereth, M. S. Spergel, and

T. E. Ward

4.43. Deterministic Transport Calculations of Dose Profiles Due to Proton Beam Irradiation

W. L. Filippone, M. S. Smith, R. T. Santoro, T. A. Gabriel, and $R$. G. Alsmiller, Jr.

4.44. Detectors for the Superconducting Super Collider, Design Concepts, and Simulation

T. A. Gabriel.

4.45. CALOR89: The Code System for Calorimeter Analysis and Design

T. A. Gabriel.

4.46. Calorimeter and Related Calculations for the Superconducting Super Collider

T. A. Gabric' 
4.47. CALOR87: HETC87, MICAP, SGS1, and SPECT, a Code System for Analyzing Detectors for Use in High Energy Physics

Experiments

T. A. Gabriel, F. S. Alsmiller, R. G. Alsmiller, Jr., B. L. Bishop,

O. W. Hermann, and J. O. Johnson. . . . . . . . . . . . 158

4.48. The Physics of Compensating Calorimetry and the New CALOR89

Code System

T. A. Gabriel, J. Brau, and B. L. Bishop . . . . . . . . . 158

4.49. Transport Calculation of Cosmogenic Nuclide Production in Stony Meteorites

T. A. Gabriel, M. Divadeenam, M. S. Spergel, T. E. Ward, and

O. Lazereth . . . . . . . . . . . . . . . . . 158

4.50. MICAP: A Program for Low Energy Neutron, Ion, and Gamma-Ray

Transport and One of Its Applications in Calorimeter Design:

Hydrogen Knock-In as a Method for Achieving Compensation

T. A. Gabriel, J. O. Johnson, anai J. Brau . . . . . . . . . . . . 159

4.51. Sources of Compensation in Hadronic Calorimeters

M. S. Goodman, T. A. Gabriel, A. Di Ciaccio, and R. Wilson . . . 159

4.52. Analysis of Neutronics Parameters Measured in Phase-II Experiment. of the JAERI/US Collaborative Frogram on Fusion Blanket Neutronics, Part I: Source Characteristics and Reaction Rate Distributions M. Nakagawa, T. Mori, K. Kosako, T. Nakamura, M. Z. Youssef, Y. Watanabe, C. Y. Gung, R. T. Santoro, R. G. Alsmiller, Jr., J. Barnes, and T. A. Gabriel . . . . . . . . . . . . . . . 159

4.53. Comparison of Calculated Results with Ixperimental Data for the Tritium Production Rates in a $\mathrm{Li}_{2} \mathrm{O}$ Assembly

R. T. Santoro, R. G. Alsmiller, Jr, J. M. Barnes, and T. A. Gabriel . 160

4.54. One-Dimensional $S_{n}$ Calculations to Evaluate the Shielding for an Alpha-Particle Charge Exchange Neutral Analyzer R. T. Santoro, W. K. Dagenhart, and J. M. Barnes . . . . . . . . . 160

\section{SPACE DEFENSIVE SYSTEMS}

4.55. Shield Optimization Program, Part II: Effects of Van Allen Belt

Radiation on SDI Weapon Platforms

J. M. Barnes, R. T. Santoro, J. O. Johnsun, J. D. Drischler,

T. A. Gabriel, and M. S. Smith . 
4.56. Recent ORNL Improvements to the HULL Hydrocode System T. J. Burns . . . . . . . . . . . . . . . 161

4.57. The Oak R.Age National Laboratory Strategic Defense Initiative Shield Of:imization Program

T. A. Gabriel, J. M. Barnes, B. L. Bishop, J. D. Drischler,

J. O. Johnson, R. A. Lillis, R. T. Santoro, and M. S. Smith . . . . 161

4.58. Shield Optimization Program, Part III: Effects of X-Ray Radiation from Nuclear Weapons on SDI Weapou Platforms

J. O. Johnson, T. A. Gabriel, J. M. Barnes, J. D. Drischler, M. S. Smith, and R. T. Santoro . . . . . . . . 162

4.59. Nuclear Weapon Radiation Effects on a Space Based Interceptor Weapon Platiorm

J. O. Johnson, M. S. Smith, and R. T. Santoro . . . . . . . . 162

4.60. The Effects of Natural and Enhanced Van Allen Belt Radiation on a Space Based Weapon Platform

R. T. Santoro, J. M. Barnes, and J. O. Johnson . . . . . . . 163

4.61. Shield Optimization Program, Part I: Executive Summary

R. T. Santoro and T. A. Gabriel . . . . . . . . . . 163

4.62. Material Response to Incident X-Rays

M. S. Smith

4.63. Shield Optimization Program, Part IV: Effects of Neutron and Gamma-Ray Radiations from Nuclear Weapons on SDI Weapon Platforms

M. S. Smith, J. O. Johnson, T. A. Gabriel, J. M. Barnes, J. D. Drischler, and R. T. Santoro . . . . . . . . . . . . 164

4.64. Shield Optinization Program, Part V: A Hydrodynamic Comparison Using HULL and PUFF-TFT for a One-Dimensional Aluminum Slab M. S. Smith, T. J. Burns, and J. O. Johnson . . . . . . . 164

4.65. Shield Optimization Program. Part VI: The Effect of Impurity Layers on the Hydrodynamic Response of a Beryllium Target to MonoEnergetic X-Rays Using the PUFF-TFT Code M. S. Smith, S. Mciveany, R. T. Santoro, and T. A. Gabriel 
xlii

4.66. Initial Effects of Nuclear Weapon X-Radiation on the Lampshade Orbital Debris Satellite Shield M. S. Smith and R. T. Santoro . . . . . . . . . . . . . . . . 165

\section{MATHEMATICAL MODELLING OF NON-LINEAR SYSTEMS INCLUDING COMBAT}

4.67. A Comparison Between the Propagators Method and the Decomposition Method for Nonlinear Equations

Y. Y. Azmy, D. G. Cacuci, and V. Protopopescu . . . . . . . . . 165

4.68. Two-Dimensional Maps Generated by Competitive Systems

Y. Y. Azmy and V. Protopopescu . . . . . . . . . . . . . 166

4.69. Stationary Fluws from a Model Boltzmann Equation

Y. Y. Azmy and V. Protopopescu . . . . . . . . . . . . . . . 166

4.70. Exact Solutions for a Semilinear Hyperbolic System with General Quadratic Interactions

V. C. Boff, V. Protopopescu, and Y. Y. Azmy . . . . . . . . . 166

4.71. Canonical Propagators for Nonlinear Systems: Theory and Sample Applications

D. G. Cacuri and V. Protopopescu . . . . . . . . . . . 166

4.72. Propagators for Nonlinear Systems

D. G. Cacuci and V. Protopopescu . . . . . . . . . . . . 166

4.73. Parabolic Systems with Nonlinear Competitive Interactions

C. Cosner, S. Lenhart, and V. Protopopescu . . . . . . . . . . . . 167

4.74. Optimal Control Theory of Load-Following and Parameter-Tracking of Nonlinear Systems: An Application of Pontryagin Maximum

Principle to Reactor Dynamics

C. March-Leuba and R. B. Perez . . . . . . . . . . . . . . . . . 167

4.75. Noise and Nonlinear Phenomena in Nuclear Systems

J. L. Munoz-Cobo and F. C. Difilippo, Editors . . . . . . . . . . 168 
xliii

4.76. Global Existence for Symmetric Discrete Velocity Models

V. Protopopescu

4.77. An Example of a Reaction-Diffusion System with Nonlinear

Competitive Interactions

V. Protopopescu ................ . 168

4.78. Combat Modeling with Partial Differential Equations

V. Protopopescu, R. T. Santoro, J. Dockery, R. L. Cox, and

J. M. Barnes . . . . . . . . . . . . . . . . . . . . . . . . 169

4.79. Two-Dimensional Combat Modeling with Partial Differential Equations

P. Rusu . . . . . . . . . . . . . . . . . 169

4.80. Mathematical Descriptions of Offensive Combat Maneuvers

R. T. Santoro, P. Rusu, and J. M. Barnes . . . . . . . . . . 169

4.81. Nonlinear Maps with Competitive Interactions: Fixed-Points,

Bifurcations, and Chaotic Attractors

D. F. Scollan, Y. Y. Azmy, and V. Protopopescu . . . . . . . . 170

\section{NUCLEAR WEAPON EFFECTS}

4.82. MASH, the Monte Carlo Adjoint Shielding Code

M. B. Emmett, W. A. Rhoades, and J. O. Johrson . . . . . . . 170

4.83. A Re-Evaluation of ${ }^{32} \mathrm{~S}(n, p)$ Cross Sections from Threshold to $5 \mathrm{MeV}$

C. Y. Fu . . . . . . . . . . . . . . 170

4.84. Fallout Facts for Nuclear-Battlefield Commanders

C. M. Haaland . . . . . . . . . . . . . . . . . . . . 171

4.85. An A proximation for Black-Body X-Ray Transport in Air

C. M. Haaland, R. T. Santoro, and J. M. Barnes . . . . . . . . 171

4.86. An Approximation for Black-Body X-Ray Transport in Air

C. M. Haaland, R. T. Santoro, and J. M. Barnes . . . . . . . . . 171

4.87. DABL:60:A Broad-Group Neutron/Photon Cross-Section Library

for Defense Nuclear Applications

D. T. Ingersoll, R. W. Roussin, C. Y. Fu, and J. E. White 
xliv

4.88. Sensitivity/Uncertainty Analysis for Free-in-Air Tissue Kerma Due to Initial Radiation at Hiroshima and Nagasaki

R. A. Lillie, B. L. Broadhead, J. V. Pace, III, and D. G. Cacuci . . . 172

4.89. Radiation Exposure Inside Reinforced Concrete Buildings at Nagasaki

W. A. Rhoades, R. L. Childs, and D. T. Ingersoll . . . . . . . . 173

4.90. New Dose Mortality Data Based on 3-D Radiation Shielding

Calculation for Concrete Buildings at Nagasaki

W. A. Rhoades, R. L. Childs, and D. T. Ingersoll . . . . . . . . . . 173

\section{RISK ASSESSMENT AND REACTOR SAFETY}

4.91. User's Guide for PRISM Arkansas Nuclear One-Unit 1: Vol. 1-Program for Inspectors Vol. 2-Program for Regulators

D. J. Campbell, V. H. Guthrie, J. R. Kirchner, J. Q. Kirkmam,

H. M. Paula, B. C. Ellison, F. M. Dycus, J. A. Farquharson, and

G. F. Flanagan ................... 174

4.92. Kisk Associated with Operation of the Oak Ridge National

Laboratory High Flux Isotope Reactor (HFIR)

G. F. Flanagan, D. Johnson, D. Buttemer, and S. Kaplan . . . . . . 174

4.93. Use of Risk Analysis Results in Support of the Final Programmatic

Environmental Impact Statement for the Disposal of the U.S. Chemical

Weapons Stockpile

G. F. Flanagan and S. Carnes . . . . . . . . . . . . 174

4.94. Risk Associated with the Demilitarization of the United States Chemical Weapons Stockpile

G. F. Flanagan, W. Fraize, and T. Kartachak . . . . . . . . 175

4.95. The Probabilistic Treatment of Potential Accidents: How, How Much, and How Often Could Chemical Agent be Released to the Biosphere?

W. E. Fraize and G. F. Flanagan . . . . . . . . . . . . . 175

4.96. A Revisit to the Passively-Safe Reactors

D. L. Moses

Section 5. ENGINEERING PHYSICS INFORMATION CENTER (EPIC)

5.0. Introduction . . . . . . . . . . . . . . . 179 
xlv

\section{RADIATION TRANSPORT DEVELOPMENTS}

5.1. Discrete Angle Biasing in Monte Carlo Radiation Transport

S. N. Cremer . . . . . . . . . . . . 183

5.2. Analytic Solution of a Five-Direction Radiation Transport Model

S. N. Cramer . . . . . . . . . . . . . . 183

5.3. Hypercube Applications of the $x y$ Geometry Nodal Method for the Neutron Diffusion Equation B. L. Kirk and Y. Y. Azmy . . . . . . . . . . . 183

5.4. An Iterative Procedure for Solving the Nodal Method Neutron Diffusion Equation on a Parallel Processor B. L. Kirk and Y. Y. Azmy . . . . . . . . . . . 184

5.5. A Parallel Approach to the Nodal Method Solution of the TwoDimensional Diffusion Equation B. L. Kirk and Y. Y. Azmy . . . . . . . . . . . . . 184

5.6. New Gamma-Ray Buildup Factor Data for Point Kernel Calculations: ANS-6.4.3 Standard Reference Data D. K. Trubey . . . . . . . . . . . . . . 184

\section{RADIATION TRANSPORT STUDIES}

5.7. The Effect of Coherent Scattering in Photon Radiation Transport Calculations

S. N. Cramer, B. L. Kirk, and J. Broadway . . . . . . . . 185

5.8. Neutron Streaming Benchmark Calculations T. Y. Lee and S. N. Cramer . . . . . . . . . . . . . 185

\section{INFORMATION ANALYSIS CENTER ACTIVITIES}

5.9. Nuclear Industry Quality, Productivity, and Innovation from the Perspective of an International Information Center B. F. Maskewitz

5.10. Technology Resuurces in Support of Programs of the Physicist in Medicine and Biology

B. F. Maskeuitz 
xlvi

5.11. Computers and Interactions Between Physicians and Health Physicists B. F. Maskewitz

5.12. Methods and Formats for ENDF/B-VI

R. W. Roussin

5.13. The Status of ENDF/B-VI

R. Roussin, C. Dunford, R. McKnight, and P. Young . . . . . . 18

5.14. RSIC After 25 Years - Challenges and Opportunities

R. W. Roussin, B. F. Maskewitz, and D. K. Trubey . . . . . . . . 187

5.15. An Overview of the Radiation Shielding Information Center (RSIC)

D. K. Trubey . . . . . . . . . . . . . . . . . . . 187

\section{TRANSFER OF COMPUTING TECHNOLOGY}

5.16. Photon Cross Sections for ENDF/B-VI

D. K. Trubey, M. J. Berger, and J. H. Habbell

5.17. Guide for Licensing Evaluations Using CRAC2: A Computer Program for Calculating Reactor Accident Consequences

J. E. White, R. W. Roussin, and H. Gilpin . . . . . . . . . . . . 188

5.18. SUSD: A Computer Code for Cross-Section Sensitivity and Uncertainty Analysis Including Secondary Neutron Energy and Angular

Distribution

K. Furuta, Y. Oka, and S. Kondo . . . . . . . . . . . . 188

\section{APPENDICES}

Personnel Changes .. . . . . . . . . . . . . . . . 193

Scientific and Professional Activities . . . . . . . . . . . . . . . 201

Conferences . . . . . . . . . . . . . . . . . . 229

Seminars at ORNL . . . . . . . . . . . . . . . . 235

Publications . . . . . . . . . . . . . . . . . . . 243

Papers Presented at Srientific Mectings and Seminars . . . . . . . . . . 265

Division Organization Chart . . . . . . . . . . . . . . . . . 293

Author Index. . . . . . . . . . . . . . . . . . . . . . . 295 
Section 1

MATHEMATICAL SCIENCES 


\title{
1.0. INTRODUCTION
}

\author{
R. C. Ward
}

Activities in the mathematical sciences during the report period continued to be divided in nearly equal proportions between basic research and collaboration with other scientists at the Oak Ridge DOE facilities. Major disciplines covered by these research and consulting activities include computer science, mathematics, and statistics.

Parallel computing remained the focus of our basic research program in computer science, again emphasizing the development of algorithms for efficiently solving important basic computational problems of science and engineering, especially matrix computations, on aavanced computer architectures. In addition a new research project has been established with the objective of understanding and characterizing the actual behavior of parallel programs on distributed-memory architectures. The project addresses the analysis of realistic and complete application codes rather than computational kernels, test problems, or single mathematical routines. Some of the recent highlights from our basic and applied computer science research have been a new algorithm for tridiagonalizing a nonsymmetric matrix, an analysis of the effect of time constraints on scaled speed-up, a parallel code for studying electron correlations in high temperature superconductors, and the development of a software package to support parallel processing on a local area network of workstations, and sponsorship jointly with the University of Tennessee of a special year devoted to numerical linear algebra year with international visitors, lecturers, and workshops.

Research in mathematics during the reporting period emphasized moving boundary problems, especially phase change problems involving heat transfer, and modeling complex phenomena using vector and parallel supercomputers. A new research program in modeling environmental waste problems was initiated and is expected to be the future focal point of our mathematical research sponsored by the DCE Applied Mathematical Sciences Subprogram. Recent highlights of our mathematics research and collaboration have been the development of a new boundary element technique that reduces computational times by an order of magnitude for numerical simulation of phenomena involving single cracks and now makes feasible simulation of multiple crack geometries, the development of a code to simulate thermal energy storage for the NASA space station, the development of a parallel code for studying lepton pair production in relativistic heavy ion collisions, and the development of a fully vectorized code for simulating 3-dimensional moving boundary problems that in: olve void formation.

In statistics, our basic research focus has continued to be in the area of computational statistics and biostatistics. Some of the highlights include the development of an algorithm for usc by computational scientists in the design and analysis of computer experiments, the development of a mathematical model that predicts hematopoietic response and associated probability of mortaiity during and following exposure to ionizing radiation, and the development of an algorithm for finding a small set of the best models from a very large number (typically thousands) of possible hierarchical models. Our applied, or collaborative, research has involved our statisticians with work in most of the ORNL divisions and major organizations in Martin Marietta Energy Systenss. Examples to illustrate the diversity of this collaborative are quality control methods for deterting flaws in currency, reliability analysis of army helicopters, life testing of irradiated materials, and time series analysis and forecasting of river flow rates. T. J. Mitchell, a researcher in our Statistics Group, was honored as the 1088 Author of the Year by Enrrgy Systems during their annual awards ceremony in May, 1080, for his paper co-authored with J. J. Beauchamp on 
variable selection in linear regression. (This research was discussed in the division's last progress report.)

Our Research Computing Facility has been upgraded and renamed the Advanced Computing Laboratory (ACL) to better reflect its computing environment, which now consists of individual workstations on a local area network with various compute, network, file, and print servers. Also, the Computer Science Department at the University of Tennessee Knoxville (UTK) has joined us in support of the ACL. Our Intel iPSC has been replaced by an Intel iPSC/2 with 64 processors and 4 MBytes of memory per processor, our Sequent has been upgraded to 12 processors with 16 MBytes of shared memory, an Ncube/4 with 16 processors and $8 \mathrm{MBytes}$ of aggregate memory has been purchased, and our DEC VAX 11/780 has been upgraded to an 11/785. Work is currently in progress to procure a multi-gigaflop research parallel computer during FY'90 for use on some of ORNL's "grand challenges." A dedicated T1 communications link has been installed to cornect our ACL with the Computer Science Department at UTK to facilitate their access to our computers and to provide ORNL a connection to SURAnet, the southeastern regional network of NSFnet. 


\title{
COMPUTER SCIENCE
}

\author{
General Parallel Computing
}

1.1

\section{A REMOTE HOST FACHITY FOR INTEL HYPERCUBES}

\section{T. H. Dunigan}

(Abaract of ORNL/TM-11068, Apri? 1989)

The structure and use of a remote bost facility for controlling application programs on an Intel hypercube are described. The facility permits an alternate UNIX host, such as a graphies workstation or supercomputer, connected by a TCP/IP network to the Intel cube manager proceseor to communicate with application programs running on the hypercube nodes. The facility supports both $C$ and FORTRAN applications.

Research sponsored by Applied Mathematical Sciences Research Program. U.S. DOE, Office of Energy Research.

\section{2}

\section{PERFORMANCE OF A SECOND GENERATION HYPERCUBE}

\section{T. H. Dunigan}

(Abutract of ORNL/TM-10881, November 1988)

The performance of four commercially available hypercube parallel processors is analyzed. Computation and communication performance for a number of low-level benchmarks are presented for the Amelek S14 hypercube, the Intel iPSC/1 hypercube, the Ncube hypercube, and the second generation Intel iPSC/ 2 hypercube.

Revearch sponsored by Applied Mathemacical Sciences Research Program, U.S. DOE Office of Energy Research.
1.3

\section{HYPERCUBE SIMULATION ON A LOCAL AREA NETWORK}

\author{
T. H. Dunigan
}

(Abatract of ORNL/TM-10685, November 1988)

We describe the structure and use of a distributed computing environment based on a hypercube programming model that run; on most UNIX (trademark of AT\&T) systems with TCP/ IP network support. The pactage rases a library of message passing routines and multiple user processes, written in either C or FORTRAN, to provide an environment for the development and testing of algorithms for hypercube parallel processors simulated by a collection of computers on a lecal area network. Each node of the simulated hypercube is a work station on the network. The simulator produces a trace file that can be used for debugging, performance analysis, or graphical display.

Research sponeored by Applied iiannematical Sciences Re search Program, U.S. DOE Office of Energy Research.

\section{4}

\section{A PARALLEL DECOMPOSITION ALGORITHM FOR STAIRCASE LINEAR PROGRAMS}

\section{R. Entriken*}

(Abatract of ORNL/TM-11011, December 1988)

As part of an extended research project on the parallel decomposition of linear programs, a parallel algorithm for Staitcase Linear Programs was designed and implemented. This class of problems encompasses a large range of planning problems and when decomposed has simple subproblem formulations and communication patterns. This makes its solution a manageable step toward our eventual goal of producing a general code that automatically exploits problem structures of various forms. 
The results presented here were derived from an implementation for a Sequent Balance $\mathbf{8 0 0 0}$ shared-memory multiprocessor. The algorithm itself is message-based but can run on either sharedor distributed-memory parallel computers.

A simple diet planning problem is used to demonstrate the principles of the algorithm's development and performance. When applied to this problem. the parallel decomposition algorithm shows promise relative to present serial optimization codes. The nonlinear optimination code MINOS 5.1 is used both as a basis for comparison and as a generic subproblem solver. The greatest room for speedup is in exploiting problem structures. The results show that decomposition can improve efficiency even with a singl $:$ processor. Examples are given where multiple processors lead to still greater efficiency.

Research sponsored by the Electric Power Research Institule; by the U.S. Air Force Office of Scientific Research; by the U.S. Department of Energr; by the Nationd Security Agency; by the National Science Foundation; by the Science Alliance Program of the State of Tenneasee; and by the Departmenc of Operations Research at Stanford Univerity.

* Stanford Liniversity, Stanford. CA.

1.5

\section{HYPERCUBE MULTIPIOCESSORS: WHAT THEY ARE AND HOW TO USE THEM}

\section{G. A. Geist}

(Abstrant of oral presentation preacnted at the University of Florida, Gaineville, FL, March 15-16, 1988)

This talk discussed the hypercube architecture and the present cornmercial implementations by $\mathrm{N}$.'UBF, and Intel. It also addressed the effects of different data mappings onto the processors and the different comm'snication patterns which develop in the netwr. '.. Finally, good and bad methods of coding comrrunication routines will be discussed.

Remearcti sponnured by Applied Mathematical Sriences He search Program. L.S. DOF, Office of Finergy Researdo.
1.6

\section{A MACHINE-INDEPENDENT COMMUNICATION LIBRARY}

\author{
G. A. Geist \\ M. T. Heath \\ B. W. Peyton \\ P. H. Worley
}

(Abetraca of paper presented at the Fourth Conference on Hypercubes, Concurnent Computers, and Applications. Monterey, CA, March 6-8, 1900)

In this report we desenibe : iibrary of routines that enables the development of portable parallel programs. The library is designed primarily to provide portable communication primitives and related system calls for distributed-memory architecture. The package need not be limited to such machines, however, and we expect that the message-passing paradigm nn which the library is based will also be useful on other parallel architectures for which data locality is $=7$ important performance consideration.

The library also provides portable routines to perform certain widely-used, high-level communication operations, such as global broadcast and global sum. Their inclusion frees users from having to develop these commonly needed capabilities or to modify them to run on different machines. Finally, the library provides an execution tracing facility that can be used to monitor performance or to aid in debugging.

Research aponsored by Applied Mathematieal Sciences Re search Program, U.S. DOE Office of Energy Research.

1.7

\section{PARALLEL COMPUTING}

\section{T. Heath}

(Abatract of article in the Oat Ridger, Sunday. Auguat 6. 1989)

Parallel computing, in which many computers are used simultaneously to solve a single problem, is one of the most significant new trends in computer technology. The fastest supercompulers available today, as well as new generations of supercomputers now being designed, all gain much of their fantastic speed by exploiting various types of parallelism. Moreover, the direction of this trend is toward even higher degreas of parallelisn using larger and larger numbers of computer processors. 
This article gives elementary examples of hom parallelism can be exploited, discusses the implications of parallelism for scientific computing, and illustrates ORNL's role in developing and using parallel computing technology.

Research sponsored by Applied Matbematical Sciences Research Progrum, U.S. DOE Office of Energy Remeand.

\section{8}

\section{PARALLEL COMPUTING: PERSPECTIVES AND PROSPECTS}

\author{
M. T. Heath
}

[Abetract of book chapler in Opportenities end Cosatrints of Perallel Computiag (in preas)]

The trend toward parallel processing is driven iy uth scientific and economic factors. At the high end of the perfornance range, parallelism is the only answer to the fundamental limits on computational speed that are being rapidly approached by today's supercomputers. In the lower and middle performance ranges, parallel architectures assembled from commodity parts, such as microprocessors and memory chips, provide the most cost effective systems. These trends are mutually supportive, as the lessons learned and software developed are transferred across systems.

Reseurch sponsored by Applied Mathernatical Sciences Research Program. U.S. DOE Ofice of Energy Research.

\section{9}

HYPERCUBE APPLICATIONS AT OAK RIDGE NATIONAL LABORATORY

\section{T. Heath}

(Abatract of oral presentation presented at the 1988 ACM Symposium on Parallel Programming, Yale Univerity, Niew Haven, CT. July 20, 1988)

To determine the viability of hypercube architectures for solving a broad spectrum of computational problems typically arising at Oak Ridge National Laboratory, a representative set of applications was chosen for implementation on : hypepcube. The projects included finite element fracture analysis, cascade sinulation, geochemical contaminant transport, image analysis, molecu- lar dynamics of po!ymers, density functional theory, and nonlinear magnetohydrodynamics. Each project was a collaborative effort of scientists and engineers from the discipline in which the problem arose and experienced hypercube users from a parallel computing research group. Results of each of these projects and an overall summary are included in this report.

Reseand sponsored by Applied Malhemacieal Sciences Phe search Program, U.S. DOE Office of Enerzy Reacarch.

\subsection{0}

\section{HYPERCUBES}

\section{T. Heath}

(Abutrect of oral presentation presented of the Conference on Computational Atomic and Nuclear Physica al One G;gallop, Oak Ridec, TN, April 15, 19e8)

This talk presented an overview of hypercube multiprocessor architectures and their use in scientific computing.

Research sponsored by Applied Malhematical Sciences Re search Program, U.S. DOE Office of Energy Research.

\subsection{1}

\section{LIMITS TO PARALLELISM}

\section{T. Heath}

(Abutrect of oral presentution presented at the IBM Workahop on Parallel Computing, San Jowe, CA, December 4-7, 1988)

We present brief remarks on the concepts of scaled speedup and the limits to parallelism implied by Amdahl's Law. It is well known that for a fixed size problem, efficiency declines as more processors are used. We point out that for many important problem classes, a similar effect results from a fixed limit on execution time (i.e., as the number of processors grows, the problem size cannot grow fast enough to ofiset the efficiency decline and still meet the overall bound on execution time).

Reacarth sponeored by Applied Machematical Sciences Rerearch Program, IJ.S. DOE Ofice of Enercy Research. 
1.12

\section{ONCE AGAIN, AMDAHL'S LAW}

\author{
M. T. Heath P. H. Worley
}

[Abstract of Comm. Assoc. Comput. Mech. 32/2, 262263 (February 1989)]

We review Amdahl's Law governing multiprocessor speedup in light of recently reported experience with massively parallel multiprocessors. We consider the interpretation of Amdahl's Law for problems of fixed size or fixed execution time, and note that in either case efficiency is ultimately limited. These results are not in conflict with the favorable experience that has been reported, since high speedups and efficiencies are attainable for sufficiently large problems and execution times.

Research sponsored by Applied Matlematical Sciences Research Program, U.S. DOE Ofice of Energy Research.

\subsection{3}

\section{MULTIPROGRAMMING A DISTRUBITED-MEMORY MULTIPROCESSOR}

\section{R. Leuze L. W. Dowdy*}

\section{K. H. Park"}

[Abstract of ORNL/TM-11064, January 1969; aleo Concurrency Practice and Experience 1, 19-33 (1969)]

The development of computing systems with large numbers of processors has been motivated primarily by the need to solve large, complex problems more quickly than is possible with uniprocessor systems. Traditionally, multiprocessor systems have been uniprogrammed, i.e., dedicated to the execution of a single set of related processes, since this approach provides the fastest response for an individual program once it begins execution. However, if the goal of a multiprocessor system is to minimize average response time or to maximize throughput, then multiprogramming must be considered.

In this paper, a model of a simple multiprocexsor system with a two-program workload is reviewed; the model is then applied to an Intel iPSC/ 2 liypercube multiprocessor with a workload consisting of parallel wavefront aigorithms for solving triangular systems of linear equations. Throughputs predirted hy the model are compared with throughputs obtained experimentally from an ac. tual system. The results provide validation for the model and indicate that significant performance improvements for multiprocessor systems are possible through multiprogramming.

Research sponsored by Applied Mathematical Sciences Research Program, U.S. DOE, Oflice of Energy Research and by the Alexander von Humboldt Fountation.

* Vanderbilt University, Nachville, TN.

\subsection{4}

\section{A BIBLIOGRAPHY ON PARALLEL AND VECTOR NUMERICAL ALGORITHMS}

\author{
J. M. Ortega* R. G. Voigt ${ }^{\dagger}$ \\ C. H. Ronine
}

(Abatract of ORNL/TM-10998, January 1989)

This technical report contains a bibliography of the current and past research papers dealing with numerical algorithms on both parallel and vector computers.

Research sponsored by Applied Mathematical Sciences Re search Program, U.S. DOE Office of Energy Research and by Nationd Aeronautice and Space Administration.

* Univenity of Virginis, Charlottesville, VA.

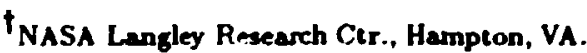

1.15

\section{A THEORETICAL INVESTIGATION OF A PARALLEL GENETIC ALGORITHM}

\author{
$\begin{array}{ll}\text { C. C. Pettey } & \text { M. R. Leuze }\end{array}$
}

(Abatract of paper presented at the Third International Conference on Genetic Algorithms, Fairfax, VA, June 4-7, 1989; Proc. 398-405, J. D. Schaffer, Ed., Morgan Kaufmann Publishers Inc., San Mateo, CA (1980)]

In the past few years the limitations of uniprocessor computing systems and the increasing availability of multiprocessors have led to investigations of parallel genetic algorithms. One algorithm in particular, called PGA, consists of a set of communicating sequential genetic algorithms. This paper includes an investigation of the theoretical allocation of trials to srhemata hy $P($;A 's in general and by a particular $\mathrm{PC} ; \mathrm{A}$ (which performs communication in a uniformly random manner) 
along with an experimenta. validation of an assumption made in the theortical investigation.

Research sponsored by the Applied Mathematical Sciences Research Program, U.S. DOE Office of Energy Research.

-Vanderbilt University, Nashville, TN.

\subsection{6}

\section{OVERVIEW OF ARCHITECTURES AND APPLICATIONS IN PARALLEL PROCESSING}

\section{H. Romine}

(Abstract of oral presentation presented at the Army Survey on High Performance Computing. Washington, DC, February 23-24, 1989)

In this talk, we survey the state of the art in parallel processing, with emphasis on both architectures and applications. A general discussion of universal topics in parallel processing will include classification of architectures (using Flynn's taxonomy), methods for evaluating performance, and general guidelines for identifying potential parallelism in existing application codes. This will be followed by a survey of some of the existing machines that exemplify each clazs of architecture, and a discussion of the advantages/disadvantages of each type of machine. Peak performance figures will be given for machines that are capable of supercomputer-class performance on problems in scientific computing. Finally, an overview of possible future developments in parallel processing will be given.

Research sponsored by Applied Mathematical Sciences Research Program, U.S. INOE Office of Energy Rewearch.

\subsection{7}

\section{THE EVOLUTION OF MULTIPROCESSING}

\author{
C. H. Romine
}

(Abstract of oral prewent ation presented at North Carolinn State University as part of the ORAU Viaiting Lecture Series, Raleigh/Durham, NC, September 12. 1988)

In order to circumvent the inherent limitations on the speed of a conventional "von Neumann" type of computer, machines are being designed that will solve problems by using multiple processing units. The evolution of working mut tiprocessors can be traced back to the Illiac IV, which was put into operation in 1972 at NASA's Ames Research Center. Since then, a large number of multiprocessors have been designch and/or built, including the Denelcor HEP, the ICL DAP, the Cosmic Cube at $\mathrm{Cal}$. Tech., the TRAC at the University of Texas, $\mathrm{Cm}^{*}$ at Carnegie-Mellon University, ZMOB at the University of Maryland, and the MPP at NASA's Goddard Space Flight Center. More recently, vendors such as Intel, NCUBE, Ametek, Floating Point Systems, and Thinking Machines Corporation have introduced new multiprocessor computers into the marketplace.

This talk will cover the history of multiprocessing, describing the advantages and disadvantages of each type of multiprocessor. The focus will be the application of multiprocessors to numerical algorithms, but only a limited knowledge of numerical analysis is required.

Revearch sponsored by Applied Mathematical Sciences Research Program, U.S. DOE Ofice of Energy Research.

\section{Dense Matrix Computations}

\subsection{8}

\section{QR FAC RORIZATION OF A DENSE MATRIX ON A HYPERCUBE MULTIPROCESSOR}

\section{E. Chu* A. George}

(A batrant of ORNL/TM-10691, February 1988)

In t'us article we describe a new algorithm for computing the QR factorization of a rectangular matrix on a hypercube multiprocessor. The scheme involves the embedding of a two-dimen-

nal grid in the hyprecube network. We employ a global communiration scheme which uses redundant computation to maintain data proxim ity, and the mapping strategy is such that for a fixed number of processors the processor idle time is small and either constant or grows linearly with the dimension of the matrix. A complexity analysis tells us what the aspect ratio of the embedder grid should be in terms of the shane of the matrix and the relative speeds of communication and computation. Numerical experiments performed on an Intel Hypercube multiprocessor support the theorctical results.

\footnotetext{
Research aponeored by Applied Mathematical Sciences Remearch Program, U.S. DOE Office of Energ: Reacarch; by Canadian Natural Scienres and Finginearing Measarch (is nril; and by Science Alliance, Univeraity of Tenneasee.
}

"Inivernity of Waterkoo. Waterlon, Ontario, Canada. 


\subsection{9}

\section{REDUCTION OF A GENERAL MATRIX TO TRIDIAGONAL FORM}

\author{
G. A. Geist
}

[Abstract of SIAM Jonmal on Matrix A aclysis and A ppli. cetions (in press); also ORNL/TM-10991, March 1989]

This report reviews recent research in finding eigenvalues of general matrices by first reducing the matrix to tridiagonal form and then applying LR iteration to this reduced form. The report also describes a new algorithm for solving this problen and shows why it is superior to the other approaches. While our results show the algorithm to be over three times faster than the EISPACK routines for finding eigenvalues, there are some matrices for which the algorithm fails. The report describes how to detect these matrices during the reduction process and how to attempt to proceed.

Research sponsored by Applied Mathematical Sciences Research Program U.S. DOE Office of Energy Research.

\subsection{0}

\section{REDUCTION OF A GENERAL MATRIX TO TRIDIAGONAL FORM USING A HYPERCUBE MULTIPROCESSOR}

\section{G. A. Geist}

(Abotract of paper presented at the Fourth Conference on Hypercubes, Concurrent Computers, and Applications, Monterey, CA, March 6-8, 1989)

Recently there has been a renewed interest in finding reliable methods of reducing general matrices to tridiagonal form. We have developed a serial reduction algorit on that appears to be very reliable in practice. In this paper we describe a parallel version of our algorithm, which has been implemented using a portable library developed at Oak Ridge National Laboratory (ORNI). The library allows the code to be portable across most comirnercial hypercubes.

The: algorithru was developed as one step in the r roress of finding eigenvalues of nonsymmetric ma' rices. Our original parallel eigenvalue routines redured the matrix to IJessentserg form and then applied QH iteration, but their performance was disapprinting. ()ur new parallel routines reduce. the matrix on tridiagonal form and then apply I. H iteratien lising an ifSS:/2, we compare the performance of the new parallel routines with our previous parallel routines and show that the new routines are nearly an order of magnitude faster. allowing us to solve much larger problems than previously attempted.

Research sponsored by Applied Mathematical Sciences Research Program, U.S. DOE Office of Energy Research.

\subsection{1}

\section{FINDING EIGENVALUES AND EIUENVECTORS OF UNSYMMETRIC MATRICES USING A DISTRIBUTED-MEMORY MULTIPROCESSOR}

\section{G. A. Geist}

(Aostract of oral presentacion presented at the Univeraity of Tenreasec, Knoxville, TN, April 11, 1988 and at the SIAM Conference on Applied Linear Algebra. Minneapolis, MN, July 11-15, 1988)

The best known method for finding the eigenvalues of dense unsymmetric matrices is the implicit double shift QR iteration. The complexity and large volume of communication required in this algoritl.m have hindered distributed-memory implementations. We will discuss several parallel implementations of this algorithm and their performance on the Intel iPSC. Recent advances in distributed-memory triangular solutions have been used to develop an algorithm for finding the eigenvectors of the matrix. While Amdahl's Law limits our present implementations of the QR iteration to a speedup of about 10 , the performance of the eigenvector routines does not suffer from the same limitation.

Research sponcored by Applied Mathematical Sciences Research Program, V.S. DOE Office of Energy Rewearch.

\subsection{2}

\section{THE UNSYMMETRIC EIGENVALUE: CAN HQR BE BEAT?}

\author{
G. A. Geist
}

(Abotract of oral presentation presented at the American Machematical Sociely Meeting, Lawrence, KS, Ortrber 29. 1988)

The IIQR algorithm is a fast and robust mothod for finding eigenvalues of gerieral real matrices. It applies the implicit double shift $\mathrm{CQR}_{\mathrm{K}}$ it- 
eraiion io an upper Fiessenberg matrux. This talk presented algorithms for reducing a general real matrix to tridiagonal form and then applying an implicit double shift LR iteration to this matrix. The methods that the algorithms employ to avoid or fix problems inherent in the approach were discussed and results were given.

Research sponsored by Applied Mathematical Sciesces Re search Program, U.S. DOE Office of Energy Rewearch.

\subsection{3}

\section{FINDING EIGENVALLES AND EIGENVECTORS OF UNSYMMETRIC MATRICES USING A DISTRIBUTED- MEMORY MULTIPROCESSOR}

\section{G. A. Geist $\quad$ G. J. Davis*}

[Abstract of ORNL/TM-10938, November 1988; aleo Perailel Compating (in preas)]

Distributed-memory parallel algorithms for finding the eigenvalues and eigenvectors of a dense unsymmetric matrix are given. While several parallel algorithms have been developed for symmetric matrices, little work has been done on the unsymmetric case. Our parallel implementation proceeds in three major steps: reduction of the original matrix to Hessenberg form, application of the implicit double-shift $Q R$ algorithm to compute the eigenvalues, and back transformations to compute the eigenvectors. Several modifications to our parallel QR algorithm, including ring communication, pipelining and delayed updating are discussed and compared. Results and timings are given.

Research sponsored by Applied Machematical Sciences Research Program. U.S. DOE Office of Energy Research.

* Georgia State Univenity, Atlanto, GA.

\subsection{4}

\section{STABILIZED GAUSSIAN REDUCTION OF AN ARBITRARY MATRIX TO TRIDIAGONAL FORM}

\author{
G. A. Geist A. Lu* \\ E. L. Wachspress" \\ (Atsutrat of ORNL/TM-11089, June 1989)
}

This report presents several ideas for improving the stability of Gaussian reduction of an arbitrary real matrix to tridiagonal form. First, we an alyze conditions under whirh reduction algorithms break down or becorre unstable. Second, we discuss how methods of threshold pivoting decrease the probability of these conditions occurring. Finally, we present new methods for recovering from brealdow $n$ when it does occur. The class of matrices that can be successfully reduced is significantly broadened by these new recovery algorithms.

Research uponsored by Applied Mathemutical Sciences Re search Program, U.S. DOE, Office of Energy Research.

"University of Tennemee, Knoxville, TN.

1.25

\section{A PARTITIONING STRATEGY FOR PARALLEL SPARSE CHOLESKY FACTORIZATION}

$$
\text { G. A. Geist E. } \mathbf{N}_{\mathbf{g}}
$$

(Abstract of ORNL/TM-10937, November 1988)

This report presents a solution to the problem of partitioning the work for sparse matrix factorization on a multiprocessor system. The goal of this partitioning strategy is to achieve load balancing and a high degree of concurrency among the processors while reducing the amount of processorto-processor data communication. The task assignment strategy is based on the structure of the elimination tree for a given ordering and can be applied to arbitrarily unbalanced trees. This is important because popular fill-reducing ordering methods, such as the minimum degree algorithm, often produce unbalanced climination trees. Results from the Intel iPSC/ 2 are presented for various finite-element problems using both nested dissection and minimum degree orderings.

Research aponeored by Applied Machematical Sciences Research Program, U.S. DOE Office of Energy Research.

\subsection{6}

\section{LU FACTORIZATION ON DISTRIBUTED-MEMORY MULTIPROCESSORS}
G. A. Geist
C. H. Fomine

(Abatract of oral presentation presented at the Univeruity of Florida, Gaineaville, Fl, March 15-16, 1988 and at the SIAM Parallel Proceasing Conferenne, Ios Angeles, CA, December 1. 4, 1987)

We discussed and compared two methots of improving the efficiency of L,U factorization with 
pivoting on distributed-memory multiprocessors. The first method uses a dynamic load balancing scheme to avoid work imbalances caused by pivoting. While this method increases the amount of communication required, it significantly reduces the execution time. The second method uses pipelining to mask the cost of pivoting. While pipelining complicates the algorithm, the execution time is slightly less than in the first method. Both algorithms have achieved efficiencies of $85 \%$ to $95 \%$ running on a 64 node iPSC hypercube.

Research sponsored by Applied Machemacical Sciences Research Program, U.S. DOE Office of Energy Research.

\section{$\mathbf{1 . 2 7}$}

\section{FINDING EIGENVALUES AND EIGENVECTORS OF UNSYMMETRIC MATRICES USING A HYPERCUBE MULTIPROCESSOR}

\author{
G. A. Geist \\ R. C. Ward \\ G. J. Davis* \\ R. E. Funderlic ${ }^{\dagger}$
}

(Abstract of paper presented at the Third Conference on Hypercube Concurrent Computers and Applications, Pastdena, CA, January 19-20, 1988; Proc. Vol. II, pp. 15771582, Geoffry Fox. Ed., May 1988)

Distributed-memory algorithms for finding the eigenvalues and eigenvectors of a dense unsymmetric matrix are given. While several parallel algorithms have been developed for symmetric systems, little work has been done on the unsymmetric case. Our parallel implementation proceeds in three major steps: reduction of the original matrix to Ilessenberg form, application of the implicit double-shift QR algorithm to compute the eigenvalues, and back transformations to compute the eigenvectors. Several modifications to our parallel $Q R$ algorithm, including ring communication and pipelining. are discussed and compared. Results and imings are given.

Research sponsored by Applied Mathemacical Scienres Research Frogram, U.S. DOE Office of Energy Resear.h.

" Cengia State Iniversity, Atlanta, GA.

'Trorth C:arolina Stake I'niveruity, Raleigh, NC.
1.28

\section{REGULARIZATION, GSVD AND TRUNCATED GSVD}

\author{
P. C. Hansen*
}

(Abstract of ORNL/TM-10779, September 1988)

The generalized singular value decomposition (GSVD) is used to anaiyze two alternative methods for solving ill-posed problems: regularization in general form and truncated SVD. We give conditions in which suitable solutions can be found, discuss the perturbation theory, and show that the optimum regularization and truncation parameters can be computed via generalized cross-validation. Our analysis also sheds light on a particular method, based on a transformation to standard form, that avoids the numerical difficulties associated with computation of the GSVD.

Research sponsored by Danish Natural Science Research Council; U.S. Air Force, Office of Scientific Research; N2tional Security Agency; National Science Foundation; Science Alliance Program of the State of Tennessee; and Applied Mathematical Sciencel Research Program, U.S. DOE Office of Energy Rewearch.

"Copenhagen University Observalory, Copenhagen K, Denmark.

\subsection{9}

\section{SOLUTION OF ILL-POSED PROBLEMS BY MEANS OF TRUNCATED SVD}

\author{
P. C. Hansen* \\ (Abatract of ORNL/TM-10772, June 1988)
}

We investigate Truncated SVD (TSVD) solutions to ill-posed least squares problems involving matrices with ill-determined as well as weltdetermined numerical rank. If a discrele Picard condition is satisfied, then in both cases the truncation parameter can be chosen such that ihe TSVD solution is satisfactory. The appropriate truncation parameter, giving the optimal signal-to-noise ratio in the solution, is the minimizer of the generalized cross-validation function.

Reacench sponsored by Danish Natural Science Research Counril; U.S. Air Forre, Offre of Srientific Research; No tionad Security Agency; National Srience Foundation; Srience Alliance Progran of the State of Tenneasere; and Applied Mathematical Sriences Reaearch Program, U.S. DOF: OMice of Finergy Remenard.

"Copenhager Iniveraity Obmervatory. Copenhagen K. Denmark. 
1.30

\section{PARALLEL TRIANGULAR SOLUTIONS AND DOWNDATING ON DISTRIBUTED-MEMORY MULTIPROCESSORS}

\author{
M. T. Heath
}

(Abotract of oral presentation presented at the American Mathematical Society Conference, Knoxville, TN, March 25, 1988 and at the SIAMI Conierence on Applied Linear Algebra, Madison, WI, May 23-26, 1988)

In this talk we review recent progress in the development of efiicient parallel algorithms for solving triangular systems and for updating or downdating triangular factorizations of matrices on distributed-memory multiprocessors. These two types of problems have a similar structure, so that algorithms for solving them are closely related. In both cases the granularity of the computations is rather fine and communication costs tend to dominate on message-passing multiprocessor systems such as a hypercube. We discuss several types of parallel algorithms for solving triangular systems, including modified cyclic algorithms, and apply them to an updating/downdating problem arising in signal processing. Empirical results obtained on commercial hypercubes are presented.

Research sponsored by Applied Mathematical Sciences Research Program. U.S. DOE Office of Energy Research.

\subsection{1}

\section{CHOLESKY DOWNDATING ON A HYPERCUBE}

\section{$\begin{array}{ll}\text { C. S. Henkel* } & \text { M. T. Heatb }\end{array}$ R. J. Plemmons*}

(Abetract of paper presented at the Third Conference on Hypercube Concurrent Computers and Applications, Pace dena. CA, January 19-20, 1988; Proc. Vol. II, pp. 1592 1598, Geofiry Fox, Fd., May 1988)

Least squares modifications associated with the addition or deletion of data often involve updating or downdating the Chnlesky factor of the observation matrix. W describe and compare parallel implementatious for the hypercube of three methorls for downdating the ('holesky factor: an orthogonal sclieme, a hypertiolic scheme, and a hybrid srheme combining the first two. The computational complexities of these algoritlims vary 'gn:ficantly, but the parallel implementations of all three are similar in communication complexity to solving triangular systems. In computational tests on Intel iPSC hypercube, the algarithms performed similarly, suggesting a preference for the orthogonal method based on stability considerations. The methods we describe can be adapted to the parallel computation of general orthogonal factorizations, but our discussion is motivated by applications in signal processing using windomed recursive least squares filtering for near real-time computations.

Research sponsored by Applied Mathematical Sciences Research Program, U.S. DOE Ofice of Energy Recearch; by the Nurlear Eagineering Fellowship Program; by the Air Force Office of Scientific Revearch; and by the Nationd Science Fouradation.

* North Carolina State Univerity, Raleigh, NC.

\subsection{2}

\section{THE INFLUENCE OF DISTRIBUTED- MEMORY ARCHITECTURES ON LINEAR ALGEBRA ALGORITHMS}

\author{
C. H. Romine
}

(Abetract of oral presentation presented at the Computer Science Seminer, Gainesville, FL, March 30, 1989)

The effective use of distributed-memory multiprocessors involves many goals, including: maximizing concurrency of operations, balancing computational load, minimizing communication vot ume, and preserving data locality. These goals are seldom complementary, and hence the algorithm designer is usually forced to make trade-off decisions - for example, sacrificing some concurrency for a reduction in communication.

We describe a collection of multiprocessor algorithms on the hypercube for the solution of several standard problems in linear algebra. These algcrithms illustrate the types of trade-offs that are made when trying to optimize overall performance on such machines. General guidelines for efficient distributed-memory computing will be presented.

Research sponsored by the Applied Machematicel Sciences Reaearch Program, U.S. DOE, Office of Energy Research. 


\subsection{3}

\section{ORTHOGONAL FACTORIZATION ON DISTRIBUTED-MEMORY MULTIPROCESSORS}

\author{
C. H. Romine A. Pothen*
}

(Abstract of oral presentation presented at the American Mlathematical Society Coaference, Knourville, TN, March 25, 1988)

We describe the design and implementation of several algorithms for orthogonal factorization on distributed-memory multiprocessors. We consider the effect that the data storage scheme has on the efficiency of orthogonal factorization. In particular, the way chat the computation is organized depends upon the initial distribution of the matrix to the processors. Our results will focus primarily on the hypercube topology, but are applicable to more general interconnection schemes. We investigate the effects of scaling and column pivoting on the parallel implementation. Factors such as the cost of column pivoting, the cost of communication, the effect of loop-unrolling, and the potential for pipelining are examined. These factors are used to model the performance of each of the factorization algorithms. Finally, the inplementation of the orthogonal factorization algorithms on an Intel iPSC is described, along with the results obtained These results are used to verify the models of exsecution time that were derived.

Research sponsored by Applied Mathematical Sciences Research Program, U.S. DOE Oflice of Energy Research.

* Penn State University, University Park. PA.

\section{Sparse Matrix Computations}

\subsection{4}

\section{SOLUTION OF SPARSE POSITIVE DEFINITE SYSTEMS ON A HYPERCUBE}
A. George*
M. T. Heath
J. Liu ${ }^{\dagger}$
E. $\mathbf{N g}$

[Abstract of ORSL/TM-10865. October 1988; also J. Comp. Appl. Hath. 27, 129 (1989)]

The solution of large sparse positive definite systems of equations typically involves four steps: ordering. data structure set-up (symbolic factorization). numerical factorization, and triangular solution. This article describes how these four phases are implemented on a hypercube multiprocessor. The role of elimination trees in the exploitation of sparsity and the identification of parallelism is explained, and pseudo-code algorithms are provided for some of the important algorithms. Numerical experiments run on an Intel iPSC multiprocessor are presented in order to provide some indication of the performance of the various algorithms.

Research sponsored by Applied Mathematical Sriences Rre search Program, L.S. DOE Office of Energy Research, and by Canadian Natural Sciences and Engineering Research Council.

* University of Waterbo, Waterlon, Ontario, Canada.

${ }^{\dagger}$ York University, Downeview, Ontario, Canarda. 
1.35

\section{COMMUNICATION RESULTS FOR PARALLEL SPARSE CHOLESKY FACTORIZATION ON A HYPERCUBE}

\author{
A. George* J. Liu' \\ E. $\mathbf{N g}_{\mathbf{g}}$
}

[Abatract of Perallel Computing 10. 287-293 (1909)]

We consider the problem of reducing data traffic among processor nodes during the parallel factorization of a sparse matrix on a hypercube multiprocessor. A task assignment strategy based on the structure of an elimination tree is presented. This assignment is a imed at achieving load balancing among the processors and also reducing the amcunt of processor-to-processor data communication. An analysis of regular grid problems is presented, providing a bound on communication volume generaled by the new strategy, and showing that the allocation scherne is optimal in the asymptotic sense. Some experimental results on the performance of this scheme are presented.

Research sponsored by Canadian Natural Sciences and Engineering Research Council; by Applied Mathematiol Sciences Research Program. U.S. DOE Office of Enercy Research; and by U.S. Air Force Office of Scientific Research.

"Universily of Waterbo, Waterloo. Ontario, Canada.

†York University, Downsview, Onterio, Canada.

\subsection{6}

\section{PARALLEL SPARSE GAUSSIAN ELIMINATION WITH PARTIAL PIVOTING}

\section{A. George* E. $\mathrm{Ng}$}

(Abetract of ORNL/TM-10866, October 1988; eleo Workshop on Supercomputers and Large-Scale Optimization: Algorithms, Software, and Applications, University of Minnesolen Minneapolis, MN, May 16-18, 1988; Proc. Special Volzme of Anneis of Operations Reseanek, Ben Rowen, Ed., North-Ho!lands (in preas)]

In this article we consiter the factorization of a sparse nonsymmetric mitrix using Gaussian elimination with partial pivoting on a multiprocessor having a globally-shared memory. The patallel algorithm inakes use of a static data structure already developed. Some numerical experiments on a Sequent Balance 8000 are presented to demonstrate the efficiency of the parallel implementation.

Researrh spronesred by Applied Mathernatical Sciences Reweasch Program, li.S. DOE, Office of Energy Rewearch.

"tinivemity of Waterko. Waterlows. Ontaritr, Canada.

\subsection{7}

\section{SOME ASPECTS OF THE SOLUTION OF SPARSE SYMMETRIC POSITIVE DEFINITE SYSTEMS ON HYPERCUBE MULTIPROCESSORS}
A. George*
E. $\mathbf{N g}_{\mathbf{g}}$

(Abstract of paper presented at the Thind Conference on Hypercube Concurrent Computers and Applications, Pace dena, CA. January 19-20, 1988)

The elimination tree of a sparse symmetric positive isfinite matrix $A$ is a tree structure as sociated with the: Cholesky factor $L$ of $A$. This tree structure is a powerful tool in the solution of sparse symmetri: positive definite systems. The elinination tree of $L$ not only describes the stiucture oi $l$. bi:t it is also useful in analyzing the parallelism available in the parallel solution of such a sysiem. In this talk botli the theoretical and practical aspects of elimination trees in the solution of positive definite systems on hypercube multiprocessors are considered.

Reseanch sponsored by Applied Machematical Sciences Re search Program. U.S. DOE Office of Energy Research.

-Univenily of Walerbo, Waterloo, Oncario, Canade.

\section{SOME SHARED MEMORY IS DESIRABLE IN PARALLEL SPARSE MATRIX COMPUTATION}
A. George*
E. $\mathbf{N g}$

[Abetract of ACM Signem Newsletter, 23(2), 9-13 (1988)]

Over the past few years a number of algorithms for solving large sparse systems of equations on distributed-memory multiproceseors have been developed. In this article the autliors point out that the properties of sparse matrix problems generally, along with the characteristics of these parallel algorithms for solving them, lead to inefficient use of memory. An example is presented to show that a relatively small amount of shared memory on an otherwise pure distributed-memory multiprozessor is very desirable when it is being used to execute these parallel algorithons.

Remearth aponanred by Applied Mathematiral Sriencea Re. seasch Program, U.S. DCEE Office of Energy Remearch and by U.S. Air Force Office of Scientific, Researrh.

\footnotetext{
* Vnivenily of Walerkm, Walerlen, Ontario, Cianada.
} 
PARALLEL SOLUTION OF SPARSE LINEAR SYSTEMS

\section{T. Heath}

(Abstract of oral presentation prevented at the University of Marylend, College Park, MD. September 29-30, 1988; at an Applicaxions Group Meeting, University of Dlinois at Urbana-Champaign. Urbana, IL, October 25, 1988; and at the State University, of New York, Buftalo, NY, November 11, 1988)

Various issues in the solution of large sparse systems of linear algebraic equations on parallel computer architectures were discussed. Parallel algorithms for the four major steps in solving a sparse positive definite linear system- ordering, symbolic factorization, numeric factorization, and triangular sclution-were presented. Special emphasis to the role played by elimination trees in exploiting sparsity and identifying potential parallelism, and numerical results for both distributedand shared-memory implementations were given.

Research sponsored by Applied Mathemacical Sciences Research Progran, U.S. DOE Office of Energy Research.

1.40

\section{PARALLEL SOLUTION OF SPARSE LINEAR SYSTEMS}

\section{T. Heath}

(Abetract of oral presencation presented at NASA Ames Research Center, Mofiet Field, CA, March 9, 1989)

We discuss various issues in the solution of large sparse systems of linear algebraic equations on parallel computer architectures. We present parallel algorithms for the four major steps in solving a sparse positive definite linear sys'em: ordering. 9 , mbolic factorization, numeric factorization, and tiiangular solution. We give special emphasis to the role played by elimination trees in exploiting sparsity and identifying potential parallelism. We give numerical sesults for both distributedmenory and shared-memory implementations.

llesearth sponmored by Applied Mathematical Sciencea Re. search Program, li.S. DOE Office of Finergy lesesarch.
1.41

\section{A FAST ALGORITHM FOR REORDERING SPARSE MATRICES FOR PARALLEL FACTORIZATION}

\author{
J. G. Lewis* B. W. Peyton
}

\section{A. Pothen ${ }^{\dagger}$}

[Abatract of ORNL/TM-11040, Jamuary 1989, ato SIAM J. Sti. Stat. Compre. 10, 1146-1173 (19e9)]

Jess and Kees (1982) introduced a method for ordering a sparse symmetric matrix $A$ for efficient parallel factorization. The parallel ordering is computed in two steps. First, the matrix $A$ is ordered by some fill-reducing ordering. Second, a parallel ordering of $A$ is computed from the filled graph that results from factoring $A$ using th- initial fill-reducing ordering. Among all orderings whose fill lies in the filled graph, this parallel ordering achieves the minimum number of parallel steps in the factorization of $\boldsymbol{A}$. Jess and Kees did not specify the implementation details of an algorithm for either step of this scheme. Liu and Mirzaian (1987) designed an algorithm implementing the second step, but it has time and space requirements higher than the cost of computing common fill-reducing orderings.

We present here a new fast algorithm that implements the parallel ordering step by exploiting the clique tree representation of a chordal graph. We succeed in reducing the cost of the parallel ordering step well below that of the fill-reducing step. Our algorithm has time and space complexity linear in the number of compressed subscripts for $L$, i.e., the sum of the sizes of the maximal cliques of the filled graph. Empirically we demonstrate running times nearly identical to Liu's heuristic Composite Rotations algorithm that approximates the minimum number of parallel steps.

Research sponsored by I;.S. Air Force Office of Sciencitir. Research; by National Science Foundacion; and by Applied Mathematical Scienres Research Program. U.S. DOE Office of Energy Research.

*Breing Computer Serviose, Seacle, WA.

${ }^{\dagger}$ Pennaylvania State University, Lniversity Park. PA. 
1.42

\section{ON THE COMPLEXITY OF SOME SPARSE MATRX FACTORIZATIONS}

\section{E. $\mathbf{N}_{\mathbf{B}}$}

(Abatract of oral presentation presented at the American Mathematical Society Conference, Knorville, TN, March 25,1988 )

Let $A$ be an $n \times \cap$ matrix derived from a twodimensional finite element mesh. If the matrix $A$ is symmetric and positive definite, and a nested dissection ordering is used, then the Cholesky factorization of $A$ can be computed using $O\left(\mathrm{n}^{3 / 2}\right)$ arithmetic operations, and the number of nonseros in the Cholesky factor is $O(n \log n)$. In this tall we show that the same complexity bounds can be attained when $A$ is nonsymmetric and indefinite, and either Gaussian elimination with partial piroting or orthogonal factorization is applied.

Research sponsored by Applied Mathematical Sciences Research Program. U.S. DOE Office of Energy Revearch.

\subsection{3}

\section{SOME IDEAS IN THE SOLUTION OF SPARSE LINEAR LEAST SQUARES PROBLEMS}

\section{E. $\mathbf{N g}$}

(Abatract of oral presentation prezented at the Univerits of Tennescee, Knoxville, TN, February 29, 1988 and as Stanford University, Stanford, CA, March 31, 1988)

The problem of solving large sparse linear least squares problems is considered. The solution method is based on orthogonal factorizations. The data structures and an algorithm for computing the orthogonal decomposition will be presented. An attractive feature of the approach is that both the orthogonal transformatinns and the triangular factor are saved. Hence multiple least squares problems which have the same coefficient matrix but different right hand sides can be solved very efficiently. Techniques for handling rank-deficient problems will be described.

Hesearch sponeored by Applied Mathematical Sciences Research Program, U.S. DOE Office of Energy Reacarch.
1.44

\section{SPARSE PARTIAL PIVOTING USING STATIC STORAGE SCHEMES}

\author{
E. $\mathbf{N}_{\mathbf{g}}$
}

(Abatrect of ord preacention preanted at the Interme tiond Matbematio and Stutietical Library. Howton, TX, Juls 7, 19e9)

Consider the problem of solving a general sparse nonsymmetric system of linear equations. The solution process typically involves computing a triangular factorization of the coefficient matrix (for example, using Gaussian elimination with partial pivoting). Existing software packages for computing such a decomposition often make uxe of dynamic storage allocations. Thus, the storage requirements are unpredictable. Furthermore, experience has shown that the overhead in storage and execution times may sometimes be large. In this talk, we describe some of our attempts in inplementing sparse Gaussian elimination with partial pivoting using fixed storage schemes. Numerical experiments comparing the static and dynamic implementations will be provided. Some outstanding problems aceociated with the static implementations will also be discussed.

Rescarch sponeored by Applied Mathematical Sciences Researdh Progrm, U.S. DOE Office of Energy Research.

\subsection{5}

\section{PARALLEL DIRECT SOLUTION OF SPARSE LINEAR SYSTEMS}

$$
\text { 2. } \mathbf{N}_{\mathbf{8}}
$$

[Aberect of ORNL/TM-11045, January 1989, aleo peper presented at a Workehop on Parallel and Vector Compuling. Austin, TX, October 17-18, 1988; Proc. Parallel Strercompating: Methodo, Algorithme, and Applicetions, pp. 157-176, G. F. Carey, Ed., John Wiley (1989)]

In this paper the direct solution of sparse linear systems on multiproceseor systems is consid. ered. Elimination trees are used as a tool for identifying and exploiting parallelism in the parallel numerical factorization of the coefficient matrix. Some open problems are described and results of some numerical experiments are provided.

Rescarch sponeored by the Applied Mathematical Sciences Research Program, U.S. DOE Office of Energy Researsh. 
1.46

\section{A COMPARISON OF SOME METHODS FOR SCLVING SPARSE NONSYMMETRIC LINEAR SYSTEMS}

\section{E. $\mathbf{N}_{\mathbf{g}}$}

(Abatract of oral presentucion presented at the SLAM Symposium on Sparse Matrices, Gleneden Beoch, OR, May 2221, (1969)

In this talk we consider the solution of sparse nonsymmetric and nonsingular systems of linear equations using Gaussian elimination with some form of row and/or column pivoting to ensure numerical stability and to preserve sparsity. The cost of the triangular factorization, in terms of execution time and storage requirement, depends very much on the choice of the data structures and the design of algorithms that manipulate these data structures. In recent years, there has been much research on the design of efficient data structures and algorithms for sparse Gaussian elimination.

Using test problems from the Harwel-Boeing sparse matrix collection, we have performed a comprehensive comparison of various methods for solving sparse nonsymmetric systems. We present our findings in this talk.

Research sponsored by the Applied Mathematical Sciences Research Program, U.S. DOE Office of Energy Research.
1.47

\section{A SCHEME FOR HANDLING RANK DEFICIENCY IN THE SOLUTION OF SPARSE LINEAR LEAST SQUARES PROBLEMS}

\section{E. $\mathbf{N}_{\mathbf{g}}$}

(Abatract of ORNL/TM-10980. November 1988)

Recently we have presented several schemes for computing sparse orthogonal factorizations using static data structures are large enough to store both the orthogonal transformations and upper triangular factor explicitly. Thus, multiple least squares problems with the same observation matrix can be solved easily. However, in order to make use of the static data structures, the orthogonal factorization is computed without column interchanges. In this article we develop an algorithm that makes use of the resulting factorization to solve rank-deficient least squares problems. The techniques used are similar to those employed by Bjorck.

Reseanch sponsored by Applied Mabematical Sciences Research Progran, U.S. DOE Ofice of Energy Research.

\section{Differential Equations}

\subsection{8}

\section{A COLLECTION OF TEST PROBLEMS FOR ORDINARY DIFFERENTIAL EQUATION SOLVERS WHICH HAVE PROVISIONS FOR ROOTFINDING}

\section{S. Thompson}

(Abatract of ACM Trans. on Mothematical Softwere (in prese)!

Several popular ordinary differential equation (ode) solvers contain provisions for locating roots of functions which depend on the solution of the ode. A collection of problems for testing such solvers is described in this report. In addition to sevesal well-known problems, the collection contains other interesting problems which have not previously appeared in the literature.

Rewesurti sponamed by Computins and Telernmmusuice tirne Diviaion.
1.49

EXPLICITLY PARALLEL ALGORITHMS FOR HYPERBOLIC PDES - A PROOF OF PRINCIPLE

\section{P. H. Worley}

(Abatract of paper presented a SIAM Annual Meeting. San Diego, CA, July 17-21, 1989)

We define a class of easily parallelized algorithms, explicilly parallel algorithms or EPAs, and show how to formulate them for specific model problems related to the numerical solution of hyperbolic PDEs in one-space dimension. We then compare their execution time to parallel imple. mentations of good serial algorithms for the same problems. The execution time is estimated analytically an!' measured empirically on an Intel iPSC:/2 lyypereube multiprocessor, demonstrating 
that the EPAs are the better parallel algorithms for both small grain and large grain parallel implementations.

Reseurch sponeored by Applied Mathematical Sciences Research Program, U.S. DOE Ofice of Energy Research.

\subsection{0}

\section{THE EFFECT OF TIME CONSTRAINTS ON SCALED SPEED-UP}

\section{P. H. Worley}

(Abetract of ORNL/TM-11031, January 1969)

Gustafson, Montry, and Benner introduced the concept of scaled speed-up to characterize the capabilities of distributed-memory MIMD multiprocessors. They argued that, for a fixed-size problem, the behavior of the speed-up of an algorithm as a function of the number of processors, the speed-up curve, can be too pessimistic a measure of a multiprocessor architecture. Instead, they measured the speed-up of algorithms when the size of the corresponding problem grew with the number of processors. They referred to the resulting function as the scaled speed-ap carve.

The scaled speed-up curve is a function of how the size of the problem is allowed to grow. We demonstrate that allowing the size of a problem to grow to fill the available memory can produce dramatically different results from allowing the size of a problem to grow subject to satisfying an upper bound on the execution time. In particular, if a constraint on the execution time is enforced, then the scaled speed-up curve is often very similar to the speed-up curve for a fixed-size problem. We show that no more than 50 processors can be used efficiently for some common problems in scientific computation when using the current generation of distributed-memory multiprocessors. For other problems, we show that the scaled speed-up curve indicales that massively parallel computers will be useful even if the execution time is constrained. In all of the cases examined, , reaningful interpretation of the scaled speed-up curve depends on a constraint on the execution time

Research oponsored by Applied Mathematical Sciences Research Pingram. U.S. DOE Office of Energy Rezearrh. Rewarch.
1.51

\section{LIMITS ON PARALLELISM IN THE NUMERICAL SOLUTION OF LINEAR PDES}

\author{
P. H. Worley
}

(Abatract of ORNL/TM-10945, Octuber 1988)

We consider approximating the solution of a linear scalar partial differential equation (PDE) at a single location in its problem domain. In previous work we described a lower bound on the amount of data required to satisfy an error bound for the approximation. Using this bound, we derive a lower bound on the parallel complexity of algorithms that approximate the solution. The lower bound is a linear function of $\log _{2} \varepsilon_{-1}$, where $\varepsilon$ is an upper bound on the error. Thus, the parallel complexity increases as $\varepsilon$ decreases, independent of ihe number of processors, the interconnection topology, and the algorithm used. We also describe how the lower bound changes when the interconnection network or the number of processors is specified.

Recent research has established that it is often possible to use a large number of processors efficiently when calculating the numerical solution of a PDE if the problem is sufficiently large. We argue that increasing the size of such a problem will usually come at the cost of increasing the execution time. We describe two examples verifying this conclusion, an algorithm-independent analysis of an elliptic PDE and an analysis of a specific algorithm for the apprcximation of a hyperbolic PDE.

Research sponeored by Applied Mithematical Sciences Research Program, U.S. DOE Office of Energy Research.

\subsection{2}

\section{LIMITS ON PARALLELISM IN THE NUMERICAL APPROXIMATION OF LINEAR PDES}

\section{P. H. Worley}

(Abutract of oral presentation presented at the Conference on Numerical Solutione of Partial Differentid Equations, Virginia Polytecthic Instituce, Blackburg, VA, September 21 27, 1988 and at the Rewearch in Multiprocesaing Special Intereat (Froup/Numeried Analysis Special Intereat Group Meeting, Anaheim, CA, (Oetober 18, 1988)

Amdahl's Law describes bounds on the efficiency of parallel algorithms for a fixed size problem as a function of the number of processers. It 
has recently been argued that this is not a fair measure of the capabilities of a multiprocessor architecture. Instead. it has been proposed that the size of the problem be allowed to increase along with the number of processors, subject to the condition that the execution time does not also increase. If the problem to be solved is a linear scalar PDE, we show that is not generally possible to increase the size of the problem without increasing the execution time. The argument easily generalizes to systems of equations, and it is reasonable to assume that most nonlinear PDEs will behave in the same way.

Researth sponsored by Applied Mathematical Sciences Research Program. U.S. DOE Office of Energy Research.

\subsection{3}

\section{INEORMATION REQUIREMENTS AND THE IMPLICATIONS FOR Parallel COMPUTATION}

\section{P. H. Worley}

(Abutract of Ph.D. Thesis in Computer Science, Stanford University, Stanford, CA, 1987)

The best serial algorithms for numerically approximating the solution of a linear partial differential equation (PDE) exploit knowledge of the solutiun oper ator. This dissert ation describes how the solution operator also influences the behavior of parallel algorithms.
Approximating the solution at a single location in the problem domain is considered. We derive a lower bound on the error in the approximation that is a function of the amount of data used and the smoothness of the data functions. From this we derive a lower bound on the parallel complexity of algorithms that approximate the solution. The lower bound on the parallel complexity is a logarithmic function of the reciprocal of an upper bound on the error. Thus, the parallel complexity increases as the error bound decreases, independent of the number of processors used. We also construet an algorithm whose parallel complexitv is of this form, proving that the form of the bound is the best possible.

The execution time of parallel algorithms is a function of both the communication costs and the parallel complexity. We describe bounds on the communication costs of parallel algorithms that are functions of the distance between collaborating processors. If the interconnection network of a given multiprocessor is a d-dimensional grid, then we prove that the execution time for algorithms that approximate the solution is bounded from below by a negative power of the error bound. Thus, if the upper bound on the error is small, then the communication costs are the dominant constraints on the optimal performance.

Research sponsored by Applied Mashematical Sciences Research Program, U.S. DOE Ofice of Energy Research.

\section{MATHEMATICS}

\section{Phase Change Problems}

\subsection{4}

\section{EFFECT OF A VOID ON CYCLIC MELTING AND FREEZING OF AN ENCAPSULATED PCM}

\section{Alexiades}

(Abatract of book chapter in Mulliphase Flow Heat and Mass Tranofer, ASME HTD-Vol. 109, Pp. 17-20, R. K. Shak, Eds., American Soriety of Mechaniral Engineers, New York (1080); alwo oral prcsentation presented at the 1989 National Heat Trandf: Conference, Philadelphia, PA, August $6.9,1989$ )

Melting and solidification processes are generally accompanied by changes in density. These induce movement in the PCM and complicate the phasm-change process considerably. In latent heat enregy storage systems being considered for the manned space station, solar energy will be used t.o inelt an encapsulated PC:N during the light part. of the ortit, and the heat. will loe withdrawn dur- ing darkness to provide continuous power to an engine. One of the promising high temperature PCMs under study is germanium encapsulated in graphite. Liquid germanium is denser than solid, so on melting the reduction in volume results in the formation of a void (vapor bubble) somewhere in the container. Since the conductivity of vapor is much smaller than that of the other phases, the void acts as a thermal barrier, altering the thermal field and thus the phase-change process itself. During freezing, the solid expands and the void diminishes. We are primarily concerned with how the void affects the cyclic charge-discharge procoss.

Results of computational experinients are presented for the case of imposed flux houndary conditions over several cliarge-dlischarge cyeles.

Research aponasored by Applied Mathematical Srieouren Re.

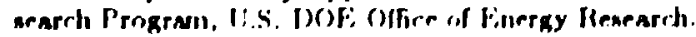


1.55

\section{SHRINKAGE EFFECTS ON SOLIDIFICATION}

\author{
V. Alexiades
}

(Abatract of paper presented at the 2nd Insernational Conference on Trende in Welding Research, Gatlisburs. TN, May 11-18: 69)

When solid is denser than liquid, the volume reduction upon solidification of an encapsulated material creates a void (vapor bubble), making the phase-change process considerably nore complicated (since mass movement is induced). The void acts as a barrier to heat transfer (due to the very small conductivity of vapor), and we are interested on how this affects the solidification process. We construct a rather simple thermal model for solidification that accounts for the presence of growth of a void inside the cavity. Results of numerical simulations are presented as examples.

Reseurch sponsored by Applied Mathematical Sciences Research Program. U.S. DOE Ofice of Energy Research.

\subsection{6}

\section{EQUILIBRIUM SIZE OF A SOLID NUCLEUS FORMING IN SUPERCOOLED LIQUID}

\section{Alexiades}

[Abstract of a chapter in Free Bosndary Problems, K.-H. Hofimann and J. Sprekels, Eda., Longman (in preas)]

Given the initial temperature, pressure and volume of a supercooled liquid in which nucleation is assumed to have occurred, the final equilibrium size and state of the solid-interface-liquid system are determined on the basis of energy conservation and thermodynamic equilibrium, taking into account surface area, curvature and pressure effects.

Research sponeored by Applied Mathematical Sciences Research Program, U.S. DOE Office of Energy Research; by Microgravity Science and Applications Division of NASA, Marshall Space Flight Center, and by Science Alliance, Ssace of Tenneswer Center of Excellence through the University of Tennessee, Knoxville, TN.
1.57

\section{A WEAK FORMULATION FOR PHASE-CHANGE PROBLEMS WITH BULK MOVEMENT DUE TO UNEQUAL DENSITIES}

\author{
V. Alexiades \\ J. B. Drake
}

(Abotract of oral presentation to be given at a meeting on Free Boundary Problems: Theory and Applicatione, Center for Research Mathemation, Montreal, Canado, Jume 13-22. ISSO)

When the densities of solid and liquid are different, a change of phase induces a volume change. forcing movement of the bulk phases. Conservation of total energy acroes the interface leads to a modified Stefan Condition which contains a term cubic in the interfacial speed. We present a weak formulation for the conservation of mass, momentum and energy and study numerically a l-phase, 1-dimensional example.

Research pponsored by Applied Mathematical Sciences Research Progrum, U.S. DOE Office of Energy Rewenth.

1.58

\section{A CONVECTIVE FLOW IN THE PRESENCE OF MELTING}
J. B. Drake
V. Alexiades

(Abatract of ora' presentation to be given at a meeting on Free Boundary Problems: Theory and Application, Cente for Research Mathematica, Montreal, Canada, June 13-22, 1990)

A numerical method for solving heat conduction and fluid flow problems that involve phase changes is described. The complications arising from materials with different liquid and solid densities are discussed and approximations developed for Marangoni stress, curvature dependent pressure jumps and void movement. Applications to both $0-g$ and $1-g$ environments are discussed, and numerical results of a 3-D simulation involving void movement are presented.

Rewearch sponeored by Applied Mathematical Sciences Rerearch Program, U.S. DOE Office of Energy Reararch. 
1.59

\section{CHANGING DOMAINS AND A SOLIDIFICATION PROBLEM WITH VOID}

\section{S. M. Lenhart \\ D. G. Wilson}

(Abstract of Quarterty of Apl. Math. (in preas); also ord presentation ptesented at the Southesetem-Atlantic Regional Conference on Difierential Equations, University of Georgia, Athens, GA, November 4-5, 1968)

We consider Laplace's equation posed in a region with a hole. The exact location and shape of this hole is not specified a priori. Dirichlet data are specified on the exterior boundary of the region and a Robin boundary condition holds on the edge of the hole. The prohlem represents a mathematical model of an equilibrium thermal state following a phase change process in which a denser solid has frozen out of its less dense liquid with the formation of a void. Since the solid is more dense than the liquid, the combined volume of frozen solid and remaining liquid is less then that of the initial liquid (which is assumed to have filled a finite container) and a vapor bubble or void results. Energy balance considerations impose two auxiliary conditions to be satisfied. We establish the existerice of admissible holes, i.e. voids, for which a solution to the problem exists and, in addition, the existence of an admissible void with minimal circumference.

Research sponscred by Applied Mathematical Sciences Research Program, U.S. DOE Office of Energy Research.

\subsection{0}

\section{PARALLEL SOLUTIONS OF A 2-D PHASE CHANGE PROBLEM ON A HYPERCUBE}

\section{H. N. Narang* J. B. Drake}

(Abatract of ORNL-6504, April 1989; aleo paper presented at the ACM Southeast Region 27th Annual Confermen, Atlanta, GA, April 5, 1989; Proc. pp. 650-656 (1986)

This report documents the model, algorithms and rexults for a heat transfer problem with phase change simulated numrrically on a hyperrube parallel computer. The temperature and enthalpy distribution in a rectangular domain with first kind boundary conditions (temperature proseribed) is found as a function of time. The model involves a non-linear partial differnntial equation witi a constitutive relation between enthajpy and temperature. The solution to the system is obtained by finite difference approximation with both explicit and implicit methods. In the implicit case, a successive over relaxation technique with red, black ordering is inplemented. The efficiency of the explicit method is compared with that of the implicit method in a multiprocessor computing environment.

The design, development, and implementation of algorithms targeted INTEL's iPSC/ 2 ypercube involving up to 64 processors. The computational workload was uniformly divided among processors as far as possible by dividing the finite difference grid into several strips and assigning each strip to a processor.

The results, depicted in terms of tables, show the effect of relaxation parameter, and stepsize on the efficiency of the parallel algorithms. This is contrasted with the sequential algorithms.

Research sponsored by Applied Mathematical Sciences Re search Program, U.S. DOE Ofice of Energy Research.

"Tuskegee University, Tuskegee, AL.

1.61

\section{SURVEY OF THE LITERATURE ON FRACTALS WITH POSSIBLE APPLICATIONS TO PHASE CHANGE PROCESSES}
L. A. Renker"
D. G. Wilson

(Abatract of ORNL/TM-10778, June 1988)

This report is a partial collection of the literature on fractals and how it applies to phase change processes, specifically solidification. Fractals can be used to model the dendrites that are formed at the interface between a liquid and a solid with restrictions hecause either dendrites or nondendritic fractals may be formed by diffusion limited solidification, depending on the presence of crystalline anisotropy. This anisotropy causes the tips of the structure being formed to remain stable, leading to the formation of a dendrite, rather than splitting to form a non-dendritic fractal. An additional type of structure is found when electrodeposition is used to model solidification. The form stion of this dense radial structure depends on the level of the voltage and the electrolyte concentration. These structures are formed at intermediale values where the cromoner from non-dendritir 
fractals in dendrites occurs. Non-dendritic fractals are $\boldsymbol{i}$ : :med at the lower ralues and dendrites are formed at the higher values. A comprehensive bibliography is included with this report.

Research sponsored by Applied Mathematical Sciences Research Program, U.S. DOE Office of Energy Research.

"Baldwin-Wallace College, Berea, OH.

1.62

\section{SIMDLA: A FORTRAN PROGRAM SIMULATING DIFFUSION-LIMITED AGGREGATION}

\author{
L. A. Renker* \\ D. G. Wilson
}

(Abatract of ORNL/TM-10876, Auguat 1988)

This report documents a FORTRAN program designed to simulate the diffusion-limited aggregation of particles. Particles are released one at a time from a growing internal boundary on a grid of possible sites and allowed to walk randomly until either they stick to the growing cluster or walk off the grid. The user may specify the grid dimension, the number of particles to be added to the cluster, the location and size of the seed cluster, and the possible directions (up to eight) in which particles are allowed to move. The output is a list of the occupied cells after all particles have been added to the cluster. A graphics post processor creates pictures from this list.

Research uponsored by Applied Mathematical Sciences Research Program, U.S. DOE Office of Energy Research.

* Ealdwin-Wallace College, Berea, OH.

1.63

\section{ITERATIVE SOLUTION OF A NONLINEAR SYSTEM ARISING IN PHASE CHANGE PROBLEMS}

\author{
M. A. Williams" \\ D. G. Wilson
}

[Abstract of SlAM J. Sci. Stat. Compzt. (in prese)]

We have investigaled the relative morits of two iterative procedures for solving the mildly nonlinear equations arising from an implicit discretization of a three-dimensional Stefan problem. The two procedures are successive uverrelaxation (SOR) and a modified preconditioned conjugate gradient method (MPCG) developed especially for this problem. The algorithms were implemented on a Cray X-MP and vectorization was a major consideration. Although MPCG converged in fewer iterations, the SOR iterations were shorter and faster. Thus, SOR required less Cray X-MP execution time. Finally, we found SOR to be more robust than MPCG.

Research sponsored by Applied Mathematical Sciences Research Program, U.S. DOE Office of Energy Research.

"Lehigh University, Bethlehem, PA.

\subsection{4}

\section{IMPLICIT FINITE DIFFERENCE SCHEMES FOR PHASE CHANGE PROBLEMS}

\section{G. Wilson}

(Abetract of oral presentation presented at the 3rd International Seminar on Free Boundary Problems and Their Applications, Univeraidad Nacional de Rosario, Rowario, Argentina, October 11-15, 1988)

C. M. Elliot has proposed an implicit discretization of the enthalpy formulation of phase change problems. The resulting mildly nonlinear, implicit equations must be solved for enthalpy and temperature fields simultaneously. We have used this scheme to model three-dimensional phase change processes in Cartesian and cylindrical polar coordinates including one that involved convection in the melt. In this talk we discuss our experience witl this scheme applied to these problems. We have used Crank-Nicholson type finite difference approximations to the partial differential equations and a corresponding discrete version of the enthalpy temperature relation, and we have used a successive overrelaxation iteration scheme with red/hlack ordering to solve the nonlinear equations. The algorithms have been vectorized for rapid execution on the ('r.y X-MP supercomputer, and if time permits, we will discuss techniques used to do this.

Research sponsored by Applied Matliematical Sriences Rewearch Program, U.S. DOF Offire of Finergy Research. 
1.65

\section{CYCLIC MELTING AND FREEZING WITH VARIABLE DENSITY}

\author{
D. G. Wilson
}

(Abstract of oral presentation presented at the 3rd International Seminar on Free Boundary Problems and Their Applications, Univerided Necional de Rosario, Rosario, Argentina, October 11-15, 1968)

We discuss the mathematical model and its discrete Sormulation (but not the computational algorithms) used to model a high temperature phase change process in a hollow metal canister. The solid phase change material is more dense than the liquid, and the change in density on freezing creates a void and also induces convection in the melt. The complete problem includes cyclic melting and refreezing, fluid flow, and void formation and movement, as well as conductive and convective heat transfer in a three-dimensional setting. We discuss only the equations used to model the phase change process. These consist of a mildly nonlinear partial differential equation (for energy redistribution), coupled with a constitutive relation for energy and temperature. This constitutes an enthalpy formulation of the phase chan ge problem. The partial differential equation contains a convective heat transfer term whose inclusion is new for phase change problems. The nor.linear discrete equations consist of a Crank-Nicholson type finite difference approximation to the partial differential equation and a discrete version of the enthalpy temperature relation.

Research sponsored by Applied Mathematical Sciences Research Prozram, U.S. DOE Office of Energy Research.

\subsection{6}

\section{AN IMPLICIT, FINITE DIFFERENCE, ENTHALPY SCHEME FOR PHASE CHANGE PROBLEMS}

\author{
D. G. Wilson
}

(Abatram of oral presentation presented at the Southeast Allant ir. Sertion of SIAM Meeting, liniversity of Tennesase. Knoxville, TN, March 17-18, 1989)

We define the enthalpy formulation of phase change problems, prosent a one-dimensional example of an implicit finite difference discretization of the formulation, and show how to solve the resulting mildly nonlinear equations for the enthalpy and temperature fields simultaneously using a "selective SOR ${ }^{n}$ iteration recommended by C. M. Elliot.

Research sponsored by Applied Mathematical Sciences Re search Program, U.S. DOE Office of Energy Rucarch.

1.67

\section{CHANGING DOMAINS AND SOLIDIFICATION PROBLEMS WITH VOIDS}

\section{G. Wilson}

(Abatract of oral presentation presented at the Inutitute for Mathematio and Its Applications, Univerxity of Minnesoen, Minnespolis, MN, February 19, 1988)

For many years solidification processes have been modeled with the convenient assumption that the densities of the solid and liquid phases are equal. Underlying this assumption is the fond hope that even if there is a density change on freezing, its effect is negligible. Recently, we at ORNL have been confronted with problems in which a substantial density change accompanies freezing. While working with these problems we have reconsidered the validity of the assumption that small density changes are ignorable. We have come to the realization that even when the density change on freezing is small, its effect may not be ignorable. We are still struggling with the consequences of this realization. In this talk we present some of the problems that have brought us to reconsider the assumption, some consequences of abandoning it and some results obtained for problems posed without it.

Research sponsored by Applied Mathematical Sciences Re search Program, U.S. DOE Office of Energy Research. 
1.68

\section{AN IMPLICIT, FINITE \\ DIFFERENCE ENTHALPY \\ SCHEME APPLIED TO A \\ MULTIDIMENSIONAL CYCLIC \\ MELTING AND FREEZING \\ PROBLEM WITH VARIABLE DENSITY}

\author{
D. G. Wilson J. B. Drake \\ R. E. Flanery
}

(Abstract of oral presentation presented at a Colloquium of the Materials Department of the Argentine Atomic Energy Commission, Buenas Aires, Argentina, October 20, 1988)

We define the enthalpy formulation of phase change problems, give one-dimensional examples of explicit and implicit finite difference discretizations of the formulation, and show how to solve the mildly nonlinear equations of the implicit formulation for the enthalpy and temperature fields simultaneously. We then discuss the use of these methods to model a multidimensional cyclic melting and freezing problem, namely a high temperature phase change process occurring in a hollow metal canister. The solid phase change material is more dense than the liquid, and the change in density on freezing creates a void and also induces convection in the melt.

Research sponsored by Applied Mathematical Sciences Research Program, U.S. DOE Office of Energy Research.

\subsection{9}

\section{PHASE CHANGE PROBLEMS WITH A VOID}

\author{
D. G. Wilson J. B. Drake \\ R. E. Flanery
}

[Abatract of chapter in Mathemetics end Industrial Prob. lemo. pp. 97-99, A. Friedman, Ed, Springer-Verlag, New York (1988)]

It is intended that the manned space station satisfy a considerable portion of its power requirements with solar energy. The station will orbit the earth in about ninety minutes and spend about two thirds of each orbit in sunlight and about one third in the earth's shadow. It will be necessary to store thermal energy during the station's exposure to the sun and to retrieve it during the transit through the earth's shadow. The thermal energy storage system considered consists of a solar collector lined with a hundred or more small metal canisters filled with a high temperature phase change material (PCM), lithium fluoride salt. The problem that we have addressed, whose solution will contribute to a stress analysis of the canistere and a prediction of the performance of the storage syetem, is the modeling of the thermal and fuid flows in a representative canister. There will be fluid flow in the liquid PCM, even in a microgravity environment, because there is a significant difference in density between solid and liquid lithium fluoride. This causes the formation of one or more voids on freezing and, of course, the disappearance of voids on remelting. $A$ heat transfer fluid, an inert gas such as helium or neon, circulates through pipes that pass through the metal canisters and carries heat aw ay to turbines, generators, etc. The continual melting and refreezing of the PCM smears out the delivery of the solar energy to the transfer fluid und hence to the heat engines beyond.

Research sponsored by the Applied Mathemntical Scienoss Research Program, U.S. DOE Office of Energy Resenrth.

\subsection{0}

\section{SUPERCOMPUTER SIMULATION OF A 3-D MELTING/FREEZING PROCESS}
D. G. Wilson
R. E. Flanery

(Abetract of oral presentation presented af the Air Force Ofice of Sciertific Research, Wachiagton, DC, March 1 , 1989)

We discuss the mathematical and computational model used to simulate a high temperature phase change process in a hollow metal canister that is an integral component of a proposed thermal energy storage system for the manned space station. The solid phase change material is more dense than the liquid, and the change in density of freezing creates a coid and also induces convection in the melt. The complete problem includes cyclic melting and refreezing, fluid flow, and void formation and movement, as well as conductive and convective heat transfer in a threedimensional setting. We discuss in detail only the simulation of the phase change process. The governing equations are a mildly nonlinear partial differential equation (for energy redistribution), coupled with a constitutive relation between energy 
and temperature. The partial differential equation contains a convective heat transfer term whose inclusion is new for phase change problems. The nonlinear discrete equations consist of a CrankNicholson type finite difterence apfroximation to the partial differential equation and a discrete version of the energy/temperature relation. The implicit difference equations and the constitutive re lation are solved simultaneously at each time slep. A successive overrelaxation iteration scheme with red/bleck ordering is used to solve the nonlinear equations. The algorithms have been vectorized for rapid execution on the Cray X-MP supercomputer, and we discuss techniques usr.d to do this.

Rescarch sponsored by Applied Mathe matical Sciences Research Program, U.S. DOE Office of binergy Research.

\subsection{1}

\section{MODELING CYCLIC MELTING AND REFREEZING IN A HOLLOW METAL CYLINDER}

\author{
D. G. Wilson \\ R. E. Flanery
}

(Abstract of ORNI-6497, September 1988)

This report documents the mathematical model and computational algoritlims used in a pair of computer programs that do energy redis- tribution calculations as part of a comprehensive simulation for thermal and structural analyses of one component of a thermal energy storage system for the manned space station. The complete problem includes cyclic melting and refreezing. fluid flow, and void formation and movement, as well as conductive and convective heat transfer in a three-dimensional setting. The problem is posed in a hollow, metal canister filled with a high temperature phase change material. The heat transfer equations discussed here consist of a pair of partial differential equations for energy transfer (one linear the otlier mildly nonlinear), coupled with a constitutive relation for energy and temperature. This constitutes a weak, "enthalpy" formulation of the phase change problem. The partial differential equations are approximated by a system of coupled Crank Nicholson-type finite difference equations. These nonlinear, implicit equations are solved for enthalpy (energy content) and temperature fields simult,uneously. A successive overrelaxation iteration scheme with red/black ordering is used to solve the nonlinear difference equations. The algorithms have been vectorized for rapid execution on the Cray X-MIP supercomputer and techniques used to do this are discussed.

Researh sponsored by NASA Advanced Solar Dynamics Program and Applied Mathematical Sciences Research Program, U.S. DOE Office of Energy Research.

\section{Applied Mathematics}

\subsection{2}

\section{PARALLEL IMPLEMENTATION OF THE BOUNDARY ELEMENT METHOD ON THE IPSC2 HYPERCUBE}

\section{J. B. Drake L. J. Gray}

(Almtrant of paper preaented at the Second International Conference on Vertor and Parallel Computing: Isoues in A pplied Hesearth and Develespment, Tromso, Norway, June 6. 10, 1088)

In this papere, the issurs assonciated with the parallel implesurutation of the boundary elersirnt inertherl (BF:Il) on a hypereculoe will be addressed. The persgrain for solving the there-dirmensirnal l,aplare cepuation is sperifieally arlapeled for plocterplating sirmulations; this ap-

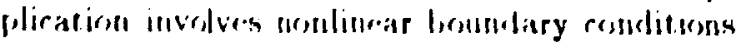

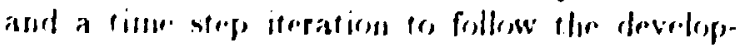

ment of the plated surface. As a consequence, the coefficient matrices of the potential and normal derivative values are formed coparately and stored. A step of the aljurithm is thus required to $c$ mbine and rearrange the system of equations into the standard form $A . l=B$. The matrix $A$ is dense and non-symmetric and recent advances in the solution of surl systems on a hypercube are employed. Fstimates of the arithmetic complexity at each step of the algorithm and a model for the communication ecosts ar.: used to study the parallel performatiere of the BE.M: ciming results are presenterl for all iumplementation on an Intel

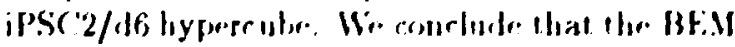
is partirularly well suited for parallel solution and

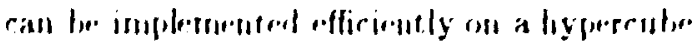

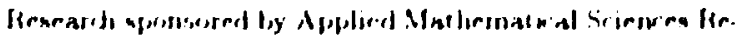

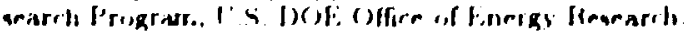




\section{MHD CALCULATIONS ON A HYPERCUBE MULTIPROCESSOR}

\author{
J. B. Drake W. F. Lawkins \\ B. Carreras" H. R. Hicks" \\ V. Lynch".
}

(Abstract of oral presentation presented at the Department of Physics, Eindhoven University of Technology, Eindhoven, The Netherlands, June 2, 1988 and at the Nationd Institule of Plasme Physics, Utrech. The Netherlands, June 3, 1988)

As part of an exploratory study of the suitability of hypercube multiprocessors for scientific computations, the nonlinear MHD code RSF was adapted for use on the iPSC hypercube. This report presents the numerical algorithm of RSF and the techniques used to obtain parallelism without sacrificing the numerical properties of the serial algorithm. A parallel method for solving multiple tridiagonal systems was developed as part of the algorithm. Timing results are presented for a sample problem.

Research sponsored by Applied Mathematical Sciences Research Program, U.S. DOE Office of Energy Research.

"Fusion Energy Division.

\subsection{4}

\section{A CAREER AS A WORKING MATHEMATICIAN - WHAT IT'S LIKE TO DO IT FOR REAL}

\section{R. E. Flanery}

(Abetract of oral presentation preseaced at the Annua Banquel of Youngarown State Chapler of Pi Mu Epsilon, Youngatown Stale Univensily, Youngrown, OH, May 19, 1989)

A career as an applied mathematician requires more than a degree in mathematics. Modeling physical processes requires a working knowt edge of an application area such as heat transfer or fluid flow. To simulate the pliysical process on a computer we first create a physical model approximating the actual physical process. This model is described using mathematical equations, and algoritlıms to solve these equations are then developed. This leads to a computar code for solving the algorithms. All of these stages can depend to some degree on the type of computer which will be used for the simulation. In most cases a 'realistic' mathematical model requires a powerful computer. Supercomputers such as the CRAY XMP, CRAY-2 or iPSC hypercube are required for very complex models. Therefore an applied mathematician must also have a good background in computer science.

In addition to the above topics I will discuss how being a mathematician at ORNL is different than being a mathematician at a university or in industry, some of the projects I have been involved with, and what I plan to be doing in the future.

Research sponsored by the Applied Machematical Sciences Research Progrem, U.S. DOE Office of Energy Research.

\subsection{5}

\section{MAGNETICALLY SUSPENDED CENTRIFUGE FOR STUDIES \\ OF NONLINEAR PHENOMENA IN STRATIFIED FLUIDS: DEVELOPMENTAL ASPECTS}
G. T. Gillies"
M. A. Lawson*
R. C. Ritter
W. F. Lawkins
S. Thompson ${ }^{\dagger}$
W. K. Sartory ${ }^{\ddagger}$

[Abatract of Rev. Sei. Instram. 60(10), 3293 (1989)]

Design studies of a novel magnetically suspended centrifuge suggest that such a device would be useful for investigating the nonlinear dynamics of interfacial waves in a rotating two-phase liquid. In its final form, the apparatus would use magnetic bearings, a directly-driven rotor, precision temperature control, and vibration isolation. The mechanical noise in the magnetic suspension coil of a preliminary apparat us was measured to be $3 \mu \mathrm{m}$ rms in a $5 \mathrm{kHz}$ bandwideh. However, these vibrations were attenuated by an active differentiator to the point where there was no observable oscillation in a $183 \mathrm{gram}$ suspended test mass.

Research sponenred by U.S. Department of Energy.

"Univensily of Virginia, Charlottesville. VA.

'Raslford Univernity, Radford, VA.

Enginearing Teelunroleggy Divinion. 


\subsection{6}

\section{BOUNDARY ELEMENT METHOD FOR REGIONS WITH CRACKS}

\section{J. Gray}

(Abatract of oral presentation presented at the Southeast Atlantic Section of SIAM Meeting, University of Tennessee. Knoxville. TN, March 17-18, 1989)

The standard boundary element technique for a region with a crack is to decompose the problem into two (or more) subregions without cracks. This is accomplished by adding internal surfaces connecting the crack to the outer boundary. These extra surfaces can substantially increase the cost (both human and computer) of performing the calculation. In addition, modelirg problems with many small cracks (e.g., concrete) becomes virtually impossible.

A new boundary element approach to cracked regions has been developed. This method avoids internal surfaces by utilizing a hypersingular ( $t$ wo derivatives of the Green's function) integral equation on the crack surface. The singular integrals are shown to have a sensible value when treated as a limit from the interior. For evaluating these integrals however, the functional approximations on the crack surface must be differentiable; an algorith $m$ for accomplishing this task will be discussed.

Computational results for two applications involving the three-dimensional Laplace equation will be presented. The first problem concerns the heat transfer between two contacting solids, and the second application is the modeling of an electroplating cell.

Research sponsored by Applied Mathematical Sciences Resez rch Program, U.S. DOE Office of Energy Research.

\subsection{7}

\section{EVALUATION OF HYPERSINGULAR INTEGRALS IN THE BOUNDARY ELEMENT METHOD}

\section{J. Gray}

[Atsetract of Computere and Mathematics with Applira. lions (in press)]

A recently developed boundary element technique for analyzing regions with cracks requires the consideration of hypersingular integrals. Although these int.grals can be evaluated analyti- cally, they require that the functional representation at collocation poirts on the crack surface be differentiable. An algorithm which accomplishes this task for three-dimensional problems utilizing standard elements, is presented herein. The entire neighborhood of a singular point is used to define the polynomial approximation, and the coefficients are determined by means of the generalized inverse of a rectangular matrix.

Research sponsored by Applied Mathematical Sciences Research Program. U.S. DOE Office of Energy Research.

\subsection{8 \\ NUMERICAL EXPERIMENTS WITH A BOUNDARY ELEMENT TECHNIQUE FOR CORNERS}

\section{J. Gray}

[Abstract of paper presented at the Eleventh International Conference on Boundary Element Methods, Cambridge, MA, August 29-31, 1989, Proc. Advences in Bosnders Elements, Vol. 1, pp. 243-251, C. A. Brebbia and J. J. Connor, Eds., Computational Mechanica Publicutions, Boston, MA (September 1989)]

A method for handling regions with corners or discontinuous boundary data has been recently pruposed by Mitra and Ingber. Using double nodes to represent the corner and an external collocation point to generate the necessary additional equation, this paper reported (for the most part successful) test results on a variety of twodimensiona! problems. In this paper, an exactly solvable Laplace equation problem, posed in a region with reentrant and non-reentrant edges, has been employed to study the behavior of this algorithm in three dimensions. As boundary surface edges give rise to multiple corner points, this problem provides a challenging test for the proposed algorithm. Experiments using several sets of external collocation points indicate that this technique is not a reliable procedure in three dimensions, yielding answers that are sen itive to the location of external nodes.

Research sponsored by the Applied Mathematical Sciences Research Program, U.S. DOE Office of Fnergy Rescarch. 
1.79

\section{BOUNDARY ELEMENT METHOD FOR REGIONS WITH THIN INTERNAL CAVITIES}

\author{
L. J. Gray
}

[Abstract of Engikeering Anclysis (in press)]

A new boundary element technique for treating thin cavities is presented. This method treats the cavity as an infinitely thin slit, but does not utilize fictitious internal boundaries to subdivide the domain. A non-singular system of equations is obtained by constructing two equations for each node on the boundary of the slit: the standard boundary element equation and the normal derivative of this equation. Although derived for Laplace's equation (in three dimensions) and tested on electroplating problems, it is expected that the method can be applied in other areas, most notably crack problems in fracture mechanies.

Research sponsored by Applied Mechematical Sciences Research Program, U.S. DOE Ofice of Health and Environmental Research; by Y-12 Development Division; and by IBMt Bergen Scienkific Centre, Norway.

\subsection{0}

\section{BOUNDARY ELEMENT METHOD FOR CONTACT HEAT TRANSFER}

\author{
L. J. Gray A. L. Askew"
}

G. E. Giles ${ }^{\dagger}$

(Abstract of paper presented at the European Boundary Element Meeting. Bruscels. Belgium, May 8-10, 1988; Proc. pp. 3.21-3.36, J. L. Migeot, Ed., Univenile-Libre de Bruxelles (1980)]

The (steady state) heat flow between two contacting solids is investigated utilizing an efficient new boundary eleinent methof for treating thin cavities. A significant advantage of this approach is that a (multidomain) decomposition of the region is avoided and the contart region remains an interior surface. Consequently, the discontinuous flux across the contact/mon-contact boundary is not solved for direcily, and no special provisions for the singularity are required. These fluxes can be accurately rotuained after the houndary integral equation lias been solved and all boundary values are known. Calculations for a circular contact region are presenters, and the results are compared against a known analytir solution. Since previous work on this method has only considered interior cavities and the contact gap is a 'surface crack,' the treatment of surface cracks will also be described.

Research sponsored by the Y-12 Development Division of Martin Marietta Enery Syatems, Lnc.

"Brown University, Providence, RI.

tComputing and Telecommunications Divieion.

1.81

\section{APPLICATION OF THE THIN CAVITY METHOD TO SHIELD CALCULATIONS IN ELECTROPLATING}

\author{
L. J. Gray G. E. Giles*
}

[Abatract of paper preanted of the 10h Boundary Element Meeting. Souchamplou, Uniled Kingdom, September 6-8, 1988; Proc. Boundery Elements $X$ Computetional Mechenics, Southampton, Endand (I988)]

In a computational model of an electroplating tank, a thin shield can be conveniently approximated as a zero-thickness 'crack'. A newly developed boundary element method for treating cracks is especially advantageous for treating complicated three-dimensional geometries. such as plating cell configurations. In particular, nonsmooth shields, i.e., wherein a normal direction is not everywhere defined on the surface, can occur in practical plating applications. This paper will describe a technique for handling non-smooth shields, and calculations for an actual plating configuration were presented.

Research sponsored by the Y-12 Development Divition of Martin Marielts Energy Systems, Inc.

- Computing and Telecommunications Diviaion.

\subsection{2}

\section{ELECTROPLATING SIMULATION}
L. J. Gray
G. E. Giles"
J. Bullock, IV ${ }^{\dagger}$
P. McKenzie ${ }^{\dagger}$

(Abstract of paper prewented at the European Conferenoe on Mathematic in Industry, Glengow, Scotland, Auguat 28-31, 1989)

A simulation of a three dimensional electroplating process whicli includes a model of the heat flow generated during the plating is presented. The stearly state temperature distribution in the cell is coupled to the electric field through the non- 
linear polarization (overvoltage) boundary conditions and the temperature dependence of the electrolyte conductivity. As the conductivity directly influences the deposition rate, variations due to temperature can have an impact on the shape of the plated part. The thermal and electrostatic problems are simultaneously solved using the boundary element method, and a simple iterative scheme is employed to satisfy the boundary conditions. A newly developed boundary element procedure for crack geometries is used to cope with the thin shielding present in the cell. Utilizing this technique, a computationally expensive and cumbersome multidomain decomposition of the region is avoided.

Research sponsored by the Applied Mathematical Sciences Research Program, U.S. DOE Office of Energy Research.

- Computing and Telecommunications Division

Y-12 Development Division.

\subsection{3}

\section{BOUNDARY ELEMENT METHOD FOR REGIONS WITH THIN INTERNAL CAVITIES. II}

\section{J. Gray G. E. Giles" M. W. Wendel}

[Abatrace of Eagineering Amalyois (in prea)]

The basic framework of a new boundary element method for the analysis of segions with cracks has been described in a previous paper. This technique is completely general and, unlike the multidomain approach, does not utilize fictitious internal boundaries to subdivide the domain. By means of example three-dimensional electroplating calculations, it will be shown that this method is a practical algorithm. In particular, these calculations will demonstrate that specialized Green's functions for symmetric regions can be incorporated into the method, and that porous cavities are easily handled.

Rewenth sponared by Computing and Telecommunice tions Division.

- Cromputing and Telecommunications Division.
1.84

\section{HYPERSINGULAR INTEGRALS IN BOUNDARY ELEMENT FRACTURE ANALYSIS}

\author{
L. J. Gray L. F. Martha*
}

A. R. Ingrafiea* [Abatract of International Josmal for Nomerical Methods
in Exgincering (in press)]

A new general purpose boundary element method for domains with cracks has been recently developed. This technique avoids the use of a multidomain decomposition by including an additional integral equation expressing the boundary condition on the crack. The principal requirement of this technique is the analytic determination of certain hypersi.ugular integrals of the Green's function which arise from this equation. In order to establish the applicability of this method for fracture, these integrals are evaluated herein for the Kelvin solution of the three-dimensional Navier equations of linear elasticity.

Research sponsored by Applied Mathematical Sciences Research Program, U.S. DOE Ofice of Energy Research; by IBM Berger. Scientific Centre; by the National Science Foundation; and by CNPq. Brazilian Government Agency.

*Cornell University, lthaca, New York.

\subsection{5}

\section{APPLICATION OF ONSAGER'S RECIPROCITY RELATIONS TO INTERFACE MOTION DURING PHASE TRANSFORMATIONS}

$$
\begin{gathered}
\text { T. Kaplan* } \\
\text { L. J. Gray }
\end{gathered}
$$

[Abatract of S. Chem. Phys. O0(2), 1133-1140 (1989)]

A number of theories have been developed for kinetics of steady-state interface unotion during a phase transformation. One requirement of such theories is that they satisfy Onsager's reciprocity relations for ccupled irreversible processes. We show how to apply Onsager's relations io a phasse transformation at a moviug planar iuterface in a two-component system. The appropriate pairs of thermodynarnic forces and conjugate fluxes are determined and used to test two proposerd theories 
31

for interface motion during solidification of binary alloys. It is shown that the Continuous Growth Model "with solute drag" of Aziz and Kaplan is inconsistent with Onsager's relations, but the Contenuous Growth Model "without solute drag" is consistent with Onager's relations.

Research sponsored by U.S. DOE Office of Expert Sciences.

"Solid Stave Division.

harvard University, Cambridge, MA.

1.86

CURVILINEAR COORDINATES FITTED TO A DISTORTED ANNULAR REGION

W. F. Dawkins

(Abstract of paper prevented al a Seminar at Odd Dominion University, Depertunent of Mechanical Engineering Norfolk, VA, November 7, 1988)

Physical problems concerning interfaces sepabating two media, where the position and veloceits of the interface are not known a priori, remain among the most useful and difficult to solve. Moden numerical methods for attacking such problems all involve one form or another of adaptive grid generation. One such class of methods is based on the use of curvilinear coordinate transformmotions such that the interface is parameterized by a fixed one of the coordinate lines. Such methods will be discussed and some recent research pertanning to distorted annular regions will be perescented. Further, the algorithms used to make calculations were implemented on an Intel Hypercube (64-node distributed memory computer), and some aspects of that experience will also be discussed.

Research sponsored by Applied Mathematical Sciences Research Program, U.S. DOE Office of Energy Research. 


\section{STATISTICS}

\section{Design and Analysis of Computational Experiments}

1.87

\section{COMPUTER EXPERIMENTS TO OPTIMIZE A COMPRESSION MOLD FILLING PROCESS}
A. Church, Jr.*
T. J. Mitchell

(Abstrect of oral presentation presented at the Workshop on Deaign for Computer Experiments, Oak Ridge, TN, November 3-5, 1988)

We describe an application of the use of computer experiments to construct a fast approximation to a computer model that simulates the compression molding of sheet molding compound (SMC) in the manufacture of an automobile hood. The model code obtains numerical solutions (using a finite element method) to the differential equations that govern the flow of the SMC as it fills the mold cavity. The main purpose of the code is to predict the position of the flow front during the modeling process. This is used in turn to determine whether "linit lines" will occur; these form when two flow fronts meet, causing a weakness in the molded part. By altering the shape and position of the "charge," the initial piece of SMC that is compressed during the process, the effects of knit lines can be reduced or eliminated. Given an executable version of the model code as a black box, we designed a 15-run computer experiment to predict the movement of the flow front as a function of the shape and position of the charge. The results of this experiment, the prediction methodology, and its effectiveness in optimizing the charge parameters will be discussed.

Research sponsored by U.S. Department of Energy.

* GenCorp, Akron, OH.

\subsection{8}

\section{A BAYESIAN APPROACH TO THE DESIGN AND ANALYSIS OF COMPUTER EXPERIMENTS}
C. Currin*
T. J. Mitchell
M. D. Morris
D. Ylvisaker ${ }^{\dagger}$

(Atratract of OKNL 6408, September 1988)

We consider the problem of designing and analyzing experiment., for prediction of the function $y(t), t \in T$, where $g$ is evaluated by means of a computer code (typically by solving complicated equations that rodel a physical system), and $T$ represents the domain of inputs to the code. We use a Bayesian approach, in which uncertainty about $\boldsymbol{z}$ is represented by a sf-atial stochastic process (random function); here we restrict attention to stationary Gaussian processes. The posterior mean function can be used as an interpolating function, with uncertainties given by the posterior standard deviations. Instead of completely specifying the prior process, we consider several families of priors and suggest son.te crose-validational methods for choosing one that performs relatively well on the function at hand. As a design criterion, we use the expected reduction in the entropy of the random vector $x\left(T^{*}\right)$, where $T^{*} \subset T$ is a given finite set of "sites" (input configurations) at which predictions are to be made. We describe an exchange algorithm for constructing designs that are optimal with respect to this criterion. To demonstrate the use of these design and analysis methods, several examples are given, including one experiment on a computer model of a thermal energy storage device and another on an integrated circuit simulator.

Research sponsored by Applied Mathematical Sciences Research Program, U.S. DOE Office of Energy Research.

*Bryn Mawr College, Bryn Mawr. PA.

'Universily of California at Los Angeles, Lo Angeles, CA.

1.89

\section{SENSITIVITY AND UNCERTAINTY ANALYSIS IN ENVIRONMENTAL MODELS}

\section{J. Downing S. Bartell*}

(Abatract of oral presentation presented at a Workehop on Deaign for Computer Experiments, Oak Ridge. TN. November 3-5, 1988)

A description and objcctives of an environinental model (FOAM) are introduced. The general definitions of uncert ainty and sensitivity analysis are disc ussed and applied to the FOA.M model. The ideas are extended to look at the model pre. dictions through time.

Research aponsored by U,S. Department of finergy.

"Posvimmomental Bicienrea I)iviniron. 


\subsection{0}

\section{A BAyESIAN APPROACH TO THE DESIGN AND ANALYSIS OF COMPUTATIONAL EXPERIMENTS}
T. J. Mitchell
M. D. Morris

(Abatract of pepper presented at the 20kh Symposium on the Interface of Computing Science and Stativica, Reaton, VA, April 21-23, 1988; Proc. Pp. 49-51, E. J. Wezman, D. T. Gantz, and J. J. Miller, Eds., American Stafiatical Aswociation. Akxandria, VA (1988)]

In a computational experiment, the data are produced by a computer program that models a physical system. The experiment consists of a set of model runs; the design of the experiment specifies the choice of program inputs for each run. This paper centers on the problem of prediction (interpoiation), the goal of which is to devise a design/analysis method which will provide predictions of model output for input values not run. We adopt a Bayesian approach as the basis for the analysis. Uncertainty about the re sponse function is quantified by choosing a class of probability distributions over the function space. This leads to design procedures based on maximizing the expected reduction in "amount of uncertainty," where the latter can be defined formally in terms of properties of the posterior distribution. Here we use as a design optimality criterion the determinant of the posterior covariance matrix of the responses at the input configurations at which we want to make predictions. This requires maximization of the deter minant of the prior covariance matrix of the responses at the design sites. We describe our computer algorithm for constructing optimal designs and give some examples of designs that it produces.

Research sponeored by U.S. Department of Energy.

\subsection{1}

\section{COMPUTER CONSTRUCTION OF OPTIMAL DESIGNS FOR PREDICTION IN COMPUTER EXPERIMENTS}

\section{T. J. Mitchell M. D. Morrix}

(Abatract of oral presentation presented at the IBM Scientific Centre, Bergen, Norway, July 18, 1988; at the University of Augaburg, Augaburg, Germany, July 22, 1988)

We consider the problem of desizning experiments for prediction of the function $y(x)$, where $y$ is evaluated by means of a computer code (typically by solving complirated equations that model a physical system), and I represents the inputs to the code. We use a Bayesian approach, in which uncertainty about is represented by a random function, i.e., a spatial stochastic process. We formulate design criteria based on maximizing the expected reduction in the entropy of the random vector $y(S)$ where $S$ is a given set of "sites" (input configurations) at which predictions are to be made. This talk will be concerned primarily with algorithms for constructing optimal designs in this setting using the entropy criterion.

Research sponsored by Applied Mathematical Sciences Research Program, U.S. DOE Office of Energr Research.

1.92

\section{EXISTENCE OF SMOOTHED STATIONARY PROCESSES ON AN INTERVAL}

\section{T. J. Mitchell M. D. Morris D. Yvisaker}

[Abstract of Slochastic Processes (in press)]

Necessary and sufficient conditions are given for there to exist a stationary process on an interval with a given stationary process as its $k^{\text {th }}$ derivative. The condition involves the norm in the reproducing kernel space asscciated with the process sought as derivative. Some explicit results are obtained when working with the Ornstein-Uhlenbeck and the linear kernels. These latter facts are useful in an adaptive Bayesian modelling of computer experimer.ts; some re.narks are given about this type of analysis.

Research sponsored by Applied Mathematical Sciences Research Progran, U.S. DOE Office of Energy Research.

"Univerity of Califormia, Los Angeles, CA.

\subsection{3}

\section{FACTORIAL SAMPLING PLANS FOR PRELIMINARY COMPUTATIONAL EXPERIMENTS}

\section{D. Morris}

(Abatract of oral presentation presented at the 1989 Joint Statiutical Meeting, Washington, DC, Auguat 6-10, 1989)

A compulational model is a representation of some physical system of interest, first expressed matliematically and then implemented in the form of a computer program; it may be viewed as a function of inpuls which, when evaluated, pro- 
duces ontputs. In this paper, we are concerned with computational models which are deterministic, complicated enough so as to make classical mathematical analysis impractical, and which have a moderate to large number of inputs. We consider the problem of designing computational experiments to determine which inputs have important effects on an output. The proposed approach is based on classical sampling ideas, involves the use of one-factor-at-a-time experimental designs, and does not rely on assumptions such as the adequacy of a low-order polynomial as an approximation to the computational model, effect sparsity, or monotonicity of outputs with respect to inputs. Prediction of the function $y(x)$, where $y$ is evaluated by means of a computer code (typically by solving complicated equations that model a physical system e.g., a chemical reaction), and $I$ represents the inputs to the code (e.g., reaction rates). We use a Bayesian approach, in which uncertainty about $y$ is represented by a random function, i.e., a spatial stochastic process. The posterior mean function can be used as an interpolating function, with uncertainties given by the posterior standard deviations. Designs are selected to minimize a measure of posterior uncertainty. The approach will be demonstrated using as examples (i) a computer model for the combustion of methane and (ii) a computer model that simulates the compression molding of sheet molding compound (SMC) in the manufacture of an automobile hood.

Research sponsored by the Applied Mathematical Sciences Research Program, U.S. DOE Office of Energy Reaeanch.

\subsection{4}

\section{BAYESIAN DESIGN AND ANALYSIS OF COMPUTER EXPERIMENTS: USE OF DERIVATIVES IN SURFACE PREDICTION}

\section{D. Morris}

(Abatract of oral presentation presented at a Workshop on Deaign for Computer Experiments, Oak Ridge. TN, November 3-5. 1988)

This talk is on our continuing research in the area of "fast predictive approximation" of a computer model. Our earlier work on a Bayesian solution is expanded for the case in which the first derivatives of the output variable with respect to some or all input variables are av silable. In addition to analysis (actual construction of the predictor based upon data collected from executions of the model), we discuss the use of Shannon's entropy as a tool in selecting optimal experimental designs.

Research sponsored by U.S. Department of Energy.

\subsection{5}

\section{COMMENT ON: DESIGN AND ANALYSIS OF COMPUTER EXPERIMENTS}

\section{D. Morris}

[Abstract of Stetiotical Science (in preas)]

The authors have provided an interesting and readable account of a statistical approach to the problem of approximating an unlinown, deterministic computer model. The approximation of unknown functions, of at least a few arguments, has received considerable attention in other specialty areas of mathematics, but is relatively new to statistics. A statistical approach brings a unique potential for dealing with uncertainty in the problem. In particular, it can lead to measures of quality for each prediction and a structure on which to base the design of efficient experiments. Techniques which are relevant for approximating computer models are particularly timely because the scientific and technical professions are quickly becoming reliant upon these as research tools. This manuscript reports some of the first serious efforts to make statistics relevant to these activities.

Research sponsored by Applied Machematical Sciences Research Program, U.S. DOE Office of Energy Research.

\subsection{6}

\section{A Bayesian approach TO THE DESILIN AND ANALYSIS OF COMPUTATIONAL EXPERIMENTS}
M. D. Morris
T. J. Mitchell

[Abetract of oral presentation presented at the First Incernational Conference-Workmhop on Optimal Deaign and Analyais of Experiments, Neuchatel, Switzertand, July 25 28, 1988)

A statistical approach to the approximation of ceterministic computational merdels will be dis- 
cussed. We regard a computational model as a complicated function mapping isput variables, from some meaningful domain, into output variables. Large-scale computational models are often prohibitively difficult to examine analytically and may require considerable computational resources for each evaluation. Hence, we seet a methodology for empirically formulating a fast predictive approximation" of the model, along with design strategies which allow us to do this in an efficient manner.

Our approach to the problem is Bayesian, basad upon the use of a stochastic process over the domain of the computational model. The talk will cover details of the prior processes we have used, a design criterion based upon expected entropy, and examples of designs and model approximations in some simple cases. Sun:- speculations on the form of optimal designs and model approximations in certain limiting situations will also be discuseed.

Research sponsored by Applied Mathematical Sciences Re. search Program, U.S. DOE Oftice of Energy Reseanch.

1.97

\section{DESIGN AND ANALYSIS OF COMPUTER EXPERIMENTS}

\author{
J. Sacks* \\ W. J. Welch ${ }^{\dagger}$ \\ T. J. Mitchell \\ H. P. Wynn
}

[Abatrart of Statiotical Science (in preas)]

Many scientific phenomena are now investigated by complex computer models or codes. A computer experiment is a number of runs of the code with various inputs. A unique feature of many computer experiments is that the output is deterministic - rerunning the code with the same inputs gives identical observations. Often, the codes are computationally expensive to run, and a commen objective of an experiment is to fit a cheaper predictor to the data. Our approach is to model the deterministic output as the realization of a stochastic process, thereby providing a statistical basis for designing experiments (choosing the inputs) for the efficient prediction. With this model, estimates of uncertainty of predictions are also available. A number of applications are discussed, and we demonstrate the methodology with an example. The example illustrates the gains in efficiency possible relative to standard least squares estimation of a response surface.

Research sponsored by U.S. DNE and NSE DMS Office of Naval Research.

* Univeraity of minois, Champaign. IL.

Univerzity of Waterloo, Waterloo, Ontario, Canade.

*City Univervity, London, England.

\section{Computational Distribution Theory}

\subsection{8}

\section{MOMENT METHODS AND THE GRAM-CHARLIER TYPE A}

\section{K. O. Bowman L. R. Shenton"}

(Abstract of paper presented at the 47th Swaion of the Internal Statiatical Inatitute, Paris, France, Aurinat 20September B, 1989)

The joint efficiency of estimatien by moments for a four-parameter Gram-Charier density involves a ratio of covariance determinants. Each element in the maximum likelihood case involves an integral, the denominator of the integrand being a quartic. In a study that came out by one of the authors several years ago, these integrals were approxinated by ratios of gencralized continuantsdetermillants with non-zero elements in the diagonal, and sul, super and sub diagonals, $(x=$
$1,2,3,4)$, i.e., determinants of nine-diagonal arrays. The computational methods at the time depended entirely on pen and paper work with some assigtance from mechanical calculators. Quadrature procedures at the time were regarded as too fragile and only to be used when error estimates were included.

Researd, eponsored by U.S. DOE

'Univenity of Georgien Athene, GA.

1.99

\section{SOLUTIONS TO JOHNSON'S SB AND SU}
K. O. Bowman
L. R. Shenton*

[Atmetrart of Commun. Staliat. Simuia. 17(2), 343 (1988)]

Simple iterative and exart molutions are de-

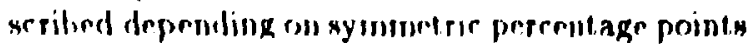


for the Johnson translation system of distributions. A condition is given for a zero skewness parameter.

Research sponsored by Applied MAsthemalical Sciences Re search Progran, U.S. DOE Office of Energy Researcil.

* University of Georgia, Athens, GA.

1.100

\section{PROPERTIES OF ESTIMATORS FOR THE GAMMA DISTRIBUTION}

\section{K. O. Bowman $\quad$ L. R. Shenton}

[Abstract of a boc Vol. 89, Marcel Dekker, Inc., New York, NY (Jamuary 1988)]

This monograph studies the subject at points where traditional exciusive mathematical approaches leave off. It is a blend of numerical analysis which is computer oriented and directed at questions involving series and the information contained therein; it brings to light much that is problematical in remainders, especially when these appear to be well defined in an existentialist fashion. It is an account of attempts to grapple with early 20 th century dedication to mathematical edifices with a slow intrusion of computational faciliiies. It should interest students, brcadly defined, who like to see science at work and in the making.

Rescarch sponsored by Applied Mathermatical Sciences Research Program. U.S. DOE Office of Energy Research.

* Univenity of Georgian Athens, GA.

1.101

SB AND SU DISTRIBUTION FITTED BY PERCENTILES: A GENERAL CRITERION

\section{K. O. Bowman $\quad$ L. R. Shenton *}

[Abutract of Cammun. Statis. .Simz/a. 18(1), 1-13(1989)]

A criterion is given to choose between Johnson's $S_{B}$ and $S_{U}$ when four percentiles are used. Also the algorithms provided make it possible to use the percentiles corresponding to the data provided, thus avoiding the intricacies of interpolatirn.

Heweardi sponmoned by Applied Machematiral Scriences Rewearch Program. US. DOCF Office of Finergy Rewearch.

- Inivemily of reosgia Athress. CiA.
1.102

\section{CONTINUED FRACTIONS AND ASYMPTOTIC SERIES IN APPLICATIONS: WITH SPECIAL REFERENCE TO PROBLEMS IN STATISTICS}

\author{
K. O. Bowman I. R. Shenton*
}

[Preface of a book, Marcel Dekker, Inc., New York, NY (April 1989)]

This book is an account of the use of continued fractions and Padé sequences as tools in the interpretation of divergent or slowly convergent series occur ring in theoretical statistics. In our early studies an obstacle we had to overcome concerned the process of developing Taylor power series for moments of statistics; for example, the expected value of the sample standard deviation from some defined population. Traditionally one or two low order terms sufficed, because later terms involved heavier and heavier algebra soon leaving behind the most ardent algehraist; the digital computer arriving in the :950s had little impact for algorithmic approaches which were not generally highly regarded, existing in a somewhat ill-defined gray area. So higher order terms in series were not available and the era of largeness of sample size was born, and the reliance from time to time on the uncertainties defined in the order of magnitude symbols (little o, big $\mathrm{O}$ ).

Also early in the present century, summation algorithms (or techniques) had few illustrations of divergent series, so a few classical cases received a lot of attention; many of them (by chance in the evolution of mathematical analysis) were such that the first few terms looked beguilingly "convergent" (the series part of In $\Gamma(x)$ for example. and the polygamma function series). So there appeared the notion of absolute error in stopping at a certain term heing related to the first term omitted; whereas this rule is sometimes true, in general it has no basis.

Our treatment is partly mathematical but pivoted on computer oriented numerical analysis (or computer oriented algebra). The increased confidence in our main algorithm for the expectation of quotients of products of sample moments was the driving force for increased knowledge of summation processes for seemingly divergent Taylor series. 'Th. study, by no means complete, and no doubt involving unavoidable errors (hopefully faw) has spanned inore thall (wo decades. 
Let it be made clear that our development of the subject has been motivated by an attempt to clarify the interpretation of divergent series in practical situations. The classical theory of rational approximation is overloaded with myraids of specialized theore ins - albeit of some interest, and exhibiting acute mathematical expertise. But very iew of these theoretical excursions amount to increased insights into the mechanics of the structure of functions and corresponding approximating functions.

Multivariate situations are not considered for there are many outstandiug problems in the univariate case. At the very least, we hope the reader will gain a new awareness of the many varieties of series and tte correspcnding summation problems.

Research sponsored by Applied Mathematical Sciences Research Progran, U.S. DOE Office of Energy Tesearch.

*Univenity of Georgia, Athens, GA.

1.103

\section{SOME PROPERTIES OF A MOMENT ESTIMATOR FOR THE INDEX PARAMETER OF NEGATIVE BINOMIAL DISTRIBUTION}

\author{
H. K. Lam* \\ L. R. Shenton ${ }^{\dagger}$
}

\section{K. O. Bowman}

[Abatract $\mathrm{n}$ paper published in Proc. of the 1988 Joint Stetiatical Meetingw, pp. 365-367, New Orleans, LA, A uguat 22-25, 1988 (1988)]

Taking the mean of the negative binomial to be $k P(1+P)$, a moment estimator of $k$ is $\left(m_{1}^{\prime}\right)^{2} /\left(m_{2}-m_{1}^{\prime}\right)$. From previous studies it is known that the series development for the mo ments of the momentor estimator $k^{*}$ appear to be markedly divergent (probably due to the singularity in the denominator). The present study shows that the moments of $1 / k^{*}$ are more stable than those of $\boldsymbol{k}^{*}$. Thus, if we wish to make inference about $k$ by using 4-moment distribution fitting, then the moments of $1 / k^{*}$ should be used, rather than those of $k^{*}$.

Fesearch sponsored by U.S. Department of Energy.

*Bell Communicalione Reacards. Ked Bank, NJ.

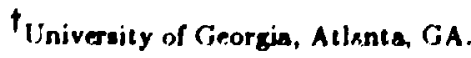

1. 104

\section{A PROBLEM WITH TRANSFORMATIONS OF STIELTJES CONTINUED FRACTIONS}
L. R. Shenton
K. O. Bowman

[Abetrect of J. Comp. Appl. Meth. (in p. =1)]

A modification of a basic periodic contimued fraction is studied in which the associated weight function (in the Stieltjes transform form) is multiplied by a positive factorable polynomial; this relates to the continued fraction form for wistan difference operations acting on the associated power series development. A proce' 'ure is given to cope with the development of the modified continued fraction; in a number of cases the partial numerators of this continued fraction turn out to have a certain periodicity of form and are rational fractions with polynomial components. In the limit they are periodic, the period (very likely) being that of the original continued fraction.

Research sponsored by Applied Machemalical Sciences Rewearch Program, U.S. DOE Office of Eneigy Research.

*Univenity of Georgie, Athens, GA. 


\section{Research in General Statistical Methods}

1.105

\section{GENERATION OF AN ORDERED SIMPLE RANDOM SAMPLE WITHOUT REPLACEMENT USING ONE RANDOM NUMBER}

\author{
Y. H. Etheridge T. Wright \\ (Abatract of ORNL/TM-10868, August 1988)
}

Nigam and Gupta presented by examples a method for the identification of the $n$ units to be included in a simple random sample (SRS) for selection from a finite universe of $N(>\mathbf{n})$ distinct and identifiable units. The method calls for the generation of only one random number to select a SRS of $\mathbf{n}$ units without having to list all $\left(\begin{array}{l}N \\ n\end{array}\right)$ possible samples - practically all other methods require the generation of between $\mathbf{n}$ and $\mathbf{N}$ random numbers. Wriglıt has given a slightly faster and generalized algorithm and demonstrated that the generalized algorithm to generate a SRS has a natural relation with Pascal's Triangle. The slight improvement in speed is a direct result of the establishment of a link with Pascal's Triangle. A program that executes the generalized algorithm is described.

Research sponsored by Applied Mathematical Sciences Research Program, U.S. DOE Office of Energy Rescarch.

\subsection{6}

\section{MAXIMUM LIKELIHOOD ESTIMATION FOR CYTOGENETIC DOSE-TESPONSE CURVES}

\section{E. L. Frome R. J. DuFrain*}

[Atmatrart of bismetrics 42, 7.3 (1986)]

In vatro dost-response curves are used to descritie the relation between chromieome aberrations and radiation dosse for human lymphocytex. The: lymphorytes are exposed to low-LET radiatirn. and the resulting dicrntric chromosome aberrations followe the Proisson distribution. The expereted yirld depends on breth the magnitude and the ermpresal distribution rif the dose. A general

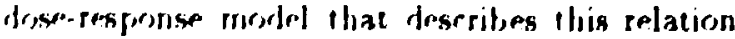
lass teren pressented using the theory of dual ra- diation action. Two special cases of practical interest are split-dose and continuous exposure experiments, and the resulting dose-time-response models are intrinsically nonlinear in the parameters. A general-purpose maximum likelihood estimation procedure is described, and estimation for the nonlinear models is illustrated with numerical examples from both experimental designs. Poisson regression analysis is used for estimation, hypothesis testing, and regression diagnostics. Results are discussed in the context of exposure assessment procedures for both acute and chronic human radiation exposure.

Research sponsored by U.S. DOE Office of Health and Envirommenta Research and by Oak Ridge Associated Unjversities.

"Oak Ridge Aseociated Universit:" Oak Ridge, TN.

1.107

\section{SURVEY DATA QUALITY CONTROL CONFERENCE: A REPORT}

\section{$\begin{array}{ll}\text { G. R. Liepins } & \text { V. R. R. Uppuluri }\end{array}$}

(Abstract of ord presentation presented at the 1988 Joint Meeting of the ASA Chapters of Tennescee, Hospital Corporation of America, Nachville, TN, April 23, 1988)

This talk was a brief summary of the Conference on Survey Data Quality Control held in Oak Ridge during April 21-22, 1988. Some higlilights of the technical presentations of the enclosed program was reported.

Research sponeores' by I.S. Department of Energy

-Enerzy Divinion.

\subsection{8}

\section{DETECTION OF ASSOCIATIONS BETWEEN DISEASES IN ANIMAL CARCINOGENICITY EXPERIMENTS}

\section{T. J. Mitche:ll \\ B. W. Turnbull*}

[Atmetract of Binmetrice (in prese)]

We present a statistiral test for the hyporsthesis of independence of two disease processes in animal earcinogenirity experistents. It is assumerred that 
the direases are occult (detectable only at death via necropsy), and progressive, in that each passes (potentially) from absent to nonlethally present to lethally present in each animal. The test is based on a series of age-stratified $3 \times 3$ tables in which the animals that die in each age interval are cross-classified into one of these three states for ach disease. Under the appropriate conditioning to remove the effect of nuisance parameters, we derive the null distribution of the data table in each interval, and in particular the distrilution of the test statistic, which is the frequency of animals that die with neither disease. (Under the conditioning in each interval, this is equivalent to the ratio of the odds for presence of one disease to the odds for presence of the other.) The test statistics from all intervals are then combined as in a Mantel-Haenszel test to yield an overall test statistic, the total number of animals that die with neither disease, which is approximately normally distributed. This test utilizes all the data, whether obtained by sacrifice or by "natural death." It avoids the biases due to lethality noted by Breslow et al. for the test based on age-stratified $2 \times 2$ tables, where the nonlethal and lethal occurrences are pooled. In a simulation experiment, we show that such biases (which usually conceal true poeitive associations and produce spurious r.agative associations) can be very severe indeed, whereas our test preserves the nominal significance level quite well. The power of the test appears to vary widely, depending on the incidence, time to onset, and rate of progressicn of each disease. In many cases, it will be necessary to combine data from several experiments in order to detect associations.

Reweardi sponsored by U.S. DOE Office of Health and Environniental Research.

"Comell Univervity. Ithaca, NY.

\subsection{9}

\section{BAYESIAN VARIABLE SELECTION IN LINEAR REGRESSION}

\section{T. J. Mitcluell J. J. Beauchamp}

(Abatract of oral presentation presented at the University of Tennemex, Department of Stativtira. Knoxville, TN, OCtober 18, 1988)

This talk will review joint work of T. J. Mitchell and the speaker concerned with the selection of predictor variables in a linear regres- sion model for the prediction of a dependent variable using a Bayesian approach. After specifying the necessary prior distributions and deriving the appropriate posterior model probabilities, methods are propesed for assessing the complexity of the response function. Examples based on a data set arising from an energy conservation study are given to demonstrate the application of the methods.

Research sponsored by U.S. Department of Energy.

\subsection{0}

\section{BAYESLAN VARIABLE SELECTION IN LINEAR REGRESSION}

\section{T. J. Mitchell J. J. Beauchamp}

(Abatract of ord presentation presented at the 1988 Joint Statiatical Meetine, New Odeans, LA, Auguat 22-25, 1968)

This paper is concerned with the selection of subsets of predictor variables in a linear regression model for the prediction of a dependent variable. It is based on a Bayesian approach, intended to be as objective as possible. A probabilicy distribution is first assigned to the dependent variable through the specification of a family of prior distributions for the unknown parameters in the regression model. The method is not fully Bayesian, however, because the ultimate choice of prior distribution from this family is aftected by the data. It is assumed that the predictors represent distinct observables; the corresponding regression coefficients are assigned independent prior distributions. For each regression coefficient that is subject to deletion from the model, the prior distribution is a mixture of a point mass at $\mathbf{0}$ and a diffuse uniform distribution elsewhere, i.e., a "spike and slab" distribution. The random error component is assigned a normal distribution with mean 0 and standard deviation $\sigma$, where $\log (\sigma)$ has a locally uniform "noninformative" prior distribution. The appropriate posterior probabilitice are derived for cach submodel. If the regression coefficients have identical priors, the posterior distribution depends only on the data and the parameter $\gamma$, which is the height of the spike divided by the height of the slab for the common prior distribution. This parameter is not assigned a probability distribution; instead, it is considered here as a parameter that indexes the members of a clase of Bayesian methords. Ciraphical methods are proposed as informal 
guides for choosing $\gamma$, for assessing the complexity of the response function and the strength of the individual predictor variables. and for assessing the degree of uncertainty about the best submodel. The dependence of the results on the scaling of the variables is discussed, and some ways to choose the scaling constants are suggested. Examples based on a large data set arising from an energy conservation study rere given io demonstrate the application of the methods.

Research sponsored by U.S. Department of Energy.

\subsection{1}

\section{SMALL-SAMPLE CONPIDENCE LIMITS FOR PARAMETERS UNDER INEQUALITY CONSTRAINTS WITH APPLICATION TO QUANTAL BIOASSAY}

\section{D. Morris}

[Abstract of Biometrics 44, 1083-1092 (1988)]

A family of methods is presented for constructing confidence limits for the parameters of a collection of distributions when a simple ordering is assumed among the parameters. The me:hods are shown to yield confidence limits that are exact or conser vative for finite samples. For discrete distributions, one of the methods produces confidence limits that are at least as tight as the limits produced by a commonly used single-sample procedure Confidence limits are demonstrated for a binomial quantal bioassay problem assuming a nondecreasing dose-response function. Recults of a simulation study show that competing asymptotic methods can produce serious discrepancies between nominal and actual coverage probabilities for binomial samples of sizes up to 30 and demonstrate that the new approach can be competitive with the asymptotic methods when the latter maintain their nominal error rates.

Newearch sponsored by Apf.ied Mathematical Sriences Rene ardi Progtam, U.S. DrJf Offire of Finergy Pesearch.

\subsection{2}

\section{SPARSE MATRXX COMPUTATIONS IN ANALYSIS OF VARIANCE}

\section{G. Ostrouchov}

(Abatract of oral preacntation presented of Kanses State University. Manhallan, KS, April 7, 1988; of the University of Tennescee, Departmene of Statistica, Knoxville, TN, February 25, 1988; at SIAM Conference on Applied Linear Algebre, Madizon, WI, May 23-26, 1988; and at the University of Tennesece as a lecture entived, "Spane Leat Squares Compulations in Stativtical Applications," Knoxville, TN, May 2-6, 1988)

Unbalanced data in analysis of variance require the explicit solution of a least squares problem. The least squares problem is sparse and can be very large, thus its solution can benefit from the use of sparse matrix techniques. The use of standard sparse matrix techniques in combination with symbolic processing that takes advantage of the special matrix structure can produce a very efficient direct method. This talk will briefly describe the analysis of variance problem and standard sparse matrix techniques for the direct solution of least squares problems. Most of the talk will concentrate on the application of sparse matrix techniques to the analysis of variance problem and symbolic processing based on model term index sets.

Researd sponsored by Applied Mashematical Sciences Research Program, U.S. DOE Office of Energy Research.

\subsection{3}

\section{STATISTICAL COMPUTING ON A HYPERCUBE}

\section{G. Ostrouchov}

(Abetract of oral presentation presented at Kansas Stale University, Manhactan, KS, April S, 1988 and at the 20th Symposium on the Interface of Computing Science and Stativtics, Reston, VA, Aprit 21-23, 1988)

A hypercisbe parallel computer is a network $2^{n}$ processors, each with only local memory, whose activities are coordinated by messages the processors send between themselves. The interronnection network corresponds to the edges of an $n$ dimensional cube with a processor at each vertex Some recent experiences and results in developing a hypercube algorithm for iterative proportional fitting of large Poisann regression problems will be discussed. The algorithm is implemented on $\approx$ 64peroressor I,NTEL, iPSC; hyperrube

Kenearti aponased by Applied Mathemational Sirienere fie.

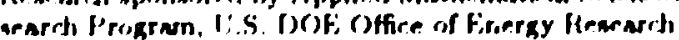


1.114

\section{ANOVA MODEL FITTING VIA SPARSE MATRIX COMPUTATIONS: A FAST DIRECT METHOD}

\section{G. Ostrouchov}

[Abatract of SIAM J. Sci. Stet. Compat. 10, 58 (1900)]

A fast and storage-nficient direct method for fitting analysis-of-variance models to unbalanced data is presented. This method exploits sparsity and rank deficiency of the model matrix and is based on orthogonal Givens factorization of a set of sparse columns of the model matrix. A class of matrices generated by index sets is defined and used to obtain results on linear dependencies between coiumns of a model matrix and fill during factorization. These results are used to develop an algorithm for the selection, ordering, and symbolic factorization of a set of sparse columns of the model matrix. This facilitates a fast and storageefficient numerical factorization and solution. $A$ comparison to both a standard direct algorithm and a general-purpose sparse least-squares algo rithm shows that the new algorithm reduces time and storage by orders of magnitude for large models and is competitive for small models.

Research sponsored by Applied Mathematical Sciences Research Program, U.S. DOE Unce of Energy Rewearch.

\subsection{5}

\section{A MODEL SEARCH PROCEDURE FOR HIERARCHICAL LOG- LINEAR MODELS}

\section{G. Ostrouchor L. L. Frome}

(Abatract of paper preanted at the 1989 Join Statiatical Meeting, Wahington, DC, August 6-10, 1989)

Large data sets crose-classified according to multiple factors are available in epidemiology and other disciplines. Their analysis often call for finding a set of best hierarchical log-linear models to serve as a basis for further analysis. This selection can be based $\mathrm{c} n$ an Akzike information type statistic. Fitting all possible mudels to find a best set is usually not feasible for as few as five factors ( 7580 possible models). By noting that the set of hierarchical models and their relationships can be represented by a graph, a graph traversal algorithm is developed that requires fitting a frac- tion of all models to find an exact subset of the best models. The algorithm classifies (in the best set/not in the best set) as many models as possible on the tesis of each fit. A data structure implementing the graph keeps track of the information required by the algorithm.

Revearch eponered by U.S. DOE and by Oak Ridge Amocisted Universities.

\subsection{6}

\section{SHRINKING TECHNIQUES FOR ROBUST REGRESSION}
R. L. Schmoyer
S. F. Arnold

[Abatract of book chepter in Contributions 10 Probability and Statiotice, Eeseyo in Honor of Ingrem Olkir, pp. 368384, L. J. Glewer, M. D. Pertman, S. S. Prese, and A. R. Sampeon, Ed., Springer-Verlag, New Yort (1989)!

The asymptotic normality of robust estimators suggests that shrinking techniques previously considered for least squares regression are appropriate in robust regression as well. Moreover, the noisy nature of the data frequently encountered in robust regression problems makes the use of shrinking estimators particularly advantageous. Asympt::ic and finite sample results and a short simulation demonstrate that shrinking techniques can indeed improve a robust estimator's performance.

Rewearch gponeored by the Applied Machematical Sciences Research Program, U.S. DOE Ofice of Energy Resenrch.

Penraylvaris State Univeraily, Univenity Park, PA.

\subsection{7}

\section{NEAR WITH REPLACEMENT SAMPLING WITHOUT REPLACEMENT USING RANKS}

\section{T. Wright}

(Abatract of oral presentation presented at the Wahing: con Statiatical Society Meeting. Weahinglon, DC, April 13, 1988; at Ohio State Univerity, Statiatics Department. Columbun, OH, May 17, 1988; and at the Univervity of Tenneace, Statistic Deparment, Knoxville, TN, January 31, 1889)

Let $U_{1}, U_{2}, \ldots, U_{N}$ be the $N$ units of a finite population and let $\left(y_{i}, x_{i}\right)$ be a vector of nonnegative components associaled with the $i^{\text {th }}$ unit, where the $x$ 's are known and the $y$ 's are unknown. A sample of fixed size $n(\angle M)$ is to be 
selected to estimate :he unknown population total $\gamma=\sum_{i=1}^{N} y_{i}$. Let $\pi_{1}$ be the probability of a sample inclusion for the $i^{\text {th }}$ unit. Many have realized improvements in efficiency by taking $\pi_{i}=n p_{i}$ where $p_{i}=x_{i} / \sum_{j=1}^{N} x_{j}$ is a normed measure of size for the $i^{\text {th }}$ unit. Such plans, called probability proportional to size ( $\pi p s$ ) sampling plans, can be difficult to execute when sampling is without replacement (wor), particularly when $n>2$.

When a suitable variable $x$, which is directly proportional to the variable of interest $y$, is not available, one may know how to rank units reasonably well relative to the unknown y values before sample selection. When such ranking is possible, we introduce a simple and efficient $\pi$ pswor sampling plan for general $n$ using the ranks as the known I measures of size. The proposed sampling plan is similar to, has the simplicity of, and has no greater sampling variance than with replacement sampling, but is without replacement. The method is compared numerically with several other methods for various populations.

Research sponsored by U.S. DOE Bacic Energy Sciences Office of Energy Rewearth.

\subsection{8}

\section{ON SOME PROPERTIES OF VARIABLE SIZE SIMPLE RANDOM SAMPLING AND A IJMIT THEOREM}

\section{T. Wright}

[Abatract of Commen. Slatisl.-Theory Meth. 17(9), 2997 (1988)]

This paper introduces a sampling plan for $f-$ nite populations herein called "variable size simple random sampling" and compares properties of estimators based on it with results from the usual fixed size simple random sampling without seplarrment. Necessary and sufficient conditions for the lim:iting distribution of the sample total (or salmple mean) to be normal are given.

Menesrcti spoitiwred by Applied Mathematical Sciences Resoarch Prigram, U.S. IDOF, Office ol Energy Research.

\subsection{9}

\section{A NOTE ON PASCAL'S TRIANGLE AND SIMPLE RANDOM SAMPLING}

\author{
T. Wright
}

[Abstract of College Mathematics Jonnal 20(1), 59-66 (1989)]

Much of the theory behind the statistical analysis of sample surveys and experiments requires that the sample be selected by random sampling. Simple random sampling without replacement is the process of selecting a set of $n$ different units from a universe of $N$ distinct and identifiabie units where each possible set of $n$ units is equally likely to be chosen; that is, has probability $1 /\left(\begin{array}{l}N \\ n\end{array}\right)$ of selection. Although the definition calls for the selection of a set of $n$ units, in practice one usually selects a simple random sample (SRS) using a unit by unit selection scheme. Unit by unit selection schemes are favored because they do not require a complete listing of all possible samples. The selection of a SRS can be challenging, particularly when $n$ (and $M$ ) are large and one has a sequential listing of the population units in a data file. As Bebbington notes, "the standard method would be to pick $n$ integers at random between $I$ and $N$, sort them, devise a method for replacing duplicates, then work through the file selecting the appropriate elements. But the method is unnecessarily costly in terms of both storage and time, particularly if $n$ is large or the operation is to be done many times, for example in a simulation study." Typically, these methods call for the generation of at least $n$ random numbers to obtain one SRS.

In 1984 Nigam and Gupta demonstrated by example how to select a SRS of size $n$ witliout having to list all possible samples and by generating only one random number. Their method requires that one think of an ordered listing of all $\left(\begin{array}{l}N \\ n\end{array}\right)$ possible samples, generate a random integer $x$ between 1 and $\left(\begin{array}{l}N \\ n\end{array}\right)$, and select the sample at the th position in the list. This article contains an improvement of their method and all interesting justification based on Pascal's triangle.

Remenrth sponnoored by the Basir Energy Srienrex Sulppro gram. U.S. DOE Office of Finergy Hewarh. 


\subsection{0}

\section{ON A NEW TEST OF EQUALTTY OF TWO PROPORTIONS BASED ON DIRECTED DIVERGENCE}

\author{
V. R. R. Uppuluri T. N. Bhargava* \\ [Abatrect of Stet. Prob. Lettro. (in preal)]
}

A new procedure for testing equality of two proportions in terms of an information-theoretic measure is presented. This measure is usually referred to as the Gini-Simpson index of diversity. In particular, use is made of the properties of the quadratic analogue of the Kullback-Liebler measure of directed divergence.

Research eponeored by Applied Mathematical Sciences Pe search Progren, U.S. DOE Ofivee of Energy Research.

* Kent Stale Univervity, Kent, OH.

\section{Applications of Statistical Methods}

\subsection{1}

\section{EQUAL OPPORTUNITISS IN SCIENCE AND ENGINEERING}

\section{K. O. Bowman}

(Abatract of oral presentuion preanted at the 1988 WATTec Conference, Knoxville, TN, Februmy 16-19, 1988)

The United States Congress enacted (Public Law 96-516) the Science and Technology Equal Opportunities Act of 1980 to promote science and engineering for under-represented groups in the population. The Law requires the National Science Foundation to prepare the report "Women and Minorities in Science and Engineering" biennially. The fourth report was due in January 1988. For the past seven years, situations of women and minorities have improved significantly, but problems still exist. By the joint effort of the $\mathrm{Na}$ tional Science Foundation and the National Research Council, a series of workshops were conducted in 1986 to understand and analyze the under-representation and carrier differentials of women and minorities in science and engineering. In this talk, we summarize the latest findings.

Research sponeored by U.S. Department of Enercy.

\subsection{2}

\section{EVALUATION OF SPECIAL NUCLEAR MATERIAL MONITORING INSTRUMENTS}
K. O. Bowman
S. A. Wallace*

(Abatrect of Intermational Vuclear Mateival Management (in prese)]

A statistical methor to protect against the loss of strategic special nuclear material (SSNM) is presented. A simplified step by step approach to the test procedure is described.

Research aponacored by U.S. DOE and the Y-12 Development Department.

*-12 Developanent Diviaion.

\subsection{3}

\section{STATISTICAL QUALITY CONTROL TECHNOLOGY IN JAPAN}
K. O. Bowman
T. Hopp
R. Kacker"
R. Lundegard*

[Abatrect of Scieatific Informetion Belletin 15(1) (1960)]

From May 17 to June 1, 1989, a survey team organized by the National Institute of Standards and Technology, visited Japan to assese research and application of statistical quality control technology. The team explored the philosophy and conduct of total quality control (TQC) in Japanese industries, government laboratories, and national agencies. The philosophy and practice of TQC in Japan is quite different from that of the U.S. industries. Our findings are similar to the findings from "R\&M 2000 Variability Reduction Process Trip to Japan," B. A. Johnson.

Research sponsored by U.S. DOE and Office of Navl Research.

* National Inatituce of Standkerds and Technology, Geithersburg, MD. 
1.124

\section{CHEMICAL EXPOSURES AND CENTRAL NERVOUS SYSTEM CANCERS: A CASE-CONTROL STUDY AMONG WORKERS AT TWO NUCLEAR FACILITIES}

A. V. Carpenter * W. D. Flanders't

E. L. Frome W. G. Tankersley*

S. A. Fry"

[Abstract of The American Josmal of Industrial Medicine 13, 351-362 (1988)]

In a nested case-control study of workers employed between 1943 and 1977 at two nuclear facilities, we evaluated the possible association of primary CNS cancers with occupational exposure to chemicals. Seventy-two white male and 17 white female workers who, according to the information on death certificates, died of primary CNS cancers were identified as cases. For each case, four controls were matched on race, sex, facility at which initially employed (cohort), year of birth, and year of hire. Each job title/department combination was subjectively evaluated for potential exposure to each of 26 chemicals or chemical groups. Although statistically significant associations were not found between CNS cancer deaths and any of the 26 chemicals, modest effects could not be ruled out for some of these chemicals such as beryllium, cutting oils, and solvents.

Research sponsored by U.S. Department of Energy.

"Oak Ridge Awocialed Universities, Oak Ridge, TN.

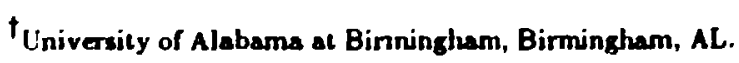

\subsection{5}

CHARACTERIZING OCCUPATIONAL DOSE DISTRIBUTIONS USING THE HYBRID LOGNORMAL DISTRIBUTION

\section{B. W. Dolan * P. G. Gröer* V. R. R. Uppuluri}

(Abatract of paper presented at the Health Physics Society Annual Meeting, Albuquerque. NM, June 25-28, 1989)

Distributions of occupational radiation exposures and doses are often thought to be lognormal. The hybrid lognormal distribution was proposed by Kumazawa and Numakunai to better model the effects of exposure limits and adminisirative. controls on the stochastic mechanism that generates occupational exposures/doses. Using the graphical procedure detailed by Kumazawa, we estimated the parameters to fit the hybrid lognormal distribution to some dose/exposure data for workers at two nuclear facilities. One data set was well represented by the lognormal distribution, the other seemed better characterized by the hybrid lognormal distribution. The lognormal behavior of the first data set may be explained by a relatively lower level of exposures, which results in a lessening of influence of regulatory limits on the stochastic exposures.

Research sponsored by U.S. DOE Office of Energy Research.

•Oak Ridge Aceociated Universities, Oak Ridge, TN.

\subsection{6}

\section{STATISTICAL GRAPHICS- THE VISUAL DISPLAY}

\section{J. Downing}

(Abatrect of oral presentation presented at the Society of Manufacturing Engineers Monthly Meeting at STIK Campus, Knoxville, TN, March 3, 1988)

Graphics in the form of charts, maps, and tables presenting statistical information began around 1700 . This talk will present a history of graphics dealing with graphical excellence and focus on problems that detract from graphical excellence such as: graphical deception, design variation, data variation, proportions and scaling, chart junk, data ink maximization, and others.

Research sponeored by U.S. Department of Enerzy.

1.127

\section{SOME OUTLIER DETECTION METHODS IN TIME SERIES ANALYSIS}

\section{J. Downing}

(Abetract of oral preacentation presented at the Univer. sity of Tennescee, Department of Statiatica, Knoxvilie, TN, February 4, 1988)

The subject of outlier detection has been dealt with by many people. The subject has grown to the point where it could really be treated as a field in its own right. The topic of outliers in time series has been a late bloomer and is now be- 
ginning to take shape - especially with the advent of the computer. This talk deals with the techniques currently used to detect outliers in time deries analysis.

Research sponsored by U.S. Department of Energy.

\subsection{8 \\ EVALUATING GOODNESS OF FIT OF POISSON REGRESSION MODELS IN COHORT STUDIES}
E. L. Frome
M. D. Morris

[A betrect of Am. Stet. 43(3), 144-147 (1989)]

Relative and absolute risk models are often used in epidemiologic studies to describe the effect of exposure on age-specific mortality rates. Poisson regression analysis is used to obtain maximum likelihood estimates of unknown parameters and to assess goodness of fit of the model. It is common practice to base both estimation and the evaluation of goodness of fit on certain marginal totals. This approach is potentially misleading for relative risk models and is totally inappropriate for absolute risk models. To illustrate the situation, we present an example using data on smoking and lung cancer. In the example, the goodnessof-fit tests based on the marginal totals indicate that neither the absolute risk nor the relative risk model can be rejected, whereas the age-specific test statistics strongly reject both models. The validity of the marginal test is based on an as sumption (i.e., of no exposure by age interaction) that is not satisfied in the example. We describe a more general approach to model evaluation that provides the analyst with summary information for various models of interest a.td provides guidance un how to identify good descriptive models.

Research sponeored by U.S. DOE Office of Healch and Environmental Remearch.
1.129

IS P A PRIME NUMBER?

SOME PROBABILISTIC

TESTS OF PRIMALITY

\author{
S. R. Jammalamadaka" \\ V. R. R. Uppuluri
}

[Abatrect of Math. Stiewtist 14, 55 (1989)]

A brief discussion of two probabilistic tests of primality is given. One of the main reasons for the new interest in large prime numbers comes from the so-called public-key cryptosystems. Chief among these is the RSA algorithm, which is also briefly outlined. The purpose of this note is to bring these ideas to a wider mathematical audience.

Research sponsored by IJ.S. DOE and the University of Californix, Sanca Barbara.

-University of California, Santa Barbara, CA.

1.130

\section{THE MIXED FUNCTION OXT: SE SYSTEM OF BLUEGILL SUNFISH, LEPOMIS MACROCHIRUS: CORRELATION OF ACTIVITIES IN EXPERIMENTAL AND WILD FISH}
B. D. Jimenez" G. H. Exull
L. S. Burtis*
B. Z. Egant

N. E. Lee

J. F. McCarthy $\$$

(Abatract of Environmental Toricalogy and Chemiotry 7 . 623-634 (1988)]

Environmental conditions can affect the metabolic transformation and accumulation of pollutants in aquatic organisms. Changes in temperature and feeding regimes had marked effects on the hepatic Odeethylation of 7-ethoxyresorufin (EROD) activity in bluegill sunfish. Animals that were denied food and acclimated for 2 weeks to 4,13 or $26^{\circ} \mathrm{C}$ water exhibited low and relatively uniform EROD activity at all temperatures. In contrast, fed fish acclimated at $26^{\circ} \mathrm{ex}$ hihited a sevenfold higher level of activity compared with those acclimated to $4^{\circ} \mathrm{C}$. Starved fish acclimated to $26^{\circ} \mathrm{C}$ were capable of EROD induction following intraperitoneal (i.p.) injection with benzola]pyrene (BaP). Doses of 10 and 20 $\mathrm{mg} \mathrm{BaP} \mathrm{kg}{ }^{-1}$ body weight increased FROD ac- 
tivity to 100 and $300 \mathrm{nmol} \mathrm{min}^{-1} \mathrm{mg}^{-1}$ respectively, compared with $10 \mathrm{nmol} \mathrm{min}^{-1} \mathrm{mg}^{-1}$ in fish injected with corn oil. Wild fish obtained from various stations along a polluted stream exhibited EROD activity levels comparable with those in laboratory fish injected with $20 \mathrm{mg} \mathrm{BaP} \mathrm{kg}{ }^{-1}$ fish. When starved fish were injected i.p. with a Purina Trout Chow extract, EROD activity was induced to levels comparable with those in laboratory-fed (Trout Chow) fish: this suggests the presence of an inducer in the Trout Chow. Exposure of fed fish to $\mathrm{pH} 5,7$, and 9 for a 2-week period at $26^{\circ} \mathrm{C}$ had no effect on the mixed function oxidase (MFO) activity in fish liver microsomes. The results of this study indicate that environmental variables can affect the levels of MFO activity. A better understanding of these effects is needed before these indicators can be used routinely as environmental monitors.

Research sponsored by L.S. Department of Energy.

* University of Tennesee, Knoxville, TN

† Millsaps College, Jackeon, MS.

'Chemical Technology Division.

$\oint_{\text {Environmental Sciences Division. }}$

1.131

\section{EIGHT BILLION MICE IS THE ANSWER. WHAT IS THE QUESTION? COMMENTARY FOR THE CITATION CLASSICS}

\section{A. Kastenbaum * K. O. Bowman}

(Abstract of Current Contents: Engineering, Technology, and Applied Science 20(42), 18 (1989); Cerrent Contents: Phyoical, Chemical, and Earth Sciences 20(42), 18 (1989); and Current Contento: Agricalture, Biology, and Environ. mental Science 20(42), 18 (1989)]

The previously published paper entitled " $\mathrm{Ta}$ bles for Determining the Significance of Mutation Frequencies," Wutation Research 0, 527-549, 1970, by . Marvin A. Kastenbaurm and K. O. Bowman was selected as (itation Classics. This paper provides answers to the question for the geneticist who has counted a total of $n$ mutations in two experimenial groups, "What is the number of mutation, $c$, in gersup, 1 that will allow ne to conclude that the mustatien frequency in group 1 is significantly greater than that of group 2?"
This note is a brief commentary on this work which provides the insights of publishing this paper.

Research sponsored by U.S. Department of Energy.

* Basye, VA.

1.132

\section{MASS LOSS AND NUTRIENT CHANGES IN DECOMPOSING UPLAND OAK AND MESIC MDXED-HARDWOOD LEAF LITTER}

\section{J. M. Kelly* J. J. Beauchamp}

[Abatract of Soil Sci. Soc. Am. J. 51, 1616-1622 (1987)]

Nylon net litterbags $(30$ by $30 \mathrm{~cm}$ ) containing $\sim 10 \mathrm{~g}$ of upland oak leaf litter were collected at 30-d intervals over a 1110-d period from two upland oak (Qvercus sp.)-mixed hardwood stands on the Cumberland Plateau of Tennessee. Statistically significant differences $(P \leq 0.01)$ in losses of mass, $\mathbf{N}, \mathbf{K}, \mathrm{Ca}$, and $\mathrm{S}$ were found between sites, whereas $\mathbf{P}$ and $\mathbf{M g}$ losses did not differ. Litterbags containing mesic mixed-hardwood leaf litter were collected at 30-d intervals over a 810-d period from mesic mixed-hardwood stands located on the same watershed study sites as the upland oak stands. Statistical comparisons indicated significant differences in mass, $\mathrm{N}, \mathrm{P}, \mathrm{K}, \mathrm{Ca}, \mathrm{Mg}$ and $S$ loss between sites. Constant rate projections of the time required for the upland oak litter weight to reach $10 \%$ of the original bag content based on 365,730 , and $1095 \mathrm{~d}$ of data were 960,1530 , and $1770 \mathrm{~d}$, respectively, at the Camp Branch Watershed and 870,1230, and $1410 \mathrm{~d}$ at the Cross Creek Watershed. Estimates of decomposition times derived from constant rate projections for both cover types were considerably less than observed values. In an attempt to obtaiis 'r re realistic projections of weight and nutrient loss, several mathematical models commonly used to exaniue decomposition data were evaluated. A morlified ex. ponential model gave the best data fit and pro vided estimates of 3240 and $2370 \mathrm{~d}$ as the time required to reach the $10 \%$ mass-semaining value for the upland oak litterhags at Camp Brancli and Cross Creek, respertively Fstimates for the mosic mixed-hardwoods were 1590 and 2490 d at Camp Branch and Ciross ('reek, respectively. "'he weright 
of most nutrients in the litterbags was modeled with limited success by the modified exponential model. However, $\mathbf{N}$ and particularly $\mathbf{P}$ weights could not be described by the model since they generally increased with time over the study $p$ riod. The results of this analysis, in addition to suggesting that a longer time is required for decomposition than is normally projected, also suggest that a single model may not be appropriate to describe all response variables. Data presented here along with the modeling work also reiterate the fact that a decay rate derived from a single species or group of species must be applied with caution over larger landscape units because of the potential differences both within and among sites.

Research sponsored by the Federal Interagency Energy/Environment Reseanch and Development Program and the Environmental Protection Agency.

-Cooperative Foreat Studies Program, Tennemee Valley Authority.

\subsection{3}

COMPARISONS OF DOSE-RESPONSE PARAMETERS FOR RADIATIONINDUCED ACENTRIC FRAGMENTS AND MICRONUCLEI OBSERVED IN CYTOKINESIS-ARRESTED LYMPHOCYTES

\section{G. Littlefield* A. M. Sayer* \\ E. L. Fome}

[Abatract of Mutegenesio $4(4), 265-270$ (1889)]

We employed cytoclialasin B to block cytokinesis in human lymphocytes exposed to $220 \mathrm{kV} X$ radiation. When $3 \mu \mathrm{g} / \mathrm{ml}$ of cytochalasin $B$ was used, most cells escaped the block whereas at 6 $\mu \mathrm{g} / \mathrm{ml}>90 \%$ of the mitogen-responsive cells became binucleate. Using the higher concentration of cytochalasin B, we observed a linear-quadratic (i.e. $Y=\gamma+\alpha D+\beta D^{2}$ ) dose dependency for $\mathrm{X}$-ray-induced micronuclei (MN) in preparations from three donors. When dose-response parameters were compared with those for total acentrics scored in first division metaphases, we observed no significant differences in estimates of the background $(\gamma)$ or linear $(\alpha)$ coefficients, but a 2 -fold reduction in the $\beta$ coefficient for $M N$. We interpret our data as providing evidence that radiation-induced nicronnclei are derived from acentric fragments ( $\mathrm{AF}$ ); that virtually all AF are recovered as $M N$ in binucleatc interphase daughter cells at low radiation doses; and that for our data, the relative proportion of AF that will be obeerved as independent MN decreases by a constant factor of approximately one-half as radiation dose increases.

Research sponsored by U.S. Department of Energy.

"Osk Ridge Associated Universities, Oak Ridge, TN.

1.134

MODULATION OF RADIATIONINDUCED CHROMOSOME ABERRATIONS BY DMSO-AN OH RADICAL SCAVENGER. 1. DOSE RESPONSE STUDIES IN HUMAN LYMPHOCYTES EXPOSED TO $220 \mathrm{kV}$ X-Rays
L. G. Littlefield ${ }^{*}$
E. E. Joiner*
S. P. Colyer*
A. M. Sayer

L. L. Rrome

[Abetract of Int. J. Radiet. Biol. 53(6), 875-890 (1988)]

Iluman $G_{o}$ lymphocytes were exposed to 220 $k V X$-radiation in the presence or absence of DMSO, an efficient selective scavenger of $\mathrm{OH}$ radicals. Our studies demonstrate that DMSO affects a concentration-dependent modulation of induced asymmetrical aberrations in human lymphocytes exposed to $\sim 3.0 \mathrm{~Gy}$, with maximum protectable fractions of approximately $70 \%$ at DMSO concentrations of $\geq 1 M$. The dose dependency for dicentrics in lymphocytes acutely exposed to X-ray doses of 0.51 to $4.98 \mathrm{~Gy}$ in the absence of DMSO is adequately described by the linear-quadratic doceresponse function $Y=\alpha D+\beta D^{2}$. Data from duplicate cultures exposed in the presence of $1 \mathrm{M}$ DMSO produce an excellent fit to the regression function modified as follows:

$$
Y(\mathrm{DMSO})=\alpha(\Delta D)+\beta(\Delta D)^{2}
$$

where the 'dose modifying' factor $\Delta=0.501$. We interpret these findings as providing evidence that OII radical-mediated lesions in DNA account for $\sim 50 \%$ of the dose dependency for dicentrics resulting from either one-t, rack or two-track events, following exponures of non-cycling cells to inoderate-to-ligh doses of low-L.ET radiation. These data may be used in additional calculations to derive an estimate of $\sim 6 \times 10^{8} \mathrm{~s}^{-1}$ for the rate of reaction of OII radicals with DNA targets involved in aberration formation.

Kenearch opononred by Applied Mathematiral Sciences Re. senrch Program, U.S. TOOF Office of Energo Rewearsh and by Oak Hidge Anorinted luivereitien.

\footnotetext{
- Oak Ridge Annercincedl Univarsitien, Oak Ridge. TN.
} 
1.135

\section{DETERMINING REGIONAL WATER QUALITY PATTERNS AND THEIR BCOLOGICAL RLLATIONSHIPS}

\author{
T. W. McDaniel ${ }^{*} \quad$ C. T. Hunsaker ${ }^{\dagger}$ \\ J. J. Beauchanup
}

[Abstract of Environmental Menegement 11(4), 507-518 (1987)]

A multivariate statistical method for analyzing spatial patterns of water quality in Georgia and Kansas was tested using data in the US Environnental Protection Agency's STORET data system. Water quality data for Georgia and Kansas were organized by watersheds. We evaluated three questions: (a) can distinctive regional water quality patterns be detected and predicted using only a few water quality variables, (b) are regional water quality patterns correlated with terrestrial biotic regions, and (c) are regional water quality patterns correlated with fish distributions? Using existing data, this method can distinguish regions with water quality very different from the average conditions (as in Georgia), but it does not discriminate well between regions that do not have diverse water quality conditions (as in Kansas). Data that are spatially and temporally adequate for representing large regions anc for multivariate statistical analysis as a available for only a few common water quality parameters. Regional climate, lithology, and biotic regimes all have the potential to affect $w$ ater quality, and terrestrial biotic regions and fish distribution do compare with regional water quality patterns, especially in a state like feorgia, where watershed characteristics are diverse. Thus, identifiable relationships between watershed cliaracteristics and water quality should allow the development of an integrated land-aquatic classification system that would be a valuable tool for resource management. Because gengraphical distributions of species may be limite. by zoogeographic and environmental factors, the recognition of patterns in fish distributions that correlate with regional water quality patterns could influence management strategies and aid regional assessments.

Researfi sponsored by U.S. Department of Energy.

- Hegional Air Pollution Control Agency. Dayton, OH.

$\dagger_{\text {Fonvironniental Sriences Division. }}$
1.136

\section{THE ROLE OF ASSUMPTIONS IN STATISTICAL MODELING}

\author{
M. D. Morris
}

(Abatract of oral presentation presented the Health Phyica Society Annual Meeting. Albuquerque, NM, June 25-28, 1989)

The process of modeling biological responses to radiation, like that of any scientific modeling activity, requires both specific evidence in the form of data and general structure in the form oi assumptions. These assumptions typically represent approximate or simplified statements about reality. To a great extent, their accuracy influences the validity of the modeling exercise, whils their specificity influences the precision of modeling. Particularly when data are sparse, or exist in contexts somewhat removed from those for which a model is needed, selection of assumptions upon which statistical modeling is based should be considered a crucial part of the scientific process. Two examples are discusses demonstrating the effect of careful and relevant (as opposed to "automatic") assumption selection.

Research oponsored by Defence Nuclear Agency.

\subsection{7}

\section{SOME EFFECTS OF RADIATION DOSIMETRY ERRORS ON AN ESTIMATED DOSE-RESPONSE RELATIONSHIP}

\section{D. Morris T. D. Jones*}

[Abatract of Healeh Phys. 56(2), 219-222 (1989)]

Estimates of mortality due to marrow injury for humans exposed to ionizing radiations are commonly based upon data taken either on radiation workers following an accident or victirns of $\mathrm{Hi}$ roshima and Nagasaki. Although these exposures are quite different, a primary source of uncertainty in the analysis of either case is the dosimetry. Various physical measurements from mockup assemblies and dose calculations are employed in order to estimate a bone marrow dose received by each individual of interest. These values are paired with the eventual result of the exposure for each individual (in this case, death or survival) in cider 
to estimate a dose-response function. This last step usually accomplished essentially as it would te in an experimental setting in which animals are intentionally exposed to varying doses of a controlled treatment; modeling techniques ranging from informal graphics to parametric statistical modeling are used to estimate parameters of interest such as the $\mathrm{LD}_{\mathbf{5 0}}$.

In the somewhat simpler modeling problem of linear regression, it has long been known that in certain cases, random errors in the independent variable can result in a biased estimate of the slope. More recently, E. S. Gilbert lias presented results of calculations of bias in the estimated slope of a linear dose-response function for dichotomous data. These calculations were specific lor the assumed distribution of true doses received by individuals and investigated the effects of certain modifications in data analysis such as the pooling of individuals by dose groups, removal of individuals with very high doses, etc. Examples of estimated bias for various case specific mortality dose-response calculations from Hiroshima and Nagasali studies were also given.

Our aim is similar to Gilbert's in that we will address the degree of estination bias (or "flattening") of a dose-response curve due to uncertainty in dose levels. However, we will not follow Gilbert's unconditional approach which requires that the distribution of doses among individuals be considered. We will specifically address the degree of distortion in the apparent dose-response relationship which exists independently of distribution of doses or statistical estimation mettod employed. Finally, our presentation is based upon a log-normal model, which allows simple, closedform expressions of the effects of uncertainty in dose on the apparent $L D_{10}$ and other quantities of interest.

Research sponsored by Delerse Nuclear Agency.

"Health and Safety ilesearch Division.
1.138

\section{HEMATOPOIETIC DEATH OF UNPROTECTED MAN FROM PHOTON IRRADIATIONS: STATISTICAL MODELING FROM ANIMAL EXPERIMENTS}

\author{
M. D. Morris T. D. Jones*
}

[Abstract of Int. J. Rediet. Biol. 55(3), 445-461 (1989)]

Most estimates of the radiation dose lethal to $\mathbf{5 0}$ per cent of a human population are based on historical data taken from well-known experiences reflecting inadequately known physical and biological conditions, or from medical procedures where individual patients received advantages of modern clinical care. It has been debated as to whether the experience of unprotected man would more closely reflect that of hospital patients or of the radicbiological studies with large animals. The issue at question is whether the apparent two-fold or more increased susceptibility of large animals to death from bone marrow damage (compared with the majority of estimates for man) is due more to true inter-species differences or the lack of medical support.

This study is an attempt to assess the radiosensitivity of unprotected man in terms of the composite animal experiments. Based upon an extensive data base containing 121 separate animal studies using 13 different species, an estimate of the mortality dose-response relationship due to a unifo:m, continuous field of photon radiation is predicted for $70 \mathrm{~kg}$ unprotected man. Man is assumed to have a level of radiation sensitivity similar to that of the species represented in the data base, after adjustinent for body weight. The mathematical model used includes fixed terms to account for effects of body weight and dose rate, and random terms reflecting inter- and intraspecies variation and experimental error. Point predictions and 95 per cenc prediction intervals are given for the $L D_{05}, L D_{10}, L D_{25}, L D_{50}, L D_{75}$, $L D_{90}$, and $L D_{95}$, for dose rates ranging from 0.01 to $0.5 \mathrm{~Gy} / \mathrm{min}$, and treatment times ranging from about $2 \mathrm{~min}$ to about $24 \mathrm{~h}$. At $1 \mathrm{cG} / \mathrm{min}$ our point prediction of the $L_{30} D_{30}$ is 299 c(iy with an associated 95 pei cen: prediction interval of (168 c(iy, 535 c(jy). The analogous values at 50 $c(i y /$ min are 183 c(iy and (103 c(iy. $326 c(i y)$.

Reaenrth sponsored by Delenar Nur:. ar Agrucy.

"Health and Siafety Menrarch Divisirn. 
STATISTICAL APPROACH TO COMBINING THE RESULTS OF SIMILAR EXPERIMENTS WITH AF? IICATION TO THE HEMATOLOGIC EFFECTS OF EXTREMELY-LOW-FREQUENCY ELECTRIC FIELD EXPOSURES
M. D. Morris
K. T. Kimball*
T. E. Aldrich ${ }^{\dagger}$
C. E. Basterly ${ }^{\dagger}$

[Abstract of Bioelectromagnetics 10, 23-34 (1989)]

A large proportion of scientific effort in investigating the possible biological effects of exposure to extremely-low-frequency (ELF) fields consists of laboratory studies on experimental animals. Most experiments in which hematologic properties are measured show no statistically significant effect due to exposure. However, some studies show significant effects, which, in general, are not clearly reproducible. A difficult question must then be addressed: Ars these relatively few indications of ELF effects statistical artifacts due to the increased risk of a type I etror in multiple studies, or is there a real biological effect that is undetected in most studies due to the relatively small sample sizes commonly used? A statistical approach for examining the accumulated results of multiple experiments which results in a single test for treatment effect is presented: The technique requires very mild assumptions, and is valid for experiments that vary widely in specific characteristics as exposure level, duration, and laboratory. The method is applied to the results of a collection of hematologic and serum chemistry experiments, and the rombined results indicate the existence of experimental effects on some end points.

Research sponsored by Assintank Secretary for Conservetion and Renewable Energy, U.S. DOE Office of Energy Storage and Distribution, Electric Energy Systema Pro gram.

"Univenity of Texas, Hounton. TX.

thealth and Safety liesearch Division.

\section{WEIGHTED VOTING MODELS}

\author{
V.R. R. Uppuluri
}

(Abatract of oral presentation presented at the East Tennessee Chapter of the American Statistical Association. Oak Ridge, TN, March 8, 1988)

In the conventional system of voting where "one person has one vote," each person asserts equal influence on the outcome. On the other hand there are voting situations in which some individuals or blocks of voters effectively cast more ballots than others. Such weighted voting systems are found in the Electoral College, in voting by stockholders in a corporation and other similar situations. In this presentation, we discuss two measured of voting power of the blocks of voters, one due to Shapley and Sluubil and the other due to Banzhaf. These indices of voting power will be illustrated. A probabilistic framework for these indices of power will also be discussed. An example where different metiods may choose different winners will be illustrated. Further, there will be a brief discussion on "approval voting."

Research sponsored by U.S. Debartment of Energy.

\subsection{1}

AN APPLICATION OF THE
MAXIMUM RATIO FOR
COMPARING DIFFERENT
PETROLEUM ESTIMATES

\section{T. Wright}

(Abatract of oral presentation presented at the 1988 Joint Statiutical Meeting. New Orleans, LA, August 22-25, i988)

Tsao and Wright (1983) introduced a simple tool called the maximum ratio that can be used when comparing several estimates $\hat{\theta}_{1}, \hat{\theta}_{2}, \ldots, \hat{\theta}_{m}$ (possibly from different sources) of the same parameter. The maximum ratio not only gives a measure of closeness among the comparable estimates, but also gives an indication of liow the rollection of estimates stands relative to the unknown true value of $\boldsymbol{\theta}$. As part of a receut Frames Update Project sponsored by the Petroleum Supply Division (PSD) of the Energy luformation Administration of the U.S. Department of Fincrgy, the maximum ratio was applied to nationwide estumates for the fuels: motor gasoluue, distillate furl, and 
reaidual fuel. This paper illustrates the application of the maximum ratio in the project as a first step towards assessing the quality (completeness) of a sampling frame.

Rewench sponeored by U.S. DOE Energy Information Adminiatration.

\subsection{2}

WHEN ZERO DEFECTIVES APPEAR IN A SAMPLE: UPPER CONFIDENCE COEFFICIENTS OF UPPER BOUNDS

\section{T. Wright}

[Abetract of Americen Stetiolicien (in prea)]

When zero defectives are observed in a sample from a finite universe, investigators and decision makers are often tempted to (and want to) declare that there are zero defectives in the finite universe with high confidence. By focusing on the more general question of upper bounds for confidence coefficients of upper confidence bounds, as a special case we are able to show explicitly that our confidence that there are zero defectives in the universe wiren zero defectives appear in the sample is bounded by the sanspling fraction. This note is meant to help reassure those who seek to help others yield not to the temptation mentioned above.

Research sponeored by Applied Mathemntical Sciences Re search Program, U.S. DOE Office of Energy Revench. 
Section 2

NUCI.EAR DATA MEASUREMENT AND EVALUATION 


\title{
2.0. INTRODUCTION
}

\author{
R. W. Peelle
}

Our primary effort is devcted to experiments using the Oak Ridge Electron Linear Accelerator (ORELA) pulsed neutron source to obtain neutron cross sections needed for efficient and safe application of nuclear technology. Additional effort is expended combining experimental results with theory to generate complete sets of evaluated cross sections which are used by engineers to design and evaluate systems that require nuclear data as input. In the US, the cooperatively evaluated and tested file of such evaluations is called ENDF/B. The first release of ENDF/B version VI is expected by early 1990, about a decade after the corresponding release of ENDF/B-V. About $60 \%$ of the evaluations were performed by our group. The new file will be quite superior to previous ones in its physics content, and will surpass many of the goals set out several years ago. However, the current body of knowledge will not be completely represented by this relcase, so future releases of ENDF/BVI revisions are anticipated.

We contributed strongly in three areas in which ENDF/B-VI is markedly improved: (a) Realistic neutron emission angular distributions as a function of neutron energy are now included to properly treat the $14-\mathrm{MeV}$ neutrons produced in a fusion reactor. This was possible because of our fundamental improvements in the pre-equilibrium part of the comprehensive models used, as implemented in our TNG nurlear model code, and because of newly extended ENDF format capabilities. (b) New resonance-region parameterizations are included for the most important fission fuel materials and for some structural materials. These are based primarily on fits to the resonance structure of new ORELA data using SAMMY, our advanced resonance fitting system. SAMMY is based on the appropriate Reich-Moore R-matrix formalism. The collaboration with Herve Derrien, now on his third visit from CEN Cadarache, France, on ${ }^{23 .} \mathrm{Pu}$ and ${ }^{241} \mathrm{Pu}$ has been particularly noteworthy. (c) Finally, more general formats have been developed to remove some severe constraints on our variance/covariance evaluations. In the first two cases the best fruits of nur effort will appear in the first release of the file. Covariance data will be incomplet 2 in this first release because of time pressure.

This year has been an exciting time at ORELA. Because of our expertise in neutron detection, staff members became involved in some "cold fusion" confirmation efforts. We didn't demonstrate any ne:utron emission. More exciting in the long run, we are working with visitors on a most interesting array of experiments:

(a) M. C. Moxon of AERE harwell, England, is making measurernents of $\eta$ (average number of neutrons emitted from fission per neutron absorbed) for ${ }^{235} \mathrm{U}$ in the thermal neutron energy range where $\eta(E)$ can affect the performance and temperature confficient of reactivity of commercial light-water reactors.

(b) Al Wasson and Roald Schrack from the National Institute of Science and Technology (formerly NBS) are remeasuring with our heip the ${ }^{10} B(n . \alpha \gamma)$ reaction so important to the use of ${ }^{10} \mathrm{~B}$ as a neutron standard. It has proven difficult to measure this cross section with good accuracy.

(c) Jïurg Srhmiedneier and Peter Riehs of the Cniversity of Viruna, Austria, are measuring the electric polarizability of the neutron with our collaboration. The teclunicgue deperuds on a precision mensurement of the cuergy rependence of the total cross 
section of ${ }^{208} \mathrm{~Pb}$, a nuclide with suitably high nuclear electric field and sufficiently few resonances to allow nuclear effects to be analyzed.

One reason these experimenters have sought out ORELA is that the elcctron linacs at NIST and at Harwell have been shut down. The other reason is that intensity and background considerations make ORELA the best choice in any case.

Users from within ORNL have also been doing unusually interesting work. Herb Mook has been checking out published results that the copper in the high temperature superconductor $\mathrm{YBa}_{2} \mathrm{Cu}_{3} \mathrm{O}_{7}$ exhibits an unusually high Debye temperature. The measured Doppler spreading of copper neutron resonances in transmission is inconsistent with this suggestion. Lester Hulett and Dave Donahue have produced a strong farasitic positron beam from ORELA and are proving its utility for experiments on unique molecular excitations and on near-surfice materials properties.

The years just ahead promise to be productive as the above experiments are completed, as we exercise an updated system for measurement and analysis of capture gamma ray cross sections in the resonance region where they have proven to be tricky to measure in medium-A nuclides, as we commission a photon multiplicity detector for low energy capture cross sections in fissile nuclides, as we carry forward a neutron emission spectrum program important to cross sections in the fusion energy region, and as we complete evaluation refinements for updates for ENDF/B-VI. Funding stringency is expected to stretch the program and delay new experiment starts not staffed by guests.

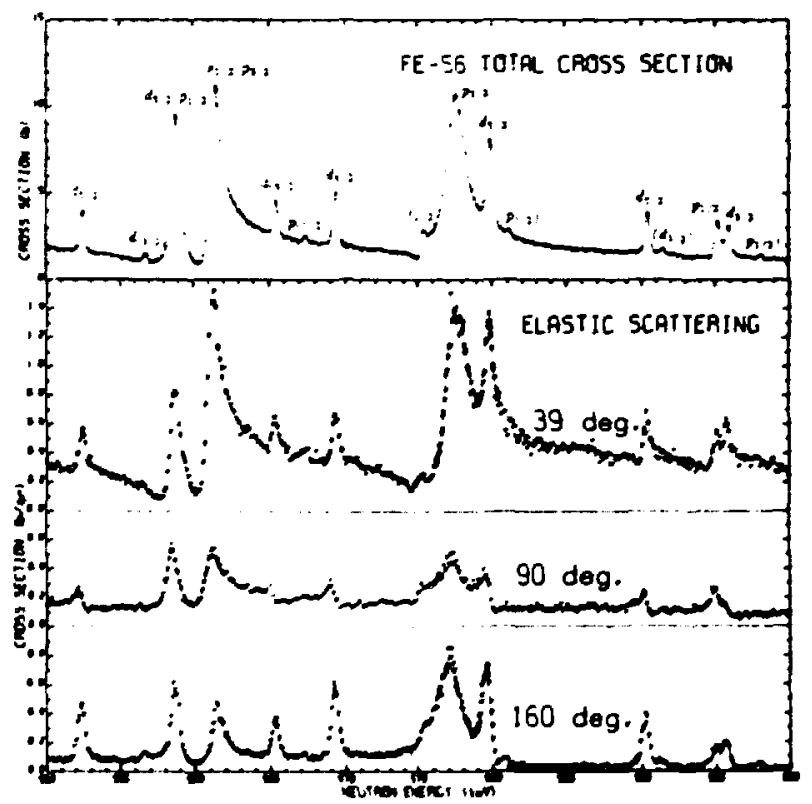

Fe-56 ORELA Total and Elastic Cross Section Data and Analysis. In the upper graph the ${ }^{56}$ F 200-m total cross section data are compared with the theoretical calculations obtained with the multileve! R-matrix code SAMMY. The spins and parities were assigned using the elastic scattering data sh.swn in the thier lower graphs. The parameters obtained from the fit to the transmission data were used to calculate the theretica' rlastic scattering cross section with the R-matrix code RFUNC. The combination of spins and parities adopted is the one which yields the best agreement with the elastic scattering data. Parentheses are used in indicate that the assignment is uncertain. 


\section{EXPERIMENTAL RESULTS}

2.1

\section{THE ${ }^{151}$ Sm BRANCHING, A PROBE FOR THE IRRADIATION TIME SCALE OF THE S-PROCESS}

\section{H. Beer R. L. Macklin}

[Atatract of Asirophys. J. 331, 1047 (1988)]

The excitation functions for the reactions $152,154,155,157 \mathrm{Gd}\left(n_{*} \gamma\right)$ have been measured over the neutron energy range of $5 \mathrm{keV}$ to $500 \mathrm{keV}$. Maxwellian averaged capture cross sections for thermal energies $k T=5$ to $100 \mathrm{keV}$ have been calculated. At $k T=30 \mathrm{keV}$ we have found: $\sigma\left({ }^{152} \mathrm{Gd}\right)$ $=1003 \pm 30 \mathrm{mb}, \sigma\left({ }^{154} \mathrm{Gd}\right)=878 \pm 27 \mathrm{mb}$, $\sigma\left({ }^{155} \mathrm{Gd}\right)=2721 \pm 90 \mathrm{mb}, \sigma\left({ }^{157} \mathrm{Gd}\right)=1355$ $\pm 39 \mathrm{mb}$. The data, in conjunction with other cross sections and solar abundances, were used to carry out an s-process analysis of the branchings in the $\mathrm{Sm}$ to $\mathrm{Gd}$ mass range. The s-process is treated in the classical as well as in the pulsed model. The solution of the classical model is contained in the pulsed :nodel as the asymptotic solution for large pulse vidths. It is shown that this solution is the cnly one which can reproduce the abundance pattern of the different branchings. Pulse durations are limited to values larger than about $3 \mathrm{yr}$.

Research sponsored by Kernfornchungazentum Karlaruhe, Institut Tür Kernphysik III, Karlaruhe I. FRG and by U.S. DOE Division of Nuclear Physics.

"Kernforachungazentrum Karbruhe, Inatitut für Kemphysik III, Karlsrube 1, Federal Republic of Germany.

\section{2}

\section{MEASUREMENT OF THE ${ }^{8}$ Rb AND ${ }^{87}$ Rb CAPTURE CROSS SECTIONS FOR \&-PROCESS STUDIES}

\section{$\begin{array}{ll}\text { H. Beer* R. L. Macklin } & \text { R }\end{array}$}

[A bat.ract of Astrophy. J. 339, 962 (1989)]

The excitation functions for the reactions ${ }^{85,87} \mathrm{Rb}(n, \gamma)$ have been measured over the neutron energy range of $175 \mathrm{eV}$ to $700 \mathrm{keV}$. Maxwellian averaged capture cross sections for thermal energies $k \cdot T=5-100 \mathrm{keV}$ have been calculaled. The data were used to carry out aprocess calculations. Solar a-process abundances were reproduced with a two component phenomenologiral model below mass number 90, a single Mux weak spencess and a pulsed s-process characterized by exponentially distributed neutron exposures. The solar s-process abundances as well as s-process abundances from Ba stars and the Murchison meteorite were studied in the frame of the AGB-models. Constraints were derived concerning the properties of the neutron pulses for a description of the empirical s-process data.

Research sponsored by Ketnforschangerentrum Karlarube, Institut fur Kernphyril III, Karlarube 1, FRG and by U.S. DOE Division of Nuclear Physia.

"Kernforschangzentrum Karbrube, Institut für Kernphysik III, Karlarube 1, Federal Republic of Germany.

\section{3}

\section{TESTS FOR "COLD FUSION" IN THE PD-D 2 AND TI-D SYSTEMS AT 40 TO 380 MPA AND - 196 TO $27^{\circ} \mathrm{C}$
J. G. Blencoe*
M. T. Naney*
D. J. Wesolowski*
F. G. Perey

[Abatract of Fasion Energy (in preas)]

Experiments have been conducted on the $\mathrm{Pd}$ $D_{2}$ and $T i-D_{2}$ systems at 40 to $380 \mathrm{MPa}$ and -196 to $27^{\circ} \mathrm{C}$ to investigate the possibility that "cold fusion" occurs in palladium and titanium deuterides generated by reaction with high-pressure $D_{2}$ gas. The experiments were performed using a $4.8 \mathrm{~mm}$ i.d. stainless steel pressure vessel that can be operated routinely at pressures as high as $400 \mathrm{MP}$.

Experimental results obtained so far range from negative to potentially significant. No sustained heat production has been olsserved in any experiment. Thermal pulses that persist briefly after pressurizing $P d$ with $D_{2}$ gas are attributable to small amounts of cheisical heat released when $P d$ and $D_{2}$ react to form palladium deuteride. No sustained neutron flux above background was observed in any $P d-D_{2}$ experiment.

On the other hand, in a ' $\mathrm{Ti}-\mathrm{D}_{2}$ experiment just completed, potentially significant results were obtalned. During this experiment, there was a period of five ennsec utive lours when count rates rose 10 appsoximately 60 coumts/hour abeve the average background rate. This detector count rate corresponds. nominally to IOOO) neutrons/lour 
emitted from the Ti- $D_{2}$ sample. However, due to several deficiencies in our neutron detection methods and equipment, we cannot demunstrate conclusively that our experimental data are valid. Consequently, we are upgrading our neutron detection equipment in preparation for a second, improved Ti- $\mathrm{D}_{2}$ experiment.

Research sponsored by U.S. DOE Division of Nuctear Phyics.

Chemistry Division.

\section{4}

\section{LEVEL DENSITIES FROM RESONANCE SPECTROSCOPY FOR $n+{ }^{86} \mathbf{K r}$ \\ R. F. Carlton* \\ R. R. Winters \\ C. H. Johnson N. W. Hills \\ J. A. Harvey}

[Abat ract of oral presentation given at the American Phyeical Society Ainnual Spring Meeting. Baltimore, MD, April 18-21, 1988: Proc. Vol. 33(4), p. 1061 (1988)]

High resolution transmission measurements on a high purity ${ }^{86} \mathrm{Kr}$ gas sample were performed at the ORELA facility over the neutron energy range of $0.015-25 \mathrm{MeV}$. From R-Matrix analysis of these data in the resolved resonance region be low $1 \mathrm{MeV}$, we have detcrınined the $n+{ }^{86} \mathrm{Kr}$ level densities for $J^{\pi}=1 / 2^{+} \cdot 1 / 2^{-}, 3 / 2^{-}$reaction channels in the 1-MeV energy interval above neutron binding. The energy dependence of the $3 / 2^{-}$ level density, for which the uncertainties are smallest, is suitably modeled by a back-shifted Fermi gas model with two adjustable parameters. This model is then used to predict the level densities for $j^{\pi}=1 / 2^{+}$and $1 / 2^{-}$. Except for an unusual nonstatistical behavior in the $500-600 \mathrm{keV}$ region for the $1 / 2^{-}$cumulative number of levels, the model is seen to give an excellent description of all three data sets. Parameters oblained are consistent with systematic trends in this mass region.

Researct sponeored by I.S. DOE Division of Nucleas Physirn.

* Mirldle Tennemser State University, Murfresoboro. TN.

tDeniaron Liniversity, (iranville, :)ll.

:Pliywirn Division.

\&

Inat rumentation and (onterrin I)ivinion.
2.5

\section{UNIFIED DESCRIPTION OF THE $n+{ }^{200}$ Bi MEAN FIELD BETWEEN $1 \mathrm{MeV}$ TO $40 \mathrm{MeV}$ VIA DISPERSION RELATIONS}

\author{
R. K. Das* R. W. Finlay*
}

\section{J. A. Harvey}

[Abetract of paper presented at the American Physical So ciety Annual Spring Meetine. Baltimore, MD, April 18-21. 1988; Proc. Vol. 33(4). p. 964 (19e8)!

The real part of the central $n+{ }^{209}$ Bi mean field is the sum of a Hartree-Fock component whose radial dependence is assumed to have a Wood-Saxon shape and whose depth is energy dependent plus a dispersive component which is determined from the imaginary part of the optical model potential. Elastic neutron scattering data from 1.5 MeV to $24 \mathrm{MeV}$, along with total cross section from 1 to $40 \mathrm{MeV}$, is used for the above analysis. The energy dependence of the geometry of the optical model is also studied.

Research sponsored by U.S. DOE Division of Nuclear Physice.

"Ohio University. Athens. OH.

\section{6}

\section{USE OF MONTE CARLO TECHNIQUES TO DERIVE YIELDS FOR $n+{ }^{12} \mathrm{C}$ MULTI-BODY BREAKUP REACTIONS}

\section{J. K. Dickens}

[Abatract of Computers in Physics 3, 62 (1989)]

A computer "experiment" using Monte Carlo sainpling methods has been designed to simulate the breaking up of ${ }^{12} \mathrm{C}$ by medium-energy neutrons into final reaction channels having 2,3 , or 4 outgoing charged particles. The calculational nuelear physics concept used in the "experiment" is one of a sequentially decaying highly-excited compound nucleus. Two methods of Monte Carlosampling, the rejection methor and the cumulativedistribution methorl, are discussed as applied in probability functions developed in the program.

Rewarth sponanred by U.S. DOF, Division of Nuclear Phynirs. 
2.7

\section{${ }^{10,11} \mathbf{B}(n, x \gamma)$ REACTIONS FOR INCIDENT NEUTRON BNERGIES BETWEEN O.1 AND $25 \mathrm{MeV}$}

\author{
J. K. Dickens \\ D. C. Larson
}

[Abatract of paper presented at the Intermational Conference on Nuclear Date for Science and Technolog. Mito, Jepan, May 30-June 3, 1988; Proc. S. Larai, Ed., Sailon Publishing Co., Led., pp. 213-215 (19a8)

Measurements have been made of gamma-ray production due to neutron interactions with samples of boron for incident neutron energies between 0.1 and $25 \mathrm{MeV}$. For ${ }^{11} \mathrm{~B}$ a 54-g sample of naturally-occurring boron in the shape of a solid cylinder was used. For ${ }^{10} \mathrm{~B}$, samples enriched to $95 \%$ in the ${ }^{10} \mathrm{~B}$ isotope were used. One sample, about $49 \mathrm{~g}$, was used to obtain $\gamma$-ray data following inelastic scattering; the other sample, of about. $15 \mathrm{~g}$, was used to delineate the production of the 478-keV gamma ray following the ${ }^{10} \mathrm{~B}(n, 0){ }^{7} \mathrm{Li}$ re action for $E_{n}$ between 0.1 and $4 \mathrm{MeV}$. The Oak Ridge Electron ! inear Accelerator (ORELA), a white source, was used to provide the incident neutrons. Data were obtained using a high-purity intrinsic-germanium photon detector positioned at 125 deg with respect to the incident neutron beam. Because the peak shapes in the raw data were substantially broadened by Doppler motion, peak yields were determined with graphic interactive methods. Except for the 478-keV gamma ray for $E_{n}<1 \mathrm{MeV}$, the $\gamma$-ray production croses sections are all small, the largest being $<200 \mathrm{mb}$.

Reacarch sponeored by U.S. DOE Diviaion of Nuclear Physica.

\section{8}

THE NE-110 SCINTILLATOR RESPONSE TO 10- TO 100-MeV CARBON IONS

\section{J. K. Dickens \\ J. W. McConnell *}

[Abstract of Nuel. Instrum. Methods in Physice Researth A281, 577 (1989)]

Measurements of the response of NF-110 organic scintillator to 13-, 34-, 44-, 70-, and 95- MeV incident carbon ions are reported.

Rewarsh aponsored by U.S. DOF, Division of Niclear Phynira.

- Physica Divinirn.

2.9

\section{A NE 110 SCINTILLATION DETECTOR FOR keV ENERGY NEUTRONS}

\author{
J. A. Harvey N. W. Hail
}

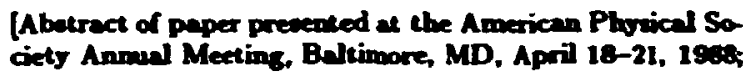
Proc. Vol. 33(4) p. 1078 (19es)]

We have developed a neutron detector with a narrow resolution function which is efficient for keV energy neutrons, has low background, and is not sensitive to overlap neutrons $(<200 \mathrm{eV}) 80$ the pulsed accelerator can be operated at a high repetition rate ( $800 \mathrm{pps})$. The detector consists of a 9 by $9-\mathrm{cm} \mathrm{NE} 110$ scintillator, $1 \mathrm{~cm}$ thick, mounted in a $0.025-\mathrm{mm}$ mylar reflecting cylindet, and epoxy-soupled on opposite edges to two 12.5cm-diameter RCA 8854 photomultipliers (PMs). Each PM is biased below the single photoelectron level, and a coincidence is required between the outputs of the two PMs to eliminate counts due to PM noise. The detector has been studied down to a neutron energy of $50 \mathrm{eV}$. The obeerved sigmalto-background ratio was 300 at $12 \mathrm{keV}$ and 30 at $2 \mathrm{keV}$. The detector has an efficiency of $40 \%$ at 15 $\mathrm{keV}$ (ten times that of a 1-cm-thick ${ }^{6} \mathrm{Li}$ glase seintillation detector) and its efficiency equals that of the ${ }^{6} \mathrm{Li}$ detector at $4 \mathrm{keV}$. The timing resolution of the deteclor is 7 nsec for $10-\mathrm{keV}$ neutrons, limited by the neutron flight time through the scintillator and is less for higher energy neutrons. Results from transmission measurements on ${ }^{238} \mathrm{IJ}$ will be presented.

Rewearch sponsored by U.S. DOE Division of Nuclear Phyrica.

\footnotetext{
"Inetrumentation and Controls Division.
} 
2.10

\section{HIGH-RESOLUTION NEUTRON TRANSMISSION MEASUREMENTS ON ${ }^{235} \mathrm{U},{ }^{230} \mathrm{Pu}$, AND ${ }^{238} \mathrm{U}$}
J. A. Harvey
N. W. Hill*
F. G. Perey
G. L. Treedt

\section{Leal ${ }^{\dagger}$}

[Abatract of paper presented at the International Conference on Nuctear Date for Science and Technolos,. Mito, Japan, May 30-June 3, 1988; Proc. S. Izareci, Ed., Saibon Publishing Co., Led., pp. 115-118 (1988)

High-resolution transmission measurements have been made on three sample thickneases of both ${ }^{235} \mathrm{U}$ and ${ }^{239} \mathrm{Pu}$ at liquid nitrogen temperacure and also on three of ${ }^{238} \mathrm{U}$ at room temperature using neutrons from the water moderated ORELA target. The data on ${ }^{235} \mathrm{U}$ and ${ }^{239} \mathrm{Pu}$ from 1 to $10,000 \mathrm{eV}$ were obtained using ${ }^{6} \mathrm{Li}$ glass scintillation detectors at 17.909 and $80.394-m$ fight paths. The ${ }^{238} \mathrm{U}$ dats from 1 to $100 \mathrm{keV}$ were obtained using a new NE 110 proton-recoil scintillation detector at a 201.558-m fight path.

Reseanch eponeoned by U.S. DOE Divivion of Nuctear Phynics.

"Inatrumentation and Controls Divition.

fUnivarity of Tennewee, Knoxville, TN.

\subsection{1}

\section{COLD FUSION NEUTRON MEASUREMENTS AT ORNL}
D. P. Hutchinson"
R. K. Richards*
C. A. Bennett*
C. C. Havener
C. H. Ma*
F. G. Perey
R. R. Spencer
J. K. Dickens
B. D. Rooney
J. Bullock, IV'

\section{G. L. Powell ${ }^{\dagger}$}

(Abalrace of paper presented at the Workehop on Cold Fuion Phenomena, Sanke Fe, NM, May 23-25, 1900; and - paper entilled "i Search for Coid Fuaion Neutron al ORELA" presented at the Spring Meeting of the American Physical Society, Baltimore, MD, May 1-4, 1989; Proc. Vol $34(1)(1980)\}$

A number of experiments were begun on Ma:rh 29, 1989 to lorik for neutron emission from a palladium cathode in an electrolytic cell using a deutrated clertrolyte. Several different electrode configurations were tried. The fast neutron detector utilized a pair of NE213 scintilla- tor/photomultiplier pairs in a shielded enclosure. This neutron detector has an efficiency of $13 \%$ and records a background count rate of 200 events per hour. At present no neutron counts above the background level have been detected.

Research epansored by U.S. Department of Energo.

*Phyaica Division.

TY-12 Developmen Division.

2.12

${ }^{63} \mathrm{Cr}\left(\mathrm{n}, \mathrm{n}^{\prime} \gamma\right)$ REACTIONS AND THE LEVEL STRUCTURE OF ${ }^{53} \mathrm{Cr}$

\section{C. Larson J. K. Dickens}

[Abetract of Plys. Rev. C 30,1736 (1909)]

Gamma-ray decay of levels in the stable isotope ${ }^{53} \mathrm{Cr}$ has been studied using ${ }^{53} \mathrm{Cr}\left(n, n^{\prime} \gamma\right)$ reactions for inciden: neutron energies between threshold and $10 \mathrm{MeV}$. Measured gamma-ray production croes sections have been compared with earlier measurements and with cros sections calculated using precompound-compound-nucleus theory. Although agreement with earlier work is generally adequate, some of the present results are at variance with earlier experimental or evaluated results. For example, for the decay of the $E_{x}=$ $1537-\mathrm{keV}$ level we are unable to explain variations in the measured branching ratios of the transition gamma rays as a function of incident neutron energy. The experimental data were analyzed within the framework of several theoretical model calculations of the level structure of ${ }^{53} \mathrm{Cr}$. Some quantitative discrepancies are discussed.

Research sponsored by U.S. DOE Division of Nuclear Phyica.

\subsection{3}

$$
\begin{aligned}
& \text { NEUTRON CAPTURE BY }{ }^{70^{\circ}} \text {, } \\
& { }^{81} \text { Br, AND }{ }^{76} \text { As } \\
& \text { R. L. Macklin } \\
& \text { [Abatrect of Nel. Sci. Eng. } 99,133(1968) \text { ] }
\end{aligned}
$$

Neutron capture up to $700 \mathrm{keV}$ was measured for arsenic and bromine and for an enriched sample of ${ }^{81} \mathrm{Br}$. Individual resonance capture was par rameterized in the first several kilmelectron-volts 
and average capture cross sections derived at the higher energies. M!axwellian average cross sections found $\mathrm{frr}{ }^{78,81} \mathrm{Br}$ and ${ }^{75} \mathrm{As}$, respectively, were 741 $\pm 30,244 \pm 10$, and $455 \pm 18 \mathrm{mb}$ for a temperature $t T=30 \mathrm{keV}$. Resonance capture integrals calculated for ${ }^{79,81} \mathrm{Br}$, respectively, were $130 \pm$ and $46.6 \pm 1.8 \mathrm{~b}$.

Research sponeored by U.S. DOE Division of Nuckenr Phyica.

\subsection{4}

\section{SEARCH FOR ${ }^{186} \mathrm{Xe}$ RESONANCE NEUTRON CAPTURE}

\section{R. L. Macklin}

(Abatract of ORNL/TM-10766, Mey 1988)

Evidence for neutron capture in ${ }^{136} \mathrm{Xe}$ at $2154-\mathrm{e}$ and 18.4keV resonances is presented and quantified in terms of limits on Breit-Wigner single level parameters. Assuming the radiation width, $32 \mathrm{meV}$, found at the $18.4 \mathrm{keV}$ resonance for all the reported resonances at higher energies, the Maxwellian average capture crnes section is calculated for a range of stellar interior temperatures $T$. For $k T=30$ keV only $0.72 \mathrm{mb}$ is found. Only one-third of this comes from the resonances above $18.4 \mathrm{keV}$ so an overall uncertainty at $k T=30 \mathrm{keV}$ of $\pm 0.11 \mathrm{mb}$ at the $68 \%$ probability level seems reasonable. Four resonanies in ${ }^{134}$ Xe were also found.

Research sponeored by U.S. DOE Divieion of Nuclear Physics.

\subsection{5}

\section{NEUTRON ABSORPTION CROSS SECTION OF URANIUM-236}
R. L. Macklin
C. W. Alexander"

(Abstract of Neel. Sei. Ens. (in prenu); dso ORNI.; rM10990. November 1988)

U-236 neutron absorption was measured as a function of neutron time-of-flight from $20 \mathrm{eV}$ to 1 $\mathrm{MeV}$. The neutron flux was monitored with a ${ }^{6} \mathrm{Li}$ glass scintillator. Average cross-sections from 3 keV to I MeV were derived. Fstimated uncertainties were less than $5 \%$ below $600 \mathrm{eV}$ and increased to $9.5 \%$ at $1 \mathrm{MeV}$. From $20 \mathrm{eV}$ to $4.2 \mathrm{keV}$ neutron energy 293 resonance peaks were parameterized.

Research sponsored by U.S. DOE !jivision of Nuclear Physics.

*Chemical Technolog Divition

2.16

\section{HIGH ENERGY RESOLUTION MEASUREMENT OF THE ${ }^{230} \mathrm{U}$ NEUTRON CAPTURE YTLLD FROM $1 \mathrm{keV}$ TO $100 \mathrm{keV}$}

\author{
Roger L. Macklin" \\ G. de Saussure \\ R. B. Perea \\ R. W. Ingle ${ }^{\dagger}$
}

[Abatract of ORNL/TM-10666, Janury 1998; alo paper entitled, "High Energy Resolution Menevurements of the U-238 Neutron Capture Yield in the Everos Region Between 1 and 100 keV," presented at the International Conference on Nuclenr Date for Science and Technology, Mito, Japan, May 30-June 3, 1988; Proc. S. Igarai, Ed., Saibon Publishing Co., Led., Pp. 71-74 (1988)]

A measurement of the ${ }^{238} \mathrm{U}$ neutron capture yieid was performed at the $150-\mathrm{m}$ flight path of the ORELA facility on two ${ }^{238} \mathrm{U}$ samples (0.0124 and 0.0031 atums/barn). The capture yield data were normalized by Moxon's small resonance method. The energy resolution achieved in this measurement frequently resulted in doublet and triplet splittings of what appeared to be single resonances in previous measurements. This resolution should allow extension of the resolved resonance energy region in ${ }^{238} \mathrm{U}$ from the present $4-\mathrm{keV}$ limit up to 15 - or $20-\mathrm{keV}$ incident neutron energy. Some 200 small resonances of the $\left({ }^{238} U+n\right)$ compound nucleus have been observed, which had not been detected in transmission measurements, in the energy range from $250 \mathrm{eV}$ to $10 \mathrm{keV}$.

Research sponsored by U.S. DOE Division of Nuclear Physics.

"Universily of Tennewee, Knoxille, TN.

Instrumencation and Controls Division. 
2.17

NEUTRON CAPTURE OF ${ }^{122} \mathrm{Te},{ }^{123} \mathrm{Te}$, R. L. Mecklin R. R. Winters"

(Abatract of ORNL-6561, July 1909)

Isotopically eariched samples of the tellurium isotopes from mass 122 to mass 126 were used to measure neutron capture in the energy range 2.6 keV to $600 \mathrm{keV}$ at the Oak Ridge Electron Linear Accelerator pulsed neutron source. Starting at $2.6 \mathrm{keV}$, over 200 Breit-Wigner resonances for each isotope were used to describe the capture data. Least-squares adjustment gave parameters and their uncertainties for a total of 1659 resonances. Capture cros sections averaged over Maxwellian neutron distributions with temperatures ranging from $k T=5 \mathrm{keV}$ to $k T=100 \mathrm{keV}$ were derived for comparison with stellar nucleosynthesis calculations. For the three isotopes shielded from the astrophysical r-prosess, ${ }^{122} \mathrm{Te},{ }^{123} \mathrm{Te}$ and ${ }^{124} \mathrm{Te}$ at $k T=30 \mathrm{keV}$ the respective values were $(280 \pm 10) \mathrm{mb},(819 \pm$ 30) $\mathrm{mb}$ and (154 \pm 6$) \mathrm{mb}$. The corresponding products of cross section and solar system abcindance are nearly equal in cloce agreement with s-process nucleosynthesis calculations.

Research sponsored by U.S. DOE Division of Nuclear Phyics.

"Denison University, Granville, OH.

\subsection{8}

\section{RESONANCE NEUTRON CAPTURE BY ARGON-40}

\author{
R. L. Macklin R. R. Winters* \\ D. M. Schmidt ${ }^{\dagger}$
}

[Abatract of Aotron. Aotrophyo. 216, 102-112 (1900)]

Neutron capture by ${ }^{40}$ Ar at resonances up to $15,0 \mathrm{keV}$ has been measured and parametrized using the single-level Breit-Wigner formula. Velocity weighted maxwellian averaged capture croes sections aic presented for use in stellar nucleosynthesis calculations for stellar temperatures from $k T=5$ to $60 \mathrm{keV}$. The results are almost constant above $k T=10 \mathrm{keV}$ because most of the resonances lir at highrr energies. At the conventional spro cess temperature $k T=30$ ke $V$ the maxwellian averaged capture cross section found is (2.79 \pm 0.15$)$ mb.

Reseanch sponeored by U.S. DOE Division of Nuclear Physica.

-Denieon University. Granville, OH.

tUniversity of Californie al Sane Barbare, Sanes Barbara. CA.

2.19

$$
\begin{aligned}
& \text { NEUTRON SCATTERING IN }{ }^{100} \text { Os } \\
& \text { POR NUCLEOSYNTHESIS RATFS } \\
& \text { OF THE ODD-A Os ISOTOPES } \\
& \text { AND NUCLEOCHRONOLOGY }
\end{aligned}
$$
M. T. MeEllistrem" R. R. Winters'
R. L. Hershbergert
2. $\mathrm{Cro}^{\S}$
R. L. Macklin
N. W. Hill"

[Abstract of Plyg. Rev. C 10, 591 (1989)]

Neutron elastic and inelastic scattering cross sections have been determined for neutrons incident on ${ }^{189} \mathrm{Os}$ at three very low energies. Cross sections for scattering to the ground state and 36.2-keV excited level have been measured for incident energies of 63.5. 73.3, and $97.5 \mathrm{keV}$. Scattering to the $69.6 \mathrm{keV}$ excited level has also been measured at $97.5 \mathrm{keV}$ incident. These measurements are combined with neutron scattering cross sections for ${ }^{183}$ Os, neutron capture cross sections, and other low energy scattering observables to provide a basis for a consistent model of all data for both odd-A Os isotopes. The properly interpreted capture rates are important for the Re/Os nucleochronology and for aprocess nucleosynthesis rate estimates. The neutron capture and inelastic scattering cross sections provide a very low energy test of statistical model flux distributions, important in confirming the validity of the statistical model at these energies; model accuracy tests at very low energies is an important issue for reaction rates used in nucleosynthesis estimates.

Research sponeored by 'J.S. DOE Division of Nuclear Physica.

*University of Kentucky, Lexingron, KY.

${ }^{\dagger}$ Denison University. Granville, $\mathrm{OH}$.

tNichol Resend, Corp., Huntaville, AL.

\$Inatitute of Atomic Physira, Beijing, Peoplea Repuldie of Chinn.

* Inatrumenemeion and Conernds Division. 
2.20

\section{${ }^{36} \mathrm{Ni}+n$ TRANSMISSION, DIFFERENTIAL ELASTIC SCATTERING AND CAPTURE MEASUREMENTS AND ANALYSIS FROM 5 TO $813 \mathrm{keV}$}

\author{
C. M. Perey \\ J. A. Harvey \\ F. G. Perey \\ N. W. Hill* \\ N. M. Larson ${ }^{\dagger}$ \\ R. L. Macklin
}

(Abetract of ORNL/TM-1081. Seplember 1983)

High-resolution neutron measurements for 58 Ni-enriched targets were made at the Oak Ridge Electron Linear Accelerator (ORELA) from 100 eV $10 \approx 20 \mathrm{MeV}$ in transmission, from $10 \mathrm{keV}$ to 5 $\mathrm{MeV}$ in differential elastic scattering, and from 2.5 $\mathrm{keV}$ to $5 \mathrm{MeV}$ in capture. The transmission data were analyzed from 10 w $813 \mathrm{keV}$ with the mut tilevel R-matrix code SAMMY which uses Bayes' theorem for the fitting process. This code provides energies and neutron widths of the resonances inside the 10- to 813-keV region as well as a possible parameterization for resonances external to that region to describe the smooth croses section from 10 to $813 \mathrm{keV}$. The differential elastic dats at different scattering angles were compared to theoretical calculations from 30 to $813 \mathrm{keV}$ using an R-matrix code based on the Blatt-Biedenharn formalism. Various combinations of spin and parity were tried to $p_{2}$ dict eroses sections for the well defined $\ell>0$ resonances, and comparison with the data then provided spin and parity assignments for most of these resonances. The capture data were analyzed from 5 to $450 \mathrm{keV}$ with a least-squares fitting code using the Breit-Wigner formula. In this energy region $30 \%$ more resonances were observed in che capture data than in the transmisoion data.

From 5 to $813 \mathrm{keV}, 477$ resonances are reported. The reduced widths of the 62 swave resonances follow the Porter-Thomas diatribution and their nearest neighbor spacings agree with the Wigner distribution. The average s-wave level spacing is equal to $13.6 \pm 0.5 \mathrm{keV}$ and the s-wave strength function to $(3.1 \pm 0.6) \times 10^{-1}$. The staircase plot of the s-wave reduced level widths and the plot of the Lorentz-weighted strength function show only a slight possibility of doorway statex. The level densities calculated with the Fermi-gas model for $\ell=0$ and $\ell>0$ resonances ase comparef with the cumulative number of observed emonances. The average :adiation widths were deduced from resonances analyzed in the three data sets below $450 \mathrm{keV}$. The mean values of the distributions of the radiation widths are equal to $2.3 \mathrm{eV}$ for the 5 -wave resonances, $0.77 \mathrm{eV}$ for the $p$-wave resonances, and $1.3 \mathrm{eV}$ for the $d$ wave resonances and the standard deviations are $1.7 \mathrm{eV}, 0.33 \mathrm{eV}$, and $0.5 \mathrm{eV}$, respectively. The correlation coefficient between the s-wave reduced neutron widths and radiation widths is equal 10 $0.66 \pm 0.11$. The average capture cross section as a function of the neutron incident energy is compared to prediction based on the tail of the giant electric dipole resonance.

Researdh aponored by U.S. DOE Dividion of Nuclear Phyics.

*Inetrumentation and Controls -iviaion.

tCompating and Telecommunicatione Division.

\subsection{1}

\section{RESPONSES OF $\mathrm{C}_{6} \mathrm{D}_{6}$ AND \\ C6FG GAMMA-RAY DETECTORS AND THE CAPTURE IN THE 1.15-keV RESONANCE OF ${ }^{\mathrm{BS}} \mathrm{Fe}$}
F. G. Perey
J. O. Johnson
T. A. Gabriel
R. L. Macklin
R. R. Winters*
J. H. 'Todd'
N. W. Hillt

(Abatract of paper preasted of the Interpational Conference on Nuclear Date for Science and Technolow, Mito, Japan, May 30-Jowe 3, 1983; Proc. S. Ifarani, Ed., Saiboa Publiahing Co., Led., pp. 379-382 (1988))

We have used the electron gamma-ray traneport code EGS to calculate responses of $C_{6} D_{6}$ and $\mathrm{C}_{6} \mathrm{~F}_{6}$ gamma-ray detectors, where the geometry of the capture experiments was carefully modelled. Very good agreement wa obtained with spectra from selected responses in the capture of neutrons by ${ }^{207} \mathrm{~Pb}$. Weighting functions based upon the calculated responses were used in measuring the capture in the $1.15 \mathrm{keV}$ resonance of ${ }^{56} \mathrm{Fe}$ relative to the capture in the $A u 4.9-\mathrm{eV}$ resonance. The neutron widch was measured to be $64.5 \pm 3 \mathrm{meV}$ with $\mathrm{C}_{6} \mathrm{~F}_{6}$ detectors and $63.0 \pm 2.5 \mathrm{meV}$ with $\mathrm{C}_{6} \mathrm{D}_{6}$ detectors. These values are in good agreement with the value of $B 1.7 \pm 0.9 \mathrm{meV}$ found from transmission measurements.

Researth sporisored by U.S. DOE Division of Nucless Physics.

\footnotetext{
- Deniemn Univernity, Granville, OH.

tInatrumeneation and Controla Division.
} 
2.22

\section{SUBTHRESHOLD AND NEAR- SUBTHRESHOLD FISSION PHYSICS MEASUREMENTS AT THE OAK RIDGE ELECTRON LINEAR ACCELERATOR FACILITY}
R. B. Peree
G. de Sausture
F. C. Difilippo
L. W. Weaton

\section{J. A. Harvey}

(Abatrect of paper prenerted at the American Neclen Society and National Burean of Standards Conference on Fifty Years with Nuclem Fiaion, Geithersburs, MD, April 2628, 1989)

This paper is an account of the work performed at the Oal Ridge Eleetron Linear Accelerator (ORELA), pertaining to the fission phenomena taking place at energies of the fissioning nucleus which are comparable to the fission barrier potential energy. In this energy region, fission crose-section measurements yield information on the physical properties of the barrier and on the nuclear states at high nuclear deformations. The ORELA facility intense pulsed neutron source and good energy resolution capabilities afford a convenient tool to carry out a program of measurements in the subthreshold and near-subthreshold regions.

Extensive and precise measurements in the actinide region were performed at the ORELA facility by an international group of researchers. These measurements were unique in many respects: fission widths and areas were determined for many previously unreported resonances in the subthreshold region and a most detailed study was performed on the physical properties of the fission barrier at high nuclear deformations.

Research sponsored by U.S. DOE Division of Nuclear Phymics.

\section{HIGH RESOLUTION FISSION CROSS SECTION MEASUREMENTS AT ORELA}
J. H. Todd

(Abatrect of peper presented at the American Nuclear Society and National Bureau of Standard Conference on Fifty Yean with Nurlear Fiesion, Gaithersburs, MD, April 2628, 1980)

Multi-level fitting of the transmission, fiesion and capture croses sections have dramatically indi- cated the need for higher resolution fission civos section measurements of the fissile nuclides. High resolution is needed for extending the resolved resonance region to higher neutron energies, investigation of theoretical phenomens and beckground determination.

New measurements of the neutron fiscion croes sections of ${ }^{235} \mathrm{U}$ and ${ }^{239} \mathrm{Pu}$ have been carried out with the whice neutron source of ORELA at a flight path of 85 meters. Fiwion chambers with fact current amplifiers and a parallel plate ${ }^{10} \mathrm{~B}$ ionization chamber were used a detectors. These measurements have resolution more nearly comparable with recent transmiasion measuremente and will aid in multi-level fits to higher nettron energies than previously feasible.

Research sponsored by U.S. DOE Divinion of Nuctear Physica

"Inetrumentation and Controls Divinion

\subsection{4}

NEW CROSS SECTION EVALUATIONS FOR REACTOR FUEL MATERIALS
L. W. Weaton
P. G. Young *
W. P. Poenitz

[Abotract of Frase. Am. Ned. Soc. 60, 606 (1900)]

There were major new evaluations for the ${ }^{-B i g} 3^{\prime \prime}$ (U-235, U-238, and Pu-239) and the "Little 2" (Pu-240 and Pu-241) for ENDF/B-VI. New and improved analysis methods and models were used for the thermal parameters, the resolved resonance region, the unresolved resonance region and the high-energy neutron region of smooth croses sections. The maturing of evaluation techniques and methods in the eleven years since ENDF/B-V has been most impressive. Appreciable amounts of new nuclear data have also become available.

Research sponsored by U.S. DOE Division of Nuclear Physics.

"Los Alamos National Leboratory, Los Alamos. NM.

† Argonne National Leboratory, Arconne, $1 \mathrm{~L}$. 
2.25

\section{$n+{ }^{10}$ Ar s- AND p-WAVT NEUTRON \\ RESONANCE SPACINGS AND STRENGTH FUNCTIONS}

\author{
R. R. Winters* R. F. Carlton ${ }^{\dagger}$ \\ C. H. Johnson
}

[Abetract of paper presented at the American Physical Society Meetine, Baltimore, MD, May 1-4, 1980; Proc. Vol. 34(4), p. 1243 (1989)\}

Using the ORELA we have measured the neutron total cross section for $n+{ }^{40}$ Ar for incident neutrons in the energy range $10 \mathrm{keV}$ to $50 \mathrm{MeV}$. Below $1500 \mathrm{keV}$ the energy resulution of the mes. surement is sufficiently good to allow an R-Matrix description of the cross section with little ambiguity in the $J^{\pi}$ assignments for the observed resonances. Our preliminary estimates for the level spacings are $(63 \pm 7),(33 \pm 3)$, and $(27 \pm 2)$ keV for $J^{\pi}=1 / 2^{+}, 1 / 2^{-}$, and $3 / 2^{-}$, respectively. The neutron strength functions for the same partial waves are $(0.77 \pm 0.25),(1.3 \pm 0.3)$ and $(0.30$ $\pm 0.06)$ in $10^{-4}$ units.

Research sponsoned by U.S. DOE Division of Nuclear Physics.

* Denison L'niversity, Granville, OH.

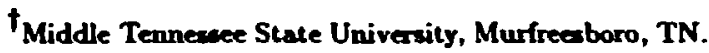

Pryyia Dirision.

\section{NEUTRON RESONANCE ANALYSES}

\subsection{6}

\section{CURRENT PLUTONIUM CROSS- SECTION EVALUATIONS IN THE RESOLVED RESONANCE RECION}

\section{H. Derrien* G. de Saussure}

[Abstract of paper presente. \& the 1988 International Reactor Physics Conference, J. on Hole, WY, September 18-21, 1988; Proc. Supplement, p. S-65 (1988)]

The resonance parameter analysis code SAMMY was used to perform consistent resonance at:alyses of several ${ }^{239} \mathrm{Pu}$ and ${ }^{241} \mathrm{Pu}$ fission and capture cross-section and transmission measurements up to $1 \mathrm{keV}$ for ${ }^{239} \mathrm{Pu}$ and up to $300 \mathrm{eV}$ for ${ }^{241} \mathrm{P}_{\mathrm{l}}$. The method of analysis, the measurement selection, and the results are discussed in this paper.

Research sponsored by U.S. DOE Division of Nuclear Phyices.

"Centre d'Eludes Nucleaires de Cadarache. France.

\subsection{7}

\section{R-MATRIX ANALYSIS OF ${ }^{230} \mathrm{Pu}$ NEUTRON CROSS SEC'TIONS IN THE ENERGY RANGE UP TO $1000 \mathrm{eV}$}

\section{H. Derrien* \\ G. de Saussiıre}

(Abutract of ORNL/TM-10986, January 1089)

The report is a description of the analysis of the ${ }^{239} \mathrm{Pu}$ neutron cross sections in the resolved resonance region using the multilevel-multichannel Reich-Moore code SAMMY. The resonance parameters were obtained in the energy range up to $1000 \mathrm{eV}$. The table of the resonance parameters is given with some statistical properties of the pazameters. Tabulated and graphical comparisons between the experimental data and the calculated cross sections are given. The results are available in ENDF, 'B-V format and will be proposed for the evaluated data libraries JEF2 and ENDF/B-VI.

Research sponsored by U.S. DOE Division of Nuclear Phynics.

"Centre d'Etudes Nucleaires de Cadarache, France.

\subsection{8}

\section{R-MATRIX ANALYSIS OF THE ${ }^{241} \mathrm{Pu}$ NEUTRON CROSS SECTIONS IN THE ENERGY RANGE THERMAL TO $300 \mathrm{eV}$}

\section{H. Derrien* G. de Saussure \\ (Abetract of ORNL/TM-11123, April 1889)}

The report is a description of the analysif of the ${ }^{241} \mathrm{Pu}$ neutron cross sections in the resolved resonance region at Oak Ridge National Laboratory (OR:") using the multilevel-multichannel Reich-Moure code SAMMY. The resonance parameters were obtained in the energy range 0 to $300 \mathrm{eV}$. The table of the resonance parameters is given with some statistical properties of the parameters. Tabulated and graphical comparison between the experimental data and the calculated cross sections are given. The results are available in ENDF/B-V format and will be proposed for the evaluated data library JFF2 and ENDF/B-VI.

Research aponasored by II.S. IOCF, Division of Nuclens Phymirn.

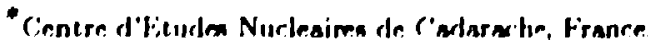




\section{R-MATRIX ANALYSES OF THE ${ }^{235}$ U AND ${ }^{239} \mathrm{Pu}$ NEUTRON CROSS SECTIONS}

\author{
H. Derrien* \\ G. de Saussure \\ N. M. Larson ${ }^{\dagger}$ \\ L. C. Leal ${ }^{t}$ \\ R. B. Perez
}

(Abatract of paper presented at the International Conference on Nuclear Date for Science and Technology, Mito, Japan, May 30-June 3, 1988; Proc. S. Lgarmi, Ed., Saikon Publishing Co., Ltd., Dp. 83-86 (1988)]

The resonance parameter analysis code SAMMY was used to perform consistent resonance analyses of several ${ }^{235} \mathrm{U}$ and ${ }^{239} \mathrm{Pu}$ fission and capture cross section and transmission measurements up to $110 \mathrm{eV}$ for ${ }^{235} \mathrm{U}$ and up to $1 \mathrm{keV}$ for ${ }^{239} \mathrm{Pu}$. The method of analysis, the measurement selection, and the resuits are briefly outlined in this paper.

Research sponsored by U.S. DOE Division of Nuclear Phyrias.

"Centre d'Etudes Nucleaires de Jadarache. France.

†Computing and Telecommunications Division.

t University of Tennessee, Knoxville, TN.

\subsection{0}

\section{REICH-MOORE AND ADLER-ADLER REPRESENTATIONS OF THE ${ }^{235} \mathrm{U}$ CROSS SECTIONS IN THE RESOLVED RESONANCE REGION}

\author{
G. de Saussure $\quad$ L. C. Leal* \\ R. B. Perez
}

(Abatract of paper to be presented at the International Conference on the Physi-s of Reactors: Operation, Design, and Compitation, Marwille, France, April 23-26, 1990)

Alternative representations of the evaluation of the ${ }^{235} \mathrm{U}$ neutron cross sections in the resolved resonance range are discussed. The resonance analysis of the cross sections was done with the code SAMMY, using the Reich-Moore formalism. We have used the POIIIA option of the code WHOPPER of IIwang to transform the ReichMoore resonance parameters describing the ${ }^{233} \mathrm{U}$ cross sections into equivalent Adler-Adler t arameters.

Wr have ales used the POLLY option of the code WHOPPER to ottain the expansion in momentum space of the cross-section representations and verified that the cross sections computed with the multiple parameters were rigorously equal to the cross sections computed with the Reich--Moore parameters.

We believe that the accurate representation of the ${ }^{235} \mathrm{U}$ evaluations with Adler-Adler parameters and the exact representation with multiple parameters in momentum space should provide useful alternatives to the Reich-Moore formalism for reactor codes, like $\mathrm{Mc}^{2}$, which use the Voigt profiles and J-functions. The evaluated Reich-Moore resonance parameters, and the equivalent AdlerAdler and multiple parameter sets, are available from the authors.

Research sponsored by U.S. DOE Division of Nuclear Physics.

*University of Tennemee, Knoxville, TN.

\subsection{1}

\section{A NEW RESONANCE-REGION EVALUATION OF NEUTRON CROSS SECTIONS FOR U.235}
G. de Saussure
L. C. Leal*
R. B. Perez
N. M. Larson ${ }^{\dagger}$
M. S. Moore

[Abstract of Nacl. Sci. Eng. 103(2), 109 (1989); also paper presental at the 1988 International Reactor Physics Conference, Jackeon Hole, WY, September 18-21, 1988; Proc. Vol. 1, pp. 293-302 (1988)]

This paper describes a new evaluation of the "resolved resonance range" for the neutron cross sections of ${ }^{235} \mathrm{U}$. Up to $110 \mathrm{eV}$ the evaluation is based on an R-matrix analysis of several fission, capture and transmission measurements. Above $110 \mathrm{eV}$ levels are not resolved anymore so that many resonances are missed; from 110 to $500 \mathrm{eV}$ most of the important resonances can be id.ntified and analyzed so that the cross section and transmission data are well represented by the proposed parameters. From 500 to $2250 \mathrm{eV}$ fictitious parameters are provided which describe fairly well the results of thick sample transmission measurements and recent fission cross-section data. We believe that such a parameterization is likely to yield a better approximation of resonance self-shielding than the current ENDF/B-V unresolved resonance treatment.

Researdi sponanred by U.S. DOE: Division of Nurlear Phynirs.

\footnotetext{
*Inivanity of Tennemere, Knoxville, TN.

†Computing and Telecommunirationa Diviaion.

I Ion Alamom Nacional Ialmontory. Iam Alamem, NM.
} 


\subsection{2}

\section{UNCERTAINTIES OE THE ENDF/B-V ${ }^{238} \mathrm{U}$ UNRESOLVED RESONANCE PARAMETERS IN THE RANGE $4 \mathrm{keV} \leq \mathrm{E} \leq 45.18 \mathrm{keV}$ \\ (MAT=1398, MF=2, MT=251)}

\section{G. de Saussure J. H. Marable}

[Abotrect of Neel. Sci. Eng. 101, 285-292 (1989)]

It is a common practice in ENDF/B to represent neutron cross sections in the unresolved resonance region by specifying the average values and distributicn laws of resonance parameters. This formalism allows the calculation of resonance selfshielding and of the variation of resonance selfshielding with temperature, two important reactor parameters. For many applications it is necessary to estimate the uncertainties in these model average resonance parameters. A possible approach to derive such uncertainties is described, using as an example the ENDF/B-V representation of ${ }^{238} \mathrm{U}$ in the range from 4 to $45 \mathrm{keV}$.

Research sponsored by U.S. DOE Division of Nuclear Physica.

* Consultana.

\subsection{3}

\section{STOCHASTIC NUCLEAR REACTION THEORY: BREIT.WIGNER NUCLEAR NOISE}

\section{G. de Saussure $\quad$ R. B. Perez}

[Abatract of peper in Noise and Nonlinear Phenomens in N'eclear Syotems, Series B: Physica Vol. 192, pr. 457-461, proceedings of the NATO Advanced Research Workehop, Universidad Politecnica de Valencis, Spain, May 23-27, 1988, J. L. Munoz Cobo and F. C. Difilippo, Eds., Plemum Preas, NY (1989)]

Our present understanding of neutron-nucleus interaction is largely based on Bohr's statistical compound nucleus model. However, theoretical developments hased on quantum corrections to Bohr's model, together with considerable improvements in neutron spectroscopy have predicted and revealed the presence of marked fluctuations in the neutron cross sections of both fissile and fertile nuclei. These fluctuations are wider than the sharp remonances associated with the compound nucleus levels and narrower than the broad structure due to the energy dependence of the neutron penetration coefficients. They, in fact, represent departures from the statistical compound nucleus model in localized energy regions, leading to an intermediate structure which is not predicted by Bohr's model. The observed enhancement of the neutron cross section is due to the presence of doorway states in the neutron channel or in the fission channels in fissile nuclei. The understanding and detection of this intermediate structure is of great relevance both in nuclear reaction theory and for the calculation of nuclear reactor parameters.

The purpose of this paper is the application of various statistical tests for the detection of the intermediale structure, which lies immersed in the Breit-Wigner "noise" arising from the superposition of many compound nucleus resonances. To this end, neutron capture cross sections are constructed by Monte Carlo simulations of the compound nucleus, hence providing the "noise" component. In a second step intermediate structure is added to the Breit-Wigner noise. The periormance of the statistical tests in detecting the intermediate structure is evaluated using mocked-up neutron cross sections as the statistical samples. Afterwards, the statistical tests are applied to actual nuclear cross section data.

Research sponsored by U.S. DOE Division of Nuclear Physics.

\subsection{4}

\section{EVALUATED CROSS SECTIONS FOR NEUTRON SCATTERING FROM NATURAL CARBON BELOW 2 MeV INCLUDING R-MATRIX FITS TO C-13 RESONANCES}

$$
\text { C. Y. Fu }
$$

\section{[Abatract of Nuel. Sei. Eng. (in preas)]}

The ENDF/B-V differential crose sections for neutron scattering from natural carbon below 2 $\mathrm{MeV}$, recommended as standards for measurements and hased on an R-matrix analysis for ${ }^{12} \mathrm{C}$ using natural carbon data, are revised to include ${ }^{13} \mathrm{C}$ resonances for high-resolution applications. The recommended ${ }^{13} \mathrm{C}$ crose sections are also based on an $R$-matrix analysis of the available. data. The 0.1520-MeV and the 1.736-MeV resonances rise above the natural carbon background by $7 \%$ and $1 \%$, pespertively. The angular distribu- 
tions of the elastically scattered neutrons from ${ }^{13} \mathrm{C}$ are generated by the R-matrix theory. The final recommended cross sectious and Legendre coefficients of angular distributions for natural carbon, adopted for ENDF/B-VI, are tabulated.

Research sponsored by U.S. DOE Division of Nuclear Physics.

\subsection{5}

\section{USE OF SAMMY IN RESONANCE ANA CYSIS OF NEUTRON DATA}

\section{N. M. Larson*}

(Abstrect of oral present thion presenled at GELINA, Geel, Belpium, November 16, :s97)

At CRELA analysis of resonance neutron cross-section data is generally performed via the computer code SAMMY. Designed for use on the ORELA VAX and/or FPS (Floating Point Systems) computers, the code is being modified for wse on the IBM 4381/P02 system at GELINA in Geel, Belgium. The various features of SAMMY and their use in the data analysis process will be described and the process of running the code will be discussed.

Research sponeored by U.S. DOE Division of Nuclear Physics.

"Computing and Telecommunications Division

2.36

\section{UPDATED USERS' GUIDE FOR SAMMY: MULTILFVEL R-MATRIX FITS TO NEUTRON DATA USING BAYES' EQUA'ION}

\section{N. M. Larson*}

\section{(Abstract of ORNL/TM1-9179/R2, June 1989)}

In 1980 the multilevel multichannel R-matrix code SAMMY was released for use in analysis of neutron data at the Oak Ridge Electron Linear Accelerator. Since that time, SAMMY has undergone significant modifications: User-friendly options have been incorporated to streamline common operations and to protect a run from common user errors. (2) The ReichMoore formalism las been extended to include an optional Ingaritlimic parameterization of the external R-matrix, for wlich any or all parameters may he varied. (3) The ability to vary sample thickness, effective temperature, matching radius, and/or resolction-broadening parameters has been incorporated. (4) To avoid loss oi information (i.e., computer round-off errors) between runs, the "covariance file" now includes precise values for all variables. (5) Unused but correlated variables may be included in the analysis.

Because of these and earlier changes, the 1980 SAMMIY manual is now hopelessly obsolete. This report is intended to be complete documentation for the current version of SAMMY. Its publication in looseleaf form will permit updates to the manual to be made concurrently with updates to the code itself, thus eliminating most of the time lag between update and documentation.

Research sponsored by U.S. DOE Division of Nuclear Physia.

"Computing and Telecommunications Division.

\subsection{7}

\section{RESONA NCE PARAMETER ANALYSIS WITH SAMMY}

\author{
N. M. Larson* F. G. Perey
}

[Abstract of paper presensed at the International Conference on Nuclear Data for Science and Technology, Mito, Japan, May 30-June 3, 1988; Proc. S. Igarasi, Ed., Saikon Publishing Co., Led., Pp. 573-576 (1988)]

The multilevel R-matrix computer code SAMMY has evolved over the past decade to become an inportant analysis tool for neutron data. SAMMY uses the Reich-Moore approximation to the mu!tilevel R-matrix and includes an optional logarithmic parameterization of the external $\mathbf{R}$ function. Doppler broadening is simulated either by numerical integration using the Gaussian approximation to the free gas model or by a more rigorous solution of the partial differential equation equivalent to the exact free gas model. Resolution broadening of cross sections and derivatives also has new options that more accurately represent the experimental situation. SAMMY treats constant normalization and some types of backgrounds directly and treats other normalizations and/or backgrounds with the introduction of user generated partial derivatives. The corle uses Bayes' method as an efficient alternative to least squares for fitting experimental data. SAMMY allows virtually any parametcr to be varied and outputs valurs, uneretainties, and covariance ina trix for all varind parameters. Vrrsions of SAMMY exist for VAX, FPS, and IBM conputres.

Renearth apounacored by IIS. DOF, Divinion of Nurlens Pliynirn.

- Compusting and Selercmumunirationa Diviaion 


\subsection{8}

URR COMPUTER CODE: A CODE TO CALCULATE RESONANCE NEUTRON CROSS-SECTION PROBABILITY TABLES, BONDARENKO SELF. SHIELDING FACTORS, AND SELF. INDICATION RATIOS FOR FISSILE AND FERTILE NUCLIDES
L. C. Leal*
G. de Saussure

R. B. Perez

(Abstract of ORNL/TM-11297, Auguet 1989)

The URR computer code has been developed to calculate cross-section probability tables, Bondarenko self-shielding factors, and selfindication ratios for fertile and fissile isotopes in the unresolved resonance region. Monte Carlo methods are utilized to select appropriate resonance parameters and to compute the cross sections at the desired reference energy.

The neutron cross sections are calculated by the single-level Breit-Wigner formalism with s, $p$, and $d$-wave contributions. The cross-section probability tables are constructed by sampling the Doppler broadened cross-section.

The various sheld-shielded factors are computed numerically as Lebesgue integrals over the cross-section probability tab!es.

Research sponsored by U.S. DOE Division of Nuclear Physics.

"University of Tennemee, Knoxvilie, TN.

2.39

\section{ANALYSIS OF THE U-235 NEUTRON CROSS SECTIONS IN THE RESOLVED RESONANCE RANGE}
L. C. Leal"
G. de Saussure

\section{R. B. Perez}

(Atsat ract of paper preserted at the American Nuclear Society and National Bureau of Standards Conference on Fifty Yeare with Nuclear Fieaion, Gaitheraburg, MD, April 2B28, 1989)

lising recent high-resolution measurements of the neutron transmission of ${ }^{23.5} \mathrm{U}$ and the spinseparaled fission cross-section data of Moore ef al., a multilevel analysis of the ${ }^{23.5}$ I; neutron cross sections was performed up to $300 \mathrm{eV}$. The Dyson Mretiod $\Delta_{3}$ statistica were used to help lorate

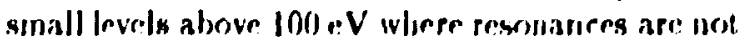

clearly resolved even in the best resolution measurements available. The statistical properties of the resonance parameters are discussed.

Research sponsored by U.S. DOE Office of Enery Re search, N-iclear Phyrica.

* Univerrity of Tennewee. Knoxville, TN.

\subsection{0}

\section{R-MATRIX ANALYSIS OF THE ${ }^{235}$ U NEUTRON CROSS SECTIONS}

\author{
L. C. Leal* G. de Saussure \\ R. B. Perez
}

[Abatract Trane Am. Necl. Soc. 56, 587 (1988)]

The ENDF/B-V representation of the ${ }^{235} \mathbf{U}$ neutron cross sections in the resolved resonance region is unsatisfactory: below $1 \mathrm{eV}$, the croses sections are given by "smooth files" (file 3) rather than by resonance parameters; above 1 $\mathrm{eV}$, the single-level formalism use i by ENDF/B$V$ necessitates a structured file 3 contribution consisting of $\geq 1300$ energy points; furthermore, information on level spins lias not been included. Indeed, the ENDF/B-V ${ }^{235} \mathrm{U}$ resonance region is based on an analysis rone in 1970 for ENDF/B-III and therefore does not include the results of highquality measurements done in the past $18 \mathrm{yr}$. This paper presents the result of an R-matrix multilevel analysis of recent measurements as well as older data. The analysis also extends the resolved resonance region from its ENDF/B-V upper limit of 81 to $110 \mathrm{eV}$.

The resonance analysis code SAMMY was used to perform a consistent resonance parameter analysis of several ${ }^{235} U$ neutron fission and capture cross-section and transmission measurements and of the spin separated data obtained from an anaiysis of the polarized neutron target measurements.

The SAMMY program uses the multilevel R-matrix Reich-Moore formalism and leads to a physically sound representation of the neutron cross sections in the resollance region. The Bayesian approach, which includes the direct introduction of experimental uncertainties, such as on sample thicknesses, broadening paiametern. etc.. allows the surecessive incorporation of new data in a consisteit manner. The option of searching on sample thicknessers, effertive sample temperatuse, and the paranieters of the 
instrumental resolution function, all consistent with predetermined uncertainty limits, leads to realistic parameter uncertainties and covariance matrices. Recent modification of the SAMMY program also permits a consistent Bayesian fit of the spin-separated fission data. The cross sections of ${ }^{235} \mathrm{U}$ up to $110 \mathrm{eV}$ were represented by a set of 260 resonances with two open fission channels in each spin state.

Researd sponsored by U.S. DOE Division of Nuclear Physica.

*University of Tennemee, Knoxville, TN.

\subsection{1}

\section{STATISTICAL PROPERTIES OF THE ${ }^{235}$ U RESONANCE PARAMETERS UP TO $300 \mathrm{eV}$}
L. C. Leal*
G. de Saussure
R. R. Winters ${ }^{\dagger}$
R. B. Perea

[Abstract of Trase. Am. Necl. Soc. 59, 341 (1989)]

An accurate resonance analysis of the ${ }^{235} \mathrm{U}$ neutron cross sections up to $300 \mathrm{eV}$ is in progress for the ENDF/B-VI files. A detailed discussion of the data base and a description of the method of resonance analysis have been given elsewhere. The purpose of this paper is to report on the statistical properties of the ${ }^{235} U$ resonance parameters and conipare our results with those of previous analyses.

The present analysis is an improvement over previous analyses bec ause of the combined use of recent spin-separated and high-energy resolution data with the $\Delta_{3}$ statistics, which facilitates the resonance analysis and improves its physical content.

Research sponeored by U.S. DOE Division of Nuclear Phynics.

- Univenily of Tennemee, Knoxville. TN.

${ }^{\dagger}$ Deniesol University, Granville, $\mathrm{OH}$.
2.42

\section{RESONANCE STRUCTURE IN THE FISSION OF $\left({ }^{235} U+n\right)$}

\author{
M. S. Moore \\ L. C. Leal ${ }^{\dagger}$ \\ G. de Saussure \\ R. B. Perez \\ N. M. Larson
}

(Abstract of paper prewented at the International Conference Fifty Years of Reseanch in Nuclear Fiasion, Berlin, West Germany, April 3-7, 1989)

A new multilevel reduced R-matrix analysis of the neutron-induced resonance cross sections of ${ }^{235} U$ has been carried out. We used as a constraint in the asalysis the angular anisotropy measurements of Pattenden and Postma, obtaining a Bohr-channel (or $\mathrm{J}, \mathrm{K}$ channel) representation of the resonances in a two-fission vector space for each spin state. Hambsch et al., have reported definitive measurements of the mass- and kineticenergy distributions of fission fragments of $\left({ }^{235} \mathrm{U}\right.$ $+n)$ in the resonance region and analyzed their results according to the fission-channel representation of Brosa el al., extracting relative contributions of the two asymmetric and one symmetric Brosa fission channels. We have explored the connection between Bohr-channel and asymmetric Brosa-channel representations. The results suggest that a simple rotation of coordinates in channel space may be the only transformation required; the multilevel fit to the total and partial cross sections is invariant to such a transformation.

Research sponsored by U.S. DOE Division of Nuclear Physics.

"Los Alamoa Nationa Laboratory, Lon Alamos, NM.

tUnivenity of Tennewsee, Knoxville, TN.

tComputing and Telecommunications Division.

\subsection{3}

\section{RFUNC - A CODE TO ANALYZE DIFFERENTIAL ELASTIC. SCATTERING DATA}

\author{
F. G. Perey
}

(Abatract of ORNL/TM-11112. March IOB9)

The code RFUNC; is used at ORF.I.A to analyze high resolution differential mastic scattering data from spin zero nurlides in thr resonance encrgy region. This report. pressuts the 
real R-function lormalism used in RFUND and gives details of how the finite size corrections are currently made. Appendix A describes the input to the code RFUNC. Appendix B describes the input to a utility code MAKPA that transforms a resonance parameter file in SAMMY format into a resonance parameter file in RFUNC format. Appendix $C$ describes the input to an adjunct code RFUNCXT that generates the contributions to the total cross section from resonances of different orbital and total angular momenta. The FORIRAN listing of the code RFUNC is given in Appendix D.

Research sponsored by U.S. DOE Divition of Nuctear Physics.
2.44

\section{ON THE UNRESOLVED RESONANCE REGION REPRESENTATION OF NEUTRON INDUCED CROSS SECTIONS}
R. B. Perez
G. de Saussure
L. C. Leal*
Roger Macklin *

(Abatract of paper presented at the 1988 Intermational Reactor Fhysic Conierence. Jactson Hole, WY, September 18-21. 1988; Proc. Vol. II, pp. 183-188 (1988)]

In this work we revie the unresolved resonance region formalism in the light of the availabitity of new high-energy resolution measurements.

Research sponsored by U.S. DOE Division of Nuclear Physics.

*University of Tennemee, Kinoxville, TN.

\section{CROSS SECTION EVALUATION AND MODELJNG}

\subsection{5}

SCINFUL: A MONTE CARLO BASED COMPUTER PROGRAM TO DETERMINE A SCINT:LLATOR FULL ENERGY RESPONSE TO NEUTRON DETECTION FOR $E_{n}$ BETWEEN 0.1 AND $80 \mathrm{MeV}$ : USER'S MANUAL AND FORTRAN PROGRAM LISTING

\section{J. K. Dickens}

(Abatract of ORNL-6462, March 1988)

This document provides a complete listing of the FORTRAM prograin SCINFUL, a program designed to provide a calculated full response anticipated for either an NE-213 (liquid) scintillator or an NE-110 (solid) scintillator. The incident design neutron energy range is 0.1 to $80 \mathrm{MeV}$. Preparation of input to the program is discussed as are important features of the output. Also included is a FORTRAN listing of a subsidiary program applicable to the output of SCINFUL. This userinteractive program is nanied SCINSPEC from which the sutput of SCINFUI, may be reformatted into a standard specteum form involving ejther equal light-unit or equal proton-energy intervals. Fxamples of input to this program and corresponding output, are given.

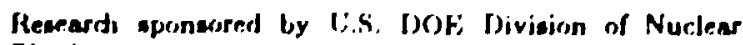
Pliynirs.

\subsection{6}

SCINFUL: A MONTE CARLO BASED COMPUTER PROGRAM TO DETERMINE A SCINTILLATOR FULL ENERGY RESPONSE TO NEUTRON DETECTION FOR $E_{n}$ BETWEEN 0.1 AND 80 MeV: PROGRAM DEVELOPMENT AND COMPARISONS OF PROGRAM PREDICTIONS WITH EXPERIMENTAL DATA

\section{J. K. Dickens}

(Abatract of ORNL-6463, April 1988)

This document provides a discussion of the development of the FORTRAN Monte Carlo program SCINFUL (for scintillator full response), a program designed to provide a calculated full response anticipated for either an NE-213 (liquid) scintillator or an NE-110 (solid) scintillator. The program may also be used to compute angleintegrated spectra of charged particles $(p, d, t$, ${ }^{3}$ IIe, and $\alpha$ ) following neutron interactions with ${ }^{12}$ (:. Extensive comparisons with a varicty of experimental data are given. 'There is generally overali good agreement ( $\leq 10 \%$ differences) of results from SCINFUL, calculations with measured detertor efficiencies for the incident design neution energy range of 0.1 to $80 \mathrm{MeV}$. Calculations of deteretor pesposises, i.e., $N\left(E_{r}\right)$ vs $E_{r}$ where $E_{r}$ is 
the response pulse height, reproduce measured detector responses th an accuracy which, at least partly, depends upon how well the experimental configuration is kncwn. For $E_{n} \leq 16 \mathrm{MleV}$ and for $E_{\mathrm{r}} \geq 15 \%$ of the maximum pulse height response, calculated spectra are within $\pm 5 \%$ of experiment on the average. For $E_{n}$ up to $50 \mathrm{MeV}$ similar good agreement is obtained with experiment for $E_{r} \geq$ $30 \%$ of maximum response. For $E_{n}$ up to $75 \mathrm{MeV}$ the calculated shape of the response agrees with measurements, but the calculation underpredicts the measured response by up to $30 \%$.

Research sponsored by U.S. DOE Divisiwn of Nuclear Physics.

\subsection{7}

\section{A SIMPLIFISD UNIFIED HAUSER- FESHBACH/PRE-EQUILIBRIUM MODEL FOR CALCULATING DOUBLE DIFFERINTIAL CROSS SECTIONS}

\section{Y. Fu}

(Abatract of paper presenter at the NEANDC Specialiats' Meeting on Pre-equilibrium Reactions, Semmerling, Aurtria, February 10-12, 1988; Proc. pp. 285-201 (1988)]

A unified Hauser-Feshbach/Pre-Equilibrium model is extended and simplified. The extension involves the addition of correlations among states of different total quantum numbers ( $J$ and $J ')$ and the introduction of consistent level density formulas for the HI-F and the P-E parts of the calculation. The simplification, aimed at reducing the computational costs, is achieved mainly by keeping only the off-diagonal terms that involve strongly correlated $2 p-1 /$ states. A correlation co efficient is introduced to fit the experimental data. The model has been incorporated into the mut tistep II-F model code TNG. Calculated double differential $(n, z n)$ crose sections at 14 and 25.7 $\mathrm{MeV}$ for iron, niobium, and bismuth are in good agreement with experimests. In use at ORNL and JAFRI, the TNG; code in various stages of development h rs been applied with success to the eval. uation of double differential $(n, x n)$ cross sections from 1 to $20 \mathrm{MeV}$ for the dominant isotopes of rhromium, manganese, iron. nirkel, coppes, and learl.

Ileseard, sponeored by US.S. DOE Divinion of Nurlear Phymica.

\subsection{8}

APPROXIMATION OF PRECOMPOUND EFEECTS IN HAUSER-FESHBACH CODES FOR CALCULATING DOUBLE DIFFERENT'AL $(n, x \mathrm{n})$ CROSS SECTIONS

\author{
C. Y. Fu
}

[Abatract of NecL Sei Eng. 100, 61 (1989)]

A simplified method for approximating precompound nuclear reaction effects in Hauser-Feshbach codes for the calculation of double difierential $(\boldsymbol{n}, \mathbf{r m})$ cross sections is presented. The method is developed from an existing quantum mechanical formula of unified compound and precompound reaction theories. The compound part of the unified formula is made identical to that of Hauser and Feshbach by applying the unified level-density formulas derived previously for the two theories. The precompound part, much more complicated than the compound part, is simplified and globally parameterized for practical purposes. Calculated double differential $(n, 2 n)$ crose sections at 14 and $26 \mathrm{MeV}$ for iron, niobium, and bismuth are shown to be in good agreement with the available experimental data. The method at various stages of development has been applied with success to the generation of evaluated files of double differential $(n, I n)$ cross sections from 5 to $20 \mathrm{MeV}$ for the major isotopes of chromium, manganese, iron, nickei, and copper.

Research sponsored by U.S. DOE Division of Nuclear Physics.

2.40

\section{PAIRING CORRECTIONS AND SPIN CUTOFF FACTORS IN EXCITON LEVEL DENSITIES FOR TWO KINDS OF FERMIONS}

$$
\text { C. Y. Fu }
$$

(Abstract of paper presentad at the Sixth Conrdination Meeting for the Program to Meet Nuclear Data Needs for Fusion Energy, Athen. OH, Septemier 10-21, 1960)

Pairing corrections in particle-hole (exciton) state-density formulas used in precompound nuclear reaction tlicorien are, strictly speaking, de. pendent on the nuclear excitation energy $V$ and the exciton number $n$. A general formula for 
$(U, n)$-dependent pairing corrections has been derived in an earlier paper for the exciton statedensity formula for one tind of fermion. In the present paper, a similar derivation is made for two kinds of Fermions. In this formulation, it is as sumed that neutions and protons occupy different sets of single particle states. It is shown that the constant-pairing-energy correction used in standard state-density formulas, such as $\boldsymbol{U}_{0}$ in Gilbert and Cameron, is a limiting case of the present general $(U, n)$-dependent results. Spin cutof faccors with pairing effects are obtained in the same derivations, thereby defining the exciton leveldensity formula for two kinds of Fermions. N'tmerical examples are shown to explain the difference in these quantities between the one-Fermion and iwo-Fermion fermulations.

Research sponsored by U.S. DOE Division of Nuclear Physics.

2.50

\section{STATUS OF THEORIES USED FOR CALCULATIONS OF LONG-LIVED ACTIVATION CROSS SECTIONS}

\author{
C. Y. Bu
}

(Abatract of paper presented at the IAEA Consultants' Meeting on Crose Sections for the Generation of Long-tived Radionuclide, Argonne. IL, Septenber 11-12, 1989)

For activation reactions that produce longlived radionuclides, the standard model codes for calculating the cross sections are multistep Ilauser-Feshbach with pre-equilibrium reaction and gamma-ray cascade capabilities. The nuclear theories required in such cones include optical models, ganima-ray strength functions, total and exciton level-density theories, and pre-equilibrium models. Recent advances and perceived weaknesses in these theories and the relevant parameters are reviewed through the examples of the ${ }^{58} \mathrm{Ni}(n, \gamma){ }^{39} \mathrm{Ni}\left(7.5 \times 10^{8} \mathrm{y}\right),{ }^{63} \mathrm{Cu}(n, p){ }^{63} \mathrm{Ni}(100$ $y)$, and ${ }^{206} \mathrm{~Pb}(n, 2 \pi)^{203} \mathrm{~Pb}\left(1.4 \times 10^{7}\right.$ y) reactions. In addition, it is shown that accuracies of the calculated results depend on the number of discrete levels uned and on the correct assignments of their spins and parities.

Rewearch aponeored by I'.S. DOF, Diviaion of Nisclear Phymirs.

\subsection{1}

\section{A REVIEW OF ACTIVATION CROSS SECTIONS IN THE ENDF/B-VI GENERAL PURPOSE FILES OF $\mathrm{Cr}, \mathrm{Pe}, \mathrm{Ni}, \mathrm{Cu}, \mathrm{AND} \mathrm{Ph}$

\author{
C. Y. Pu
}

(Abatrect of pape - presented at the NEANDC Specialiets Meeting on Neutron Activetion Crom Sections for Fimion and Fusion Funerg Applicutions, Argonnen I., September 13-15, 1800)

Leolopic evaluations for ${ }_{50,52,53,54} \mathrm{Cr}$,
$54,56,57,5 \mathrm{Fe}$, $58,60,61,62,64 \mathrm{Ni},{ }^{63,65} \mathrm{Cu}$, and
$206,207,208 \mathrm{~Pb}$ are included in ENDF/B-VI for the first time. These general purpose files, all by the ORNL evaluation group, naturally account for many activation croses sections. In this review, we first took the $\mathbf{3 3}$ activation reactions for these moterials from the high priority CSEWG list for fusion reactor design and checked for their presence in the general purpose files. It was immediately obvious that an evaluation for ${ }^{204} \mathrm{~Pb}$, omitted due to its low abundance, needs to be added as it would contain three high priori, setivation cross sections, namely, ${ }^{204} \mathrm{Fo}(n, \gamma),(n, p)$, and $(n, 2 n)$. Among the three, ${ }^{204} \mathrm{~Pb}(n, \gamma){ }^{205} \mathrm{~Pb}$, having the very long half life of 14 million years, is particularly important for consideration of radioactive waste disposal. Another reaction with long-lived (0.3 million years) daughter, ${ }^{61} \mathrm{Ni}(n, 2 p)^{60} \mathrm{Fe}$, ifnored in the general purpose evaluation because of its small croes section, should aiso be added. The remainder of the activation cross sections are reviewed in terms of the experimental data base and the evaluation methods. Most of them have been significantly improved over ENDF/B$V$ through either detailed resonance analysis (e.g., capture croses sections) or least-squares averaging (e.g., dosimetry reactions).

Research sponsored by U.S. DOE, Division of Nuclear Phyaice. 
2.52

\section{ENDF/B-VI EVALUATIONS FOR ISOTOPES OF $\mathrm{Cr}$, Pe, Ni, AND Cu, AND FOR NATURAL Pb}

\author{
$\begin{array}{ll}\text { D. M. Hetrick* C. Y. Pu } & \text { C }\end{array}$ \\ D. C. Larson
}

[Abatrect of paper prenented at the ANS Eighth Topical Meeting an Techoologs of Pecion Eners. Selt Lale City. UT, October 9-13, 19s8; Fevion Tech. 15(2), 453 (March 1989)

ENDF/B-VI is the first version of the ENDF library to pay particular attention to fusion date needs. Structural materials exist in significant amounts in various locations of proposed fusion reactors. Thus, the evaluations must be adequate to meet a wide variety of needs, including neutron transport, heating, radiation damage, etc. Much work, both experimental and theoretical, has gone into the structural material evaluations, and this paper highlights the major improvements over F.NDF/B-V.

Research sponsored by U.S. DOE Divition of Nuclear Physices.

- Computing and Telecommunications Diviaion.

2.53

\section{DESCRIPTION OF EVALUATION OF DEP PERFORMED FOR ENDF/B-VI}
D. C. Lareon
C. Y. Fu

(Abatract of paper presented at the IAEA Specialiats' Meeting on the International Nucles Date Library for Fu sion Reactor Technology, Vienne, Austria, November 1619, 1987)

An evaluation of data for neutron induced reactions on natural lead was performed for ENDF/ B-VI and is briefly reviewed. The evaluation is hased on experimental data and results of model calculations which reproduce the experimental data. Evaluated data are given for neutron induced reaction cross sections, angular and energy distributions, and for gammarray production cross scetions associated with the reactions. File 6 formats are used to represent energy-angle correlated data. Uncertainty files are included for the major cross sections. Much of the present evaluation is taken over from ENDF/B-IV and ENDF/B-V; emphasis is placed on updates for ENDF/B-VI.

Remeards eponerored by U.S. DOE Division of Nuclear Phynir.n.
2.54

\section{SOME NOTES ON THE \\ CALCULATION OF ENERGY-ANGLF \\ CORRELATED DISTRIBUTIONS \\ WTTH TNG AND THEIR \\ REPRESENTATION IN FILE 6 FORMATS}

\author{
$\begin{array}{ll}\text { D. C. Larson } & \text { C. Y. Pu }\end{array}$ \\ D. M. Hetrick*
}

[Abatract of paper presented at the IAEA Specialiats' Meeting on the Incernutional Nucler Dela Library for Fu son Reactior Technolog, Viena, Autris, November 1618, 1967; Proc. p. 220, V. Goula, Ed. (Auguat 1969)]

The model code TNG has been extensively used in evaluation work of structural materials for ENDF/B-VI performed at Oak Ridge National Laboratory. A new aspect of ENDF/B-VI is the use of File 6 formats for energy-angle correlated data. Such data are generally calculated, anchored by experimental data. In this informal note we outline how the TNG results are calculated and entered in the File 6 formats.

Research spomsored by U.S. DOE Division of Nuclear Phyics.

* Computing and Telecommunications Divition.

\subsection{5}

\section{DESCRIPTION OF EVALUATION POR $54,56,57,58$ Pe \\ PERFORMED FOI ENDF/B-VI}

\author{
$\begin{array}{ll}\text { D. C. Larson } & \text { C. Y. Tu }\end{array}$ \\ D. M. Hetrick"
}

[Abutract of paper premented at the IAEA Specialials' Meeting on the International Nuclew Date Library for Fu. sion Reactor Technokgy, Vienna, Auatria, Nuvember $16-$ 18, 1987; Proc. p. 204, V. Goulo, Ed. (August 1989)]

Isotopic evaluations for $54,56,57,58 \mathrm{Fe}$ performed for ENDF/B-VI are briefly reviewed. The evaluations are based on analysis of experimental data and results of model calculations which reproduce the experimental data. Evaluated data are given for neutron induced reaction cross sections, angular and energy distributions, and for gamma. ray production cross sections associated with the reactions. File 6 formats are used to represent energy-angle correlated data and recoil spectra. Uncertainty files are included for ihe major cross sections. A detailed evaluation is given for ${ }^{36} \mathrm{Fe}$. and results of calculations for tlie major reartions are used for evaluations of the minor isolopes, with 
particular attention paid to inelastic seattering to the low-lying levels in ${ }^{57} \mathrm{Fe}$.

Research aponeored by U.S. DOE Division of Nuclenr Physica.

"Computing and Telecummanications Diviaion

\subsection{6}

\section{DESCRIPTION OF EVALUATION FOR ${ }^{30,00,61,82, \mathrm{AA}_{\mathrm{Ni}}}$ \\ PERFORMED FOR ENDF/B-VI}

$$
\begin{aligned}
& \text { D. C. Larson D. M. Hetrick" } \\
& \text { C. Y. Du }
\end{aligned}
$$

[Abatract of paper presented at the IAEA Specialists' Menting on the Intermational Nuclea Data Library for Fuion Reactor Technolog, Vienne, Avetrie, November 1618. 1987; Proc. p. 195, V. Goula, Ed. (Auguat 1969))

Isotopic evaluations for $38,60,61,62,64 \mathrm{Ni}$ performed for ENDF/B-VI are brielly reviewed. The evaluations are based on analysis of experimental data and results of model calculations which re produce the experimental data. Evaluated data are given for neutron induced reaction cross sections, angular and energy distributions, and for gamma-ray production cross sections aseociated with the reactions. File 6 formats are used to represent energy-angle correlated data and recoil spectra. Uncertainty files are included for the major cross sections. Detailed evaluations are given for $58,60 \mathrm{Ni}$, and results of calculations for the major reactions are used for evaluations of the minor isotopes.

Research aponeored by U.S. DOE Diviajon of Nucles Phyrics.

"Computing and Telercommunications Division.

\section{$\mathbf{2 . 5 7}$}

\section{DESCRIPTION OF EVALUATION FOR ${ }^{63,05} \mathrm{Cu}$ PERFORMED FOR ENDF/B-VI}

\section{U. C. Larson D. M. Hetrick*}

$$
\text { C. Y. Fu }
$$

(Abatrace of paper prewented at the IAEA Speciatints Weet ing on the International Nuclear Dace Library for Fu. nion Reartisi Tecilusilogy. Viennn, Austria. November 1tr 18. 1987; Pror. p. 212, V. Gros!o, Fd. (Auguat 1980)]

Isotopic evaluations for ${ }^{33,6,3}$ Cu performed for ENDF/B-VI are briefly peviewnd. The evali- ations are based on analysis of experimental data and results of model calculations which reproduce the experimental data. Evaluated data are giver: for neutron induced reaction cross sections, angular and energy distributions, and for ganma-ray production cross sections associated with the reactions. File 6 formats are used to represent energyangle correlated data and recoil spectra. Uncertainty files are included for the major croses sections. Full evaluations are given for ${ }^{63,65} \mathrm{Cu}$.

Resenth sposeored by US. DOE Division of Nudear Phyrics.

* Compertirs and Telecommunications Divition.

\subsection{8}

\section{DESCRIPTION OF EVALUATION FOR ${ }^{50,52,53,54} \mathrm{Cr}$ PERFORMED FOR ENDF/B-VI}
D. C. Larson
D. M. Hetrick"

$$
\text { C. Y. Pu }
$$

(Abutract of peper preserted at the IAEA Specialiats' Meeting on the Indernational Nucleor Dast Library for Fusion Reactor Technology, Vienna, Auatrix, November 1618, 1987: Proc. p. 187, V. Goula, Ed. (August 1989)]

Isotopic evaluations for ${ }^{50,52,53,54} \mathrm{Cr}$ performed for ENDF/B-VI are briefly reviewed. The evaluations are based on analysis of experimental data and results of model calculations which reproduce the experimental data. Evaluated data are given for neutron induced reaction cross sections, angular and energy distributions, and for gemma-ray production cross sections associated with the reactions. File 6 formats are used tc represent energy-angle correlated data and recoil spectra. Uncertainty files are included for the major cross sections. Detailed evaluations are given for ${ }^{32,53} \mathrm{Cr}$, and results of calculations for reactions with large crosc sections are used for evaluations of the minor isotopes.

Researd sponsored by V.S. DOE Division of Nuclear Physirs.

- Computing and Telecommunirations Division. 
2.59

\section{STRUCTURAL AND SHIELDING MATERIAL EVALUATIONS FOR ENDF/B-VI}

\author{
D. C. Larson $\quad$ P. G. Young *
}

[Abatract of Trans. Am. Neel Soc. 60,606 (1909)]

Following completion of ENDF/B-V several problems and shortcomings were apparent in evat uations for important shielding and structural materials. Lack of energy balance was a new problem which arose from use of nonisotopic evaluations and lack of checking by evaluators. Discrepancies between evaluated and measured neutron er ission spectra resulted from omission of a precompound component in the theoretical analysis of the data. Difficulties experienced by users in obtaining accurate KERMA and radiation damage function quantities were due prinarily to format shortcomings. These problems and potential solutions were discussed by the General Purpose Evaluation Subcommittee of CSEWG in the early 1980's. Improvements in formats and nuclear model codes, and use of isotopic evaluations were some of the techniques implemented to insure improved evaluations for ENDF/B-VI. New developments in experimental techniques provided improved data upon which to bencinmark the evaluations. In this paper we outline major improvements and provide a list of materials upgraded for ENDF/B-VI.

Research sponsored by U.S. DOE Division of Nuclear Physics.

*Los Alamos National Laboratory, Lo Alamos, NM.

\subsection{0}

\section{TEST OF THE ENDF/B UNRESOLVED RESONANCE FORMALISM FOR U-235}
L. C. Leal"
R. B. Perez
G. de Saussure
R. Q. Wright ${ }^{\dagger}$

[Abalise of Trans. Am. Neel. Soc. 60,620 !1989)]

It is common practice in ENDF/B to represent neutron crose sections in the unresolved resonance region by specifying the average values and distribution laws of resorsance parameters. This formalism allows the calculation of resonance selfshielding and of its variation with temperatuse.
The purpose of this paper is to present a test of the validity of the formalism by comparing selfshielding factors computed with the ENDF/B unresolved formalism, with values computrd with the resolved resonance parameters recentl; tvaluated for the neutron cross sections of U-235 up to j.,0 eV.

Research sponsored by U.S. DOE Division of Nuclear Phyices.

*Univerity of Tenuence, Kooxville, TN.

tComputing and Telecommunications Divition.

2.61

\section{Covariance Data For ENDF/B.VI}

R. W. Peelle

[Abstract of Trens. Am. Neel. Soc. 60,608 (1980]]

Since the development of ENDF/B-V, more experimenters have included covariance information with their results, and techniques for data evaluation that include covariance information that have been developed. For ENDF/B-VI these developments and the extended ENDF-6 covariance formats make possible inclusion of covariance data for pressure vessel dosimetry, fusion reactor activation, fast reactor core physics, etc.

Covariance data for cross sections that are output from model or fitting codes can be represented implicitly in ENDF/B-6 formats in terms of the model parameter covariance matrix and the tabulated derivatives of cross sections with respect to the model parameters. Also for ENDF/B-VI, all new covariance evaluations of smoo' $h$ crose sections will include a minimum rarianc component to increase validity and relieve numerical problems encountered by data-base adjusters.

ENDF/B-VI evaluated covariance files can meet most requirements for uncertainty propagation and data adjustment because of broadened format cpabilities and because of the increased experience with covariance data. However, the first release of ENDF/B-VI will not contain all the needed information.

The author acknowledges the large contributions by members of the CSEW'; Covariance Subcommittee, Doug Muir in particular.

Researdi sponeored by U.S. DOF: Division of Nuclear Pliymics. 


\section{NEUTRON STANDARD DATA}

\author{
R. W. Peelle A. Conde*
}

(Abatrect of paper presened at the International Comference on Nuclear Dace Lor Science and Techoolog. Mito. Japan, May 30-Jane 3, 1808; Proc. S. Igarei, Ed, Sailon Publialing Co.. Led., Pp. 100s-1012 (19se)]

The neutron standards are reviewed with emphasis on the evaluation for ENDF/B-VI. Also divcussed are the neutron spectrum of ${ }^{232} \mathrm{CT}$ spontaneous fission, activation croes sections for neutron fux measurement, and standards for nentron energies greater than $20 \mathrm{MeV}$. Recommendations are made for future work.

Researdh aponsored by U.S. DOE Divicion of Neclear Physice.

-Uppara Univercity. Upparal, Sweden.

\subsection{3}

\section{EXTENDED COVARIANCE DATA FORMATS FOR THE ENDF/B-VI DIFERENTIAL DATA EVALUATION}

\author{
R. W. Peelle \\ D. W. Muir*
}

[Abetract of paper prewented at the Specialists' Meetin on the Applieation of Critieal Experiments and Operating Data to Care Design vis Formal Methods of Crons Section Dale Adjuitment, Jackecen Hole, WY. Seplember 23-24, 1988; Proc. NEACRP-L-307, pp. 305-315 (1988)]

The ENDF/B-V included crows rection covariance data, but covariances could not be éncoded for all the important data types. New ÉNDF-6 covariance formats are outlined including those for croce-file (MF) covariances, resonance parameters over the whole range, and recondary energy and angle distributions. One "late entry" format encodes covariance data for crose sections that are output from model or fitting codes in terms of the model parameter covariance matrix and the tabulated derivatives of cross reetions with respect to the model parameters. Another new format yields multigroup crom section variances that increase as the group width decreases. When evaluators use the new format, the files can be processed and used for improved uncertainty propagation and data combination.

Reecarch sponeored by U.S. DOE Division of Nucles Phroics.

"Los Alamos Nacional Leboratory, Los Alamoa, NM.

\section{COVARIANCES OF PHYSICAL CONSTANTS}

\author{
F. G. Perey
}

(Abetract of ord presentalion presended at the NCSL Anowl Conferenoe and Symposinm, Wathingtoe, DC, Anget 14-18, 18:8)

In many modern engineering-system studies the uncertainties in some tey performance parameters are dominaled by the uncertainties in the physical constants of the components of the system and are very strongly affected by the covariances of these phyrical constants. About 10 years ago this wrs realized as the case for fast nuelear reactor atudies, and the data bases rere modified to include covariances of the key physical constants. Experimentalists who provide nuclear data for applications are now increasingly describing the covariances in their measured data. The problems of determining and reporting the complete covarances matrix of measured data will be discussed.

Research aponeored by U.S. DOE Divicion of Nuctear Plysica.

\subsection{5}

\section{A STOCHASTIC METHOD FOR NUCLEAR POWER PLANT DIAGNOSIS}
R. B. Perea
R. T. Wood*

(Abatract of paper in Noice and Nonbinear Phesomene in Neclear Syotems, Series B: Phyrice Vol. 192, pp. 451-456, proceeding of the NATO Advanced Research Wortubop. Univenidad Polilecrice de Valenos, Spain, May 23-27, 19as, J. L. Munos Cobo and F. C. Diflippo, Eda., Plenum Pres, NY (1200)]

The value of using neutron noise descriptors, especially the power spectral densities (PSD3) of ex-core detectors, to monitor core barsel motion and fuel element vibrations in pressurized rater reactors (PWRs) is well established. In the midfrequency range (1-20 Hz), PWR neutron noive is dominated by vibration peaks in the neutron PSD that result from the motion of reactor internals. $A$ qualitative examination of such deacriptors from a 1150-MWE Weatinghouse PWR plant, revealed significant changes in the plant's noise signature (PSD) as its first fuel cycle proceeded. The purpose of this work is to use a mathematical model of the interaction between small mechanical mo- 
tions and the core meutronics to provide a tool for quantitative investigation of the PSD structure in the vibrational frequency range.

The purpose of weutron noise analysis is to provide information on plant conditions by the study of pomer traces from ex-core detectors. Due to the action of the coolant flow through the core and pump induced vibrations, the core barrel, which is bolted at the top of the reactor vessel, performs a pendular motion with a natural frequency determined by the mechanical parameters and constraints of the system. In addition, exter- nal forces deform the cylindrical shape of the core barrel by inducing vibrational modes which are excited by the turbulense of the coolant flow and by the "table-shaker" effect of the core barrel motion. As a result of the various mechanical motions, the detector response will vary in a manner proportional to the importance function at the location of the disturbance.

Revearch aponeored by U.S. DOE Divicion of Nuclear Phyice.

*University of Temenee, Knoxville, TN.

\section{REVIEWS}

\subsection{6}

\section{BLECTRON LINEAR ACCELERATORS FOR FAST NEUTRON DATA MEASUREMENTS IN SUPPORT OF FUSION ENERGY APPLICATIONS}

\author{
K. H. Bockhof \\ O. A. Wasson't \\ A. D. Carleon't \\ D. C. Larson \\ [f.betract of Noel. Sei. Eng. (in preas)]
}

The interest in neutron data for energy applications has moved over the past $\mathbf{4 0}$ years from thermal/epithermal data to data in the eV/keV range (following or anticipating the general trend of technological development from thermal to fast fission reactors) to the $\mathrm{MeV}$ energy range to include fusion reactors. While the support for further improvement of data for fiscion reactors has weakened, the interest in neutron data for the development of terrestrial fusion power sources is rising, irrespective of the uncertain time schedule for the successful realization of such reactors. For this application, neutron data are mainly wanted from the keV region to about $14 \mathrm{MeV}$, which implies an extension of the energy region intensively inveatigated for fission reactor interests to substantially higher energies.

Research sponsored by U.S. DOE Division of Nuclear Physice.

"CBNM, Gell, Belgium.

'NIST, Gaither' 'urg, MD.

\subsection{7}

DECAY heat MEASUREMENTŚ AIJD THE LOSS-OT,-COOLANT ACKIDENT

\section{J. K. Dichens}

(Abarnat of cral presentation given at the Department of Nuclear Engincering, Univenity of Tennemee, Kmoxville, TN, October 26, 1938)

About fifteen years ago serious concern about the consequences of an hypothetical loseof-coolant accident at a Light Water Reactor prompted several experiments in the United States having the goal of measuring the heat source due to decay of fission products built up in the fuel. The experiment completed at ORNL is described and results from this experiment are compared with other U.S. experiments and with recently reported experiments done in Japan. The summation technique used to calculate this energy source, called "decay heat," is described. Application of these concepts to the accidents at Three-Mile lo land and at Chernobyl is discuseed.

Reseasch eponeored by U.S. DOE Diviaion of Nuclear Phyrices.

\subsection{8}

REACTOR SAFETY AND THE STATUS OF COMMERCIAL NUCLEAR POWER IN THE UNITED STATES

\section{J. K. Dickens}

(Abotract of ord presentation preaented at Ohio University, Athens, Ohio, April 6-7, 1969)

Of all the major industrial activitien in the United States that of cummercial electricity production ty nuclear power is probably the most widely discussed in public forums today. Scarcely 
a day passes without a report on some aspect of nuclear power in our nows media. A review of the (brief) history of commercial nuclear power especia'ly in the context of other social changes in this country provides a reasonable explanation for present-day interest in this topic. Indeed, several paradoxes concerning nuclear power provide clues tr its current status as a viable contributor to our society. As an example of a paradox, nuclear power has been a substantial growth industry for fifteen years, and yet companies involved in this industry have been considered as poor investments for nearly all classes of investors. As a second example of a paradox, nuclear power is perceived by many as the most dangerous commercial undertaking ever conceived, and yet the actual experience in terms of accidental human deaths of injuries indicates that nuclear power is at present the safest industry in the United States. As a third example of a paradox, there is a strong demand for professional and skilled workers to fill many job openings in nuclear power production, and yet it is well known that there have been no new nuclear power plants initiated in this cou itry for over seven years. Indeed, this last fact provides a fourth example of a paradox in that this cessation of growth at the production level has occurred despite federal governmental recognition of the need to increase this nation's self-sufficiency in energy production and the concomitant recognition and support of the role of nuclear power in trying to fulfill this goal.

Research sponsored by U.S. DOE Division of Nuclear Physics.

\subsection{9}

\section{NEUTRON RESEARCH AT TH O OAK RIDGE ELECTRON LIPJEAR ACCELERATOR}

\section{J. K. Dickens}

(Abatract of oral presentation pi esented at Ohio Univeraity, Athens, Ohio, April 6-7, 1989)

The Oak Ridge Electron Linear Accelerator (ORELA) is a multi-purpose facility used principally as a source of neutrons having energies between thermal and $80 \mathrm{MeV}$. A description of the facility will be presented. Research with neutrons at ORELA lias been divided about equally between basic physics and applications. For the former, for example, there have been recent studies leading to (a) an estimate of the age of the universe, and (b) some relationships between the optical model of neutron scattering and the shell model potential describing single-particle orbits in ${ }^{10} \mathrm{Ca}$. For applied research effort has been directed toward obtaining data to be incorporated into the U.S. Evaluated Nuclear Data File (ENDF). Some current research in high-resolution in-beam gamma-ray spectroscopy, $(n, X \gamma)$ resctions, will be discussed, in particular some results for ${ }^{53} \mathrm{Cr}$ that are not yet understood from basic principles.

Research eponsored by U.S. DOE Division of Nuctear Physic.

2.70

\section{ENDF/B-VI NUCLEAR DATA EVALUATIONS FOR FUSION APPLICATIONS}

\author{
C. L. Dunford ${ }^{*}$ D. C. Larson \\ P. G. Young ${ }^{\dagger}$
}

[Abatract of paper presented at the ANS Eighth Topical Meeting on Technolony of Fusion Energy, Salt Lake City, U1, October 9-13, 1988; Fuion Teeh. 15(2), 429-439 (1969)]

The next release of the EN DF/B data library planned for 1989 contains improved data evaluations of interest to the fusion neutronics community. New data formats permit inclusion of energy-angle correlated particle emission spectra and recoil nucleus energy spectra. Enhanced for:nats for covariance information have been developed. Many new isotinpic evaluations will lead to improved energy conservation and kerma factor calculations. Improved nuclear model calculations will provide reliable particle emission data whee experimental information is sparse. Improved Bayesian fitting codes will provide more accurate evaluations for data rich reactions such as $\operatorname{Li}(n, n t) \alpha$. nll of the most important fusion material evaluations contain these new features.

Research sponsored by U.S. DOE Division of Nuclear Physics.

*Brockhaven National Laboralory, Upton, NY.

tLos Alamos National Laboratory, Los Alamon, NM. 
2.71

\title{
REPORTS ON NUCLEAR DATA ACTIVTTIES FOR THE DOE NUCLEAR DATA COMMITTEE
}

\author{
D. C. Larson
}

(Abatrect of BNL-NCS-40911, p. 132, May 1988; and of BNL-NCS-12382, p. 136, April 1969)

These reports were prepared for the DOE Nuclear Data Committee and cover work performed at ORNL since January 1987 in areas of nuclear data of relevance to the U.S. applied nuclear energy program. These reports were mostly generated ihrough a review of abstracts of work completed to the point of being subjected to some form of $p$. slication in the open literature, formal ORNL reports, ORNL technical memoranda, progress reports, or presentations at technical conferences. As much as possible we have reproduced the complete abstracts of the original publications with only minor editing. In a few cases progress reports were written specifically for this publication. The editor has selected the materials to be included in these reports on the basis of percetved interest of DOE Nuclear Data Committee members and cannot claim completeness.

Rewearch sponsored by U.S. DOE Division of Nuclear Phyrics. 
Section 3

INTELLIGENT SYSTEMS 


\title{
3.0. INTRODUCTION
}

\author{
R. C. Mann
}

The Center for Engineering Systems Advanced Research (CESAR) located in the Intelligert Systens Section continued to serve as a focal point for research in autonomous mobile robots and intelligent systems for unstructured operational environments. Publications indicate significant progress in several areas including machine learning, asynchronous production systems, and multi-sensor integration. Particular emphasis was placed on a thorough investigation of the robustness of robot hardwvare and software modules integrated into the CESAR mobile robot prototype HERMIES-IIB. This continuing effort underlined the importance of our continued commitment to mobile robot prototypes that can be used to generate quantitative data to characterize the performance of innovative methods for autonomous robots.

HERMIES-III, our latest mobile robot, was delivered and has been undergoing successful functionality tests. The robot features a seven degree-of-freedom research manipulator (CESARm) developed at ORNL, mounted on a platform with omni-directional steering. HERMIES-III can sense its three-dimensional surroundings through a laser range camera, stereo pairs of CCD cameras, and an array of sonar transducers. The on-board computer architecture includes a hypercube multi-processor, and multiple 68020 processors in four VME systems, a configuration that was designed to support full robot autonomy. The system represents a unique testbed for our research in combined mobility and manipulation, intelligent multi-sensor systems, concurrent computing, and machine reasoning, HERMIES-III and HERMIES-IIB also constitute a unique facility for pioneering research in multiple cooperating autonomous robots.

The section's modeling and simulation activities continued to address several domains, including energy-economy modeling, nuclear waste isolation, and radiation dosimetry. Automated sensitivity analysis represents one of the common themes in these diverse applications.

The Division's Human Factors Group joined our section during this reporting period. Significant accomplishments are reported in the areas of human-computer interactions, cognitive modeling of human operators in nuclear power plants, and human factors for usage of advanced instrumentation and controls in the nuclear industry. The challenging area of human interactions with mobile robots that have a high degree of autonomy may serve to enhance the integration of the human factors work into the section. 


\section{AUTONOMOUS MOBILE ROBOTS}

3.1

\section{ROBOT NAVIGATION RESEARCH AT CESAR}
D. L. Barnett* S. S. Iyengart
G. de Saussure
A. Sabharwal'
F. G. Pin
C. R. Weisbin

(Abatract of paper to be preanted at the 18th IEEE Conference on Decivion and Control, Tampa, FL, Decenber 13-15, 1989)

A considerable amount of work has been re ported on the problem of robot navifation in known static terrains. Algorithms have been proposed and implemented to search for an optimum path to the goal, taking into account the finite size and shape of the robot. Not as much work has been reported on robot navigation in unknown, unstructured, or dynamic environments.

A robot navigating in an unknown environment must explore with its sensors, construct an abstract representation of its global environment to plan a path to the goal, and update or revise its plan based on accumulated data obtained and processed in real-time.

The core of the navigation program for the CESAR robots is a production system developed on the expert-systent-shell CLIPS which runs on an NCUBE hypercube onboard the robot. The production system can call on C-compiled navigation procedures. The production rules can read the sensor data and address the robot's effectors. The architecture was found efficient and flexible for the development and testing of the navigation algorithms; however, in order to process intelligently unexpected emergencies, it was found necessary to be able to control the production system through externally generated asynclironous data. This led to the design of a new asynchronous production system, APS, which is now being developed on the robot.

This paper will review some of the na "ation algorithms developed and tested at CES $\Lambda \mathrm{H}$ and will discuss the need for the new APS and how it is being integrated into, the robot architecture.

Researds sponsored by U.S. DOE Office of Baeic Energy Sciences.

"Computing and Telecommunicationa Division.

'Louigiam Slate University. Baton Rouge, LA.
3.2

\section{THE USE OF AN BXPERT SYSTEM FOR AUTONOMOUS ROBOT CONTROL}

\author{
D. L. Barnett* G. de Sausoure \\ P. P. Spelt
}

(Abotract of oral presentation presented at the American Society for Information Scienoe Meetint, Amn Arbor, MI. May 15-18, 1983)

This paper describes the use of an expert system for control of HERMIES-IIB, a mobile antonomous mbot developed at the Center for Engineering Systems Advanced Research (CESAR). An expert system was considered desirable since the knowledge is in the form of rules that are eary to understand and modify. CLIPS, the expert system shell used in creating the knowledge bace, accomodates access and management of information from a database through user-defined ' $C$ ' routines linked to the shell; therefore, the robot is able to mate high-level decisions and to direet both data acquisition and information processing at a lower level. Each decision can be explained by retrieving the sequence of rules which led to that decision.

Future experiments are planned using an expanded version of the present expert system to demonstrate HERMIES' ability to diagnoce and correct a proces control problem. By experimentation the robot will learn a sequence of panel manipulations which will correct the diagnowed problem.

Research sponeored by U.S. DOE Ofice of Baxic Energ Sciences.

"Computing and Telecommunications Division.

\section{3}

ROBOT NAVIGATION RESEARCH USING THE HERMIES MOBILE ROBOT

$$
\text { D. L. Barnett* F. G. Pin }
$$

(Abstract of paper to be prevented at the 1900 IEEE Conference on Robotice and Automation. Cíncinnati, OH, May 13-18, 1990)

In recent years robot navigation has attracted much attention from researchers around the world. Not only are theoretical studien being simulated on 
sophisticated computers, but many mobile robots are now used as test vehicles for these theoretical studies. Various algorithms have been perfected for navigation in a known static environment; but navigation in an unknown and dynamic environment poses a much more difficult problem for researchers. Many different methodologies have been developed for autonomous robol navigation, but each methodology usually has a particular type of environment in which it does not work well.

At the Center for Engineering Systems Advanced Research (CESAR) at Oak Ridge National Laboratory, research is focusing upon autonomous bavigation in unkrown and dynamic environments using the series of HERMIES mobile robots for testing algorithms. The research approach is one that has an expert system for high level planning interfaced with $C$ routines for implementing the plans, and for quick processing of data requested by the expert system. In using this approach, the navigation is not restricted to one methodology since the expert system can activate a rule module for the methodology best suited for the current situation. Modules can be added as they are developed and tested and can be interfaced with the rule base. The rule base remains small, fast, effcient, and easy to understand and maintain. The C code is also small, easy to maintain, and modular with each routine performing a very specific function. Modules can be developed for navigating from a map, searching for a target, exploring, potential-field navigation, navigation using edgedetection, intense sensing of trouble spots, etc. This paper will address this particular approach that is now being researched and developed using the HERMIES-IIB robot.

Research sponsored by U.S. DOE Office of Basic Energ Scienres.

-Computing and Telecommunications Division.
3.4

\section{SPATIAL REA JONING IN THE TREATMENT OF SYSTEMATIC SENSC.R ERRORS}

\author{
M. Beckeruan \\ J. P. Jones \\ R. C. Minn \\ L. A. Parkas" \\ c. E. Johnston ${ }^{\dagger}$
}

[Ahatract of pape presented at the SPIE Cooference on Sensor Fusion: Spativl Reaconing and Scene Interpretacion. Cambridge, MA, November 6-11, 1988; Proc. Vol. 1003, pp. $388-349$ (1863)!

In processing ultrasonic and visual sensor data icquired by mobile robots systematic, errors can occur. The sonar errors include distortions in size and surface orientation due to the beam resolution and false echoes. The vision errors include, among others, ambiguities in discriminating depth discontinuities from intensity gradients generated by variations in surface brightness. Is this paper we present a methodology for the removal of systematic errors using data from the sonar sersor domain to guide the processing of information in the vision domain, and vice versa. During the sonar data processing some errors are removed from 2D navigation maps through pattern analyses and consistent-labelling conditions, using spatial reasoning about the sonar beam and object characteristics. Others are removed using visual information. In the vision data processing vertical edge segments are extracted using a Canny-like algorithm and are labelled. Object edge features are then constructed from the segments using statistical and spatial analyses. A least-squares method is used during the statistical and spatial analyses. A least-squares method is used during the statistical analysis, and sonar range data are used in the spatial analysis.

Research sponeored by U.S. DOE Office of Nuclear Energy and by the Office of Technoloy Support Prozrame.

"Comell University, Jthra, NY.

'Univenity of Kenturky, Lexington, KY. 
3.5

\section{ROBUST PERFORMANCE OF MULTIPLE TASKS BY AN AUTONOMOUS ROBOT}

\author{
M. Beckerman \\ D. L. Barnett" \\ J. R. Einstein \\ J. P. Jones \\ P. F. Spelt \\ C. R. Weisbin
}

(Abatract of paper presented at the Wortuhop on Integretion of AI and Robotic Sydews, Scoltadale, AZ, May 19, 1900)

There have been many successful mobile robot experiments, but very fen papers have appeared that examine the range of applicability, or robustness, of a robot system. The purpose of this paper is to determine and quantify robustness of the Hermies-11B experimental capabilities.

Research eponsored by U.S. DOE Ofice of Buxic Energy Sciences.

*Compoting and Telecommunications Divicion.

\section{6}

\section{WORLD MODELLING AND MULTI- SENSOR INTEGRATION FOR A MOBILE ROBOT}

\author{
M. Beckerman I. A. Barkas" \\ J. P. Jones R. C. Mann \\ C. W. Glover
}

[Abutract of paper presented at the ANS Third Topical Meeting on Robotica and Rernote Syatems, Charketton, SC, March 13-16, 1809; Proc. Section 11-2, pp. 1-8 (1900)

In this paper we present a strategy for the reduction of systematic errors through the fusion of ultraconic range and visual intensity informar tion. This is done within a framework consisting of several world models, integration procedures, and senser models which include appropriate sensorspecific signal and information procesaing algo rithms. Among the world models are linked lists of geometric features extracted from the intensity images, and 2D Cartesian mape built from the conar range data. Spatial reasoning was used in the fusion of ultraconic data from multiple scans, and in the analysis of visual edge information following exchange of range and angular information acrose the sensor domains.

Research sponeored by U.S. DOE Office of Nuclear Energy, Technology Support Programe and by the Office of Baic Energy Sciences.

"Cornell Univerity, Ithaca, NY.

3.7

\section{TREATMENT OF SYSTEMATIC ERRORS IN THE PROCESSING OF WIDE ANGLE SONAR SENSOR DATA FOR ROBOTIC NAVIGATION}
M: Becherman
E. M. Oblow

[Abstrect of ORNL/TM-10763, April 1988; abo IEEE Thene. Robotice $\boldsymbol{O}$ Antomation (in prem))

A methodology has been developed for the treatment of systematic errors which arise in the processing of sparse sensor data. We present a detailed application of this methodology to the construction from wide-angle sonar sensor data of navigation maps for use in autonomous robotic navigation. In the methodology we introduce a four-valued labelling scheme and a simple logic for label combination. The four labels, conflict, occupied, empty and unknown, are used to mark the cells of the navigation maps; the logic allows for the rapid updating of these maps as new information is acquired. The systematic errors are treated by relabelling conflicting pixel assignments. Most of the new labels are obtained from analyses of the characteristic patterns of conflict which arise during the information processing. The remaining labels are determined by imposing an elementary consistent-labelling condition.

Research sponsored by U.S. DOE Office of Baic Energy Sciences and Office of Technolozy Support Program.

\section{8}

\section{AN APPROACH TO ELEMENTAL TASK LEARNING}

\section{P. F. R. Belmans*}

(Abetrect of paper to be presented at the International Society for Optical Engincers, Applicatione of Artificial Intelligence, Orlando, FL, April 16-20, 1000)

Today, great interest is granted to the development of learning abilities for machines. For our research carried out in the field of robotics, this means the capability by a robot of "understanding" and performing new tasks by observation of a human operator. This feature is useful in recovery procedures, when a mobile roisut is unable to solve a given problem; the hunan operator takes over the control, e.g. performa necessary actionn to reset the robot in a normal situation. If this recovery is properly analyzed and learned by the 
robot, it would then be possible for it to solve similar situations in the future.

Research sponsored by U.S. DOE Office of Banic Energ Soiences.

* On post-doctoral tellowship enigmonent at ORNL moder NATO and CEA/UGRA/ROL sponsonhip

3.9

\section{OBJECT RECOGNITON FOR THE HERMISS ROBOT}

\section{K. W. Bowyer"}

(Abetract of ORNL/TM-10035, October 1908)

The HERMIES-IIB robot in the CESAR lab at ORNL is used in research related to vision, multi-sensor integration, coordinated arm movement, path planning, parallel algorithms, and learning. This report describes the development of a visual object recognition system for HERMIES. This system is implemented on an NCUBE system, an MIMD hypercube parallel computing system. In general, the input to the object recognition system will be an image containing one or more instances of the known different types of obstacles, and the output will be the location(s) of the obstacle(s) relative to the camera. Based on assumptions about the known geometry of the obstacles, the viewing conditions, the relevant range of vieving distances, and the image resolution needed, it is proposed that the obstacles can be recognized by the presence of roughly rectangular outlines in the image. The object recognition system uses Hough transforms to find rectangular outlines in the image and then uses a sequence of constraints to eliminate those outlines which could not be due to obstacles.

Research sponcored by U.S. DOE Office of Nuclear Energy. Technolngy Support Program.

"Univenily of South Florida, Tampa, FL.
3.10

\section{A DEMONSTRATION OF AUTONOMOUS NAVIGATION AND MACHINE VISION USING THE HERMIIS-IIB ROBOT}
B. L. Burts
D. L. Barnett*
J. P. Jones
S. M. Killought

(Abatrack of paper prevented al the Southentem Regional Medic Leaderhip Coumcil Coaference on Microcoupeters and Artificid Intellifsuce in Communication Techaologies, Univerity of Tenneswee at Chattanooge, Chattemoog, TN, October 29-31, 1937)

In earlier published work, the authors developed and demonstrated expert system control of an autonomous robot navigating in unknown environments and dealing with unexpected events such as moving obstacles. The limitations of this earliet work included insufficient on-board computing capability, restrictions on the sensor suite to only sonar transducers, and a geometrically specified goal location.

In this paper, we describe advances to our mobile robot series (currently HERMIES-IIB) to include 8 NCUBE procesors onbourd, (computationally equivalent to $8 \mathrm{VAX} 11 / 780$ 's) operating in parallel, and augmentation of the sensor suite with cameras to facilitate on-board vision analysis and goal finding. The esential capabilities of the expert aystem deacribed in our earlier paper have been ported to the on-board HERMIES-IIB computers thereby eliminating off-board computation. We describe a successful experiment in which a robot is placed in an initial arbitrary location without prior specification of the room contents, successfully discovers and navigates around stationary and moving obstacles, picks up and moves small obstacles, searches for a control panel, and reads the meters found on the panel. The experiment will be expanded to include more complex on-board vision analysis and expert system control in dynamic environments as well as research in robot learning ar the robot attempts to adjust the meter reading to a particular value without a priori knowledge of the control panel system dynamics.

Hesearh aponeored by U.S. DOE, Office of Basic Energy Sciences.

"Computing and Telecommunications Division.

$\dagger_{\text {Instrumenation and Concrol Division. }}$ 
3.11

\section{ONGOING RESEARCH USING HERMILS - THE HOSTILE ENVIRONMENT ROBOTIC MACHINE INTELLIGENCE EXPERIMENT SERITS}

\author{
B. L. Burlss P. F. Spelt
}

(Abotract of paper preaseded of the Materials Handlins

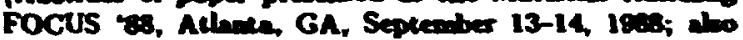

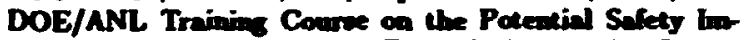
pect of New and Emergios Technolngies on the Operetion of DOE Nudear Fecitives, Idaho Fal, ID, Auguat 20-Seplember 1, 1908)

The Cenler for Engineering Systems Advanced Research (CESAR) was established in 1983 at Oak Ridge National Laboratory as a Department of Energy (DOE) Center of Excellence in the study of intelligent machines. The major emphasis of the research at CESAR is in basic research related to the operation of autonomous mobile robots in unatructured environments. The long-range goal of the research is to develop intelligent machise systems capable of replacing bumans in potentially hazardous environment such as surveillance and maintenance functions in high radiation, deep underground or undersea areas. Currently research is underway and/or has beels publiched in the areas of: real-time expert sytems with dynamic replanning, rnodeling and control of light-weight, compliant, redundant manipulators mounted un a mobile vebicle; model-based scene understanding using multiple seneors, dynamic task albeation among heterogeneous recources, and machine learning.

Research sponsored by U.S. DOE Ofice of Basic Energy Sciences.

\subsection{2}

\section{AUTOMATIC LEARNING BY AUTONOMOUS MOBILE ROBOT}

\author{
G. de Saussure \\ P. F. Spelt \\ S. M. Killough" \\ F. G. Pin \\ C. R. Weisbin
}

(Abaract of paper presenced al the ANS Third Topical Meeting on Robrotica and Remole System., Charleston, SC, March 13-16, 1849; Proc. Section 6-1, pp. 1-5 (1960)]

This paper deacribes recent research in auto matic learning by the autonornous mobile robot
HERMIES-IIB at the Center for Engineering Systerns Advanced Research (CESAR). By acting on the environment and observing the consequences during a set of training examples, the robot learns a sequence of successful manipulations on a simulated control panel. The robot learns to classify panel configurations in order to deal with new configurations that are not part of the original training set.

Researdh apococod by US. DOE Otioe of Benic Beng Sciences.

- Pusion Eneros Divicion.

\subsection{3}

\section{NAVIGATION AND LEARNING EXPERDMBNTS BY AN AUTONOMOUS ROBOT}

\author{
G. de Saussure C. R. Weibin
}

P. F. Spelt

[Abatrect of paper prewented at the Second interantioan Symposium, Robotic and Maronfeturing Research, Ed ucation, and Application, Alboquerque, NM, November 16-18, Ires, Proc. pp. 935-948 (1909))

Developing an autonomous mobile robot capable of navigation, surveillance and manipulation in complex and dynamic environments is a key research activity at CESAR, Oak Ridge Nor tional Laboratory's Center for Engineering Syrtems Advanced Research. The latest series of completed experiments was performed using the autonomous mobile robot HERMIES-IIB (Hotile Environment Robotic Machize Intelligence Experiment Series II-B). The next section deacribes HERMIES-IIB and some of its major components required for autonomous operation in unstructured, dynamic environments. Section 3 outlines some ongoing research in autonomous navigation. Section 4 discusees our newest research in machine learning concepts. Section 5 describes a succeas ful experiment in which the robot is placed in an arbitrary initial location without any prior specification of the content of its environment, successively discovers and navigates around stationary or moving obstacles, picks up and moves small obstacles, searches for a control panel, and performs a learncd sequence of manipulation on the panel devices. The last section outlines soine future directions of the program.

Reweardh aponcored by U.S. DOE Offuce of Bacic Energy Sciences. 
3.14

\section{NON-LINBAR SURPACE FITTING OF LASER RANGE MMAGES USING A HYPDRCUBE CONCURRENT COMPUTER}

\author{
M. Finley* \\ (Abotract of ORNL/TM-1119s, Jume 1800)
}

Segmentation and non-linear surface fitting with laser range images are attempted on a hypercube concurrent processor. Lon-level software acquires range images onto the hypercube. A substantially modified existing range image edge detector works in the hypercube image analysis environment to accomplish segmentation. The Levenberg-Marquardt algorithm implements the non-linear least equares fitting of the surfaces.

Problems were found with each part of the approach. The edge detection algorithm is unnecessarily complex and yields an all too cluttered edge map. The fitting routine is limited by hardware in the number of surfaces that it can process. Finally, the fitting approach requires input eatimates of the parameters which do not appear to be generalizable for any image, thus keeping the process from being totally automated. Other research in surface fitting on serial computers bas yielded results which may be helpful additions to solve the problems of automation.

Research sponsored by U.S. DOE Office of Baxic Energ Sciences.

*Transylvaria Univesily, Lexington, KY.

\subsection{5}

\section{NAVIGATION PLANNING USING QUADTREES}

\section{R. C. Fryxell*}

(Abetract of ORNL/TM-10481, November 1987)

An algorithm is presented for planning a 2-D collision-free path for a mobile robot in an unstruetured work environment. The algorithm acoumes the existence of a pixel map of all or part of the environment, where each pixel is either on (implying blocked) or of (implying clear). The goal is to compute a "reasonable path" between two points in a minimal amount of time, and this is achieved through a "compressed" representation of the pirel map using a modified quadtree data structure. The algorithm has been coded in the $C$ programming language, and the results of tests made on "realistic" indoor environments are presented. A discussion on how the environmental maps were obtained from sonar range-finder data is also included.

Rewearch eponmed by Albion College, Albion, MI, by Greak Lales Colleges Amociation. Ann Arbor. MI, and by U.S. DOE Otice of Bacic Enery Sciences.

- Abion College, Albion, MI.

3.16

\section{THE MAN-ROBOT SYMBIOSIS PROJECT}

\author{
W. R. Hamel* F. G. Pin
}

(Abetrect of paper presented at the 1908 WATTec Coaference, Knoxville, TN, February 16-19. 1908)

Remote operations which have been used to protect man from hazardous radioactive environments for nearly $\mathbf{4 0}$ years significantly reduce effective work proficiencies. In fact, the best master/slave manipulator systems in existence, while able to perform very complex tasts remotely, reduce work efficiency by factors of five to eight. The ultimate goal in remote operations is to perform necessary tasks at least as quickly as humans could do them with direet contact, and in some cases to hopefully be able to perform them even faster than manual operation allows. It is believed that the key to achieving such levels of performance is the integration of effective automation and intelligent control into the operation of advanced teleoperators. ORNL is pursuing this premise in a new research project called "Man-Robot Symbiosis." As the name implies, man-robot symbicois is the next step beyond advanced telerobotics in which sensor, computers, and graphics will be integrated to accomplish cooperative man-machine control and intelligence in a dynamic and highly fluid way. The "symbiotic" system will facilitate cooperation to the mutual advantage of both the man and machine, and will ultimately facilitate the dynamic optimization of the division of work between the two.

Research sponeored by U.S. Department of Eutergy.

"Inatrumentation and Controls Division. 
3.17

\section{SMELTING NETWORKS FOR REAL TIME COOPERATTVE planning in the presence OF UNCERTAINTIES}

\author{
S. S. Iyengar" S. Gulati* \\ J. Barhen'
}

(Abetreat of peper preconted at the Applications of Artif cill hivelligence VI Comberence, Odando, FL, April 12-14. 1903)

The accelerated emphasis on research and development in masively parallel architectures, biological and neurocomputing, connectioniat machines, etc., and their feasibility in near future, promises the AI community an opport unity for re covering from the "Al Trap" and the scepticism related to its applicability and delivercbility for large scale applications. Given the gargantuan computational capabilities, an area where AI can be put to work extensively is the development of rule-based strategies for cooperative problem solving and planning in complex, spatially diatributed systems and presence of uncertainties. This paper discusses the applicability and limitations of the existing AI paradigm in designing algorithms to do real time, onboard tack planning for the multiple target tracking problem.

Research eponsored by U.S. DOE Office of Baic Every Sciences.

-Loviciana State Univerity, Baton Rouge. LA.

Jet Propulsion Laboravery, Paadena, CA.

\subsection{8}

\section{ASYNCHRONOUS PRODUCTION SYSTEMS FOR CONTROLLING AUTONOMOUS MOBILE ROBOTS}

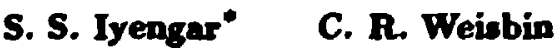 \\ F. G. Pin
}

(Abatsact of paper presented at the sht International Conpreas of Cybemetio and Syatem, Hurker Collete, New York, June 11-15, 1989)

Today, the robotics and automation community is teing swept by broad, pervasive technological demands. The successful deployment of Intel. ligent Computing Systens for robotice and proceas control application requires an efficient integration of traditional expert system technologies with real time response and control capabilities. To address such requirements, researchers at Oak Ridge National Lab and LSU have been working on a control architecture called Asynchronous Production System (APS), which is a rule based inference engine capable of monitoring and processing asynchronous, real-time information. The primary motivation of the APS architecture is to provide a unique mecharism for designing rule-based expert systems capable of dynamic and rapid interaction with the environment. This paper elaborates on the implementation results of the APS in a multiprocessor environment.

Research aponeored by U.S. DOE Ofice of Bacic Ewery Sciences.

-Loniciana State Univeruits. Balon Ronge. LA.

\subsection{9}

\section{THE U.S. DOE/NE UNIVERSTY PROGRAM IN ROBOTICS FOR ADVANCED REACTORS}

\author{
R. C. Mann
}

(Abatraca of poper presented at the 1808 WATTec Conference, Knox ville, TN, February 16-19, 19es)

The U.S. Department of Energy has provided support to four universities and the Oat Ridge $\mathrm{Na}$ tional Laboratory in order to pursue research leading to the development and deployment of an advanced robotic system capable of performing tasks that are hazardous to humans, that generate significant occupational radiation exposure, and/or whose execution times can be reduced if performed by an automated system. The goal is to develop a generation of advanced robotic systems capable of performing surveillance, maintenance, and repair tasks in nuclear facilities and other hazardous environments.

The development of general purpose robots to perform skilled labor tasks in restricted environments is expected to have extensive payback in areas of energy systems (nuclear and fossil units), chemical plants, fire fighting, apace operationa, underwater artivities, defense, and other hazardoun activities.

Rewearch aponsored by U.S. DOE Office of Technology Support Progrems. 


\section{THE DEVELOPMENT OF AN INTELLIGENT NUCLEAR MAINTENANCE ROBOT}

\author{
R. C. Mann W. R. Hamel* \\ C. R. Weisbin
}

(Abstrect of paper presented at the IEEE 1983 Intermetionel Conference on Robotics and Avtomation, Philndetphic, PA, April 25-20, 19ed; Proc. Vol. 1, Pp. 621-623 (19e8)]

The U.S. Department of Energy has provided support to four universities and the Oak Ridge National Laboratory (ORNL) in order to pursue research leading to the development and deployment of robotic system(s) for advanced nuclear power stations. This paper describes the scope of the program and the R\&D efiort contributed by ORNL.

Research sponsoned by U.S. DOE Offor of Besic Energ Sciences and Otice of Technoloss Support Prodans.

*Instrumentation and Controls Diviaion

\subsection{1}

\section{AN INTELLIGENT INTEGRATED SENSOR SYSTEM FOR THE ORNL MOBILE ROBOT}
R. C. Mann
J. P. Jones
M. Beckerman
C. W. Glover
L. Farkas"
J. Han
E. Wacholder
J. R. Einstein

(Abutrect of peper presented at the Third IEEE Incerneiiond Symposium on Inelligent Control, Artington, VA, A ugust 24-28, 1988; Proc. H. E. Stephunou, A. Meyatel. and J. Y. S. Isuh. Eds., pp. 170-173 (1989)]

The mobile robot prototypes developed at the ORNL Center for Engineering Systems Advanced Research (CESAR) are equipped with sonar sensors and CCD cameras that are used to support autonomous navigation and simple inspection tasks in an a priori unknown and unstructured dynamic environment. A laser range camera is being added in the sensor suite of HERMIES III, a mobile robot that is currently being built. This paper summarizes work directed at extracting information from data collected with these sensors and integrating it, in order to produce reliable descriptions of the robot's environment. Specifically, the paper describes the integration of two-dimensional vision and sonar range information. All operations are carried out onboard the mobile robot using a 16-processor hypercube computer.

Resenrch sponered by U.S. DOE Otice of Benic Emery Sciences and Ofice of Technolosy Support Program.

* Cornell University. Ithaca, NY.

\subsection{2}

\section{U.S. DEPARTMENT OF ENERGY NUCLEAR ENERGY UNTVERSTTY PROGRAM IN ROBOTICS FOR ADVANCED REACTORS}
R. C. Mann
D. Tesar'
R. C. Gonnalez"
J. S. Tuleakot
D. K. Wehe

(Abotrect of DOE/OR-e4/R1, Jub I8s3)

The U.S. Department of Energy has provided support to four universities and the Oak Ridge National Laboratory in order to pursue research leading to the development and deployment of advanced robotic systems capable of performing tasks that are hazardous to humans, that generate significant occupational radiation exposure, and/or whose execution times can be reduced if performed by an automated system. The goal is to develop a generation of advanced robotic systems capable of economically performing surveillance. maintenance, and repair tasks in nuclear facilitios and other hazardous environments.

This program features a unique teaming arrangement among the Univervities of Florida, Michigan, Tennessee, Texas, and the Oak Ridge National Laboratory, and their industrial partners, Combustion engineering, Odetics, Gulf State Utilities, Florida Pow.er and Light Company, Remotec, and Telerobotics International. Each of the universities and ORNL have ongoing activities and corresponding facilities in areas of R\&D related to robotics. This program is designed to take full adva:: dge of a balance of these existing resourr $\approx$ at the participating institutions.

Research sp.nessed by U.S. DOE, Office of Technoloso Support Programs.

- Univenity of Tennewce, Knoxville, TN.

Univenity of Texas, Austin, TX.

Univereity of Florida, Gainesville, FL

\$Univenity of Mirhigan, Ann Artorr. MI. 


\subsection{3}

\section{RESEARCH IN MOBILE ROBOTICS AT ORNL/CESAR}

\author{
R. C. Mann C. R. Weisbin \\ F. G. Pin
}

(Abatract of paper presented at the 1900 IEEE Internoiond Conference on Sytem Enjineering, Devton, OH. Augual 24-28, 1969, Proc. pp. 367-371 (1969)]

This paper reviews cngoing and planned research with mobile autonomous robots at the Oak Ridge National Laboratory (ORNL), Center for Engineering Systems Advanced Research (CESAF). Specifically we report on results obtained with the robot HERMIES-IIB in navization, intelligent sensing, learning, and on-board paral lel computing in support of these functions. We briefly summarize an experiment with HERMIESIIB that demonstrates the capability of smooth transitions between robot autonomy and tele operation. This experiment results from collaboration among teams at the Universities of Florida, Michigan, Tennessec, and Texas: and ORNL in a program targeted at robotics for advanced nuclear power stations. We conclude by 8 mmarizing ongoing R\&D with our new mobile robot HEFMIES-III which is equipped with a seven degree-of-freedom research manipulator arm.

Research sponsored by U.S. DOE Office of Buic Energ Sciences and the Ofice of Nuclear Energy.

\subsection{4}

\section{ADAPTIVE OPTIMAL CONTROL OF UNCERTAIN NONLINEAR SYSTEMS: ON-LINE MICROPROCESSOR-BASED ALGORITHM TO CONTROL MECHANICAL MANIPULATORS}

\section{March-Leuba" \\ R. B. Perez}

(Abatract of ORNL/TM-10764, May 1988)

This report presents an adaptive optimal control algorithm for uncertain nonlinear systems. A variational technique based on Pontryagin's Maximum Principle is used to track the system's unknown terms and to calculate the optimal control. The reformulation of the variational terhnique as an Initial Value Problem allows this microprocessor-based algorithm to perform on-line model-updating and control. To validate the algo- rithm, a system representing a two-link mechanical manipulatcr is simulated. In the control model, the coupling and friction terms are unknown. The robot's task is to follow a prescribed trajectory and to pick up an unknown mass.

Resench eponeored by U.S. DOE Ofice of Bexic Eners Sciences.

"University of Tennewee, Kmorville, TN.

\subsection{5}

\section{OPTIMAL CONTROL OF UNCERTAIN NONLINEAR SYSTEMS: AN APPLICATION TO A TWO-LINK ROBOTIC ARM}
C. March-Leuba*
R. B. Peres

(Abatract of paper preseated at the 27 ch IEEE Confereace on Decision and Contcol, Anetin, TX, Decenaber 7-9, 1800)

This paper presents an edaptive optimal control algorithm for uncertain nonlinear systems. A variational technique based on Pontryagin's Maximum Principle is used to track the system's unknown terms and to caiculate the optimal control. The reformulation of the variational technique as an initial value problem allows this microproceseorbased algorithm to perform as a cloced-loop controller by updating the model and controlling the system on-ine. To validate the algorithm a system representing a two-link mechanical manipulator:; simulated. In the control model, the coupling and friction terms are unknown. The robot's task is to follow a prescribed trajectory and to pick up an unknown mase.

Research sponsored by U.S. DOE Office of Bacic Enerty Sciences and Ofice of Technolocy Support Propoum.

"Inetrumenetion and Controle Division.

\subsection{6}

\section{A PROBA BILISTIC-PROPOSITIONAL FRAMEWORK FOR THE O-THEORY INTERSECTION RULE}

\section{E. M. Oblow}

[Abatract of J. General Syoteme 13, 187 (1987)]

In a previous paper, $O$-theory (OT), a hybrid uncertainty theory was proposed for dealing with problems of uncertainty in logical inference. The foundations of one of the concepts introduced, the 
OT intersection operator, are explored in this paper. The developments rely solely on set-theoretic and probability notions which are the distinguishing features of this operator's role in the theory.

The OT intersection rule has as its basis Dempster's rule of combination which ties it closely to Dempster-Shafer theory. In this paper the OT rule will be shown to be based more fundamentally on classical probability theory. To demonstrate this, possibility sets are interpreted in a propositional framework and mass assignments are converted to the probabilistic form originally proposed by Dempster. These changes are used to show that the OT intersection rule can be derived from first principles in a probability theory of propositions. Since this derivation does not require conditional probabilities, it can be used as alternative to Bayes' theorem for combining conjunctive information consistently. Dempster's rule will be shown to be a special case of the OT intersection rule. It too will be derived using probability theory.

The formal connection between mass and probability presented originally by Dempster and used here in a propositional framework, makes distinctions between DST and probability theory less consiquential. DST is still seen to be a generalizatior of the concept of probability, but it is also seen to fit within a probabilistic-propositional framework.

Researdh sponsored by U.S. DOE Office of Bacic Energy Sciences.

\subsection{7}

\section{FOUNDATIONS OF O-THEORY: MEASUREMENTS AND RELATION TO FUZZY SET THEORY}

\section{E. M. Oblow}

[Abstract of Int. J. General Syutems 14, 357-378 (1988)]

A measurement framework for O-theory, an algebra of random sets, is presented. The framework is probabilistic and derived from experimental measurements of evidential support. It is used to quantify the probabilistic masses needed in $\mathrm{O}$. theory. This framework allows a connection to be made between this random set Igebra and the possibilistic form of fuzzy set theory. Instances in which these two different representations of the same rxperimental data are equivalent are ex- plored. The relationships bet ween some basic operators in both theories are then discussed, along with their connectior to Dempster-Shafer theory. Finally, several illustrative examples of deductive inferencing under uncertainty are solved. The equivalence of the representations of the results in both uncertainty tbeories if demonstrated.

Research sponsored by U.S. DOE Ofice of Basic Energy Seiences.

\subsection{8}

\section{SUPDTTRACKS, SUPBRTRACK FUNCTIONS AND CHAOS IN THE QUADRATIC MAP}

\section{E. M. Oblon}

[Abetract of Phys. Lettrs. A 128(8), 406 (1968)]

Chaotic behavior for the quadratic map is conjectured to be a bounding pheinmenon based on state-space trajectories starting from the maximum in the map. These trajectories, called sapertrucks, have no sensitivity to initial conditions. The chaotic regime is found to be characterized recursively by these trajectories as functions of the map parameter.

Research sponeored by U.S. DOE Office of Bacic Energ Sciences.

\subsection{9}

\section{MAN-ROBOT SYMBIOSIS: A FRAMEWORK FCR COOPERATTVE INTELLIGENCE AND CONTROL}
L. E. Parker
F. G. Pin

[Abatract of paper preaented at the SPIE Conference on Senuor Fusion: Spatial Reaconing and Scene Interpretation, Cambridze, MA, November 6-11, I9es; Proc. Vol. IOns, pp. 94-103 (1888)]

The man-robot symbiosis concept has the fundamental objective of bridging the gap between fully human-contrnlled and fully autonomous systems to achieve true man-robot cooperative control and intelligence. Such a system would allow improved speec, accuracy, and efficiency of task execution, while retaining the man in the loop for innovative reasoning and decision-making. The symbiont would have capaisilities for supervised and unsupervised learning, allowing an in. 
crease of expertise in a wide task domain. This paper describes a robotic system architecture facilitating the symbiotic integration of teleoperative and automated modes of task execution. The architecture reflects a unique blend of many disciplines of artificial intelligence into a working system, including jcb or mission planning, dynamic tack allocation, man-robot communication, automated monitoring, and machine learning. These disciplines are embodied in five major components of the symbiotic framework: the Job Planner, the Dynamic task Allocator, the Presenter/Interpreter, the Automated Monitor, and the Learning System.

Resenuch eponeored by U.S. DOE Office of Basic Energy Sciences.

\section{$\mathbf{3 . 3 0}$}

\section{WORKSHOP ON HUMAN. MACHINE SYMBIOTIC SYSTEMS}

\section{Editors:}

\author{
L. E. Parker \\ C. R. Weisbin
}

(Abutract of proceedinge of che 1988 Workhop on HumenMachine Symbiotic Sytiem, Oak Ridge, TN, December $5-6,1988)$

This report presents the proceedings of the 1988 Workshop on Human-Machine Symbiotic Systems. Held December 5-6, 1988 in Oak Ridge, Tennessee, the workshop served as a forum for the discussion of reveral critical issues in human-machine symbiosis: human-machine communication, autonomous task planning and execution monitoring for heterogeneous agents, dynamic task allocation, human-machine system architecture, and machine learning vis experience and buman observation. The presentation of overview papers by invited keynote speakers provided a background for the breakout, session discussions in these five areas. The full paprese furnished by the speakers are included in the proceedings, along with written summaries of the group discussions that report scsuion conclusions and recommendations for future work.

Research oponsored by U.S. DOE Ofice of Basic Energy Seiences.

\subsection{1}

\section{REVIEW OF THE 1988 WORKSHOP ON HUMAN-MACHINE SYMBIOTIC SYSTEMS}

\author{
L. E. Parker \\ C. R. Weisbin
}

(Abetract of paper presented at the Workwhop on Integretion of AI and Robotic Systems, Scottedale, AZ, May 19, 1980)

This report presents a review of the 1988 Workshop on Human-Machine Symbiotic Systems. Held December 5-6, 1988 in Oak Ridge, Tennessee, the workshop served as a forum for the discussion of several critical issues in human-machine symbiosis: human-machine communication, autonomous task planning and execution monitoring for heterogeneous agents, dynamic task allo cation, human-machine system architecture, and machine learning via experience and human observation. The presentation of overview papers by invited keynote speakers provided a background for the breakout session discussions in these five areas. A summary of the conclusions and recommendations for the future work resulting from the workshop is reported.

Research sponsored by U.S. DOE Office of Basic Energy Sciences.

3.32

\section{AUTONOMOUS MOBILE ROBOT RESEARCH USING THE HERMIES-III ROBOT}
F. G. Pin
M. Beckerman
P. F. Spelt
J. T. Robinson*

\section{R. Weisbin}

(Abatract of paper presented at the '89 IROS International Conference on Intelligent Rohot and Systems, "The At conomou Robot and Its Application," Tsukube, Japan, September 3-7, 1989)

This pape: reports on the status and future directions in the research, development and experimental validation of intelligent control techniques for autonomous mobile robots using the IERMIES-III robot at the Center for Engineering Systems Advanced Research (CESAR) at Oak F'Jge National Laboratory (ORNL). HERMIESIII is the fourth robot in a series of increasingly more sophisticated and capable experimental 
test beds developed at CESAR. HERAIIES-III is comprised of a battery powered, onmi-directional wheeled platform with a stion degree-of-freedom manipulator arm, video carri ras, sonar range sensors, laser imaging scanne: and a dual computer system containing up to 128 NCUBE nodes in hypercube configuration. All electronics, sensors, computers, and communication equipment required for autonomous operation of HERMIIES111 are located on board along with sufficient battery power for three to four hours of operation. The paper first provides a more detailed description of the IIERMIES-Ill characteristics, focussing on the new areas of research and demonstration nov: possible at CESAR with this new test-bed. The initial experiniental program is then described with emphasis placed on autonomous performance of human-scale tasks (e.g., valve manipulation, ise of tools), integration of a dexterous manipulator and platform motion in genmetrically complex environments, and effective use of multiple cooperating robots (HERMIES-IIB and HERMIES-III). The paper concludes with a discussion of the integration prob!ems and safety considerations necessarily arising from the set-up of an experinental program involving human-scale, multi-autonomous mobile robots performance.

Researth sponsored by U.S. DOE Office of Basic Enersy Sciences.

"Instrumejuation and Controls Division.

\subsection{3}

\section{RECENT CESAR RESEARCH ACTIVITIES IN SENSOR BASED REASONING FOR AUTONOMOUS MACHINES}

\author{
F. G. Pin \\ G. de Saussure \\ P. F. Spelt \\ S. M. Killough* \\ C. R. Weisbin
}

(Abatract of paper presented at the IEEE International Workshop on Intelligent Robots and Systems, Tokyo, Japan, Octotser 31-November 2, 1988; Pruc. 417-421 (1988)]

This paper describes recent rescarch activities at the Center for Enginecring Systems Advanced Research (CESAR) in the area of senso? based reasoning, with ernphasis being given to their application and implementation on our IIFRMIFS-IIB autonomous mobile vehicle. These artivities, including navigation and exploration in n-prior unknown and dynamic environments, goal recogni- tion, vision-guided manipulation and sensor-driven machine learning are discussed within the frameworr of a scenario in which an autonomous robot is asked to navigate through an unknown dynamic environment, explore, find and dock at the panel, read and understand the status of the panel's meters and dials, learn the functioning of a process control panel, and successfully manipulate the control devices of the panel to solve a maintenance emergency problem. A demonstration of the successful implementation of the algorithms on our HERMIES-IIB autonomous robot for resolution of this scenario is presented. Conclusions are drawn concerning the applicability of the methodologies to more general classes of problems and implications for future work on sensor-driven reasoning for autonomous robots are discussed.

Research sponsored by U.S. DOE Office of Basic Energy Sciences.

-Instrumentations and Controls Division.

\subsection{4}

\section{DESIGN OF AN OBSTACLE AVOIDANCE SYSTEM FOR AIMS}

\author{
D. B. Reister M. W. Noakes"
} [A batract of paper presented at the SPIE Conference on
Senecr Fucion: Spatial Reaconing and Scene Interpretation,
Cambridge MA, November 6-11, 1988; Proc. Vol. 1006,
pp. 124-131 (1988)]

The Advanced Integrated Maintenance System (AIMS) is a remote maintenance system that uses a dual-arm, teleoperator to control the Advanced Servomanipulator (ASM). Although, the ASM was designed for nuclear fuel reprocessing, it can provide a testbed for space telerobotics (space servicing and assembly). The objective of this project is to design and implement a generalized capability for automatic path-planning and obstacle avoidance during motion of the manipulator transporter (overhead crane and main manipulator shaft) from one work location in the hotcell to another The work is to be accomplished in two phases. In the first phase, the transporter will move in a known world. In the second phase, sensor data will be used to update and verify the world nodel. This paper describes the development of a systenti for phase one and describes the plans for phase two. 
Before the design of the phase two system, an extensive program of experiments will be conducted using three types of sensors: laser rangefinders, phased array sonar, and television cameras.

Rewearch sponsored by U.S. DOE Office of Bacic Energy Sciences.

* Inatrumentation and Controls Division.

\section{$\mathbf{3 . 3 5}$}

\section{LEARNING BY AN AITTONOMOUS ROBOT AT A PROCESS CONTROL PANEL}

\author{
P. F. Spelt G. de Saussure \\ D. Lyness* F. G. Pin \\ C. R. Weisbin
}

[Abatract of IEEE Expert (im preas)]

The Center for Engineering Systems Advanced Research (CESAR) was founded at Oak Ridge National Laboratory (ORNL) by the Department of Energy's Office of Energy Research/Division of Engineering and Geoscience (DOE-OER/DEG) to conduct basic research in the area of intelligent machines. Within this framework, CESAR has undertaken several research activities in the field of machine learning. In this paper, we describe our approach to a class of machine learning which involves autonomous concept formation using feedback from trial-and-error learning. Our formulation is being experimentally validated using an autonomous robot, learning tasks of control panel monitoring and midu: inulation in effective process control. The C'IIPS Expert System which resides in a hypercube computer aboard the robol, and the knowledge base used by the robot in the learning process are described in detail. Benchmark testing of the learning process on a robot/control panel emulator system consisting of two interacting computers is presented, along with results of sample problems illustrating machine learning and robot performance improvement. Conclusions are drawn concerning the appiicability of the system to a more general class of learning problems, and implications for future work on machine learning for autollomous robots are discussed.

Resenrch sponsored by U.S. DOE Office of Basic Enerc Sciences.

* Iowa Stale Univenily, Amea, IA.

\subsection{6}

\section{PATH-PLANNING IN A KNOWN ENVIRONMENT WITH UNEXPECTED OBSTACLES: POTENTIAL APPLICATION TO AN AUTOMATIC ALARM TESTING ROBOT}

N. Sreenath

(Abueract of ORNL/TM-10726, May 1988)

The feasibility of using an expanded version of a path planning algorithm in the development of an Automatic Alarm Testing Robot (AATR) was investigated. Use of an AATR for security tasks implies the requirement that communication exists between the robot and the security systems or personnel except in the event of a site emergency or mechanical failure aboard the robot. Furthermore, the execution of the actual alarm testing needs to be carried out in narrow, predefined time windows. Thus the path planning algorithm for such a robot should be capable of not only taking the robot from one point to another in a known environment with unexpected (a priori unknown) obstacles in real time, but also time-synchronization with the security systems. Here we propose such an algorithm which uses a breadth-first search technique and discuse its implementation onboard the HERMIES IIB robot. A demonstration of the algorithm in a hard real-time environment is presented and some future desirable erhancements of the algorithm are discussed.

Reseanch sponeored by U.S. DOE Oflice of Baxic Energy Sciences.

\section{$\mathbf{3 . 3 7}$}

\section{PARALLELISM IN PRODUCTION SYSTEMS}
A Sabharwal"
S. S. Iyengar*
G. de Saussure
C. R. Weisbin

(Abetract of paper presented at the Applications of Artifcial Invelligence VI Conference, Orlando, FL, April 12-14, 1988)

Production systems have found considerable popularity in the areas of artificial intelligence and expert system development. Part of their popularity stems from their proven use as convenient tools for knowledge representation and logic program- 
ming. Unturtunately, their obvious advantages are accomr anied by a series drawback - being computationally very expensive, production systems tend to be inherently slow.

In this paper, an $n$-ary tree architecture is proposed which effectively combines the production-level and condition-level parallelism inherent in the match process.

Research sponsored by U.S. DOE Office of Becic Energy Sciences.

"Louisiana State University, Baton Rouge, LA.

\section{$\mathbf{3 . 3 8}$}

\section{ASYNCHRONOUS PRODUCTION SYSTEMS}
A. Sabharwal*
S. S. Iyengar*
C. R. Weisbin
F. G. Pin

[Abatract of Krowledge-Besed Systems 2(2), 117-127 (1989)]

Intelligent computing systems for aerospace, robotics and process control applications require an integration of traditional expert system technologies with real-time response and control capabilities. To address such requirements, the Asynchronous Production System (APS) is presented, which is a rule-based inference engine capable of monitoring and processing asynchronous, realtime information. The primary motivation of the proposed APS is to provide a unique and convenient mechanism for designing rule-based expert systems capable of dynamic and rapid interaction with their environments. This paper elaborates on the architectural and operational characteris tics of the APS and addresses issues pertinent to implementation of the APS in a multi-processor environment.

Research sponsoret by U.S. DOE Office of Basir: Energy Sciences.

- Louisiana State University, Baton Rouge, LA.

\subsection{9}

\section{ASYNCHRONOUS PRODUCTION SYSTEMS FOR REAL-TIME EXPERT SYSTEMS}
A. Sabharwal*
S. S. Iyengar*
C. R. Weisbin
F. G. Pin

[Abatract of paper preaented at A vignon '88 Eighth International Workehop on Expert Systems and Their Applications, Nantere, France, May 30-June 3, 1988; Proc. PP 113-146 (1988)]

Intelligent Computing Systems for Autonomous Mobile Robots and Process Control applications require an integration of traditional expert system technologies with real-time response and control capabilities. To address such requirements, we propose an Asynchronous Production System (APS), which is a rule-based inference engine capable of monitoring and processing asynchronous, real-time information. The primary motivation of the proposed APS is to provide a unique and convenient mechanism for developnient of rulebased expert systems capable of dynamic and rapid interaction with their environments. This paper elaborates on the architectural, operational and implementational ramifications of Asynchronous Production Systems. To facilitate its description, an example of an APS-based distributed expert system is presented to handle the task of autonomous navigation in the presence of unexpected, moving obstacles in a mobile robot.

Research sponsored by U.S. DOE Oftice of Basic Energy Sciences.

*Louisiana State Univervity, Baton Rouge, LA.

\section{A TWO-PC SIMULATION FOR DEVELOPMENT AND TRAINING OF A LEARNING EXPERT SYSTEM IN AN AUTONOMOUS MOBILE ROBOT}

\author{
P. F. Spelt E. Lyness* \\ G. de Saussure
}

[Abstract of Simulation Josmal (in press)]

The Center for Engineering Systems Advanced Remearch (CESAR) at Oak Ridge National Laboratory conducts basic research in the area of intelligent machiues. Recer'ly at CESAR 
a learning expert system was created to operate on board an autonomous robot in real tine at a process control panel. In this paper we present the development and use of a two-computer concurrent simulation system used to create, evaluate and train this learning system. The simulation system uses graphics to display the current status of the process being simulated, and the same program which does the simulating also drives the actual control panel process. We discuss the value of using a computerized simulator to train a learning computer.

Research eponsored by U S. DOE Ofice of Bexic Enery Sciences.

- lowe Stale Univeraity, Amea, IA.

\subsection{1}

\section{DYNAMICS FOR SPACE BASED ROBOTIC MANTPULATION}

\section{N. Sreenath P. S. Krishnaprasad}

[Abatract of paper presented at the 26th IEEE Conference on Decivion and Concrol, Los Angeles, CA, December 9-11, 1987; Proc. Vol. II, p. 1417 (1987)]

In this paper, we present new results on the Hamiltonian atructures of planar multibody sye tems. The models we obtain are reduced models accounting for the symmetry group of rigid motions in the plane. We give some new controllability and stabilizability theorems for anticulating such multibody systems. The models of this paper provide a natural framework for questions of inverse kinematics arising in space based robotic manipulation. We find some very interesting applications of holonomy in this context.

Research eponeored by U.S. DOE Office of Baic Enero Sciences.

"Univenity of Maryland, Colleze Park, MD.
3.42

\section{INTELLIGENT ROBOTICS AND REMOTE SYSTEMS FOR THE NUCLEAR INDUSTRY}

$\begin{array}{cc}\text { D. K. Wehe* } & \text { J. C. Lee* } \\ \text { W. R. Martin * } & \text { R. C. Mann } \\ \text { W. R. Hamel } & \text { J. S. Tulenkot }\end{array}$

[Abatrect of Naclear Enginetriag and Design 113, 259-267 (1969)\}

The nuclear industry has a recognized need for intelligent, multitask robots to carry out tasks in harsh environment. From 1986 to the present, the number of robotic systems available or under development for use in the nuclear industry has more than doubled. Presently, artificial intelligence (AI) plays a relatively small role in existing robots used in the nuclear industry. Indeed, the lack of intelligence has been labeled the "Achilles

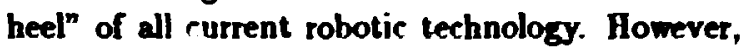
larger-scale efforts are underway to make the muttitask robot more sensitive to its environment, more capable to move and perform useful work, and more fully autonomous via the use of AI. In this paper, we review the terminology, the history, and the factors which are motivating the development of robotics and remove systems; discuss the applications related to the nuclear industry; and, finally, examine the state of the art of the technologies being applied to introduce more autonomous capabilities. Much of this latter work can be classified as within the artificial intelligence framework.

Research sponeored by I'.S. DOE Office of Bacic Energ Research.

"Univenily of Michign, Ann Arbor, MII.

IInstrumentation and Controls Division.

Univensity of Florida, Gainesville, FL. 
3.43

\section{RESEARCH IN AUTONOMOUS ROBOTICS AT ORNL USING HERMISS-III}

\author{
C. R. Weisbin \\ B. L. Burks \\ J. R. Einstein \\ R. R. Feesell* \\ W. W. Manges" \\ D. H. Thompson*
}

(Abatred of paper preanted at the 1960 ASME Preanue Venels and Piping Conference, Hooolutu, HI, July 23-27, 1900)

HERAIIES-III is an autonomous robot comprised of a seven degree-of-freedom (DOF) manipulator designed for human seale tasks, a laser range finder, a sonar array, an omni-directional wheel-driven chasis, multiple cameras, and a dual computer system containing a 16-node hypercube expandable to 128 nodes. The curreat experimental program involves performance of human-scale tasts (e.g-, valve manipulation, use of cools), integration of a dexterous manipulacor and platform motion in geometrically complex enviropments, and effective use of multiple cooperating robots (HERMIES-IIB and HERMIES-III). The environment in which the robots operate has been designed $t o$ include multiple valves, pipes, meters, obstacles on the floor, valves occluded from view, and multiple paths of differing navigation complexity. The ongoing research program supports the development of autonomous capability for HERMIES-IIB and III to perform complex navigation and manipulation under time constraints while dealing with imprecise sensory information.

Rewearch sponeored by U.S. DOE Oflice of Bavic Enerpor Seiences.

* Instrumeneacion and Controls Divicion.
3.44

\section{HERMIIS-III: A STEP TOWARD AUTONOMOUS MOBILITY, MANIPULATION AND PERCEPTION}

\author{
C. R. Weisbin \\ B. L. Burks
}

J. R. Binstein

R. R. Feezell

W. W. Manges"

D. H. Thompron*

(Abatrect of paper presenled al the NASA Conference on Spece Telerobotica, Pavedean, CA, Janery 31-February 2. 1909)

HERMIES-III is an autonomous robot comprised of a seven degree-of-freedom (DOF) $\mathrm{ma}$ nipulatior designed for human scale tasks, a laser range finter, a sonar array, an omni-directional wheet-drivei: chassis, multiple cameras, and a dual computer system containing a 16-node hypereube expandable to 128 nodes. The current experimental program involves performance of human-scale tacks (e.g., valve manipulation, use of tools), integration of a dexterous manipulator and platform motion in geometrically complex environments, and effective use of multiple cooperating robots (HERMIES-IIB and HERMIES-III). The environment in which the robots operate bas been designed to include multiple valves, pipes, meters, obstacles on the floor, valves occluded from view, and multiple paths of differing navigation complexity. The ongoing research program supports the development of autonomous capability for IIERMIES-IIB and III to perform complex navigation and manipulation under time constraints, while dealing with imprecise sensory information.

Research sponsored by U.S. DOE Office of Basic Energy Science.

- Inetrumeneation and Controls Division. 
3.45

\section{AUTONOMOUS MOBILE ROBOT NAVIGATION AND LEARNING}

\author{
C. R. Weisbin G. de Saussure \\ J. R. Einstein E. Heer* \\ F. G. Pin
}

[Abatract of Competer Megarine 22(8), 29-35 (Jume 1909)]

This paper reviews ongoing and planned research for mobile, autonomous robots including aspects of navigation, exploration, recognition and learning, as performed at the Oak Ridge National Laboratory (ORNL), Center for Engineering Systems Advanced Research (CESAR). We begin with a discussion of the HERMIES-IIP robot, a selfpowered, wheel-driven platform used primarily for navigation and surveillance research. The vehicle contains an on-board, 16-node NCUBE hypercube parallel processor interfaced to effectors and sensors through a Motorola 68020 processor. HERMIES-IIB uses a phased sonar array and mut tiple camera to sense its environment.

The software for HERMIES-IIB control is next described including detailed discussion of its expert system brain, constructed to handle numerical and symbolic information processing dealing with real-time decision making (including consideration of unexpected events).

The capability for HERMIES-IIB to perform intelligently depends vitally on its capability for perception. We review completed CESAR research pertaining to on-board vision processing, combinatorial geometry representation of range information, and algorithms to resolve conflict among seemingly disparate sonar data. Techniques to systematically integrate information from multiple sensors in a consistent way are described. Our efforts to incorporate the capability for HERMIESIIB to improve its performance through autonomous machine learning are described, and experimental results are presented. A complete experiment is then described, and the results presentud, which illustrate HERMIES-IIB capability for $d y$ namic replanning.

The paper concludes with a discussion of HERMIES-III, and the associated research program. HERMIES-III is comprised of a seven degree-of-freedom manipulator designed for human scale tasks, a laser iange finder, an omnidirectional, wheel-driven chassis, in addition to multiple cameras, sonar arrays, and a 16-node hy-

percube expandable to 64 nodes. The current experimental program involves performance of actual human-scale tasks (e.g., valve manipulation, use of rools), integration of dexterous manipulators and platform motion in geometrically complex environments, and effective use of multiple cooperating robots (HERMIES-IIB and HERMIESIII). The environment in which the robots operate include multiple valves, pipes, meters, obstacles on the foor, absence of an accurate world model, valves occluded from view, multiple paths of differing complexity, and tasks subject to fixed time constraints. The research described supports the development of autonomous capability for HERMIEC IIB and III to perform complex navigation and manipulation in time constrained, emergency environments while dealing with imprecise information.

Research sponsored by U.S. DOE Ofice of Bmic Energy Sciences.

* Heer Acecciales, LaCenada CA.

\subsection{6}

\section{THE ROBOTICS AND INTELLIGENT SYSTEMS PROGRAM AT ORNL}

\section{R. Weisbin W. R. Hamel* \\ $\begin{array}{ll}\text { D. P. Kuban' } & \text { S. A. Meachamt }\end{array}$}

F. G. Pin

[Abetrect of Robotice 7(2), 161-11I (April 1989)]

This paper describes the Robotics and Intelligent Systems Program (RISP) at the Oak Ridge National Laboratory (ORNL). RISP is a focal point at ORNL for studies in robotics, teleoperations, and related aspects of intelligent machines, such as artificial intelligence, neural network -, and parallel computing. RISP research is intended to minimize human error through automation of repetitive, full, and routine tasks; to minimize human risk by allowing effective remote operatiors in hazardous environments; to optimize cooperation of humans and machines; and to cope effectively with emergency situations where fast response is casential.

Research eponeored by U.S. DOE, U.S. Deparment of DePense, and Nationd Aeronausical and Space Adminiatraijon.

Inatrumentation and Controls Divition.

tORN, Enginerring (RLD) Division.

Fuel Rerycle Division. 


\section{CONCURRENT COMPUTING}

\subsection{7}

\section{COMPUTING THE HOUGH TRANSFORM ON AN MIMD HYPERCUBE}

\author{
K. W. Bowyer $\quad$ J. P. Jones \\ C. H. Lake*
}

(Abetract of paper presented at the 6h Scandanavian Image Analyas Conference, Oulu, Fuland, Jane 19-22, 1909)

The computational expense of Hough transforms has prompted researchers to investigate the feasibility of parallel implementations. Most such work to date has dealt with special-purpose architectures or with implementations for SIMD machines such as the MPP or GAPP. This report considers the problem of efficiently implementing Hough transforms on an MIMD hypercube architecture. Beginning with a general analysis of how the data structures might be partitioned to allow a parallel computation, we formulate algorithm and data structure partitioning strategies appropriate to the architecture and then discuss modifications to optimize the performance for a particular hypercube system (the NCUBE). We also present the results of implementing and benchmarking Hough transforms on a 64-processor NCUBE.

Research sponsored by the Air Force Office of Scientific Research, National Science Foundation, U.S. DOE Office of Nuclear Energy, the Ofice of Technology Support Program, and the Ofince of Basic Energy Science.

"Univensity of South Floride, Tampe FL.

\subsection{8}

\section{A CONCURRENT APPROACH TO MODEL-BASED MOTION DETECTION}

\section{H. I. Clıristensen*}

(ALatract of ORNL/TM-11051, January 1989)

In robot-vision it is important to be able to acquire and describe dynamically changing scenes. A token based method for real-time motion detection is described here. This method uses a prediction-verification scheme to cope with amhiguous motion patterns. To improve performance it is investigated how the application maj be implemented on a concurrent architecture like a hypercube. An implementation based on the SPMD approach is presented together with some experimental results. The results demonstrate the systems ability to handle ambiguous situations. Timing results irdicate the system has a potential for tracking of less than 10 objects or more than $\mathbf{4 0}$ objects, while the advantage of using a concurrent architecture for 10-40 objects is very small.

Rewearch eponsored by U.S. DOE Ofice of Bacic Energo Researd.

* Aalborg Universits, Aalborg. Denmart.

\subsection{9}

\section{CONCURRENT SPATIO-TEMPORAL MMAGE ANALYSIS}

\section{H. I. Christensen*}

(Abatract of paper presented at the Fourth Conference on Hypercubes, Concurrent Computers, and Applications, Monterey, CA, March 6-8, 1809)

A new method for motion detection is introduced. The method is based on extraction of global/long-term motion descriptors from lowresolution images while detailed/short term motion descriptors are extracted from higher resolution images. This approach can be characterized as a method of least effort, i.e., as much information as possible is extracted from low resolution images.

An experimental implementation based on the method of least effort is reported for a hypercube architecture (NCUBE Corp., Beverton, OR). Results indicating the generality of the approach are also presented. The implementation allows discussion and measurement of scaling properties for the method and a comparison to performance on a pyramid architecture.

Research eponsored by U.S. DOE Office of Nuciear Energy and by Danish Terhnical Rewearch Council.

-Aalborg University, Aalborg, Denmark. 
$\mathbf{3 . 5 0}$

\section{CONCURRENT MULTI-RESOLUTION MMAGE ANALYSIS}

H. I. Chriatemsen*

J. P. Jones

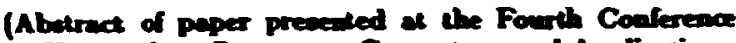
an Hypercuber, Coscurrent Completers, and Applicatione, Monterey, CA, March 6-8, 1909)

Analysis at multiple resolutions can simplify many image analysis problems. In such methods, - coarse-to-fine search strategy replaces an exhanstive search for features within a single resolution image. Coarse resolution images are used for extraction of global information in a hypotheais formation proces. Analysis of fiver resolution images are subsequently used for hypothesis verification or rejection. The multiresolution hierarchy of images form a data strueture known as a pyramid, and a tightly coupled synchronous multiprocensor architecture (the pyramid machines) bas been developed for the andysis of auch struetures.

In this paper, methods for mukiresolution image analysis uaing the more general hypercube architecture are discussed, and a particular implementation is presented. The implementation has been designed to proceses pyramide of images of at most arbitrary size composed of pixels of arbitrary storage clase on hypercubes of arbitrary dimension. An efficient dereferencing strategy permits the construction of a broed clase of efficient algorithms for traversing the pyramid. An experimental implementation on a bypereube multiproceser (NCUBE Corp., Beverton, OR) is presented. The implementation demonstrates almont linear scaling properties. We discuss these properties and compare performance to pyramid archilectures.

Revearch aposeoned by U.S. DOE Ofice of Nuclear Enery Technology Support Programs and by Danich Technical Research Counci.

* Aalborg Univeraily, Aalbort. Denmark.
3.51

\section{A TOOL TO AID IN MAPPING COMPUTATIONAL TASKS TO A HYPERCUBE}

\author{
J. R. Einstein C. W. Glover \\ M. Dickens"
}

(Abatract of paper prevented at the Fourth Conference on Hypercubes, Concurnent Compoters, and Applications. Monterey. CA, March 6-8, 1980)

We deseribe a tool to aid in static scheduling of precedence-constrained tasks on nodes of a hypercube computer. The tool is useful where the sequential program is suitable for large-grained de composition, i.e., where meseage-passing times are negligibly small compared to task execution times. The tool is also useful for small sets of task, or for quasi-periodic sets of tacks, even where mesagepassing times are not negligible. Quasi-periodic sets of tasks arise when the computation is dominated by loops over a group of tasks which are the same or nearly the same for each iteration of the loop - for example, programs for the dynamica and kinematics of robotic manipulators.

Research sponeret by U.S. DOE Ofince of Beaic Energ Sciences, Nuclenr Energ. Technology Support Program.

"Maceachuetes Insitute of :xhnolog, Cambridze, MA.

\subsection{2}

\section{DESIGN AND IMPLEMENTATION OF A MULTI-SENSOR FUSION ALGORITH: ON A HYPERCUBE COMPUTER ARCHITECTURE}

\author{
C. W. Glower
}

(Abatract of paper to be presented at the Senecor Fuaion II: Human and Machioc Strategies SPIE's Advances in Intelligent Robotica, Philadelphie, PA, November 5-10, 1909)

A multi-censor integration (MSI) algorithm written for sequential single proceseor computer architecture has been transformed into a coneurrent algorithm and implemented in parallel on a multi-proceseor hypercube computer architecture. This paper will present the philosophy and methodoloiges used in the decomposition of the sequential MSI algorithm, and its transformation into a parallel MSI algorithm. The parallel MSI algoritlim was implemented on a NCUBE ${ }^{T M}$ hypersube computer. The performance of the parallel 
MSI algorithm has been measured and compared against its sequential counterpart by running test case scenarios through a simulation program. The simulation program allows the user to define the trajectories of all players in the scenario, and to pick the sensor suites of the players and their operating characteristics. For, example, an air-to-air engagement scenario was used as one of the test cases. In this scenario, two friend aircrafts were being attacked by six foe aircrafts in a pincer maneuver. Both the friend and foe aircrafts launch missiles at several different time points in the engagement. The sensor suites on each aircraft are dual mode RADAR, dual mode IRST, and ESM sensors. The modes of the sensors are switched as needed throughout the scenario. The RADAR sensor is used only intermittently, thus most of the MSI information is obtained from passive sensing. The maneuvers in this scenario caused aircraft and missile to constantly fly in and out of sensors field-of-view (FOV). This resulted in the MSI algorithm to constantly reacquire, initiate, and delete new tracks as it tracked all objects in the scenario. The objective was to determine performance of the parallel MSI algorithm in such a complex environment, and to determine how many multi-processors (nodes) of the hypercube could be effectively used by an aireraft in such an environment. For the scenario just discussed, a 4-node hypercube was found to be the optimal size and a factor two in speedup was obtained. This paper will also discus the design of a completely parallel MSI algorithm. targets.

Research sponeored by U.S. Air Force Wright Aeronautical Laboratory and U.S. LOE Office of Basic Energy Sciences.

\section{MULTI-SENSOR INTEGRATION ON THE NCUBE ${ }^{\text {TM }}$ HYPERCUBE COMPUTER}

\section{W. Glover}

(Abstract of paper presented at the Third Conference on Hypercube Concurrent Computers and Applicalions. Pacadena, CA, January 19-20, 1988; Proc. Vol. II, Pp. 1236-1246, Geoflyy Fox. Ed. (1988)]

This paper presents the quantitative performance results obtained from converting a large sequential Multi-Sensor Integration (MSI) program into a concurrent form and executing the program on an NCUBE hypercube computer for several teat case scenarios. Each sensor tract was assigned to a different node of the hypercube where computationally intensive calculations were performed. A 4-node hypercube was found to be the optimal size for the test case scenario discussed here, where speed-up factors of 1.5 to 1.7 were obtained. A completely concurrent MSI algorithm is also presented.

Research sponsoned by U.S. DOE OTice of Bucic Energ Sciences and by U.S. Air Force Wright Aeoronavical Laboratoriea.

\subsection{4}

\section{CONCURRENCY IN MOBILE ROBOTICS AT ORNL}

\section{J. P. Jones}

(Abatrect of oral presentation presented at the beternional Wortahop on Concurrent Computer Vivion, Aalborg. Denmart, January 25-27, 1900)

The Center for Engineering Systems Advanced Research (CESAR) was established in 1983 by the U.S. Department of Energy at the Oak Ridge National Laboratory to perform basic research and development in autonomous mobile and manipulative robots for energy-related applications. Early in the program it was recognized that autonomy implied the solution, in real-time, of a variety of complex problems in mathematical modeling, sensor data processing, and multisensor integration. This realization led to the study of concurrent computing, focusing specifically on issues related to hypercube concurrent multicomputers.

In this talk we p: ssent the hardware architecture of HERMIES-IIB, our first mobile robot with an on-board concurrent computer, and its successor, presently under development, IIFRMIES-III. We discuss the organization of tasks in the present system and present some options for the future.

Research sponsosed by U.S. DOE Office of Bacic Energy Research. 


\subsection{5}

\section{CONCURRENT COMPUTER VISION} ON A HYPERCUBE MULTICOMPUTER

\section{J. P. Jones}

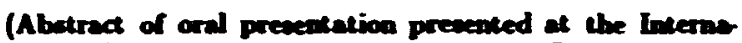
tiond Wortshop on Concurrent Computer Vinion, Aallbot. Denmart, January 25-27, 1989)

This decade has witnessed the development and commercialization of the first generation of practical general-purpose multicomputers (tightly-coupled distributed-memory concurrent maltiprocessors). One of the most popular commercial-grade architectures is the hypercubeconnected multicomputer, which has been successfully used in a wide variety of applications from physics to artificial intelligence.

Here we present a brief review of the properties of hypercube multicomputers, with specific reference to the NCUBE, Inc. (Beaverton, OR) machine and a discussion of the "loosely synchronous" programming model. Since the issues in concurrent programming reduces in many cases to the treatment of memory and communications, we discuss in detail graycodes, the distributed data structures, and low-level communications algorithms in the aforementioned system. We conclude with a generalization of memory albeation in support of multi-resolution image processing and compare the expected performance of the hypercube, to pyramid computers.

Research sponsored by U.S. DOE Office of Bacic Enery Research.

\subsection{6}

\section{A CONCURRENT ON-BOARD VISION SYSTEM FOR A MOBILE ROBOT}

\section{J. P. Jones}

(Abatract of paper presented at the Third Conference on Hypercube Concurrent Computers and Applications, Pacedena, CA. January 19-20, 1988; Proc. Vol. II, Pp. 1022-1032, Geofiry Fox, Ed. (1988)]

Robot vision algorithms have been implemented on an 8-node NCUBE-AT hypercube system on-board a mobile robot (HERMIES) developed at Oak Ridge National Laboratory. Images are digitized using a framegrabber mounted in a VME rack. Image processing and analysis are per- formed on the hypercube system. The vision system is integrated with robot navigation and control softrare, enabling the robot to find the front of a mockup control panel, move up to the panel, and read an analog meter. Among the concurrent algorithms used for image analysis are a new component labseling algorithm and a Hough transform algorithm with lood balancing.

Research aponsored by U.S. DOE Office of Bmic Energy Seiences.

\subsection{7}

\section{THE BUTTLRFLY ACCUMULATOR AND ITS APPLICATION IN CONCURRENT COMPUTER VISION ON HYPERCUBE MULTCOMPUTERS}

\section{J. P. Jones}

(Abetrect of oral presentation presented at the Inermitiond Wottabop on Concurreat Competer Viaion, Aalbort. Denmark, Januery 25-27, 1989)

In concurrent programs the accumulation of data from all processors to a single location (gather) or the distribution of data from a single location to all processors (seatter) is frequently useful. An efficient algorithm exists for hypercube multicomputers which performs gather-scatter simultaneously to all processors in $\lg (\mathrm{N})$ time. Because of the isomorphism of the communications graph of this algorithm to that of the fast Fourier transform, we call this algorithm the "butterfly accumulator."

In this talk we demonstrate by example the generality of the butterfly accumulatot by giving concurrent solutions for a variety of problems taken from image processing and analyois, including image scaling, histogramming, component labeling, relaxation labeling, simple load balancing, fast transforms, and some selected neural networks. We conclude with a discuseion of the relationship of this communications algorithm to memory allocation in distributed multi-resolution images.

Rewench sponeored by U.S. DOE Office of Bavic Energ Research. 


\subsection{8}

\section{PARALLFi RELAXATION MATCHING}

\section{J. P. Jones K. M. Clinard"}

(Abatrect of paper presented at the 17th Wortubop on Applied Imagery Pretern Recognition, Wahington, DC, November 2-4. 1988)

Model based vision systems require correspondence between expectation and observation. One strategy for establishing this correspondence is relaxation matching. In relaxation matching, region features in segmented images and geometric relationships between regions are evaluated with respect to expectations established by a model. A set of possible matching regions are determined for each region of the model. These candidate matches are refined by a relaxation procedure until one or more have a highty likely assignment, which is than fixed. Other candidate matches are then reevaluated in this context. The procedure is repeated until all model regions have firm assignments.

Research sponsored by U.S. DOE Ofice of Buic Eners Sciences.

*Inirensity of Tennemee, Knoxville. TN.

\section{A COMPUTER VISION SYSTEM FOR A HYPERCUBE CONCURRENT ENSEMBLE}

\section{J. P. Jones R. C. Mann E. M. Simpson*}

(Abstrect of ORNL/TM-10679, Augual 1988)

A system of image processing and analysis utilities for a general-purpose hypercube copology concurrent multiprocessor system is described. The purpose of the system is to provide an efficient development and run-time environment for theoretical and experimental inquiry into computational vision for mobile and manipulative sobots. An additional objective is to provide an environ ment with rich support for tlie development of concurrent computer vision algorithms. A number of principles for programming concurrent systems are embodied in the implementation of this system. In particular, all significant computation is performed hy the hypercube proper, reserving the host proceseor exclusively for input/output func- tions. One consequence of this principle is that applications are developed by viewing the concurrent multiprocessor as a single computet with a special internal structure, rather than as a number of independent machines. Applications derive from a single source, rather than many, reducing development time without sacrificing run-time efficiency. This document serves as an introduction to the image processing and analysis system, its principles of operation, auc' as a reference manual for its use.

Researdh sponased by U.S. Departmeat of Energy and U.S. Army Human Enjinedine Laboratory.

*Univesity of Weat Flaride, Penacola FL.

3.60

\section{SENSOR INTEGRATION USTNG CONCURRENT COMPUTING ON- BOARD THE ORNL MOBILE ROBOT}
R. C. Mann
J. P. Jones
M. Beckerman
C. W. Glover
L. Parkas*
J. R. Binstein

(Abarach of paper presented at the IEEE 1909 Intern:tional Conference on Robotios and Automation Scotedale, AZ, May 14-19. 1989)

The mobile robot prototypes developed at the ORNL Center for Engineering Systems Advanced Research (CESAR) are equipped with sonar sensors, CCD cameras and a laser range camera that are used to support autonomous navigation and simple inspection tasks in an a priori unknown and unstructured dynamic environment. This paper summarizes work directed at extracting information from data collected with these sensors and integrating it, in order to produce reliable descriptions of the robot's environment. The approach consists in studying difierent world models and mappings among them, sensor models and parallel algorithms for sensor information processing, and appropriate integration strategies. Specifcally, the paper describes the inlegration of two dimensional vision and sonar range information and the integration of laser range and luminance images. All operations are carried out on-board the mobile robot using a 16-procesoor hypercube. computer.

Researsh sponeored by U.S. DOE Oflice of Bamir. Fnergy Sciences and Office of Nisclear Energy.

"Cornell I'niversity, Ithera, NY. 


\section{OPTIMIZATION FOR INTELLIGENT SYSTEMS}

3.61

\section{YET ANOTHER NON-LINEAR APPROACH TO LINEAR- PROGRAMMING}

\section{J.-C. Culioli}

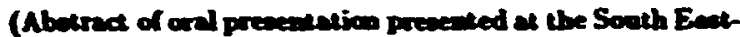
ar Allaric Section of SIAM Meeting, Knowille. TN, Mand 18, 1900)

The recent succes of Karmartar's algarithm and of many related interiot-path methods has revived the application of non-linear techniques to Linear Programming. In this talk, we consider another non-linear approach. We use an augmented Lagrangian primaj-dual method and build several variants for which we discuss convergence to optimality and implementation issues.

Reweard aponsoned by U.S. DOE and by U.S. Air Frece Wingt Aeroamitienl Leboratory.

\subsection{2}

\section{DECOMPOSITION/COORDINATION ALGORITHMS IN CONVEX STOCHASTIC OPTIMIZATION}

$$
\text { J.-C. Culioli G. Cohen }
$$

(Abotract of paper presented at the SIAM Conference on Applied Linear Algebre, Minnenpolis, MN, July 11-15, 1998)

Large scale systems are often imbedded in a stochastic environment. Theit dimenaionality usually prevents a global solution technique. Decompocition algorithms usually assume differentiabitity and separability of the global criterion. The deterministic Auxiliary Problem Principle propoeed by $\mathbf{G}$. Cohen does not require these properties. It also enables to solve the sub-problems separately with heterogeneous algorithmic techniques. We present here a generalization to the stochar tic case. We addrea "price decomposition" and "rewource alkucation decomponition," state almost sure convergence results, and present come numer. ical tests on sequential and parallel computer..

Reecerch eponeored by U.S. DOE Office of Milieary Applications and Office of Bacic Enercy Sciences and by U.S. Air Force Wright Aeronautical Laboratories.

-Ecole Nasionale des Mines de Paris, Codex, France.
3.63

\section{DECOMPOSITION/COORDINATION ALGORITHMS IN STOCHASTIC OPTIMIZATION}

$$
\text { J.-C. Culioli G. Cohen* }
$$

[Abatract of SIAM Joenel of Optimization (in preas)]

In this paper, we consider an extension to the situation of stochastic programming of the Auxiliary Problem Principle formerly introduced in a deterministic setting to serve as a general framework for der:omposition/coordination optimization algorithms. The idea is based upon that of the stochastic gradient, that is, independent noise realizations are considered successively along the iterations. As a consequence, deterministic subproblems are solved at each iteration whereas iterations fulfill the two tasks of coordination and stochastic approximation at the same time. Coupling cost function (expectation of some performance index) and deterministic coupling constraints are considered. Price (dual) decompositior. (encompassing extensions of the Uzawa and A) row-Hurwicz algorithms to this stochastic case) are studied as well as resource allocation (primal decomposition).

Recearch aponeored by U.S. DOE and Air Fonce Wriche Aeronantical Laboratory.

-Ecole Nationale des Mines de Paris, Cedex, France.

\subsection{4}

\section{NCUBE IMPLEMENTATION OF SOME HEURISTICS AND AN OPTIMAL ALGORITHM FOR LARGE-SCALE ASSIGNMENT PROBLEMS}

$$
\begin{array}{cc}
\text { J.-C. Culioli } & \text { C. W. Glover } \\
\text { J. P. Jones } & \text { C. Roe }
\end{array}
$$

(Abotract of paper presented at the Fourth Conference on Hypercubes, Concurrent Computers and Applications. Monierey, CA, March 6-8, 1989)

We consider the standard Assignment problem: mini,nize: $z=\sum_{i} \sum_{j} c_{i j} x_{i}$ j subject to $\sum_{i}$ $x_{i j}=1$ and $\sum_{j} x_{i j}=1$, where $1 \leq i \leq n, 1 \leq j \leq$ $m$, and $r_{i j} \in 0,1$, and $c_{i j} \geq 0$. We are specially interested in large scale problems such that $n$ and $m \geq 100$. We describe and compare several ap- 
proaches, some of them being well-known heuristics to initiate a Transportation problem such as the Least Cost Rule and Vogel's Approximation Method, others being optimal methods. गich as the Hungarian Method. For each algorithm, our study focuses on two major issues; the parallel implementation strategies on hypercube concurrent computers and the solution optimality given the computational load. We describe implementations on an NCUBE (Beaverton, OR) hypercube. We also propose some decision scenarios using a combination of different heuristics in order to get as fast as possible sub-optimal solutions.

Reseanch sponsoned by U.S. DOE Ofice of Nuclear Eneroy, Technolosy Support Program and by U.S. Air Force Wright Aeroantical Laboratory.

*Lopola University, New Orelanas, LA.

\subsection{5}

\section{A NEW ALGORITHM FOR LINEAR PROGRAMMING THAT IS EASY TO IMPLEMENT: APPLICATION TO THE TRANSPOFTATION PROBLEM}

\section{J.-C. Culioli V. Protopopescu}

(Abatract of ORNL/TM-10929, September 1988)

We proposc a new algorithm for linear pro gramming and apply it to solve some transportation problems. We prove the convergence of the algorithm under reasonable sufficient conditions. The main motivation of this new linear-programming solver is its extreme simplicity and its parallel structure which allows for straightforward implementations on personal computers as well as on more sophisticated machines. We give in the Appendix a version of the algorithm programmed in $\mathrm{C}$ in less than 30 lines. We also comment in the Appendix the relationship of our algorithm with some models of neural networks.

Research eponsored by U.S. DOE Office of Basic Energy Sciences.

\subsection{6}

\section{AN ALGORITHM FOR LINEAR PROGRAMMING THAT IS EASY TO IMPLEMENT}

\section{J.-C. Culioli V. Protopopescu}

[Abatrect of Appl. Math. Lett. 2(2), 125 (1900)]

We present a new linear-programming algorithm that is simple, effective, fully parallelizable, and inmediately implementable on personal computers as well as on more sophisticated machines. Sufficient conditions are found that guarantee the convergence of the algorithm. Sorme applications are given showing that the applicebility of the at gorithm is actually wider than indicated by the theoretical results.

Research sponsored by U.S. DOE and by the U.S. Air Forte Wright Aeronatical Laboralory.

\subsection{7}

\section{BIPURCATING OPTIMIZATION ALGORTHMS AND THEIR POSSTBLE APPLICATION}

\section{J.-C. Culioli V. Protopopescu}

(Abatract of ORNL/TM-10976, November 1989)

A new clars of optimization algorithms for linear and nonlunear problems is proposed. The algorithms are based on sigmoidic updatings and can be looked upon as nonlinear multidimensional maps. The bifurcation and chaotic regimes of thcse maps are analyzed and their poseible applications in optimization problems are indicated.

Research eponsored by U.S. DOE Ofice of Beeic Energy Sciences. 


\section{SIGMOIDIC LINEAR AND NON-LINEAR OPTIMIZATION ALGORITI!MS}

\section{J.-C. Culioli V. Protopopescu}

(Abatract of paper preaented at the Intermational Neural Network Society Annmal Meeting, Bocton, MA, September 6-10, 1988)

Since Hopfield and Tank have proposed their neural network based computation method to solve optimization rombinatorial problems, a significant amount of work has been Jone to apply it to a great number of difficult problems, with various degrees of success. Here, we address continuous optimization and propose neural network models for linear and non-linear optimization.

Reseanch sponsored by US. DOE Office of Bacic Energy Sciences and Office of Military Application.

3.69

\section{A NEK CLASS OF OPTIMIZATION ALGORITHMS WITH BIFURCATIONS AND CHAOTIC BEHAVIOR}

\section{J.-C. Culioli V. Protopopescu}

(Abatract of paper presented at the SIAM Conference on Applied Linear Algebra, Minneapolis, MN, July 11-15, 1988)

We introduce a new class of optimization algorithms that use prarameter dependent sigmoidic functions to limit the sequence of iterates. For Linear-Programming, the proposed scheme converges to a close approximation of the solution for small parameter values. For the (constrained or unconstrained) non-linear case, the optimal solution is a stable fixet point of the algorithm. In both cases, if the design parameter is "very small," the sequence of iterates shows bifurcations and ultimately chaotic behavior. We conjecture that this typical property could be used to sompute very quickly a satisfactory approximation of the sought optimal solution.

Revearch wroneored by U.S. DOE Office of Benic Energy Sciences and Office of Milicary Applications and by U.S. Air Force Wriche Aeronautical Latioratorien.

\section{A LINEAR-PROGRAMMING METHOD INSPIRED BY THE NEURAL NETWORKS FRAMEWORK}

\author{
J.-C. Culioli V. Protopopescu
}

(Abetract of paper presented at the IEEE International Conference on Neural Networts, San Diego, CA, July 2427. 1988)

We propose a discrete numerical algorithm for linear programming that has common features with non-linear optimization algorithms and with neural network models. the "computation ener $g y$ " of our algorithm is not quadratic, but linear, which greatly simplifies the model. The colloctive computational properties are obtained by dividing the neurons in two classes: primal and dual. The algorithm is parallelisable, easier to code than most classical linear-programming algorithms, and implementable in VLSI. The relationship with other neural network models is discussed.

Rewearch sponeored by U.S. DOE Office of Banic Energy Sciences and Office of Military Applications and by U.S. Air Force Wrighe Aeronatical Laboratories.

\subsection{1}

\section{STABILITY AND CHAOS IN A NUMERICAL SCHEME INSPIRED BY NEURAL NETWORKS}

\section{J.-C. Culioli V. Protopopescu}

(Abatract of oral presentation presented at the Wurkehop of the AAAS in Corvallis, OR, June 20, 1988)

We address some new non-linear optimization algorithms based on a neural network model with sigmoidic updating. The iteration used to minimize the unconstrained convex function $F(x)$ is realized through the non-linear mapping: $x(k+1)$ $=h(x(k))$ with $h(x)=2 x /\left(1+\exp \left[F^{\prime}(x) / T\right]\right)$, where $T$ is a positive parameter and $F$ is the derivative of $F$. When varying $T$, we observe the full scenario of hifurcations and chaotic behavisit of the mapping and we investigate the Lyapunor exponents and the stability properties acocialed to it. We show that, in some cases, the cyclic behavior can be used to get fast approximations of the sought optimal solution. We also vary $T$ (a form of annealing scheme) in order to find the solution before entering chaos.

Research oponeored by U.S. DOE Office of Basic Energy Science and Office of Military Applications and by U.S. Air Force Wright Aeronautical Lalorntroties. 
3.72

\section{NEURAL NETWORKS MODELS FOR LINEAR PROGRAMMING}

\author{
J.-C. Culioli \\ V. Protopopescu \\ C. Britton* \\ N. Ericson
}

(Abatract of paper to be presented at the International Joint Conference on Neural Networks, Wachington, DC, Jamuary 15-19, 1990)

The purpose of this paper is to present a neural network that solves the general Linear Programming (LP) problem. In the first part, we recall Hopfield and Tank's circuit for LP and show that although it converges to stable states, it does not, in general, yield admissible solutions. This is due to the penalization treatment of the constraint. In the second part, we propose an approach based on Lagrange multipliers that converges to primal and dual admissible solutions. We also show that the duality gap (measuring the optimality) can be rendered, in principle, as small as needed.

Research opontored by U.S. Air Force Wright Aeronamical Leboratory and U.S. DOE Office of Bacic Energy Sciences.

*Ir urumentation and Controle Divition.

\subsection{3}

\section{A NEURAL NETWORK FOR BOUNDED LINEAR PROGRAMMING}

\section{J.-C. Culioli V. Protopopescu C. Britton * N. Ericson*}

(Abatract of paper to be presented at the Intermational Joint Conference on Neural Networks, Washington, DC, Jemuary 15-19, 1990)

The purpose of this paper is to describe a neural network implementation of an algorithm recently designed at ORNL to solve the Transportation and the Asaignment Problems, and, more generally, any explicitly bounded linear program.

Recearch sponeored by U.S. Air Force Wright Aeronautical Lethoratory and U.S. DOE Office of Baxic Energy Sciences.

\footnotetext{
*Instrumentution and Controls Division.
}

3.74

\section{A COMPARISON STUDY OF OPTIMIZATION METHODS FOR THE BIPARTITE MATCHING PROBLEM (BMP)}

\author{
M. Goldstein * N. Toomarian' \\ J. Barhen
}

[Abatract of paper presented at the IEEE International Corference on Neural Networks, San Diego, CA, July 2427, , 388; Proc. Vol. II, pp. 267-273 (1988)]

A comparison study of optimization methods for the bipartite matching problem (BMP) with independent random distances between the points is presented. It is concluded that a variant of simulated annealing, developed at ORNL, is the most promising for the BMP.

Research eponsored by U.S. DOE Ofice of Basic Energy Sciences.

* On leave from Nuclear Research Centre-Negev, Imad.

Omnimet Communications Services, Los Angeles, CA.

Iet Propultion Leboralory, Pasadena, CA.

\subsection{5}

\section{A CONCURRENT NEURAL NETWORK ALGORITHM FOR THE TRAVELING SALESMAN PROBLEM}

\section{N. Toomarian*}

[Abatract of paper presented at the Third Conference on Hypercube Concurrent Computers and Applications, Paudens, CA, Jamuary 19-20, 1988; Proc. Vol. II, pP. 1483-1490, Geofiry Fox, Ed. (Mey Is88)!

A binary neuromorphic data structure is used to encode the N-city Traveling Salesman Problem (TSP). In this representation the computational complexity, in terms of number of neurons, is reduced from Hopfield and Tank's $O N^{2}$ ) to $O\left(\log _{2} M\right)$. A continuous synchronous neural network algorithm, in conjunction with the Lagrange multiplier, is used to solve the problem. The algorithm has been implemented on the NCUBE hypercube multiprocessor. This algorithm converges faster and has a higher probability to reach a valid tour than previously available results.

Research sponeored by U.S. DOE Office of Basic Enercy Sciences and Office of Military / pplications.

- Omninet Communicatione Services, Los Angelen, CA. 


\section{A NEURAL NETWORK-BASED OPTIMIZATION ALGORITHM FOR THE WEAPON-TARGET ASSIGNMENT PROBLEM}

\section{E. Wacholder}

(Abetract of ORNL/TM-11025, Februm 1309)

A neural networt-based algorithm was developed for the Weapon-Target Assignment Problem (WTAP) in Ballistic Missile Defense (BMD). An optimal assignment policy is one which allocates targets to weapon platforms such that the total expected leakage value of targets surviving the defense is minimized. This involves the minimization of a non-linear objective function subject to inequality constraints specifying the maximum number of interceptors available to each platform and the maximum number of interceptors albwed to be fired at each target as imposed by the Battle Management/Command Control and Communications $\left(\mathrm{BM} / \mathrm{C}^{3}\right)$ system. The algorithm consists of solving a system of $O D E$ trajectories and variables. Simulations of the algorithm on PC and VAX computers were carried out using a simple numerical schems. In all the battle instances tested, the algorithm has proven to be stable and to converge to solutions very close to global optims. The time to achieve convergence was consistently lese than the time constant of the network's processing elements (neurons). This implie that rast solution can be realized if the algorithm is implemented in hardware circuits. Three series of battle scenarios are analyzed and discussed in this report. Input data and results are presented in detail. The main advantage of this algorithm is that it can be adapted to either a special-purpose hardware circuit or a general-purpose concurrent mechine to yield fast and accurate solutions to difficult decision problems.

Research sponeored by the Office of Military Applications of the U.S. Department of Energy and by the U.S. Air Force Wrighe Aetonmitical Laboratorie.

\section{AN EXTENSION OF THE HOPFIELD TANK MODEL FOR SOLUTION OF THE MULTIPLE TRAVELING SALESMEN PROBLEM}

\author{
D. Wacholder J. Han \\ R. C. Mann
}

[Abetract of paper presented at the IEEE International Conference on Neural Network, Sin Diego, CA, July 2427, 1908; Proc. Vol. II, pp. 305-324 (1983)]

We developed an efficient neural network algorithm for solving the Multiple Traveling Salesmen Problem (MTSP). A new transformation of the N-City, M-salesmen MTSP to the standard Traveling Salesmen Problem (TSP) is introduced. The transformed problem is represented by an expanded version of Hopfield-Tank's neuromotphic city-position map with $(\mathbf{N}+\mathbf{M}-1)$-city and a single fictitious salesman. The dynamic model as sociated with the problem is based on the Basic Differential Multiplier Method (BDMM). The algorithm was successfully tested on many problems with up 2030 cities and 5 salesmen. In all test cases, the algorithm always converged to valid solutions.

Research oponeored by U.S. DOE Ofice of Basic Eners Sciences and Office of Military Applications.

\subsection{8}

\section{A NEURAL NETWORK ALGORITHM FOR THE MULTIPLE TRAVELING SALESMEN PROBLEM}

$$
\begin{gathered}
\text { E. Wacholder J. Han } \\
\text { R. C. Mann }
\end{gathered}
$$

(Abatract of ORNL/TM-10799. November 1988; aleo Biol. Coberw. 61, 11-19 (1989)]

We developed an efficient neural network algorithm for solving the Multiple Traveling Saleamen Problem (MTSP). A new travisformation of the N-city. M-salesmen MTSP to the standard Traveling Salesmen Problem (TSP) is introduced. The transformed problem is represented by an expanded version of Ilopfield-Tank's neuromorphic city-position map with $(\mathbf{N}+\mathbf{M}-1)$-city and a single fictitious salesman. The dynamic model aseo ciated with the problem is based on the Basic Differential Multiplier Method (BDMM) which evalu- 
ates Lagrange multipliers simultaneously with the problem's state variables. The algorithm was successfully tested on many problems with up to 30 cities and five salesmen. In all test cases, the algorithm always converged to valid solutions. The great advantage of this kind of algorithm is that it can provide solutions to complex decision making problems directly by solving a system of ordinary differential equations. No learning steps, logical if statements or adjusting of parameters are required during the computation. The algorithm can therefore be implemented in hardware to solve complex constraint satisfaction problems such as the MTSP at the speed of analog silicon VLSI devices or possibly future optical neural computers.

Research sponsored by U.S. DOE Office of Basic Enery Sciences and Office of Military Applications.

3.79

\section{A NEURAL NETWORK ALGORITHM FOR THE TARGET-WEAPON ASSIGNMENT PROBLEM}

\section{E. Wacholder J. Han}

R. C. Mann

[Abatract of paper presented at the International Neurad Network Society Annul Meeting, Boston, MA, September 6-10, 1988; Proc. Vol. 1, Supplement 1, p. 147 (1988)]

A neural network algorithm was developed to solve the problem of Weapon-Target Assignment
(WTA) for space-based Kinetic Energy Weapons (KEWs) against Intercontinental Ballistic Missiles (ICBMs). We consider here only the static varsion of this problem for a completely known attack scenario within a given time. The analytical model is an extended version of the Hopfield-Tank model combined with the Basic Differential Multiplier Method (BDMM) to solve for LaGrange mut tipliers simultaneously with the problem's state variables. Results obtained shor the validity of the proposed model for large-scale WTA problems. Computation time can be extremely short with hardware implementation using neuromorphic devices or possibly future optical neutral computers.

Research sponsored by U.S. DOE Ofice of Bavic Enery Sciences and Ofice of Military Applications.

\section{AUTOMATED METHODS FOR SENSITIVITY AND UNCERTAINTY ANALYSIS}

\subsection{0}

\section{GRESS ADJOINT MATRIX REDUCTION ALGORITHMS}

\section{J. E. Horwedel*}

[Abstract of Trans. Am. Nuel. Soc. 60, 590 (1989)]

GRESS is an efficient automated soft ware system for calculating sensitivities of model results to input data in existing FORTRAN 77 computer models. The GRESS ADGEN option calculates first derivatives of selected inodel results with respect to an almost unlimited arnount of data by application of the adjoint method. However, data storage has been a major limiting factor. The data storage requirement for an ADGEN application was more than 332 megabytes for a code that executes in 1 minutes on a VAX 8600 computer. The purpose of this paper is to describe two new algorithms that Jramatically reduce the data storage requirement and execution time for application of the ADGEN option.

Research sponeored by U.S. DOE Office and Nuclear Regulatory Commission.

\footnotetext{
-Computing and Telecommunications Division.
} 
3.81

EXAP-PRECOMPILER FOR THE GRESS AND ADGEN AUTOMATED SENSITIVITY CALCULATION SYSTEMS

J. E. Horwedel* F. G. Pin B. A. Worley

[Abetract oi Trane. Az. Neel. Soc. 56, 301 (1988)]

In recent years, two automated systems have been developed that add derivative taking capability to existing FORTRAN codes. The derivative information is used to calculate sensitivities of model results to input data. The two systems, GRESS and ADGEN, solve the forward and adjoint sensitivity problems, respectively. Both systems are based on a precompiler that automates the calculation of derivatives of the real variable on the left-hand side (LHS) of each equation with respect to the real variables on the right-hand side (RHS) of each equation. This paper describes the features of the extended arithmetic proceaser (EXAP) precompilet that makes the automation of differentiation capab.lity practical.

Revench sponeoned by U.S. DOE Ofice of Salt Repocitory Project.

*Computing and Telecommumications Diviaion

\subsection{2}

\section{GRESS VERSION 0.0} USER'S MANUAL

\author{
J. E. Horwedel* \\ B. A. Worley \\ E. M. Oblow \\ F. G. Pin

$$
\text { R. Q. Wright" }
$$

(Abstract of ORNL/TM-10835, October 1988)

This manual provides a deacription of the Grar dient Enhanced Software System (GRESS) and explains how to use it to enhance FORTRAN 77 models for gradient calculations. The use of the Extended Arithmetic F'roceseor (EXAP) as the precompiler for GREs, is presented. A complete deacription of how to enhance a source code for forward propagation of derivatives using the calculus chain rule is provided. On option, EXAP can be used to generate derivatives and store them on a direct access device for subsequent solution of the numerical adjoint equations. Programming information is also provided to aid in the installation and maintenance of the software.

Revench eponeored by U.S. DOE Otice of Defense Wuste and Environmental Restoration.

- Computing and Telecommunications Divicion

\subsection{3}

\section{COMPARISON OF RESULTS BASED ON A DETERMINISTIC VERSUS A STATISTICAL UNCERTAINTY ANALYSIS}

R. E. Maerker

$$
\text { (Abatract of ORNL/TM-10773, Mas 1983) }
$$

A deterministic method for performing an uncertainty analyais is applied to the results of a system of three codes previously analyzed by purety statistical means. This new method propagates parameter joint probabilities through computer models to obtain the distributions of model re sults by linear extrapolation from a pool of several gradient-enhanced reference runs. Results employing various options in the method are tested against those from the earlier statistical treatment. Both point-by-point and cumulative distribution comparicons were generally performed. This effort leads to the conclusion that in the present application 15 gradient-enhanced runs provide virtually as much uncertainty information as 200 statistical executions of the code system, and this feature of the analysis is expected to remain generally valid for most types of problems. The advantage of significant reduction in the number of reference runs required by the deterministic method is partially offset by the longer execution times of the enhanced versions of the coden, howsver. Thus efficient derivative-taking algorithms will be neeescary to insure cost effectiveness.

Research sponsored by U.S. DOE Ofice of Nucleer Wate Isoletion. 
3.84

DEVELOPMENT AND APPLICATION

OF A NEW DETERMINISTIC METHOD FOR CALCULATING COMPUTER MODEL RESULT UNCERTAINTIES

\author{
R. E. Maerker \\ B. A. Worley
}

[Abatract of Traws. Am. Nech. Soc. 60, 591 (1969)]

Interest in research into the field of uncertainty analysis has recently been stimulated as a result of a need in high-level waste repository de sign assessment for uncertainty information in the form of response coinplementary cumulative distribution functions (CCDF's) to show compliance with regulatory requirements. The solution to this problem must obviusly re:; on analysis of computer code model which, however, employ parameters that can have large uncertainties.

The motivation for the research presented here is a search for a method involving a deterministic uncertainty analysis approach which could serve as an improvement over those methods that make exclusive use of statistical techniques.

A deterministic uncertainty analysis (DUA) approach based on the use of first derivative information is the method studied in the present procedure. Since deterministic sensitivity methods have been shown in the past to be a cost efficient and practical means for determining derivative and sensitivity information, the possibility arises that the DUA approach could lead to a substantial reduction in the number of model runs compared to conventional analysis.

Rewearch sponeored by U.S. DOE Office of Fusion Energy and Defence Nuclee Agency.

$3.85^{\circ}$

\section{ANALYTICAL METHODS FOR LARGE-SCALE SENSITIVITY ANALYSIS USING GRESS AND ADGEN}

\section{F. G. Pin}

(Abatract of paper presenced at the 1988 Woskshop on High Level Wacte Disposal, Stockholm, Sweden, April 11-14, 1988)

Sensitivity analysis is an established methodology uned by researchers in almost every field to gain essential insight in design and modeling stud- ies and in performance assessments of complex systems. Conventional sensitivity analysis methodologies, however, have not enjoyed the widespread use they deserve considering the wealth of information they can provide, partly because of their prohibitive cost or the large initial analytical investment they require. Automated systems have recently been developed at ORNL to eliminate these drawbacts. Compilers such as GRESS and ADGEN now allow automatic and cost effective calculation of sensitivities in FORTRAN computer codes. In this paper, these and other related tools are described and siseir impact and applicability in the general areas of modeling, performance assessment and decision making for radioactive waste isolation problems are discussed.

Research sponsored by U.S. DOE National Low-Level Wate Managemeat Progren.

\subsection{6}

\section{SENSITIVITY ANALYSIS OF TWO-PHASE FLOW PROBLEMS}

\author{
N. Toomarian* E. Wacholder \\ S. Kaizerman ${ }^{\dagger}$
}

[Abatract of Neel. Sei. Eng. 99, 53 (1988)]

The mathematical derivation and application of two deterministic sensitivity analysis methods, the direct approach of sensitivity (DAS) and the adjoint sensitivity method (ASM), are presented for two-phase flow problems. The physical problems investigated are formulated by the transient one-dimensional two-phase flow diffusion model, which consists of a system of four coupled quasilinear first order partial differential equations. The DAS method provides the sensitivity coefficients of all primary dependent variables at each time and space lecation with respect to a single input parameter. On the other hand, the ASM method provides the sensitivity coefficients of a single response function at a specified time and space lo cation with respect to all input parameters.

The systems of governing equations of both sensitivity methods developed possess the same chararteristic directions as those of the original physical model. Therefore, the same numerical methods for the solution of these equations have been selected as for the solution of the physical problem, i.e., Turner sclieme and Modified Turner (NAIAD) scheme. Special techniques to incorpo- 
rate the boundary conditions of the ASM governing equations for each numerical scheme were developed. The sensitivity coefificients computed by both methods have been verified against results from standard parametric studies. Two sample problems are thoroughly investigated. The first problem considers the transient fuid behavior in a uniformly heated channel subjected to an inlet flow decay. The second problem considers the transient fiuid response within the same channel when a pressure step change at the channel inlet is imposed. Both methods predict satisfactorily the seneitivity coefficient behavior in space and time in comparieon with parametric st udies, even when a moving boiling boundary exists within the flow field. Certain coefficients in thr; thermodynamic correlations of the liquid density and the liquid saturation enthalpy, as well as the boundary conditions of the problems, were found to be the most "sensitive" input parameters in both problem investigated. Some input parameters of minor significance in the steady-atate conditions were found to be very anfluential during the transient and vice-veras. The behavior of moet of the sensitivity coefficients, in space and time, cannot be timated without a systematic sensitivity analysis method.

Reveirch sponeored by U.S. DOE Ofice of Bavic Energ Seiences.

-Omninet Communications Services, Los Angeles, CA.

tIreel Inatitute of Technology, Irreel.

3.87

\section{A TORTRAN PRECOMPILER AND SYSTEM FOR AUTOMATING THE IMPLEMENTATION OF THE FOEWARD AND ADJOINT DETERMINISTIC SENSITIVITY METHODS INTO EXISTING FORTRAN COMPUTER CODES}

\section{B. A. Worley}

(Abatract of oral preseneation presenled af Los Alamos Netiond Laboratory, Los Alamos, NM. April 27, 1088)

This talk reports on the development of a Gradient-Enhanced Software System (GRESS) that makes une of computer calculus precompit ers to automate the implementation of deterministic sensitivity analysis into existing FORTRAN computer models. This automation removes the considerable programming effort reçuired for im- plementation of the forward and adjoint sensitivity methods by directly changing the model. Deterministic sensitivity theory, the GRESS system, and sample problem results will be addressed.

Rewearch eponeored by U.S. DOE National Lon-Lewd Wate Management Program Otice of Defence Wete and Eavironmental Restoration.

\subsection{8}

\section{CALCULATION AND USE OF COMPUTER MODEL DERIVATIVES}

\author{
B. A. Worley
}

(Abatract of paper pereanted at the SIAM Confereace on Optimiention, Bocton, MA, April 3-5, 1939 and at a Wortuhop an Desion for Computer Experimente, Oal Ridge, TN, Nowember 3-5, 1888)

The calculation of derivatives of computer model results with respect to model data is performed using GRESS, a system of routines that enhances existing FORTRAN computer codes with the capability to analytically compute derivatives of interest in addition to normal model results. The feasibility and cost of calculating derivatives by both the direct chain rule and the adjoint options within GRESS and exemplified by the application of GRESS to a large-scale computer code that models radionuclide transport. The use of the derivative information for performing sensitivity analysis, in the formation of responee surfaces, and in optimization applieations is presented.

Revearch sponsored by U.S. DOE National Lon-Level Weate Management Progrun.

\subsection{9}

\section{ADGEN - ADJOINT GENERATOR} FOR COMPUTER MODELS
B. A. Worley
F. G. Pin
J. E. Horwedel"
E. M. Oblow

(Abatrect of ORNL/TM-11037, May 1000)

This paper presents the development of a FORTR $A N$ compiler and an acsociated oupporting software library called ADGEN. ADGEN reads FORTRAN models as input and produces an enhanced version of the input model. The enhanced version reproduces the original model 
calculations but also has the capability to calculate derivatives of model results of interest with respect to any and all of the model data and input parameters. The method for calculating the derivatives and sensitivities is the adjoint metbod. Partial derivatives are calculated analyti:ally using computer calculus and saved as elenrents of an adjoint matrix on direct assess storage. The total derivatives are calculated by solving an appropriate adjoint equation. ADGEN is applied to a major computer model of interest to the Lom-Level Waste Community, the PRESTO-II model. PRESTO-II sample problem resulus reveal that ADGEN correctly calculates derivatives of a response of interest $w$ ith respect to 3000 parameters. The execution time to create the adjoint matrix is a factor of 45 times the execution ime of the reference sample problem. Once this matrix is determined, the derivatives with respect to $\mathbf{3 0 0 0}$ parameters are calculated in a factor of 6.8 that of the reference model for each response of interest. For a single response, the total factor of 51.8 compares to a factor of $\mathbf{3 0 0 0}$ for determining these derivatives by parameter perturbations. The automation of the implementation of the adjoint technique for calculating derivatives and sensitivities eliminates the costly and manpowerintensive task of direct hand-implementation by reprogramming and thus makes the powerful adjoint technique more amenable for use in sensitivity analysis of existing models.

Research sponsored by U.S. DOE National Lom-Level Waste Manajement.

"Computing and Telecommunications Division.

\section{DETERMINISTIC SENSITIVITY AND UNCERTAINTY ANALYSIS FOR LARTE SCALE COMPUTER MODELS}

\author{
B. A. Worley \\ F. G. Pin \\ E. M. Oblow \\ R. E. Maerker \\ J. E. Horwedel* \\ R. Q. Wright"
}

techniques when large-scale modeling codes are being analyzed. These methods are able to estimate sensitivities, generate response surfaces, and estimate response probability distributions given the input parameter probability distributions. Be cause the statistical methods are computationally costly, they are usuelly applied only to problems with relatively small parameter sets. Deterministic methods, on the other hand, are very efficient and can handle large data sets, but generally require simpler models because of the considrable programming effort required for their implementation. The first part of this paper reports on the development and availability of two systems, GRESS and ADGEN, that make use of computer calculus compilers to automate the implementation of deterministic sensitivity analysis capability into existing computer models. This automation removes the traditional limitation of deterministic sensitivity methods. This second part of the paper describes a deterministic uncertainty analysis method (DUA) that uses derivative information as a basis to propagate parameter probability distributions to obtain result probability distributions.

Research possoned by U.S. DOE Natioal Lom-Leved Wate Management Program Ofice of Defence Weate and Environmental Rescoracion.

*Computing and Telecommunications Division.

IAbatract of paper presented at the Tenth Annual DOE Low-Level Radioactive Wacte Management Cronference, Denver, CO, Auguat 30-September 1, 1988, jession II: Site Performance Axceament, Proc. p. 126 (December 1988); and at the NEA Probabilistic Syatem Awesument Coden User Group Meeting. Paris, France, November 29 December 2, 1988]

The fields of sensitivity and unerrtainty analysis have traditionally beell doininated by statistical 


\section{ADVANCED CARBOLS PROGRaM}

\subsection{1}

\section{AN INTELLIGENT SMMULATION BNVIRONMENT FOR CONTROL SYSTEM DESIGN}

\section{J. T. Robinson}

[Abetrect of paper presented at the 1900 Enctern Multiconference, Taupe, FL, Mard 26-31, 1800 Proc. Alouees in A/ and Stivelation 20(4), pp. 20-32 (March 1909); doo 7th Pover Plane Dyanmica, Control, and Teatine Sympoinm, Kmoxville, TN, May 15-17, 19es, Proc. Pp. $3.01-3.12$ (Mas 1809)!

The Oak Ridge National Laboratory is currently asisting in the development of advanced control systems for the next generation of muclear power plants. This paper presents a prototype interactive and intelfigent simulation environment being developed to support this effort. The environment combines tools from the field of Artificial Intelligence; in particular object-oriented programming, a LISP programming environment, and a direct manipulation user interface; with traditional numerical methods for simulating combined continuous/diecrete procenses. The resulting environment is highly interactive and eary to use. Models may be created and modified quickly through a window oriented direct manjpulation interface. Models may be modified at any time, even as the simulation is running, and the results observed immediately via real-time graphics.

Research sponsoned by U.S. DOE Ofive of Reactor Tech nologies Derdoponent.

\subsection{2}

\section{AN INTELLIGENT DYNAMIC SIMULATION ENVIRONMENT: AN OBJECT-ORIENTED APPROACH}

\section{J. T. Robinson R. A. Kisner"}

(Abetract of peper prewentad at the Third IEEE Incerne tiond Sympocium on Intellicent Control, Artington, VA, Auguat 24-28, 1968; Proc. H. E. Stephanou, A. Meyotel, and J. Y. S. Luh, Eds., pp. $687-692$ (1989)]

This paper presents a prototype simulation environment for nuclear power plants which illus trates the application of object-oriented program. ming to process simulation. Systems are modeled using this technique as a collection of objects which communicate via message passing. The environment allows users to build simulation models by selecting iconic representations of plant components from a menu and connecting them with the aid of a mouse. Models can be modified graphically at any time, even as the simulation is rusning, and the results observed immediately via real-time graphics. This prototype illuatrates the we of object-oriented programming to create a highly interactive and automated simulation environment.

Researds apowered by U.S. DOE Ofiee of Reactor Tech nologio Developenen.

*Inatruncatation and Controls Division

3.93

\section{AN APPLICATION OF OBJECT ORIENTED PROGRAMMING TO PROCESS STMULATION}

\section{J. T. Robinson P. J. Otaduy}

:Abetrect of peper preceated at che Nationel Mecting of the American Inatilute of Chemical Engiveas, Denre, CO, Angue 22-25, 1838)

This paper discuses the application of objectoriented programming to dynamic simulation of continuous procences. Procesces may be modeled using this technique as a collection of objects which communicate with each other via menage pasing. Arriving measages invoke methods that describe the atate and/or dynamic behavior of the receiving object. The objects fall into three broed categories (1) actual plant components such as pumps, pipes, sad tanks, (2) plant systems such as flow loops, and (3) simulation control and interface objects. This technique differs from traditional approaches to process simulation, in which the process is represented by either a system of differential equations or a block diagran. of mathematical operators. The use of objects minimizes the representational gap between the model and the actual process. From the users point of view, construetion of a simulation model becomes equivalent to drawing a plant schematic.

As an example application, a package developed for the simulation of nuclear power plante is deacribed. The package allows users to build simulation models by selecting iconographic repre- 
sentations of plant components from a menu and connecting them with a mouse. Objects for generating and optimizing a mathematical model of the system and for controlling the simulation are automatically generated. This example illustrates the use of object-oriented programming to creute - highly interactive and automated simulation environment.

Reacend sponered by US. DOE Otice of Reactor Tech nologies Developonent.

\subsection{4}

\section{VOI DISTRIBUTIONS IN BUBBLY PLOW TAROUGH YAWBD ROD ARRAYS}

\section{J. T. Robinson N. B. Todreas" D. Ebeling-Koning ${ }^{\dagger}$}

[Abetrad of Int J. Mehiphese Fow 14, G45-652 (ISes)]

In this communication, obeervations of void distribution in bubbly flom through an array of rods inclined relative to the average flow direction are presented. The experiments reveal a strong dependence of roid distribution on bubble size and rod arrangement. It is postulated that this dependence is due to the entrapment and migration of bubbles in vortices behind the rods. Not surprisingly, attempts to simulate the experimental results with a six-equation porous-body model in which localized phenomenon such as vortices have been averaged out were unsuccessful.

Research sponered by U.S. DOE Ofice of Buic Energ Sciences and Masachnets Inatitute of Technolog.

"Meanchusette Inatiule of Technolos, Cambridze, MA.

tWeatinghouse Electric Corporation, Pittuburch, PA.
3.95

\section{himRarchical Control of a NUCLEAR REACTOR USING UNCERTAIN DYNAMICS TECHNIQUES}
L. A. Rovere*
P. J. Otaduy
C. Britton'
R. B. Perea

[Abstract of paper presented at the Thind IEEE Interm. tiond Sympocium on Ineellitent Control, Arington, VA, Augua 24-26, 1968; Proc. H. E. Stephanou, A. Mejued, and J. Y. S. Liu, Ed., pp. 713-718 (1909)h

Recent advances in the nonlinear optimal control area are opening new possibilities towards its implementation in process control. Algorithms for multivariate control, hierarchical decomposition, parameter tracking, model uncertainties, actuator saturation efiects and physical limits to state variables can be implementied on the basis of a consis tent mathematical formulation.

In this paper, good agreement is shown be tween a centralized and a hierarchical implementation of a controller for a hypothetical nuclear power plant subject to multiple demands.

The performance of the hierarchical distributed system in the presence of localized subsystem failures is analyzed.

Research sponered by U.S. DOE Office of Reactor Technologies Development.

-Comision Necioinal Energia Alomica, Argentina.

tInstrumentation and Controls Divition.

3.96

DEVELOPMENT OF AN UNCERTAIN MODEL BASED NONLINEAR OPTIMAL PRESSURE CONTR OL SYSTEM FOR A NUCLEAR REACTOR
L. A. Rovere*
P. J. Otaduy
C. March-Leubat

(Abutract of paper presented at the IEEE 1988 Nuclear Science Symponium, Orlando, FL, November 9-11, 1988)

Using nonlinear optimal control theory for uncertain systems, an algorithm has been developed to control reactor primary pressure and estimate the imbalance between power source and heat sink by observation of the pressure signal alone.

The algorithm was tested with a simulation of ORNL's l!igh Flux Isolope Reactor (IIFIR) 
primary pressure boundary. The BFIR operates in a feed-and-bleed "solid" mode, where constant epeed pressurizing pumps supply make-up fiow and a bleed valve adjusts the syatem's letdown for. The controller's internal model of the process is purposely los detailed than that of the simulation but incorporates a time dependent term to account for unknown effects. Using Pcintryagin's Meximum Principle and the appropriate Hamit tonian a control algorithm is generated that from the difference between the meacured preseure and the internally computed one, it not only generates the correet blend valve control signal needed to fotlow a time dependent pressure setpoint but it also identifies the magnitude and temporal behavior of the energy inventory in the primary system.

Resennch sponsored by U.S. DOE Otice of Reactor Tectnologies Development.

"Comicion Nacioinal Energia Alomica. Argendian.

tThe Univeries of Tennescee, Knaxille, TN.

\subsection{7}

\section{ESTIMATING FINDING RATES FOR U.S. CRUDE OII}
D. S. Christiansen"
D. B. Reister

(Abatrect of ORNL/TM-10007, October 19e0); alo Energs (in prewe)]

In this report we provide extimates of both exploratory and developmental finding rate equer tions for crude oil for six onshore regjoms in the lower 48 United States. We entimate the finding rate in two different ways - one based on the relationship between the finding rate and cumulative reserve additions (s "derivative approach") and one based on the relationship between cumulative reserve additions and cumulative drilliag (an "integral approach"). We develop the integral approach according to two difierent methods. We inveatigate differences in finding rates between approaches and between regions. The regional data set is included as an appendix.

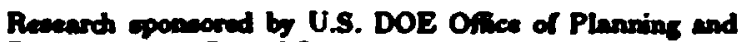
Eavironmete Ofice of Posal Easow.

* Abion Colleze, Albion. MI.

\subsection{8}

\section{THE AGGREGATE PRODUCTION PROFILE FOR U.S. CRUDE OIL}

\section{S. Christiansen* \\ D. B. Reister}

(Abatract of ORAL/TM-10s57, October 1938; aloo Resonres and Energs Jownal (in prean)]

In this paper we estimate the aggregate production profile for U.S. crude oil for the period 1961 to 1985 . Using data on reserves, we find that there is a significant lag between the time that reserves are added and the time production peaks. We attribute this long lag to a system of production controls that existed until about 1970. When we re-eatimate over the period from 1970 to 1985 , we find that there is a much shorter period between reserve additions and peak production. We consider an alternative approach baced on drilling data that gives similar results for the entire sample period but difierent results for the subperiod 1970 . 1985. We discuse the factors that are responsible for the general shapes of the profiles we eatimate.

Research sponcored by U.S. DOE-Fomil Enero, Ofice of Planning and Environment and by the Facults Research Participation Progem adminitiered by Oak Ridge Asecialed Univenities.

-Albion College, Albion, MI. 


\section{OIL VULNERABILITY AND INTERMEDIATE PRICE FLUCTUATIONS: A PRELIMINARY ASSESSMENT AND PROPOSAL}
T. R. Curlee*
D. B. Reister

(Abatract of ORNL/TM-11259, September I989)

Most of the work on oil vulnerability has focused on the implications and appropriate public and private responses to short-term oil price spikes. This paper focuses on the posibility that the world oil market is inherently more prone to large intermediaie price fuctuations that are often triggered by short-term disturbances.

The purposes of this paper are (1) to pose the question of whether large and repeated intermediate price swings are a likely outcome, given our knowledge of the world oil market, (2) to address bow the effectiveness of policy instruments designed to mitigate the impacts of oil vulnerabitity may change in a world in which large intermediate oil price fluctuations are expected, and (3) to propose future research that would address in greater detail the potential for, and appropriate responses to, large intermediate price swings on the world and domestic oil markets.

The paper concludes that there are plausible conceptualizations under which oil prices may fluctuate over the intermediate term. It is suggested that a major focus of future work should be on how the structure of the oil market may itself change over time, causing oil prices to go up and down. The paper also concludes that the implications for such price swings may be significant for both the types and quantities of mitigation measures held by the public and private sectors. Finally, it is argued that the current world oil market provides a valuable opportunity for studying how oil prices msay fluctuate over the interniediate term and how U.S. energy policies can beat be structured to copy with oil vulnerability.

Researsh sponsored by U.S. Department of Energy.

- rinergy Division.

\section{ENERGY TECHNOLOGY RED: WHAT COULD MAKE A DIFPERENCE?}

\author{
W. Tulkerson" \\ A. T. Crane \\ S. I. Auerbaght \\ A. M. Perry* \\ D. B. Kash\$
}

C. W. Hagan, Jr."

(Abstract of ORNL6541/VI, May 1989)

The objective of this study was to survey both energy technologies and crosecutting areas of science and technology in order to identify important R\&D needs and opportunities in the context of the U.S. and world energy situations. The imperative for RkD was judged against its potential for fixing current energy system problems; for providing a robust set of options for coping with, taking advantage of, or encouraging future energy circumstances; and for creating unanticipated opportunities.

Fesearch sposecred by U.S. Depertment of Eners.

* Energa Divicion.

Enviconmental Sciences Divition.

tOfice of Technology Ameacment, Wahington, DC.

\$University of Oklahome, Normen, OK.

3.101

\section{GLOBAL WARMING - AN ENERGY TECHNOLOGY RED CHALLENGE}
W. Mulkerson"
A. M. Perry"
D. B. Reister
D. E. Kash
A. T. Crane
S. I. Auerbach $\S$

[Abstract of Science 246, 868 (1989)]

Two major uncertainties preclude gaining a clear pisture of future energy technology needs: (i) growth of energy demand, particularly of oil, and (ii) the seriousness and urgency of the changing greenhouse effect and of other environmental, health, and safety problems. To allow for these uncertainties, a broad research and development (R\&D) effort is needed; one that is balanced with respect to emphasis on improved energy sources and improved efficiency of energy end-use and conversion. A recent Oak Ridge National Laboratory (ORNL) survey of energy teclinology R\&D 
revealed a rich variety of opportunities for improving both end-use and supply technologies. It also concluded that the U.S. effort is sufficiently broad since combined public and private sector investments are covering most of the promising energy technology options at some level.

Neverthelors the effort is inadequate. None of the nonfossil energy sources are yet ready to substitute competitively at the scale necessary to reduce $\mathrm{CO}_{2}$ emissions. To correct this inadequacy, a three prong R\&D strategy is required: improve the efficiency of energy conversion and use, improve nonfossil energy sources, and improve technologies tailored to the needs of developing nations.

Reseench sponsored by U.S. DOE.

Enerar Divicion.

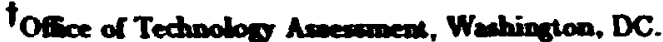

tUniverity of Othabome, Norman, OK.

\$Environmental Sciences Divicion.

\subsection{2}

\section{ENERGY TECHNOLOGY RED: WHAT COULD MAKE A DIPFERENCE}

\section{B. Reister}

(Abetract of paper preseated at che Techoolong-Buned Coosfidence Buildins: Fners and Environment, Sante Pe, NM, July 9-14, 1809; and at the MIT Wortebop on Energy and Environumenel Modeline and Policy Analycis, Cambridge, MA, July 31-Angut 1, 1800)

Two major uncert ainties cloud the future energy system: the level of energy demand and the consequences of the greenhouse effect. Energy technology R\&D provides technological alternatives for the future ener $\mathrm{g}$ system. The Oak Ridge National Laboratory (ORNL) recently completed a broad survey of energy technology R\&D. The survey revealed a rich variety of options for both end-use technologies and energy supply technologies. However, none of the nonfowil energy sources are ready to substitute for fomil fuels at the scale necessary to mitigate the potential consequences of the greenhouse effect.

Research sponsored by U.S. Department of Energy.
3.103

\section{FUTURE EMISSIONS OF CARBON DIOXIOE: CAN WE MAKE A DRAMATIC REDUCTION?}

\author{
D. B. Reister
}

(Abotract of paper presented at the WATTec Conference, Knoxville, TN, Februry 16, 1999)

The hot and dry summer of 1988 aroused public concern about the impacts of increaced concentrations of greenhouse gases in the atmoephere. Scientists agree that higher concentrations will result in higher temperatures, climate changes, higher sea levels, and economic damage. However, scientists do not agree on the magaitude or timing of the changes.

During the next 100 years, the level of emis sions of carbon dioxide will depend on world population, per capita GNP, energy efficiency, and energy supply. World population and per capita GNP are expected to increase. Reduction of future emissions of carbon dicuxide requires increases in energy efficiency and increases in the use of atternative ener gy sources (nuclear power, biomas, and advanced sources [solar and fusion]). Reforestation and the substitution of natural gas for coal can make a small reduction in future emissions.

Reseanch sponsored by U.S. DOE Office of Fonil Enerd and Ofice of Planning and Eavironmens.

\subsection{4}

\section{THE REFIACEMENT COST INTEGRATION PROGRAM USER'S GUIDE}

\section{B. Reister}

(Abetract of ORNL/TM-11124, March 1989)

The Oak Ridge National Laboratory and subcontractor ICF-Lewin Energy have developed a set of computer models to forecast the replacement cost of domestic crude oil. The REPCO model forecasts the replacement cost in the lower 48 states. The Aretic Economics Model forecasts the replacement cost in Alaka. The two models of the replacement cost system forecast domeatic oil supply curves (schedules of the amount of oil available at various costo). The Replacement Coat Integration Program (RCIP) 
integrates the output from the two models to forecast the annual discoveries and production of domestic crude oil.

RCIP is a user-friendly menu-driven program that is designed to run on an IBM-PC. RCIP allows the user to conveniently edit the input parameters, to calculate the results, and to display the output. In addition, the user can easily stcre a scenario on a dist and retrieve a scenario from the disk. The built-in output editor allows the user to choose an aggregation scheme for the regional results and retrieve a scenario for comparison. The ortput can be directed to a file or to the screen.

This user's guide for RCIP contains detailed directions for installing and operating RCIP. The guide discusses all of the parameters on the input sereens of the model. The format and units of the output files of the model are reviewed in Appendix B.

Resenrch sponeored by U.S. DOE Office of Fomil Eners and Ofince of Oil, Gas, Shate, and Special Technology Gar.

\subsection{5}

\section{REPLACEMENT COST INTEGRATION PROGRAM MODEL DESCRIPTION}

\section{B. Reister}

\section{(Abatract of ORNL/TM-11132, March 1989)}

The Oak Ridge National Laboratory and subcontractor ICF-Lewin Energy have developed a set of computer models to iorecast the replacement cost of domestic erude oil. The Replacement Cost model forecasts the replacement cost in the lower 48 states; the Arctic Economics Model forecasts the replacement cost in Alaska. The two models of the replacement cost system forecast domestic oil supply curves (schedules of the amount of oil available at various costs). The Replacement Cost Integration Program (RCIP) integrates the output from the two models to forecast the annual discoveries and production of domestic crude oil.

RCIP is a user-friendly, menu-driven program designed to run on an IBM personal computer. RCIP allows the user to conveniently edit the input parameters, to calculate the results, and to display the output. In addition, the user can easily store a scenario on a diak and retrieve a scenario from the disk. The built-in output editor allows the user to choose an aggregation scheme for the regional results and retrieve a scenario for comparison. The output can be directed to a file or to the screen.

This Model Description provides an introduction to the models and presents a mathematical description of the oil supply model and of the drilling model.

Revesuch eponsored by US DOE Office of Fosil Enery and Ofice of Planning and Eavironmeat.

\subsection{6}

\section{REPLACEMENT COST INTEGRATION PROGRAM MODEL OVERVIEW}

\author{
D. B. Reister
}

(Abatreet of ORNL,TM-11042, Febreary 1989)

The Oak Ridge National Laboratory and its subcontractor ICF-Lewin Energy have developed a set of computer models to forecast the replacement cost of crude oil. The REPCO model forecasts the replacement cost in the lower 48 states. The Aretic Economics Model forecasts the replacement cost in Alasta. The two models of the replacement cost system forecast domestic oil supply curves (schedules of the amount of oil available at various costs). The Replacement Cost Integration Program (RCIP) integrates the output from the two models to forecast the annual discoveries and production of domestic crude oil.

RCIP is a user-friendly menu-driven program that is designed to run on an IBM-PC. RCIP allows the user to conveniently edit the input parameters, to calculate the results, and to display the output. In addition, the user can easily store a scenario on a disk and retrieve a scenario from the disk. The built in output editor allows the user to choose an aggregation scheme for the regional renults and retrieve ascenario for comparison. The output can be directed to a file or to the screen.

This Model Overview provides an introduction to the models and presents some typical results.

Research sponeored by U.S. DOE Office of Fonil Energy and Ofice of Oil, Gan, Shale, and Special Technolog Gas. 


\section{$3.10 t$}

\section{VALIDATION OF THE REPLACEMENT COST INTEGRATION PROGRAM}

\author{
D. B. Reister \\ D. S. Christionsen*
}

(Abatract of ORNL/TM-11046, Februmy 1989)

The Oak Ridge National Laboratory and its aubcontractor ICF-Lewin Fnergy have developed a set of computer models to forecast the replacement cost of domestic crude oil. The model REPCO forecasts the replacement cost in the lower 48 states. The Arctic Economics Model (AEM) forecasts the replacement cost in Alasta. The two models of the replacement cost system forecast domestic oil supply curves (achedules of the amount of oil available at varions prices). The Replecement Cost Integration Program (RCIP) integrates the output from the two models to forecast the annual discoveries and production of domestic crude oil.

RCIP has been developed over several years. The most recent version was completed and delivered to DOE in January 1988. In the summer of 1987, a project was begun to validate RCIP. To validate RCIP, a historical data base was developed for the period 1970-1986, and the data base was used to estimate the parameters in the drilling model and to estimate regional finding rates. The data base includes regional data on drilling footage tor exploratory and developmental wells, on additions to proved reserves from exploratory and developmental activities, and on production.

Research sponeored by U.S. DOE Offec of Planning and Environment.

* Albion College, Albion, MI.

3.108

\section{THE REPLACEMENT COST INTEGRATION PROGRAM, AN ENGINEERING-ECONOMIC MODEL OF OIL SUPPLY}
D. B. Reister
D. S. Christiansen"

(Abatract of paper to be presented at the IAEE 1989 North American Conference, Los Angeles, $C A$, Ochober 16-18, 1989; Proc. Pp. 89-88 (1089)]

The Replacement Cost Integration Program (RCIP) forecasts the annual additions to proven reserves and production of domestic crude oil.
The inputs to RCIP include parameters (discount rate, tax rate, and supply elasticity), expected oil prices, and process model output. The process model output consists of capital costs, operating costs, and production leveis for 31 regions. For each region and year, RCIP calculates expected profits and forecasts the level of reserve additions. Two production profiles relate reserve additions to future oil production. The parameters in the model were estimated using data for 1970 to 1986. Results are presented for two sets of prices: high and low. For the period from 1987 to 2020, the cumulative production of oil increases by 64 percent from the low case to the high case, while the cumulative additions to proven reserves increase by more than a factor of three.

Research sponsored by U.S. DOE Fowil Enery Office of Oil, Gas, Shale, and Special Technoloys Gas.

* Albion Colleze, Albion, MI.

3.109

THE RAMS COAL MODEL: VOLUME I - MODEL OVERVIEW VOLUME II - USER'S GUIDE VOLUME III - MODEL DESCRIPTION
D. B. Reister
J. V. Conopask*
P. E. Mihlmester*

(Abstract of ORNL/TM-10686/V1, ORNL/TM-10686/V2, and ORNL/TM-10686/V3, February 1988)

The RAMS coal model was developed to aid the planning functions of the Office of Fowsil Energy of the Department of Energy. The model was designed to simulate the market penetration of processes that use coal to produce liquid fuels. To simulate market penetration, we assume that the least cost process will have the largest share of the market. To evaluate the future income and costs for a process, we nee.t wo estimate the prices of all of the inputs and outputs for all of the coal proceses and for all of the competing processes. After a literature review, we identified 26 types of energy, by-products, and factors of production that are potential inputs or outputs for a process. The coal model simulates the supply and demand for the 26 types of goods.

The coal model input-output (I-O) structure has 13 sectors to produce the first 24 goods (the last 2 factors, labor and capital, are exogenous inputs). The first 10 sectors produce the first 10 goods, which are either primary or secondary forms of energy. Sector !l, the liquid fuels sec- 
tor, produces refined patroleum products and byproducts, the goods from 11 to 23 . Sector 12 produces good 24, materials. The final sector, sector 13 , is the trade sector.

Volume I provides an overview of the RAMS coal model. After an introduction to the RAMS family of models, we discuss some of the key design issues for the coal model. To demonstrate the flexibility of the model, we adjusted the parameters and input variables of the model in an attempt to reproduce the base case scenario published in the most recent National Energy Policy Plan (NEPP). The results demonstrated that the model can create a scenario that is quite similar to the NEPP scenario.

Volume II is a user's guide for the RAMS coal model. After a discussion of how to install and operate the model, the report provides a guided tour of the five input data sets and the six output data sets. One of the input data sets can be edited using LOTUS 1-2-3. The model has a menu-driven output editor that displays graphical output for all of the variables in the model.

Volume III presents the equations for the core model and for all of the existing satellite models. The supply models (oil, gas, and coal) calculate the I-O coefficients for the first 6 sectors. The liquid fuels model calculates the coefficients for sector 11. The end-use model calculates the coefficients for sector 12 . The remaining sectors (7 to 10$)$ have fixed coefficients. The overall architecture of RAMSCOAL features a core model and a family of satellite models. The core model is a general equilibrium model of the supply and demand for the 23 types of energy and hy-products. Given I-O coefficients for each of the first $12 \mathrm{sec}$ tors and the levels of imports and exports, the core model balances supply and demand and forecasts prices and quantity of production for the 23 types of energy and by-products. The I-O coefficients for each sector are forecast by the satellite models.

Research sponsored by U.S. DOE Ofice of Fowil Energy.

-Applied Management Sciences, Inc, Oak Ridge, TN.

\section{DOSIMETRY}

3.110

\section{PRESSURE VESSEL FLUE'VCE ANALYSIS AND NEUTR.ON DOSIMETRY}

\author{
F. B. K. Kam* \\ M. L. Williams' \\ R. E. Maerker \\ F. W. Stallmann* \\ [Abstract of ORNL/TM-10651 (NUREG/CR-5049), De- \\ cember 1587]
}

A review of the various methodologies used by industries and research institutes for reactor pressure vessel (RPV) fluence determination shows that most oiganizations employ an analysis sequence consisting of three steps. These include transport calculations, dosimetry measurements, and a statistical procedure to combine the calculations and measurements to arrive at a fluence value which has a smaller uncertainty than the original calculations. An accurate determination of damage fluence accumulated by the RPV as a function of space and time is essential in order to ensure the vessel integrity for both pressurized thermal shock transients and end-of-life considerations. The desired accuracy for neutron expo- sure parameters such as displacements per atom or neutron fluence (E $>1.0 \mathrm{MeV}$ ) is of the order of $\pm 10 \%$ to $\pm 15 \%(1 \sigma)$. These types of accuracies can only be obtained realistically by validation of the entire analysis sequence in benchmark experiments. This report identifies a standardized procedure based on benchmarked calculations, data, and dosimetry measurements, which could be used by organizations performing RPV fluence determinations. Another purpose of this report is to provide supporting documentation for any proposed regulatory guide on this subject.

Rosearch aponeored by U.S. Nuclear Regulatory Commic sion, Office of Nuclear Regulatory Research.

* Research Reactors Division.

†Luisians Stake Univensily, Baton Rouge, LA. 
3.111

\section{ANALYSIS OF THE VENUS-3 EXPERIMENTS}

\author{
R. D. Maerker
}

(Abatrect of ORNL/TM-11106, NUREG/CR-5338, Auguat 1909)

The results of applying a hybrid superposition-synthesis calculational method to a mockup of a three-dimenaional geometry involving a partial length shield arsembly (PLSA) at the VENUS-3 facility in Mol, Belgium, are described. Comparisons of transport calculations using the method and many measurements involving nickel, indium, and aluminum dosimeters indicate agreement generally to within five percent if effects of inaccuracies in the dosimeter cross sections are minimized and proper orientation of the coordinate system used in the synthesis procedure is observed. These conclusions bode well for the success of this melhod in solving neutron transport problems involving the use of PLSAs in light water reactors to reduce core leakage in pressurized thermal shock programs.

A second report describing the experimental details of the measurements will serve as companion documentation to this one and will be furnished by the Studiecentrum voor Kernenergie/ Centre d'Etude de l'Energie Nucléaire, Mol, Belgium.

Research sponcorrd by Division of Engineering, Office of Nuciear Regulwory Research, U.S. Nucken Reguletory Commiscion.

\subsection{2}

THE LEPRICON CODE SYSTEM: CONSOLIDATION OF TRANSPORT ANALYTICAL AND UNFOLDING PROCEDURES IN LWR PRESSURE VESSLL DOSIMETRY

\section{R. E. Maerker}

[Abutract of Trane. Am. Necl. Soc. 57, 225 (1989)]

The Least-Squares Dlectric Power Research Institute Consolidation (LEPRICON) program code system has been developed over the past 10 years to provide a complete analysis of light water reactor (LWR) pressure versel dosimetry. The system incorporates nine modules. All but one of the modules treat various aspects of neutron transport from the core through the reactor internals to dosimetry locations in the downcomer and/or reactor cavity regions and to critical fuence locations in the pressure vessel. The LEPRICON adjustment module, on the other hand, performs a state-of-the-art least-squares analysis of the results from the transport modules, a procedure often referred to as spectral unfolding or the combining of integral and differential data. In terms of development, the adjustment module alone required $\sim 70 \%$ of the total effort. The results from the LEPRICON system thus represent prior and adjusted fluences in each of 38 groups from 0.1 to $20 \mathrm{MeV}$, along with their corresponding standard deviations, at critical locations in the pressure vessel.

Results of applying the LEPRICON system to two different power reactors have appeared in the literature, and the conclusion was reached in both cases that the accumulated pressure vessel fuences could be determined to within $\sim 10 \%$. One of the major discoveries was a consistent adjustment of $\sim 8 \%$ downward in the iron inelastic cross section in the 3- to 8-MeV region. An update to the Mod3 evaluation of ENDF/B-V natural iron, spurred by these LEPRICON findings as well as from other sources, has recently been performed, and the resulting improvements in the agreement between calculations and measurements for all 37 benchmarks involving iron penetration, as well as in the above reactor analyses, provide strong evidence for the superiority of this new evaluation and the consistency of the LEPRICON adjustment procedure. As a consequence, this set has been added to the ELXSIR library for future calculations.

In conclusion, the complete LEPRICON methodology provides a comprehensive and accurate analysis of LWR dosimetry and, as a result, has been recommended as serving the needs of the U.S. Nuelear Regulatory Commiseion for extended pressure vescel lifetime nonitoring.

Research aponsored by U.S. Nuclear Regulatory Commieion Office of Nuclear Regulatory Researd. 


\subsection{3}

BENCRMARK VERIFICATION OF A METHOD FOR CALCULATING LEAKAGE FROM PLSA MODIPIBD CORES
R. E. Maerker
P. D'hondt*
L. Leenders*
A. Fabry

[Abotract of Trens. Am. Neel Soc. 00, 545 (1909)]

The results of applying a hybrid superposition-synthesis calculational method to a mockup of a three-dimensional geometry involving a partial length shield assembly (PLSA) at che VENUS3 facility in Mol, Belgium, are degrribed. Comparisons of transport calculations using the method and many measurements involving nickel, indium, and aluminum dosimeters indicate agreement geterally to within five persent if effects of inacerracies in the dosimeter crosesections are minimized and proper orientation of the coordinate system used in the synthesis procedure is observed. These conclusions bode well for the success of this method in solving neutron transport problems involving the use of PLSAs in light water reactors to reduce core leakage in pressurized thermal shock programs.

Reseanch eponeored by U.S. DOE Office and Nuclem Rezulatary Conamiecion.

"SCK,CEN, Mol, Belgium.

\subsection{4}

\section{ANALYSIS OF THE VENUS-3 EXPERIMENTS}
R. E. Maerker
P. D'hondt*
L. Leenders*
A. Fabry*

(Abatrat of paper lo be presented at the Seventh ASTMEURATOM Sympocium on Resctor Donimetry in Stre bourg, France, Auguet 27-31, 1980)

Over the past reveral years, plant life extension programs have been implemented in many U.S. plants. One method of pressure veasel (PV) fluence rate reduction being used in several of the older reactors involves partial replacement of the oxide fuel with metallic rods in those peripheral as semblies located at critical azimuths. This substitution extends axially over a region that depends on the individual plant design, but covers the most critical PV weld and plete locations which may be subject to pressurized thermal shock. In order to andyze the resultirg PV dosimetry using these partial length shield asemblies (PLSA), a relatively simple but accurate method needs to be formulated and qualified that treats the axially asymmetric core leabage.

Comparisons of the equivalent fission fluxes involving all the dosimeters indicate agreement generally to within $5 \%$ when proper orientation of the coordinate system used in the synthesis procedure is obeerved. The method is apparently very accurate (i.e., the agreement lies generally within the measurement uncertainties) for detector locations of greatest concern in PLSA geometries. In particular, the agreement behind the PLSA is comparable to that behind an unmodified ascembly. In conclusion, the success of the proposed method bodes well for the accuracy of future anatyes of on-line plants using PLSAs.

Researd sponeored by U.S. Nuclear Regulatory Commie sion Office of Nucles Regulesory Recearch and U.S. De partment of Enexp.

"SCK/CEN, Mol, Belpjum. 


\section{HUMAN-COMPUTER INTERACTIONS}

\subsection{5}

\section{HUMAN FACTORS IN THE NAVAL AIR SYSTEMS COMMAND: COMPUTMR-BASED TRAINING}

\author{
T. L. Seamster" \\ C. E. Snydert \\ M. Terranova \\ W. J. Walker? \\ D. T. Jonest
}

(Abetrect of paper preanted at the Human Factors Society 32nd Annual Meeting, Anabeim, CA, October 24-23, 1808 Proc. Vol. 2, pp. 1005-1009 (1803),

Military standards applied to the private sectos contracts have a substantial effect on the quality of Computer Based Training (CBT) systems procured for the Naval Air Syatem Command. This study evaluated atandards regulating the fot lowing areas in CBT development and procuremeat: interactive training systems, cognitive task analysis, and CBT hardware. The objective was to develop some high-level recommendations for evolving standards that will govern the next generation of CBT systems. One of the key recommendations is that there be an integration of the instructional system development, the human factors engineering, and the software development standards. Recommendations were also made for tack analysis and CBT hardware standards. Recommendations were also made for task analysi and CBT hardware atandards.

Rewearch spossored by U.S. Depertment of Enercy and Navl Air Syatem Command.

-Carlor Aesociales, Inc., Fairfax, VA.

tData Syatem Resench and Development Division.

INaval Air Syoteme Command, Weahinglon, DC.
3.116

TEAMWORK AND DATA DELIVERABLES: A MAP TO SOFTWARE AND TRAINING DESIGN INTEGRATION
C. E. Sayder*
D. E. Smitht
J. Davis?
N. E. Clapp, Jr. ${ }^{\dagger}$
M. Terranova
P. G. Guthriet
J. L. Tinneyt
S. Webstert
G. B. Paynet

[Abstract of peper to be presented at the Interwervice/lindusty Training Systems Conference, Fort Worth, TX, Norember 16, 1909]

A team of training technologists has devetoped an acquisition structure to help the Navy Airborne W.apons Training Program in managing the development of desk-top training systems by the private sector. The goal of the structure is to meet the special requirements of the users: NAVAIR managers, Navy trainers, and the fleet. Developing interactive training systems requires the application of three engineering disciplines: software engineering, instructional design, and human factors engineering. Each discipline has an acsociated set of military standards and Data Item Descriptions (DIDs), but these usually have not been applied wgether in the acquisition of training systems. The cost involved in using three sets of standards and DIDs becomes prohibitive, especially for procuring desk-top computer-based systems. The alignment of the three development processes is not specified by existing gujdelines. This paper offers a way to specify the objectiven of training and to ensure that they are met during the software development process; it also suggests how to integrate human factors engineering up-front at an affordable level that includes the recognition of cognitive and effective factors in the training process. Ultimately: it specifies software documentation and development that should lead to generic programs that can be maintained and supported by the procuring organization. The acquisition structure is summarized in a matrix that aligns the review of documentation supporting each engineering process in an integrated development cycle. The matrix is a map of the planning, analysis, design, and development phase that coordinates the data deliverablen for training, human 
factors, and software development. Only through the successful integration of the three disciplinessoftware engineering, instructional design, and human factors engineering - can future training goals be reached.

Research sponsored by U.S. Department of Energ.

*Dale Syatems Recearch and Development Diviaion

IInstrumentation and Controls Diviaion.

the University of Tennessea, Knowrille, TN.

\subsection{7}

\section{A PART-TASK TRAINER IN THE NAVAL AIR SYSTEMS COMMAND}

\section{E. Snyder* M. Terranova}

(Abatrect of paper to be presented at the American Nudear Society Topical Meeting on Advances in Human Faccors Research on Man/Compuler Internctions: Nuclear and Beyond, Nachville, TN, June 10-14, 1990,

Military preparedness is becoming more dependent upon human-computer interaction. Interactive computer systems are playing major roles in training the military. Simulation of the total system, as physically realistic as budgets allow, has been the military's approach to training. Several factors now lead to consideration of pr.rt-task trainers for single, complicated tasks. One factor is the difficulty of maintaining simultaneous delivery of the weapons system and the complete simulator. Another is the need for on-the-job refresher training in distant fields of operation. Because part-task trainers are in a sense modules, they increase the maintainability and adaptability of the total system and the acceptability of partial simulation when operational systems are very complex. However, part-task trainers do have drawbacks. Separating skilks in pist-task trainers may give the impression of simple tasks and may not portray the complexity of military operations. User arceptability and training effectivenes are subject to question with partial simulation. "How real is real enough?" and "How do we identify the escential elements of a task?" have no easy answers.

The introduction of new weapons on an existing aircraft offers a special case where part-task trainers may be appropriate. The configuration of shore-based aircraft simulators may not include the new weapon in time for initial training, or the weapon might be deployed to the flect before the aircrews are scheduled for shore-based simulator training. Because the trainees are already expert pilots, they have no delusions about the complexity of their environment and should be able to accept a trainer that covers only tasks new to total system operation. The Naval Air Systems Command has therefore developed a part-task trainer for a new airborne weapon, the Stand-off Land Attack Missile. The trainer has been deployed to the fleet and is slated for shore-based training. This provides an opportunity to use instructional design and human factors methodology to fieldtest the efiectiveness and acceptability of part-task trainers. The Department of Energy's Oak Ridge training technology team will gather data that evaluates the trainer's instructional design and acceptability features. This evaluation will provide criteria for future decisions regarding coinputeraided training options for complex tasks.

Research sponeored by U.S. Department of Enersy.

"Date Syatem Research and Developanent Diviaion.

\subsection{8}

\section{DYNAMIC ALLOCATION OF RESPONSIBILITY BETWEEN OPERATORS WITH DIFEERENT MODELS OF SYSTEM INEGRMATION USING COMPUTER-MEDIATED COMMUNICATION}

\section{Terranova}

[Abatract of Ph.D. Theais, Old Dominion Univeraity, Norfolk, VI, Diesertacions Abetracts International (in prese)]

This research focused on the performance of human operators in systems with high levels of automation. In these systems the use of more than one operator has the potential to optimize the contribution of human performance. In this study, specific consideration was given to different models of system representations and operator communication requiremsents. The results demonstrated that the communication of the operators is vital to this team effort.

An experimental design was utilized which allowed dynamic coritol between two human operators in a process control simulation. Control and failure detection responsibilities were shared between the operatorn using computer-mediated communication. Each of the operators, in teams composed of a primary and support operator, was 
provided with either the same or different representations of the process. The results demonstrated a positive relationship between the ability of the teams to stabilize the process and the amount of communication they exchanged. Communication dropped significantly during seasions that included system failures. When failures were present the performance of the teams in optimizing the cystem and minimining system takeovers was significantly degraded. The drop in communication was indicative of the increased cognitive worklosd of diagnosing failures. Atthough there were no differences in the ability of the teams to control the process or detect failures based on the syscem representations that the operators used, the way in which they utilized information did vary with this factor. This information utilization was significantly better for the teams composed of a primary operator utilizing an alphanumeric representation and a support operator utilizing a graphic representation. Comparisons were made with similar research which used individuals ratber than teams (Coury \& Pietras, 1986). These comparisons revealed that the teams in the present study were not as effective in optimizing the process or detecting failures. These differences may be attributed either to population differences, workload requirements of team communication and coosdination, of actual differences in the performance of teams versus individuals.

Reseurch sponeored by U.S. Depertmen of Energy.
3.119

\section{COGNITIVE TASK ANALYSIS: TECHNIQUES APPLIED TO AIRBORNE WEAPONS TRAINING}
M. Terranova
C. E. Saydert
T. L. Senmater
L. E. Treitlext

(Abatract of paper wo be presemed at the 33ird Amonal Homan Fectoos Society Meeting, Denver, CO, October 16-2, 1900)

This is an infroduction to cognitive tadk andyais as it may be used in Naval Air Syatem Command (NAVAIR) training development. The focus of a cognitive tant analyais is human knowledge, and its methods of analyais are those developed by cognitive poychologiets. This paper explains the role that cognitive tack analysis and presents the finding from a preliminary cognitive tats analysis of airborne weapons operaton. Cognitive task analyais is a collection of powerful techniques that are quantitative, computational, and rigorous. The techniques are currently now in vide we in the training community, so examples of this methodology are presented along with the results.

Reseandh eponeored by U.S. Nary and U.S. Departmeat of Erenty.

"Cieron Amociates, lec., Fiarvax, VA.

'Dace Syatem Reveach and Development Diviaion

Univerily of Tenomee, Knoxville, IN.

\section{COGNITIVE MODELING/OPERATOR ROLES}

\subsection{0}

\section{FEASIBLITY OF QUANTITATIVE PERTORMANCE MEASURES FOR EVALUATING NUCLEAR POWER PLANT OPERATORS}

\author{
R. J. Carter E. M. Connelly \\ P. A. Krois'
}

(Abatract of papar preacated at the $8 \mathrm{th}$ Sympocium on the Trainine of Nucler Fecility Persannel, Gatinburs. TN, April 24-27, 1909, Proc. V.A.S.1-V.A.S.19 (April 1969)]

A more valid measure of team performance in nuelear power plants is needed. A study is deacribed which was oriented towards evaluating the feasibility of syntheaizing performance mear sures by deriving measures for crews responding to an off-normal event in a full-scope simulator.
The thesis was that performance accesment is baced on the subjective judgment of training instructors. The procedure used to synthesize the performance measure consiated of: identification of the factors believed to be important to performance ancument, development of example crew performances and ratinge on eacb be instructors, and derivation of the meacuse by capturing the instructors' ancuement rules. A performance measure was derived which explains nearly all of the variance of the instructors' team performance at sessments. There is reason to believe that this method of aynthesizing measures can be spplied to other events.

Research eponsored by U.S. Depertment of Ener of and U.S. Nuclear Regulatory Commicaion.

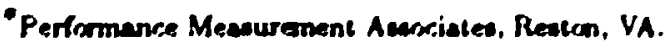

†Computer Technolog Amariates, Cioloreds, Spring, CO.
} 


\subsection{1}

\section{OPERATOR ROLE DEFINITION AND HUMAN SYSTEM INTEGRATION}

\author{
H. E. Knee \\ J. C. Schryver
}

[Abatract of paper preanted at the The Power Plane Dynamice, Control and Teating Sympocinum, Knowvilte, TN, May 15-17, 19a9; Proc. p. 5.01 (May 1909)]

This paper discusses operator role definition and human-ystem integration from a perspective of systems engineering and allocation of functions. Current and traditional allocation of tasts/functions can no longer be applied to syotems that are significantly more sophisticated and dynamic than current system desigms. For such advanced and automated designs, explicit attention must be given to the role of the operator in ofder to facilitate eificient system performance. Turthermore, such systems will include intelligent attomated systems which will aupport the cognitive activities of the operator. If such systems share responsibility and control with the human operator, these computer-based asciatants/acociates should be viewed as inteligent team members. As such, factors such as trust, intentions, and expectancies among team members must be considered by the systems designer. Such design considerations are discused in this paper.

This paper also discusece the area of dynamic allocation of functions and the need for models of the human operator in support of machine forecast of human performance. The Integrated Reactor Operator/System (INTEROPS) model is dicusced as an example of a cognitive model capable of functioning beyond a rule-baced behavioral structure.

Recearch eponcored by U.S. DOE Office of Technolong Support Programe.
3.122

\section{AN OPERATOR MODEL-BASED FILTERING SCHEME}

\author{
R. S. Sawhey* \\ J. C. Sehryve
}

(Abatract of paper to be presented at the American Noclear Societs Topical Meeting on Adrances in Hewnen Feccors Reverrch on Man/Computer Interentions: Nedear and Beyoud, Naluville, TN, June 19-14, 1990)

This paper presents a LISP-based diagnostic model for off-normal events developed at Oak Ridge National Laboratory (ORNL) under the Advanced Controls Program (ACTO). Although the diagnostic model was intended to serve as an embedded module of a cognitive model of the hut man operator, one application of this model could be to asist con' rol room operators in correctly responding to off-normal events by providing a rapid and accurate assesament of alarm patterns and parameter treads. The sequential filter model is comprised of two diatinct subcomponents - an alarm analysis followed by an analysis of plant signals. During the alarm andyris phace, patterns of annunciated alarms are evaluated to generate bypotheses of possible initiating events in order of likelihood of occurrence. The parameter analysis further evaluates each member of the hypothesis with respect to the current trends of state variables to validate/reject (in the form of increased/decreased certainty factor) the given hypothesis. Updated analysis also incorporates old hypotheses into the first stage filter.

The model is unique in the sense that it incorporates information not utilized by oiher models in diagnosing initiating events for off-normal scenarios. In particular, there are three types of information which hold the opportunity to improve the prioritization of hypotheses. Firat, the model has the ability to perform alarm-to-alarm mapping to modify alarm confidence factors based on expectations derived from the annunciated alarm patterns. The basis for this mapping is a correlation matrix that apecifies dominant correlations between an annuneiated alarm and other relevant alarms. Second, the model performs an unannunciated alarm analysis to verify and modify the results of the annunciated alarm analysis. The as sumption is that there is valuable information content contained in unannunciated alarms that may be useful in generating more accurate hypotheses. Lant, a parameter analyois procedure is developed that incorporates the distance between expected 
and obwerved parameter trends to modify the confidence factors. The basis for the distance metric is a eet of trend tree structures developed for each parameter/initiating event pair.

This model is an improvement over the knowledge-based component of the Integrated Resetor Operator/Syatem (INTEROPS) model aloo developed at ORNL. The new diagnoutic model is a more fexible model which integrates the alarm analynis and the parameter analynie into a single diagmoetic model.

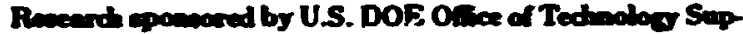
pat Progren.

"Uhivaries of Temmer, Keorvitie, IN.

\subsection{3}

\section{A BYPOTHESTS GENERATION MODBT OF INITIATING EVENTS FOR NUCLEAR POWER PLANT OPERATORS}
R. S. Sawher"
J. C. Sehryver
A. E. Knee
R. L. Dodds"

[Abetrect of Theses. Am. Neel. Soc. CO, 179 (1900)]

The goal of exiating alarm filtering model is 10 provide the operator with the moat accurate at cesment of patterns of annunciated alarm. Some models are based on event-tree analyia such as DuPont's Diagnanis of Multiple Alarm (DMA). Other models focus on improving hypothesis generation by deemphaxizing alarm not relevent to the current plant scenario. Many wuch models utilize the Alarm Filtering Syotem (AFS) as a bavis of dynamic prioritization. The Lipp-baced alarm analyris model presented here was developed for the Advaneed Controls Program (ACTO) at Oak Ridge National Laboratory (ORNL) to dynamically prioritize hypotheses vis an AFS by incorporating an unannunciated alarm andyeis with other plant-baced concepts.

The objective of this effort is to develop an alarm analysis model that would allow greater flexibility and more accurate hypotheris generetion than the prototype fault diagnonis model utilized in the Integrated Reactor Operator/Syatem Model (INTEROPS). INTEROPS is a time-baced predictive model of the nuclear powrer plant operator which utilizes alarm information in a manner similar to the human operatos. This is achieved by recoding the knowledge base from the PC- based expert system shell to a common Lisp structure providing the ability to easily modify both the manner in wiich the tnowledge is atructured * well as the logic by which the program performs fault diagnosis. The Liop-based larm anat yois logic incorporates (a) alanm-to-darm domain mapping and (b) utilizes information from alarms that have not annunciated.

Resench pposcored by U.S. DOE Otioe of Tedunologs Sup part Proguen.

*Univerity of Tencemee, Kroxville, TN.

3.124

OPBRATOR MODELBASBD DESIGN AND EVALUATION OP ADVANCBD SYSTEMS: COMPUTATIONAL MODELS

\section{J. C. Sehryver}

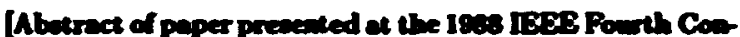

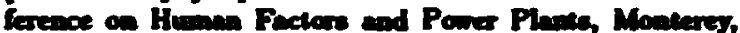
CA, Jue 5-9, 190, Proc. pp. 121-127 (19:3),

A multi-level operator modeling effort is recommended to provide broad support for integrated deaign of advanced control and protection systems for new nuclear power plants (NPP). A con uputotional model is described which accounts for the cognitive activities of the operator by 2 -abining the adventages of network simulation and knowledge-baced aimulation. The Integrated Reactor Operator/System (INTEROPS) model is a timebased simulation model which can be coupled with, and control, a thermal-hydraulice model of a nuclear power plant. The INTEROPS model can provide fine-grained evaluation of allocation of functions during the iterative deaign phase of advanced systems.

Research eponeored by U.S. DOE Oflice of Technolog Support Propram. 


\subsection{5}

OPERATOR MODEL-BASED DESIGN AND EVALUATION OF ADVANCED SYSTEMS: CONCEPTUAL MODELS

\section{J. C. Sehryver}

[Aberact of paper prewented at the 1908 IFES Fourth Coor

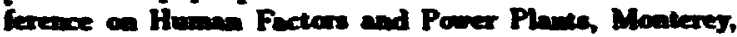
CA. Jome 5-9, 19at Proc. pp. $115-120$ (1908)'

A multi-level operator modeling approach is recommended to provide broad support for the integrated design of advanced control and protection syatems for new neclear power plants. Preliminary design should address the symbiosis of antomated systems and human operator by giving careful attention to the roles assigned to these two system elements. A conceptual model of the operator role is developed in the context of a command-controlcommunication problem. According to this approach, joint responsibility can be realized in at least two ways: sharing or allocation. The inherent stabilities of different regions of the operator role space are considered. It is conjectured that the "supervisor" role may prove to be leos efiertive than an "equal partner" role, because the latter maximizes the degree of engagement for the operator.

Reweand spononed by U.S. DOE Orice of Tectuolog Seppart Prograne.

\section{USAGE OF ADVANCED INSTRUMENTATION A,JD CONTROLS - THE NUCLEAR INDUSTRY}

\subsection{6}

\section{DIGITAL INSTRUMENTATION AND INTERACTIVE COMPUTER SYSTEMS: A SURVEY OF THE USE OF ADVANCED TECHNOLOGY IN THE NUCLEAR INDUSTRY}

\section{R. J. Carter}

(Abatrect of paper presented of the American Inotitute of Chemical Engineers Summer National Meeting, Philaddphin, PA, Anguat 20-23, 1800)

Similar to the chemical industry, the nuclear utilitie have utilined analog instrumentation and controls (I\&C) in their power plants since the first facility went on-line in the late 1950 's. Unlike moot of the chemical industry, however, the nuclear utilities have begun to make the transition to digital I\&C. This is due in part to: 1) analog components and syatems are becoming obsolete and unavailable, and 2) it seems easier to handle the multiple (and cometimes conflieting) goals of efficiency, reliability, economic operation, and safety during the operation of a nuclear power plant with digital technology.

$A$ survey of the use of advanced I\&C in the nuclear industry was conducted by Oak Ridge National Laboratory for the U.S. Nuclear Regular tory Commiseion (NRC). The purpose of the survey wa to identify the current, planned, and potential future uses of digital and interactive computer systems in power plant control rooma and technical support centers. The survey was conducted at the U.S. and Canadian nuclear vendore and six utilities who have begun to use advanced technology/systems. The survey investigated such topics as: the utilization of computens, digital I\&C, artificial intelligence, and expert systems; computer-generated displays; cathode-ray tube and gas plasma displays; coftware validation and verification; usefulnes and operability; and human factors. The conference paper and the presentation describe the aurvey and results therefrom. The viewpoints and opinions expreseed are those of the author and do not necemarily reflect thove of the U.S. NRC.

Research sponsored by NRC Office of Nuclear Regulatory Research and U.S. Department of Energy.

\subsection{7}

\section{HUMAN FACTORS ASPECTS OF ADVANCED INSTRUMENTATION IN THE NUCLEAR INDUSTRY}

\section{R. J. Carter}

(Abetract of paper preanted of the 7th Power Plant Dynamics, Cons rol and Teatine Symposium, Knoxvills, TN, May 15-17, 1989; Proc. pp. 14.01-14.12 (May 1909)]

The use of advanced/digital inatrumentation systems in the nuclear power industry is the wave of the future. These systems will probably be utilized in the life extension of plants. It has been 
demonstrated in other industries that digital instrumentation provides almost error-free performance that is three to four orders of magnitude better than analog components performing the same function. With the increase in sophistication in the operation of modern nuclear power plants that is needed to handle the multiple (and sometimes conficting) goals of efifiency, reliabit ity, economic operation, and safety, the nuclear industry will be driven to the use of advanced instrumentation.

The point has been reached where the isures in using advanced inotrumentation in nuclear power plants are not hardware and coftwase re liablity or performance, but rather the interface between the instrumentation system and the human. Unfortunately, until recently the human factors iswes had not been identified.

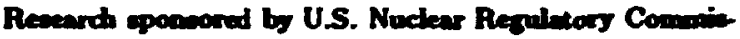
ion.

3.128

\section{HUMAN FACTORS SURVEY OF ADVANCED INSTRUMENTATION AND CONTROLS}

\section{R. J. Carter}

(Abatract of peper to be presented at the 17th Water Roactor Safety Information Meeting, Rockvile, MD, October 23-25, 1989)

The nuelear power industry has used analoy instrumentation and controls (I\&C) in their control rooms and technical support centers since the first nuclear power plant went on-line in the late 1950's. Even today the industry, as a whole, has been slow to implement advanced/digital I\&C. The utilization of digital I\&C appears, however, to be the wave of the future because most of the ana log components and systems are becoming obsolete and no longer available. These advanced syztems will also probably be utilized in the life extension of nuclear plants. It has been demonstrated in other induntrie that digital I\&C provides almost erros-free performance that is three-to-four orders of magnitude better than analog components performing the same function. With the increace in cophistication in the operation of modern nuclear power plants that is needed to handle the multiple (and sometimes conflicting) goals of efficiency, reliability, economic operation, and safety, the nuclear industry will be driven to the use of advanced I\&C.

Research sponeored by U.S. Nuclear Regulatory Commininon and 11.S. DOE.

\subsection{9}

HUMAN FACTORS ISSUES RELATED TO EXPEFT SYSTEMS FOR ELECTRIC POWBR PLANT CONTROL ROOMS

\section{R. J. Carter R. E. Uhrig*}

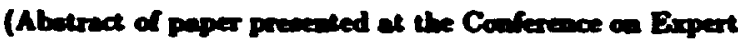

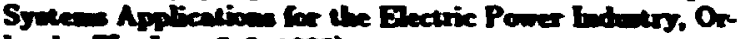
lada, FL, Jise 5-a, 1000)

The primary purpose of expert aystem is to represent the knowledge of experts and make the expertive available to the human 20 that it can contribute to improved performance. In order to achieve this objective, human factors principles must be incorporated into the desigm. Two surveys oriented towards identifying the human factors insues related to expert aystems were conducted. This paper describes the recults from those surveye. It diacuenes the buman factors inues under four main categories, the knowledge bace of the expert aystem, the human-expert ayotem interface, organizational support, and related topics (e.g., training, workload, and performance under stress).

Reweanch rponeored by U.S. Depurtment of Enersy and Nodear Regulatars Commiesion.

*Iastrumentation and Coatrols Divinion. 


\section{HAZARDOUS WASTE MANAGEMENT}

\subsection{0}

\section{DESIGNING A HAZMAT INCIDENT MANAGEMENT SYSTEM FOR FACILITISS WITH WIDELY VARYING BMERGENCY ORGANIZATION STRUCTURBS}
R. J. Carter
C. L. Basterly*

[Abatrect of paper premented at the American Inetitute of Chericel Engineas' sth Biennial Hatardoes Materid Spill Conference, Chicago, IL, May 16-18, 1808, Proc. pp. seo-5et (Miny 19:8)]

Oak Ridge National Labotatory is currently conducting a research program for the United States Air Foree, the purpose of which is to assiat them in their emergency planning for HAZMAT apills. This paper describes the fint ino tasks in the program. These tanks are oriented towards: determining the extent of the hazardous materials (HAZMAT) problem and establishing plans directed toward HAZMAT incident management.

Research sponsored by U.S. Air Force.

-Healch and Salesy Reacerch Division.

\subsection{1}

A GENBRC HAZARDOUS WASTE MANAGEMENT TRAINING PROGRAM

\section{R. J. Carter B. Karnofaky*}

(Abatract of paper presented at the American Nuclear Society Topical Meeting on Nuclear and Hazardon Waste Manazement, Pwco, WA, September 1I-15, 1903)

The Resource Conservation and Recovery Act (RCRA) is the primary federal law governing the generation, transportation, and dispoeal of hazardous waste. All nuclear power facilities have proceses which produce waste chemicals that are now regulated under this law and its amendments. Some of the more demanding aspects of the RCRA regulation are the training requirements outlined in title $\mathbf{4 0}$ of the Code of Federal Regulations, Section 264.16. This section requires semi-annual training of personnel involved in the use, storage, transport, and disposal of hazardous waste. Because the definition of harardous waste includes relatively inert materials, this training requirement impacts many of the personnel at a nuclear facility.

Oak Ridge National Laboratory is developing a generic, i.e., applicable to all major commands and installations, three-hour RCRA training program for the United States Air Force which is directed towards people reoponaible for day-to-day use, storage, traneport, and disposal of hazardous materials and wastes, and first-line supervisors of these operations. The program bighlights major compliance requirements and chronologically follows a hazardous material from its arrival onbase to its departure through a disposal contract. It focuses on both individual responsibilities and the importance of teammork. Most of the training program also appears to be applicable to the nuclear power industry.

Research sponeored by U.S. Air Force.

- Envioamental Resources Center, Fasetteville, NC. 


\section{RELIABILITY}

3.132

\section{ASSESSMENT OF CRITICAL COMPONBNT UNAVAUABIITY IN LQUID METAL REACTORS}

\author{
K. H. Koger J. Haire* \\ B. L. Humphry J. R. Mrinneschmidt \\ K. Setoguchit R. Natit
}

[Abetract of Necl Teel es, 251 (1900), alo These Am. Wuel Soe. S6, $300(1803)]$

Avalability information contained within the Centralized Reliability Date Organization (CREDO) liquid-metal reactor (LMR) data base is presented, and the availability critical items lists are developed. Individual components are ranked in prioritized lists from wort to beat performers from an availability standpoint. A vailability, as wed bere, is asumed to be an inherent characteristic of the component and is not necessarily ascumed to be related to plant operability.

A major observation is that of $\sim 5000 \mathrm{com}-$ ponents analyzed at each reactor site, a few components have a much higher unavailability factor than the average. In particular, 15 components contribute 93,77 , and $87 \%$ of the total aystem unavailability for the Experimental Breeder Reactor II, the Fast Flux Test Facility, and the Japanese Experimental Fast Reactor (JOYO), respectively. Critical components common to all three sites are mechaoical pumpa and electromagnetic pumps. By identifying components in this way, sile personnel will be more efficient in their attempts at increasing overall system availability; i.e., attention can be focused on components that have a high contribution to overall system unavailability.

All three sites demonatrate that low maintainability (i.e., long repair times) is about twice a likely to contribute to unavailability of component than unreliability (i.e., high failure rates).

The analyces were conducted using data and information from CREDO, the largeat repori:orv of LMR component reliability data in the world. The system is cosponeored by the U.S. Department of Energy and the Power Reacior and $\mathrm{Nu}$ clear Fuel Development Corporation of Japan. The CREDO data base contains information on a population of $>20,000$ components and atdresses $\sim 1500$ events (i.e., abnormal component occurrences). A conservative estimation is that cotal component operating hours approaches 2.2 billion hours.

Resenrth sponsored by U.S. DOE Office of Technoloy Support Program and the Power Renctor and Nuctenr Fuel Developonene Corporacion of Japen.

"Fuel Recycle Division

tCompoting and Telecommonications Divivion.

tPomer Reactor and Nudear Puel Developanem Corpore. tion, Toljo, Jepan.

\subsection{3}

\section{DEVELOPMENT OF IRREDDOM/ CREDO DATA BASE FOR LMFBR PSA}
R. Nakai"
K. Setoguchi*
H. Yasuda*
H. 2. Knee

(Abatrad of paper presented at the UAEA Technical Committee Meeting on Evaluation of Reliability Dats Sources, Vienne, Austrik, February 1-5, 1988)

A probabilistic safety aseessment (PSA) is being performed for the liquid-metal cooled fast breeder reactor (LMFBR), MONJU, which is currently in the construction phase. FBR Reliability Evaluation Database ior Operation and Maintenance (FREEDOM) has been developed at the Power Reactor and Nuclear Fuel Development Corporation (PNC) for the collection, storage, maintenance, and evaluation of the data to support the MONJU PSA. The FREEDOM system includes the data contents and structure of the Centralized Reliability Data Organization (CREDO). The CREDO system was initially developed in the United State at Oak Ridge National Laboratory (ORNL), and is jointly sponsored by the U.S. Department of Energy (DOE) and PNC of Japan. Data have been collected from the experimental fast reactor, JOYD, the Fast Flux Test Facility (FFTF), and the Experimental Breeder ReactorII (EBR-II). In addition, data have also been collected at several important lest facilitie in the U.S. and Japan.

The utilization of the CREDO database has recently been initiated for the MONJU PSA. This paper describes the structure of the database and the approacts of CRE.DO application to the 
LMIFBR PSA. For better use of reliability data, the effects of component boundary, component failure modes and failure severity were investigated A failure trend analysis was performed to examine the applicability of these data to the MONJU PSA. The accumulation of operational experience in LMFBRs and a detailed investigation of the existing reliability, availability, and maintainability (RAM) data would contribute to the reduction of the uncertainty of PSA.

Research sponsored by U.S. DOE Office of Technology Support Programs and the Power Resctor and Nuclear Fuel Development Corp., Tokjo, Japan.

"Power Renctor and Nuclear Fuel Development Corp. Tokjo, Japar

\subsection{4}

\section{INTERACTION OF CREDO WTTH THE EBR-II PRA DEVELOPMENT}

\author{
M. S. Smith W. A. Ragland*
}

[Abetract of Traw. Am. Neel. Soc. 60, 411 (1909)]

The National Academy of Sciences review of DOE Class 1 reactors recommended that the Experimental Breeder Reactor II (EBR-II), operated by Argonne National Laboratory, develop a level 1 PRA and make provisions for level 2 and level 3 PRAs based on the results of the level 1 PRA. The PRA Analysis Group at ANL will utilize the Centralized REliablity Data Organization (CREDO) at the Oak Ridge National Laboratory to support the PRA data needs. CREDO contains many years of empirical liquid metal reactor component data from EBR-II. As of June 1989, there were over 22,000 components (engineering records) and 1918 event records within the CREDO database, of which 2182 engineering and 417 event records wre contributed by EBR-II.

Reseanch sponsored by U.S. DOE Office of Technoloy Support Programs and Office of Basic Energy Sciences.

*Argonne National Laboracory, Argonne, IL. 
Section 4

NUCLEAR ANALYSIS AND SHIELDING 


\title{
4.0. INTRODUCTION
}

\author{
D. T. Ingersoll
}

The Nuclear Analysis and Shielding Section is comprised of four groups whose major research activities include: (1) methods development, analysis, and experiments for fission reactor radiation transport and shielding, (2) methods development and analysis for fission reactor core physics, (3) development of computer methods and models for high-energy radiation and particle transport and detection, and (4) mathematical modeling and computer analysis of ground- and spaced-based defensive systems. (Note: The Department of Energy has categorized some of the work for the reactor programs as "Applied Technology" so explicit references to this work cannot be included in this report.)

The long-established expertise established in the reactor shielding group continued to draw support from a diversity of projects including: (1) characterization of the neutron fluence at the pressure vessel of the 30-year-old Halden reactor in Norway, (2) prediction of neutron and gamma-ray transport in the beam tubes of the proposed Advanced Neutron Source (ANS), design analysis for the high-temperature gas-cooled rexctor program, and (4) prediction of gamma-ray dose rates in a proposed demonstration fuel reprocessing plant. Several neutronics analyses were also performed to surport the restart of the ORNL High Flux Isotope Reactor. The joint U.S./Japan program of shielding experiments supporting the liquid-metal-cooled reactor program was mostly idled due to the extended shutdown of the Tower Shielding Facility.

Activities in the newly reestablished reactor physics group focused on core performance and design analyses for the ANS, including control concepts, fuel grading options, and suclear heating. A new effort was initiated which will include methods development, benchmark analysis, and design methodology verification for the gas-cooled reactor concept for the New Production Reactor program. Also, a novel application of artificial intelligence methodology was used to investigate geometry optimization of the cold source for the ANS.

Projects in the high-eneigy physics area included further developments and improvements to physics models and nuclear data base for the HETC high-energy radiation and particle transport code. Particular emphasis was given to coordinating specific research, design, fabrication, and testing capabilities at ORNL which could contribute toward the large detector projects associated with the Superconducting Super Collider (SSC) program. A successfi! proposal was developed to establish at ORNL a specialized center for SSC detector development which will involve the active participation of laboratories and universities in the southeast region of the U.S. The center is expected to provide direct support of at least one SSC detector project, especially for the hadron calorimeter stage of the detector, and basic research support of general high-energy detection systems.

The ORNL Fast Track Program, which was initiated earlier to support the Strategic Defense Initiative (SDI), successfully predicted and demonstrated the performance of ultra-lightweight shie!ds for spaced-based platforms subjected to hypervelocity projectiles. Related programs under the SDI umbrella included nptimization studies for radiation threat shields and the effects of radiation on specific space platform components. Work also continued on the further development of the TORT three-dimensional discrete ordinates code and its application to the dose assessment program for personnel at Hiroshima and Nagasaki. Related work was performed to improve adjoint Monte Carlo methods used to predict the radiation hardness of armored vehicles. New analytic models using coupled nonlinear partial differential equations were developed to descritse the behavior of 
combative forces. Numerical solutions to the system of equations were used to test and improve the models, which include the effects of spatial troop movement, attrition and reenforcement, battlefield length, etc. 


\section{RADIATION TRANSPORT AND PHYSICS FOR FISSION REACTORS}

4.1

\section{ARTIFICIAL INTELLIGENCE SEARCH TECHNIQUES FOR OPTIMIZATION OF THE COLD SOURCE GEOMETRY}

\author{
Y. Y. Azmy
}

[Abetrect of Trene Art. Nacl. Soc. 57, 97 (1968)]

We draw an analogy between the problem of optimizing the gemetric shape of the cold source and a state space search, then we use a simple artificial intelligence (AI) search technique to determine the optimum cold source shape based on a two-group, r-z diffusion model. We implemented this AI design concept in the computer program AID, which consists of two modules, a physical model module and a search module, which can be independently modified, improved, or made more sophisticated. The physical model equations are solved using a special-purpose two-group, $r-z$ nodal diffusion code. The search module in AID uses a nearest-neighbor strategy; at each !evel of the search, the search module generates all permis sible states from the current state using the production rules, evaluates each one, then compares the resulting cold leakage. If none is larger than the cold leakage of the current state, the search terminates surcessfully. Otherwise, the state with the largest cold leakage is taken to be the current state and the search proceeds to the next level.

In order to demonstrate the capabilities of this new approach, we obtain optimum cold source shapes on two meshes, with a variety of initial states. The utility of our new approach is undercored by the large improvement in performance of the cold source determined by the nearest-neighbor algorithm whose uncommon shape is practically impossible to foresee as a candidate design based on pure physical and engineering intuition.

Research sponsored by U.S. DOE Office of Basic Eners Sciences.
4.2

\section{A STATE SPACE SEARCH TECHNIQUE FOR CPTIMIING THE SHAPE OF A COLD NEUTRON SOURCE}

\author{
Y. Y. Axmy
}

[Abrtract of NecL. Sai. Eng. (in prem)]

We develop a novel approach for optimining the geometrical shape of an object designed to extremize a set of performance criteria, and we apply it to the problem of optimizing the shape of a cold neutron source. First, we draw an anat ogy between the shape optimization problem and a state space saarch, which is the fundamental problem in Artificial Intelligence applications. Then, we describe our implementation of this new approach into a computer code, DAIT, in which the physical model is represented by a two-group, $r-2$ geometry nodal diffusion method, and the search is conducted via a truncated breadth-first algorithm. This algorithm reduces to the traditional Nearest Neighbor algorithm if we truncate the search breadth at one. We establish the accuracy of the nodal diffusion method solution on the meshes of interest in this work, then we establish the adequacy of the diffusion approximation iteelf via comparieons with transport theory solutions. Then, re investigate the dependence of the optimum shape and its value on several physical and rexch parameters via several numerical experiments. Finally, we show that starting from different initial statso, the came final optimum state can be obtained if we increase the search breadth sufficiently.

Fresearch sponsored by Defense Advenced Revearch Projects Agency and by U.S. DOE OIfice of Basic Enerd Sciences. 


\section{3}

\section{GEOMETRICAL SHAPE OPTIMIZATION \\ OF A COLD NEUTRON SOURCE USING ARTIFICIAL INTELLIGENCE STRATEGIZS}

\section{Y. Y. Azmy}

[Abatract of paper presented at the ANS Topical Meeting on Advances in Nuclear Fngineering Computation and Rtdiation Shielding, Sante Fe, NM, April 9-13, 1989; Proc. Vol. 2, pp. 67:1-67:12 (1989)]

A new approach is developed for optimizing the geometrical shape of a cold neutron source to maximize its cold neutron outward leakage. An analogy is drawn between the shape optimization problem and a state space search, which is the fundamental problem in artificial intelligence applications. The new optimization concept is implemented in the computer code DAIT in which the physical model is represented by a two group, $r$ $z$ geometry nodal diffusion method, and the state space search is conducted via the nearest neighbor algorithm. The accuracy of the nodal diffusion method solution is established on meshes of interest and is shown to behave qualitatively the same as transport theory solutions. The dependence of the optimum shape and its value on several physical and search paramecers is examined via numerical experimentation.

Research sponsored by U.S. DOE Office of Basic Energy Sciences.

4.4

\section{A PARALLEL ALgorithM FOR SOLVING THE MULTIDIMENSIONAL NEUTRON TRANSPORT EQUATION}

\author{
Y. Y. Azmy
}

(Abetract of paper presented at the SIAM Conference on Applied Linear Algebra, Minneapolis, MN, July 11-15, 1988)

The standard inner iteration procedure, commonly used to solve the discrete or dinates form of the neutron transport equation, is ccmposed of incependent sweeps of the mesls along the various discrete directions. In particular, in the $S_{n}$ approximation, there are $N \equiv n(n+2) / 8$ discrete ordinates per quadrant in angle space, so that for each corner at which faces with vacuum boundary conditions (i.e., known incoming angular flux) intersect there are $N$ independent processes (sweep); that can execute simultaneously. Since the decomposition of the iteration process into parallel subprocesses occurs in a natural way, the solution obtained from the paralkel algorithm is exactly equivalent to its sequential counterpart. We used this algorithm to solve a simple test problem on the Intel iPSC hypercube using the zeroth and first order nodal transport methods and measured the speedup and efficiency as a function of the various parameters in the problem. Speedups larger than $\mathbf{3 0}$ (for $\mathbf{4 0}$ independent processes) and efficiencies in excess of $70 \%$ were consistently achieved throughout our investigation.

Research sponsored by U.S. Department of Energy and by Defense Advanced Reararch Projects Agency.

4.5

$$
\begin{aligned}
& \text { MULTIDIMENSIONAL NODAL } \\
& \text { TRANSPORT METHODS FOR } \\
& \text { MULTIPLE-INSTRUCTION } \\
& \text { MULTIPLEDATA, DISTRIBUTED } \\
& \text { MEMORY MACHINES }
\end{aligned}
$$

$$
\text { Y. Y. Azmy }
$$

[Abatrect of Trans. Am. Necl. Soc. 56, 292 (1988)]

It has become evident in recent years that any significant improvements in the performance of future computers must be rooted in advanced architectures. In this paper we present the implementation of the zeroth and first-order twodimensional nodal transport methods on the hypercube. Our objective is to study the effect of the various parameters in a simple test problem on speedup and efficiency in order to determine the cases most suitable for solving on a distributed memory (DM) machine, such as the hypercube.

In general, discrete ordinates methods have the potential for coarse-grain parallelization because of the complete independence within each inner iteration between the mesh sweeps in the various distinct discrete directions. The parallel solution strategy for each iteration consisted of simultaneous sweeps of the mesh, in the standard sense, performed by the nodes in a preassigned order (static scheduling).

The main feature of this strategy is the very coarse parallelization achieved. This minimizes the number of messages exchanged between 
the independent processes, thus improving the efficiency. However, it also limits the largest number of processes that can be executed simultaneously to a few hundred, far below the potential number of processors on DM machines. Another characteristic of this strategy is the very mell balanced computational load.

We solved several test problems with vacuum boundary conditions on all external edges in order to study the effect of several parameters on the speedup and the efficiency. For all cases considered, efficiency decreases monotonically, but is consistently above $70 \%$. Our results indicate that, for the same problem, the lowest efficiency of the first-order method was $\sim 10 \%$ higher than that of the zeroth method because the sweeps consume a larger fraction of the total CPU time in the former case. For similar reasons we found that; for a given number of processors, $P$, doubling the number of mesh cells in each direction slightly improved the efficiency. Also, we found that, for a given $P$, increasing the number of independent processes by increasing the order of angular quadrature results in a higher efficiency. For similar reasons, one would expect threedimensional methods for problems with purely vacuum boundary conditions to be more efficient than two-dimensional methods.

Research sponsored by U.S. DOE Office of Basic Energy Sciences.

4.6

\section{ACCURACY OF TRANSPORT CALCULATIONS FOR THE ADVANCED NEUTRON SOURCE}

\section{Y. Y. Azmy}

[Abetract of Trens. Am. Neel. Soc. 57, 293 (1988)]

Due to the very compact core geometry, high fuel enrichments, and low $\mathrm{D}_{2} \mathrm{O}$ moderator/coolant cuntent within the core of the ANS reactor, the thermal $f$ ix gradient in the core, as well as the epithermal and fast flux gradients in the large $\mathrm{D}_{2} \mathrm{O}$ pool reflector, are extremely large. This raises questions aboust the adequacy of diffusion calcula. tions for this problem, and also about tlie accuracy of the DORT transport calculations performed to verify the diffusion calculations; in this paper we consider the latter question.
We have roughly estimated the accuracy of the multiplication factor, the fission source, and pointwise scalar flux, obtained from the transport calculations of the ANS reactor, using as reference the corresponding quantities resulting from $h^{2}$ extrapolation of two fine-mesh calculations. Our results indicate that the group scalar flux is inarcurate at locations of very low flux levels. This inaccuracy has almost no effect on the multiplication factor, however, and a relatively small effect on the fission source. These, as well as other integral quantities, such as the heating rates, for example, can be expected to be accurate even on relatively coarse meshes due to the penultimate observation.

Research sponcored by U.S. DOE Office of Basic Energ Sciences.

\section{7}

\section{COMPARISON OF THE DIFFUSION AND TRANSPORT CALCULATIONS FOR THE ADVANCED NEUTRON SOURCE REACTOR}

\author{
Y. Y. Azmy F. C. Difilippo
}

[Abatract of paper prewented at the 1988 Intermational Ro actor Physica Conference, Jackeon Hole, WY, September 18-21, 1986; Proc. Vol. II, pp. 183-194 (18e8)]

We performed six-group two-dimensional, $r$ z criticality calculations for a previous candidate design for the Advanced Neutron Source (ANS) reactor using a diffusion theory code (BOLD.VENTURE) and a transport code (DORT). Several quantities of practical interest in the design of the ANS were evaluated from the converged solutions and compared to one another. The relative difference between the diffusion and tsansport estimates of these quantities was also compared to a recently obtained estimate of the accuracy of the transport calculation itself in order to evaluace the diffusion approximation. Our results confirmed that most diffusion calculated quantitie are sufficiently accurate with the error mostly arising from the finite size of the computational cells ratlier than from the underlying theory.

Remearch ofrounored by U.S. DOE Office of Basic Energy Sriances. 
4.8

\section{CONVERGENCE PROPERTISS OF ITERATIVE ALGORITHMS FOR SOLVING THE NODAL DIFPUSION EQUATIONS}

\author{
Y. Y. Axmy \\ B. L. Kirk
}

(Abatrect of paper to be presented at the Intermational Conference on the Phyice of Reactos: Operation, Deaign, and Compratation, Marweille, France, April 23-26, 1990)

A five-point seheme has been derived for the nodal diffusion method in two-dimensional Cartesian geometry. An unaccelerated, iterative procedure based on this scheme was derived and implemented on serial as well as parallel computers. In addition, a partial SOR methos was developed and shown to provide significant reduction in the number of iterations required to achieve a converged solution. In this work we present and validate expressions for the spectral radius and the number of iterations required for convergence, as a function of the mesh size for a model problem. Aleo we present preliminary results for a full SOR method that indicates an even better performance.

Recearch sponeored by U.S. DOE Ofice of Magnetic Pusion.

\section{9}

\section{THREE-DIMENSIONAL CALCULATIONS OF NEUTRON STREAMING IN THE BEAM TUBES OF THE ORNL HYIR REACTOR}

\section{R. L. Childs* W. A. Rhoades}

\section{L. $\mathbf{R}$. Williams}

\begin{abstract}
(Abutract of paper preaented at the 7th International Conference on Radiation Shielding, Bournemouth, UK, Sep rember 12-16, 1988; Proc. Vol. II, pp. 400-418 (1969)]
\end{abstract}

The streaming of neutrons through the beam tubes in the High Flux Isotope Reactor at Oak Ridge National Laboratory has resulted in a reduction of the fracture toughnes of th: reactor veasel. As a result, an evaluation of vescel integrity was undertaken in order to determine if the reactr, can he operated again. As a part of this evaluation, three-dimensional neutror transport calculations were performed to obtain fluxes at pointe of interest, in the wall of the vescel. By comparing the calculated and measured activation of doaimetry specimens from the vessel surveillance program, it was determined that she calculated flux shape was satisfactory to transpose the surveillance data to the locations in the vesel. A bias factor was applied to correct for the average C/E ratio of 0.69 .

Resench sponvored by U.S. Departinent of Energy.

- Compreting and Telecommanications Division

\subsection{0}

\section{DETPRMINATION OF KINETICS PARAMETERS USING STOCHASTIC METHODS IN A ${ }^{252}$ CE DRIVEN SYSTEM}

\section{F. C. Difilippo}

(Abotrect of peper in Neive and Nonbinear Phenomena in Neclear Syoteme, Series B: Phyica Vol. 192, Pp. 99-106, proceedine of the NATO Adranced Revearch Workisop. Univernidad Politecnica de Valencia, Spain, May 23-27, 18as, J. L. Mumoe Cobo and F. C. Dillippo, Ed., Plenom Preas, NY (1890)]

Safety analysis and control system design of nuclear systems require the knowledge of neutron kinetic related parameters like effective delayed neutron fraction, neutron lifetime, time between neutron generations and subcriticality margirs. Many methods, deterministic and stochastic, are being used, some since the beginning of nuclear power, to meastise these important parameters. The method based on the use of the ${ }^{252} \mathrm{Cr}$ neutron cource has been under intense study at the Oak Ridge National Laboratory, both experimentally and theoretically, during the last years. The increasing demand for this isotope in industrial and medical applications and new deaigns of advanced bigh flux reactors to produce it make the isotope available as neutron source (only few micrograms are necessary). A thin layer of ${ }^{252} \mathrm{Cr}$ is deposited in one of the electrodes of a fisaion chamber which produces pulses each time the ${ }^{252} \mathrm{Cf}$ disintegrates via $\alpha$ or spontaneous fission decay; the smaller pulses ascociated with $\alpha$ decay can be easily discriminated with the important result that we know the time when $\nu_{\text {c neutross are injected }}$ into the system (number of neutrons per fiscion of ${ }^{252} \mathrm{Cf}$ ). Thus, a small ( $\mathrm{few} \mathrm{cm}^{3}$ ) and nonintrusive device can be used as a random pulsed neutron source with known natural properties that do not depend on biaces aseociated with more complex interrogating devices like accalerators. This paper presents a general formalism that relates the kinetics parameters with stochastic descriptors linat naturally appear because of the random nature of ti.e production and transport of neutrons.

Research aponsored by I:S. DOE Office of Basir Energy Sciences. 
411

\section{THBORETICAL ANALYSIS OF REACTIVTYY MEASUREMENT WITH THE ${ }^{252} \mathrm{CP}$ SOURCE RATTO METHOD}

\section{F. C. Difilippo}

(Abutract of Imermational Meating on Safas Margin in Criticality Safay, Sm Prancinos, CA, Nowember 28-30, 1800; Proc. Pp. 16-23 (1800)']

Stochatic descriptors are calculated using the concepts of detector field of view and an equivalent source of noive with results that are compatible with a more bacic formaliom based on a probabilitic analycis of Markovian processes. The model expansion of the detectors' fields of view are further used to relate reactivities to observables measured with the ${ }^{252} \mathrm{Cf}$ ratio method. Results from the analysis of experiments are shown together with a numerical analysis of spatial effects.

Rewearch eponeored by U.S. DOE Ofitee of Benic Enery Sciences.

\subsection{2}

\section{HARMONIC ANALYSIS OF STOCHASTIC DESCRIPTORS AND THE INTERPRETATION OF ${ }^{262}$ CF NEUTRON SOURCE EXPERIMENTS}

\section{F. C. Difilippo}

[Abatract of Necl. Sei. Eng. (in prea)]

The harmonic expansion of the detector field of view in terms of direct and adjoint kinetic eigenfunctions together with the use of modified version of Schottky prescription of the equivalent source of noise make possible the harmonic analysis of stochastic descriptors used in the measurement of kinetic parameters. The results are general in the sense that they do not depend on the approximations of the Boltzmann operator, on the distribution of fissile materials, or on the multiplicity of the neutron cource that drives the subcritical sys tem. The results are applied to anslyze the particular case of a system driven by a ${ }^{252} \mathrm{C}$ f source, with the californium deposited in one of the electrodes of a fisajon chamber, and monitored by two sets of neutron detectors. This analysis is oriented towards the interpretation of experiments to mear sure reactivitie with the stocliastic method of the
${ }^{252} \mathrm{Cr}$ source (ratio method). The theory is then used to synthesize stochastic observables using a simple approximation of the Boltzmann operator, i.e., two-point kinetics, which show the general features of the spatial effects observed experimentally.

Research sponsored by U.S. DOE Office of Busic Enery Sciences.

\subsection{3}

\section{NEUTRON KINETICS OF HIGH- INTENSE FISSION NEUTRON SOURCES}

\section{F. C. Difilippo}

(Abatract of paper to be preasented at the International Conference on the Phycics of Renctoss: (Pperalion, Decipp and Computation, Marseille, France, Afril 23-26, 1990)

Fission neutron sources capsble of producing thermal neutron flux pealis of the order $10^{20} \mathrm{~m}^{-2} \mathrm{~s}^{-1}$ and releasing lou power $(\lesssim 300$ MW) require a small and undermoderated core (core volume $V_{c} \lesssim 50 \mathrm{~L}$ with heavy water coolant) and very low absorbing reflectors (e.g., $\mathrm{D}_{2} \mathrm{O}$ ). Consequently, the neutron kinsties of highly intense fission neutron sources lave the following characteristic problems: There is production of photodelayed neutrons with aceciated questions about the transport of $\boldsymbol{\gamma}$-rays in a very small core; prompt decay constants $\alpha_{m}$ can be of the order of precursor decay constants $\gamma, s 0$ the usual $1 / \nu$ poisoning calculation of $\alpha_{m}$, kinetic eigenfunction $\chi_{m}$ and kinetic mean life $\ell_{m}$ might not be correct; the kinetic distortion is very intense, and the strong space-dependent neutron spectra raise questions about the validity of point-kinetics mudels. Furthermore, a general formalism for the calculation of lumped parameters is desirable in order to have an appropriate reference for lese accurate models. Time-dependent equations for the vector flux $\psi(\vec{\mu}, t), \vec{\mu} \epsilon[\vec{r}, \vec{\nu}]$ that make allowance for transport effects of the $\gamma$ 's that induce photoneutron emission in $\mathrm{D}_{2} \mathrm{O}$ were derived.

Assuming there are I delayed neutron groups $i=1$ to $I$ and $J$ pliotodelayed neutrons groups ( $i$ $=I+1$ to $I+D$, the kernels, $K_{i}$ are introduced, $K_{i}\left(\vec{r}^{\prime}, \vec{r}\right)=\delta\left(\vec{r}^{\prime}-\vec{r}\right)$ for $i \leq 1$, whereas the $K_{i}$ for $i>$ I accounts for the transports of $\gamma$ 's.

In the context of the kinetics of highly intense neutron sources, the conclusions of this work are the following: 
1. Rigorous equations for consistent definitions of kinetic parameters were obtained; they include the transport of $\boldsymbol{\gamma}$-rays.

2. The prompt approximation for the calculation of time eigenvalue $\alpha$ is not correct in near-critical cases.

3. Because of the kinetic disturtion, there is a large difference between static and kinetic mean lives. The difference increases with subcriticality.

4. Point kinetics is a reasonable approximation ( 10\% error) if proper lumped parameters are used.

The experience provided by the previous results is being used to generate lumped kinetic parameters of the Advanced Neutron Source reactor for use in controlrelated calculations.

Reseanch sponsoned by U.S. DOE Office of Bacic Enersy Sciences.

\subsection{4}

\section{FLATTENING THE POWER DISTRIBUTION OF THE ADVANCED NEUTRON SOURCE REACTOR}

\section{F. C. Difilippo}

[Abetract of Trens. Am. Ned. Soc. 56, 563 (1988)]

The goal of the advanced neutron source (ANS) project is to design an ultrahigh thermal neutron flux $\left(\sim 10^{16} / \mathrm{cm}^{2} \cdot \mathrm{s}\right)$ scattering facility with a reactor as a source of neutrons. Efficient design requires the combination of a low absorbing reflector and a small (relative to the neutron slowing down length) core. The results of this combination are a larger than usual average powes density (comparcd to other high flux reactors) and a highly skewed distribution of the power density if the fuel is uniformly loaded. Both of these characteristics and thermohydraulic limits make necessary the use of some remedial technique to flatten the power distribution. We have developed instead an iterative numerical technique to calculate a fuel distribution that will reduce the power peaking factor at a prescribed time during the life of the core. We liave used this numerical technique to calculate a fuel distribution that would fiatten the power distribution at the middle of core life of a candidate design for the ANS. The maximum loral peak-tc-average power factor at that time is 1.14. At beginning of life it is 1.59 and at end of life it is 1.64. These values should be compared with a value of 3.67 at beginning of life for a uniform loading.

The powcr distribution as a function of space and time was used to compute thermal-hydraulic para zeters. The maximum fuel temperature is $\sim 360^{\circ} \mathrm{C}$, well below the maximum value of $\sim 385^{\circ} \mathrm{C}$ specified by the ANS project office.

In conclusion, the procedure developed in this paper to reduce the power peaking factor was successfully applied to the design of the fuel distribution within the ANS reactor core. It provides for automated rigorous, quantitative redistribution of fuel, which should provide conve-gence to an optimized fuel distribution more rapidly than a qualitative human decision procedure.

Research sponsored by U.S. DOE Office of Basic Energ Sciences.

\subsection{5}

REACTOR PHYSICS CALCULATIONS FOR THE CONTROL OF THE ADVANCED NEUTRON SOURCE REACTOR

\section{F. C. Difilippo M. Abu-Shehadeh*}

R. B. Perez

(Abetract of paper preanted at the 1868 International Reactor Phyaica Conferenoe, Jackeon Hole, WY, September 18-2I, 1988; Proc. Vol. II, 195-201 (1988)]

Efficient production of extremely high fluxes requires compact cores with consequent high po er densities and initial excess reactivities. Strong space dependent neutron spectras and limited access to the small core are other characteristics that make the design of the control system of these type of facilities an interesting problem. We present calculations of (a) the worths of ${ }^{10} \mathrm{~B}$ to reduce the initial excess reactivity, (b) the worth of Hf and B control rods, and (c) the neutron lifetimes, for the case of candidate designs for the Advanced Neutron Source reactor.

Research iponwored by U.S. DOE, Office of Basic Energy Sriences.

- Universily of Tennemes, Knoxville, TN. 
4.16

\section{TWO-POINT AND TWO-ENERGY GROUP KINETICS MODEL OF THE ANS REACTOR}

\author{
F. C. Difilippo M. Abu-Shehadeh*
}

R. B. Pere:

[Abatrect of Trase. Am. Necl Soc. 59, 347 (19e9)]

The production of extremely high thermal neutron Guxes $\left(\sim 10^{20} / \mathrm{m}^{2} \cdot s\right)$ with reasonable power levels $(\sim 300 \mathrm{MW})$ requires the combination of a very undermoderated core and a lowabsorbing moderator/reflector (like $\mathrm{D}_{2} \mathrm{O}$ ). Candidace designs for the reactor of the advanced neutron source (ANS) project bave in common the strong coupling between two neutron fields with very different spectra: predominance of epithermal neutrons in the core and thermal neutrons in the reflector. Because there are large variations of the neutron spectre within a few centimeters in a small core, concerns might arise regarding the validity of the conditions under which the point kinetic approach is valid, i.e., the factorization of the flux as the product of two functions: one depending on phase space $\mu(r, v)$ and the other depending only on the time $t$. In this paper, we present a two-point (core and reflector) and twoenergy group fast and thermal model used to calculate power transients in order to detect conditions under which one-point kinetics is not a valid approximation. The equations for the core were written under the hypothesis that the source of fast neutrons in the core is mainly the fission process and the neutron decay of fiesion products, i.e., we neglected the fast neutrons coming from the reflector and the photoneutron production inside the core. The balance equations for the reflector state that fast neutrons in the reflector are neutrons that come from the core and photodelayed reutrons produced in the $\mathrm{D}_{2} \mathrm{O}$ by the gamma coming from the core and that the thermal neutrons in the reflector come mainly from the moderation proceas in the reflectinr; i.e., we neglected the thermal neutrons coming from the core. In summary, the coupling in the fast and thermal groups is produced by neutrons that go mainly from the core to the reflector and from the reflector to the core, reapectively. The model was applied to compute transients corresponding to practical problems of start-up: The reactor is critical and in equilibrium for $t \leq 0$; for $t>0$ a positive reactivity is inserted to produce a fast and monotonir, rise in power without overshoot. This is accomplished by the following reactivity profile: a ramp to a value $A$ between 0 and $t_{1}$, constant $A$ between $t \mid 1$ and $t_{2}$, and an exponential decay to zero for $t>t_{2}$.

The results show a systematic dependence of the power profile on the way the change in reactivity is made, especially if the reactivity transient is severe, i.e., as $t_{2}$ of $A$ increases. This type of power transient is then sensitive to spatial effects not described by the point kinetics approximation.

Resenrch eponsored by U.S. DOE Ofice of Bacic Enery Sciences.

"Univesily of Tennesce, Ynoxville, TN.

\subsection{7}

\section{RADIATION TRANSPORT \\ CALCULATIONS FOR THE ANS BEAM TUBES}

\author{
W. W. Engle, Jr. R. A. Lillie \\ C. O. Slater
}

[Abstract of Trand. Am. Neel. Soc. 57, 294 (1888)]

The Advanced Neutron Source (ANS) facility will incorporate a large number of both radial and no-line-of-sight (NLS) beam tubes to provide very large thermal neutron fluxes to experimental facilities. Coupled two- and three-dimensional radiation transport calculations were performed to obtain thermal and fast (damage) neutron and gamma-ray scalar fluxes in these beam tubes to allow comparison of the ANS single- and split-core deaigns. For experimental locations far from the reactor cores, angular fux data are required; however, for cloce-in experimental locations, the sealar fluxes within each beam tube provide a credible timate of the various signal to noise ratios. The ratios of the thermal to fat scalar neutron fluxes (scalar neutron fluxes below and above $3.05 \mathrm{eV}$ ) were found to be greater for the NLS beam tubes for both core deaigns since these tubes do not "view" either core; and for both the radial and NLS tubes, the thermal to fast ratios were found ton be greater at all distances from the beam tube entrance and increase much more rapidly with increasing dietance for the split-core design. Very little difference wa obtained for the thermal to gamma-ray acalar flux ratios as a function of core deaign.

Hesearch sponeored by U.S. DOE Office of Basic Enercy Sriences. 
4.18

\section{ADVANCED NEUTRON SOURCE DESIGN: BURNOUT HEAT FLUX CORRELATION DEVELOPMENT}

\author{
W. R_ Gambill* \\ T. Mochizukit
}

[Abotract of Trano. Am. Nuel. Soc. 57, 293 (19e8)]

In the Advanced Neutron Source (ANS) reactor fuel element region, heat fluxes will be ef evated. Early designs corresponded to average and estimated hot spot fluxes of 11-22 and 21$22 \mathrm{MW} / \mathrm{m}^{2}$. In either event, the development of a satisfactory burnout heat flux correlation is an important element among the many thermal hydraulic design issues, since the critical power ratio will depend in part on its validity. Relatively little work in the area of subcooled flow burnout has been published over the past 12 years.

We have compared seven burnout correlations and modifications thereof with several sets of experimental data. The best overall agreement between the correlations tested and these data is currently provided by a modificaticn of Thorgenson's correlation.

Research sponeored by U.S. DOE Office of Basic Energ Sciences.

"Chemical Technology Divicion.

Kinki Univerity, Oakle, Japan.

\subsection{9}

\section{PRELIMINARY ANS REACTOR COLD SOURCE GAIN FACTOR CALCULATIONS FOR LIQUTD DEUTERIUM AND LIQUID NITROGEN-15}

\section{L. Henderwon}

(Abatract of ORNL/TM-10824, November 1988)

Individual energy group gain factors are computed for liquid nitrogen-15 and liquid deuterium cold source moderators using simple one-dimensional slab and spherical geometry calculational models. The energy spectrum of the neutron source is asumed to be that of a thermalized maxwellian flux at $20^{\circ} \mathrm{C}$. The slab genmetry calculations indicate that the optimum thickness for neutron transmiscion through a slab given an isotropic incident flux is for wavelengths above $.6 \mathrm{~nm}$, approximately $.20 \mathrm{~m}$ for liquid deuterium and between .28 and $.32 \mathrm{~m}$ for liquid nitrogen-15. The gain factors at $.8 \mathrm{~nm}$ corresponding to these thicknesses are 15.5 for liquid deuterium and 3.50 for liquid nitrogen-15. The spherical geometry analysis showed that the cold neutron current below 10 $\mathrm{meV}$ of $1.36 \mathrm{n} / \mathrm{m}^{2}-\mathrm{s}$ for the neutron component entering the cavity of a .16-m-thick liquid deuterium spherical shell exceeds the neutron leakage current of $1.08 \mathrm{n} / \mathrm{cm}^{2}-8$ from a .38-m-diameter liquid deuterium solid sphere. However, the cold neutron factors for the neutron entering the void region are considerably lower than for the solid sphere case.

Research sponeored by U.S. DOE Office of Baxic Energy Sciences.

\subsection{0}

\section{SOME PRELIMINARY DESIGN CONSIDERATIONS FOR THE ANS REACTOR COLD SOURCE}

\section{L. Henderson}

[Abetrect of Trans. Am. Nucl. Soc. 56, 561 (18e8)]

The performance of a poesible isotopic liquid nitrogen-15 $\left({ }^{15} \mathrm{~N}\right.$ ) (operating at $65 \mathrm{~K}$ ) versus a liquid deuterium (operating at $20 \mathrm{~K}$ ) cold neutron moderator has been investigated by computing the enhancement or gain in the cold neutron leakage fraction relative to the initial cold neutron source fraction for each moderator. One-dimensional slab and spherical geometry calculational models are used for the transport calculations to obtain the cold neutron group leakage fractions. An isotropic incoming boundary flux (left boundary for the alab) having a thermalized Maxwellian energy distribution at $20^{\circ} \mathrm{C}$ is used the cource condition. $A \quad 0.20$ m-thick liquid ${ }^{15} \mathrm{~N}$ slab moderator and a 0.15 -m-thick liquid deuterium slab moderator are considered. Liquid ${ }^{15} \mathrm{~N}$ and liquid deuterium spherical moderators with diameters of $0.34,0.42$, and $0.50 \mathrm{~m}$ are also investigated.

The transmitted gain factors at $0.8 \mathrm{~nm}$ for the liquid ${ }^{15} \mathrm{~N}$ and the liquid deuterium slab moderators are approximately 3.0 and 15.0 . The reflected gain factor values at $0.8 \mathrm{~nm}$ are approximately 4.0 for liquid ${ }^{15} \mathrm{~N}$ and 21.0 for 
liquid deuterium. Gain factor values for a 0.42 m-diameter sphere at $0.8 \mathrm{~nm}$ are approximately 6.0 for liquid ${ }^{15} \mathrm{~N}$ and 32.0 for liquid deuterium. Though the liquid ${ }^{15} \mathrm{~N}$ values are considerably below those computed for liquid deuterium, liquid ${ }^{15} \mathrm{~N}$ represents a viable option should safety related issues prohibit the use of liquid deuterium as a moderating material.

Rewearch epomored by U.S. DOE Office of Basic Energ Seiences.

\subsection{1}

\section{PRELIMINARY ONE-DIMENSIONAL NEUTRONICS SCOPING STUDY FOR A CALITORNIUM-252 COLD NEUTRON SOURCE MODERATING DEVICE}
D. L. Hendermon
R. G. Alsmiller, Jr.

[Abotract of Nech. Inotrem. Methods Phys. Res. (in preas)]

One-dimensional neutron transport calculations have been performed for a proposed Californium Cold Neutron Source experimental facility. The computations indicate that a peak cold neutron flux (flux below $10 \mathrm{meV}$ ) of $1.4 \times 10^{13}$ neutrons $/ \mathrm{m}^{2}-\mathrm{s}$ can be attained from a $500 \mathrm{mg}$ Californium neutron source. The cold neutron exit (leakage) current from the spherical cryostat containing the liquid deuterium is $2.2 \times 10^{12}$ neutrons/m $\mathbf{m}^{2} \mathbf{s}$.

Research sponeored by U.S. DOE Office of Basic Enera Sciences.

\section{TIME-DEPENDENT SINGLE-COLLISION KERNELS FOR INTEGRAL TRANSPORT THEORY}
D. L. Henderson
C. W. Maynard*

[Abetract of Neel. Sei. Eng. 102, 172 (1889)]

Time-dependent integral traneport equation single-collision kernels for one-dimensional geometries corresp onding to the steady state single-collision kernels found in the available literature have been calculated by making use of the Lapiace transform technique, simple geometrical transformation relationships and point kernel integrations. Using the convolution theorem, the time-dependent scalar flux is obtained by convoluting the singlecollision kernel with the time-dependent source. Using the multiple collision formulation of the integral transport solution, isotropic sources that are delta distributions in time are considered in several examples. Analytical solutions for the uncollided and first collided scalar fluxes are obtained for a point source at the origin of an infinite medium and finite sphere of radius $R$ and for a boundary source having an isotropic angular distribution directed into a semi-infinite medium and into a slab of thickness B. A closed form solution is obtained for the simple problem of uniformly distributed sources within an infinite medium.

Research sponsoned by Sandia National Laboratories and Lawrence Livemnore National Laboratory.

- Univessily of Wisconsin, Madieon, WI.

\subsection{3}

\section{GENERATION OF BROAD-GROUP NEUTRON/PHOTON CROSS- SECTION LIBRARIES FOR SHIELDING APPLICATIONS}
D. T. Ingersoll
R. W. Roussin
C. Y. Fu
J. E. White

LAbatract of paper presented at the ANS Topical Meeting on Advances in Nuclear Engineering Computation and Radiation Shieiding, Sante Fe, NM, April 9-13, 1989; Proc. Vol. 1, pp. 21:1-21:9 (1988)]

Two new multigroup crose-section libraries have been generated from ENDF/B-V data for two specific shielding applications. The first library was prepared for use in sodium-cooled reactor systems and is available in both broad-group (61. neutron, 23-photon) and few-group (22-neutron, 10-photon) energy structures. The second library was prepared for use in defense-related radiation shielding applications and is available in a broadgroup (46-neutron, 23-photon) energy structure. The establishment of sper ications for the libraries, especially the specific group structures and energy weighting functions, was an important part of the generation of both libraries.

Rewearch sponecred l,y U.S. DOE: Licuid Metal Converter Reacira Progran and by Defense Nuclear Agency. 


\section{A USER'S GUIDE TO MICAP: A MONTE CARLO IONIZATION CHAMBER ANALYSIS PACKAGE}

\author{
J. O. Johnson T. A. Gabriel
}

(Abatrect of ORNL/TM-10340, January 1968)

A collection of computer codes entitled MICAP - A Monte Carlo Ionization Chamber Analysis Package has been developed to determine the response of a gas-filled cavity ionization chamber in a mixed neutron and photon radiation environment. In particular, MICAP determines the neutron, photns, and total response of the ionization chamber. The applicability of MICAP encompasses all aspects of mixed field dosimetry analysis including devector design, pre-experimental planning and post-experimental analysis.

The MICAP codes include: RDNDF for reading and processing ENDF/B-formatted crosesection files, MICRO for manipulating microscopic cross section data se's, MACRO for creating macroscopic crose-section data sets, NEU. TRON for transporting neutrons, RECOMB for calculating correction data due to ionization chamber saturation effects, HEAVY for trans porting recoil heavy ions and charged particles, PECSP for generating photon and electron croes section and material data sets, PHOTPREP for generating photon source input tapes, and PHO. TON for transporting photons and electrons. The codes are generally tajlored to provide numerous input options, but whenever possible, default values are supplied which yield adequate results. All of the MICAP codes function independently and are operational on the ORNL IBM 3033 computer system.

Research sponeored by Defence Nuclear Agency.

\section{DESIGN CALCULATIONS FOR THE ANS COLD SOURCE}

\author{
R. A. Lillie \\ R. G. Alsmiller, Jr.
}

(Abatract of paper preanted at the International Collabo-
ration on Advanced Neviron Sources (ICANS-DX) Confer-
ence, Los Alamos, NM, October 3-T, 1988)

The calculational procedure, based on discrete ordinates transport methods, that is being used to carry out design calculations for the Advanced Neutron Source cold source is described. Calculated results on the gain in cold neutron flux produced by a liquid deuterium cold source are compared with experimental data and with calculated data previously obtained ai the Institute Max von Laue-Paul Langevin in Grenoble, France. Calculated results are also presented that indjcated how the flux of cold neutrons vary with cold source parameters.

Researd sponsored by U.S. DOE Office Bacic Energy Sciences.

4.26

\section{CROSS-SECTION PROCESSING METHODOLOGY USED TO DESIGN THE ADVANCED NEUTRON SOURCE REACTOR}

\author{
R. T. Primm, III
}

[Abotract of Trans. Am. Necl. Soc. 57, 292 (1988)]

Studies for the Advanced Neutron Source reactor have shown that the following conclusions regarding crous-section processing can be made. The use of a 27-group data library as a starting point appears to offer sufficient resolution in energy. The thermal flux depression in traverting from coolant to fuel is insignificant. If fewgroup diffusion calculations are to be performed with only one thermal group, then multiple, spatially dependent croar-section rets are needed for all principal nuclides. To account properly for thermalized neutrons that return to the care from the reflector, intracore plenum, or core central hole, one should use a fine-group, two-dimensional transport represeritation to collapse crose-section data.

Research sponsored by U.S. DOE Office of 3wic Energ Sciences. 


\section{GENERATION OF LUMPED FISSION PRODUCT CROSS SECTIONS FOR HIGH BURNUP, HIGHLY ENRICHED URANIUM FUEL}

\author{
R. T. Primm, III \\ N. M. Greene*
}

[Abatract of Trese. Am. Necl. Soe. 38, 592 (1908))

To estimate the Advanced Neutron Source reactor's performance as a function of time, it is necessary to model fuel depletion and the buildup of fission product poisons. An exact calculation of fission product buildup is not feasible, since there are several hundred fission product nuciides and concentrations that wonld have to be computed at a few hundred points in the reactor. Consequently, a simplified treatment in which a few fission products are represented explicitly and the remainder grouped into two lumped fission products is utilized. A methodology which accounts for the ANS reactor's short length, high-burnup, highly enriched U-235 fuel cycle was developed. Previous data were inadequate due to revised nuclear data, changes in the expected reactor fuel cycle, and the need for data in energy ranges previously not investigated.

Research sponsored by U.S. DOE Office of Basic Energy Sciences.

"Computing and Telecommunications Division.

\subsection{8}

\section{REACTOR PHYSICS STUDIES OF VARIOUS ADVANCED NEUTRON SOURCE REACTOR CORE CONEIGURATIONS}

\author{
R. T. Frimm, III
}

[Abatrace of Trans. Am. Nucl. Soc. 50, 346 (1989)]

The VENTURE diffusion theory code was utilized to perform calculations with crose-section data f:om ENDF/B-V. The parameters varied in the study were total fueled core volume, number of elements in the core (number of vertical splits), and vertical distance between annuli (height of the split). The cores with higher fuel volume but smaller plenum thickness have greater reactivity. The more compact cores (both in terms of total fueled volume and plenum height) have higher efficiencies, i.e., for constant fission power among all designs, the lower volumes would yield a higher peak thermal flux in the reflector. These calculations must be coupled with thermal-hydraulic analyses before selecting a specific geometry.

Research aponeored by US. DOE Ofice of Beaic Energy Sciences.

\subsection{9}

\section{THE DORT TWO-DIMENSIONAL DISCRETE ORDINATES TRANSPORT CODE}
W. A. Rhoades
R. L. Childs*

[Abetrect of Nech Sci. Exg. (CCA), p. 88 (1968)]

DORT determines the flux or fiuence of particles throughout one- and two-dimensional geometric systems due to sources generated as a result of particle interaction with the medium or incident upon the system from extraneous sources. The principal application is to the deep-penetration transport of neutrons and photons. Criticality (ktype and search) problems can be solved. Numerous printed edits of the ruists are available and results can be transferred to output files for subsequent aralysis.

Research eponeored by Defense Nuclear Agency.

"Computing and Telecommunications Division

\subsection{0}

\section{3-D DISCRETE ORDINATES CalCUlations With parallel- VECTOR PROCESSORS}

\author{
W. A. Rhoades R. E. Flanery
}

(Abatract of peper presented at the ANS Topical Meetins on Advances in Nuclear Engineering Computation and Ra diation Shielding, Sane Fe, NM, April 9-13, 1969; Proc. Vol. 2, pp. 69:1-69:11 (1089)]

This study applies the concepts of parallel processing to a large three-dimensional discretc ordinates transport problem in a production CrayCTSS environment. In noncurved geometry, the fluxes in various directions can be calculated in parallel, anj the problem was reatructured accordingly. In addition, natural paralleliam in thr acceleration of the fluxes was exploited. The degree of success hinged upon coping with delays encountered in attaching more than one processor to a single job. 
Due to memory limitations on the system used, the largest contemporary production problems could not be run. However, when the corresponding amount of work was simulated, practical cost benefits of $24 \%$ were demonstrated on a 2processor Cray X-MP. A modest benefit in real time was also noted, $13 \%$ under the moet fevorable conditions, although real time measurements are to be viewed with skepticism when obtained in the face of random competition from other wears.

A test program was helpful in understanding the time-dependent and task-dependent opportonities for parallelism. It is shown that a good eatimate of the cost benefits could have been obtained in advance from such information.

The parallel features had only a slight impact on the overall structure of the code, and the intention is to leave them in the production version under user control. It is likely that they can be adapted to future macrotasking systems without difficulty.

Resenuch sponsored by Defence Nuclear Agency.

\subsection{1}

\section{USER'S MANUAL FOR FERD-PC}

\section{B. D. Rocney}

(Abstrect of ORNL/TM-11028, April 19e9)

FERD-PC is a modified version of the unfolding code FERD, rewritten in Mierocoft FORTRAN and optimized for use on an IBM-PC or compatible computer. It can be used to correct observed pulse-height distributions for the notideal response of a pulse-height spectrometer and has been successfully applied to the analysis of data from the Tower Shielding Facility at Oak Ridge Nativulal Laboratory (ORNL). Modifications to the original code have been incorporated to optimize program execution and run time, while still maintaining most input and output options supported by the original code. In addition, several changes have been made to include an iteractive binning code, a flux integration code, and a plotting utility, which supports enhanced color graphics (EGA) and a HP 7475A or equivalent plotter. This manual describes thase changes and enhancemento, and covers essential information to set up and run FERD-PC. Sample problems are included to illuetrate input and output procedures using various options of the code.

Reaenrsh sponasrad by U.S. DOE Energy Programs Division.
4.32

REATOR PHYSICS METHODS FOR THE PRECONCEPTUAL CORE
DESIGN OF THE A DVANCED
NEUTRON SOURCE

\section{J. M. Ryskamp* F. C. Difilippo} R. T. Primm, III

[Abstract of Trase. Am. Neel. Soc. 57, 200 (1908)]

Idaho National Engineering Laboratory (INEL) and Oak Ridge National Laboratory (ORNL) have been jointly wotking to develop and evaluate preconceptual reactor core configurations for the advanced neutron source. This paper reviews the reactor phyrics methods used to compute reactor parameters and demonstrates that the labotatories achieve good agreement on these parameters.

The single- and split-core reactor concepts examined are similar. Both have very small cores $(\leq 35 \ell)$ inside a large tank of heavy water. These small, high-leakage, hard-spectrum cores are diffcult to analyze, requiring advanced reactor physics methods. Reactor physics analyes were performed with ENDF/B-V crosesection data.

Four-group croses sections were calculated in different regions. In particular, the one thermal group ${ }^{235} \mathrm{U}$ cros sections have significant variation depending on the distance from the core periphery. Cron-section exposure dependence was also examined and included for some nuclides. Both laboratories primarily analyzed two-dimensional $(r, z)$ core models with four-group diffusion theory (PDQ-7 for INEL and BOLD-VENTURE for ORNL). Excellent agreement wis achieved on moet parameter by INEL and ORNL for both core concepts. Difference in efficiencies between the single and the split-core designs is largely related to the difference in core volumes.

In summary, the parameters computed by INEL and ORNL are close and accurate enough, however, for the two preconceptual reactor designs to be compar ad on a consistent basis.

Research sponeored by U.S. DOE Ofice of Bacic Enero Seiences.

"Idaho Nacional Encincering Laboratory, Idaho Falls, ID. 


\section{DOS-HEATING6: A GENERAL CONDUCTION CODE WITH NUCLEAR MEAT GENERATION DERIVED FROM DOT-IV TRANSPORT Calculations}

\author{
M. L. Williams" A. Yũcel* \\ S. Nadlorny"
}

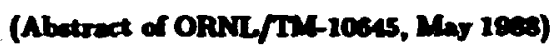

The HEATING6 beat conduction code is modified to (a) read the maltigroup particle furces from a two-dimemsional DOT-IV mentron-photon traneport calculation, (b) interpolate the filuxes from the DOT-IV variable (optional) meah to the HEATING6 control volume mesh, and (c) fold the interpolated furces with kerma factors to obtain - unclear heating source for the heat conduction equation. The modified HEATING6 is placed as a module in the ORNL discrete ordinates syatem (DOS) and has been renamed DOS-HEATING6. DOS-HEATING6 provices the capability for determining temperature dietributions due to noclear heating in complex, multi-dimenoional aytems. All of the original capabilities of HEATING6 are retained for the nuclear heating calculation; e.g., generalized boundary conditions (convective, radiative, finned, fixed temperature or beat flux), temperature and opace dependent thermal propertien, steady-otate or transient analyois, general geometry description, etc.

The numerical techniques used in the code are reviewed, and the user input instructions and JCL to perform DOS-HEATING6 calculations are presented. Finally a sample problem involving soupled DOT-IV and DOS-HEATING6 calculations of a complex space-reactor configuration is deacribed, and the input and output of the calculations are listed.

Rewearch sponsored by U.S. DOE Office of Nuclear Enercy.

-Louiciana Stale Univerily, Bacon Rouge, LA.

\section{IMPACT OF ${ }^{235} \mathrm{U}$ (MAT 9235) \\ EVALUATION ON kef OF THERMAL SYSTEMS}

\author{
R. Q. Wright* M. L. Williamst \\ C. O. Slater
}

[Abetrect of Trass. Am. Necl. Soc. 59, 343 (1900)]

The inpect on teff of thermal systems due to a proposed new evaluation of ${ }^{235} U$ neutron cross sections for ENDF/B-VI (tentatively referred to as MAT 9235) was studied. There is considerable interest in the new evaluation because of the potential impact of the croes-section changes on teff of low- and high-enriched uranium sytems. The crose-section changes proposed include the resolved and unsesolved resonance regions, the thermal $\bar{\nu}$, the fission spertrum, and $\eta$. Of particular interest is the change in $\eta$ below $1.86 \mathrm{eV}$. Several thermal benchmarks were calculated wa ing ENDF/B-V and ENDF/B-VI evaluations of ${ }^{235} \mathrm{U}$ crose sections. The results shomed that relative to the ENDF/B-V evaluation, the MAT 9235 (ENDF/B-VI) evaluation decreases $\Delta k / k$ by about $0.26 \%$ for the low-enriched benchmarks and about $0.35 \%$ for the high-enriched benchmarks. Values of the MAT $9235 \eta$ below $1.86 \mathrm{eV}$ were found to differ from ENDF/B-V values by -1.1 to $3.5 \%$. (Note that subsequent to the submis sion and acceptance of the paper, further revivions were made to the fission and capture cross nertions. The revised calculations showed the change in $\Delta k / k$ was eswentially $0 \%$ for the high-enriched benchmarks and about $0.1 \%$ for the low-enriched benchmarks. While there were some changes in $\eta$ below $1.86 \mathrm{eV}$, the differences still lay within the range stated above.)

Research sponsored by U.S. DOE.

"Computing and Telecomnunications Division.

tLouisiana State Univerrity, Beton Rouge, LA. 


\section{HIGH ENERGY RADIATION AND PARTICLE TRANSPORT}

4.35

\section{INCLUSION OF CORRELATIONS \\ IN THE EMPIRICAL SFLECTION OF INTRANUCLEAR CASCADE NUCLEONS FROM HIGH-BNERGY HADRON-NUCLEUS COLLISIONS}

\author{
F. S. Alsmiller \\ R. G. Alsmiller, Jr.
}

[Abdract of ORNL/TM-11052, Jamang 1809, doo Na:L

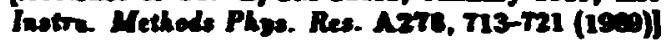

The very high energy (5 GeV to $20 \mathrm{TeV}$ ) hadron-nucleus differential particle production moded found in the Monte Carb transport code ILUKA 87 has been adapted for inclusion in the transport code HETC88. The empirical selection of intranuclear cascade nucleons has heen modified to provide simple correlations with the randomly selected number of hadron-nucleon collisions. A andard method of calculating the excitation energy of the compound nucleus preceding an added evaporation step by ascuming the particles are produced in a one-dimensional nuclear well is appliet. This method, coupled with the above correlations, leads to improved correlations of the excitation energy with the $A$ and $Z$ of the compound nucleus, and then to greatly improved distributions of the residual nuclei following evaporation. The frequency distributions of low energy $(\beta<0.7)$ charged particles show good agreement with experiment for $200 \mathrm{GeV}$ protons incident on emulsions. Average multiplicities of shower and grey particles after evaporation for protons and pions incident on reveral elemente are also compared with experiment.

Research eponeored by U.S. Depertment of Energy.

\subsection{6}

\section{THE HIGH-ENERGY TRANSPORT CODE HETC88}

R. G. Alsmiller, Jr. F. S. Alsmiller
T. A. Gubriel
O. W. Lermann*

\section{J. M. Barnes"}

(Abstract of paper presented at the Workehop on Calorimetry for tine Superconducting Super Collider, University of Alabama, Tuecalooas, AL, March 13-17, 1889)

An upgraded version, HETC88, of the previously available IIigh-Energy Transport Code
HETC is briefly described. In the upgraded code, the particle production model from hadronnucleus nonelastic collisions at energies greater than 5 geV has been revised. At nuckeon and pion energies below $5 \mathrm{GeV}$, HETC88 is not different from the code previoudy available. In particular, provision is still made to allow neutrons with energies $\leq 20 \mathrm{MeV}$ to be traneported by one of the available codes designed for kom-energy nettron transport.

Calculated results for the longitudinal diatribution of the fux of neutrons with energy $\geq 40$ $\mathrm{KeV}$ in the Tevatron tunnel when $900 \mathrm{GeV}$ prolons interact with $N_{2}$ in a warm section are prerented and compared with experimental data. Some diagreements between the calculated and meacured neutron flux are found. For $20 \mathrm{TeV}$ procons incident on a large cylindrical iron target, calculated "star" density results from HETC88, FLUKA87, CASIM, and MARS10 are also compared.

Research spopered by U.S. DOE OAlice of Hidh Energ and Nincler Physics.

* Compoting and Telecommonications Divicion

4.37

\section{THE MODIPISD HIGH-ENERGY TRANSPORT CODE HETC AND DESIGN CALCULATIONS FOR THE SSC}

R. G. Alsmiller, Jr. F. S. Alsmiller

T. A. Gabriel O. W. Hermann"

B. L. Bishop"

[Abatrect of Trese. Am. Nucl. Soc. s6, 270 (19e8)]

The proposed superconducting super collider (SSC) will have two circulating proton beams, each with an energy of $20 \mathrm{TeV}$. In ordet 10 perform detector and ahield deaign calculations at these higher energies that are as aciurate as possible, it is necessary to incorporate in the calculations the best available information on differential particle production from hadron-nucleus collisions. In this paper, the manner in which this has been done in the high-energy transport code (HETC) is deacribed, and calculated results obtained with the modified code are compared with experimental data.

Revearch eponeored by U.S. DOE Office of High Energ and Nucles Phyoirs.

"Computing and Telecommunicatione Division. 


\section{CaLCULATEd INCLUSTVE NEUTRON PRODUCTION FROM $400 \mathrm{GeV}$ PROTCN-NUCLEUS COLLISIONS}

\author{
R. G. Alsmiller, Jr. F. S. Alsmillex \\ O. W. Hermann
}

(Abatract of ORNL/TM-11257, Anget 1969)

Calculated inclusive neutron production from $400 \mathrm{GeV}$ proton-r.ucleus collisions is presented and compared with experimental data. Target nuckei $\mathrm{B}, \mathrm{Be}, \mathrm{Cu}$, and $\mathrm{Pb}$ are considered, and the comparisons cover the laboratory ener $8 y$ range of 20 to $400 \mathrm{GeV}$ and angular range 0.7 to $10 \mathrm{mr}$. Boderately good agreement between the calculated and experimental data is found, but the agreement in the case of $\mathrm{Be}, \mathrm{Cu}$, and $\mathrm{Pb}$ is significantly betier than in the case of $B$.

Researdy sponsoned by U.S. DOE Office of High Enerd Phyica.

"Computing and Telecmmmunicacions Diviaion

THEORETICAL STUDIES OF HADRONIC CALORIMETRY FOR HIGH LUMINOSITY, HIGH ENERGY COLLIDERS

\section{J. E. Brau* T. A. Gabriel}

[Abetract of Nacl. Inotram. Methode A270, 40 (1989); leo ORNL/TM-10903, January 1989]

Experiments at the high luminosity, high energy colliders of the future will require optimization of the state of the art of calorimetry design and colsatruction. During the past few years, the understanding of the basic phenomenology of hadron calorimeters has advanced through parallel theoretical and experimental investige iions. Tr: important underlying proceace are reviewed to set the framework for the presentation of recent calculations of the expected performance of silicon detector baned hadron calorimeters. Such devices employing uranium are expected to achieve the compensation condition (that is, $\mathrm{e} / \mathrm{h} \approx 1.0$ ) based on the understanding that has been desived from the uranium-liquid argon and uraniumplastic scintillator systems. In fact, even lead- silicon calorimeters are found to achieve the attractive value for the $\mathrm{e} / \mathrm{h}$ ratio of 1.16 at $10 \mathrm{GeV}$.

Researen sponsored by U.S. DOE, Ofice of Hidh Energ and Nuder Phyice.

-Uniresiar of Oregos, Eugene, OR.

4.40

\section{PROSPECTS FOR AND TESTS OF HADRON CALORIMETRY WITH SHICON}

\author{
J. E. Brau* T. A. Gabriel \\ P. G. Rancoita ${ }^{t}$
}

(Abotract of ORWL/TM-105s, Mach 1800)

Hadron calorimetry with silicon may provide crucial capabilities in experiments at the high luminosity, high energy colliders of the feture, particularly Jue to silicon's fast intrinsic speed and abeolute calibration. The important underlying proceses of our understanding of hadron calorimeters are reviewed to set the framemork for the presentation of recent calculations of the expected performance of silicon detector based hadron calorimeters. Such devices employing uranium are expected to achieve the compensation condition (that is, the ratio of the most probable electron signal to hadron signal $(e / h)$ is $\approx 1.0$ ) baced on the understanding that has been derived from the uranium-liquid argon and uranium-plaatic scintit lator nystems. In fact, even lead-silicon calorimeters are found to achieve the attractive value for the e/h ratio of 1.16 at $10 \mathrm{GeV}$. An experimental test of these predictions is underway at CERN by the SICAPO Collaboration.

Research sponsored by U.S. Department of Enercy.

- Univenity of Oregon, Eugene, OR.

INFN, Milan, Icaly. 


\section{THE CALCULATION OF COSMOGENIC NUCLIDE ABUNDANCE IN STONY METEORITES}
M. Divadeenam*
O. Lazereth"
T. A. Gabriel
M. S. Spergel

\section{T. E. Ward*}

(Abatred of orel presentation presented at a Sympocium on the Cosmic Abeondances of Motter, Minneapoli, MN, Augut 15-17, 1988)

In our calculations, use is made of Monte Carlo techniques for identifying nuclear collisions and specific nuclear reactions as well as establishing the transport of the incident nucleon and its generated nucleons. It is possible to use an integrated calculational approsch in predieting the coemogenic nuclide production rate and depth de pendence. In particular, the High Energy Intramuclear and Internuclear Cascade Transport Code, HETC, is used to ealculate directly the nuclide production rate due to spallation. When the neutron source results generated with HETC is linked to the low ener gy neutron transport code, MORSE, the neutron-induced cosmogenic nuclide, profiles can be culculated without resorting to extrapolations of excitation functions.

An incident cosmic ray spectrum to $200 \mathrm{GeV}$ has been utilized in the calculation to predict the induced cosmogenic spectra. The neutron source spectra energy at the surface and at select depths in a 1-m diameter meteorite has been examined. In particular, one of the four componitional models has been utilized: $\mathbf{L}$. Chondrite, II. Chondrite, Aubrite and C. Chondrite. Spallation and neutron-induced production similarly will be examined in detail for frequently measured radiogenic nuclides such as ${ }^{22} \mathrm{Na},{ }^{36} \mathrm{Cl},{ }^{53} \mathrm{Mn}$, ${ }^{59} \mathrm{Ni}$ and ${ }^{80} \mathrm{Co}$. It is seen that the spallation contribution dominates near the surface with neutron-induced contributions dominating deep within the meteorite. Since cosmogenic nuclide ratios are insensitive to incident flux normalization, select ratios are examined to give insight into inherent properties of tive meteorite, such as preatmospheric size.

Researth aponsonred by U.S. DOE Office of High Energy and Nuclear Physica.

-Brockliaven National Laboratory, Upton, NY.

†York College, Jamaica, NY.
PRODUCTION RATES OF COSMOGENIC NUCLIDES IN STONY METEORITES
M. Divadeenam*
O. Laxereth"
T. A. Gabriel

T. E. Ward*

(Abutract of paper presented at the American Nucleor Society and National Bureen of Stand de Conference on Fifty Years with Nedeer Fivion, Gaithereburs, MD, April 2626, 1989)

Monte Carlo calculations of $\mathrm{Al}^{26}$ and $\mathrm{Mn}^{53}$ production due to spallation induced by cosmogenic protons in model meteorite composition similar to L Chondrite has yielded predictions which are consistent with the oboerved decay rates in $L$ Chondrile stony meteorites. The calculated $\mathrm{Al}^{26}$ production rate $(54 \mathrm{dpm} / \mathrm{kg})$ in a $1-\mathrm{m}$-diameter meteorite is within 1/2 S.D. of the mean ( $49 \pm 11$ $\mathrm{dpm} / \mathrm{kg}$ ) taken from 100 bulk determinations in $\mathrm{L}$ Chondrite samples compiled. Similarly calculated average value for $\mathrm{Mn}^{53}(223 \mathrm{dpm} / \mathrm{kg})$ is consistent with one S.D. off the mean in the widely scattered $\mathrm{Mn}^{53}$ data (362 $\pm 113 \mathrm{dpm} / \mathrm{kg}$ ) compiled.

Reseanch eponered by U.S. DOE Office of High Energy and Nuclea Phyica.

-Brookharen Nacional Laboratory, Upton, NY.

Yort College, Jamaica, NY.

4.43

\section{DETERMINISTIC TRANSPORT CaLCULATIONS OF DOSE PROFILES DUE TO PROTON BEAM IRRADIATION}
W. L. Filippone*
M. S. Smith
R. T. Santoro
T. A. Gabriel
R. G. Alsmiller, Jr.

[Abatract of Trene. Am. Neel. Soe. 56, 273 (1988)]

In this paper we present discrete ordinates solutions to the Spencer-Lewis equation for protons. In its present form, our code calculates the energy deposition profile and primary proton flux in $x, y$ geometry due to proton beam irradiation. Proton energies up $100.4 \mathrm{GeV}$ are permissible. 
Below this value, protons lose energy primarily through coulomb collisions with electrons. This is modeled using the continuous slowing down approximation (CSDA). The required stopping powers are obtained from the computer code SPAR, which has been included in the main program a subroutine. Nuclear coulomb collisions are modeled uaing Rutherford scattering with the screening parameter given by Moliere. Due to the extreme anisotropy of proton coulomb scattering, the SMART scattering matrix technique has been applied. This method allows modeling of a large number of mall angular deflections by relatively few large deffection. Currently, the code treats only primary protons. Cros sections for these reactions are obtained from the NCDATA code.

An analytic first, collision source is used to model range straggling. Sontce particlea with the same energy are given alightly different stopping powers to simulate straggling from energy low fluctuations. These values are chosen such that the average stopping power and the percentage of range straggling are preserved.

Dose profiles have been calculated for several source and target geometries. The SN and Monte Carlo results are in excellent agreement, except near the peak. The discrepancy is probably due to diamond difference errors, differences in the treatment of range straggling. and considering HETC calculations do not include multiple nuelear scattering.

Research sponsored by US. Department of Defene; by Air Force Weapons Leboratory; and by the Univerity of Arizona, Tueson, AZ.

"Univensity of Arizona, Tuceon, 12.

\section{DETECTORS FOR THE SUPERCONDUCTING SUPER COLLIDER, DESIGN CONCEPTS, AND SIMULATION}

\section{T. A. Gabriel}

(Abatract of ORNL/7'M-11189, June 1980; aleo paper preented at the Workehop on Calorimetry for the Supereonducting Super Collider, Univeruity of Alabama, Tuacabous, AL, March 13-17, 1960)

The physics of compensation calorimetry is ieviewed in the light of the needs of the Superconducting Super Collider (SSC) detectors. The four major detector types: liquid argen, scintillator, room temperature liquids, and silicon are analyzed with respect to some of their strengths and weaknesses. Finally, general comments are presented which reflect the reliability of simulation code systems.

Research sponsored by IIS. DOE Ofice of High Energ and Nuclear Physica.

\subsection{5}

\section{CALOR89: THE CODE SYSTEM FOR CALORIMETER ANALYSIS AND DESIGN}

\section{T. A. Gabriel}

(Abutract of paper presented at the Workhop on Caborimeing for the Superconductiog Super Collider, Univeruity of Alabame, Tuscaloosen, AL, March 13-17, 1900)

As part of a strong experimental high energy physics program, a substantial effort must be involved in calculational analysis of the detector system. This calculational capability must be fundamentally sound and based on previous interchange bet ween theoretical calculations and experimental test programs. The CALOR89 system for analyzing calorimeters offers a solid approach for investigating all facets of detector systems and has been used in many calculational studies. CALOR89 is one of two major code systems recommended for analysis of SSC detector systems.

Research sponsored by U.S. DOE Office of High Enesty and Nuclear Phyica.

\subsection{6}

\section{CALORIMETER AND RELATED CALCULATIONS FOR THE SUPERCONDUCTING SUPER COLLIDER}

\section{T. A. Gabriel}

\section{(Absetrace of ORNL/TM-10SBA, Fabruary 1980)}

There are four topics to be covered in this paper. The first topic deals with the programs, the models, and the cross sections in the CALOR code package. Secondly, a discussion on compensating calorimetry as it applies to $\mathrm{Fe} / \mathrm{Si}$ and $\mathrm{U} / \mathrm{Si}$ calorimetry will be given. Thirdly, calculated results obtained on $\mathrm{U} / \mathrm{Si}$ calorimeters using the CAIOR system will be presented and discussed. 
Finally, the calculations that the Oak Ridge $\mathrm{Na}$ tional Laboratory are carrying out dealing with radiation damage at the proposed Superconducting Super Collider (SSC) are deseribed.

Research sponsored by U.S. DOE Otice of High Enerd and Nuclear Phyica.

\subsection{7}

\section{CALOR87: HETC87, MICAP, EGS4, AND SPECT, A CODE SYSTBM FOR ANALYZING DETECTORS FOR USE IN HIGH ENBRGY PHYSICS EXPERMMENTS}

\section{T. A. Gabriel}

R. G. Alsmiller, Jr.

O. W. Hermann"

\section{F. S. Alomiller}

B. L. Bishop"

J. O. Johnson
[Abatrect of paper preseated at the Wortabop on Deteocor Simulation for the SSC, Argonne National Laboratory. Auguet 24-28, 1987; Proc. pp. 50-60 (19e0)]

A brief history of CALOR (BETC, EGS, MICAP, SPECT) is presented to indicate the evolution of this code system. Details concerning the current modifications and additions to the highenergy transport code, HETC, are also presented and new comparisons with experimental data are included to verify tbe new physics improvements.

Rescarch sponeored by U.S. DOE Otice of Hith Eaeng Phyrics and Nuclear Phyice.

-Computing and Telecommanicationa Divicion.

4.48

\section{THE PHYSICS OF COMPENSATING CALORIMETRY AND THE NEW CALOR89 CODE SYSTEM}

\author{
T. A. Gabriel J. Brau" \\ B. L. Bishop
}

(Abstract of ORNL/TM-11060, March 1909, deo paper presented at the IEEE 1988 Nuclear Science Symposium, Orlando, FL, November \&-11, 1988; Proc. IEEE Trane. on Nuclear Science 37(1), 14-17 (1909)]

Much of the understanding of the physics of calorimetry has come from the use of excellent radiation transport codes. A new understanding of compensating calorimetry wa introduced four years ago following detailed atudiea with a new CALOR syotem. Now, the CALOR syotem has

again been revised to reflect a better comprehension of high energy nuclear collisions (HETC88) by incorporating a modified high energy fragmentation model from FLUKA87. This revision will allow for the accurate analysis of calorimeters at energies of 100's of GeV. Presented in this paper is a discussion of compensating calorimetry, the new CALOR system (CALOR89), the revisions to HETC, and recently generated calorimeter related data on modes of energy deposition and secondary neutron production ( $\leq 50 \mathrm{MeV}$ ) in infinite iron and uranium blocks.

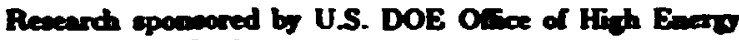
and Noder Phrica.

-Univesiity of Orepon, Engede, OR.

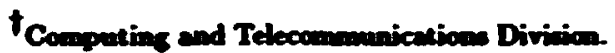

4.49

\section{TRANSPORT CALCULATION OF COSMOGENIC NUCLIDE PRODUCTION IN STONY METEORITES}
T. A. Gabriel
M. S. Spergelt
M. Divadeenam*
o. Lasereth*

\section{T. E. Ward*}

(Abetreat of paper premented at the American Phyical Society Meetinz, Santa Fe, NM, October 12-15, 19s8)

Cosmogenic nuclide production rate is calculated with the high energy nucleon and meson transport code IIETC for spherical Chrondite Stony Meteorites of difierent sizes. The galactic proton flux (at solar flares) with proton energy ranging from $40 \mathrm{MeV}$ to about $200 \mathrm{GeV}$ is sampled via Monte Carlo technique for determining the incident proton energy and intensity on the meteorites. The coumogenic nuclide production rate is calculated as a function of depth for different meteorite sizes. The eatimation of the nuclide production due to low energy neutron transport is being undertaken with the help of transport code MORSE. The HETC predicted conmogenic nuclide production rates will be presented.

Research eponeored by U.S. DOE Office of High Energy and Nucles Physics.

"Brookheven National Laboratory, Upton, NY.

'York College, Jamaice, NY. 
4.50

\section{MICAP: A PROGRAM FOR LOW ENERGY NEUTRON, ION, AND \\ GAMMA-RAY TRANSPORT AND ONE OF ITS APPLICATIONS IN CALORIMLTER DESIGN: HYDROGEN KNOCK-IN AS A METHOD FOR ACHISVING COMPENSATION}

\section{T. A. Gabriel J. O. Johnson J. Brau"}

(Abetrnct of paper preverted at the Wortabop on Deteolor Simolation for the SSC, Argenene National Laboratory. A co 24-23, 1987; Proc. pp. 252-208 (1900))

A new code system, MICAP, has been developed for the tranoport and production of lowenergy ( $<20 \mathrm{MeV}$ ) neutrons, photons, and light and heavy ions. The results generated by this code syatem compare favorably with a wide variety of experimental data. Because of this success, MICAP can be used as a valuable tool in helping to analyze calorimeter systems. In particular, MICAP can be used a valuable tool in belping to analyze calorimeter systems. In particular, MICAP is used in this paper to determine the practicality of hydrogen tnock-in as a method of achieving compensation in a uranium/silicon calorimeter, i.e., $e / h=$ !. The results indicate that compensation is probabiy posible but shower fluctuations may be increased.

Research spmecred by U.S. DOE Office of Hich Energ Phrice and Nuclear Physics.

"Univenily of Tennewee, Knoxville, TN.

\subsection{1}

\section{SOURCES OF COMPENSATION IN HADRONIC CALORIMETERS}
M. S. Goodman*
T. A. Gabriel
A. Di Ciaccio ${ }^{\dagger}$
R. Wileon"

[Abatract of OR NL/TM-10004, December 198s; aleo Niel. Inotrem. Methods in Phys. Reocerch A270, 141 (1900)]

Monte Carlo simulations are presented using the CALOR code syotem to study the deaign c! a large hybrid hadron calorimeter system employing a warm liquid active medium (letramethylsilane, $\left.\mathrm{Si}\left(\mathrm{CH}_{3}\right)_{4}\right)$ and uranium plates in addition to a conventional Fe/plastic system. In the system de scribed here, the uranium provides partial compensation by suppreasing the electromagnetic cascade produced by incident electrons due to sampling ineficiencies. The results of the simulations also indicate that significant compensation is achieved (given small enough saturation) due to low energy recoil protons produced in collisions with low energy (1-20 MeV) cascade and fision neutrons in the sctive medium. Both compensetion mechaniums are important to belp balance the rexponse of a calorimeter to incident electrons and hadrons, that is, to achieve a ratio of pule heights $(e / h \approx 1)$ which will lead to the beat energy resolution.

Reacarch pomoned by U.S. DOE Oxice of Hidh Enets and Nucles Phyicica.

-Bell Communication Receardh, Morriatom, NJ.

tCERN/LNFN/Univarits Tor Vergere," Rome, Ifaly.

\$Harvard Univasity, Cambridse, MA.

4.52

\section{ANALYSIS OF NEUTRONICS \\ PARAMETERS MEASURED IN PHASE-I EXPERMMENTS OF \\ THE JAERI/US COLLABORATIVE PROGRAM ON FUSION BLANKET NEUTRONICS. PART I: SOURCE CHARACTERISTICS AND REACTION RATE DISTRIBUTIONS}
M. Nakagawa*
T. Mori"
K. Koanko"
T. Nakamura"
M. 2. Youseer
Y. Watanabe
C. Y. Gung ${ }^{\dagger}$
R. T. Santoro
R. G. Alsmiller, Jr. J. Barnest T. A. Gabriel

[Abatrect of Froion Eng. Design 0, 315 (1900)]

Fusion blanket neutronica parameters ineasured in the Phave II acsembly have been analyzed at both JAERI and the US. Both pasties have analyzed the experiments independently by using different nucleas date and calculational methods baced on 3-D Monte Carlo and 2-D $S_{n}$ codes. This part includes the results of the anal. ysis on the cource characteristic in the ansembly and the reaction rate diatributions in the 
test zone consisting of $\mathrm{Li}_{2} \mathrm{O}$ with and without a beryllium muliplier. The source characterization has been made by measuring the neutron spectrum and various reaction rates. These re actions include ${ }^{58} \mathrm{Ni}(\mathrm{n}, 2 \mathrm{n}),{ }^{38} \mathrm{Ni}(\mathrm{n}, \mathrm{p}),{ }^{27} \mathrm{~A}(\mathrm{n}, a)$, ${ }^{93} \mathrm{Nb}(\mathrm{n}, 2 \mathrm{n}),{ }^{197} \mathrm{Au}(\mathrm{n}, 2 \mathrm{n})$, and ${ }^{197} \mathrm{Au}(\mathrm{n}, \gamma)$. The ratios of calculated to measured values are compared among both countries and the different nuclear data used. Considerable discrepancies have been observed for the ${ }^{58} \mathrm{Ni}(\mathrm{n}, 2 \mathrm{n}),{ }^{58} \mathrm{Ni}(\mathrm{n}, \mathrm{p})$, and ${ }^{93} \mathrm{Nb}(\mathrm{n}, 2 \mathrm{n})$ reactions depending on which nuclear data was used, while good agreement is seen for the reactions ${ }^{197} \mathrm{Au}(\mathrm{n}, 2 \mathrm{2n})$ and ${ }^{27} \mathrm{~A}(\mathrm{n}, a)$. The distributions of these reaction rates in the test zone have also been analyzed to examine the pre diction accuracy of neutronics parameters in a breeder zone. Using the recently measured croces sections at the FNS resulted in a significant reduction in the discrepancies for most reaction rates.

Research sponeored by U.S. Department of Eners.

-Japan Atomic Energs Revearch Institute, Toloi-mura, Ibaraki-ken, Japan.

'Univenity of California a Los Angeles, Las Angeles, CA.

†Computing and Telecommunications Divition.

\section{COMPARISON OF CALCULATED RESULTS WITH EXPERIMENTAL DATA FOR THE TRITIUM PRODUCTION RATES IN A Li2 $O$ ASSEMBLY}
R. T. Santoro R. G. Alsmiller, Jr. J. M. Barnes" T. A. Gabriel

[Abstract of Nicl. Sci. Eng. (in prewe); aleo ORNL/TMI1181, May 1989]

The Monte Carlo codes MORSE and MCNP have been used to calculate the tritium production from $\approx 14 \mathrm{MeV}$ neutron reactions in a $\mathrm{Li}_{2} \mathrm{O}$ assembly. Tritium production from neutron reactions with ${ }^{6} \mathrm{Li}$ and ${ }^{7} \mathrm{Li}$ uuclei were calculated along the central axis of a $0.60-\mathrm{m}$ djameter by 0.60 -m-long assembly and four additional assemblies where sheets of stainless steel type $\mathbf{3 0 4}$ and polyethylene were placed in front of the $\mathrm{Li}_{2} \mathrm{O}$ to simulate first wall and coolant materials. The calculated data are compared with measured data obtained at the Fusion Neutron Source at the Japan Atomic Energy Research Institute. The calculated data reproduce the measured data in shape, but differ from the measured data by 10-20\% in the case of ${ }^{6} \mathrm{Li}$ and as much as $30 \%$ at some spatial locations for the ${ }^{7} \mathrm{Li}$ tritium production.

Resenrch sponsored by U.S. DOE Ofice of Bacic Energ Sciences.

*Computins and Telecomumunications Divicion.

\subsection{4}

ON-DIMENSIONAL $S_{\mathfrak{n}}$ CALCULATIONS TO EVALUATE THE SHISLDING FOR AN ALPHA-PARTICLE CHARGE EXCHANGE NEUTRAL ANALYZER

\author{
R. T. Santoro W. K. Dagenhart*
} J. M. Barnes ${ }^{t}$

(Abatract of ORNL/TM-10649, January 1888)

One-dimensional discrete ordinates calculations have been carried out to estimate the effectiveness of both ${ }^{-}$concrete and stainlesteel plus borated water shadow shields in reducing the neutron and gamma-ray background levels at an alpha-particle charge exchange neutral analyzer. The system is proposed for studying the alpha particle and plasma characteristics of fusion machines with ignited plasmas such as the CIT. A stainless. steel-borated water shield was determined to be effective in reducing background counting rates.

Research sponsored by U.S. Department of Enercy and U.S Air Force Weapons Laboratory.

"Fusion Energy Division.

†omputing and Telecommuniracions Division. 


\section{SPACE DEFENSIVE SYSTEMS}

4.55

\section{SHILLD OPTIMIZATION PROGRAM, PART II: BFPECTS OF VAN ALLEN BELT RADIATION ON SDI WEAPON PLATFORM;}
J. M. Barme:
R. T. Santoro
J. O. Johneon
J. D. Drischler
T. A. Gabriel
M. S. Smith

(Abatrect of ORWL/ML-10557, December 1998)

The effects of both natural and man-made Van Allen Belt (VAB) radiation at an altitude of $500 \mathrm{~km}$ are presented for various components of a prototypic apace-based interceptor (SBI) weapon platiorm. The weapon platiorm is deacribed in detail and represents the authors' concept of such a system. The calculated results show that the SBI platform will survive long term (10 years) exposure to natural VAB protons and electrons. Horever, when the electron belts are enhanced by the detonation of a nuclear weapon, high levels of radiation can be expected in componepts mounted on or pear the surface of the spacecraft. These dose levels are sufficient enough to produce damage in the mont sensitive components.

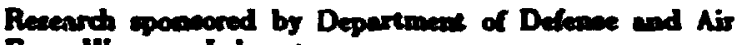
Farce Weapose Laborntary.

"Computing and Telecomansications Diviaion.

\subsection{6}

\section{RECENT ORNL IMPROVEMENTS TO THE HULL HYDROCODE SYSTEM}

\section{T. J. Burns}

(Abotract of ORNL/TM-1000s, Mey 1900)

Modifications to the HULL hydrocode syztem are deacribed. The modificarions concern the problem generator of the system, KEEL. Revised algorithm for implementing combinatorial geometry, defining reference geometries, material "packaging." and reatarting two- and three-dimensional calculations are described. The user input neceasary to exercise the added/improved features is outlined and examples illustrating the revised capabilities are provided.

Revearch oponeored by Departmant of Defence and Air Forer Weapons Laboralory.

\subsection{7}

\section{THE OAK RIDGE NATIONAL LABORATORY STRATEGIC DEFENSE INITIATIVE SHIELD OPTMMIZATION PROGRAM}
T. A. Gabriel
J. M. Barnes"
B. L. Bishop*
J. D. Drischler
J. O. Johnson
R. A. Lillie
R. T. Santoro
M. S. Smith

[Abetract of ORNL/TM-10631, April 1938 and Execucive Sumeny (ORNL/TM-10629, April 1983) by R. T. Sentoro and T. A. Gabiell

Scoping studies have been completed to ertimate the radiation induced damage in silicon based electronic components carried on a satellite. The analyses were completed for natural (Van Allen belt protons and electrons, solar flares, and galactic coemic rays) and man-made (nuclear and directed neutral particle beam weapons) radiation expected to be encountered by an SDI satellite or weapon platform. The Van Allen belt protons, depending on altitude and orbital inclination, were found to be the most stressing natural radiation threat. Nuclear weapon radiation, depending upon the weapon yield and distance of the detonation from the satellite, and neutral particle beam radiation were found to terminally deatroy electronic compopents. Calculations were also made to estimate the amount of local shielding required to extend miscion performance. These shields were optimized for minimum weight for specified damage thresholds. All of the calculations were carried out assuming the presence of a thin primary shield tailored specifically for survivability against an incident low mases kinetic energy weapon projectile and which affords minimal protection againat energetic radiation.

Research sponsored by U.S. Department of Defence and Air Force Weapons Laboratory.

"Computing and Telecommunications Divicion. 


\section{SHIELD OPTIMIZATION PROGRAM, PART III: EFFECTS OF X-RAY RADIATION FROM NUCLEAR WEAPONS ON SDI WEAPON PLatforms}
J. O. Johneon
T. A. Gabriel
J. M. Barnes"
J. D. Drischler
M. S. Smith
R. T. Santoro

(Abatrect of ORNL/TM-10985, March 1999)

Initial studis have been completed to estimate the radiation induced damage to the silicon based electronic components and other sensitive areas (fuel tants) carried on a representative Space Based Interceptor (SBI) weapon platiorm. The SBI weapon platform model used in the atudies represents the author's concept of such a system. The analysis was completed for the blackbody $\mathrm{X}$ ray radiation environment emanating from a nuclear weapon detonation in space and considered blackbody temperatures in the range of 1 to 25 $k e V$ and exterior wall loadings in the range of 1 to $10 \mathrm{cal} / \mathrm{cm}^{2}$.

The results indicate the dose to the sensitive components within the exterior bull of the platform was insufficient to cause any damage at a 1 to $3 \mathrm{cal} / \mathrm{cm}^{2}$ exterior wall loading. At higher (5 to $10 \mathrm{cal} / \mathrm{cm}^{2}$ ) exterior wall loadings, some of the Kinetic Kill Vehicle (KKV) computers and sensors begin to receive dces sufficient enough to cause damage. The effects of shielding from within the platform architecture is seen by the different dose levels received in the KKVs and fuel tanks. Because of the low-doce levels to the components within the exterior hull of the platform, no additional shielding is required for these components and for low exterior wall loadings. However, sencors and electronic components on the surface or outside of the exterior hull will require some form of X-ray shielding to survive the effects of a nuclear weapon detonation yielding a wall loading of $1 \mathrm{cal} / \mathrm{cm}^{2}$ or greater. All of the calculations were carried out for an unshielded SBI weapon platform to determire the radiation levels for which shieljing must be designed to insure survivability of the electronic systems.

Research sponsored by Depertment of Defense and Air Force Weapons Laboracory.

"Computing and Telecommunications Division.

\section{NUCLEAR WTAPON RADIATION EFFECTS ON A SPACE BASED INTERCEPTOR WEAPON PLATFORM}

\author{
J. O. Johnoon M. S. Smith \\ R. T. Santoro
}

(Abotract of paper to be presented at the 1890 HISART Conference, Navil Poetgeduate Sdwool, Monterey. CA, February 12-16, 1900)

Initial studies have been completed to estimate the dose to the various electronic compoments and sensitive areas of an ORNL computer model of a representative Space Based Interceplot (SBI) weapon platform due to an exo-atmoopheric nuclear weapon detonation. In particular, the damage resulting from incident neutrons, gammerays, and $\mathrm{X}$-rays genetated by the meapon detonstion was ascessed for the critical electronic components and for materials whose ehemizal/physical properties might degrade.

The neutron and gamma-ray source speetra employed in this study include a deuteriumtritium fusion reaction spectrum, a pure ${ }^{235} \mathrm{U}$ fiesion spectrum and a prompt fission gamma-ray spectrum. Blackbody radiation spectra at temperatures (KT) of 2, 5, 10, and 20 keV were used with surface loadings in the range of $1-10 \mathrm{cal} / \mathrm{cm}^{2}$ considered. An actual weapon spectrum may be constructed with a combination of the fusion and fission neution and gamma-ray source spectra and the blackbody source spectra at various temperatures. Nuclear detonations directly above, directly in front, and at a 45 degree angle to the front and top faces of the weapon platform were considered to aseses shielding effects inherent in the platform geometry. The nuclear weapon detonation was modeled such that the incident radiation spectra could be acsumed monodirectional. The MORSE code was used to perform all neutron and gamma-ray calculations, and the EGS4 code was used to perform all X-ray calculations.

Initial results indicate neutron and gammaray dose and dose rate levels to the rensitive components within the SBI weapon platform may exceed design limits if the weapon is detonated within a critical radius. For example, the 1962 Starfish event had a yield of $1.4 \mathrm{MT}$, of which I MT was fisaion yield. Al a distance of $91.4 \mathrm{~km}$ from the device, the $X$-ray surface loading is 1 $\mathrm{cal} / \mathrm{cm}^{2}$. Using this distance and yield, the total dose in the $C^{3}$ bay eritical box is $136 \mathrm{rads}(\mathrm{Si}$; from 
primzary aeutrons, primary ge ima rays (prompt gamma rays), and secondary gamma rays. The dose rate assuming a 40 nanosecond pulse width, $\dot{\gamma}$, is $i .7 \times 10^{9} \mathrm{rads}(\mathrm{Si}) / \mathrm{sec}$. Consequently, the limiting factor with respect to damage is the dose rate.

The X-roy dose to the sensitive components within the exterior hull of the piatform wes not suficient to cause any damage at a $1 \mathrm{cal} / \mathrm{cm}^{2}$ exterior wall loading. However, if a 40 manocecond pulse width is asoumed, dose rates to the sens:tive components in the range of $10^{8}-10^{12} \mathrm{rads} / \mathrm{sec}$ would oceur and be large enough to cane damage. Consequently, the doee rate beesmes the primary mode of faihure even though the lotal dowe is not large enough to cause any damage to the remsitive components. At higher wall badings, come of the KKV computers and sensors begin to receive dowes large enough to cause damage. Furthermore, the majority of the dose to the internal components of the SBI platform came from blactbody devices with temperatures greater than $10 \mathrm{keV}$. The low temperature devices will yield a higher flux of $X$ rays, but the incident energy will be insufficient to cause permanent damage to the internal electronic components.

Reseanch sponeored by U.S. Air Fonce and U.S. DOE.

\subsection{0}

\section{THE EFFECTS OF NATURAL AND ENHANCED VAN ALLEN BELT RADIATION ON A SPACE BASED WEAPON PLATFORM}

\section{R. T. Santoro J. M. Barnes"}

\section{J. O. Johnson}

(Abalract of paper in be presented at the 1900 HEART Conference, Nevel Postereduate Sthool, Monleney, CA, Fr bruary 12-16, 1990)

Monte Carlo radiation transport calculations have been performed to eatimate the effects of natural (protons and electrons) and weapon enhanced (electron) Van Allen Belt (VAB) radiation on a space deployed weapon platform. (The satellite configuration adopted for this study represents the authors' concept of such a system.) The purpose of this study was to evaluate the long term and transient effects of these radiation modes on the components of a satellice deployed in a circular orbit at an altitude of $500 \mathrm{~km}$ and inclination angle of 0 -deg. These tinds of satellites must survive long-term (up to 10 years) exposure to the natural radiation environment and the effects of enhanced radiation introduced when nuclesr weapons are detonated in spece.

This paper describes the piatform/weagon system and its components. the Van Allen Belt spectra, the rrethods of calculation, and numme rizes the rediation damage to platform components from VAB protons and electrons and enhanced electron belt radiation.

Researd apomand t. U.S. Air Foxce.

*Computior end Teleconmmiontion Divinion

4.61

\section{SHILLD OPTIMIZATION PROGRAM PART I: EXECUTIVE SUMMARY}
R. T. Santoro
T. A. Gabriol

(Abetract of ORNL/IM-11143, June 1900)

Detailed atudies have been completed to es timate the radiation-induced damage in senoitive electronic components carried on a space-baned interceplor weapons platform. The architecture of the interceptor wa devined in be as realiatic as ponible. The analynes were completed for natural (Van Allen belt protons and electrons at an orbital altitude of $500 \mathrm{~km}$ ) and man-made (nuclear weapons: neutron, gamma-rays, X-rays, and pumped electron belts) radiation expected to be encountered by a SDI platform.

Studies have aleo been completed to detoxmine the hydrodynamic responses of beryllium mirror surfaces to mono-energetic $X$-rays 91 and $2 \mathrm{keV}$ ). This work included the effects of impurities which are introduced into the surface during machining.

Finally, a compariesn of the HULL and PUFF. TFT codes are presented which includes the thermo-mechanical reaponae of an Al slab to $5 \mathrm{keV}$ black body radiation.

Research aponeored by Department of Defence and Air Force Wrighe Aeroneutical Leboratory. 
4.62

\section{MATERIAL RESPONSE TO INCIDENT X-RAYS}

\author{
M. S. Smith
}

(Abstrad of paper to be presented at the 1990 HEART Conference, Naval Pestgraduate School, Monterey, CA, February 12-16, 1990)

This paper discusses results of hydrodynamic calculations to assess the thermo-mechanical response of various materials to energy loadings from incident X-rays. A beryllium mirror surface impurity study was performed to determine what effect surface impurities contribute to the material response. A second stuly, the LAMPSHADE survivability analysis, was conducted to ascertain whether the satellite debris shield would survive various $X$-ray energy loadings.

Research sponsored by Air Force Weapons Laboratory.

\subsection{3}

SHIELD OPTIMIZATION PROGRAM, PART IV: EFFECTS OF NEUTRON AND GAMMA-RAY RADIATIONS FROM NUCLEAR WEAPONS ON SDI " "EAPON PLATFORMS
M. S. Smith
J. O. Johnson
T. A. Gabriel
J. M. Barnes*
J. D. Drischler
R. T. Santoro

(Abetract of ORNL/TM-10975, March 1989)

Initial studies have been completed to estimate the radiation induced damage in silicon based electronic components onboard a representative Space Pased Interceptor (SBI) weapnn platform. The SBI weapon platform model. used in the studies represents the author's concept of such a system. The analysis was completed for neutrons and gamma rays emanating from a nuclear wearon detonation in space.

Results indicate dose levels to the sensitive components wienin the SBI weapon platform may exceed design limits if the weapon is detonated within a critical radius. For example, a 1962 Starfish detonation at a distance of $91.4 \mathrm{~km}$ from the SBI weapon platform generates a total dose in the central instrument bay of $9 B A \mathrm{rads}(\mathrm{Si})$. The dose rate, $\gamma$, assuming a 40 nsec deposition time, is $1 \times 10^{10} \mathrm{rads}(\mathrm{Si}) / \mathrm{sec}$. Al, of the calculations were car ried out for an unshielded SBI weapon platform to detrermine the radiation levels for which shielding must be designed to ensure survivability of the electronic systems.

Research sporsored by U.S. Department of Defense and Air Force Weapons Leboralory.

* Computing and Telecammunications Divasion.

\subsection{4}

\section{SHIELD OPTIMIZATION PROGRAM, PART V: A HYDRODYNAMIC COMPARISON USING HULL AND PUFF-TFT FOR A ONE-DIMENSIONAL ALUMINUM SLAB}

\author{
M. S. Smith T. J. Burns \\ J. O. Tohnson \\ (Abstract of ORNL/TM-11160, June 1989)
}

Results from the PUFF-TFT code vere compared with thuse from HULL for a 1-dimensional aluminum slab subje ted to $X$-rays from a 5 -keV blackbody source. The source spectra was equivalent to a 1.5-Megaton thermonuclear device detonated at a distance of $10 \mathrm{~km}$. Code predictions of density, internal energy, fracture locations, and axial stress were examined. With the exception of the magnitude of the axial st:ess, results from both codes were in agreement as to che characteristics of the material response.

Research sponsored by Department of Defense and Air Force Wenpons Laboratory.

\subsection{5}

SHIELD OPTIMIZATION PROGRAM, PART VI: THE EFFECT OF IMPURITY LAYERS ON THE HYDRODYNAMIC RESPONSE OF A BERYLLIUM TARGET TO MONO-ENERGETIC X-RAYS USING THE PUFF-TFT CODE
M. S. Smith
5. McNeany*
R. T. Santoro
T. A. Gabriel

(Abat ract of ORNL/TM-1 1038, March 1989)

Calculations were performed using the PUFF. TFT code to determine the thermo-mechanical response arising from incident mono-energetic $X$. rays on a beryllium substrate with a thin $(300 \AA)$ impurity layer. The impurities were introduced 
into the material during typical fabrication processes. Responses were calculated for 5 nanosecond square wave pulses of monoenergetic $X$-rays ( 1 and $2 \mathrm{keV}$ ) with fluence levels corresp nding to surface londinge of 1 and $5 \mathrm{cal} / \mathrm{cm}^{2}$. The presence of these surface impurities was found to significantly alter the thermal response of the medium. As the concentration of surface impurities increased, the energy deposition increased and the temperature gradient increased. In some cases, additional impurities resulted in a phase change in the medium. For the moot part, the mechanical reoponse was unaffected by the surface impurity concentration.

Revenrch eponosed by U.S. DOE Otice of Hid Energ and Nadear Phyice.

* Bninecrias Technolos Divicion
4.66

\section{INITIAL EFFECTS OF NUCLEAR WEAPON X-RADIATION ON THE LAMPSHADE ORBITAL DEBRIS SATELLITE SHIFLD}
M. S. Smith
R. T. Santoro

(Abetract of ORNL/TM-11321, September 1909)

One-dimensional thermal-hydrodynamic calculations have been carried out to estimate the response of the lead bumper plate and tantalum liquidation screen of the LAMPSHADE orbital debris satellite shield. The mass loss fraction in the olid, liquid, and vapor phases as a function of time after irradiation for several typical incident $x$-ray spectra and fluences were calculated using the PUFF-TFT code. The material loseses did not exceed $2 \%$ and fracture and spallation were confined to the surface region with no apparent reduction in the performance of these cornponents against incident debris.

Research eponeored by U.S. DOE and Air Force Weapon Leboratons.

\section{MATHEMATICAL MODELLING OF NOIV-LINEAR SYSTEMS INCLUDING COMBAT}

\subsection{7}

\section{A COMPARISON BETWEEN THE PROPAGATORS METHOD AND THE DECOMPOSITION METHOD FOR NONLINEAR EQUATIONS}

\author{
$\begin{array}{ll}\text { Y. Y. Azmy } & \text { D. G. Cacuci* }\end{array}$ \\ V. Protopopescu \\ [Abatract of ORNL/TM-11316 (in preas)]
}

Recently, s sew formalism for solving nonlinear problems has been formulated. The formalism is baced on the construction of advanced and retarded propagators that generalize the rustomary Green's functions in linear theory. One of the main advantages of this formaliam is the powibility of transforming nonlinear differential equations ints nonlinear integral equations that are usually easier to handle theoretically and computationally. The aim of this paper is ro compare, on an example, the performances of the propagator method with other methods used for nonlinear equations, in particular, the decomposition method. The propagator method is stable, accurate, and efficient for all initial values and time intervals considered, while the decomposition method is unstable at large time intervals, even for very conveniently chosen initial conditions.

Research sponsored by U.S. DOE Office of Energy Rerearch.

"Univenity of California al Santa Barbara, Santa Barbara CA. 
4.68

\section{TWO-DIMENSIONAL MAPS GENERATED BY COMPETITIVE SYSTEMS}

\section{Y. Y. Army V. Protopopescu}

(Abetract of ORNL/TM-11026, Februmy 1969)

By discretizing time and space in a PDE model for competitive systems, we derive the corresponding discrete maps. We analyze these maps from an analytical and numerical viewpoint with emphasis on the military interpretation of the system. For the one-species map we study the effect of convection on the bifurcation behavior and we find periodic solutions. For the two-species map, we limit our analysis to an illustrative example, but we indicate the general procedure.

Reseanch spomeored by Defenese Advanced Research Projects Agency, Application and Computation.

4.69

\section{STATIONARY FLOWS FROM A MODEL BOLTZMANN EQUATION}

\section{Y. Y. Azmy V. Protopopescu}

[Abetract of peper presented at the 16th Ineernational Symposium on Rarefied Ga Dymamics, Pandens, CA, Juls 10-16, 1988, Proc. Progress in Astronatecis and Aeronesties, Vol. 118, pp. 15-28 (1969)]

The one-dimensional Boltzmann equation model of laniro and Lebowitz is generalized by allowing the collision frequency to be an arbitrary positive function of position and by including more general boundary conditions. Conservation laws and explicit solutions are derived, and macroscopic quantities obtained from the stationary BhatnagarGrose-Krook (BGK) variant of the model are compared with available experimental data.

Research sponeored by U.S. Departmenx of Energy.

4.70

\section{EXACT SOLUTIONS FOR A SEMILINEAR HYPERBOLIC SYSTEM WITH GENERAL QUADRATIC INTERAC'TIONS}

$$
\begin{aligned}
& \text { V. C. Boff* V. Protopopescu } \\
& \text { Y. Y. Azmy } \\
& \text { [Abatract of Navo Cimente D (in presa)] }
\end{aligned}
$$

The approximate conservation laws for a binary gas mixturs with quadratic interactions can be written as a system of two semilinear hyperbolic equations. By using the method of characteristics the study of this system is reduced to the study of a system of nonlinear ordinary differential equations, for which we find several classes of exact solutions. Exact integrability conditions are found for ODE systems of generalized LotkaVolterra type. Some situations are also considered, in which the characteristic method cannot be applied and exact solutions are obtained via an operator method.

Rewearch sponsored by Defense Adranced Research Projects Agency Applicution and Computation and U.S. Departinent of Bnergy.

* Bologor Itealy.

4.71

\section{Canonical propagators for NONLINBAR SYSTEMS: THEORY AND SAMPLE APPLICATIONS}
D. G. Cacuci*
V. Protopopescu

(Abatract of peper preachted at the Thind Intermational Workehop on Macheration Appects of Fhid and Pleame Dynamics, Salice Terme, Ilab, Seplember 26-30, 1868)

A new canonical formalism for solving general nonlinear systems is presented. Fundsmental to this formalism is the construction of forward (advanced) and backward (retarded) propagators that yield the problem's solution eractly, by propagating volume, surface, and initial cources. These propagators also satisfy reciprocity and semi-group properties. Therefore, they represent a generalization to nonlinear systems of the Green's functions from linear theory. Several examples are presented to illustrate the application of the algorithm as well as its numerical advantages over alte:native methods.

Research oponeored by Department of Defence Joint Chiefs of Staff and U.S. Degurtment of Enercy.

* University of Califormia, Santa Barbara, CA.

4.72

\section{PROPAGATORS FOR NONLINEAR SYSTEMS}

\section{G. Cacuci* V. Protopopascu}

[Abetract of J. Phyoics A 22, 2399 (1989)]

A canonical formalism based on forward and hackward propagators is developed for problems described by systems of general nonlinear equations. These propagators are shown to yield the 
problem's solution by propagating exactly the bulk/surface/initial sources. They naturally generalize to nonlinear problems the Green's functions of linear theory. Unlike the customary Green's functions, though, the forward and backward propagators depend parameterically and nonlinearly on the problem's solution; however, the propagators themselves satisfy linear equations that can, in principle, be solved by methods of linear theory. Three examples, comprising both scalar and vector problems, are presented to highlight the main points underlying the application of this format ism.

Research sponsorad by U.S. Department of Energy.

*University of Celifornia, Sante Barbara, CA.

4.73

\section{PARABOLIC SYSTEMS WITH NONLINEAR COMPETITIVE INTERACTIONS}

\section{Cosner* S. Lenhart V. Protopopescu}

[Abetract of ORNL/TM-11071, January 1989; ale IMA J. of Applied Math. (in preas)]

A parabolic system with nonlinear local or nonlocal interactions is considered, with specific applications to the description of competition/rombat situations. Global existence and comparison results are proven under suitable assumptions on the interaction terms. Comparisons with solutions of the "equivalent" ordinary difierential (space independent) model, that has been previously used in modelling combat, are of special interest. The stationary state of such a system - corresponding to a stalemate - is also considered. Under certain conditions on the interaction, domain size and boundary conditions, a uniqueness result is obtained for the stationary state. A simple example shows that these conditions are sharp and uniqueness may be lost when they are not fulfilled.

Research sponeornd by L.S. DOE and Defenve Advanced Research Projects Agency.

"Universily of Miami, Miarru, FL.
4.74

OPTIMAL CONTROL THEORY OF LOAD-FOLLOWING AND PARAMETERTRACKING OF NONLINEAR SY STEMS: AN APPLICATION OE PONTRYAGIN MAXIMUM PRINCIPLE TO REACTOR DYNAMTCS
C. March-Leubs*
R. B. Peres

(Abatract of ORNL/TM-10652, December 1987)

A demand-following and parameter-tracking algorithm has been developed which utilies the Pontryagin Maximum Principle (PMP). Starting from a variational principle, we have derived the methodology for the construction of a Hamiltonian function, with the analytical properties required by the application of the Pontryagin Maximum Principle method to Free Terminal Time optimization problems. A crucial result has been the conversion of the two point boundary value problem, typical of the Pontryagin Maximum Principle method for nonlinear systems, into a noniterative initial value problem. The introduction of sensor signals information, as a set of differential equations complementing the model's equation, allows the reformulation of parameter tracking algorithms as control optimization problems, where the demands are the plant signals, and the controls are the time-varying plant parameters. The present algorithm allows correction for the time delays which affect the information flowing from the plant. The control algorithm presented in this paper has been validated against a nonlinear model of a nuclear power plant with time-varying parameters.

Research sponsored by U.S. DOE Office of Reactor Physice and Shielding.

"Univesity of Tennemee, Knoxville, TN. 
NOISE AND NONLINEAR PHENOMENA IN NUCLEAR SYSTEMS

\section{Editors: \\ J. L. Munoz-Cobo* F. C. Difilippo}

[Abetract of book, Series B: Phyices Vol. 192, Plewom Prea, New York (196) ] ]

The main goal of the workshop was to facilitate and encourage the application of recent devetopments in the physical and mathematical sciences to the analysis of determiniatic and stochactic processes in nuclear engineering. in contrat with the rapid growth (triggered by computer developments) of nonlinear analyais in other branches of the physical seiences, the theoretical analysis of nuclear reactors is still bened on linearized models of the neutronies and thermal-hydraulic feedback loop, an approach that ignores some intrinsic nonlinearities of the real syetem. The subject of noise was added because of the importance of the noise technique in detecting abnormalities amociated with perturbations of aufficient amplitude to generate nonlinear procesces.

Thirty-five presentations were discused by participants from fourteen countries and internetional organizations at the Polytechnical University of Valencia (Spain). A crow-fertilization of ideas was obeerved; for example, new stochastic tools to detect nonlinear proceses in nuclear power plants are being developed, whereas the paradigm of noise perturbations in a distorted potential well is being applied to analyze jumping processes in the internal vibrations of nuclear reactors. The papers presented at the meeting fall into some of the following categories:

- Basic methodology to analyze nonlinear deterministic and stochastic processes.

- Deterministic analysis of nuclear dynamics, transition to chaces.

- Noise theory and its application to the surveillance and diagnosis of nulcear systems. The Organizing Committee gratefully acknowledges the financial support of the following institutions: Scientific Afiairs Division of NATO, Dirucceión General de Investigación Científica y Tecnica of Spain, and the Polytechnical University of Valencia. Their contributions made the workshop possible.

Res earch uponuored by NATO Office of Scientific Aftairs.

"Polylectrnical Univerrily of Valenóa, Valencia, Spain.

\subsection{6}

\section{GLOBAL EXISTENCE FOR SYMMETRIC DISCRETE VELOCITY MODELS}

\section{Srotopopeseu}

[Abatract of paper in the Proceedings of the Wortshop on Discrete Models Lettixe Ges Dymomics and Foundations of Hydredymaics, Torino, Inth, Seplember 19-24, 1988, p. 258, World Scientific, Singapore (1999)]

For symmetric discrete velocity models, some generalizations of previous global existence proofs are given to include bounded geometries, continuous models, and stationary states with no momentum conservation. Additional results and simpler proofs are obtained for completely symmetric models.

Research sponsoned by Organization of the Joint Chicfs of Staf, Department of Defene.

\subsection{7}

\section{AN EXAMPLE OF A REACTION- DIFPUSION SYSTEM WITH NONLINEAR COMPETITIVE INTERACTIONS}

\section{Protopopescu}

(Abatract of paper preasted at the 11 ith International Confereace on Tranaport Theory, Blackeburg. VA, May 22-26, 1989)

An example of a parabolic system with nonlinear local or nonlocal interactions is considered, with specific applications to the description of competition situations. Under suitable assumptions on the interaction terms, one can derive global existence as well as a comparison result between the PDE solutions and the "lumped" (space-integrated) ODE solutions. The uniqueness issue for the stationary state of the system - corresponding to a stalemate - is also considered. The discrete version of the system is then constructed and some preliminary results for the two-species one-index map and for the one-species two-index map are discussed.

Research iponsored by Defense Advanced Research Projects Agency Applications and Computation and U.S. Departmene of Enercy. 
COMBAT MODELING WTTH PARTIAL DIPFERENTIAL EQUATIONS

\author{
V. Protopopescu R. T. Santoro \\ J. Dockery * L. Cox' \\ J. M. Barnest
}

(Abotrect of ORNL/MK-10056, Noneaber 1857)

A new analytic model based on coupled nonlinear partial differential equations is proposed to describe the temporal and spatial evolution of oppoing forces in combat. Analytic deacriptions of combat have been developed previoualy uing retatively simplex models baned on ordinary difierential equations (e.s., Lancheater's equations of combet) that capture only the global temporal ravition of the forces, but not their spatial movement (advance, retreat, flanking maneuver, etc.). The rationale for analytic models and, particularly, the motivation for the present model are reviewed. A detailed description of this model in terms of the mathematical equations together with the ponible and plausible military interpretation are presented. Numerical solutions of the nonlinear differential equation model for a large variety of parameters (battlefield length, initial force ration, initial apatial distribution of forces, boundary conditions, type of interaction, etc.) are implemented. The computational methods and computer program are deacribed and the results are given in tabular and graphic form. Where posible, the reaults are compared with the predictions given by the traditional Lanchester equations. Finally, a PC program is deacribed that veses data downloaded from the mainframe computer for rapid analysis of the various combat scenarios.

Research eponeored by U.S. DOD Organizetion of the Joint Chich of Staff, Command, Conerol, and Commanicationas.

-Organization of the Joine Chinefo of Star, Command, Conenol, and Communications, The Pentrgen, Wahingen, DC.

tComputing and Telecommunicutions Division.

\section{TWO-DMMENSIONAL COMBAT MODELING WITH PARTIAL DIFFERENTIAL EQUATIONS}

\author{
P. Rusu"
}

(Abatrect of CANL/TM-10973, December 1968)

The system of partial differential equations introduced to raodel combat in one spatial dimension has been extended to include two spatial dimensions and has been numerically integrated to demonstrate its capability to decribe maneuver. The analysis of a turning maneuver is demonstrated.

Research eponored by Defere Adranced Research Projects Agency Applications and Competation.

-Univesies ef Coloredo, Boalder, CO.

\subsection{0}

\section{MATHEMATICAL DESCRIPTIONS OF OPFENSIVE COMBAT MANEUVERS}

\author{
R. T. Santoro P. Rusu* \\ J. M. Barnes ${ }^{\dagger}$
}

(Abatract of ORNL/TM-11000, January 1809)

Mathematical descriptions of several forms of offensive maneuvers have been calculated uing a recently developed tro-dimensional nonlinear partial differential equation (PDE) model for describing confliet between opposing forces. Engagement cenario wherein the attacking force(s) employs the frontal attack, turning movement, envelopment, or infiltration against a fixed defensive force are presented for various combinations of troop and firepower ratios. The time and spatjal dietributions of the forces are displayed in graphical form along with approximate attrition rates as a function of battle duration. The results eatablish that the PDE iormalism replicates these maneuver forms within the constraints and present development of the model.

Research sponeored by Defence Advanced Research Projects Agency Applications and Computation.

-Univenity of Colorada, Boulder, CO.

†Computing and Telerommunication. Division. 
4.81

NONLINEAR MAPS WTTH COMPETITIVE INTERACTIONS: FIXED-POINTS, BIFURCATIONS, AND CHAOTIC ATTRACTORS

$$
\begin{aligned}
& \text { D. F. Scollan* Y. Y. Azmy } \\
& \text { V. Protopopescu }
\end{aligned}
$$

(Abetract of ORVL/TM-11315, Seplember 1989)

We examine several aspects of the dynamical behavior of competitive systems modeled by nonlinear maps. In the second chapter of this report, we analytically investigate some features of spe cial cases of such maps, and we use the symbolic manipulator MACSYMA to derive expressions for the linearized systems' eigenvalues. These enable us to determine the linear stability of some of the fixed and periodic points of these maps, and in some cases, yield the regions of attraction in phase space.

In Chapters 3-5 we extend the analysis to a more general form of competitive systems. The two-species one-index system and the one-species two-index map are numerically explored in search for interesting bifurcation and/or chaotic regimes over a large range of parameters. We will show that the system (1-1-4) with linear attrition displays stable, interesting behavior for a large re gion of parameter space even if the quadratic selfrepression is taken very small. We will also show that small quadratic self-repression combined with bilinear attrition generally results in steady-state solutions with one species zero or in unbounded iterates.

In Chapter 6, we describe the method used to obtain an approximate estimate of the correlation dimenaions of the chactic attractor presented in Chapter 3. We also propose a new method to evaluate the correlation dimension of a cheotic attractor using statistical analysis methods.

Resenurch sponecred by Defense Advanced Research Projects Agency Application and Compatation and U.S. DOE.

*Penn State Univerity, University Park, PA.

\section{NUCLEAR WEAPON EFFECTS}

\subsection{2}

\section{MASH, THE MONTE CARLO ADJOINT SHIELDING CODE}

\author{
M. B. Emmett $\quad$ W. A. Rhoades \\ J. O. Johneon
}

(Abatrant of paper presented at the ANS Topical Meeting on Advances in Nuclear Encineering Computation and Re diation Shielding, Sante Fe, NM, April 9-13, 1989; Proc. Vol. 1, pp. 42:1-42:10 (19e0)]

The Monte Carlo Adjoint Shielding (MASH) code calculates protection factors for armored mit itary vehicles by coupling a forward discrete ordinates calculation with an adjoint Monte Carlo calculation. The discrete ordinates calculation determines the fluence on a coupling surface surrounding the vehicle due to an external neutron/photon source. The Monte Carlo calculation determines the effectiveness of the fluence at that surface in causing a response in a detector within the vehicle, i.e., the "dowe importance" of the surface fluence. A coupling code folds the fluence together with the done importance, giving the desired dose response. This coupling code can also rotate the vehicle, move it to different distances from the source, and perturb the energy response of the detector.

Research eponeored by Defenee Nuclear Agency.

* Computing and Telecommunication Divicion.

4.83

\section{A RE-EVALUATION OF ${ }^{32} \mathrm{~S}(n, p)$ CROSS SECTIONS FROM THRESHOLD TO $5 \mathrm{MeV}$}

\section{Y. Fu}

(Abatract of paper presented at the ANS Topical Meetin on Advances in Nuclear Engineering Computation and Re diation Shielding, Sante Fe, NM, April o-13, 1989; Proc. Vol. 1, Pp. 17:1-17:11 (1089)]

Two evaluations of the ${ }^{32} S(n, p)$ reaction crows sections, presently being used for the Nagasaki and Hiroshima dosimetry studies, are of low quality and are in serious disagreement. The present reevaluation of these croses sections was intended to be the most advanced possible and was based on 
an exhaustive dala search that uncovered an additional data set, a Bayrs-theorem code for combining experimental data with uncertainties, a special procedure for including the effects of energy resolutions in data averaging, and the application of the much-improved ENDF/B-VI standards. Correlations in the experimental data were evaluated and tested for their effects but were mat noed for the final results because such correlations caused erross in the presence of resonances. The new results below $5 \mathrm{MeV}$ (the upper limit of the present need) were combined with the ENDF/B-V dosimeiry data above $5 \mathrm{MeV}$ to generate a complete file, including covariances from threshold to $20 \mathrm{MeV}$. These point data were processed into two VitaminE 174-group sets, one with a constant energy weighting and the other with a Marmell-1/Efirsion weighting.

Research aponsored b, Defene Nuclear Agency.

\subsection{4}

\section{FALLOUT FACTS FOR NUCLEAR- BATTLEFIELD COMMANDERS}

\author{
C. M. Harland \\ [Abetract of ORNL/TM-11193 (in preas)
}

The basic phenomenology of radioactive falt out from nuclear detonations in described nonmathematically for military commanders who may become engaged in nuclear battle. Subjects include a description of fallout debris, the process of formation and deposition, and hazards to perronnel. Comparicons are made between initial nucleas radiation and radiation from fallout. A radiation sate and exposure forecasting method is presented for situations where the source of fallout and rate of decay are unknown. Additional research is recommended for (1) development of improved methods for estimating casualties resulting from combined exposure to initial nuciear radiation and falk out radiation; and (2) improving the eatimate of the K-factor (how much of the radioactive material comes to the ground as early fallout).

Rresearch sponeored by U.S. Department of Enercy.
4.85

\section{AN APPROXMMATION FOR BLACK-BODY X-RAY TRANSPOFT IN AIR}

\author{
C. M. Halaut R. T. Santoro \\ J. M. Barnes"
}

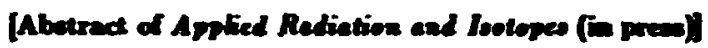

This paper deseribes a cimplified method for approximating the air-traneported $x$-ray spectrum from nuedear detosations in the atmoophere. The carly fireball of a nuclear detonation emits intenese I-ray radiation that can be approcimated by the apectrum from a black-bods at a temperature of around $10^{-7}-10^{-8} \mathrm{~K}$. $A$ number of procedures have been devived for calculating the traneport of photos and neutron in varions media $T$. bles have been prepared that provide the x-ray flux at a diatance $R$ from a muclear detonation in the atmonphere for a limited et of parameters. The method deacribed here reduces computational time such that a deak-top computer can provide results that match detailed Monte Carlo calcule tions within $\pm 10 \%$ for a wide range of condition. The approximation requires a computation time of 1-2 seconds on a deat-top computer with numerical coprocessor, whereas the comparable calcula tion using the Monte Carlo code typically requiren 10-15 seconds on a Cray computer.

Reseanch eponored by U.S. Air Ponce and U. S. DOE OAive of Becic Bantr Sciences.

"Computing and Telecommanication Diviaion

\subsection{6}

\section{AN APPROXIMATION FOR BLACK-BODY X-RAY TRANSPORT IN AIR}

\section{M. I Iaaland R. T. Santoro J. M. Barne:"}

(Abstract of ORNL/TM-10310, Auguat 1998)

A simplified method is presented for approximating the air-transported $x$-ray spectrum from nuclear detonations in the atmosphere. Computational time is reduced such that a deak-top computer can provide results that match, within $\pm 10 \%$ for a wide ranse of conditions, Monte Carlo 
results which require about the same computation time on a Cray. The method consists of (1) representing the black-body contineons spectrum by discrete emiscion windows, (2) conoulting tables of photon buildup factors for the diverete enision energies, (3) unfolding the buildup photons by using air berma respone function and an esumption for redietribeting these photons to windows of lower energs, and (4) applying colofi and weighting factors to improve the correlation with the Monte Carb benchmart caleulations.

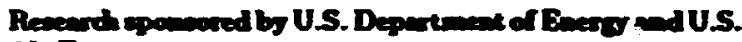
Air Ponce.

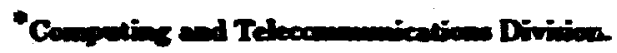

\subsection{7}

DABL69: A BROAD-GROUP NEUTRON/ PHOTON CROSS-SECTION LIBRARY FOR DEPENSE NUCLBAR APPLICATIONS
D. T. Ingerwoll
R. W. Rousin
C. Y. Pu
J. E. White
(Abetrect of ORNu/TuL10ses, Jese Ised)

A new multigroup crom-section librery has been generated from ENDF/B-V data for we in defense-related radiation shielding applieations. The library is available in a broad-group (46neutron, 23-photon) energy structure and is der. ignated DABL68. The extablishment of epecificetions for the library, expecially the apecific group structure and energy weighting functions, we an important part of the generation of the library. The energy group structure containa some special tailoring to the oxygen and nitrogen crow rections and improved energy realution in the range of 100-1000 keV where the iron croms section is highly structured. The library contains mont materials of importance in defence-related shielding problem and includes several reference cource spectra and responef functions. The library is available in two formats including the commonly used ANISN format and the more versatile AMPX mater format.

\subsection{8}

SENSITIVITY/UNCERTAINTY ANALYSIS FOR FREE-IN-AIR TISSUE KERMA DUE TO INITUL RADIATION AT HIROSHMA AND NAGASAKI
R. A. Lille
B. L. Broadhead"
J. V. Pace, III*
D. G. Cacucit

[Abotract of Ned. Sci Bng. 100, 105 (1800)]

Uncertaindy eximates and cross correlations by range/survivor have beea calculated for the Hirohima and Nagacali free-in-air (FIA) tione brrme obtained from tro-dimencioas air/ground tranoport calculations. The uncertainties due to modeling parameter and baic nuclear trasuport date uncertainties mere calculated for 700-, 1000. and 1500-m ground ranges. Only the FIA tiane kerms due to initial rediation wes trealed in the andysia; the uncertainties anociated with terrain and building chielding and phantom attenuation were not considered in this atudy. Uncertainties of 20\% were oblained for the prompt neutron and secondary gamm kerme and 30\% for the prompt gamma kerma at both cities. The une" "ainties on the total prompt kerma at Hirochims and Na gacali are $\sim 18 \%$ and $15 \%$, respectively. The eatimated uncertaintie vary oaly alightly by ground range and are fairly high correlated. The cotal prompt kerma uncertainties are dominated by the cecondary gamma uncertainties, which in turn are dominated by the modeling parameter uncertaintien, particularly those anociated with the weapon yield and radiation cources.

Reseanch eponeored by U. S. DOE Ofice of Environmental Rewench and Development.

\footnotetext{
- Compotins and Telecommunications Diviaion.

Univereity of California af Santa Berbere, Santa Barbere, CA.
} 
$4 \times$

\section{RADLATON EXPOSURE INSIDE RENTORCED CONCRETE BUILDINGs at NagasakT}

\author{
W. A. Rloodes R. L. Child: \\ D. T. Ingared
}

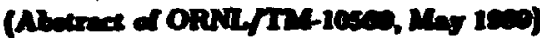

The biologieal efiects on the reideate of fir-

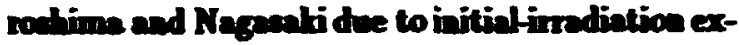
ponse during the muelear attecks of Word Whr II were recogaived immedietely $\boldsymbol{\omega}$ an important onver of information. Aiter the war, an extesive efort gothered data concening the beations of intivituals at the tine of the attack and their

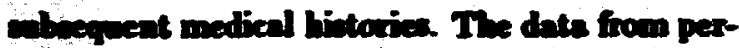
cond located in reinforced concrete buildiags are particalarty sigifieant, since large groups of oeenpanter received radiation injury withont couplicetion due to blat and thermal efecte.

In order to correlate the radiation does with phyiologieal effecte, the dome to each individad and be calculated. Beough information about the conctruction of the building wos areilable of ter the war to allow a radiation traseport model to be condructed, bot the sccurate calculation of pewetration into weh large, thict-wnlled threodimencional struetures wes beyond the seope of computing lechnology until recently. Now, the anilability of Cray vector compaters and the development of a specially-conatructed diecrete ordinates traneport code, TORT, have combined to allow the succesifol completion of auch a etudy.

This document deacribes the radiation traneport calculations and tabulates the resulting dose by cource component and individual cace beation.
An extensive uncertainty analysis is also included. These data are to be used in another study as input to a formal statistical analyais, reoulting in a new value for the LD50 doce, i.e., the doee at which the mortality rist is 50\%.

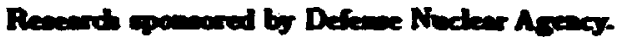

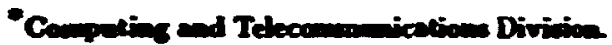

\subsection{0}

\section{NEW DOSE MORTALTY DATA BASBD ON 3-D RADIAMION SHIILDING CALCULATION POR CONCRETE BUILDINGS AT NAGASAXI}
W. A. Rhoudes
1. I. Chids"
D. I. Ingerwal

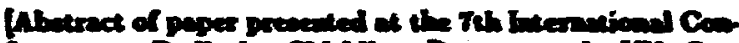

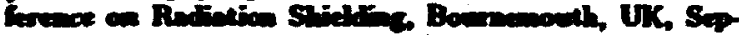

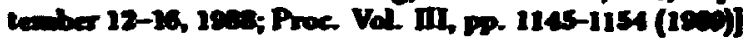

The analycis of radiation dones received during the World War II attack on Nagacali provides an important source of biomedieal information. More than 40 years after the war, it has been ponjble to male a sativfactory calculation of the dones to personnel inside reinforced concrete boilding by une of a 3-dimencional diverete ordinates code, TORT. The results mere need to deduce a wew value of the LD50 parameter that is is good agreement with tradistional values. The new discrete ordinates coftrure appears to have potential application to conventional radiation tranoport caf culations a well.

\footnotetext{
Reward mponored by Defene Nuclear Acency.

"Compotion and Telecommunications Divicion.
} 


\section{RISK ASSESSMENT AND REACTOR SAFETY}

4.91

USER'S GUIDE FOR PRISM ARKANSAS NUCLEAR ONE-UNTT 1: VOL. 1-PROGRAM POR INSPECTORS VOL 2-PROCRAM FOR REGULATORS
D. J. Camplen"
J. R. Kirehne:
H. M. Paula*
v. H. Guthrie*
J. Q. Kirloman"
B. C. Ellieon"

P. M. Dyeus" J. A. Rarquhareon*

\section{G. F. Flanagan}

(Abutrect of ORNL/TM/10004/VIE2 (NUREG/CR-5021, Valk 1 \& 2), Mand 1804

These uner's guides are derigned to teach NRC inepectors and NRC regulators how to access probabilistic risk ascexsment information from the two Plant Rick Status Information Management Syatem (PRISIM) programs developed tor Artaneas Nuclear One - Unit One (ANO-1). Volume 1 describes how the PRA information available in Vercion 1.0 of PRISIM is useful for planning inspections. Using PRISIM, inepectors can quictly access PRA information and ue that information to update risk analysis results, reflecting a plant's status at any particular time. Volume 2 deacribes how the PRA information available in Version 2.0 of PRISIM is useful as an evaluation tool for regulatory activities. Using PRISIM, regulatore can both acces PRA information and modify the information to aweas the impact these changes may have on plant safety. Both volumes are stand-alone documents; each volume presents several sample computer seasions designed vo lead the ueer through a variety of PRISIM applications used to obtain PRA-related information for monitoring and controlling plant risk.

Research eponeored by U.S. Nuckear Regulecory Comminsion, Ofice of Nuclear Regulationy Resench.

* JBF Aesociales, Inc., Knowville, TN.
4.92

\section{RISK ASSOCLATBD WITH \\ OPERATION OF THE OAK RIDGE \\ NATIONAL LABORATORY HIGH \\ FLUX ISOTOPE REACTOR (HFIR)}
G. F. Managan
D. Johnoon"
D. Buttemer"
S. Kaplan*

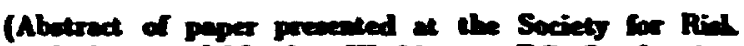

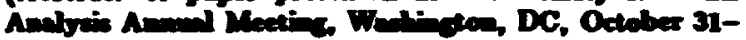

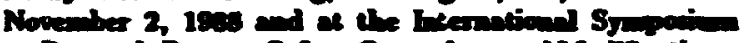

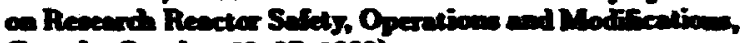
Concde, October 23-27, 1600)

A level 1 Probabiliatic Rist Amecoment (PRA), excluding external eveate, we performed on HFIR. Thi was the fint PRA on a Department of Bnergy research reactor. Several unique features of BFIR resulted in new initiating erente not found in the standard commercial nuclear reactor. Mout of these events involved core flow blockages and fuel manufacturing and inspection errors. Innovative techniques mere developed to quantify these sequences. As a result of the PRA calculations, changes were made in the HFIR design and operating procedures which revulted in increaced confidence in the safety of the reactor. The HFIR PRA has provided a quantative framowork for making riak baced management decicions for future (pont restart) deaign modifieations.

Reweand eponeored by U.S. Departwest of Enersy.

"Picland, Lowe and Garid, Inc., Newport Beach, CA.

\subsection{3}

USE OF RISK ANALYSIS RESULTS IN SUPPORT OF THE PINAL PROGRAMMATIC ENVIRONMENTAL IMPACT STATEMENT FOR THE DISPOSAL OF THE U.S. CHEMICAL WEAPONS STOCKPILE
G. F. Flanagan
S. Carnes"

(Abotract of peper prenented at the Nationd Meeting of the American Inatitule of Chemical Enginass, Deaver, CO, Anguat 22-25, 1028)

Risks ancociated with the disposal of the U.S. Chemical Weapons Stockpile were a major input 
into the selection of the programmatic environmentally preferred alternative as stated in the objectives of the President's Council on EnvironmenEl Quality regulations implementing the National Environmental Poliey Act (NEPA). The alternatives considered were presented. Five measures of rish were used in the comparion of alternatives. Several risk mitigation measures mete identified and the U.S. Army agreed to implement several of these. The final comparison between alternatives are presented in the paper. The no-action alternative pooed the largest riak for all the five rish meacures. The on-site diepocal alternative and the regional dieposal alternative reonlted in the lead riak for all the riak measures when all the uncertainties are included.

In addition to the overall programmatic riat, rish asociated with each alterative at each site are presented. In this case, the continued storage (no-action) alternative still results in the largest rist for all the risk measures. However, the on-site dipposal alternative does not necenarily result in the lowest riak of the posible alternatives for all ites if the r.ncertainty in the analysis is considered.

Other factors, in addition to the riaks to heman health, had to be considered in making the final selection of an environmentally preferred at tenative. These factors, the logic argumente used, and methodology developed were described.

Revenuch sposeored by U.S. Army Program for Chemical Munitions.

* Enerra Divicion.

\section{RISK ASSOCIATED WITH THE DEMILITARIZATION OF THE UNITED STATES CHEMICAL WEAPONS STOCKPILE}

\section{G. F. Flanagan W. E. Fraize" T. Kartachak ${ }^{\dagger}$}

(Abutrect of Reliability Enpineering and Syotem Safety (in prese); and sleo paper presented at the Society for Riak Analyais Annual Meeting, Washington, DC, October 31November 2, 1388 and at the Air Poltution and Watc Merecement Control Awocietion 82nd A nnwal Meeting and Exhibition, Anaheim, CA, June 25-30, 1880)

A full scope probabilistic risk ascessment was performed in order to determine the public risk aceciated with the congreasionally mandated unitary chemical weapons demilitarization program.
The method to be used for destruction of the chemical agents is high temperature incineration. The rist assessment was specifically used to assist in determining if any emvironmentally preferred alternative exioted defined by regulations implementing the $N$ r tional Environmental Policy Aet. The alternatives examined included the construction and operation of a single national incinerator with agent shipped by rail, two regional incinerations with rail trameportation of agent, eight on-aite incinerators beated at the current eight U.S. Army depot atorage bocations, and the no action alternative defined for this study as continued atorage for 25 years. Accidents realting from handling, tranportation, and plant operation, well as; thowe resulting from external events were considened. Several meanures of riak were analyzed and innovative methods of displaying the rewalts to the general public were uned in the final reports. Results indicated that the continued storage af ternative presented the largeat risk with the onsite incineration alternative presenting the lead risk. Expected fatalities ranged from greater than $10^{-2} / \mathrm{yr}$ for the continued storage alternative to less than $10^{-4} / \mathrm{yr}$ for the on-site dispoeal alternatives. Braujmum fatalities were greater than 10,000 for the sontinued storage alternative at some sites.

As a result of the risk aveasment and the lom likelihood for successful response to accidents occurring off-site, the on-site incineration alternative was seleeted as the environmentally preferred alternative.

Revard sponsored by U.S. Amms.

- MITRE Corp., Melear, VA.

U.S. Army, Aberdeen, MD.

4.95

\section{THE PROBABILISTIC TREATMENT OF POTENTIAL ACCIDENTS: HOW, HOW MUCH, AND HOW OFTEN COULD CHEMICAL AGENT BE RELEASED TO THE BIOSPHERE!}

\author{
W. E. Traize* G. F. Flanagan
}

[Abetreat of Environmentel Profensionel (in preal)]

The rationale for, and the approach used in, the risk analyais of the Army's Chemical Stockpile Disposal Program (CSDP) is described. The 
papa iscusses the major features of the CSDP that affected the design of the rist analyais, principal among which are the wide range of potentially risky activities, the use of multiple sites connected by transportation corridors, the lon probability high consequence nature of moat hasards, the lack of a historical data bace of actual secidents involving chemical agent releane, and the presence of energetic materials (explosives) in conjunction with the chemieal agent. The importance of communicating the revults of the rict analynic to a broed audience spectrum and the impact those conaiderations had on the choice of rich mewenres and the wey they wae portrajed is dexcribed.

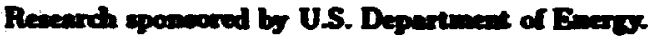

- Mritre Copporation, Mdear, VA.
4.96

\section{A REVISIT TO THE; PASSIVELY-} SAFE REACTORS

\author{
D. L. Moses
}

(Abativet of ond presentulion presented to the Chat.

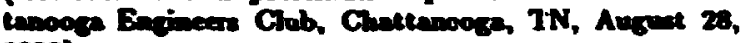
1800)

International activity in the development of pascively-aafe reactors is reviewed over the past two years. Rexitor options and their development status are noted.

Rewend spoumosed to U.S. Depertmeat of Enesy. 
Section 5

ENGINDERING PHYSICS AND MATHEMATICS INIORMATION CBNTERS 


\title{
5.0. INTRODUCTION
}

\author{
R. W. Roussin
}

Information analysis and data management, important activities of the division since its inception, comprise a significant fraction of the division's program. Under the managernent umbrella of Engineering Physics Information Centers (EPIC), we opereted two specialized centers during the reporting period, October 1, 1987 - August 31, 1989. The Radiation Shielding Information Center (RSIC), founded in FY 1963, has served the international scientific cummunity as a technical institute for shielding design and analysis. The Technical Data Management Center (TDMC) was founded in FY 1978 to perform various tasks in support of Nuclear Regulatory Commission (NRC) programs in technical data management and computer code development, standardization and maintevance. Residual tasks started within TDMC were completed during FY 1988.

\section{Radiation Shielding Information Center (RSIC)}

During this reporting period, RSIC continues to be widely used as attested by current user-statisties. Usage reported at the end of FY 1989 showed that the total number of requests processed during the year (3419) exceeded those in FY 1988 (3244) by 5\%. The statistics indicate that the individual user is requesting more products and/or services; activities required to fill the requests increased by $4 \%(12,660$ vs 12,194). The increased usage is largely attributed to waste : nanagewent . und defense-related programs.

Eotablished to promote the exchange of shielding technology, RSIC acts as a resource base for both government and civilian agencies in the United States and in foreign countries and performs such diverse functions as testing, assembling, and distributing computer codes; preparing, testing and distributing multigroup cross-section libraries, both fine-group and broad-group; collecting and distributing other types of data bases; hulding seminars to educate the shielding community of particular techniques, especially computerbased techniques for solving radiation transport or nuclear data problems; belping to establish shielding benchmark problems and shielding standards; providing bibliographic information; and generally providing problem assistance to requesters. Staff members also engage in radiation transport developments and perform radiation transport studies, often in collaboration with staff members in EPMU as well as other institutions.

The RSIC scope includes the physics of interaction of radiation with matter; radiation production, protection, end transport; radiation detectors and measurements; engineering design techniques; shielding materials properties; computer codes useful in research and design; and nuclear data compilations. The goals of RSIC are to function as a technical inotitute to provide information (computer codes, technical advice, bibliographic and other (ata,) upon request; collect, evaluate, enrich, distill, and repackage iniormation to extend the state of the art, bringing into the public domain technology more isesble and more valuable than the sum of the input; and to initiate and effect research and development in appropriate areas of need.

During this reporting period work has continued in tine developinent of fine- and broadgroup cross-section data libraries. The 174 neutron, 38 gamma ray group VITAMIN-E general- purpose cross-section library based on ENDF/B-V now contains 94 materials. It has been used with success for fusion neutronics, fast reactor core and shielding applications, and various other radiation transport calculations within the division and extemally. During the reporting period, broad-group cross-section libraries for fast reactor 
shielding (VELM61 and VELM22) and defense applications (DABL69) were derived from VITAMIN-E and made available through RSIC.

RSIC has continued to participate in the work of the Cross Section Evaluation Working Group (CSEWG), with the Methods and Formats Subcommittee chaired by the RSIC director. Other staff members are actively involved with the ANS in developing radiation protection and shielding standards and standards for scientific computer programming and its documentation.

In the area of computing technolozy, RSIC now makes available 816 computer code packages and 146 data packages. The RSIC Newsletter is currently mailed monthly to 1650 people. A total of 98 persons (22 foreign, 76 domestic) came during the reporting period for an orientation visit and /or to use the center's facilities, an average of 49 persons/year.

In terms of reciprocity in international information exchange, a significant percentage of all incoming computing technology (codes and data) comes from foreign contributors, with Jar'n and France continuing in the lead. Much of the new technology is of domestic interest.

The RSIC computer-based information system (SARIS), containing more than 23,000 literature references, is used in support of internal research activities and user services. Seven consecutive indexed bibliographies have been published and data files have been constructed in preparation for publication of the eighth in the series. SARIS, formerly accessible nationally on the now-defunct DOE RECON system, je currently accessible for a search on demand service.

RSIC interacts daily with, and contimues to have the support of, its user community. Participants contributed their publications and 184 transmissions of technology as follows: 80 new computer codes and data libraries; 20 replacements of existing code/data packages with newly-frozen versions; 20 new hardware versions or other extensions to existing code/data pachages; 39 updates to improve existing code/data packages; and 25 corrections to fix errors in existing code/data packages.

Announcement of availability was made for 141 transportable, tested packages of new or revised computer code systems and data libraries, including contributions received in the previous fiscal year. It should be noted that the same evaluation, computer testing, and packaging is followed for updates to existing code pacloges as for new technology.

\section{Technical Data Management Center}

TDMC was established in July 1978 to serve NRC programs with goals and priorities determined by an advisory committee of NRC technical staff members chaired by the contract monitor within the Division of Technical Information and Document Control of the NRC Office of Administration. Its primary purpose was to support NRC programs by assisting in verification, validation, documentation, and standardization of computing technology (including codes and other technical data) used in the licensing and regulatory processes. All tasks assigned by the contract monitor in FY 1987 were completed.

A subtask included calculations to generate data for the proposed ANS-6.4.3 standard, "Gamma-Ray Attenuation Coefficients and Buildup Factors for Engineering Materials," a collaboration with the ANS-6 Standards Subcommittee. The working group has selected data sets from NIST and from Japan with other data serving as validation. The G$P$ buildup factor coefficients were determined in Japan. The G-P technology has been 
implemented in the QAD- CGGP code system which is available from RSIC as is the 1988 data base as a data library. A report of the compilation effort, including discussion of the technical issues and the draft standard, has been published as ORNL/RSIC-49.

When the new photon cross section library PHOTX became available, the Japanese colleh vrators recalculated the buildup factors for the elements molybdenum to uranium. They gave careful attention to the behavior near the $K$ edge where the buildup factors become extremely large. The latest draft of the standard also includes correction factors to compensate for the neglect of coherent scattering based on calculations of collaborators in India. Coefficieuts for the Taylor buildup factor function have recently been published in WAPD-TM-1628. A selection of the data will be made on the basis of sufficient accuracy to include in the standard. The final draft is expected to be released for ANS-6 approval in early 1990. 


\section{RADIATION TRANSPORT DEVELOPMENTS}

5.1

\section{UISCRITE ANGLE BIASTNG IN MONTE CARLO RADIATION TRANSPORT}

\section{S. N. Cramer}

(Abatrect of ORNL/ASTC-50, May 1806)

An angular bianing procedure is presented for ose in Monte Carlo radiation traneport with divcretised scattering angle data. As in more general ctudies, the method is shown to reduce statiatical veight fluctuations when it is combined with the exponential traneformation. This discrete dats application hes a simple andytic form which is probiem independent. The rewults from a sample problem illustrate the variance reduction and eficiency characteristics of the combined biasing procedures, and a large neutron and gamma-ray integral experiment is also calculated. A propocal is given for the possible code generation of the biasing parameter $p$ and the preferential direction $\bar{\Omega}_{0}$ ased in the combined biasing sechemes.

Reweand sponered by Defense Noclenr Agency.

5.2

\section{ANALYTIC SOLUTION OF A FIVE-DIRDCTION RADIATION TRANSPORT MODEL}

\section{S. N. Cramex}

[Abetract of Trans. Am. Nweh. Soe. 87, 232 (1903)]

To teat certain apatial and angular-dependent Monte Carlo biacing techniquies, a one-dimensional, one-ener gy, two-media, five-direction radiation transport model has been devieed for which an analytic solution exists. Although this solution is too long to be conveniently expressed in an explicit form, it can be eavily evaluated on the smalleat of computers. The salient features of the solution are that it involves only a $4 \times 4$ determinant, a quadratic equation, and two sets of four linear algebraic equations. The Monte Carlo simulation of this transport model is very simple and also applicable to small computers. This model gives an analyat the ability to leat certain features of biasing techniques and code generation of bias ing parameters, involving directional dependence and changes in crow-ection media through many mean-free-paths of particle penetration. The comparison of results with an exact solution eliminates many of the uncertainties and mout of the expense asociated with the colution of realistic calculstions wing general Monte Carbo codes on mainframe computers.

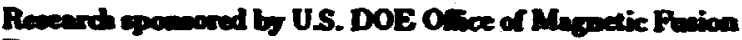
Bung.

\section{3}

\section{hypgRcube appications of THE कथ GEOMPTRY NODAL MBTHOD FOR THE NEUTRON DIPTUSION EQUATION}

\section{B. I. Kint Y.Y. Asmy}

[Abatrect of Trase. Am. Neel Soc. 57, 104 (18as)]

Solving the one-group neutron diffusion equar tion by the sodal method requires finding the solution of a syatem of three equations: the z- and 5 current contimity and the conservation equations. This requires matrix inversion. For a very coanse mesh size, the solution may take only a few seeonds. As the meah gets finer, the aystem of equations grows in the number of unknowns resulting in a matrix of very high order. On serial computers, matrix invervion is limited by the amount of memory. The CPU time for large syotem of equations can also become probibitive. Thun, a new parallelized iterative algorithm is developed.

We have derived and teated an algorithm that is truly parallel, but its application on the hypetcube is greatly reatrained by meanage pacoing. Our reoults augzeat that there is an upper limit on the number of proceacons that may be used on hypercube. If follows that extremely large epeedupe characteriatic of hypereube implementation cannot be expected and that relatively low efficiencies are produced unles very large problems ( $M$ $\sim 100$ ) are solved. It may be that parallel procersors with shared memory are more euitable for the problem.

Reserach aponeored by U.S. DOE Ofites of Magnetic Puaion Enersy. 
5.4

\section{AN ITERATIVE PROCBDUR: FOR SOLVING THE NODAL METHOD NEUTRON DIFPUSTON BQUATION ON A PARALLI PROCBSSOR}

\author{
B. L. Kirk Y. Y. Army
}

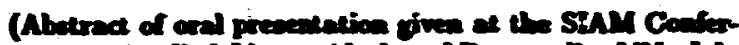

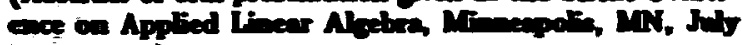
11-15, 1800)

Nodal methods have been shown to be a powerful computational tool in solving the nentron diffusion equation. Solving the nodal equations uins matrix invercion subroutines heavily taxes the computer on menory and CPU time, even for relatively coance meches. We derive an iterative procedure that iterates on the cell-averaged and the cell-eurface fuxces. In each iteration, we wolve a set of tridiagonal matrix equations reprecenting row or columne of computational cells. Since these are uncoupled, they can be colved in parallel. We solved a teat problem and compared the required CPU time uxing DGTSL (LINPACK) and the new iterative method. For all methes finer than $12 \times 12$ the latter required shorter CPU time than the former; for example, on an $18 \times 18$ mech 696.4 and 1609.9 s were required, respectively. In addition, the inversion method for a $20 \times 20$ mech (matrix order 1160) requires storage of abcut 1.4 MW, posibly exceeding the capacity of a serial computer. The same computer can solve much finer meshes with our secheme.

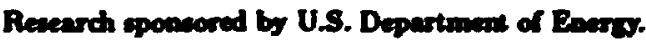

5.5

\section{A PARALLEL APPROACH TO THE NODAL METHOD SOLUTION OF THE TWO-DLMENSIONAL DIFTUSION EQUATION}

\section{B. L. Kirk Y. Y. Asmy}

(Abaract of peper preanead at the Amariena Nuelear Sodety Topied Mating on Advence in Nucleser Enginow. ins, Computation, and Radiation Shideling, Sane Fe, NM, Aprl o-13, 1000; Proc. Vol. 1, pp. 23:1-23:12 (1000)]

The neutron difiusion equation in two-dimensional geometry is solved using the nodal method formaliom. Three syotems of equations arice in the solution: the s-current continuity equations, the g-current contimuity equations, and the batance of nentrons equations. An iterative procedure is developed to solve for the surface fiures and cell-averaged fluxes by de-coupling the set of balance equations from the two current continnity systems. The iteration is initiated in the balance equation which sopplies the starting extimates of the cell-averaged fluxes. These estimates are fed inlo the uncoupled, tridiagonal sets of securrent and g-current equations which can be solved independently of each other, 20 that the surface fluxes can be compuled. The resulting aurface fluxes are subatituted into the balance equations to update the cell-averaged fiuxes. These updated values become the new iterates.

The independent mature of the $z$ and 5 current contimity equations becomes the focal point of paralleliom. The algorithm is implemented on two types of parallel machines-the Intel iPSC/2 hypercube which betongs to the clas of diatributed memory procesors, and the Sequent balance 8000 which is a shared memory computer. Reanlts indicate the algorithm to be better suited for a shared memory environment.

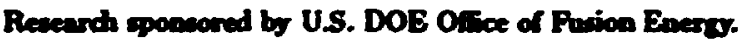

\section{6}

\section{NEW GAMMA-RAY BUHDUP RACTOR DATA POR POINT KGRNEL CALCULATIONS: ANS-6.4.3 STANDARD REPERENCE DATA}

$$
\text { D. K. Trubey }
$$

(Abutract of ORNL/RS1C-40, September 1838)

An American Nuclear Society Standards Committee Working Group, identified as ANS 6.4.3, has developed a cet of evaluated gamma-ray isotropic pointecurce buildup factors and attenuation coefficients for a standard reference dats bace. The largely unpublished set of buildup face ton calculated with the moments method bas been evaluated by recalculating key values with Monte Carlo, integral transport, and discrete ordinates methods. Additional buildup factor data were obtained from PALLAS code results. Attention has been given to frequently neglected procesen such as bremetrahlung and the effect of introducing a 
tione phantom behind the shield. The proposed draft standard, provided as an appendix, contains data for a sonrce energy ranse from 15 beV to 15 MeV and for 23 elements and 3 mixtures (water, air, and concrete). The buildup factor date are also represented as coefificients for the G-P fitting function. Tables giving correction factors for multiple seattering in tissue are also provided.

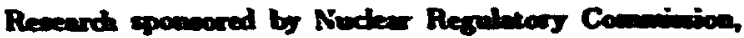

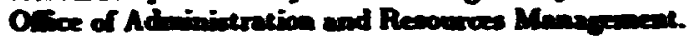

\section{RADIATION TRANSPORT STUDIES}

5.7

\section{THE BPFECT OF COAIBRNT SCATTBRING IN PEOTON RADIATION TRANSPOET calculations}

\author{
S. N. Cramer B. L. Kirt \\ J. Broadway" \\ (Abotract of ORNL/RSTC-51, Aprit 19e0)
}

The efeet of incloding or excluding the coherent scattering crom section in the Monte Carlo analysis of photon penetration in water has been atudied. Source energies from $80 \mathrm{keV}$ down to $20 \mathrm{keV}$ mere inveatigated. The inclusion of coherent scattering bas the effect of degrading the photon population with depth of penetration, but at the came time dispersing the population ove a larger volume than when it is excluded from the scattering model.

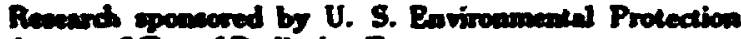

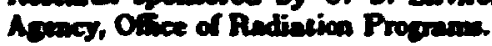

"U.S. Envionmentel Protoction A geacy, Monteoners, AL.
5.8

\section{NEUTRON STREAMING BeNCHMARK CALCULATIONS}

\author{
T. Y. Lee* \\ S. N. Cramer
}

(Abutreat of ORNL/RSTC-52, Angere 1800)

Computational techniques nod to analyse nentron streaming through cavities and ducts are decribed. The configurations are simplified geometries of atreaming paths whict can be found in nuelear fincion and fusion reactors. The reaults obtained using available computational toots are compared to each other.

Research sponsored by U.S. DOE Obloe of Nuclear Banow.

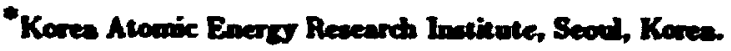

\section{INFORMATION ANALYSIS CENTER ACTIVITIES}

\section{9}

\section{NUCLEAR INDUSTRY QUALTTY, PRODUCTIVITY, AND INNOVATION FROM THE PERSPECTTVE OF AN INTERNATIONAL WNORMATION CENTER}

\section{B. F. Mackewitz}

(Abatrect of ord presentation given at the Anumal Congress of the South African Aceciation of Phyiciets in Madicin and Biology, Cape Town, Africa, Mardi 2-4, 19es)

In the aftermath of the Chernobyl accident. the prevailing attention of the international nuclear community is focused on the safe operation of the world's nuclear power plants. QA, man- machine interaction, early warning syatems, and eflectively organized eriais centers are increasingly receiving attention. At the same time, the nations which lack natural energ; resources are increasingly dependent on the nuclear option, and the productivity of their nuclear power plants is of greal importance.

The paper examines the USA situation in general. Then, through the unique "window" furnished by an international information center, briefly review quality, productivity, and innovetion appects of the nuclear industry in ceveral foreign countries including China (PRC), the Soviet Union, Crechoulovakia, and Hungary.

Reseaph sponsored by U.S. Department of Enarky. 
5.10

\section{TECHNOLOGY RESOURCES IN SUPPORT OF PROGRAMS OF THE PHYSICIST IN MEDICINE AND BIOLOGY}

\author{
B. F. Maskewits
}

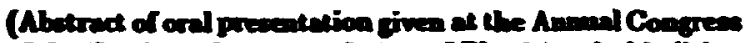

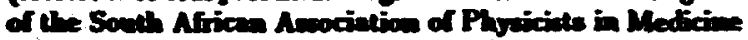

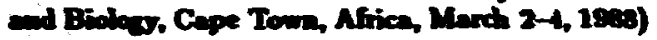

This paper will present a brief overview of methodology in use in USA energy research and derelopenent program for rodiation protection and other expects of health phyrics. It will aloo cover some appects of biomedieal computing with emplasis on quality mourance.

A diacusion of cocperative efforts to advance the state of the art in radiation protection, traneport, and shielding will be included in the pre entation. Technology resources (itterature, computer codes, data libraries, etc.) available from the Radiation Shielding Information Center (RSIC) for we in rewarch areas covered in the 28th SAAPMB Congress will be emphasized.

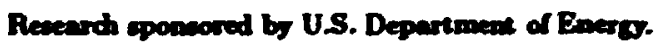

\subsection{1}

\section{COMPUTERS AND INTERACTIONS BETWEEN PHYSICIANS AND HEALTH PHYSICISTS}

\section{B. R. Maskewitz}

(Abatract of ord presentation given at the Summer School co Consputers in Medied Resenrch, South African Medical Reseand Council, Cape Town, Africe, Februmy 20-March 1, 1808)

Interaction of the physician and the health physicist within the medical biophysics commulnity of the United States (USA) is, in general, often cooperative and sometimes collaborative. It is aleo complimentary, with the health physicist in a research environment formatting his research reoults to facilitate usage in the physician's domain, often in the form of computer-readable data. The interaction of the physician and the medical physjciat, especially in nuclear medicine areas, is reletional, collaborative, and supportive. The use of computers in clinical applications oflen implies a partnerchip of physician and medical physicist and a mutualy supportive interaction between phyaician and bealth physicist.

This paper will examine interections above as related to the use of computers, with emphasis on activities in nuclear medicine. Related technical information and computing technology resources produced by health phyciciets and other scientiets in support of medical and biophysics research will be cited. Computer uage for other medical applications will be briefly reviewed from the appect of cofimare systems.

The experiences of the Society of Nuclear Medicine (SNM) in the U.S.A. (memberchip including phyicians, medical physicists, bealth physicids, radiologists, ete. in which the Society eeds to strengthen the interactions within its veried memberahip for a unified efiont to advance the state of the art in the efiective use of compaters in clinical and other medical applications will be explored. Related paralled efforts of the American Society for Computer Medicine (SCM) will also be brielly reviewed.

Conclusions will be drawn concerning the success/failure of the experimental efforts described and the current state of the art will be briefly summarized.

Research sponsored by U.S. Department of Dnerer.

5.12

\section{METHODS AND FORMATS FOR ENDF/B-VI}

$$
\text { R. W. Rousoin }
$$

[Abetract of Trane. Am. Nech Soc. 60, 617 (1800)]

The formats for ENDF/B-VI have been drematically revised (though Vereion $V$ formats had been extremely reaistant to change). These changes are due to the recognition of come inherent limitations in the format in terms of providing a true representation of the physics of interaction of particles with matter.

The format manual, "Data Formats and Procedures for the Evaluated Nuclear Data File, ENDF ${ }^{n}$ has been completely rewritten and the final version is expected to be icaued during 1989. Draft copies are available to evaluators and potential procesuors of ENDF/B-VI. The new format, designated ENDF-6, allows higher incident energies, adds more complete descriptions of the ditributions of emitted particles, and provides for 
incident charged particles and photons by parttioning the ENDF library into sublibraries. De cay data, fission product yield data, thermal newtron scattering data, and photo-atomic data have also been formally placed in sublibraries. Repre sentations of the resonance region via R-function and R-matrix have been introduced and emittedparticle-correlated energy-angle distributions are allowed. Covariance formats for secondary distributions and isomer production are provided.

Reseach apopersed by US. DOE Otice of Puido Every and Defene Noder Agacy.

\subsection{3}

\section{THE STATUS OF ENDF/B-V}
R. Rouscin
C. Dunford"
R. MeKnight
P. Youngt

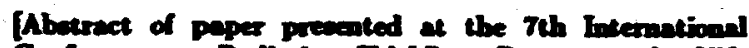
Conference os Aldition Shicking, Bourwemonth, UK, Seplanber 12-16, 1989, Proc. VoI III, Pp. 1125-1134 (1909))

A new version of the United States evaluated nuclear data file, ENDF/B-VI, is presently under development. Major empharis is being placed on correcting some long-etanding nuclear data problems that adversely affect applied calculations for both fiesion and fusion sactors. The paper reviews modifications to the formats and utility codes, outline the evaluation activities, discusces the data testing programs, and projects a date for the unrestricted releave of the new library.

Research uponeored by Defence Nuclear A gency and U.S. DOE Ofice of Fucion Enery.

Brookhaven National Laboratory, Upton, NY.

Argonne National Laboralory, Arconne, II.

† Los Alamos Netional Laboracory, Los Alemos, NM.
5.14

\section{RSIC AFTER 25 YEARS - CHALLENGES AND OPPORTUNITIES}

\author{
R. W. Ronasin B. F. Makewits
}

D. K. Trubey

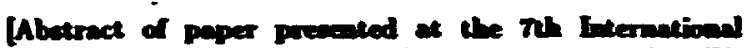

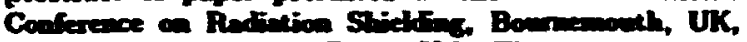
Seplember 12-16, 19as, Proc. VoL III, pp. 1135-1142 (1909)]

The Radiation Shielding Information Center (RSIC) observed its 25th year is operation in 1967. During that time aumerow changes bave occurred in the government programs that eponor RSIC and in the radiation tranoport commanity which it serves. The continned need for RSIC is evident from the steady volume of requests and interactions with the community. It in a contional challenge to adjust and adapt our operation to rospond to the demands placed on RSTC by sponsors and users. Cooperation between sponsons, weers, and the RSIC atafi is the key to keeping RSIC a the focus of activities in the international radiation transport commanity.

Researd sponsored by U.S. DOE Olice of Fusion Energ.

\subsection{5}

\section{AN OVERVIEW OE THE RADIATION SHIBLDING INPORMATION CENTER} (RSIC)

\section{K. Trubey}

[Abourect of Health Phyoies 57(3), 497-400 (1900)]

The Radiation Shielding Information Center (RSIC) at Oak Ridge National Laboratory, an an integral part of its information analysis activities, collects, makes operable packages, and distributes computer code software and data packages to nuclear engineering and health physicists engaged in shielding derign or doce calculations. The various codes are designed for calculations related to rer diation from reactors, radioisolopes, weapons and accelerators and to radiation occurring in opace. The Cenler uses the word "package" to mean all the items needed to utilize a code effectively. The package normally includes documentation deacribing the theory and code operation (contributor's report plus RSIC abstract and notes) and one or more reels of tape or diskettes on which is written 
the source program, oparatias program, inpat and ontpel for a sample problem, data libraries, and anciliary rontines. Thongh most of the software and date are contributed to RSTC by its user com- monity, the Center does some solitrare development and has been very actively involved in data library generation for user applications.

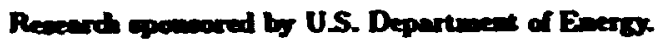

\section{TRANSFER OF COMPUTING TECHNOLOGY}

5.16

\section{PHOTON CROSS SECTIONS POR ENDE/B-VI}

\section{K. Trubey M. J. Berger" J. A. Fubbel"}

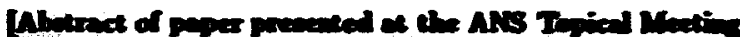

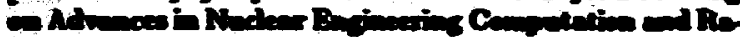

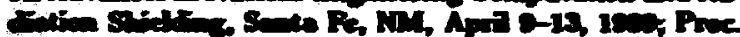

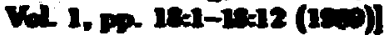

The PHOTX library of the National Inatitute of Standards and Techuoloss will be and as the basis of Pile 23 (photion cross nectiona) for ENDF/B-VI. The PHOTX library is beed on an experimental data beve conciuting of 21,000 data points from 512 literature cources for 82 eleswents. An important diflerence from eartict compilation is that renormalization of pholo-fiect eroes eeclions is not performed.

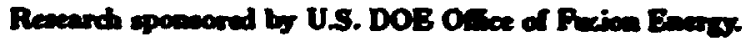

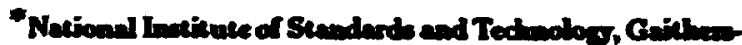
bos. MD.

\subsection{7}

\section{GUDDE FOR LICENSING EVALUATIONS USHNG CRAC2: A COMPUTRR PROGRAM FOR CaLCULATING REACTOR ACCIDENT CONSEQUENCES}

\section{J. E. White R. W. Rousin 11. Gilpin"}

(Abolreat of ORNL/TDMC-3, NUREG/CR-525, December isas)

$A$ verzion of the CRAC2 computer code applicable for use in analyes of consequences and risks of reactor accidents in cace work for environmental stacements has been implemented for use on the Nuclear Regulatory Comminion
Data General MV/8000 computer gratem. Inpat peeparation is facilitated through the nee of an incerective computer program which operates on a IBU personal competer. The resulting CAAC2 inpat deck is tranemitted to the MV/6000 by wing

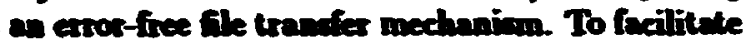
the we of CRAC2 af NRC, relerant backfround matierial on inpent requirements and model decriptions has been extracted from foen reports "Calcalations of Reactor Accident Consequences," Verion 2 NUREG/CR-2325 (SANDBI-1994) and CRAC2 Model Descriptions," NUREG/CR2552 (SAND62-0342), CRAC Calculations for Accident Sections of Euvironmental Statements," NUREG/CR-2901 (SAND82-1693), and Senotivity and Uncertainty Studies of the CRAC2 Compuler Code," NUREG/CR-4038 (ORNL 6114). When this background information is combined with indructions on the input proceseor, this report provides a self-eontained guide for preparing CRAC2 input dats with a specific orientation loward applications on the MV/8000.

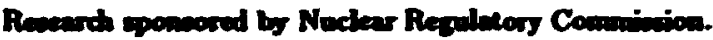

- NRC Sumerer Stadeat.

\section{SUSD: A COMPUTER CODE FOR CROSS-SECTION SENSITIVITY AND UNCERTAINTY ANALYSIS INCLUDING SECONDARY NEUTRON ENERGY AND ANGULAR DISTRIBUTIONS}

$$
\begin{gathered}
\text { K. Buruta" Y. Ola" } \\
\text { S. Kondo* }
\end{gathered}
$$

(Abatract of ORNL/TR-88/18, November 19as)

A crow-ection rensitivity-uncertainty analysis code SUSD has been developed. This code calculates sensitivity coefficients for one- and two- 
dimesaional traneport problem bened on the firet order perturbation theory. Variance and standard deviation of detector tespones or deciog parameters can be obtained wing crom-ection corariance matrices. In meutron tramport probleme thin coie - able to perform sencitivity-ancertainty analywes for eecondary angular diutribution (SAD) or eee- ondary energy distribetion (SED). Some peripheral program, which prepare croos-ection data neceseary for the SUSD code, aloo have been developed, and they are described in this report.

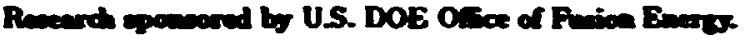

"Univesies of Tolyo, Takso, Japon 


\section{APPENDICES}




\section{PERSONNEL CHANGES}

\section{NEW STAFF MEMBEFS}

\section{A. Sciontific Stat}

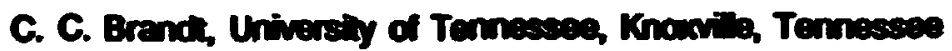

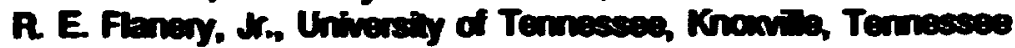

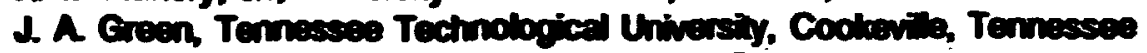

M. R Leures, Duto Univesily, Dutrem, North Cerolina

P. J Oondey. Univendy of Forida, Cainosilia, Forida

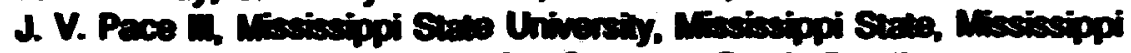

B. W. Poyton, Clemeon Uhivariny, Clomeon, South Cardina

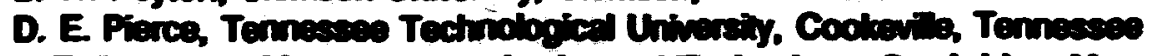

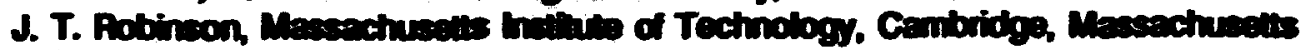

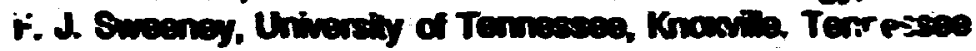

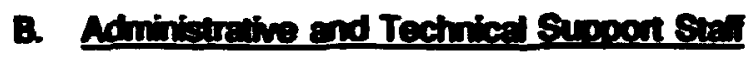

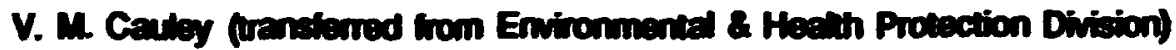

C. R. Kodwel franciened hom Publestions Division)

A. A Leavold (co-op)

G. D. Otiver (co-op)

J. L Paister (co-cp)

M. D. Russein (co-op)

A L Vanitun (co-op)

D. H. Wood (co-op)

\section{STAFF TRANSFERS OUT AND TERMINATIOAS}

\section{a sionific sten}

J. Bathen (other employmenl)

B. L Burks (uranterred to Central Menagement Ofices)

D. G. Cacuci (other employment)

Y. H. Etheridge (other conployment)

G. F. Flanagan (tranderred to Rescerch Reactors Divition)

M. Goldetein (complotion of axigrment)

E. C. Habent (tranterred to Phycios Divition)

D. L Henderson (other comployment)

C. C. Jorgensen (other employmem)

J. J. Manning (retired)

B. F. Mackewilz (retired)

D. L Moses (trenterred to Encineoring Technology Divition)

J. T. Robineon (tranterred to Intrumentation 8 Controb Divition)

L E. Parker (leave of abience)

D. L 8olby (iransicred to Central Management Organization)

A. D. Solomon (other employment)

N. Sreenath (other employment)

E. Wacholder (completion of aseignment)

C. R. Woibbin (other employmont) 


\section{B. Adminstratie and Tectrical Supoot Staf}

N. S. Currence pransierred to Heath and Safety Research Division)

A. B. Gustin (rotirement)

B. L Humphys (completed co-op assignment)

J. K ingersell (other employment)

S. E. Johnston (completed co-op assignment)

D. M. Jolley (completed co-op assignment)

K H. Kogar (complated co-op assignment)

M W. Nay (other employmeni)

G. D. Otiver (comploted co-pp essigrinany)

J. J. Roberts (completed co-op assignument)

M. D. Pussell (complated co-op assignment)

C. H. Zeigler furansfarred to Solid State Division)

\section{DISTMEYSHED SCEZNISTS}

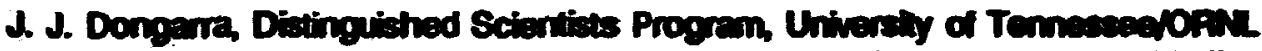

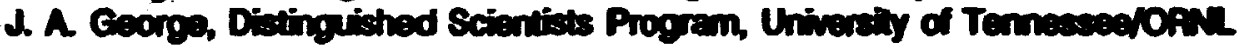

\section{CUESTS AND SHORT-TERM APPONTIENIS}

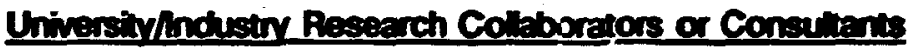

\section{A Scientific Staf}

J. Batow, Pem State Universidy, Universiy Pack, Penneytania

G. L Bibro, North Caroina State Universiy, Ralaigh, North Carclina

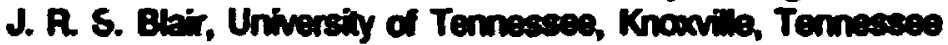

W. J. Book, Oktahoma State University, Stilwater, Oktahoma

C. L Bowman, Ball Systems Enginearing Division, Sen Diego, Callomia

C. R. Bovell, Physics Applications, Inc., Hivon, Tennesceos

D. C. Cacuci, Universily of Cellomia-Santa Batbara, Santa Babara, Callomia

B. W. Cher, Universiny of Tennesee, Kroxville, Tennescee

K. Chatani, PNC, Japan

C. C. Coener, Seilemployed, Miami, Florida

A. Coseic, CEA - CENSactay, France

B. Couny, Universiny of Maseachueetus, Amhorst, Mascachusents

G. J. Davis, Gaorgia Btate Universily, Altanta, Goorgia

E. Deplante, Maesachusens Inethute of Technology, Cambridge, Maseachunets

H. Demien, CEN, Cadaracha, France

J. J. Dorning, Univeriny of Virginia, Chartottesvilu, Virginia

A. E Fundertic, North Cacolina Etate Universiny

J. B. Fuscell, JBF Ascociates, Knowilib, Tennesece

G. H. Colub, Stantord Univeriny, Etantord, Callomia

P. Hase, Concord Aseociates, Inc., Knoxville, Temneaseo

K. F. Hancen, Maseachuseus Insthute of Technology, Cambridge, Maseactusects

A. M. Haralick, University of Wachington, Beatib, Wahington

E. Heer, Heer Asecciaces, LaCanada, Califomia

8. 8. Hyengar, Loubitana 8tate, Beton Rouge, Loulitians

C. M. Jencen, University of Oklahoma, Norman, Oktahoma

M. 8. Jones, Viginia Polytechnic Inathuse \& State Universiny, Blacksburg, Virginla

O. A. Karakashian, O. A, University of Tennesece, Knoxville, Tennescese

F. Kentesz, Bell-employed, Oak Aidge, Tennescee 
D. Knesid, Universiny of Texes, Austin, Austin, Texes

A. L. Muri, Universidy of Florida, Gainesvila, Fboida

T-Y. Lee, Korea Advenced Energy Reseench hstitute, South Korea

J. E. Laiss, SelEmployod, Gethersburg Maytand

M. R Leune, Vanderbil Universily, Nashilie, Tennessee

d. W. H. Lin, York Uhiversity, Toronta, Ontario

B. J. Machenner. Univershy of Tennessee, Khonvila, Tennessee

B. F. Maskewiz, SelFemployed Knowila, Tenneseed

N. Moray, Uhiversity of Uno's, Utbara, tinots

M. C. Mowor, UKAEA, AEREFtanwei, Oxon, England

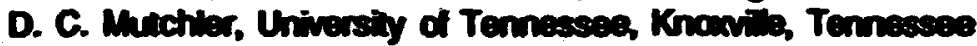

B. Nownam, Los Abmos National Laboratory, Los Alamos, Now Maxico

B. Otham, Texes Techical Uhiversiy, Lubbock, Texes

d. Orega University of Virginia Chadottesila, Viginia

N. Ohtani, PNC, Jepan

J. F. Paimer, Oltahoma Stete Uhivareiy, St water, Otahoma

\&. Planczer, Science \& Technology, ha, Oak Ridg, Tennesese

H. E Pople, Oltahome Srab Universiy, Sinweder, Olderoma

M d Rabins, Odtahoma State Universily, Stimeter, Otahoma

M. Rahman, Bangladech Aromic Energy Commiseion

M. Raa, Old Dominion Universiny, Nortolk, Viginia

K N. Roid, Oltahoma State Uhiversily, Stimater, Ottahoma

J. P. Reihs, Technische Universiks Wien, Kateplate, Austua

HJ. Schmiedmayer, Technieche Universitat Wien, Kaleplatr, Austra

S. Sertin, University of Tennessea, Knovvila, Tennessee

$K$ Sotoguchi, PNC, dapan

L R. Shention, Universiny of Coorgia, Athene, Coorgia

$K$ Shibata, Japen Alomic Energy Rosearch hwethete, Tolcaitura, Jepen

W. E. Snyder, North Carolina Stado Universily, Rabigh, North Carolina

A. D. Solomon, Sellemployed, Omer, terael

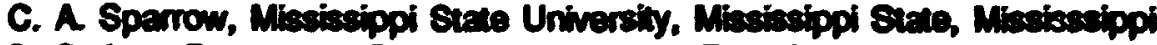

D. Steiner, Renseotaer Polytechnic instihute, Troy, Now Yodk

R. Stchler, Kaman Science Corporation, Abuquerque, Now Moxico

8. Stojenovic, Universiny of Cincinnati, Cincinnati, Ohio

K Storjohann, Dantsche Forschung Sgomeinechait, Maing, Weat Comany

J. B. Svirshy, Universiy of Tennesseg, Knoxille, Tennesces

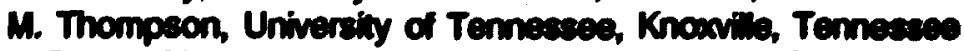

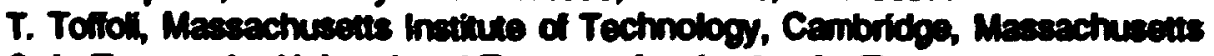

8. L. Tounogh, Univerity of Texas at Ausin, Alutin, Texes

1. Treiter, Universiny Uf Tenneseces, Knoxville, Ternescese

B. W. Tumbull, Comell University, thaca, Now Yook

C. C. Vining. Univerity of Florida, Cainevevile, Florida

M. Vose, University of Tennessee, Knoxvile, Tenneseses

E. L. Wachepreas, Universiny of Tenneasee, Knowille, Tennescese

H. Watanabe, Univerify sf North Carolina-Chapel Hill, Chaped Hit, North Carolina

M. F. Wheder, Univereity of Houston, Houston, Texas

M. A. Walliams, IBM, Kingoton, New York

M. L. Williams, Louisiana 8tate Unwertity, Baton Rouge, Loulciana

A. R. Yager, Self-employed, New York, New York 
B. Tectrical Suppoit

L S. Abbott, Tec-Com Company, Knoxvile, Tennessee

J. A. Etheridge, Roanoke College, Salem, Virginia

F. B. Hedspeth, University of Tennessee, Knonvila, Tennessee

H. P. Hiriyannaiah, North Carolina State Universiy, Rabigh, North Carolina

B. L Humphys, University of Tennessee, Knonille, Tennessee

D. M. Jolloy, University of Tennessee, Knonville, Tennessee

P. Ruse, Universiny of Colorado, Boulder, Colorado

P. L Stanfield, Universiky of Kentucky, Lexington, Kentuchy

C. Graduate Students

M. H. AbuShehadeh, Universily of Temessee, Knomila, Tennessee

M. E. Brediey, University of Vrginis, Charlottesvile, Virginia

G. L Cecuci, Univereity of Tennesees, Knowilla, Tennesses

C-X Chen, Universizy of Tennesese, Knowille, Tennessee

H. L. Christeinsen, Aaborg University, Aebong. Denmak

E. Chu, Universty of Waterbo, Waterioo, Ortaio, Canada

J. A. Davis, Universiny of Tenneseee, Knowilie, Tennessee

H. D. Embrechts, Kathotieke Universilen, Lewven, Belginm

R. E. England, University of Tennessee, Knowillo, Tenmessee

K Gates, University of Washington, Seatlo, Washington

J. A. Ceorge, University of Tennessee, Knoxvile, Tennessee

L M. T. Gomes, University of Tennessee, Knoxvile, Temessese

J. Graham, Louisiana State University, Baton Rouge, Louisiana

P. A Graham, Lovisiana State Universiny, Baton Rouge, Louisiana

E S. Groton, Universiny of Tennessee, Knoxvile, Tennessee

S. Gulat, Lousiana State University, Baton Rouge, Louisiana

D. Hantey, University of Tennessee, Knoxvile, Tennessee

V. G. Hedge, Louisiana State Universiny, Baton Rouge, Louisiana

C. S. Henkel, North Carolina State University, Raveigh, North Carolina

L C. Leal, University of Tennessee, Knoxvile, Tennessee

R. L. Macklin, Universiny of Tennessee, Knoxvilie, Tennessee

C. March-Leuba, University of Tennessee, Knoxille, Tennesses

L 8. Ostrouchov, University of Tennesese, Knoxville, Tennesses

L. E. Palko, University of Tenneseve, Knoxville, Tennesece

N. Rao, Louisiana State Univereity, Baton Rouge, Louisiana

A. 8. Sabhanwal, Louisiana 8tato Univereity, Baton Pouge, Louisiana

R. S. Sawhney, University of Tennessee, Knoxville, Tennessee

R. C. Thomton, Old Dominion Univerity, Nortolk, Virginia

A. K. VanCleave, University of Tennescee, Knoxville, Tennessee

D. Creat Lakes College Association Faculy Research Particination

D. S. Chrietianeen, Albion College, Abion, Michigan

R. R. Winters, Denison Univereity, Granville, Ohio

E. Great Lakes College Association Science Semester Program

T. 8. Attari, Denison University, Granville, Ohio

8. 8. Khawaja, Coe College, Cedar Rapids, lowa 
F. Oak Ridoe Associated Universtios - Faculy Reseerch Paticioans

K. Bowyer, University of Socith Florida, Tampa, Frrida

J. C. Courtney, Louisiana Stri.e University, Baton Rouge, Louisiana

G. J. Davis, Georgia State L'nivensity, Altanta, Georgia

H. N. Narang, Tuskegee University, Aubum, Alabama

D. A Poplawski, Michigan Technological University, Houghton, Michigan

A Poy, Arizona State Universily, Tempe, Arizona

K Sigmon, University of Florida, Cainesvila, Florida

P. F. Speth, Wabash Collega, Craviordsville, Indiana

C. W. Steidloy, Central Washington Iniversity, Elensburg, Washington

V. S. Sunderean, Emony University, Altenta, Georgia

S. Thompeon, Racta.d Universily, Radtord, Virginia

C. Oak Ridge Associeted Univorities - Post-Doctorel Progren

P. F. R. Beimans, Institit National de Recherche en Iniormetipie ef en Atometique, Crein, France

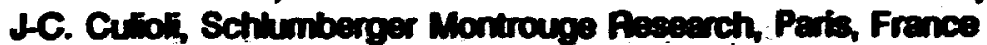

J. H. Han, University of Manylend, Colege Paik, Manytand

N. Tocmarian, Technion, leraid

H. Cak Ridge Associated Universines - Undergradute Roseanch Paticipans

D. P. Katzman, University of Pennsylvenia, Phiadelphia, Pennsylvania

J. L. Painter, East Tennessee State Universily, Johnesn Ciy, Tennessee

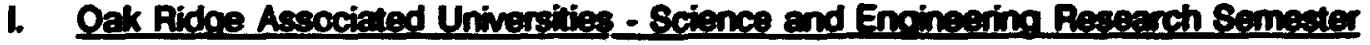

T. K Aledge, Ohio Northem University, Ada, Ohio

S. Autommarchi, Florida Allantic Universiby, Boca Raton, Fiorida

L S. Auvi, Adereon-Broaddus Colloge, Phippi, West Virginia

V. K Bansal, Comell Universiny, thace, Now York

$K$ A Bary, Brigham Young Univereity, Provo, Utah

R. Brunkhorst, Loras College, Dubuque, lowa

R. A. Cacheiro, Tennesees Technological Universily, Cookeville, Tenneseee

8. M. de Rada, Southem Miesissippi Univertity, Matioeburg, Miesicsippi

N. D. Drochak, Miami University, Oxford, Ohio

R. Even, Comell University, thaca, Now York

L A. Farkas, Comell Universiny, thaca, New York

M. C. Finley, Traneytvania Univereity, Lexington, Kentu 'cky

C. W. Flake, Clemson University, Clemeon, South Carolina

K H. Gaiser, Michigan Technological Univereihy, Houghton, Michigan

K J. Herzog, Northem Illinois Universihy, De Kalb, Ilino's

T. Heywood, Univereity of Florida, Gainesville, Florida

A M. Jijina, Illinois Inatitute of Technology, Chicago, Ilinois

L J. Kelleher, Ohio Northem Univertety, Ada, Ohio

K L Knight, Meredith College, Raleigh, Nurth Carolina

C. M. Lewder, Southem Illinois Univereity, Edwardoville, Illinois

E. Lazaridis, University of Pennoylvania, Philadelphia, Penneytvania

I. L Lough, Rhode laland College, Providence, Ahode laland

E. 8. Lyness, lowa State University, Ames, lowa

M. 8. Madanat, Cheyney Univereity of Pennoywania, Choyney, Pennoytvania

K L Marrs, Universiny of MiseourtRolla, Rolla, Misecouri

P. Marquis, Tuth University, Mediord, Maseachusetts 
J. W. Paimer, State Universidy of New York-Butialo, Butalo, Now York

R. R. Powell Alegheny Colloge, Meadville, Pennsylvania

C. Roe, Loyola University, New Orleens, Lovisiana

J. A. Sandberg Utah State University, Logar, Utah

D. L Senderson, Lawrence instiute of Technology, Southifid, Michigan

D. F. Scollen, Pennsylvaria State Universily, University Park, Pennoytvania

C. M. Shepperd, University of Michigan, Amn Abor, Nichigen

D. M. Sminh, Davis \& Elins College, Elkins, West Virginia

Hd. Wh Universiy of Caliomia, Los Angoles, Caliomia

J. Oak Rides Associated Uhiversitios - Precticum Assignments

S. Epperson, University of Forida, Gainesvila, Florida

J. P. Hannon, Universiny of Vinginia, Chantotteserla, Virginia

S. L. Hruska, Jacksonvile State University, Jackoonima, Alabama

K Oak Pidge Associated Uhiversios - Professional Intemshio Progrem

J. A. Etheridge, Roanoke Cologe, Salem, Virinia

M. S. Siminan, Universiy of Missouriflolla, Rolla, Mrssour

L DOE Sciance and Engror Reseach Semester Progrem

C. Currin, Byn Mawr, Byn Maw, Pennsylvania

C. S. Henkel, North Carolina State University, Raleigh, North Carolina

S. P. Kho, Truman Collega, Chicago, Iniois

J. L. Kng, Universiny of Tennessee, Knowvile, Tennessee

C. M. Lance, Michigan State Universily, East Lansing, Michigan

L A. Renker, Baldwin-Wallace College, Berea, Ohio

M. Teacher Research Program

L W. Daniels, Oak Ridge Schools, Oak Ridge, Tenneseee

D. J. Dodd, Haywood County Board of Education, Jackeon, Tennessee

I. J. Meletiou, Knox County School System, Knoxville, Tennesees

N. U.S. Miltan Senice Academ Research Assochates

J. A. Dietz, U.S. Naval Academy, Annapolis, Manland

J. R. Dundas, U.8. Naval Academy, Annapolis, Maryland

T. M. Mathis, U.8. Naval Acadomy, Annapolis, Manyland

C. A. Mocougan, U.8. Milltary Academy, Weet Point, Now York

D. McVay, U.8. Mintary Acadomy, Weat Point, New York

T. E. Shuearchyk, U.8. Naval Academy, Annapolis, Maryland

C. 8. Vanni, U.8. Naval Academy, Annapolis, Manyland

o. Soocial Program for Gined High-School Students

D. A. Huff, Oak Ridge High School, Oak Ridge, Tennessee 


\section{P. ORNLUT Speciel Year on Numerical Lneer Nobre}

L Adams, Universiy of Washington, Seadla, Washington

J. Barlow, Pern State Universiny, Univensiy Park Pernsivivaia

A. Biorck, Linkoping Universiy, Linkoping Swoden

C. Bectia, Virginia Pohtechnic Instiute and State University, Beckeburg Viginia

J. R Bunch, University of Callomiasen Diego, La Jolla, Cerlomia

R. Byers, Univereily of Kenses, Lamrence, Kanses

T. F. Colemen, Comeil University, thece, Now Youk

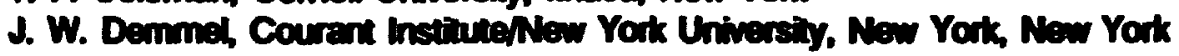

R. Entriken, Staniord Universiuy, Stanford Calfornia

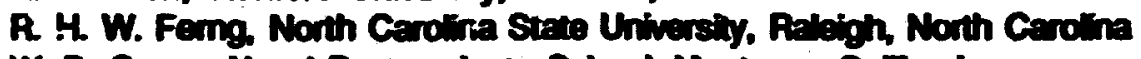

W. B. Grage Naval Postgraduete School, Monterey, Caliomia

C. P. Hansen, Universiny of Tennessee, Kroxvila, Tennesese

C. Golub, Stantord Uhiversty, Staniond, Camomia

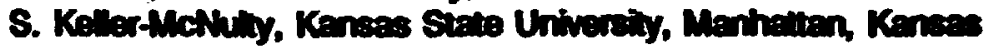

D. Knkaid, Universiyy of Texese-Austin, Austin, Texes

V. Koma, Maseachuectis inetitus of Technology, Cambridge, Maseachusedts

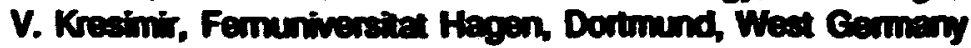

R. LeVeque, Uhiversiny of Tennessea, Kronvilio, Tenneseses

J. Lu, Yotk Univorsily, Ontario, Canada

T. H. Olesen, Christien Mich, Bergen, Norway

J. Ortega, University of Viginia, Chatomesvila, Virinia

H. Park, University of Minmescta, Minneapolis, Minnesota

B. Parteth Universiny of Calfomia, Berkeley, Cellomia

T. Peierts, Comell University, thaca, Now York

R. J. Plemmons, North Caroina State Universiny, Rabigh, North Caroina

A Pothen, Pennoytvania State Universily, Univerciny Patk, Pennoytrania

M. Saunders, Stantord University, Stanford, Callfomia

W. D. Shoaff, Florida Instiute of Technology, Neboume, Florida

D. Sorensen, Argome National Laboratory, Aggonne, Minois

C. W. Stewart, Universiny of Mayland, College Park, Mantand

C. O. Svenseon, Linkoping University, Lnkoping Sweden

K. Veselic, Fomunivereitat Hagen, Cortmund, Wout Cormany

B. R Vona, Universiny of Texae-Austin, Auntin, Texas

D. Wamer, Clemson Universiny, Clemeon, socth Carolina

S-Y. Xas, University of Temnesese, Knoxvilla, Tennescese

D. Young, Universiny of Texae-Auntin, Austin, Texas 


\section{SCIENTIFIC AND PROFESSIONAL ACTIVITIES}

October 1, 1987 - August 31, 1989

\section{Alexiades}

\section{Invited papers}

- "Shrinkage Effects on Solidification," 2nd International Conference on Trends in Welding Research, Gatlinburg, TN, May 1989.

- "Effect of a Void on Cyclic Melting and Freezing of an Encapsulated PCM," 26th National Heat Transfer Conference, Philadelphia, PA, August 1989.

Professor

- Mathematics Department, University of Tennessee, Knoxville, TN.

\section{Award}

- UTK-ORNL Science Alliance Research Incentive Award, 1986-1989.

\section{Reviewer}

- Mathematics Reviews

Referee

- Applicable Analysis

- SIAM Journal of Mathematical Analysis

- Nonlinear Analysis

- International Journal of Heat and Mass Transfer

- ASME Journal of Heat Transfer

- NSF and DOE Proposals

F. S. Alsmiller

Member

- Superconducting Super Collider Task Force on Radiation Levels in the SSC Interaction Regions.

Invited paper

-- "The Modified High-Energy Transport Code HETC and Design Calculations ior the SSC," (with R. G. Alsmiller, Jr., T. A. Gabriel, O. W. Hermann, and B. L. Bishop) American Nuclear Society Meeting, San Diego, CA, June 12-16, 1988.

R. G. Alsmiller, Jr.

Chairman

-. DOE Advisory Panel on Accelerator Radiation Safety for the Review of the Continumus Electron Beam Accelerator Facility (CEBAF). 
Member

- National Committee of Radiological Protection Scientific Committee 5? on Conceptual Basis of the Calculation of Dose Distributions.

- Superconducting Super Collider Task Force on Radiation Levels in the SSC Interaction Regions.

Invited paper

- "The Modified High-Energy Transport Code HETC and Design Calculations for the SSC," (with F. S. Alsmiller, T. A. Gabriel, O. W. Hermann, and B. L. Bishop) American Nuclear Society Meeting, San Diego, CA, June 12-16, 1988.

Referee

- Nuclear Science and Engineering

- Physics in Medicine and Biology

- Health Physics

- Fusion Nuclear Technology

Y. Y. Aziny

Chairman

- Neutron Transport and Thermalhydraulics Session, 1989 American Nuclear Society Annual Meeting, Atlanta, GA, June 1989.

Referee

- Nuclear Science and Engincering

J. J. Beauchamp

Invited paper

- "Bayesian Variable Selection in Linear Regression," (with T. J. Mitchell) 1988 Joint Statistical Meet'ngs, New Orleans, LA, August 22-25, 1988.

Invited lecture

- "Bayesian Variable Selection and Linear Regression," Department of Statistics, University of Tennessee, October 18, 1988.

A wards

-- 1088/89 Technical Communication Award by the East Tennessee Chapter of the Society for Technical Communication.

-. 1080 Martin Marietta Team Award for Publications (with T. J. Mitchell).

Instructor

Oak Ridge Graduate School of Biomedical Sciences, University of Tennessee, Knoxville, TN. 
Referee

- Water Resources Bulletin

- Soil Science Society of America Journal

- Canadian Journal of Fisheries and Aquatic Sciences

K. O. Bowman

Member

- Committee of History of Statistics, video presentation of Sesquicentinnial, American Statistical Association.

- Advisory Committee on Equal Opportunities in Science and Engineering, National Science Foundation.

- Advisory Board, Journal of Statistical Computation and Simulation.

- International Editorial Bcard, Communications in Statistics.

- Proposal Review Panel, National Science Foundation.

- Nominating Committee, Oak Ridge Chapter, Sigma Xi, 1988-1989.

\section{Chair}

- Subcommittee on Scientists and Engineers with Disabilities, Equal Opportunities in Science and Engineering, National Science Foundation.

- Coordinating Committee on Equal Opportunities in Statistics, American Statistical Association.

Invited papers

- "Solutions to Johnson's $S_{B}$ and $S_{U}$ " (with L. R. Shenton) Commun. Statist.-Simula. Computa., 17(2), 343-348.

- " $S_{B}$ and $S_{U}$ Distributions fitted by Percentiles: A General Criterion," (with L. R. Shenton) Commun. Statist.-Simula. Computa., 18(1), 1-13.

- Commentary, "Eight Billion Mice is the Answer. What is the Question?" to appear in Citation Classics section of Current Contents, October 16, 1989.

Contributing editor

- Current Index to Statistics

Organizer

- Open Meeting, ASA Annual Meeting, New Orleans, August 1988.

- Open Meeting, ASA Annual Meєuing, Washington, DC, August 1989.

Award

- Distinguished and Sustained Contributions to Science Award, AWIS, 1088.

-- Technical Achievement Award, Martin Marietta Energy Systerns, Inc., 1988. 


\section{Reviewer}

- Science, American Association for the Advancement of Science

- Research Proposal. National Science Foundation.

\section{Referee}

- Communications in Statistics

- Computational Statistics $\&$ Data Analysis

- American Statistician

- Journal of Statistical Computation and Simulation

- Psychometrika

R. J. Carter

\section{Invited papers}

- "Digital Instrumentation and Interactive Computer Systems: A Survey of the Use of Advanced Technology in the Nuclear Industry," American Institute of Chemical Engineers Summer National Meeting, Philadelphia, PA, August 21-23, 1989.

- "Human Factors Aspects of Advanced Instrumentation in the Nuclear Industry," Institute of Electrical and Electronics Engineers Symposium on Power Plant Dynamics, Control, and Testing, Knoxville, TN, May 15-17, 1989.

- "Review Criteria for Human Factors Aspects of Advanced Controls and Instrumentation," (with R. E. Uhrig), Nuclear Safety Research Review Committee's Subcommittee Meeting on Human Factors and Reliability, Brookhaven National Laboratory, December 1-2, 1988.

- "Designing a HAZMAT Incident Management System for Facilities with Widely Varying Emergency Organization Structures," (with C. E. Easterly), American Institute of Chemical Engineers Hazardous Material Spills Conference, Chicago, IL, May 16-19, 1988.

\section{Session organizer}

- Hands-On Demonstrations of Computer Models, ANS Topical Meeting on Advances in Human Factors Research on Man/Computer Interactions: Nuclear and Beyond.

\section{J-C. Culioli}

\section{Award}

- Ph.D. Thesis was awarded the first prize in Theoretical Automatic Control by the AFCET, Paris, France (French Association for Cybernetics and Systems) (March 1988). 
G. de Saussure

Member

- Organizing Committee for the Topical Conference "Fifty Years with Nuclear Fission," National Bureau of Standards, Washington, DC, April, 26-28, 1989.

- International Program Committee International Topical Meeting on the Physics of Reactor Operation, Design, and Computation: Physor '90 (1990).

Assistant technical chairman

- ANS/ENSA International Conference, October 30 - November 4, 1988.

Honorary professor

- Nuclear Engineering Department, University of Tennessee.

Referee

- Nuclear Science and Engineering

- The Physical Review

- Nuclear Safety

J. K. Dickens

Member

- American Nuclear Society Standards Working Group 5.1, Denzy Heat Standard.

- American Nuclear Society Standards Working Group 5.2, Fission Product Yields.

- Nuclear Energy Agency Task Force on Decay Heat Predictions.

- University Nuclear Engineering Research Program, Review Panel.

- Oak Ridge National Laboratory Spcakers' Bureau.

- Oak Ridge Associated Universities DOE Traveling Lecture Program.

- Oak Ridge Associated Universities Tennessee Visiting Scientist Roster.

Correspondent

- Designated by IAEA Working Group on Fission Product Nuclear Data as correspondent on worldwide experimental measurements of fission product decay heat (1977-continuing).

Invited paper

-- "Review of New Integral Determinations of Decay Heat," Proceedings of a Specialists' Meeting on Data for Decay Heat Predictions, Studsvik, Sweden, September 7-10, 1087, NEACRP-302 'L,' NEANDC'-245 ' $U$ ' (OECD, Paris, 1087) p. 100. 


\section{Invited lectures}

- "Decay Heat Measurements and the Loss of Coolant Accident," Nuclear Engineering Department, University of Tennessee, October 26. $19 S 5$.

- "Neutron Research at the Oak Ridge Electron Linear Accelerator," Physics Department, Ohio University, Athens, Ohio, April 7, 1989.

- "Reactor Safety and the Status of Commercial Nuclear Power in the U.S.," Public forum presentation, Ohio University, Athens, Ohio, April 7, 1989.

\section{Referee}

- Nuclear Science and Engineering

- The Physical Review

- Nuclear Instruments and Methods

\section{F. C. Difilippo}

\section{Member}

- Program Committee Reactor Physics Division American Nuclear Society.

\section{Invited lecture}

- "Determination of Kinetics Parameters Using Stochastic Method in a ${ }^{252}$ Cf Driven System," University of Michigan, October 1988.

- "Determination of Kinetics Parameters Using Stochastic Method in a ${ }^{252}$ Cf Driven System," Institut of Nuclear Technology, Madrid, Spain, May 1988.

\section{Invited papers}

- "Reactor Physics Methods for the Preconceptual Core Design of the Advanced Neutron Source," American Nuclear Society 1988 Winter Meeting, November 26-30, 1989, San Francisco, CA.

- "Determination of Kinetics Parameters Using Stochastic Method in a ${ }^{232}$ Cf Driven System," NATO Advanced Research Workshop, Valencia, Spain, May 1988.

\section{Co-organizer}

- Chairman, editor NATO Advanced Research Workshop on "Noise and Nonlinear Phenomena in Nuclear Systems," Valencia, Spain, May 1088. (Proceedings edited by Plenum, NATO ASI Series).

- ORNL Workshop on Scientific and Industrial Applications on Free Electron Laser, June 12-13, 1989.

\section{Organizer}

-- Special Session of Winter 1988 American Nuclear Society Meeting about the Advanced Neutron Source. 


\section{Award}

- Best paper award Reactor Physics Division. Winter 1958 ANS Meeting: co-author.

\section{Fellow}

- Elected Fellow American Nuclear Society, November 1988.

\section{Listed}

- International Directory of Nuclear Criticality Safety Personnel.

\section{Referee}

- Nuclear Science end Engineering

J. B. Drake

\section{Member}

- Computing User Advisory Committee.

- Computing User Advisory Steering Committee.

Invited paper

- "Application of Parallel Computing to Scientific Problems," Tuskegee University, February 1989.

W. W. Engle, Jr.

\section{Chairman}

- Membership Committee, Radiation Protection and Shielding Division, American Nuclear Society, through July 1988.

\section{Award}

- Elected American Nuclear Society Fellow.

\section{Special assignment}

- On assignment at the OECD-NEA Data Bank, Saclay, France, August 1988 through June 1990.

\section{G. F. Flanagan}

\section{Member}

- American Nuclear Society Board of Directors 1987-1900.

-. American Nuclear Society Finance Committee 1089-1992.

-.. American Nuclear Society Nuclear Reactor Safety Division Executive Committee.

-. Society for Risk Analysis (Charter).

Board of directors

$$
\text { Amcrican Nuclear Society (elected). }
$$




\section{Treasurer}

- Nuclear Reactor Safety Division - American Nuclear Soriety (elected).

Invited papers

- DOE-ER "HFIR PRA" Tutcial.

- DOE-ER "HFIR Safety and Operations" Tutorial.

- American Institute Chemical Engineer (AICHE) - Risks of Chemical Weapon Demilitarization.

- American Air Pollution and Waste Management - Risks Associated with Chemical Weapon Demilitarization.

Award

- Martin Marietta Energy System Technical Achievement Award.

Editorial board

- Risk Analysis

E. L. Frome

Member

- American Statistical Association Committee on Statistics and the Environment.

- Statistics Test Panel for Data Management Systems in Genetic Toxicology Sponsored by U.S. EPA.

- Organizing Committee for the American Statistical Association Conference on Radiation and Health (Chairman in 1985).

- ORAU/University of North Carolina Research Planning Group for DOE Health and Mortality Studies.

Associate professor

- Department of Biostatistics, University of North Carolina, Chapel Hill, NC.

Associate editor

- The American Statistician, 1983-present.

\section{Referee}

- The American Journal of Epidemiology

- Statistics in Medicine

- The American Statistician

- Radiation Research 
C. Y. Fu

Member

- Cross Section Evaluation Working Group (CSEWG).

- NEANDC Activation Cross Section Working Group.

\section{Invited papers}

- "Status of Theories Used for Calculations of Long-Lived Activation Cross Sections," IAEA Consultants Meeting on the Generation of Long-Lived Radioactive Nuclides, Argonne, IL, September 11-12, 1989.

Referee

- Nuclear Science and Engintering

T. A. Gabriel

\section{Spokesperson}

- ORNL High Energy Physics Task Force.

\section{Member}

- Task Force on Radiation Levels in the Experimental Halls at the SSC (DOE).

- Steering Committee of the Southern Association for High Energy Physics (SAHEP).

\section{Publicity chairman}

- Organizing Committee for the Southeastern Conference on the Superconducting Super Collider (SSC).

\section{Invited papers}

- "Calorimeter and Related Calculations for the SSC" the Second ORAU Workshop on the SSC, Atlanta, GA, May 12-13, 1988.

- "Detectors for the Superconducting Super Collider, Design. Concepts, and Simulation," Workshop on Calorimetry for the Superconducting Super Cuilider, University of Alabama, Tuscaloosa, AL, March 13-17, 1989.

- "Theoretical Studies of Hadronic Calorimetry for High Luminosity and High Energy Colliders," (with J. Brau) International Conference Advanced Technology and Particle Physics, Villa Olmo, Lake Como, Italy, June 15, 1988 (NIM, A279, 40-56 (1989).

- "The Modified High-Energy Transfort Code, HETC, and Design Calculations for the SSC," (with R. G. Alsmiller, Jr., F. S. Alsmiller, O. W. Hermann, and B. L. Bishof; 1988 Annual Meeting of the ANS, San Diego, CA, June 12-16, 1988.

-- "CALOR83: The Code System for Calorimeter Analysis and Design," Workshop on Calorimetry for the Superconducting Super Collider, University of Alabama, Tuscaloosa, AL, March 13-17, 1989. 
- "Silicon Calorimetry, A Calculational Perspective," Workshop on Calorimetry for the Superconducting Super Collider, University of Alabama, Tuscaloosa, AL, March 13-17, 1989.

- "Methods Used in the MORSE Code for Calculational Speedup," Workshop on Calorimetry for the Superconducting Super Collider, University of Alabama, Tuscaloosa, AL, March 13-17, 1989.

- "The High-Energy Transport Code HErC88," (with R. G. Alsmiller, Jr., F. S. Alsmiller, O. W. Hermann, and J. M. Barnes) Workshop on Calorimetry for the Superconducting Super Collider, University of Alabama, Tuscaloosa, AL, March 13-17, 1989.

Referee

- Nuclear Sizience and Engineering

- Nuclear Instruments and Methods

- Nuclear Technology/Fusion

C. W. Glover

\section{Organizer}

- Aerospace Applications of Artificial Intelligence (AAAIC) Robotics and Control Section Organizer.

\section{Invited lectures}

- "Neural Networks for Pattern Classification," ORNL Fusion Energy Division Seminar.

- "Neural Networks and Adaptive Resonance Theory (ART 1)," Ohio Neural Networks Society.

- "Applications of Neural Networks at ORNL," Ohio Neural Networks Society; also University of Tennessee Nuclear Engineering Seminar.

\section{J. Gray}

\section{Invited talk}

- "Boundary Element Method for Regions with Cracks," SIAM Meeting, Knoxville, TN, March 17-18, 1989.

Invited paper

- "Evaluation of Hypersingular Integrals in the Boundary Element Method," Computers and Mathematics with Applications, accepted 1989.

Editorial board

- Engineering Analysis with Boundary Elcments

- Electrosoft

- Boundary Elemerts: Abstracts and Newsletters

\section{Referee}

- National Science Foundation 
C. M. Haaland

Advisor

- National Council for Fadiation Protection and Measurements Scientific Committee \#63, Radiation Exposure Control in a Nuclear Emergency, Lewis V. Spencer, chairman.

\section{Member}

- Hypersonic Glide Vehicle Mission Analysis Working Group.

\section{Invited paper}

- "Fallout Facts for Nuclear-Battlefield Commanders," Armed Forces Radiobiology Research Institute, Bethesda, MD, September 9, 1988.

\section{Referee}

- Journal of Civil Deferse

- Journal of Health Physics

J. A. Harvey

\section{Member}

- ORNL Acceleraturs and Radiation Sources Review Committee.

- Panel on Basic Nuclear Data Compilations National Research Council.

Labor coordinator

- Engineering Physics and Mathematics Division.

Safety officer

- Engineering Physics and Mathematics Division.

Radiation control officer

- Engineering Physics and Mathematics Division.

Chairman

- Executive Committee, Electromagnetic Isotope Enrichment Facility Users Group, 19\&?.

\section{Referee}

- Physical Review $C$ and Physical Review Letters

- Nuclear Science and Engineering

M. T. Heath

\section{Member}

- Board of Directors, ACM-SIGNUM.

- Martin Marietta Technical Achievement Award Committee. 
- Organizing Committee, Fifth Distributed Memory Computing Conference Series.

- Organizing Committee, Fourth Hypercube Concurrent Computers Conference.

Referee

- SIAM Journal Scientific and Statistical Computing

- IMA J. Numer. Anal.

- Mathematical Programming

- Nuclear Science and E'ngineering

- IEEE Computer

- IEEE Trans. on Computers

- Concurrency: Practice and Experience

Proposal reviewer

- National Science Foundation

Chairman

- Steering Committee, Distributed Memory Computing Conference Series

Vice-chairman

- ACM SIGNUM (Special Interest Group on Numerical Mathematics)

- SIAG-LA (SIAM Activity Group on Linear Algebra)

Invited lectures

- Workshop on Vector and Parallel Computing, Umea, Sweden, 1988.

- Danish Summer School in Supercomputing, Copenhagen, Denmark, 1989.

D. T. Ingersoll

Member

- Nuclear Energy Agency Committee on Reactor Physics (NEACRP)

- ORNL Research Reactors Users Group.

- ORNL Nuclear Energy Panel.

Vice-chair/chair-elect

- Radiation Protection and Shielding Division, American Nuclear Society.

Chairman

- Shielding Data Testing Subcommittee, Cross-Section Evaluation Working Group (CSEWG).

- U.S.-JASPER Shielding Program Working Group. 


\section{Reviewer}

- DOE Nuclear Engineering Research Program.

B. L. Kirk

\section{Membership chairman}

- American Nuclear Society, Radiation Protection and Shielding Division.

\section{Member}

- American Nucicar Society (ANS-10 Standards Committee).

- Society for Industrial and Applied Math Special Interest Group on Supercomputing.

H. E. Knee

\section{Member}

- American Nuclear Society

-- EPRI Chapter $10 \mathrm{Man} / \mathrm{Machine}$ Interface Requirements Committee.

Technical chairman

- ANS Topical Meeting on Advances in Human Factors Research on ManComputer Interactions: Nuclear and Beyond.

D. C. Larson

Member

- Department of Energy Nuclear Data Committee.

- NEANDC International Task Force on Evaluation Cooperation.

- Evaluation Methods Subcommittee, Cross Section Evaliation Working Group (CSEWG).

- Resonance Region Subcommittee, CSEWG.

\section{Invited papers}

- "ENDF/B-VI Nuclear Data Evaluations for Fusion Applications," (with C. Dunford and P. Young) Eighth Topical Meeting on Technology of Fusion Energy, Salt Lake City, UT, October 9-13, 1988.

- "Structural and Shielding Material Evaluations for ENDF/B-VI," (with P. Young) American Nuclear Society Winter Meeting, San Francisco, CA, November 26-30, 1989.

- "Electron Linear Accelerators for Fast Neutron Data Measurements in Support of Fusion Energy Applications," (with K. Bockhoff, A. Carlson, $O$. Wasson, and J. A. Harvey) for special issue of Nuclear Science and Engineering on Neutron Sources for Fusion Technology. 


\section{Chairman}

- Working Group A, Office of Nuclear Physics Committee on Nuclear Data for Fusion Reactor Applications.

- Working Group 2, IAEA Specialist's Meetings on Fusion Evaluated . Nuclear Data Library.

- Co-chairman of General Purpose Evaluations Subcommittee, CSEWG.

Proposal reviewer

- Office of Nuclear Physics, Department of Energy, Low Energy Nuclear Physics.

Referee

- Nuclear Science and Engineering

M. R. Leuze

Invited papers

- "Independent Set Orderings for Parallel Matrix Factorization by Gaussian Elimination," Parallel Computing 10, 177-191 (1989).

- "Multiprogramming a Distributed-Memory Multiprocessor," (with L. W. Dowdy and K. Y. Park) Concurrency: Practice and Experience 1 (1989).

W. E. Lever

\section{Member}

- American Statistical Association Biometrics Society.

R. A. Lillie

\section{Chairman}

- ORNL Reactor Experiments Review Committee.

\section{Referee}

- Nuclear Technology

- Nuclear Technology/Fusion

- Journal of Fusion Energy

- Nuclear Science and Engineering

- Nuclear Instruments and Methods

R. L. Macklin

Fellow

- American Association for the Advancement of Science.

- American Physical Society. 
Referee

- Nuclear Science and Engineering

- Physical Review

R. E. Maerker

\section{Member}

- ASTM Eí0.05 Subcommittee Member Ad-Hoc Committee to Prepare Guide on LWR-RPV' Surveillance Analysis for NRC.

- American Nuclear Society.

- American Society for Testing and Materials.

Invited paper

- "The LEPRICON Code System: Consolidation of Transport Analytical and Unfolding Procedures in LWR Pressure Vessel Dosimetry," Trans. Am. Nucl. Soc. 57, 225 (1988).

\section{Referee}

- Nuclear Science and Engineering

- Journal of Nuclear Materials, Amsterdam, Holland

R. C. Mann

Member

- Advisory Committee for the Universidad del Turabo, Puerto Rico, Engineering Curriculum Development.

Invited papers

- "Sensor Integration Using Concurrent Computing On-Board the ORNL Mobile Robot," IEEE R\&A Conference, Scottsdale, AZ.

- "Research in Mobile Robotics at ORNL/CESAR," IEEE International Conference Systems Engineering, Dayton, $\mathrm{OH}$.

- "Multi-Sensor Integration for a Mobile Robot," International Workshop on Robot Hands, Dubrovnik, Yugoslavia.

Chairman

- Review Team for the Robntics Part of the DOE Environmental Restoration and Waste Management Program Plan.

Reviewer

- IEEE Trans. Systems, Man, Cybernatics

- IEEE Computer

- IEEE J. Robotics \& Automation

- DOE SBIR Program 


\section{T. J. Mitchell}

\section{Invited papers}

- A Bayesian Approach to the Design and Analysis of Computational Experiment," Thirty-Third Conference on the Design of Experiments in Army Research, Development and Testing, Newark, DE, October 21-23, 1987.

- "A Bayesian Approach to the Design and Analysis of Computer Experiments," (with M. D. Morris) 20th Symposium on the Interface of Computing Science and Statistics, Reston, VA, April 21-23, 1988.

- "A Bayesian Approach to the Design and Analysis of Computer Experiments," (with M. D. Morris) First Internationa! ConferenceWorkshop on Optimal Design and Analysis of Experiments, Neuchatel, Switzerland, July 25-28, 1988.

- "Bayesian Variable Selection in Linear Regression," (JASA Theory and Methods Special In ited Paper) Joint Statistical Meetings, New Orleans, LA, August 22-25, 1988.

- "Computer Experiments to Optimize a Compression Mold Filling Process, Workshop on Design of Computer Experiments, Oak Ridge, TN, November 3-5, 1988.

- "Design and Analysis of Computer Experiments in Chemistry and Chemical Engineering," (with M. D. Morris) 1989 Gordon Research Conference on Statistics in Chemistry and Chemical Engineering, New Hampton, NH, July 31-August 4, 1989.

\section{Invited talks}

- "A Bayesian Approach to the Design and Analysis of Computational Experiments," (with M. D. Morris) Thirty-Third Conference on the Design of Experiments in Army Research, Development and Testing, Newark, DE, October 21-23, 1987.

- "A Bayesian Approach to the Design and Analysis of Computer Experiments," (with M. D. Morris) 20th Symposium on the Interface of Computing Science and Statistics, Reston, VA, April 21-23, 1988.

- "Computer Construction of Optimal Designs for Prediction in Computer Experiments," (with M. D. Morris) IBM Scientific Centre, Bergen, Norway, July 1988.

- "Computer Construction of Optimal Designs for Prediction in Computer Experiments," (with M. D. Morris) University of Augsburg, Augsburg, Germany, July 1088.

- "A Bayesian Approach to the Design and Analysis of Computational Experiments," (with M. D. Morris) First International ConferenceWorkshop on Optimal Design and Analysis of Experiments, Neuchatel, Switzerland, July 25-28, 1088.

- "Computer Construction of Optimal Designs for Prediction in Computer Experiments," (with M. D. Morris) First International ConferenceWorkshop on Optimal Design and Analysis of Experiments, Neuchatel, Switzerland, July 25-28, 1088. 
- "Computer Construction of Optimal Designs for Prediction in Computer Experiments," (with M. D. Morris) University of Reading, Reading. England, August 1988.

- "Design and Analysis of Computer Experiments in Chemistry and Chemical Engineering," (with M. D. Morris) 1989 Gordon Research Conference on Statistics in Chemistry and Chemical Engineering, New Hampton, NH, July 31-August 4, 1989.

\section{Awards}

- 1988 Martin Marietta Energy Systems Publication Award (2).

- 1988 Martin Marietta Energy Systems Author of the Year.

\section{D. Morris}

\section{Member}

- Editorial Board - Journal of Quality Technology

\section{Invited papers}

- "Two-Stage Factor Screening Procedures Using Multiple Grouping Assignments," Commun. Statist.-Theor. Meth. 16(10), 3051-3068 (1987).

- "A Bayesian Approach to the Design and Analysis of Computational Experiment," (with T. J. Mitchell) Thirty-Third Conference on the Design of Experiments in Army Research, Development and Testing, Newark, DE, October 21-23, 1987.

- "A Bayesian Approach to the Design and Analysis of Computer Experiments," (with T. J. Mitchell) 20th Symposium on the Interface of Computing Science and Statistics, Reston, VA, April 21-23, 1988, Eds. E. J. Wegman, D. T. Gantz, and J. J. Miller, pp. 49-51, American Statistical Association, 1988.

-- "Book Review of 'Cyclic Designs' by J. A. John," Technometrics 30(120), 455-456 (1988).

\section{Invited talks}

- "A Bayesian Approach to the Design and Analysis of Computational Experiments," (with T. J. Mitchell) Thirty-Third Conference on the Design of Experiments in Army Research, Development and Testing, Newark, DE, October 21-23, 1987.

- "A Bayesian Approach to the Design and Analysis of Computer Experiments," (with T. J. Mitchell) 20th Symposium on the Interface of Computing Science a.d Statistics, Reston, VA, April 21-23, 1988.

- "Computer Construction of Optimal Designs for Prediction in Computer Experiments," (with T. J. Mitchell) IBM Scientific Centre. Bergen. Norway, July 1088.

- "Computer Construction of Optimal Designs for Prediction in Compruter Experiments," (with T. J. Mitchell) University of Augshurg. Augshurg. Germany, July 1088. 
- A Bayesian Approach to the Design and Analysis of Computational Experiments," (with T. J. Mitchell) First International ConferenceWorkshop on Optimal Design and Analysis of Experiments, Neuchatel, Switzerland, July 25-28, $198 S$.

- Computer Construction of Optimal Designs for Prediction in Computer Experiments," (with T. J. Mitchell) First International ConferenceWorkshop on Optimal Design and Analysis of Experiments, Neuchatel. Switzerland, July 25-28, 1988.

- Computer Construction of Optini:1 Designs for Prediction in Computer Experiments," (with T. J. Mitchell) University of Reading. Reading, England, August 1088.

- "Bayesian Design and Analysis of Computer Experiments: Use of Derivatives in Surface Prediction," Workshop on Design for Computer Experiments, Oak Ridge, TN, November 3-5, 1988.

- "The Role of Assumptions in Statistical Modeling," Health Physics Society Annual Meeting, Albuquerque, NM, June 28, $10 S 9$.

- "Factorial Sampling Plans for Preliminary Computational Experiments," 1989 Joint Statistical Meetings, Washington, DC, August 6-10, 1989.

- -Design and Analysis of Computer Experiments in Chemistry and Chemical Engineering," (with T. J. Mitchell) 1089 Gordon Research Conference on Statistics in Chemistry and Chemical Engineering, New Hampton, NH, July 31-August 4, 1980.

Reviewer

- Technometrics

D. L. Moses

\section{Chairman}

- Oak Ridge Research Reactor Subcommittee of the ORNL Reactor Operations Review Committee.

Recording secretary

- MHTGR Subgroup of the New Production Reactor Physics Technical Working Group (Interlaboratory).

\section{Treasure:}

-.. Oak Ridge/Knoxville Section of the Anerican Nuclear Society.

Invited talk

-.. .A Revisit to the Passively Safe Reactors," Cliattanooga Engineers Club, Augist 28, 1089.

E. $\mathbf{N g}$

\section{Invited lectures}

"Recrut Developments in the Direct Solution of Sparse Fonsymunetrir Limear Systrums," Departurut of Computer Srience, Vauciegbilt

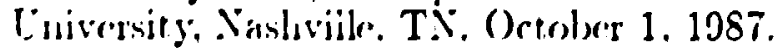


- "Direct Solution of Sparse Linear Systems on Multiprocessors," Minisymposium on Sparse Matrix Computation on Vector and Parallel Computers, SIAM 35th Anniversary Meeting, Denter, CO, October 12$15,1987$.

- "Direct Solution of Sparse Nonsymmetric Linear Systems," Gatlinburg International Conference on Numerical Liner Algebra, Fairfield Glade, TN, October 19-23, 1987.

- "Recent Developments in the Direct Solution of Sparse Nonsymmetric Linear Systems," Department of Computer Science, University of Tulsa, Tulsa, OK. November 23, 1987; also Department of Mathematics, Oklahoma State University, Stillwater, OK, November 24, 1987.

- "Some Ideas in the Solution of Sparse Linear Least Squares Problems," Departments of Computer Science and Mathematics, The University of Tennessee, Knoxville, TN, February 29, 1988.

- "On the Complexity of Some Sparse Matrix Factorizations," AMS Sectional Meeting, Knoxville, TN. March 25-26, 1988.

- "Some Ideas in the Solution of Sparse Linear Least Squares Problems," Systems Optimization Laboratory, University of Stanford, Stanford, CA, March 31, 1988.

- "On the Parallel Direct Solution of Sparse Linear Systems," Workshop on Methods and Algorithms for PDS's on Advanced Processors, Austin, TX, October 17-18, 1988.

- "A Comparison of Some Methods for Solving Sparse Nonsymmetric Linear Systems," SIAM Symposium on Sparse Matrices, Gleneden Beach, OR, May 22-24, 1989.

- "Sparse Partial Pivoting Using Static Storage Schemes," IMSL, Inc., Houston, TX, July 7, 1989.

\section{Invited papers}

- "Parallel Algorithms for Matrix Computations," (with A. Geist and M. Heath) Pp. 233-251 in The Characteristics of Parallel Algorithms, Editors, R. Douglass, D. Gannon, and L. Jamieson, MIT Press, Cambridge (1987).

- "Experiments with Method of Lines Solvers on a Shared-Memory Parallel Computer," (with S. Thompson and P. G. Tuttle) pp. 161-166 in Advances in Computer Methods for Partial Differential Equations - VI, Editor, R. Vichnevetsky. IMAC'S (1987).

- "Sparse Cholesky Factorization on a Local-Memory Multiprocessor," (with A. George, M. Heath, and J. Liu) pp. 58-75 in Parallel Processing and Medium-Scale Multiprocessors, Editor, A. Wouk, SIA.M (1989).

- "Solution of Sparse Positive Definite Systems on a Hypercube," (with A. George, MI. Heath, and J. Liu) to appear in Journal of Computation and Applied Mathematics (1080); also ORNL/TM-10865.

- "Parallel Sparse Gaussian Elimination with Partial Pivoting," (with A. George) in Pror. of the Workshop on Supercamputers and Large. Scale Optivization: Algorithms Softurare Applications. Univorsity of Minnesotia. May 10-18. 1088: to appear as a spectial volume of Annals of Operations Research. North-Hollasids; also OR.NL/TM-108GC. 
- "Parallel Direct Solution of Sparse Linear Systems," in Parallel Supercomputing: Methods. Algorithms, and Applications, G. F. Carey, Ed.. pp. 157-176 (19S9).

\section{G. Ostrouchov}

\section{Invited talks and lectures}

- "Sparse Matrix Computations in Analysis of Variance, ${ }^{n}$ presented at the University of Tennessee, in conjunction with the Numerical Linear Algebra Year and the Department of Statistics, Knoxville, Tennessee, February 25, 1988.

- "Statistical Computing on a Hypercube," presented at Kansas State University, Manhattan, Kansas, April 5, 1988.

- "Sparse Matrix Computations in Analysis of Variance," presented at Kansas State University, Manhattan, Kansas, April 7, 1988.

- "Statistical Computing on a Hypercube: Iterative Proportional Fitting," 20th Symposium on the Interface of Computing Science and Statistics, Reston. VA, April 21-23, 1988.

- -Sparse Matrix Computations in Statistics," a lecture at A Short Course on Numerical Solution of Least Squares Problems, in conjunction with the Numerical Linear Algebra Year, Ḱnoxville, TN, May 2-6, 1988.

- "Sparse Matrix Computations in Analysis of Variance," Third SIAM Conference on Applied Linear Algebra, Madison, WI, May 23-26, 1988.

Associate editor

\section{- Journal of Statistical Computation and Simulation}

\section{Organizer and chair}

- Minisymposium on Matrix Computations in Statistics." Third SIAM Conference on Applied Linear Algebra, Madison, WI, May 23-26, 1988.

R. W. Peelle

\section{Member}

- Cross Section Evaluation Working Group (CSEWG) Executive Committee.

- Program Committee, September 1988 A.NS Reactor Physics Division Topical Mleeting.

- Program Committee, 1085 NBS Conference in honor of the 50th Anniversary of the Discovery of Fission.

- C.S. member to International Nuclear Data Committee.

-. International Arlvisory Committee for 1001 Jülich Conference on Nuclear Data.

Invited paper

".imuton Standard Crosss Sections," 1088 Mito Conference on Niuclear Data for Sriener de Terhnology. Mlito, Japan. June 1083. 


\section{Chairman}

- CSEWG Covariance Data Subcommittee.

Reviewer

- Small Businr is Incentive research grant programs of the Department of Energy Office of Energy Research and of the National Science Foundation.

\section{Referee}

- The Physical Review

- Nuclear Science and Engineering

F. G. Perey

\section{Member}

- Nuclear Energy Agency Nuclear Data Committee (NEANDC).

\section{Chairman}

- NEANDC Task Force to resolve discrepancies in the Fe $1.15 \mathrm{keV}$ resonance parameters.

Referee

- Nuclear Science and Engineering

- Physical Review

- Nuclear Physics

R. T. Primm, III

Member

- Executive Committee Local Section, American Nuclear Society.

- Technical Program Commitiee, 1990 Annual Meeting of American Nuclear Society.

- Reactor Operation Review Committee - TSF.

V. A. Protopopescu

Member

-. Editorial Board of the Series Advances in Mathematics for Applied Sciences, World Scientific.

Invited lecture

- "Propagators for Nonlinear Systems: Theory and Applications," (with D. G. Cacuci) 3rd International Workshop on Mathematical Aspects of Fluid and Plasma Dynamics, Salice Terme, Italy, September 1088.

Invited papers

"Combat Modeling with Partial Differential Equations," FY 1087 Annual Meeting of the Applied and Computational Mathematirs Program, DARPA, Washington, DC, October 1987. 
- -Global Existence Theory for Symmetric Discrete Velocity Models," International Workshop on Discrete Velocity Models, Lattice Gas Dynamics. and Foundations of Hydrodynamics, Torino. Italy, September $198 s$.

-- Combat Modeling with Partial Differential Equations, Workshop on Attrition Modeling in Large-Scale Simulations. Dallas, TX. February 1989.

- An Example of Reaction-Diffusion Systems with Competitive Interactions," 11th International Conference on Transport Theory, Blacksburg, VA, May 1989.

\section{Proposal referee}

- Office for Basic Energy Science.

- Natural Sciences and Engineering Research Council of Canada.

\section{Reviewer}

- Mathematical Reviews

\section{Referee}

- Journal of Statistical Physics

- International Journal for Intelligent Systems

- Transport Theory and Statistical Physics

- Nuclear Science and Engineering

- SIAM Journal of Applied Mathematics

- Computer

W. A. Rhoades

Invited paper

-- $S_{n}$ Transport Calculations on Vector and Parallel Processors," Trans. Am. Nucl. Soc. 55, 320-321, Los Angeles, CA, November $195 i$.

Referee

-.. Nuclear Science and Engineering

R. W. Roussin

Member

Panel on Reference Nuclear Data, representative for American Nuclear Soriety, Radiation Protertion and Shielding Division.

A.SS 6.1.2: Shielding Cross Sections Committer.

Shiclding Data Testing and Applications Subrommitter, Cross Section Evaluation Working Group (C'SEWG).

OR.L Fedrat Crerlit Luion Supervisory Committer. 


\section{Chairman}

- Methods and Formats Committee, Cross Section Evaluation Working Group (CSEWG).

- Photon Interaction Evaluation Subcommittee, CSEWG.

- Nominations Committee, American Nuclear Society Radiation Protection and Shie]ding Division.

- Division coordinator, United Way campaign.

\section{Referte}

- Nuclear Science and Engineering

- Nuclear Technology/Fusion

-- Trans. of Symposia on Space Nuclear Power Systems

D. L. Selby

Member

- American Nuclear Society.

- Spectrum-90 Organizing Committee.

- WATTec 1989 Presidents Program Committee.

Vice-chairman

- 1990 Summer Meeting of the American Nuclear Society.

\section{Invited paper}

- Test, Research, and Training Reactor Meeting, October 1987.

M. S. Smith

\section{Executive committee}

- Oak Ridge/Knoxville Section of American Nuclear Society.

\section{Terranova}

Member

- Human Factors Society, Computer Systems Group, Metropolitan Chapter \& Tidewater Chapter.

- Association for Computing Machinery, Special Interest Group on Humaz Computer Interaction.

- American Psychological Society.

- IEEE Society for Systems, Man, and Cybernetics.

- IEEE Society for the Social Implications of Technology.

Theses

-. Dynamic Allocation of Pesponsibility Between Operators with Different Morlels of System Information lising Computer-Mediated 
Communication," Doctoral Dissertation, Old Dominion University, 1988 (in press); also submitted to Dissertations Abstracts International.

\section{Invited presentations}

- "The Individual and Organizational Impacts of Computer Systems," presentation made for the Senior Executive Seminar: Information Resources Management in the Navy Reserves, August 18, 1989.

- "Cognitive Skills for Complex Tasks," presentation made for the Data Systems R\&D Program Advisory Committee, June 6, 1989.

- "Innovations in Cognitive Task Analysis: Techniques for Operators of Airborne Weapons Systems," presentation made for the First Annual Airborne Weapons Training Technology Review, March 30, 1989.

D. K. Trubey

Session organizer

- Special Session on Radiation Protection Standards, ANS Winter Meeting, November 1089.

Theses

- JJANE, A New Information Retrieval System for the Radiation Shielding Information Center," University of Tennessee, August 1988.

ANS representative

- ANSI Committee N17: Research Reactors, Reactor Physics, and Radiation Shielding.

\section{Chairman}

- ANS-6: Radiation Protection and Shielding Subcommittee, Standards Committee, American Nuclear Society.

- ANS-6.4.3: Standards Working Group, Gamma-Ray Attenuation Data.

Computer security officer

- Engineering Physics and Mathematics Division.

SI standards coordinator

- Engineering Physics and Mathematics Division.

Referee

-- Nuclear Science and Engineering

V. R. R. Uppuluri

Invited papers

"Some Results on the Best of Pairerl Comparison," (with R. C. Hanumara aurl E. P. (Quim) Applied Mathematics and Computation 28, 247-268 (1988). 
- "Is p a F rime Number? Some Probabilistic Tests for Primality," (with S. R. Jammalamadaka) Mathematical Scientist 14, 55-61 (1989).

Board member

- PRISM (Partnership for Resources and Initalities in Science and Mathematics), sponsored by ORNL and ORAU.

Associate editor

- Journal of Risk Analysis, Plenum Press, Inc.

President

- Tennessee Mathematics and Computer Sciences Foundation, Inc.

-- East Tennessee Chapter of the American Statistical Association.

Council member

- East Tennessee Chapter of the Society for Risk Analysis.

Organizing committee

- Seminar/Workshop on Theory and Pragmatics of Data Quality Control, April 21-22, 1988, Oak Ridge, TN.

- Session on Consensus Methods at the 1989 Joint Statistical Meetings, March 19-22, 1989, Lexington, $\mathrm{KY}$.

Committee member

- American Statistical Association Committee for Nuclear Regulatory Research.

R. C. Ward

Member

- SIAM Council.

- Organization Committee for Gatlinburg X Meeting.

- Advisory Committee, 1989 Sparse Matrix Symposium.

- ORNL Working Group on Computing.

- Computer Science Site-Review Team, NSF Science \& Technology Centis.

Invited paper

- "Matrix Computations on Parallel Computers," American Mathematics Society, 94th Annual Meeting, Atlanta, GA, January 6-9, 1988.

Chairman

-- SIAM Activity Group on Liner Algebra.

Co-chairman

... UTK/ORNL Special Year in Numerical Linear Algebra. 


\section{Organizer}

- ORNL Workshop I on Parallel Computing.

- ORNL Workshop II on Parallel Computing.

- Minisymposium on Influence of Architecture Upon Linear Algebra Algorithms, SIAM Annual Meeting, San Diego, CA, July 17-21, 1989.

Proposal reviewer

- NSF and DOE.

\section{Fellow}

- American Assoriation for Advancement of Science.

\section{R. Weisbin}

\section{Member}

- DOE Representative to the Department of Defense, Joint Directors of Laboratories, Robotics Technology Panel.

- Program Committee, IEEE International Conference on Robotics and Automation, 1988-1989.

- U.S. Robotics Representative to International Program Committee for "Supercomputing in Nuclear Applications."

- Martin Marietta Corporate Headquarters Steering Committee on Artificial Intelligence.

- IEEE Control Society: Commiitee on Intelligent Controls.

\section{Chairman}

- 1988 Workshop on Man-Machine Symbiotic Systems: Program Chairman.

- $10 \varepsilon 9$ Chairman: Symposium on Robotics for the 8th International Congress of Cybernetics and Systems.

- (Program) Second International Conference on Artificial Intelligence.

Director

-- Robotici and Intelligent Systems Program (RISP).

- Section Head, Mathematical Modeling and Intelligent Control.

-- Center for Engineering Systems Advanced Research (CESAR).

Associate editor

-... IEEE Expert Intelligent Systems and Their Application

Techuical editor

Transaction.s on Robotic.s and Automation 
Associate professor

- Artificial Intelligence, Computer Science Department, University of Tennessee, Knoxville, TN.

Management course

- Regional Advanced Management Program.

Special assignments

- Topical Meeting on Nuclear Code Development, Tokyo, Japan, 1987.

- NATO Workshop on Mobile Robotics, Lisbon, Portugal, 1987.

Referee

- IEEE Transactions on Robotics and Automation

- Journal of Robotic Systems.

- IEEE Expert

Proposal referee

- National Science Foundation

- Department of Energy

Professional and academic honors

- IEEE Society Certificate of Appreciation.

- Who's Who in Technology Today: 6th Edition.

L. W. Weston

Co-chairman

- Fvaluation Committee of CSEWG (Cross Section Evaluation Working Group).

Invited paper

- "New Cross Section Evaluations for Reactor Fuel Materials," American Nuclear Society 1980 Winter Meeting, San Francisco, CA, November 2630, 1989.

\section{T. Wright}

\section{Chairman}

- American Statistical Association Advisory Committee to the U.S. Bureau of the Census (1988).

Invited participant

- Workshop of Demograplyy of Scientists and Engineers with Disabilities, organized by American Statistical Association, AAAS and NSF.

- Defense Personnel Planning Workshop, organized by Cornmittee on National Statistics of the National Academy of Sciences. 


\section{Invited lectures}

- "Near With Replacement Sampling Without Replacement Using Ranks," The Washington Statistical Society, Washington, DC, April 13, 1988.

- "Near With Replacement Sampling Without Replacement Using Ranks," Statistics Department, Ohio State University, Columbus, OH, May 17, 1988.

- "Randomization, Simple Random Sampling, and Pascal's Triangle," NSF Summer Research Program for College Students in Mathematical Sciences, Mathematics Department, University of Tennessee, Knoxville, TN, July 15, 1988.

- "Near With Replacement Sampling Without Replacement Using Ranks," Statistics Department, University of Tennessee, Knoxville, TN, January 31, 1989.

- "Some Basic Concepts in Probability Sampling from a Finite Universe," NSF Summer Research Program for College Students in Mathematical Sciences, Mathematics Department, University of Tennessee, Knoxville, TN, June 28, 1989.

\section{Editorial board}

- The American Journal of Mathematical and Management Sciences.

Board of directors and treasurer

- Tennessee Mathematics and Computer Sciences Foundation.

Associate professor

- (Mathematics) Knoxville College, Knoxville, TN.

- (Statistics and Mathematics) University of Tennessee, Knoxville TN.

Reviewer

- Mathematical Reviews

- National Science Foundation

Honor

- Elected to Membership to the International Statistical Institute, 1989.

Referee

- The American Journal of Mathematical and Management Sciences

- The American Statistician

-- Communications in Statistics

-. Metrika 


\section{CONFERENCES}

1987

"Short Course on Parallel Computing," September 28-October 2, 1987, Oak Ridge, Tennessee

Organizer. M. T. Heath

Attendees: $\quad 26$

Sponsor: $\quad$ ORNL, Mathematical Sciences Section

"Workshop on the Solution of Sparse Systems of Equations," October 5-9, 1987, Knoxville, Tennessee

Organizers: $\quad$ R. C. Ward and J. A. George

Attendees: $\quad 40$

Sponsors: $\quad$ ORNL, Mathematical Sciences Section, DOE Applied Mathematical Sciences Research Program, Science Alliance Program of the University of Tennessee, National Security Agency, National Science Foundation, U.S. Air Force Office of Scientific Research

"Gatlinburg X Conference on Numerical Algebra," October 18-23, 1987, Fairfield Glade, Tennessee

Co-Chairmen:

R. C. Ward (ORNL) and G. W. Stewart (University of Maryland)

Attendees: $\quad 100$

Sponsors: $\quad$ ORNL, Mathematical Sciences Section, DOE Applied Mathematical Sciences Research Program

\section{8}

"Workshop on Eigenvalues and Singular Values," January 11-15, 1988, Knoxville, Tennessee

Organizers: $\quad$ R. C. Ward and J. A. George

Attendees: $\quad 45$

Sponsors: $\quad$ ORNL, Mathematical Sciences Section, DOE Applied Mathematical Sciences Research Program, Science Alliance Program of the University of Tennessee, National Security Agency, National Science Foundation, U.S. Air Force Office of Scientific Research 
${ }^{4235} \mathrm{U}$ and ${ }^{230} \mathrm{Pu}$ Resonance Prescriptions," February 1988

Organizers: $\quad$ G. de Saussure and M. Moore (LANL)

Attendees: $\quad 30$

Sponsor: $\quad$ ORNL, Nuclear Data Measurement and Evaluation Section

"Training Workshop for the PUFF/TFT Computer Codes," March 2931, 1988, Oak Ridge, Tennessee

Organizer: M. S. Smith

Attendees: $\quad 9$

Sponsor: ORNL

"Workshop on Matrix Least Squares Problems," April 4-8, 1988, Knoxville, Tennessee

Organizers: $\quad$ R. C. Ward and J. A. George

Attendees: $\quad 35$

Sponsors: $\quad$ ORNL, Mathematical Sciences Section, DOE Applied Mathematical Sciences Research Program, Science Alliance Program of the University of Tennessee, National Security Agency, National Science Foundation, U.S. Air Force Office of Scientific Research

"Theory and Pragmatics of Data Quality Control Workshop," April 2122, 1988, Oak Ridge, Tennessee

Organizers: $\quad$ V. R. R. Uppuluri, G. E. Liepens, and D. M. Flanagan

Attendees: $\quad$ Expect $\sim 70$

Sponsors: Data Systems Research and Development Program

"NATO Advanced Research Workshop on Noise and Nunlinear Phenomena in Nuclear Systems," May 23-27, 1988, Valencia, Spain

Organizers: J. L. Munoz-Cobo and F. C. Difilippo

Attendees: $\quad 40$

Sponsors: $\quad$ NATO, Spanish Government, ORNL 
"Design for Computer Experiments," November 2-5, 1988, Oak Ridge, Tennessee

Organizer: $\quad$ T. J. Mitchell

Attendees: $\quad 40$

Sponsor: $\quad$ National Science Foundation, Air Force Office of Scientific Research, U.S. DOE Applied Mathematical Sciences Research Program and ORNL

"ENDF/B-VI Phase I Review Workshop," December 5-8, 1988, Oak Ridge, Tennessee

Organizer: $\quad$ D. C. Larson

Attendees: $\quad 30$

Sponsor: $\quad$ ORNL, Nuclear Data Measurement and Evaluation Section

"DOE/NE Robotics for Advanced Reactors - First Concerted Robot Experiment," December 14, 1988, Oak Ridge National Laboratory, Oak Ridge, Tennessee

Organizer. $\quad$ R. C. Mann

Attendees: $\quad 35$

Sponsor: ORNL

1989

"Organizing Workshop for the Southern Association for High Energy Physics," February 24, 1989, Oak Ridge, Tennessee

Organizers:- Hugh Brashear, T. A. Gabriel, and J. Gumnick

Attendees: $\quad 60$

Sponsors: ORAU and ORNL

"1989 CESAR/CEA Workshop on Autonomous Robots," May 30-June 1, 1880, Oak Ridge, Tennessee

Organizer: $\quad$ F. G. Pin

Attendees: $\quad 20$

Sponsor: $\quad$ Center for Engineering Systems Advanced Research (CESAR) 
"Robotics and Intelligent Interfaces Program, The Second International Conference on Industrial and Engineering Applications of Artificial Intelligence and Expert Systems," June 6-9, 1989, Tullahoma, Tennessee

Organizer: $\quad$ F. G. Pin

Attendees: $\quad 320$

Sponsors: $\quad$ The University of Tennessee Space Institute, Association for Computing Machinery - Sigart, Cooperating Organizations: American Association for Artificial Intelligence, IEEE - Computer Society, Oak Ridge National Laboratory, Oak Ridge Associated Universities, UTRC - Hertel Events, National Society of Professional Engineers.

"Workshop on the FITCAL Calorimeter for the SSC," April 18, June 1, July 14, August 31, September 22, 1989

Organizers: Hugh Brashear, T. A. Gabriel, and J. Walker

Attendees: $\quad 15$

Sponsors: $\quad$ ORNL and University of Florida

"Workshop on Scientific and Industrial Applications of Free Electron Laser," June 12-13, 1989, Oak Ridge National Laboratory, Oak Ridge, Tennessee

Organizers: $\quad$ F. C. Difilippo and R. B. Perez

Attendees: $\quad 30$

Sponsor: $\quad$ ORNL

"Workshop on a Hybrid Central Tracking Detector for the SSC," July 25, August 2, August 17, 1989

Organizers: Hugh Brashear, T. A. Gabriel, and A. L. Goshaw

Attendees: $\quad 20$

Sponsors: Duke University and ORNL

"Workshop on the FAST Detector for the SSC," September 7-8, 1989, Oak Ridge, Tennessee

Organizers: $\quad$ Hugh Brashear, T. A. Gabriel, and Larry Price

Attendees: $\quad 60$

Sponsors: $\quad$ ANL and ORNL 
"Workshop on a Silicon Calorimeter for the SSC," September 11, 1989 Organizers: Hugh Brashear, W. Bugg, and T. A. Gabriel Attendees: $\quad 15$

Sponsor: ORNL and University of Tennessee

"Workshop on CALOR89," September 20-21, 1989, Oak Ridge, Tennescee

Organizer: T. A. Gabriel

Attendees: $\quad \sim 35$

Sponsor: ORNL 


\section{SEMINAFS AT ORNL}

M. Beckerman was seminar coordinator during the period covered by this report. The following seminars were be!d during October 1987 through August 1989.

V. Alexiades, Engineering Physics and Mathematics Division, "Nonsymmetric, Strongly Coupled, Quasilinear, Parabolic Systems"

B. J. Allen, Lucas Heights Research Laboratory, Australia, "Neutron Diagnosis and Therapy: A Physicist's Incursion into Medicine"

R. G. Alsmiller, Jr., C. West, R. T. Primm, III, and F. C. Difilippo, Engineering Physics and Mathematics Division, "The Advanced Neutron Source"

J. L. Barlow, Computer Science Department, The Pennsylvania Stat.- University, University Park, PA, "Algorithms for Equality-Constrained Least-Squares Problems"

K. Barry, Brigham Yount University, Provo, LT, "Graphical Representation of Sparse Matrix Elimination Trees on a Sun Workstation"

A. Björck, Department of Mathematics, Linköping University, Linköping, Sweden, "Stability Analysis of the Method of Seminormal Equations for Linear LeastSquares Problems"

V. Boffi, University of Bologna, Italy, "Dynamical Systems from Nonlinear Particle Transport"

D. Boley, Computer Science Department, University of Minnesota, Minneapoiis, MN, "The Nonsymmetric Eigenproblem-A Prototype Hypercube Implementation"

J. Borenstein, Department of Mechanical Engineering, The University of Michigan, Ann Arbor, MI, "The Vector Field Histogram - Fast Obstacle Avoidance for Mobile Robots"

B. Bradley, University of Virginia, Charlottesville, VA, "A Survey of Methods in the Optimal Control of the Stefan Problem"

C. A. Brau, Physics Department, Vanderbilt University, Nashville, TN, "Free-Electron Laser Technology"

R. Byers, Department of Mathematics, University of Kansas, Lawrence, KS, "A Torlbox for Very Special Eigenvalue Problems"

D. Cain, State University of New York at Oswego, "A Graphics Package for Displaying Sparse Matrices on in Sun Workstation"

I. Cavers, Department of Computer Science University of British Colimluia, "Using Deficiency Mleasure for Tiebreaking the Minirnum Degree Algorithm" 
M. R. Celis, IMSL, Inc., Houston TX, “A Trust-Region Strategy for Nonlinear Equality Constrained Optimization ${ }^{n}$

W. Celmaster, D. Fanton, and Richard Schaff, BBN Advanced Computers, Inc., "The Butterfy GP1000 Parallel Processor"

B. W. Char, Department of Computer Science, The University of Tennessee, Knoxville, TN, "Automatic Old-Fashioned Analysis of Numerical Stability"

H. I. Christensen, Laboratory of Image Analysis, Aalborg University, Denmark, "Monitoring Railway Crossings in Real Time: An Application in Computer Vision"

A. Cleary, University of Virginia, Charlottesville, VI, "The Solution of Narrowly Banded Linear Systems on Parallel Computers by Direct Methods"

T. Coleman, Department of Computer Science, Cornell University, Ithaca, NY, "Parallel Algorithms for Solving Systems of Nonlinear Equations on a Hypercube Computer"

T. Coleman, Department of Computer Science, Cornell University, Ithaca, NY, "On the Convergence Analysis of Algorithms for Constrained Optimization"

T. Coleman, Department of Computer Science, Cornell University, Ithaca, NY, "Exploiting Sparsity in Large-Scale Optimization"

J.-C. Culioli, Engineering Physics and Mathematics Division, "Sigmoid Functions: A Delicate Tool to Design Some Optimization Algorithms"

C. Currin, Bryn Mawr College, Bryn Mawr, PA, "Design and Analysis of Computational Experiments"

H. Davis, Stanford University, Stanford, CA, "Characterizing the Synchronization Behavior of Parallel Programs"

Eduardo n'Azevedo, Department of Computer Science, University of Waterloo, Ontario, "On Optimal Triangulation for Piecewise Linear Approximation"

F. C. Difilippo and R. T. Primm, Engineering Physics and Mathematics Division, and C. D. West, Engineering Technology Division, "The Advanced Neutron Source (ANS)"

D. L. Donohue and L. D. Hulett, Jr., Analytical Chemistry Division, "Experimental Applications of the Positron Facility at ORELA"

L. Dowdy, Department of Computer Science, Vanderbilt University, Nashville, TN, "On the Partitioning of Multiprocessor Systems"

D. Downing, Engineering Physics and Mathematics Division, and S. Bartell, Euvironmental Sciences Division, "Sensitivity and Uncertainty Analysis in Environmental Models"

J. V. Draper, Fuel Recycle Division, "Teleoperator Human-Machine Interface Research at the Remote Systems Development Section"

.v. R. Draper, University of Wisconsin, Madison, WI, "Simple and Intricate Two-Level Designs" 
R. E. England, Department of Computer Science, The University of Tennessee, "Cliques and Their Separators in Triangulated Graphs"

R. Entriken, Department of Operations Research, Student - Stanford University, Stanford, CA, "The Parallel Solution of the Stochastic Capacity Expansion Problem

L. Etheridge, Roanoke College, Salem, VA, "Graphical Animation of Message-Passing Parallel Algorithms"

R. E. Flanery, Jr., Engineering Physics and Mathematics Division, Multitasking and Multiprocessing on the CRAY-2n

R. N. Fleischman, Independent Consultant, "Development and Verification of a Model of the Cognitive Contents of Displays"

P. Fua, SRI International, Menlo Park, CA, "Objective Functions for Model-Based Vision"

K. Gates, Applied Mathematics Department, University of Washington, Seattle, WA, "A Fast Variation of Cuppen's Method which Computes only the Eigenvalues of a Symmetric Matrix"

G. A. Geist, Engineering Physics and Mathematics Division, "Finding Eigenvalues and Eigenvectors of Unsymmetric Matrices Using a Distributed-Memory Multiprocessor"

G. A. Geist and E. Ng, Engineering Physics and Mathematics Division, "A Partitioning Strategy for Sparse-Matrix Factorization on Multiprocessors"

J. A. George, UT/ORNL Distinguished Scientist, "On the Complexity of Sparse LU and QR Factorization of Finite-Element Matrices"

M. E. Glicksman, Rensselaer Polytechnic Institute, Troy, NY, "Kinetics of Dendritic Growth"

G. Golub, Computer Science Department, Stanford University, Stanford, CA, "The Theory of Moments in Linear Algebra"

S. W. Hammond, Computer Science Department, Rensselaer Polytechnic Institute, "Arcinitecture and Operation of a Systolic Engine for Finite Element Computations"

C. Hansen, INRIA, Paris, France, "CAD-Based 3-Dimensional Computer Vision: The Automatic Generation of Recognition Strategies"

P. C. Hansen, Copenhagen Unjversity Observatory, Kobenhavn K, Denmark, "Truncated .JD Solutions to Ill-Posed Problems With Ill-Determined Numerical Rank"

C. T. Harston, Department of Computer Science, The University of Tennessee, Chattanooga, TN, "Neural Networks Used in Robotics Control"

J. B. Hayter, Solid State Division, "Transport and Application of Cold Neutrons from ANSR" 
M. T. Heath, Engineering Physics and Mathematics Division, "Parallel Matrix Computations on Hypercubes"

R. L. Iman. Sandia National Laboratory, Albuquerque, NM, "Bayesian Models for Time To Recovery and Initiating Event Frequency for Loss of Off-Site Power Incidents at Nuclear Power Plants"

A. R. Ingraffea, Department of Civil and Environmental Engineering, Cornell University, Ithaca, NY, "Interactive Computer Simulation of Fracture Processes"

E. Jessup, Department of Computer Science, Yale University, "Parallel Solution of the Symmetric Tridiagonal Eigeuproblem"

L. Kellerher, Ohio Northern University, Ada, $\mathrm{OH}$, "A Graphical Interface for a Log-Linear Model"

S. Keller-McNulty, Department of Statistics, Kansas State University, Manhattan, KS, "Error-Free Sparse Least Squares"

T. Kerlin, Department of Nuclear Engineering, The University of Tennessee, Fnoxville, TN, "Vuclear Engineering at The University of Tennessee"

A. I. Khuri and G. G. Vining, Department of Statistics, University of Florida, Gainesville, FL, "Some Recent Developments in Response Surface Methodology"

D. R. Kincaid, University of Texas at Austin, "Software and Numerical Experiments for Nonsymmetric Preconditioned Conjugate Gradient Methods"

V. Klema, Laboratory for Information and Decision Systems, Massachusetts Institute of Technology, Cambridge, MA, "Robust Statistics Computations on Parallel Computing Machines"

K. Knight, Meredith College, Raleigh, NC, "Use of Meet-in-the Midlle Problem in Cryptanalysis of Closed Cryptosystems"

S. Kundu, Louisiana State University, Computer Science Department, "Rule Discovery from Examples"

E. Lazaridis, University of Pennsylvania, Philadelphia, PA, "The Use of Derivative Information in the Analysis of Multidimensional Computation Experiments"

M. R. Leuze, Vandertilt University, Nashville, TN, "Independent Set Orderings for Parallel Matrix Factorization by Gaussian Elimination"

R. Leveque, Departments of Mathematics and Applied Mathematics, University of Washington, Seattle, WA, "Cartesian Ǵ:id Methods for Compressible Flow in Irregular Regions"

K. Lindenberg, Department of Chemistry and Institute for Nonlinear Science, University of C'alifornia, San Diego, CA, "Effects of Colored Soise on Lincar and Nonlinear Dynamical Systerns" 
K. Lindenberg, Department of Chemistry and Institute for Nonlinear Science, University of California, San Diego. CA, "How Does Energy Migrate in Molecular Aggregates?"

J. W. H. Liu, Department of Computer Science, York University, Ontario, "Some Practical Aspects of Elimination Trees in Sparse Factorization"

I. Lough, Rhode Island Coliege, Providence, RI, “Generation of Tracefiles for DistributedMemory Machines"

V. Lumelsky, Department of Electrical Engineering, Yale University, New Haven, CT, "Robot Motion Planning Amids: Unknown Obstacles"

B. J. MacLennan, Computer Science Department, The University of Tennessee, "Field Computation: A Basis for Neural, Optical and Molecular Information Processing"

J. M. J. Madey, Department of Physics, Duke University, Durham, NC, "Infrared FreeElectron Laser Light Sources for University and Laboratory Research"

L. K. Mansur, Metals and Ceramics Division, "On a Possible Mechanism for Accelerated Embrittlement in Pressure Vessel Steels"

M. Marx, Physics Department, SUNY, Stonybrook, NY and SSC/CDG, Lawrence Berkeley Laboratory, CA "Alternative Approach to Superconducting Supercollider (SSC) Detectors"

T. Miki, Power Reactor and Nuclear Fuel Development Corporation, Japar, "Japanese Artificial Intelligence Technology and Research"

T. J. Mitchell, Engineering Physics and Mathematics Division, "Computer Construction of Optimal Designs for Prediction in Computer Experiments"

T. J. Mitchell, Engineering Physics and Mathematics Division, "Computer Experiments to Optimize a Compression Mold Filling Process"

T. J. Mitchell and J. J. Beauchamp, Engineering Physics and Mathematics Division, "Bayesian Variable Selection in Regression"

T. J. Mitchell and M. D. Morris, Engineering Physics and Mathematics Division, "A Bayesian Approach to the Design and Analysis of Computational Experiments"

D. A. Mitta, Human Factors Laboratory, Virginia Polytechnic Institute \& State University, Blacksburg, VA, "Expert System Usability"

R. Monaco, Politecnico di Torino, Torino, Italy, "Discrete Models in Kinetic Thenry of Gases Analysis and Applications"

M. D. Morris, Engincering Physics and Mathematics Division, "Bayesian Design and Analysis of Computer Experiments: Use of Derivatives in Surface Prediction"

M. D. Morris, Engineering Physics and Mathematirs Division, "A Bayesian Approach to the Design and Analysis of Computational Experiments" 
D. Mutchler, Computer S̄cience Department, The University of Tennessee, Knoxville, TN, "Distributed Algorithms for Maintaining the Consistency of a Replicated File in the Face of Network Partitioning"

M. Mutrie, University of Waterloo, "Towards a Symbolic System for Floating-Point Error Analysis"

H. Nagao, College of Engineering, University of Osaka Prefecture, Japan, "Ons the Jackinife Statistics for Canonical Correlation Coefficients"

E. Ng, Engineering Physics and Mathematics Division, "Some Ideas in the Solution of Sparse Linear Least-Squares Problems"

E. $\mathrm{Ng}$, Engineering Physics and Mathematics Division, "Direct Solution of Sparse Linear Systems on Multiprocessors"

T. H. Olesen, Christian Michelseı Institute, Bergen, Norway, "Vectorized Dissection on the Hypercube"

G. Ostrouchov, Engineering Physics and Mathematics Division, "Iterative Proportional Fitting of Log-Linear Models on a Hypercube"

G. Ostrouchov, Engineering Physics and Mathematics Division, "Sparse-Matrix Computations in Analysis of Variance"

H. Park, Department of Computer Science, University of Minnesota, Minneapolis, MN, "Jacobi Algorithms for Eigenproblems on a Hypercube"

T. Peierls, Department of Computer Science, Cornell University, "Sparse Gaussian Elimination in Essential Time"

F. G. Perey, Engineering Physics and Mathematics Division, "Measured and Calculated Capture Detector Response Functions"

I. Petr, Lniversity of California, Berkeley, and Technical University of Prague, Czechoslovakia, "Computer Modeling of International Relations: Alternative Defense Systems and Prospects for Winding Down"

B. W. Peyton, Boeing Computer Services, Seattle, WA, "A Fast Implementation of the Jess and Kees Algorithm Based on Clique Trees"

R. J. Plemmons, Departments of Computer Science and Mathematics, North Carolina State University, Raleigh, NC, "Parallel Least Squares Algorithms in Signal Processing"

D. A. Poplawski, Department of Computer Science, Michigan Technological University, Houghton, MI, "Two Aspects of Parallel Program/Architecture Characterization"

R. Powell. Allegheny College, Meadville, PA, "A Debugging Interface for a Heterogeneous Network"

L. Renker. DOE Science and Engineering Research Participant, "Survey of the Literature on Fractals with Poxsible Applications to Phase Change Processes" 
J. Roning, Oulu University, Oulu, Finland, "Model-Based Visual Navigation System for a Mobile Robot ${ }^{n}$

P. Rusu, University of Colorado, Boulder, $\mathrm{CO}$, "On the Wave-Particle-Like Solutions of Nonlinear Equations"

B. N. Sanders, RichardsonSmith, Inc., Worthington, $\mathrm{OH}$, "An 'Experiment' in Interdisciplinary Design"

M. Saunders, Department of Operations Research, Stanford University, Stanford, CA, "Maintaining the Rank of Sparse LU Factors"

A. D. Solomon, ORNL Consultant, Omer, Israel, "Simulation of a PCM-Impregnated Sensible Heat Wall"

A. D. Solomon, ORNL Consultant, Omer, Israel, "The Simulation of a Thermal Performance of a Structure Containing Distribute Phase Change Material"

V. S. Sunderam, Department of Mathematics and Comp/Science, Emory University, Atlanta, GA, "PVM: A Framework for Parallel Distribited Computing"

S. Thompson, Mathematics and Statistics Department, Radford University, VA, "A New Approach for Handling Discontinuities Arising in the Solution of Differential Equations with State-Dependent Delays"

S. Ting, Massachusetts Institute of Technology, "In Search of the Basic Building Blocks of Naturen

R. I. Van Hook, Environmental Sciences Division, "Global Environmental Studies"

C. Vaughan, University of Virginia, "The SSOR Preconditioned Conjugate Gradient Method on Parallel Computers"

K. Vest lić, Fernuniversität, Hagen, Germany, "On an Eigenreduction Algorithm for Definite Matrix Pairs"

E. Wachspress, Department of Mathematics, The University of Tennessee, Knoxville, TN, "Iterative Solution of Sylvester's Equation"

E. Wachspress, Department of Mathematics, The University of Tennessee, Knoxville, TN, "Essential Sparse LU Factorization"

C. G. Wagner, The University of Tennessee, Knoxville, TN, "Consensus for Belief Functions and Related Uncertainty Mes ;ures"

N. J. Wahls, Vanderbilt University, Department of Computer Science, Nashville, 'TN, "A Conceptual Framework for Distributed Debugging"

R. M. Waldman, Atomic Energy Commission of Argentina, Buenos Aires, "Safety Analysis of MTR Cores"

H. Watanabe, Departmest of Computer Science, lniversity of North Carolina, Chapel Hill, NC, "VLSI Fuzzy Logic Inference Processor for Real -Time Control" 
S. M. Watt, IBM T. J. Watson Research Center, Yorktown Heights, NY, "The Scratchpad System for Algorithmic Mathematics"

D. G. Wilson, Engineering Physics and Mathematics Division, "An Implicit, Finite Difference, Enthalpy Scheme and its Application to a Multidimensional Cyclic Melting and Freezing Problem"

D. G. Wilson, Engineering Physics and Mathematics Division, "Changing Domains and Solidification Problems with Voids"

P. H. Worley, Engineering Physics and Mathematics Division, "Limits on Parallelism in the Numerical Approximation of Linear PDEs"

T. Wright, Engineering Physies and Mathematics Division, "Near Probability Proportional to Size (xps) Sampling with Replacement Which is Without Replacement Based on Ranks" 


\section{PUBLICATIONS}

\section{ALEXIADES, $\mathrm{V}$.}

"Effect of a Void on Cyclic Melting and Freezing of an Encapsulated PCM," book chapter in Multiphase Flow Heat and Mass Transfer, ASME HTD, Vol. 109, pp. 17-20, R. K. Shak, Ed., American Society of Mechanical Engineers, New York, 1989. (1.54)t

"Void Formation in Solidification," book chapter in Eifferential Equations, Vol. 118, pp. 17-23, C. M. Dafermas, G. Ladas, and G. Papanicohou. Eds., Lecture Notes in Pure and Applied Math., Marcel Dekker, New York, 1989. (1987)

\section{ALEXIADES, V., AND A. D. SOLOMON}

"The Formation of a Solid Nucleus in Supercooled Liquid, Part II," J. of Non-Equilibr. Thermodyn. 14, 99-109 (1989). (1987)

\section{AJEXIADES, V., A. D. SOLOMON, AND D. G. WILSON}

"I'he Formation of a Solid Nucleus in Supercooled Liquid, Part I,"J. of NonEquilibr. Thermodyn. 13, 281-300 (1988). (1987)

\section{ALEXIADES, V., AND R. WICHNER*}

"Calculation of the Specific and Volumetric Enthalpies of Ge-Si Alloys," ORNL/TM-10548 (April 1988).

\section{ALSMILLER, F. S., AND R. G. ALSMILLER, JR.}

"Inclusion of Correlations in the Empirical Selection of I.tranuclear Cascade Nucleons from High-Energy Hadron-Nucleus Collisions," ORNL/TM-11032 (January 1989); also Nucl. Instrum. Methods P3ys. Res. A278, 713-721 (1989). (4.35)

ALSMILLER, R. G., JR., F. S. ALSMILLER, AND O. W. HERMANN* "Calculated Inclusive Neutron Production from $400 \mathrm{GeV}$ Proton-Nucleus Collisions," ORNL/TM-11257 (Áugust 1989). (4.38)

\section{AZMY, Y. Y.}

"The Weighted Diamisnd Difference Form of Nodal Transport Methods," Nucl. Sci. Eng. 98, 29 (1988). (1987)

t In most cases, the number shown in parentheses following the publication corresponds to the number of an abstract included in this report. In some cases, the number is a year, which means the abstract was fublished in an earlier progress report published that year. If neither an abstract number nor a year appears, then an abstract was not available for this report.

* Not a member of Fnginecring Physics and Mathernatics Division. 
"Comparison of Three Approximations to the Linear-Linear Nodal Transport Method in Weighted Diamond-Difference Form," Nucl. Sci. Eng. 100, 190 (1988). (1987)

\section{AZMY, Y. Y., AND V. PROTOPOPESCU}

"Twu-Dimensional Maps Generated by Competitive Systems," ORNL/TM$110 \approx 6$ (February 1989). (4.68)

\section{BARHEN, J., N. TOOMARIAN, AND V. PROTOPOPESCU}

"Optimization of the Computational Load of a Hyyercube Supercomputer Onboard a Mobile Robot," Applied Optics 26(23), 5007 (1987). (1987)

BARNES, J. M., * R. T. SANTORO, J. O. JOHNSON, J. D. DRISCHLER, T. A. GABRIEL, AND M. S. SMITH

"Shield Optimization Program, Part II: Effects of Van Allen Belt Radiation on SDI Weapon Platforms," ORNL/TM-10957 (December 1988). (4.55)

\section{BECKERMAN, M., AND E. M. OBLOW}

"Treatment of Systematic Errors in the Processing of Wide Angle Sonar Sensor Data for Robotic Navigation,"ORNL/TM-10763 (April 1988). (3.7)

\section{BEER, H., * AND R. L. MACKLIN}

"The ${ }^{151} \mathrm{Sm}$ Branching, A Probe for the Irradiation 'lime Scale of the sProcess," Astrophys. J. 331,1047 (1988). (2.1)

"Measurement of the ${ }^{85} \mathrm{Rb}$ and ${ }^{87} \mathrm{Rb}$ Capture Cross Sections for s-Process Studies," Astrophys. J. 339, 962 (1989). (2.2)

BOWMAN, K. O., T. HOPP,* R. KACKER, * AND R. LUNDEGARD* "Statistical Quality Control Technology in Japan," Scientific Information Bulletin 15(1) (1990). (1.123)

\section{BOWMAN, K. O., AND L. R. SHENTON*}

"Properties of Estimators for the Gamma Distribution," book, Vol. 89, Marcel Dekker, Inc., New York, NY, January 1988. (1.100)

"Sums of Powers of Binomial Coefficients," Commun. Statist.-Simula. 16(4), 1189 (1987). (1987)

"Solutions to Johnsun's $S_{B}$ ind $S_{U}$, "Commun. Statist.-Simula. 17(2), 343 (1988). (1.99)

" $S_{B}$ and $S_{U}$ Distribution Fitted by Percentiles: A General Criterion," Commun. Statist.-Simula. 18(1), 1-13 (1989). (1.101) 
"Continued Fractions and Asymptotic Series in Applications: With Special Reference to Problems in Statistics," book, Marcel Dekker, Inc., New York, NY, April 1989. (1.102)

BOWMAN, K. O., L. R. SHENTON, * AND H. K. LAM*

"Simulation and Estimation Problems Associated with the 3-Parameter Gamma Density," Commun. Statist.-Simula. 16(4), 1147 (1987). (1987)

BOWYER, K. W.*

"Object Recognition for the HERMIES Robot," ORNL/TM-10935 (October 1988). (3.8)

BRAU, J. E., * AND T. A. GABRIEL

"Theoretical Studies of Hadronic Calorimetry for High Luminosity, Higt. Energy Colliders," ORNL/TM-10903 (January 1989); also Nucl. Instrum. Methods A279, 40 (1989). (4.39)

BRAU, J. E., * T. A. GABRIEL, AND P. G. RANCOITA*

"Prospects for and Tests of Hadron Calorimetry with Silicon," ORNL/TM10954 (March 1989). (4.40)

BURKS, B. L., G. de SAUSSURE, C. R. WEISBIN, J. P. JONES, AND W. R. HAMEL*

"Autonomous Navigation, Exploration, and Recognition Using the HERMIES-IIB Robot," IEEE Expert 2(4), 18 (1987). (1987)

BURNS, T. J.

"Recent ORNL Improvements to the HULL Hydrocode System," ORNL/TM10995 (May 1989). (4.56)

CACUCI, D. G., * R. B. PEREZ, AND V. PROTOPOPESCU

"Duals and Propagators: A Canonical Formalism for Nonlinear Equations," J. Math. Phys. 29(2), 353 (1988). (1987)

CACUCI, D. G., * AND V. PROTOPOPESCU

"Propagators for Nonlinear Systems," J. Physics A 22, 2399 (1989). (4.72)

CAMPBELL, D. J.,* V. H. GUTHRIE, * J. R. KIRCHNER,* H. M. PAULA," If. C. ELLISON, * F. M. DYCUS,*. J. A. FARQUHARSON,* AND G. F. FLANAGAN

"User's Guide for PRIS!M Arkansas Nuclear One - Unit 1: Volume 1, Program for Inspectors," ORNL/TM-10604/V1 (NUREG/CR-5021, Vol. 1) (March 1988). (4.91) 
CAMPBELL, D. J.,* V. H. GUTHRIE,* J. R. KIRCHNER,* J. Q. KIRKMAN, * H. M. PAULA,* B. C. ELLISON,* F. M. DYCUS,* AND G. F. FLANAGAN

"User's Guide for PRISIM Arkansas Nuclear One - Unit 1: Volume 2, Program for Regulators," ORNL/TM-10604/V2 (NUREG/CR-5021, Vol. 2) (March 1988). (4.91)

CARPENTER, A. V.,* W. D. FLANDERS, * E. L. FROME, P. COLE,* AND S. A. FRY*

"Brain Cancer and Nonoccupational Risk Factors: A Case-Control Study Among Workers at Two Nuclear Facilities," Am. J. Public Health 77, 11801182 (1987). (1987)

CARPENTER, A. V., W. D. FLANDERS, * E. L. FROME, D. J. CRAWFORD-BROWN,* AND S. A. FRY*

"Central Nervous System Cancers and Radiation Exposure: A Case-Control Study Among Workers at Two Nuclear Facilities," Journal of Occupational Medicine 29, 601-604 (1987). (1987)

CARPENTER, A. V.,* W. D. FLANDERS, $*$ E. L. FROME, W. G. TANKERSLEY, * AND S. A. FRY*

"Chemical Exposures and Central Nervous System Cancers: A Case-Control Study Among Workers at Two Nuclear Facilities," The American Journal of Industrial Medicine 13, 351-362 (1988). (1.124)

CHRISTENSEN, H. I.*

"A Concurrent Approach to Model-Based Motion Detection," ORNL/TM11051 (January 1989). (3.48)

CHRISTIANSEN, D. S., * AND D. B. REISTER

"Estimating Finding Rates for U.S. Crude Oil," ORNL/TM-10897 (October 1988). (3.97)

"The Aggregate Production Profile for U.S. Crude Oil," ORNL/TM-10857 (October 1988). (3.98)

CHU, E.,* AND A. GEORGE*

"QR Factorization of a Dense Matrix on a Hypercube Multiprocessor," ORNL/TM-10691 (February 1988). (1.18)

COSNER, C.," S. LENHART, AND V. PROTOPOPESCU

"Parabolic Systems with Nonlinear Competitive Interactions," ORNL/TM11071 (January 1989). (4.73)

"Transport Equations with Second Order Differential Collision Operators," SIAM J. Math. Anal. 19, 797 (1988). (1987) 
CRAMER, S. N.

"Discrete Angle Biasing in Monte Carlo Radiation Transport," ORNL/RSIC50 (May 1988); Nucl. Sci. Eng. 98, 279 (1988). (5.1), [Journal Article was in 1987 Progress Report]

CRAMER, S. N., B. K. KIRK, AND J. BROADWAY*

"The Effect of Coherent Scattering in Photon Radiation Transport Calculations," ORNL/RSIC-51 (April 1989). (5.7)

\section{CULIOLI, J.-C., AND V. PROTOPOPESCU}

"A New Algorithm for Linear Programming That Is Easy to Implement: Application to the Transportation Problem," ORNL/TM-10929 (September 1988). (3.65)

"Bifurcating Optimization Algorithms and Their Possible Application," ORNL/TM-10976 (November 1988). (3.67)

"An Algorithm for Linear Programming That Is Easy to Implement," Appl. Math. Lett. 2(2), 125 (1989). (3.66)

CURLEE, T. R., * AND D. B. REISTER

"Oil Vulnerability and Intermediate Price Fluctuations: A Preliminary Assessment and Proposal," ORNL/TM-11259 (September 1989). (3.89)

CURRIN, C., $*$ T. J. MITCHELL, M. D. MORRIS, AND D. YLVISAKER *

"A Bayesian Approach to the Design and Analysis of Computer Experiments," ORNL-6498 (September 1988). (1.88)

\section{DEPIANTE, E. V.*}

"A Symbolic Generator of State Equations for Modeling of Dynamic Systems Based on the Electrical Network Analog Paradigm," ORNL/TM-10979 (March 1989).

\section{DERRIEN, H., * AND G. DE SAUSSURE}

"R-Matrix Analysis of ${ }^{230} \mathrm{Pu}$ Neutrnn Cross Sections in the Energy Range up to $1000 \mathrm{eV}, "$ ORNL/TM-10986 (January 1989). (2.27)

"R-Matrix Analysis of the ${ }^{241} \mathrm{Pu}$ Neutron Cross Sections in the Energy Range Thermal to $300 \mathrm{eV}$," ORNL/TM-11123 (April 1989). (2.28)

DE SAUSSURE, G., L. C. LEAL,* R. B. PEREZ, N. M. LARSON,* AND M. S. MOORE*

"A New Resonance-Region Evaluation of Neutron Cross Sections for U-235," Nucl. Sci. Eng. 103(2), 109 (1989). (2.31) 
DE SAUSSURE, G., AND J. H. MARABLE*

"Uncertainties of the ENDF/B-V ${ }^{238} \mathrm{U}$ Unresolved Resonance Parameters in the Range $4 \mathrm{keV} \leq \mathrm{E} \leq 45.18 \mathrm{keV}$ (MAT=1398, MF=2, MT=251), ${ }^{n}$ Nucl. Sci. Eng. 101, 285-292 (1989). (2.32)

DICKENS, J. $\mathrm{K}$.

'SCINFUL: A Monte Carlo Based Computer Program to Determine a Scintillator Full Energy Response to Neutron Detection for $E_{n}$ Between 0.1 and $80 \mathrm{MeV}$ : User's Manual and FORTRAN Program Listing," ORNL-6462 (March 1988). (2.45)

"SCINFUL: A Monte Carlo Based Computer Program to Determine a Scintillator Full Energy Response to Neutron Detection for $\mathbf{E}_{n}$ Between 0.1 and $80 \mathrm{MeV}$ : Program Development and Comparisons of Program Predictions with Experimental Data," ORNL-6463 (April 1988). (2.46)

"Use of Monte Carlo Techniques to Derive Yields for $n+{ }^{12} \mathrm{C}$ Multi-Body Breakup Reactions, ${ }^{n}$ Computers in Physics 3, 62 (1989). (2.6)

\section{DICKENS, J. K., AND J. W. McCONNELL*}

"The NE-110 Scintillator Response to 10- to 100-MeV Carbon Ions," Nucl. Instrum. Methods in Physics Research A281, 577 (1989). (2.8)

DIFILIPPO, F. C.

"Correlation of the Signals from Detectors in the Presence of a Stochastic Neutron Field," Nucl. Sci. Eng. 99, 28 (1988). (1987)

DRAKE, J. B., A. K. HUDSON, E. JOHNSON,* G. A. PFEFFER,* D. W. NOID,* AND S. THOMPSON

"Molecular Dynamics of a Model Polymer on a Hypercube Parallel Computer," Comput. Chem. 12(1), 15 (1988). (1987)

DUNIGAN, T. H.

"Performance of a Second Generation Hypercube," ORNL/TM-10881 (November 1988). (1.2)

"Hypercube Simulation on a Local Area Network," ORNL/TM-10685 (November 1988). (1.3)

"A Remote Host Facility for Intel Hypercubes," ORNL/TM-11068 (April 1989). (1.1)

EISENSTAT, S. C., ${ }^{*}$ M. T. HEATH, C. S. HENKEL, ${ }^{*}$ AND C. H. ROMINE

"Modified Cyclic Algorithms for Solving Triangular Systems on DistributedMemory Multiprocessors," SIAM J. Sci. Statist. Comput. O(3), 589 (1988). (1887) 
ENTRIKEN, R.

"A Parallel Decomposition Aigorithm for Staircase Linear Programs," ORNL/TM-11011 (December 1988). (1.4)

ETHERIDGE, Y. H., AND T. WRIGHT

"Generation of an Ordered Simple Random Sample Without Replacement Using One Random Number," ORNL/TM-10868 (August 1988). (1.105)

FINLEY, M.*

"Non-Linear Surface Fitting of Laser Range Images Using a Hypercube Concurrent Computer," ORNL/TM-11198 (June 1989). (3.14)

FROME, E. L., AND R. J. DuFRAIN*

"Maximum Likelihood Estimation for Cytogenetic Dose-Response Curves," Biometrics 42, 73 (1986). (1.106)

FROME, E. L., AND M. D. MORRIS

"Evaluating Goodness of Fit of Poisson Regression Models in Cohort Studies," Am. Stat. 43(3), 144-147 (1989). (1.128)

\section{FROSALI, G.,* AND V. PROTOPOPESCU}

"Can the Mixed Norm Be Used in Kinetic Theory," Bull. U.M.I. (7) 1-B, 999 (1987). (1987)

FRYXELL, R. C.*

"Navigation Planning Using Quadtrees," ORNL/TM-10481 (November 1987). (3.15)

FU, C. Y.

"Approximation of Precompound Effects in Hauser-Feshbach Codes for Calculating Double Differential (n,xn) Cross Sections," Nucl. Sci. Eng. 100, 61 (1988). (2.48)

FU, C. Y., AND D. M. HETRICK*

"Calculations of Double Differential ${ }^{184} \mathrm{~W}(n, x n)$ Cross Sections at $25.7 \mathrm{MeV}$ by the TNG Code," NEA Data Bank, NEANDC-253"U" INDC(NEA)7 (February 1989). (1987)

FULKERSON, W.,* S. I. AUERBACH, * A. T. CRANE,* D. E. KASH, A. M. PERRY,* D. B. REISTER, AND C. W. HAGAN, JR.*

"Energy Technology R\&D: What Could Make a Difference?" ORNL-6541/V'1 (May 1989). (3.100) 
FULKERSON, W., D. B. REISTER, A. M. PERRY,* A. T. CRANE,* D. E. KASH, *' S. I. AUERBACH, *

"Global Warming - An Energy Technology R\&D Challenge," Science 246, 868 (1989). (3.101)

FURUTA, K., ${ }^{*}$ Y. OKA, * AND S. KONDO,*

"SUSD: A Computer Code for Cross-Section Sensitivity and Uncertainty Analysis Including Secondary Neutron Energy and Angular Distributions," ORNL/TR-88/18 (November 1988). (5.18)

GABRIEL, T. A.

"Calorimeter and Related Calculations for the Superconaucting Super Collider," ORNL/TM-10996 (February 1989). (4.46)

"Detectors for the Superconducting Super Collider, Design Concepts, and Simulation," ORNL/TM-11199 (June 1989). (4.44)

GABRIEL, T. A., J. M. BARNES,* B. L. BISHOP,* J. D.DRISCHLER, J. O. JOHNSON, R. A. LILIIE, R. T. SANTORO, AND M. S. SMITH "The Oak Ridge National Laboratory Strategic Defense Initiative Shield Optimization Program," ORNL/TM-10631 (April 1988). (4.57)

\section{GABRIEL, T. A., J. E. BRAU,* AND B. L. BISHOP*}

"The Physics of Compensating Calorimetry and the New CALOR89 Code System," ORNL/TM-11060 (March 1989). (4.48)

GEIST, G. A.

"Reduction of a General Matrix to Tridiagonal Form," ORNL/TM-10991 (March 1989). (1.19)

\section{GEIST, G. A., AND G. J. DAVIS*}

"Finding Eigenvalues and Eigenvectors of Unsymmetric Matrices Using a Distributed-Memory Multiprocessor," ORNL/TM-10938 (November 1988). (1.23)

\section{GEIS', G. A., A. LU, * AND E. L. WACHSPRESS*}

"Stabilized Gaussian Reduction of an Arbitrary Matrix to Tridiagonal Form," ORNL/TM-11089 (June 1989). (1.24)

GEIST, G. A., AND E. G. NG

"A Partitioning Strategy for Parallel Sparse Cholesky Factorization," ORNL/TM-10937 (Norrmber 1988). (1.25) 
GEIST, G. A., AND C. H. ROMINE

"LU Factorization Algorithms on Distributed Memory Multiprocessor Architectures," SIAM J. Sci. Stat. Comput. 9(4), 639 (1988). (1987)

GEORGE, A.,* M. T. HEATH, AND J. LIU*

"Sparse Cholesky Factorization on a Local-Memory Multiprocessor," SIAM J. Sci. Stat. Comput. 9(2), 327 (1988). (1987)

GEORGE, A.,* M. T. HEATH, J. LIU,* AND E. NG

"Solution of Sparse Positive Definite Systems on a Hypercube, ${ }^{n}$ ORNL/TM10865 (October 1988); also J. Comp. Appl. Math. 27, 129 (1989). (1.34)

\section{GEORGE, A.,* AND J. LIU*}

"The Evolution of the Minimum Degree Ordering Algorithm," SIAM Reviev 31, 1-19 (March 1989). (1987)

GEORGE, A.,* J. LIU,* AND E. G. NG

"Communication Results for Parallel Sparse Cholesky Factorization on a Hypercube," Parallel Computing 10, 287-298 (1989). (1.35)

\section{GEORGE, A.,* AND E. NG}

"Parallel Sparse Gaussian Elimination with Partial Pivoting," ORNL/TM10866 (October 1988). (1.36)

"On the Complexity of Sparse $Q R$ and $L U$ Factorization of Finite Element Matrices," SIAM J. Sci. Stat. Comput. 8, 849-861 (September 1988). (1987)

"Some Shared Memory is Desirable in Parallel Sparse Matrix Computation," ACM Signum Newsletter 23(2), 9-13 (1988). (1.38)

GILLIES, G. T., ${ }^{*}$ M. A. LAWSON,* R. C. RITTER, * W. F. LAWKINS, S. THOMPSON,* AND W. K. SARTORY*

"Magnetically Suspended Centrifuge for Studies of Nonlinear Phenomena in Stratified Fluids: Developmental Aspects," Rev. Sci. Instrum. 60(10), 3293 (1989). (1.75)

GOODMAN, M. S.,* T. A. GABRIEL, A. Di CIACCIO,* AND R. WILSON*

"Sources of Compensation in Hadronic Calorimeters," ORNL/TM-10904 (December 1988); also Nucl. Instrum. Meth. A278, 441 (198n). (4.51)

GOPINATH, D. V.,* K. V. SUBBAIAH,* AND D. K. TRUBEY "Gamma Ray Transport in Shield-Tissue Composite System and the Buildup Factor Implications," Nucl. Sci. Eng. 97, 362 (1987). (1987) 
HAALAND, C. M.

"Forecasting Radiation Rates and Exposure from Multi-Aged Fallout," Health Phys. 53(6), 613 (1987). (1987)

HAALAND, C. M., R. T. SANTORO, AND J. M. BARNES*

"An Approximation for Black-Body X-Ray Transport in Air," ORNL/TM10810 (August 1988). (4.86)

HANSEN, P. C.*

"Solution of IIl-Posed Problems by Means of Truncated SVD," ORNL/TM10772 (June 1988). (1.29)

"Regularization, GSVD and Truncated GSVD," ORNL/TM-10779 (September 1988). (1.28)

HEATH, M. T.

"Parallel Computing," Oak Ridger, Sunday, August 6, 1989. (1.7)

HEATH, M. T., AND C. H. ROMINE

"Parallel Solution of Triangular Systems on Distributed-Memory Multiprocessors," SIAM J. Sci. Statist. Comput. 9(3), 558 (1988). (1987)

HEATH, M. T., AND P. H. WORLEY

"Once Again, Amdahl's Law," Comm. Assoc. Comput. Mach., 32/2, 262263 (February 1989). (1.12)

HENDERSON, D. L.

"Preliminary ANS Reactor Cold Source Gain Factor Calculations for Liquid Deuterium and Liquid Nitrogen-15," ORNL/TM-10824 (November 1988). (4.19)

HENDERSON, D. L., AND C. W. MAYNARD*

"Time-Dependent Single-Collision Kernels for Integral Transport Theory," Nucl. Sci. Eng. 102, 172 (1989). (4.22)

HIRAYAMA, H.,* AND D. K. TRUBEY

"Effects of Incoherent and Coherent Scattering on the Exposure Buildup Factors of Low-Energy Gamma Rays," Nucl. Sci. Eng. 99, 145 (1988). (1987)

HORWEDEL, J. E.,* B. A. WORLEY, E. M. OBLOW, F. G. PIN, AND R. Q. WRIGHT*

"GRESS Version 0.0 User's Manual," ORNL/TM-10835 (October 1988). (3.82) 
INGERSOLL, D. T., R. W. ROUSSIN, C. Y. FU, AND J. E. WHITE "DABL69: A Broad-Group Neutron/Photon Cross-Section Library for Defense Nuclear Applications," ORNL/TM-10568 (June 1989). (4.87)

JAMMALA MADAKA, S. R., * AND V. R. R. UPPULURI

"Is $p$ a Prime Number? Some Probabilistic Tests of Primality," Math. Scientist 14, 55 (1989). (1.129)

JIMENEZ, B. D., $*$ L. S. BURTIS, * G. H. EZELL, * B. Z. EGAN,* N. E. LEE, ${ }^{*}$ J. J. BEAUCHAMP, AND J. F. MCCARTHY*

"The Mixed Function Oxidase System of Bluegill Sunfish, Lepomis Macrochirus: Correlation of Activities in Experimental and Wild Fish," Environmental Toaxicology and Chemistry 7, 623-634 (1988). (1.130)

JOHNSON, J. O., AND T. A. GABRIEL

“A User's Guide to MICAP: A Monte Carlo Ionization Chamber Analysis Paclage," ORNL/TM-10340 (January 1988). (4.24)

JOHNSON, J. O., T. A. GABRIEL, J. M. BARNES, ${ }^{*}$ J. D. DRISCHLER, M. S. SMITH, AND R. T. SANTORO

"Shield Optimization Program, Part III: Effects of X-Ray Radiation from Nuclear Weapons on SDI Weapon Platforms," ORNL/TM-10995 (March 1989). (4.58)

JONES, J. P., R. C. MANN, AND E. M. SIMPSON*

"A Computer Vision System for a Hypercube Concurrent Ensemble," ORNL/TM-10679 (August 1988). (3.59)

KAM, F. B. K.,* R. E. MAERKER, M. L. WILLIAMS, AND F. W. STALLMAN*

"Pressure Vessel Fluence Analysis and Neutron Dosimetry," ORNL/TM10651 (NUREG/CR-5049) (December 1987). (3.110)

KAPLAN, T.,* M. J. AZIZ,* AND L. J. GRAY

"Application of Onsager's Reciprocity Relations to Interface Motion During Phase Transformations," J. Chem. Phys. 90(2), 1133-1140 (1989). (1.85)

KASTENBAUM, M. A.,* AND K. O. BOWMAN

"Eight Billion Mice Is the Answer. What Is the Question? Commentary for the Citation Classics," Current Contents, Engineering, Technology, and Applied Science 20(42), 18 (1989); Current Contents: Physical, Chemical, and Earth Sciences 29(42), 18 (1989); Current Contents: Agriculture, Biology, and Environmental Science 20(42), 18 (1989). (1.131) 


\section{KELLY, J. M.,* AND J. J. BEAUCHAMP}

"Mass Loss and Nutrient Changes in Decomposing Upland Oak and Mesic Mixed-Hardwood Leaf Litter," Soil Sci. Soc. Am. J. 51, 1616-1622 (1987). (1.132)

KIRK, B. L.

"Update - A FORTRAN 77 Source File Manipulator," ORNL/TDMC-4 (NUREG/CR-4478) (January 1989). (1987)

KOGER, K. H., J. HAIRE,* B. L. HUMPHRYS, J. F. MANNESCHMIDT,* K. SETOGUCCHI, AND R. NAKAI*

"Assessment of Critical Component Unavailability in Liquid Metal Reactors," Nucl. Tech. 85, 251 (1989). (3.132)

LARSON, D. C.

"Reports on Nuclear Data Activities for the DOE Nuclear Data Committee," BNL-NCS-40911, p. 132 (May 1988) and BNL-NCS-42382, p. 136 (Apri) 1989). (2.71)

LARSUN, D. C., AND J. K. DICKENS

${ }^{c: 53} \mathrm{Cr}\left(n, n^{\prime} \gamma\right)$ Reactions and the Level Structure of ${ }^{53} \mathrm{Cr},{ }^{n}$ Phys. Rev. $C$ 39, 1736 (1989). (2.12)

LARSON, N. M.

'Updated Users' Guide for SAMMY: Multilevel R-Matrix Fits to Neutron Data Using Bayes' Equation," ORNL/TM-9179/R2 (June 1989). (2.36)

LEAL, L. C., G. DE SAUSSURE, AND R. B. PEREZ

"URR Computer Code: A Code to Calculate Resonance Neutron CrossSection Probability Tables, Bondarenko Self-Shielding Factors, and SelfIndication Ratios for Fissile and Fertile Nuclides," ORNL/TM-11297 (August 1989). (2.38)

LEE, T. Y.,* AND S. N. CRAMER

"Neutron Streaming Benchmark Calculations," ORNL/RSIC-52 (August 1989). (5.8)

LEUZE, M. R., L. W. DOWDY, AND K. H. PARK*

"Multiprogramming a Distributed-Memory Multiprocessor," ORNL/TM11064 (January 1989); also Concurrency Practice and Experience 1, 19-33 (1989). (1.13)

\section{LEWIS, J. G.,* B. W. PEYTON, AND A. POTHEN*}

"A Fast Algorithm for Reordering Sparse Matrices for Parallel Factorization," ORNL/TM-11040 (January 1089); also SIAM J. Sci. Stat. Comput. 10, 1146-1173 (1989). (1.41) 
LILLIE, R. A., B. L. BROADHEAD,* J. V. PACE, II,* AND D. G. CACUCI*

"Sensitivity/Uncertainty Analysis for Free-in-Air Tissue Kerma Due to Initial Radiation at Hiroshima and Nagusali," Nucl. Sci. Eng. 100, 105 (1988).

(4.88)

LITTLEFIELD, L. G., * E. E. JOINER,* S. P. COLYER,* A. M. SAYER,* AND E. L. FROME

"Modulation of Rudiation-Induced Chromosome Aberrations by DMSO-AN OH Radical Scavenger 1: Dose Response Studies in Human Lymphocytes Exposed to $220 \mathrm{kV}$ X-Rays," Int. J. Radiat. Biol. 53(6), 875-890 (1988). (1.134)

\section{LITTLEFIELD, L. G., A. M. SAYER, AND E. L. FROME}

"Comparisons of Dose-Response Parameters for Radiation-Induced Acentric Fragments and Micronuclei Observed in Cytokinesis-Arrested Lymphocytes," Mutagenesis 4(4), 265-270 (1989). (1.133)

MACKLIP, R. L.

"Neutron Capture by ${ }^{79} \mathrm{Br},{ }^{81} \mathrm{Br}$, and ${ }^{75} \mathrm{As},{ }^{,}$Nucl. Sci. Eng. 99, 133 (1988). (2.13)

"Search for ${ }^{136} \mathrm{Xe}$ Resonance Neutron Capture," ORNL/TM-10766 (May 1988). (2.14)

MACKLIN, R. L., AND C. W. ALEXANDER*

"Neutron Absorption Cross Section of Uranium-236," ORNL/TM-1C999 (November 1988). (2.15)

MACKLIN, R. L., AND R. R. WINTERS*

"Neutron Capture of ${ }^{122} \mathrm{Te},{ }^{123} \mathrm{Te},{ }^{124} \mathrm{Te},{ }^{125} \mathrm{Te}$, and ${ }^{126} \mathrm{Te}$," ORNL-6561 (July 1989). (2.17)

MACKLIN, R. L., R. R. WINTERS,* AND D. M. SCHMIDT*

"Resonance Neutron Capture by Argon-40," Astron. Astrophys. 216, 109112 (1989). (2.18)

MACKLIN, ROGER L.,* R. B. PEREZ,* G. de SAUSSURE, AND R. W. INGLE*

"High Energy Resolution Measurement of the ${ }^{238} \mathrm{U}$ Neutron Capture Yield from $1 \mathrm{keV}$ to $100 \mathrm{keV}$," ORNL/TM-10666 (January 1988). (2.16) 
MAERKER, R. E.

"Comparison of Results Based os a Deterministic Versus a Statistical Uncertainty Analysis," ORNL/TM-10773 (May 1988). (3.83)

"Application of the LEPRICON Methodology to LWR Pressure Vessel Surveillance Dosimetry," Reactor Dosimetry; Methods, Applicetions, end Standardization STP 1001, 40-414 (May 1989). (1987)

"Analysis of the VENUS-3 Experiments," ORNL/TM-11106, NUREG/CR5338 (August 1989). (3.111)

MAIENSCHEIN, F. C.

"Engineering Physics and Mathematics Division Progress Report for Period Ending September 30, 1987," ORNL-6425 (December 1987).

MANN, R. C., R. C. GONZALEZ,* D. TESAR,* J. S. TULENKO,* AND D. K. WEHE*

"U.S. Department of Energy Nuclear Energy University Program in Robotics for Advanced Reactors," DOE/OR-884/R1 (July 1988). (3.22)

\section{MARCH-LEUBA, C.; * AND R. B. PLREZ}

"Optimal Control Theory of Load-Following and Parameter-Tracking of Nonlinear Systems: An Atpplication of Pontryagin Maximum Principle to Reactor Dynamics," ORNL/TM-10662 (December 1987). (4.74)

"Adaptive Optimal Control of Uncertain Nonlinear Systems: On-Line Microprocessor-Based Algorithm to Control Mechanical Manipulators," ORNL/TM-10764 (May 1988). (3.24)

MCDANIEL, T. W.,* C. T. HUNSAKER, * AND J. J. BEAUCHAMP "Determining Regional Water Quality Patterns and Their Ecological Relationships," Environmental Management 11(4), 507-518 (1987). (1.135)

McELLISTREM, M. T.; R. R. WINTERS,* R. L. HERSHERGER,* Z. CAU,* R. L. MACKLIN, AND N. W. HILL*

"Neutron Scattering in ${ }^{189}$ Os for Nucleosynthesiz Rates of the Odd-A Os Isotopes and Nucleochronology," Phys. Rev. C 40, 591 (1989). (2.19)

\section{MITCHELL, T. J., AND J. J. BEAUCHAMP}

"Bayesian Variable Selection in Lirear Regression," J. Am. Stat. Assoc. 83(404), 1023 (1988). (1987)

MITCHELL, T. J., AND D. S. SCOTT*

"A Computer Program for the Design of Group Testing Experimentz," Commun. Statist. Theory Meth. 16(10), 2943 (1087). (1987) 
MORRIS, M. D.

"Two-Stage Factor Screening Procedures Using Multiple Grouping Assignments," Commun. Stetist.-Theory Meth. 16(10), 3051 (1987). (1987)

"Small-Sample Confidence Limits for Parameters Under Inequality Constraints with Application to Quantal Bioassay," Biometrirs 44, 1083-1092 (1988). (1.111)

MORRIS, M. D., AND T. D. JONES*

"A Conjiarison of Dose-Response Models for Death from Hematological Depression in Different Species," Int. J. Radia. Biol. 53(3), 439 (1988). (1987)

"Some Effects of Radistion Dosimetry Errors on an Estimated Dose-Response Relationship," Irealth Phys. 56(2), 2ı9-2222 (1969). (1.137)

"Hematopoietic Death of Unprotected Man from Photon Irradiations: Statistical Modeling from Animal Experiments, ${ }^{n}$ Int. J. Radiat. Biol. 55(3), 445-461 (1989). (1.138)

MORRIS, M. D., K. T. KIMBALL, T. E. ALDRICH,* AND C. E. EASTERIY*

"Statistical Approach to Combining the Results of Similar Experiments with Application to the Hematologic Effects of Extremrly-Low-Frequency Electric Field Exposures," Bioelectmmagnetics 10, 23-34 (1989). (1.139)

MUNOZ-COBO, J. L.," A ND F. C. DIFILIPPO, Editors

"Noise and Nonlinear Phenomena in Nuclear Systems," book, Series B: Physics, Vol. 192, Plenum Press, New York (1989). (4.75)

NAKAGAWA, M.,* T. MORI,* K. KOSAKO,* T. NAKAMURA,* M. Z. YOUSSEF, ${ }^{*}$. WATANABE, * C. Y. GUNG, * R. T. SANTORO, R. G. ALSMILLER, JR., J. RARNES,* AND \%. A. GABRIEL

"Analysis of Neutronics Parameters Measured in Phase-II Experiments of the JAERI/US Collaborative Program on Fusion Blanket Neutronjes. Part I: Source Characteristics and Reaction Rate Distributions," Fusion Eng. Design 9, 315 (1989). (4.52)

NARANG, H. N., * AND J. B. DRAKE

"Parallel Solutions of a 2-D Phase Change Problem on a Hypercuhe," ORNL 650 (A pril 1989). (1.60)

NG, E.

"A Scheme for Handling Rank Deficiency in the Solution of Sparse Linear Least Squares Problems," ORNL/TM-10980 (November 1988). (1.47) 
"Parallel Direct Solution of Sparse Linear Systems," ORNL/TM-11045 (January 1989). (1.45)

\section{OBLOW, E. M.}

"A Probabilistic-Propositional Framework for the O-Theory Intersection Rule," J. General Systems 13, 187 (1987). (3.26)

"Foundations of O-Theory: Measurements and Relation to Fuzzy Set Theory," Int. J. General Systems 14, 357-378 (1988). (3.27)

"Supertracks, Supertrack Functions and Chaos in the Quadratic Map," Phys. Lettrs. A 128(8), 406 (1988). (3.28)

\section{ORTEGA, J. M.,* AND C. H. ROMINE}

"The ijk Forms of Factorization Methods. II. Parallel Systems," Parallel Compet. 7, 149-162 (June 1988). (1987).

\section{ORTEGA, J. M., * R. G. VOIGT, * AND C. H. ROMINE}

"A Bibliography on Parallel and Vector Numerical Algorithms," ORNL/TM10998 (January 1989). (1.14)

\section{OSTROUCHOV, $G$.}

"ANOVA Model Fiating via Sparse Matrix Computations: A Fast Direct Method," SIAM J. Sci. Stat. Comput. 10, 58 (1989). (1.114)

PARKS, C. V., * B. L. BROADHEAD,* O. W. HERMANN,* J. S. TANG,* S. N. CRAMER, J. C. GAUTHEY, B. L. KIRK, AND R. W. ROUSSIN

"Assessment of Shielding Analysis Methods, Codes, and Data for Spent Fuel Transport/Storage Applications," ORNL/CSD/TM-246 (July 1988). (1987)

PEREY, F. G.

"RFUNC - A Code to Analyze Differential Elastic-Scattering Data," ORNL/TM-11112 (March 1989). (2.43)

PEREY, C. M., F. G. PEREY, J. A. HARVEY, N. W. HILL, * N. M. LARSON,* AND R. L. MACKLIN

${ }^{458} \mathrm{Ni}+n$ Transmission, Differential Elastic Scattering and Capture Measurements and Analysis from 5 to $813 \mathrm{keV}$," ORNL/-TM-10841 (September 1988). (2.20)

\section{PROTOPOPESCU, V.}

"On the Fokker-Planck Equation with Force Term," J. Phys. A20, 1239 (1987). (1987) 
PROTOPOPESCU, V., R. T. SANTORO, AND J. DOCKERY*

"Combat Modeling with Partial Differential Equations," Eur. J. Oper. Res. 38, 178 (1989). (1987)

PROTOPOPESCU, V., R. T. SANTORO, J. DOCKERY,* R. L. COX, AND J. M. BARNES*

"Combat Modeling with Partial Differential Equations," ORNL/TM-10636 (November 1987). (4.78)

REISTER, D. B.

"The Link Between Energy and GNP in Developing Countries," ASSET 9(1), 20 (1987). (1987)

"Replacement Cost Integration Program Model Overview," ORNL/TM-11042 (February 1989). (3.108)

"Replacement Cost Integration Program Model Description," ORNL/TM11132 (March 1989). (3.105)

"The Replacement Cost Integiation Program User's Guide," ORNL/TM11124 (March 1989). (3.104)

"A Compact Model of Oil Supply Disruptions," Resources \& Energy 10, 111183 (1988). (1987)

REISTER, D. B., AND D. S. CHRISTIANSEN*

"Validation of the Replacement Cost Integration Program," ORNL/TM11046 (February 1989). (3.107)

\section{REISTER, D. B., J. V. CONOPASK,* AND P. E. MIHLMESTER*}

"The RAMS Coal Model, Volume I - Model Overview," ORNL;TM10686/V1 (February 1988). (3.109)

"The RAMS Coal Model, Volume II - User's Guide," ORNL/TM-10686/V2 (February 1988). (3.109)

"The RAMS Coal Model, Volume III - Model Description," ORNL/TM10686/V3 (February 1988). (3.109)

\section{RENKER, L. A.,* AND D. G. WILSON}

"Survey of the Literature on Fractals with Possible Applications to Phase Change Processes," ORNL/TM-10778 (June 1988). (1.61)

"SIMDLA: A FORTRAN Program Simulating Diffusion-Limited Aggregation," ORNL/TM-10876 (August 1988). (1.62) 
RHOADES, W. A., AND R. L. CHILDS*

"The DORT Two-Dimensional Discrete Ordinates Transport Code," Nucl. Sci. Eng. Computer Code Abstrect, p. 88 (1988). (4.29)

RHOADES, W. A., R. L. CHIIDS, * AND D. T. INGERSOLL "Radiation Exposure Inside Reinforced Concrete Buildings at Nagasaki," ORNL/TM-i0569 (May 1989). (4.89)

ROBINSON, J. T., N. E. TODREAS, * AND D. EBELING-KONING* "Void Distributions in Bubbly Flow Through Yawed Rod Arrays," Int. J. Multiphese Flow 14, 645-652 (1988). (3.94)

ROONEY, B. D.

"User's Manual for FERD-PC," ORNL/TM-11028 (April 1989). (4.31)

\section{RUSU, P.*}

"Two-Dimensional Combat Modeling with Partial Differential Equations," ORNL/TM-10973 (December 1988). (4.79)

SABHARWAL, A.,* S. S. IYENGAR,* C. R. WEISBIN, AND F. G. PIN

"Asynchronous Production Systems," Knowledge-Based Systems 2(2), 117127 (1989). (3.38)

SANTORO, R. T., R. G. ALSMILLER, JR., J. M. BARNES, * AND T. A. GABRIEL

"Comparison of Calculated Results with Experimental Data for the Tritium Production Rates in a $\mathrm{Li}_{2} \mathrm{O}$ Assembly," ORNL/TM-11181 (May 1989). (4.53)

\section{SANTORO, R. T., P. RUSU,* AND J. M. BARNES*}

"Mathematical Descriptions of Offensive Combat Maneuvers," ORNL/TM11000 (January 1989). (4.80)

\section{SANTORO, R. T., W. K. DAGENHART,* AND J. M. BARNES*}

"One-Dimensional $S_{n}$ Calculations to Evaluate the Shielding for an AlphaParticle Charge Exchange Neutral Analyzer," ORNL/TM-10649 (January 1988). (4.54)

\section{SANTORO, R. T., AND T. A. GABRIEL}

"Executive Summary: The Oak Ridge National Laboratory Strategic Defense Initiative Shield Optimization Program," ORNL/TM-10629 (April 1988). (4.57)

"Shield Optimization Program, Part I: Executive Summary," ORNL/TM11143 (June 1989). (4.61) 
SCHMOYER, R. L.

"Linear Interpolation with a Nonparametric Accelerated Failure-Time Model, J. Am. Stat. Assoc. 83, 441 (1988). (1987)

\section{SCHMOYER, R. L., AND S. F. ARNOLD*}

"Shrinking Techniques for Robust Regression," book chapter in Contributions to Probability and Statistics, Bsseys in Honor of Ingram Olkir, pp. 368-384, L. J. Gleser, M. D. Periman, S. S. Press, and A. R. Sampson, Eds., SpringerVerlag, New York (1989). (1.116)

SCOLLAN, D. F., Y Y. Y. AZMY, AND V. PROTOPOPESCU

"Nonlinear Maps with Competitive Interactions: Fixed-Points, Bifurcations, and Chaotic Attractors," ORNL/TM-11315 (September 1989). (4.81)

SMTTH, M. S., T. J. BURNS, AND J. O. JOHNSON

"Shield Optimization Program, Part V: A Hydrodynamic Comparison Using HULL and PUFF-TFT for a One-Dimensional Aluminum Slab, " ORNL/TM11160 (June 1989). (4.64)

SMITH, M. S., J. O. JOHNSON, T. A. GABRIEL, J. M. BA RNES, * J. D. DRISCHLER, AND R. T. SANTORO

"Shield Optimization Program, Part IV: Effects of Neutron and Gamma-Ray Radiations from Nuclear Weapons on SDI Weapon Platforms," ORNL/TM10975 (March 1989). (4.63)

SMITH, M. S., S. McNEANY,* R. T. SANTORO, AND T. A. GABRIEL

"Shield Optimization Program, Part VI: The Effect of Impurity Layers on the Hydrodynamic Response of a Beryllium Target to Mono-Energetic X-Rays Using the PUFF-TFT Code," ORNL/TM-11038 (March 1989). (4.65)

\section{SMITH, M. S., AND R. T. SANTORO}

"Initial Effects of Nuclear Weapon X-Radiation on the LAMPSHADE Orbital Debris Satellite Shield," ORNL/TM-11321 (September 1989). (4.66)

SREENATH, $\mathbf{N}$.

"Path-Planning in a Known Environment with Unexpected Obstacles: Potential Application to an Automatic Alarm Testing Robot," ORNL/TM10726 (May 1988). (3.36)

TOOMARIAN, N.," E. WACHOLDER, AND S. KAIZERMAN* "Sensitivity Analysis of Two-Phase Flow Probiems," Nucl. Sci. Eny. 99, 53 (1988). (3.86) 
TRUBEY, D. K.

"New Gamma-Ray Buildup Factor Data for Point Kernel Calculations: ANS-

6.4.3 Standard Reference Data," ORNL/RSIC-49 (September 1988). (5.6)

"An Overview of the Radiation Shielding Information Center (RSIC)," Health Physics 57(3), 497-498 (1989). (5.15)

WACHOLDER, E.

"A Neural Network-Based Optimization Algorithm for the Weapon-Target Assignment Problem," ORNL/TM-11025 (February 1989). (3.76)

WACHOLDER, E., J. HAN, AND R. C. MANN

"A Neural Network Algorithm for the Multiple Traveling Salesmen Problem," ORNL/TM-10799 (November 1988); also Biol. Cybern. 61, 11-19 (1989). (3.78)

WElIE, D. K., J. C. LEE, * W. R. MAI TIN,* R. C. MANN, W. R. HAMEL, J. S. TULENKO*

"Intelligent Robotics and Remote Systenis for the Nuclear Industry," Nuclear Engineering and Design 113, 259-267 (1989). (3.42)

WEISBIN, C. $\mathbf{R}$.

"Real-Time Control: A Significant Test of AI Technologies:" IEEE Expert 2(4), 16 (1987). (1987)

WEISBIN, C. R., G. DE SAUSSURE, J. R. EINSTEIN, E. HEER," AND F. G. PIN

"Autonomous Mobile Robot Navigation and Learning," Computer Magazine 22(6), 29-35 (June 1989). (3.45)

WEISBIN, C. R., W. R. HAMEL, D. P. KUBAN, ${ }^{*}$ S. A. MEACHAM, AND F. G. PIN

"The Robotics and Intelligent Systems Program at ORNL," Robotica 7(2), 101-111 (April 1989). (3.46)

WHITE, J. E., R. W. ROUSSIN, AND H. GILPIN*

"Guide for Licensing Evaluations Using CRAC2: A Computer Program for Calculating Reactor Accident Cotsequences," ORNL/TDMC-3, NUREG/CR-5264 (December 1988). (5.17)

\section{WILLIAMS, M. L.,* A. YÜCEL,* AND S. NADKARNY*}

"DOS-HEATING6: A General Conduction Code with Nuclear Heat Generation Derived from DOT-IV Transport Calculations," ORNL/TM10645 (May 1988). (4.33) 
WILSON, D. G., J. B. DRAKE, AND R. E. FLANERY

"Phase Change Problems with a Void," in Mathematics and Industrial Problems, pp. 97-99, A. Friedman, Ed., Springer-Verlag, New York (1988). (1.69)

\section{WILSON, D. G., AND R. E. FLANERY}

"Modeling Cyclic Meltirg and Refreezing in a Hollow Metal Cylinder," ORNL6497 (September 1985 (1.71)

\section{WINTERS, R. R., AND R. L. MACKLIN}

"Resonance Neutron Capture by ${ }^{20,22} \mathrm{Ne}$ in Stellar Environments," Astrophysical Journal 329, 943 (1988). (1987)

WORLEY, B. A.

"Deterministic Uncertainty Analysis," ORNL-6428 (December 1987). (1987)

WORLEY, B. A., F. G. PIN, J. E. HORWEDEL, AND E. M. OBLOW "ADGEN - ADjoint GENerator for Computer Models," ORNL/TM-11037 (May 1989). (3.89)

WORLEY, P. H.

"Limits on Parallelism in the Numerical Solution of Linear PDEs," ORNL/TM-10945 (October 1988). (1.51)

"Information Requirements and the Implications for Parallel Computation," Ph.D. Thesis in Computer Science, Stanford University, Stazford, CA (1987). (1.53)

"The Effect of Time Constraints on Scaled Speed-Up," ORNL/TM-11031 (Jamuary 1989). (1.50)

WRIGHT, T.

"A Note on Optimal Allocation and an Ordering of the Family of Beta Prior Distributions," Commun. Statist.-Theory Meth. 17(1), 123 (1988). (1987)

"On Some Properties of Variable Size Simple Random Sampling and a Limit Theorem," Commun. Statist. Theory Meth. 17(9), 2997 (1988). (1.118)

"A Note on Pascal's Triangle and Simple Random Sampling," College Mathematics Journal, 20(1), 59-66 (1989). (1.119) 


\section{PAPERS PRESENTED AT SCIENTIFIC MEETINGS AND SEMINARS}

Southeastern Regional Medic Leadership Council Conference on Microcomputers and Artificial Intelligence in Communication Technologies, University of Ternessee at Chattenooge, Chattanooga, TN, October 29-31, 1987

BURKS, B. L., D. L. BARNETT,* J. P. JONES, AND S. M. KILLOUGH,* "A Demonstration of Autonomous Navigation and Machine Vision Using the HERMIES-IIB Robot." (3.10) $f$

Oral Presentation, GELINA, Geel, Belgium, November 16, 1987

LARSON, N. M., "Use of SAMMY in Resonance Analysis of Neutron Data" (2.35)

IAEA Specialists' Meeting on the Internitional Nuclear Data Library for Fuion Reactor Technology, Vienna, Austria, November 16-18, 1987; Proc. V. Goulo, Ed. (August 1989)

LARSON, D. C., AND C. Y. FU, "Description of Evaluation of "att Pb Performed for ENDF/B-VI," (did not appear in proceedings). (2.53)

LARSON, D. C., C. Y. FU, AND D. M. HETRICK," "Description of Evaluation for ${ }^{34,56,57,58}$ Fe Performed for ENDF/B-VI, p. 204." (2.35)

LARSON, D. C., C. Y. FU, AND D. M. HETRICK," "Some Notes on the Calculation of Energy-Angle Correlated Distributions with TNG and Their Representation in File 6 Formats," p. 220. (2.54)

LARSON, D. C., D. M. HETRICK, * AND C. Y. FU, "Description of E vluation for ${ }^{38,61,61,62,64} \mathrm{Ni}$ Performed for ENDF/B-VI," p. 195. (2.56)

LARSON, D. C., D. M. HETRICK, * AND C. Y. FU, "Description of Evaluation for ${ }^{30,52,33,54}$ Cr Performed for ENDF/B-VI," p. 187. (2.58)

LARSON, D. C., D. M. HETRICK,* AND C. Y. FU, "Description of Evaluation for ${ }^{63,65} \mathrm{Cu}$ Performed for ENDF/B-VI," p. 212. (2.57)

SIAM Parallel Processing Conference, Los Angeles, CA, December 1-4, 1987 GEIST, G. A., AND C. H. ROMINE, "LU Factorization on DistributedMemory Multiprocessors." (1.26)

26th IEEE Conference on Decision and Control, Los Angeles, CA, December 9-11, 1987; Proc. (1987)

SREENATH, N., AND P. S. KRISHNAPRASAD," "Dynamics for Space Based Robotic Manipulation," Vol. II, p. 1417. (3.41)

* Not a member of Engineering Physics and Mathematics Division.

† The number shown in parentheses following the publication corresponds to the number of an shstract included in this report. If a number does not appear, then no abstract was available for this report. 
94th Annual Meeting of the American Mathematical Society, Atlenta, GA, January 6-9, 1988

WARD, R. C., "Matrix Comfutations on Parallel Computers."

Trind Conference on Hypercube Concurrent Computers and Applications, Pesedene, CA, Janzary 19-20, 1988; Proc. Vol. II, Geoffry Pox, Bd, (Mey 1988)

GEIST, G. A., WARD, R. C., G. J. DAVIS, ${ }^{*}$ AND R. E. FUNDERLIC,* "Finding Eigenvalues ar,d Eigenrectors of Unsymmetric Matrices Using a Hypercube Multiprocessor," pp. 1577-1582. (1.27)

GEORGE, A., * AND E. NG, "Some Aspects of the Solution of Sparse Symmetric Positive Definite Systems on Hypercube Multiprocessors," (did not appear in proceredings). (1.37)

GLOVER, C. W., Multi-Sensor Integration of the NCUBE ${ }^{\mathrm{TM}}$ Hypercube Computer," pp. 1236-1246. (3.53)

HENKEL, C. S.," M. T. HEATH, AND R. J. PLEMMONS," "Cholesky Downdating on a Hypercube, pp. 1592-1598. (1.31)

JONES, J. P., "A Concurrent On-Board Vision System for a Mobile Robot," pp. 1022-1032. (3.56)

TOOMARIAN, N., "A Concurrent Neural Network Algorithm for the Traveling Salesman Problem," pp. 1483-1490. (3.75)

IAEA Technical Committec Meeting on Evaluation of Reliability Date Sorrces, Vienna, Austria, Pebruary 1-5, 1988

NAKAI, R. ${ }^{*}$ K. SETOGUCHI, ${ }^{*}$ H. YASULA, ${ }^{*}$ AND H. E. KNEE, "Development of Freedom/Credo Data Base for LMFBR PSA." (3.133)

Oral Presentation, University of Ternessee, Department of Statistics, Knorville, TN, Pebruary 4, 1988

DOWNING, D. J., "Some Outlier Detection Methods in Time Series Analysis." (1.127)

NEANDC Specialists' Meeting on Pre-equilibrium Reactions, Semmerling, Austria, February 10-12, 1988; Proc. (1988)

FU, C. Y., "A Simplified Unified Hauser-Feshbach/Pre-Equilibrium Model for C'alculating Double Differential Cross Sections," pp. 285-291. (2.47)

1988 WATTec Conference, Knoxville, TN, February 16-19, 1988

BOWMAN, K. O., "Equal Opportunities in Science and Engineering." (1.121)

HAMEL, W. R.," AND F. G. PIN, "The Man-Robot Symbiosis Project." (3.16)

MANN, R. C., "The U.S. DOE/NE University Program in Robotics for Advanced Reactors." (3.19) 
Oral Presentation, Institute for Mathematics and Its Applications, University of Minnesota, Minneapolis, MN, Friruary 19, 1988

WILSON, D. G., "Changing Domains and Solidification Problems with Voids." (1.67)

Oral Presentation, University of Tennessee, Department of Statistics, Knorville, TN, Pebruwry 25, 1988

OSTROUCHOV, G., "Sparse M-trix Computations in Analysis of Variance." (1.112)

Oral Presentation, University of Tennessee, Knoxville, TN, February 29, 1988

NG, E., "Some Ideas in the Solution of Sparse Linear Least Squares Problems." (1.43)

Summer School on Computers in Medical Research, South African Medical Research Council, Cape Town, Africa, Pebruary 29-March 1, 1988

MASKEWITZ, B. F., "Computers and Interactions Between Physicians and Health Physicists." (5.11)

Annual Congress of the South African Association of Physicists in Medicine and Biology, Cape Town, Africa, March 2-4, 1988

MASKEWITZ, B. F, "Nuclear Industry Quality, Productivity, and Innovation from the Perspec: $\cdots$ of an International Information Center." (5.8)

MASKEWITZ, B. F., "Technology Resources in Support of Programs of the Physicist in Medicine and Biology." (5.10)

Society of Manufacturing Engineers Monthly Meeting, STIK Campus, Knoxville, TN, March 9, 1988

DOW NING, D. J., "Statistical Graphics - The Visual Display." (1.126)

Oral Presentation, East Tennessee Chapter of the American Statistical Association, Oak Ridge, TN, March 8, 1988

UPPULURI, V. R. R., “Weighted Voting Models.” (1.140)

Oral Presentation, University of Florida, Gainesville, FL, March 15-16, 1988

GEIST, G. A., "Hypercube Multiprocessors: What They Are and How To Use Them." (1.5)

GEIST, G. A., "LU Factorization on Distributed-Memory Multiproressors." (1.26)

American Mathematical Society Conference, Knoxville, TN, March 25, 1988

HEATH, M. T., "Parallel Triangular Solutions and Downdating on DistributedMemory Multiprocessor3." (1.30) 
NG, E., "On the Complexity of Some Sparse Matrix Factorizations." (1.42)

ROMINE, C. H., AND A. POTHEN, "Orthogonal Factorization on Distributed-Memory Multiprocessors." (1.33)

Oral Presentation, Stanford University, Stanford, CA, March 91, 1988

NG, E., "Some Ideas in the Solution of Sparse Linear Least Squares Problems." (1.43)

Oral Presentation, Kansas State University, Manhattan, KE, April 5-7, 1988

OSTROUCHOV, G., "Statistical Computing on a Hypercube." (1.113)

OSTROUCHOV, G., "Sparse Matrix Computations in Analysis of Variance." (1.112)

Oral Presentation, University of Tennessee, Knoxville, TN, April 11, 1988

GEIST, G. A., "Finding Eigenvalues and Eigenvectors of Unsymmetric Matrices Using a Distributed-Memory Multiprocessor." (1.21)

1988 Workshop on High Level Waste Disposal, Stockholm, Sweden, April 11-14, 1988

PIN, F. G., "Analytical Methods for Large-Scale Sensitivity Analysis Using GRESS and ADGEN." (3.85)

Applications of Artificial Intelligenie VI Conference, Orlaizdo, FL, April 12-14, 1988

IYENGAR, S. S.," S. GULATI, * AND J. BARHEN, * "Smelting Networks for Real Time Cooperative Plannirg in the Presence of Uncertainties." (3.17)

SABHARWAL, A., ${ }^{*}$ S. S. IYENGAR, * G. DE SAUSSURE, AND C. R. WEISBIN, "Parallelism in Production Systems." (3.37)

Washington Statistical Society Meeting, Washington, DC, April 19, 1988

WRIGHT, T., "Near with Replacement Sampling without Replacement Using Ranks." (1.117)

Conference on Computational Atomic and Nuclear Physics at One Gigaflop, Oak Ridge, TN, April 15, 1988

HEATH, M. T., "Hypercubes." (1.10)

American Physical Society Annual Spring Meeting, Baltimore, MD, April 18-21, 1988; Proc. Vol. 33(4) (1988)

CARLTON, R. F., ${ }^{*}$ R. R. WINTERS," C. H. JOHNSON, ${ }^{*}$ N. W. HILL, AND J. A. HARVEY, "Level Densities from Resonance Spectroscopy for $n+$ ${ }^{86} \mathrm{Kr}, "$ p. 1061. (2.4) 
DAS, R. K., ${ }^{*}$ R. W. FINLAY, ${ }^{*}$ AND J. A. HARVEY, "Unified Description of the $n+{ }^{209} \mathrm{Bi}$ Mean Field Between $1 \mathrm{MeV}$ to $40 \mathrm{MeV}$ via Dispersion Relations," p. 964. (2.5)

HARVEY, J. A., AND HILL, N. W., "A NE 110 Scintillation Detector for keV Energy Neutrons," p. 1078. (2.9)

20th Symposizm on the Interface of Computing Science and Statistics, Reston, VA, April 21-29, 1988; Proc. E. J. Wegman, D. T. Gantz, and J. J. Miller, Eds., American Statistical Association, Alexandria, VA (1988)

MITCEELL, T. J., AND M. D. MORRIS, "A Bayesian Approach to the

Design and Analysis of Computational Experiments," pp. 49-51. (1.90)

OSTROUCHOV, G., "Statistical Computing on a Hypercube," (did not appear in proceedings). (1.113)

1988 Joint Meeting of the ASA Chapters of Tennessee, Hospital Corporation of America, Nashville, TN, April 29, 1988

LIEPINS, G. R., * AND V. R. R. UPPULURI, "Survey Data Quality Control Conference: A Report." (1.107)

IEEE 1988 International Conference on Robotics and Automation, Philadelphia, PA, April 25-29, 1988; Proc. Vols. 1,2,9 (1988)

MANN, R. C., W. R. HAMEL," AND C. R. WEISBIN, "The Development of an Intelligent Nuclear Maintenance Robot," Vol. 1, pp. 621-623. (3.20)

Oral presentation, Los Alamos National Laboratory, Los Alamos, NM, April 27, 1988

WORLEY, B. A., "A FORTRAN Precompiler and System for Automating the Implementation of the Forward and Adjoint Deterministic Sensitivity Methods Into Existing FORTRAN Computer Codes." (3.87)

Lecture, University of Tennessee, Knoxville, TN, May 2-6, 1988 OSTROUCHOV, G., "Sparse Least Squares Computations in Statistical Applications." (1.112)

European Boundary Element Meeting, Brussels, Belgium, May 8-10, 1988; Proc. J. L. Migeot, Ed., Universite-Libre de Bruxelles (1989)

GRAY, L. J., A. L. ASKEW, * AND G. E. GILES," "Boundary Element Method for Contact Heat Transfer," pp. 3.21-3.36. (1.80)

American Society for Information Science Meeting, Ann Arbor, MI, May i5-18, 1988

BARNETT, D. L.," G. DE SAUSSURE, AND P. F. SPELT, "The Use of an Expert System for Autonomous-Robot Control." (3.2) 
Workshop on Supercomputers and Large-Scale Optimization: Algorithms, Software, and Applications, University of Minnesota, Minneapolis, MN, May 16-18, 1988; Proc. Special Volume of Annals of Operations Research, Ben Rosen, Ed., NorthHollands

GEORGE, A.," AND E. NG, "Parallel Sparse Gaussian Elimination with Partial Pivoting." (1.36)

American Institute of Chemical Engineers' 9th Biennial Hazardous Material Spills Conference, Chicago, IL, May 16-19, 1988; Proc. (May 1988)

CAKTER, R. J., AND C. E. EASTERLY, "Designing a HAZMAT Incident Management System for Facilities with Widely Varying Emergency Organization Structures," pp. 589-597. (3.130)

Oral Presentation, Statistics Department, Ohio State University, Columbus, OH, May 17, 1988

WRIGHT, T., "Near with Replacement Sampling without Replacement Using Ranks." (1.117)

SIAM Conference on Applied Linear Algebra, Madison, WI, May 29-26, 1988

HEATH, M. T., "Parallel Triangular Solutions and Downdating on DistributedMemory Multiprocessors." (1.30)

OSTROUCHOV, G., "Sparse Matrix Computations in Analysis of Variance." (1.112)

NATO Advanced Research Workshop on Noise and Nonlinear Phenomena in $\mathrm{Nu}$ clear Systerin, Universidad Politecnica de Valencia, Spain, May 23-27, 1988; Proc. Noise and Nonlinear Phenomena in Nuclear Systems, Series B: Physics Vol. 192, J. L. Munoz-Cobo and F. C. Difilippo. Eds., Plenum Press, NY (1989)

DE SAUSSURE, G., AND R. B. PEREZ, "Stochastic Nuclear Reaction Theory: Breit-Wigner Nuclear Noise," pp. 457-461. (2.33)

DIFILIPPO, F. C., "Determination of Kinetics Parameters Using Stochastic Methods in a ${ }^{252}$ Cf Driven System," pp. 99-106. (4.10)

PEREZ, R. B., AND R. T. WOOD,* "A Stochastic Method for Nuclear Power Plant Diagnosis," pp. 451-456. ( 2.65)

Avignon '88 Eighth International Workshop on Expert Systems and Their Applications, Nanterre, France, May 90-June 9, 1988; Proc. (1988)

SABHARWAL, A., S. S. IYENGAR,* C. R. WEISBIN, AND F. G. PIN, "Asynchronous Production Systems for Real-Time Expert Systems," pp. 113146. (3.39)

International Conference on Nuclear Data for Science and Technology, Mito, Japan, May 30 June 3, 1988; Pror. S. Igarasi, Ed., Saikon Publishing Co., Ltd. (1988)

DERRIEN, H., G. DE SAUSSURE, N. M. LARSON, ' L. C. LEAL, * AND R. B. PEREZ, "R-Matrix Analyses of the ${ }^{235} \mathrm{U}$ and ${ }^{239} \mathrm{Pu}$ Neutron Cross Sections," PP. $8386 .(2.20)$ 
DIFILIPPO, F. C., "Flattening the Power Distribution of the Advanced Neutron Source Reactor," p. 563. (4.14)

FILIPPONE, W. L., ${ }^{*}$ M. S. SMITH, R. T. SANTORO, T. A. GABRIEL, R. G. ALSMILLER, JR., "Deterministic Transport Calculations of Dose Profiles Due to Proton Beam Irradiation," p. 273. (4.43)

HENDERSON, D. L., "Some Preliminary Design Considerations for the ANS Reactor Cold Source," p. 561. (4.20)

HORWEDEL, J. E., * F. G. PIN, AND B. A. WORLEY, "EXAP - Precompiler for the GRESS and ADGEN Automated Sensitivity Calculation Systems," p. 301. (3.81)

KOGER, K. H., J. HAIRE, * B. L. HUMPHRYS, J. F. MANNESCHMIDT,* K. SETOGUCHI, AND R. NAKAI, "Assessment of Critical Component Unavailability in Iiquid Metal Reactors," p. 388. (3.132)

LEAL, L. C.,* G. DE SAUSSURE, AND R. B. PEREZ, "R-Matrix Analysis of the ${ }^{235} U$ Neutron Cross Sections," p. 587. (2.40)

PRIMM, R. T., III, AND N. M. GREENE, "Generation of Lumped Fission Product Cross Sections for High-Burnup, Highly Enriched Uranium Fuel," p. 592. (4.27)

Oral Presentation, Workshop of the AAAS, Corvallis, OR, .Iune 20, 1988 CULIOLI, J.-C., AND V. PROTOPOPESCU, "Stability and Chaos in a Numerical Scheme Inspired by Neural Networks." (3.71)

16th International Sympusium on Rarefied Gas Dynamics, Pasadena, CA, July 1016, 1988; Proc. Progress in Astroneutics and Aeronautics, Vols. 116-118 (1989)

PROTOPOPESCU, V., AND Y. Y. AZMY, "Stationary Flows from a Model Boltzmann Equation," Vol. 118, p. 15-28. (4.69)

SIAM Conference on Applied Linear Algebre, Minneapolis, MN, July 11-15, 1988 AZMY, Y. Y., "A Parallel Algorithm for Solving the Multidimensional Neutron Transport Equation." (4.4)

CULIOLI, J.-C., AND G. COHIEN,"* "Decomposition/Coordination Algorithms in Convex Stochastic Optimization." (3.62)

CULIOLI, J.-C., AND V. PROTOPOPESCU, "A New Class of Optimization Algorithms with Bifurcations and Chaotic Behavior." (3.69)

KIRK, B. L., AND Y. Y. AZMY, "An Iterative Procedure fcr Snlving the Nodal Method Neutron Diffusion Equation on a Parallel Processor." (5.4)

GEIST, G. A., "Finding Eigenvalues and Eigenvectors of Unsymmetric Matrices Ứng a Distributed-Memory Multiprncessor." (1.21) 
DICKENS, J. K., AND D. C. LARSON, ${ }^{10,11} \mathrm{~B}(n, \tau \gamma)$ Reactions for Incident JNeutron Energies Between 0.1 and $25 \mathrm{MeV}$," pp. 213-215. (2.7)

HARVEY, J. A., N. W. HILL, ${ }^{*}$ F. G. PEREY, G. L. TWEED, * AND L. LEAL, ${ }^{*}$ "High-Resolution Neutron Trensmission Measurements on ${ }^{25 \mathrm{U}},{ }^{239} \mathrm{Pu}$, and ${ }^{238} \mathrm{U}$, " pp. 115-118. (2.10)

LARSON, N. M., * AND F. G. PEREY, "Resonance Parameter Analysis with SAMMY," pp. 573-576. (2.37)

MACKLIN, ROGER L., * R. B. PEREZ, G. DE SAUSSURE, AND R. W. INGLE, "High Energy Resolution Measurements of the ${ }^{238} \mathrm{U}$ Neutron Capture Yield in the Energy Region Between 1 and $100 \mathrm{keV}, "$ pp. 71-74. (2.16)

PEELLE, R. W., AND H. CONDE, “Neutron Standard Data," pp. 10051012. (2.62)

PEREY, F. G., J. O. JOHNSON, T. A. GABRIEL, R. L. MACKLIN, R. R. WINTERS, ${ }^{*}$ J. H. TODD, ${ }^{*}$ AND N. W. HILL, ${ }^{*}$ "Responses of $C_{6} D_{6}$ and $\mathrm{C}_{6} \mathrm{~F}_{6}$ Gamma-Ray Detectors and the Capture in the 1.15-keV Resonance of ${ }^{56} \mathrm{Fe}$." pp. 379-382. (2.21)

Cral Presentation, Department of Physics, Eindhoven University of Technology, Eindhoven. The Netherlands, June 2, 1988 and at the National Institute of Plasma Physics, Utrecht, The Neth 'rlands, June 9, 1988

DRAKE, J. B., W. F. LAWKINS, B. CARRERAS, ${ }^{*}$ H. R. HICKS, ${ }^{*}$ AND V. LYNCH," "MHD Calculations on a Hypercube Multiprocessor." (1.73)

1988 IEEE Fourth Conference on Human Factors and Power Plants, Monierey, CA, June 5-9, 1988; Proc. (1988)

SCHRYVER, J. C., "Operator Model-Based Design and Evaluation of Advanced Systems: Conceptual Models," pp. 115-120. (3.125)

SCHRYVER, J. C., "Operator Model-Based Design and Evaluation of Advanced Systems: Computational Models," pp. 121-127. (3.124)

Second International Conference on Vector and Parallel Computing: Issues in Applied Research and Developmert, Tromso, Norway, June 6-10, 1988

DRAKE, J. B., AND L. J. GRAY, "Parallel Implementation of the Boundary Element Method on the iPSC2 Hypercube." (1.72)

1988 Summer Meeting of the American Nuclear Society, San Diego, CA, June 1216, 1988; Trans. Am. Nucl. Soc. 'i6 (1988)

ALSMILLER, R. G., JR., F. S. A LSMILLER, T. A. GABRIEL, O. W. HERMANN,* AND B. L. BISHOP," "The Modified High-Energy Transport Code HETC and Design Calcularions for the SSC," p. 270. (4.37)

AZMY, Y. I'., "Multidimensional Nodal Transport Methods for MultipleInstruction Multiple-Data, Distributed Memory Machines," p. 202. (4.5) 
Oral Presentation, NSF Summer Progrem for College Students, Mathematics De$p$ tment, University of Tennessee, Kroxville, TN, July 15, 1988

WRIGHT, T., "Randomization, Simple Random Sampling, and Pascal's Triangle."

Oral Presentation, IBM Sicientific Centre, Bergen, Norway, July 18, 1988

MITCHELL, T. J., AND M. D. MORRIS, "Computer Construction of Optimal Designs for Prediction in Computer Experiments." (1.91)

1988 ACM Symposium on Parallel Programming, Yale University, New Haven, CT, July 20, 1988

HEATH, M. T., "Hypercube Applications at Oak Ridge National Laboratory." (1.9)

Oral Presentation, University of Augsbury, Augsburg, Germany, July 22, 1988 MITCHELL, T. J., AND M. D. MORRIS, "Computer Construction of Optimal Designs for Prediction in Computer Experiments." (1.91)

IEEE International Conference on Neural Networks, San Diego, CA July 24-27, 1988; Proc. Vol. II (1988)

CULIOLI, J.-C., AND V. PROTOPOPESCU, "A Linear-Programming Method Inspired by the Neural Networks Framework," (not published in proceedings). (3.70)

GOLDSTEIN, M., N. TOOMARIAN, * AND J. BARHEN, "A Comparison Study of Optimization Methods for the Bipartite Matching Problem (BMP)," pp. 267-273. (3.74)

WACHOLDER, E., J. HAN, AND R. C. MANN, "An Extension of the Hopfield Tank Model for Solution of the Multiple Traveling Salesmen Problem," pp. 305-324. (3.77)

First International Conference-Workshop on Optimal Design and Analysis of Experiments, Neuchatel, Switzerland, July 25-28, 1988

MORRIS, M. D., AND T. J. MITCHELL, "A Bayesian Approach to the Design and Analysis of Computational Experiments." (1.96)

NCSL Annual Conference and Symposium, Washington, DC, August 14-18, 1988 PEREY, F. G., "Covariances of Physical Constants." (2.64)

Symposium on the Cosmic Abundances of Matter, Minneapolis, MN, August 15-17, 1988

DIVADEENAM, M., * T. A. GABRIEL, O. LAZERETH, * M. S. SPERGEL, AND T. E. WARD," "The Calculation of Cosmogenic Nuclide Abundance in Stony Meteorites." (4.41) 
National Meeting of the American Institute of Chemical Engineers, Denver, CO, August 22-25, 1988

FLANAGAN, G. F., AND S. CARNES, "Use of Risk Analysis Results in Support of the Final Programmatic Environmental Impact Statement for the Disposal of the U.S. Chemical Weapons Stockpile." (4.93)

ROBINSON, J. T., AND P. J. OTADUY, "An Application of Object Oriented Programming to Process Simulation." (3.93)

1988 Joint Statistical Meetings, New Orleans, LA, A ugust 22-25, 1988; Proc. (1988) LAM, H. K.,* L. R. SHENTON, ${ }^{*}$ AND K. O. BOWMAN, "Some Properties of a Monuent Estimator for the Index Parameter of Negative Binomial Distribution," pp. 365-367. (1.103)

MITCHELL, T. J., AND J. J. BEAUCHAMP, "Bayesian Variable Selection in Linear Regression," (did not appear in proceedings). (1.110)

WRIGHT, T., "An Application of the Maximum Ratio for Comparing Different Petroleum Estimates," (did not appear in proceedings). (1.141)

Third IEEE International Symposium on Intelligent Control, Arlington, VA, August 24-25, 1988; Proc. B. E. Stephanou, A. Meystel, and J. Y. S. Luh, Eds. (1989) MANN, R. C., J. P. JONES, M. BECKERMAN, C. W. GLOVER, L. FARKAS, * J. HAN, E. WACHOLDER, AND J. R. EINSTEIN, "An Intelligent Integrated Sensor System for the ORNL Mobile Robot," pp. 170-173. (3.21)

ROBINSON, J. T., AND R. A. KISNER, "An Intelligent Dynamic Simulation Environment: An Object-Oriented Approach," pp. 687-692. (3.92)

ROVERE, L. A.," P. J. OTADUY, C. BRITTON, * AND R. B. PEREZ, "Hietarchical Control of a Nuclear Reactor Using Uncertain Dynamics Techniques," pp. 713-718. (3.95)

DOE/ANL Training Course on the Potential Safety Impact of New and Emerging Technologies on the Operation of DOE Nuclear Facilities, Idaho Falls, ID, August 29-September 1, 1988

BURKS, B. L., AND P. F. SPELT, "Ongoing Research Using HERMIES The Hostile Environment Robotic Machine Intelligence Experiment Series." (3.11)

Tenth Annual DOE Low-Level Radioactive Waste Management Conference, Denver, CO, A ugust 30-September 1, 1988; Proc. Session II: Site Performance Assessment (December 1988)

WORLEY, B. A., F. G. PIN, E. M. OBLOW, R. E. MAERKER, J. E. HORWEDEL, * AND R. Q. WRIGHT," "Deterministic Sensitivity and Uncertainty Analysis for Large-Scale Computer Models," p. 126. (3.90) 
10th Boundary Element Meeting, Southampton, United Kingdom, September 68, 1988; Proc. Bonndary Elements X, Computational Mechanics, Southampton, England (1988)

GRAY, L. J., AND G. E. GILES, “Application of the Thin Cavity Method to Shield Calculations in Electroplating." (1.81)

International Nexral Network Society Annwal Meeting, Boston, MA, September 610, 1988; Proc. (1988)

CULIOLI, J.-C., AND V. PROTOPOPESCU, "Sigmoidic Linear and NonLinear Optimization Algorithms." (3.68)

WACHOLDER, E., J. HAN, AND R. C. MANN, “A Neural Network Algorithm for the Target-Weapon Assignment Problem," Vol. 1, Supplement 1, p. 147. (3.78)

American Nuclear Society Topical Meeting on Nuclear and Hazardous Waste Management, Pasco, WA, September 11-15. 1988

CARTER, R. J., AND B. KARNOFSKY, “A Generic Hazardous Waste Management Training Program." (3.131)

ORAU Visiting Lecture Series, North Carolina Stnte University, Raleigh/Durham, NC, September 12, 1988

ROMINE, C. H., "The Evolution of Multiprocessing." (1.17)

Tth International Conference on Radiation Shielding, Boumemouth, UK, September 12-16, 1988; Proc. Vols. I, II, and III (1989)

CHILDS, R. L., * W. A. RHOADES, AND L. R. WILLIAMS, "Three-Dimensional Calculations of Neutron Streaming in the Beam Tubes of the ORNL HFIR Reactor," Vol. II, pp. 409-418. (4.8)

RHOADES, W. A., R. L. CHILDS, AND D. T. INGERSOLL, “New Dise Mortality Data Based on 3-D Radiation Shielding Calculation for Concrete Buildings at Nagasaki," Vol. III, pp. 1145-1154. (4.80)

ROUSSIN, R. W., B. F. MASKEWITZ, AND D. K. TRUBEY, "RSIC After 25 Years - Challenges and Opportunities," Vol. III, pp. 1135-1142. (5.14)

ROUSSIN, R., C. DUNFORD,* R. MCKNIGHT,* AND P. YOUNG, "The Status of ENDF/B-VI," Vol. III, pp. 1125-1134. (5.13)

Materials Handling FOCUS '88, Atlanta, GA, September 19-14, 1988

BURKS, B. L., AND P. F. SPELT, "Ongoing Research Using HERMIES The Hostile Environment Robotic Machine Intelligence Experiment Series." (3.11)

1988 International Reactnr Physics Conference, Jackson Hole, WY, September 18 21, 1988; Proc. Vols. I, II, and III (1988)

AZMY, Y. Y., AND F. C. DIFILIPPO, "Comparison of the Diffusion and Transport Calculations for the Advanced Neutron Srource Reartor," Vol. II, pp. 183 194. (4.7) 
DERRIEN, H., * AND G. DE SAUSSURE, "Current Plutonium Cross-Section Evaluations in the Resolved Resonarce Region," Supplement, p. S-65. (2.26)

DE SAUSSURE, G., L. C. LEAL, * R. B. PEREZ, N. M. LARSON, * AND M. S. MOORE "A New Resonance-Region Evaluation of Neutron Cross Sections for U-235," Vol. I, pp. 293-302. (2.31)

DIFILIPPO, F. C., M. ABU-SHEHADEH," AND R. B. PEREZ, "Reactor Physics Calculations for the Control of the Advanced Neutron Source Reactor," Vol. II, pp. 195-201. (4.15)

PEREZ, R. B., G. DE SAUSSURE, L. C. LEAL, * AND ROGER MACKLIN, "On the Unresolved Resonance Region Representation of Neutron Induced Cross Sections," Vol. III, pp. 183-188. (2.44)

Workshop on Discrete Models Lattice Ges Dynamics and Foundetions of Hydrodynamics, Torino, Italy, September 19-24, 1988; Proc. World Scientific, Singapore (1989)

PROTOPOPESCU, V. "Global Existence for Symmetric Discrete Velocity Models," p. 258. (4.76)

Specialists' Meeting on the Application of Critical Experiments and Operating Date to Core Design via Formal Methods of Cross Section Data Adjustment, Jackson Hole, WY, September 29-24, 1988; Proc. NEACRP-L-S07 (1988)

PEELLE, R. W., AND D. W. MUIR, * "Extended Covariance Data Formats for the ENDF/B-VI Differential Data Evaluation," pp. 305-315. (2.63)

Conference on Numerical Solutions of Partial Differential Equations, Virginia Polytechnic Institute, Blackburg, VA, September 24-27, 1988

WORLEY, P. H., "Limits on Parallelism in the Numerical Approximation of Linear PDEs." (1.52)

Third International Workshop on Mathematical Aspects of Fluid and Plasme Dy. namics, Salice Terme, Italy, September 26-90, 1988

CACUCI, D. G.," AND V. PROTOPOPESCU, "Canonical Propagators for Nonlinear Systems: Theory and Sample Applications." (4.71)

Oral Presentation, University of Maryland, College Park, MD, September 29-90, 1988

HEATH, M. T., "Parallel Solution of Sparse Linear Systems." (1.39)

International Collaboration on Advanced Neutron Sources (ICANS-IX) Conference, Los Alamos, NM, October 3-7, 1988

LILLIE, R. A., AND R. G. ALSMILLER, JR., "Design Calculations for the ANS Cold Source." (4.25) 
Americen Nuclear Society Bighth Topical Meeting on Techn, 7 of Pusion Energy, Salt Lake City, UT, October 9-19, 1988; Proc. Pusion Tech. 15(2) (March 1989) DUNFORD, C. L., D. C. LARSON, AND P. G. YOUNG, " "ENDF/B-VI Nuclear Data Evaluations for Fusion Applications, ${ }^{n}$ p. 429-439. (2.70)

HETRICK, D. M., * C. Y. FU, AND D. C. LARSON, "ENDF/B-VI Evaluations for Isotopes of $\mathrm{Cr}, \mathrm{Fe}, \mathrm{Ni}$, and $\mathrm{Cu}$, and for $\mathrm{Natural} \mathrm{Pb},{ }^{n} \mathrm{p}$. 453. (2.52)

Ird International Seminar on Free Boundary Problems and Their Applications, Universidad Nacional de Rosario, Rosario, Argentina, Octoler 11-15, 1988

WILSON, D. G., "Cyclic Melting and Freexing with Variable Density." (1.65)

WILSON, D. G., "Implicit Finite Difference Schemes for Phase Change Problems." (1.64)

American Physical Society Meeting, Sente Pe, NM, October 12-15, 1988

GABRIEL, T. A., M. DIVADEENAM, * M. S. SPERGEL, T. E. WARD,* AND O. LAZARETH, "Transport Calculation of Cosmogenic Nuclide Production in Stony Meteorites." (4.49)

Workshop on Parallel and Vector Computing, Austin, TX, October 17-18, 1988; Proc. Parallel Supercomputing: Methods, Algorithms, and Applications, G. . Carey, Ed., John Wiley (1989)

NG, E., "Parallel Direct Solution of Sparse Linear Systems," pp. 157-176. (1.45)

Research in Multiprocessing Special Interest Group/Numerical Analysis Special Interest Group Meeting, Anaheim, CA, October 18, 1988

WORLEY, P. H., "Limits on Parallelism in the Numerical Approximation of Linear PDEs." (1.52)

Oral Presentation, Department of Statistics, University of Tennessee, Knoxville, TN, October 18, 1988

MITCHELL, T. J., AND J. J. BEAUCHAMP, "Bayesian Variable Selection in Linear Regression." (1.109)

Colloquium of the Materials Department of the Argentina Atomic Bnergy Commission, Buenos Aires, Argentina, October 20, 1988

WILSON, D. G., J. B. DRAKE, AND R. E. FLANERY, "An Implicit, Finite Difference Enthalpy Scheme Applied to a Multidimensional Cyclic Melting and Freezing Problem with Variable Density." (1.68)

Human Factors Society 92nd Annual Meeting, Ansheim, CA, October 24-28, 1988; Proc. Vols. 1 and 2 (1988)

SEAMSTER, T. L., C C. E. SNYDER, * M. TERRANOVA, W. J. WALKER, AND D. T. JONES," "Human Factors in the Naval Air Systems Command: Computer Based Training," Vol. 2, pp. 1095-1090. (3.115) 
Oral Presentation, Applications Group Meeting, University of Illinois at UrbanaChempaign, Urbana, IL, October 25, 1988

HEATH, M. T., "Parallel Solution of Sparse Linear Systems." (1.39)

Oral Presentation, Department of Nuclear Engineering, University of Tennessee, Knoswille, TN, October 26, 1988

DICKENS, J. K., "Decay Heat Measurements and the Loss-of-Coolant Accident." (2.67)

American Methemetical Society Meeting, Lewrence, KS, October 29, 1988

GEIST, G. A., "The Unsymmetric Eigenvalue: Can HQR Be Beat?" (1.22)

1988 Winter Meeting of the American Nuclear Society, Washington, DC, October s0-November S, 1988; Trans. Am. Nucl. Soc. 57 (1988)

AZMY, Y. Y., "Accuracy of Transport Calculations for the Advanced Neutron Source, p. 293. (4.6)

AZMY, Y. Y., "Artificial Intelligence Search Techniques for Optimization of the Cold Source Geometry," p. 97. (4.1)

CRAMER, S. N., "Analytic Solution of a Five-Direction Radiation Transport Model," p. 232. (5.2)

ENGLE, W. IV., JR., R. A. LILLIE, AND C. O. SLATER, "Radiation Transport Calculations for the ANS Beam Tubes," p. 294. (4.17)

GAMBILL, W. R.," AND T. MOCHIZUKI,* "Advanced Neutron Source Design: Burnout Heat Flux Correlation Development," p. 298. (4.18)

KIRK, B. L., AND Y. Y. AZMY, "Hypercube Applications of the XY Geometry Nodal Method for the Neutron Diffusion Equation," p. 104. (5.3)

MAERKER, R. E., "The LEPRICON Code System: Consolidation of Transport Analytical and Unfolding Procedures in LWR Pressure Vessel Dosimetry," p. 225. (3.112)

PRIMM, R. T., III, "Cross-Section Processing Methndology Used to Design the Advarsced Neutron Source Reactor," p. 292. (4.26)

RYSKAMP, J. M.., F. C. DIFILIPPO, AND R. T. PRIMM, III, "Reactor Physics Methods for the Preconceptual Core Design of the Advanced Neutron Source," p. 290. (4.32)

Society for Risk Analysis Annual Meeting, Washington, DC, October \$1-November 2, 1988

FLANAGAN, G. F., W. FRAIZE, AND T. KARTACHAK," "Risk Associated with the Demilitarization of the United States Chemical Weapons Stnckpile. (4.94) 
FLANAGAN, G. F., D. JOHNSON, * D. BUTTEMER, * AND S. KAPLAN, * "Risk Associated with Operation of the Oak Ridge National Laboratory High Flux Isotope Reactor (HFIR)." (4.92)

IBEE Internetional Workshop on Intelligent Robots and Systems, Tokyo, Japen, October 31-November 2, 1988; Proc. (1988)

PIN, F. G., G. DE SAUSSURE, P. F. SPELT, S. M. KILLOUGH, ${ }^{*}$ AND C. R. WEISBIN, "Recent CESAR Research Activities in Sensor Based Reasoning for Autonomous Machine," pp. 417-421. (3.33)

17th Workshop on Applied Imagery Pettern Recognition, Washington, DC, November $2-4,1988$

JONES, J. P., AND K. M. CLINARD," "Parallel Relaxation Matching." (3.58)

Workshop on Design for Computer Experiments, Oek Ridge, TN, November 3-5, 1988

CHURCH, A., JR., AND T. J. MITCHELL, "Computer Experiments to Optimize a Compression Mold Filling Process." (1.87)

DOWNING, D. J., AND S. BARTELL," "Sensitivity and Uncertainty Analysis in Environmental Models." (1.89)

MORRIS, M. D., "Bayesian Design and Analysis of Computer Experiments: Use of Derivatives in Surface Prediction." (1.94)

WORLEY, B. A., "Calculation and Use of Computer Model Derivatives." (3.88)

Southeastern-Atlantic Regional Conference on Differential Equations, Oniversity of Georgia, Athens, GA, November 4-5, 1988

LENHA RT, S. M., AND D. G. WILSON, "Changing Domains and a Solidification Problem with Void." (1.59)

SPIE Conference on Sensor Fusion: Spatial Reasoning and Scene Interpretation, Cembridge, MA, November 6-11, 1988; Proc. (1988)

BECKERMAN, M., J. P. JONES, R. C. MANN, L. A. FARKAS, * AND S. E. JOHNSTON, "Spatial Reasoning in the Treatment of Systematic Sensor Errors," Vol. 1003, pp. 338-349. (3.4)

PARKER, L. E., AND F. G. PIN, "Man-Robot Symbiosis: A Framework for Cooperative Intelligence and Control," Vol. 1006, pp. 94-103. (3.29)

REISTER, D. B., AND M. W. NOAKES," "Design of an Obstacle Avoidance System for AIMS," Vol. 1006, pp. 124-131. (3.34) 
Seminer, Old Dominion University, Depertment of Mechenical Engineering, Norfolk, VA, November 7, 1988

I.AWKINS, W. F., "Curvilinear Coordinates Fitted to a Distort.d Annular Region." (1.86)

IEEE 1988 Nuclear Science Symposium, Orlerdo, FL, November 9-11, 1988; IBEE Trens. on Neclear Seience 37 (1) (1989)

GABRIEL, T. A., J. BRAU, * AND B. L. BISHOP, "The Physies of Compensating Calorimetry and the New CALOR89 Code System," pp. 14-17. (4.48)

ROVERE, L. A., * P. J. OTADUY, AND C. MARCH-LEUBA, "Development of an Uncertain Model Based Nonlinear Optimal Pressure Control System for a Nuclear Reactor," (did not appear in proceedings). (3.96)

Oral Presentetion, State University of New York, Buffelo, NY, November 11, 1988 HEATH, M. T., "Parallel Solution of Sparse Linear Systems." (1.39)

Second International Symposium, Robotics and Manufacturing Research, Education, ard Applications, Albuquergue, NM, November 16-18, 1988; Proc. (1989)

DE SAUSSURE, G., C. R. WEISBIN, AND P. F. SPELT, "Navigation and Learning Experiments by an Autonomous Robot," pp. 935-948. (3.13)

NEA Probabilistic System Assessment Codes User Group Meeting, Paris, Prance, November 29-December 2, 1988

WORLEY, B. A., F. G. PIN, E. M. OBLOW, R. E. MAERKER, J. E. HORWEDEL, * AND R. Q. WRIGHT," "Deterministic Sensitivity and Uncertainty Analysis for Large-Scale Computer Models." (3.80)

Oral presentation, IBM Workshop on Parallel Computing, San Jore, CA, December $1-7,1988$

HEATH, M. T., "Limits to Parallelism." (1.11)

1988 Workshop on Human-Machine Symbiotic Systems (Proceedings), Oak Ridge, TN, December 5-6, 1988

PARKER, L. E., AND C. R. WEISBIN, Editors, "1988 Workshop on HumanMachine Symbjotic Systems." (3.30)

27th IEEE Conference on Decision and Control, Austin, TX, December 7-9, 1988 MARCH-LEUBA, C.," AND R. B. PEREZ, "Optimal Control of Uncertain Nonlinear Systems: An Application to a Two-Link Robotic Arm." (3.25)

International Workshop on Concurrent Computer Vision, Aalborg, Denmark, Jan. uary 25-27, 1989

JONES, J. P., "Concurrency in Mobile Robotics at ORNL." (3.54) 
JONES, J. P., "The Butterfly Accumulator and Its Application in Concurrent Computer Vision ou Hypercube Multicomputers." (3.57)

JONES, J. P., "Concurrent Computer Vision on a Hypercube Multicomputer." (3.55)

Oral Presenta: on, Statistics Department, The University of Tennessee, Knoxville, TN, January \$1, 1989

WRIGHT, T., "Near With Replacement Sampling Without Replacenıent Using Ranks." (1.117)

NASA Conference on Space Telerobotics, Pasadena, CA, Jenuary 91-February 2, 1989

WEISBIN, C. R., B. L. BURKS, J. R. EINSTEIN, R. R. FEEZELL, W. W. MANGES, * AND D. H. THOMPSON, "HERMIES-III: A Step Toward Autonomous Mobility, Manipulation and Perception." (3.44)

WATTec Conference, Kroxville, TN, February 16, 1989

REISTER, D. B., "Future Emissions of Carbon Dioxide. Can We Make a Dramatic Reduction?" (3.103)

Oral presentation, Army Survey on High Performance Computing, Washington, DC, February 28-24, 1989

ROMINE, C. H., "Overview of Architectures and Applications in Parallel Processing." (1.16)

Oral presentation, Air Force Office of Scientific Research, Washington, DC, March 1, 1989

WILSON, D. G., AND R. E. FLANERY, "Supercomputer Simulation of a 3-D Melting/Freezing Process." (1.70)

Fourth Conference on Hypercubes, Concurrent Computers, and Applications, Monterey, CA, March 6-8, 1989

CHRISTENSEN, H. I.," "Concurrent Spatio-Temporal Image Analysis." (3.49)

CHRISTENSEN, H. I., AND J. P. JONES, "Consurrent Multi-Resolution Image Analysis." (3.50)

CULIOLI, J.-C., C. W. GLOVER, J. P. JONES, AND C. ROE," "NCUBE Implementation of Some Heuristics and an Optimal Algorithm for Large-Scale Assignment Problems." (3.64)

EINSTEIN, J. R., C. W. GLOVER, AND M. DICKE!SS," "A Tool to Aid in Mapping Computatioral Tasks to a Hypercube." (3.51)

GEIST, G. A., "Reduction of a General Matrix to Tridiagonal Form Using a Hypercube Multiprocessor." (1.20) 
GEIST, G. A., M. T. HEATH, B. W. PEYTON, AND P. H. WORLEY, “A Machine-Independent Communication Library." (1.6)

Oral presentation, NASA Ames Research Center, Moffett Field, CA, March 9, 1989 HEATH, M. T., "Parallel Solution of Sparse Linear Systems." (1.40)

ANS Third Topical Meeting on Rototics and Remote Systems, Charleston, SC, March 19-16, 1989; Proc. (1989)

BECKERMAN, M., L. A. FARKAS,* J. P. JONES, R. C. MANN, AND C. W. GLOVER, "World Modelling and Multi-Sensor Integration for a Mobile Robot," Section 11-2, pp. 1-8. (3.6)

DE SAUSSURE, G., P. F. SPELT, S. M. KILLOUGH,* F. G. PIN, AND C. R. WEISBIN, "Automatic Learning by an Autonomous Mobile Robot," $\because$ retion 6-1, pp. 1-5. (3.12)

Workshop on Calorimetry for the Supereonducting Super Collider, Oniversity of Alabama, Tuscaloosa, AL, March 19-17, 1989

ALSMILLER, R. G., JR., F. S. ALSMILLER, T. A. GABRIEL, 0. W. HERMANN, * AND J. M. BARNES, " "The High-Energy Transport Code HETC88." (4.36)

GABRIEL, T. A., "CALOR89: The Code System for Calorimeter Analysis and Design." (4.45)

GABRIEL, T. A., "Detectors for the Superconducting Super Collider, Design Concepts, and Simulation." (4.44)

Southeast Atlantic Section of SIAM Meeting, University of Ternessee, Knoxville, TN, March 17-18, 1989

GRAY, L. J., "Boundary Element Method for Regions with Cracks." (1.76)

WILSON, D. G., "An Implicit, Finite Difference, Enthalpy Scheme for Phase Change Problems." (1.66)

Oral Presentation, Souih Eastern Atlantic Section of SIAM Meeting, Knoxville, TN, March 18, 1989

CULIOLI, J.-C., "Yet Another Non-linear Approach to Linear-Programming." (3.61)

1989 Eastern Multiconference, Tampa, FL, March 28-31, 1989; Proc. Advances in AI and Simulation 20(4) (March 1989)

ROBINSON, J. T., "An Intelligent Simulation Environment for Control System Design," pp. 28-32. (3.91)

Computer Science Seminar, Gainesville, FL, March 90, 1989

ROMINE, C. H., "The Influence of Distributed-Memory Architectures on Linear Algebra Algorithms." (1.32) 
SIAM Conference on Optimization, Boston, MA, April 9-5, 1989

WORLEY, B. A., "Calculation and Use of Computer Model Derivatives." (3.88)

International Conference Fifty Years Research in Nuclear Fission, Berlin, West Germany, April 8-7, 1989

MOORE, M. S., ${ }^{*}$ L. C. LEAL, ${ }^{*}$ G. DE SAUSSURE, R. B. PEREZ, AND N. M. LARSON, " "Resonance Structure in the Fission of $\left({ }^{235} \mathrm{U}+n\right) . "(2.42)$

ACM Southeast Region 27th Annual Conference, Atlanta, GA, April 5, 1989; Proc. (1989)

NARANG, H. N.,* AND J. B. DRAKE, "Parallel Solutions of a 2-D Phase Change Problem on a Hypercube," pp. 650-656. (1.60)

Oral presentation, Ohio Oniversity, Athens, OH, April 6-7, 1989

DICKENS, J. K., "Neutron Research at the Oak Ridge Electron Linear Accelerator." (2.69)

DICKENS, J. K., "Reactor Safety and the Status of Commercial Nuclear Power in the United States." (2.68)

ANS Topical Meeting on Advances in Nuclear Engineering Computation and Radiation Shielding, Sante Fe, NM, April 9-19, 1989; Proc. Vols. 1 and 2 (1989)

AZMY, Y. Y., "Geometrical Shape Optimization of a Cold Neutron Source Using Artificial Intelligence Strategies," Vol. 2, pp. 67:1-67:12. (4.3)

EMMETT, M. B., W. A. RHOADES, AND J. O. JOHNSON, "MASH, The Monte Carlo Adjoint Shielding Code," Vol. 1, pp. 42:1-42:10. (4.82)

FU, C. Y., "A Re-Evaluation of ${ }^{32} S(n, p)$ Cross Sections from Threshold to 5 $\mathrm{MeV}$, "Vol. 1, pp. 17:1-17:11. (4.83)

INGERSOLL, D. T., R. W. ROUSSIN, C. Y. FU, AND J. E. WHITE, "Generatio.. of Broad-Group Neutron/Photon Cross-Section Libraries for Shielding Applications," Vol. 1, pp. 21:1-21:9. (4.23)

KIRK, B. L., AND Y. Y. AZMY, "A Parallel Approach to the Nodal Method Solution of the Two-Dimensional Diffusion Equation," Vol. 1, pp. 23:1-23:12. (5.5)

RHOADES, W. A., AND R. E. FLANERY, "3-D Discrete Ordinates Calculetions with Parallel-Vector Processors," Vol. 2, pp. 69:1-69:11. (4.30)

TRUBEY, D. K., M. J. BERGER, * AND J. H. HUBBELL," "Photon Cross Sections for ENDF/B.VI," Vol. 1, pp. 18:1-18:12. (5.16)

8th Symposium on the Training of Nuclear Facility Personnel, Gatlinburg, TN, April 24-27, 1989; Proc. (April 1989)

CARTER, R. J., E. M. CONNELLY,* AND P. A. KROIS,* "Feasibility of Quantitative Performance Measures for Evaluating Nuclear Power Plant Operators," pp. V.A.5.1 - V.A.5.19. (3.120) 
American Nuclear Society and National Bureau of Standards Conference on Fifty Years with Nuclear Fission, Gaithersburg, MD, April 26-28, 1989

DIVADEENAM, M., * T. A. GABRIEL, O. LAZARETH, * M. S. SPERGEL, * AND T. E. WARD, "Production Rates of Cosmogenic Nuclides in Stony Meteorites." (4.42)

LEAL, L. C., G. DE SAUSSURE, AND R. B. PEREZ, "Analysis of the ${ }^{235} U$ Neutron Cross Sections in the Resolved Resonance Range." (2.39)

PEREZ, R. B., G. DE SAUSSURE, F. C. DIIILIPPO, L. W. WESTON, AND J. A. HARVEY, "Subthreshold and Near-Subthreshold Fission Physics Measurements at the Oak Ridge Electron Linear Accelerator Facility." (2.22)

WESTON, L. W., AND J. H. TODD, * "High Resolution Fission Crnss Section Measurements at ORELA." (2.23)

American Physical Society, Baltimore MD, May 1-4, 1989; Proc. Vol. 94(4) (1989) HUTCHINSON, D. P., ${ }^{*}$ R. K. RICHARDS, ${ }^{*}$ C. A. BENNETT, ${ }^{*}$ C. C. HAVENER, ${ }^{*}$ C. H. MA, F. G. PEREY, R. R. SPENCER, J. K. DICKENS, B. D. ROONEY, "A Search for Cold Fusion Neutrons at ORELA." (2.11)

WINTERS, R. R., * R. F. CARLTON, ${ }^{*}$ AND C. H. JOHNSON, ${ }^{*} n+{ }^{10}$ Ar sand p-Wave Neutron Resonance Spacings and Strength Functions," p. 1243. (2.25)

2nd International Conference on Trends in Welding Research, Gatlinburg, TN, May $14-18,1989$

ALEXIADES, V., "Shrinkage Effects on Solidification." (1.35)

IEEE 1989 International Conference on Robotics and Automation, Scottsdale, AZ, May $14-19,1989$

MANN, R. C., J. P. JONES, M. BECKERMAN, C. W. GLOVER, L. FARKAS,* AND J. R. EINSTEIN, "Sensor Integration Using Concurrent Computing OnBoard the ORNL Mobile Robot." (3.60)

7th Power Plant Dynamics, Control, and Testing Symposium, Knoxville, TN, May 15-17, 1989; Proc. (IA 19y 1989)

CARTER, R. J., "Human Factors Aspects of Advanced Instrumentation in the Nuclear Industry," pp. 14.01-14.12. (3.127)

KNEE, H. E., AND J. C. SCHRYVER, "Operator Role Definition and Human System Integration," p. 5.01. (3.121)

ROBINSON, J. T., "An Intelligent Simulation Environment for Control System Design," pp. 3.01-3.12. (3.91) 
Workshop on Integration of AI and Robotic Systems, Scottsdale, AZ, May 19, 1989 BECKERMAN, M., D. L. BARNETT,* J. R. EINSTEIN, J. P. JONES, P. F. SPELT, AND C. R. WEISBIN, "Robust Performance of Multiple Tasks by an Autonomous Robot." (3.5)

PARKER, L. E., AND C. R. WEISBIN, "Review of the 1988 Workshop on Human-Machine Symbiotic Systems." (3.31)

Oral Presentation, Annual Banquet of Youngstown State Chapter of Pi Mu Epsilon, Youngstown State University, Youngstown, OH, May 19, 1989

FLANERY, R. E., “A Career as a Working Mathematician - What It's Like to Do It for Real." (1.74)

11th Intemational Conference on Transport Theory, Blacksburg, VA, May 22-26, 1989

PROTOPOPESCU, V., "An Example of a Reaction-Diffusion System with Nonlinear Competitive Interactions." (4.77)

SIAM Symposium on Sparse Matrices, Gleneden Beach, OR, May 22-24, 1989

LEWIS, J. G., * PEYTON, B. W., AND A. POTHEN,* "A Fast Algorithm for Reordering Sparse Matrices for Parallel Factorization."

NG, E., "A Comparison of Some Methods for Solving Sparse Nonsymmetric Linear Systems." (1.46)

PEYTON, B. W., "A Tie-Breaking Scheme for the Minimum Degree Algorithm."

Oral Presentation, Institute of Atomic Energy, Department of Nuclear Physics, Beijing, China, May 29,1989

WHITE, J. E., "Multigroup Cross Section Processing Methods Used at Oak Ridge National Laboratory."

Workshop on Cold Fusion Phenomena, Santa Fe, NM, May 29-25, 1989

HUTCHINSON, D. P., R. K. RICHARDS, C. A. BENNETT, C. C.

HAVENER,* C. H. MA,* F. G. PEREY, R. R. SPENCER, J. K. DICK-

ENS, B. D. ROONEY, J. BULLOCK, IV, * AND G. L. POWELL," "Cold Fusion Neutron Measurements at ORNL." (2.11)

Joint USDOE/Japan Workshop on Fus n Neutronics, Osaka University, Japan, May 24-25, 1989

WHITE, J. E., "Current Activities of RSIC Related to Fusion Neutronics Codes and Data."

The Third International Conference on Genetic Algorithms, Fairfax, VA, June \&-7, 1989; Proc. J. D. Schaffer, Ed., Morgan Kaufmann Publishers, Inc., San Maten, CA (1989)

PETTEY, C. C., AND M. R. LEUZE, "A Theoretical Investigation of a Parallel Genetic Algorithm," pp. 398-405. (1.15) 
1989 Annual Summer Meeting of the Americen Nuclear Society, Atlante, GA, June 1-8, 1989; Trans. Am. Nucl. Soc. 59 (1989)

DIFILIPPO, F. C., M. ABU-SHEHADEH, * AND R. B. PEREZ, "Two-Point and Two-Energy Group Kinetics Model of the ANS Reactor," p. 347. (4.16)

LEAL, L. C., * G. DE SAUSSURE, R. R. WINTERS, * AND R. B. PEREZ, "Statistical Properties of the ${ }^{235} \mathrm{U}$ Resonance Parameters up to $300 \mathrm{eV}$," $\mathrm{p}$. 341. (2.41)

PRIMM, R. T., III, "Reactor Physics Studies of Various Advanced Neutron Source Reactor Core Configurations," p. 346. (4.28)

WRIGHT, R. Q. * M. L. WILLIAMS, * AND C. O. SLATER, "Impact of ${ }^{235} \mathrm{U}$ (MAT 9235) Evaluation on $k_{\text {eff }}$ of Thermal Systems, ${ }^{n}$ p. 343. (4.34)

Conference on Expert Systems Applications for the Electric Power Industry, Orlando, FL, June 5-8, 1989

CARTER, R. J., AND R. E. UHRIG," "Human Factors Issues Related to Expert Systems for Electric Power Plant Control Rooms." (3.129)

8th International Congress of Cybernetics and Systems, Hunter College, New York, June 11-15, 1989

IYENGAR, S. S., C. R. WEISBIN, AND F. G. PIN, "Asynchronous Production Systems for Controlling Autonomous Mobile Robots." (3.18)

6th Scandanavian Image Analysis Conference, Oulu, Finland, June 19-22, 1989 BOWYER, K. W., * J. P. JONES, AND C. H. I.AKE, * "Computing the Hough Transform on an MIMD Hypercube." (3.47)

Air Pollution and Waste Management Control Association 82nd Annual Meeting and Exhibition, Anaheim, CA, June 25-90, 1989

G. F. FLANAGAN, W. FRAIZE, W., * AND T. KARTACHAK," "Risk Associated with the Demilitarization of the United States Chemical Weapons Stockpile." (4.94)

Oral presentation, Health Physics Society Annual Meeting, Albuquerque, NM, June 25-28, 1989

MORRIS, M. D., "The Role of Assumptions in Statistical Modeling." (1.136)

DOLAN, B. W.," P. G. GRÖER,* AND V. R. R. UPPULURI, "Characterizing Occupational Dose Distributions Using the Hybrid Lognormal Distribution." (1.125)

Oral presentation, NSF Summer Program for College Students, Mathematics De. partment, University of Tennessee, Knoxville, TN, June 28, 1989

WRIGHT, T., "Some Basic Concepts in Probability Sampling From a Finite Universe (Population)." 
Oral presentation, International Mathematics and Statistical Library, Houston, TX, July 7, 1989

NG, E., "Sparse Partial Pivoting Using Static Storage Schemes." (1.44)

Technology-Based Confidence Building: Energy and Environment, Santa Fe, NM, July 9-14, 1989

REISTER, D. B., "Energy Technology R\&D: What Could Make a Difference?" (3.102)

SIAM Annual Meeting, San Diego, CA, July 17-21, 1989

WORLEY, P. H., "Explicitly Parallel Algorithms for Hyperbolic PDEs - A Proof of Principle." (1.49)

1989 ASME Pressure Vessels and Piping Conference, Honolulu, HI, July 28-27, 1989

WEISBIN, C. R., B. L. BURKS, J. R. EINSTEIN, R. R. FEEZELL, W. W. MANGES, * AND D. H. THOMPSON, "Research in Autonomous Robotics at ORNL Using HERMIES-III." (3.43)

MIT Workshop on Energy and Environmental Modeling and Policy Analysis, Cambridge, MA, July S1-A ugust 1, 1989

REISTER, D. B., "Energy Technology R\&D: What Could Make a Difference?" (3.102)

Oral Presentation, Gordon Research Conference on Statistics in Chemistry and Chemical Engineering, New Hampton, NH, July \$1-August \&, 1989

MITCHELL, T. J., AND M. D. MORRIS, "Design and Analysis of Computer Experiments in Chemistry and Chemical Engineering."

1989 National Heat Transfer Conference, Philadelphia, PA, August 6-9, 1989

ALEXIADES, V., "Effect of a Void on Cyclic Melting and Freezing of an Encapsulated PCM." (1.54)

1989 Joint Statistical Meetings, Washington, DC, August 6-10, 1989

HU, P. S., * WRIGHT, T., AND S.-P. MIADO,* "A Comparative Study of Six Data Sources' Ability for Estimating Interstate Motor Carrier VMT."

MORRIS, M. D., "Factorial Sampling Plans for Preliminary Computational Experiments." (1.93).

OSTROUCHOV, G., AND E. L. FROME, "A Model Search Procedure for Hierarchical Log-Linear Models." (1.115)

American Institute of Chemical Engineers Summer National Meeting, Philadelphia, PA, A ugust 20-2S, 1989

CARTER, R. J., "Digital Instrumentation and Interactive Computer Systems: A Survey of the Use of Advanced Technology in the Nuclear Industry." (3.126) 
1989 IEEE International Conference on Systems Engineering, Dayton, OH, August 24-26, 1989; Proc. (1989)

MANN, R. C., C. R. WEISBIN, AND F. G. PIN, "Research in Mobile Robotics at ORNL/CESAR," pp. 367-371. (3.23)

Oral Presentation, presented to the Chattanooga Engineers Club, Chattanooga, TN, A ugust 28, 1989

MOSES, D. L., "A Revisit to the Passively-Safe Reactors." (4.96)

European Conference on Mathematics in Industry, Glasgow, Scotland, August 28S1, 1989

GRAY, L. J., G. E. GILES, ${ }^{*}$ J. BULLOCK, IV* AND P. McKENZIE, * "Electroplating Simulation." (1.82)

Eleventh International Conference on Boundary Element Methods, Cambridge, MA, August 29-91, 1989; Proc. Advances in Boundary Elements, Vol. 1, C. A. Breb. bia and J. J. Connor, Eds., Computational Mechanics Publications, Boston, MA (September 1989)

GRAY, L. J., "Numerical Experiments with a Boundary Element Technique for Corners," pp. 243-251. (1.78)

17th Session of the Internal Statistical Institute, Paris, France, August 29-September 6,1989

BOWMAN, K. O., AND L. R. SHENTON," "Moment Methods and the Gram-Charlier Type A." (1.98)

NOTE: The following meeting is included here because it was erroneously left out of the 1987 Progress Report.

Workshop on Detector Simulation for the SSC, Argonne National Laboratory, August 24-28, 1987

GABRIEL, T. A., F. S. ALSMILLER, R. G. ALSMILLER, JR., B. L. BISHOP,* O. W. HERMANN, ${ }^{*}$ AND J. O. JOHNSON, "CALOR87: HETC87, MICAP, EGS4, and SPECT, A Code System for Analyzing Detectors for Use in High Energy Physics Experiments." (4.47)

GABRIEL, T. A., J. O. JOHNSON, AND J. BRAU," "MICPAP: A Program for Low Energy Neutron, Ion, and Gamma-Ray Transport and One of Its Applications in Calorimeter Design: Hydrogen Knock- ${ }^{-} .1$ as a Method for Achieving Compensation." (4.50) 
NOTE: The following meetings/seminars will be held after August 31, 1989. However, since the papers/abstracts were cleared through the Laboratory Records Department before August 31, 1989, they are being included in this report.

'89 IROS International Conference on Intelligent Robot and Systems, “The Autonomous Robot and Its Application," Tsubube, Jepan, September S-7, 1989

PIN, F. G., M. BECKERMAN, P. F. SPELT, J. T. ROBINSON,* AND C. R. WEISBIN, "Autonomous Mobile Robot Research Using the HERMIES-IH Robot." (3.32)

IAEA Consultants' Meeting on Cross Sections for the Generation of Long-Lived Redionuclides, Argonne, IL, September 11-12, 1989

FU, C. Y., "Status of Theories Used for Calculations of Long-Lived Activation Cross Sections." (2.50)

NEANDC Specialist's Merting on Neutron Activation Cross Sections for Fission and Fuion Bnergy Applications, Argonne, IL, September 19-15, 1989

FU, C. Y., "A Review of Activation Cross Sections in the ENDF/B-VI General Purpose Files of $\mathrm{Cr}, \mathrm{Fe}, \mathrm{Ni}, \mathrm{Cu}$, and $\mathrm{Pb} . "$ (2.51)

Sixth Coordination Meeting for the Program to Meet Nuclear Data Needs for Pusion Energy, Athens, OH, September 19-21, 1989

FU, C. Y., "Pairing Corrections and Spin Cutoff Factors in Exciton Level Densities for Two Kinds of Fermions." (2.48)

IAEE 1989 North American Conference, Los Angeles, CA, October 16-18, 1989; Proc. (1989)

REISTER, D. B., "The Replacement Cost Integration Program, An Engineering-Economic Model of Oil Supply," pp. 89-98. (3.108)

99rd Annual Human Factors Society Meeting, Denver, CO, October 16-20, 1989 TERRANOVA, M., R. L. SEAMSTER, C. E. SNYDER,* AND 1. E. TREITLER," "Cognitive Task Analysis: Techniques Applied to Airborne Weapons Training." (3.119)

17 th Water Reactor Safety Information Meeting, Rockville, MD, October 29-25, 1989

CARTER, R. J., "Human Factors Survey of Advanced Instrumentation and Controls." (3.128)

Internatinnal Symposium on Research Reactor Safety, Operations and Modifications, Canada, October 29-27, 1989

FLANAGAN, G. F., D. H. JOHNSON, D. BUTTEMER,* AND S. KAPLAN," "Risks Associated with the Operation of the Oak Ridge National Laboratory High Flux Isotope Reactor (HFIR)." (4.82) 
Sensor Fusion II: Human and Machine Strategies SPIE's Advances in Intelligent Robotics, Philadelphia, PA, November 5-10, 1989

GLOVER, C. W., "Design and Implementation of a Multi-Sensor Fusion Algorithm on a Hypercube Computer Architecture." (3.52)

Interservice/Industry Training Systems Conference, Forth Worth, TX, November 16,1989

SNYDER, C. E., N. E. CLAPP, JR., D. E. SMITH,* M. TERRANOVA, J. DAVIS, * J. L. FINNEY, * P. G. GUTHRIE,* G. B. PAYNE,* AND S. WEBSTER, "Teamwork and Data Deliverables: A Map to Software and Training Design Integration." (3.116)

1989 Annwal Winter Meeting of the American Nuclear Society, San Francisco, CA, November 26-90, 1989; Trens. Am. Nucl. Soc. 60 (1989)

CARLSON, A. D., W. P. POENTITZ, * G. M. HALE, * AND R. W. PEELLE, "The Neutron Cross-Section Standards Evaluations for ENDF/B-VI," p. 604.

HORWEDEL, J. E., "GRESS Adjoint Matrix Reduction Algorithms," p. 590. (3.80)

LARSON, D. C., AND P. G. YOUNG, "Structural and Shielding Materiai Evaluations for ENDF/B-VI," p. 606. (2.58)

LEAL, L. C., * G. DE SAUSSURE, R. B. PEREZ, AND R. Q. WRIGHT, "Test of the ENDF/B Unresolved Resonance Formalism for U-235," p. 620. (2.60)

MAERKER, R. E., P. D'HONDT, * L. LEENDERS, * AND A. FABRY, "Benchmark Verification of a Method for Calculating Leakage from PLSA Modified Cores," p. 545. (3.113)

MAERKER, R. E., AND B. A. WORLEY, "Development and Application of a New Deterministic Method for Calculating Computer Model Result Uncertainties," p. 591. (3.84)

PEELLE, R. W., "Covariance Data for ENDF/B-VI," p. 608. (2.61)

ROUSSIN, R. W., "Methods and Formats for ENDF/B-VI," p. 617. (5.12)

SAWHNEY, R. S., J. C. SCHRYVER, H. E. KNEE, AND H. L. DODDS,* "A Hypothesis Generation Model of Initiating Events for Nuclear Power Plant Operators," p. 179. (3.123)

SMITH, M. S., AND W. A. RAGLAND," "Interaction of CREDO with EBRII FRA Development," p. 411. (3.134)

WESTON, L. W., P. G. YOUNG, * AND W. P. POENITZ," "New Cross Section Evaluations for Reactor Fuel Materials," p. 606. (2.24) 
International Meeting on Safety Margirs in Criticality Safety, San Prencisco, CA, November 26-90, 1989; Proc. (1989)

DIFILIPPO. F. C., "Theoretical Analysis of Reactivity Measurement with the ${ }^{252}$ Cf Source Ratio Method," pp. 16-23. (4.11)

18th IEEE Conference on Decision and Control, Tampe, FL, December 15-15, 1989 BARNETT, D. L., G. DE SAUSSURE, S. S. IYENGAR, * F. G. PIN, A. SABHARWAL, * AND C. R. WEISBIN, "Robot Navigation Research at CE SAR." (3.1)

International Joint Conference on Neural Networks, Weshington, DC, Januery 1519,1990

CULIOLI, J.-C., V. PROTOPOPESCU, C. BRITTON, * AND N. ERICSON,* "A Neural Network for Bounded Linear Programming." (3.73)

CULIOLI, J.-C., V. PROTOPOPESCU, C. BRITTON, ${ }^{*}$ AND N. ERICSON, "Neural Networks Models for Linear Programming." (3.72)

1990 HEA RT Conference, Naval Postgreduate School, Monterey, CA, Pebruery 1216,1990

JOHNSON, J. O., M. S. SMITH, AND R. T. SANTORO, "Nuclear Weapon Radiation Effects on a Space Based Interceptor Weapon Platform." (4.59)

SANTORO, R. T., J. M. BARNES, * AND J. O. JOHNSON, "The Effects of Natural and Enhanced Van Allen Belt Radiation on a Space Based Weapon Platform." (4.60)

SMITH, M. S., "Material Response to Incident X-Rays." (4.62)

International Society for Optical Engineers, Applications of Artificial Intelligence, Orlando, FL, April 16-20, 1990

BELMANS, P. F. R., "An Approach to Elemental Task Learning." (3.8)

International Conference on the Physics of Reactors: Operation, Design, and Computation, Marseille, France, April 28-26, 1990

AZMY Y. Y., AND B. L. KIRK, "Convergence Properties of Itcrative Algorithms for Solving the Nodal Diffusion Equations." (4.8)

DE SAUSSURE, G., L. C. LEAL," AND R. B. PEREZ, "Reich-Moore and Adler-Adler Representations of the ${ }^{235} \mathrm{U}$ Cross Sections in the Resolved Resonance Region." (2.30)

DIFIIIPPO, F. C., "Neutron Kinetics of High-Intense Fission Neutron Sources." (4.13) 
1990 IBEE Conference on Robotics and Autometion, Cincinneti, OH. May 19-18, 1990

BARNETT, D. L.," AND F. G. PIN, "Robot Navigation Research Using the HERMIES Mobile Robot." (3.3)

American Nuclear Society Topical Meeting on Advences in Humen Factors Research on Man/Computer Interections: Nuclear and Beyond, Neshwille, TN, June 10-14, 1990

SAWHNEY, R. S., * AND J. C. SCHRYVER, "An Operator Model-Based Filtering Scheme." (3.122)

SNYDER, C. E.," AND M. TERRANOVA, "A Part-Task Trainer in the Naval Air Systems Tommand." (3.117)

Pree Boundary Problems: Theory and Applications Meeting, Center for Research Merhematics, Montreal, Careda, June 19-22, 1.990

ALEXIADES, V., AND J. B. DRAKE, A. Weak Formulation for PhaseChange Problems with Bulk Morement Due to Unequal Densities." (1.57)

DRAKE, J. B., AND V. ALEXIADES, "A Convective Flow in the Presence of Melting." (1.58)

Seventh ASTM-EURATOM Symposium on Reactor Dosimetry, Strasbourg, Prence, August 27-91, 1990

MAERKER, R. E., P. D'HONDT,* L. LEENDERS, * AND A. FABRY,

"Analysis of the VENUS-3 Experiments." (3.114) 


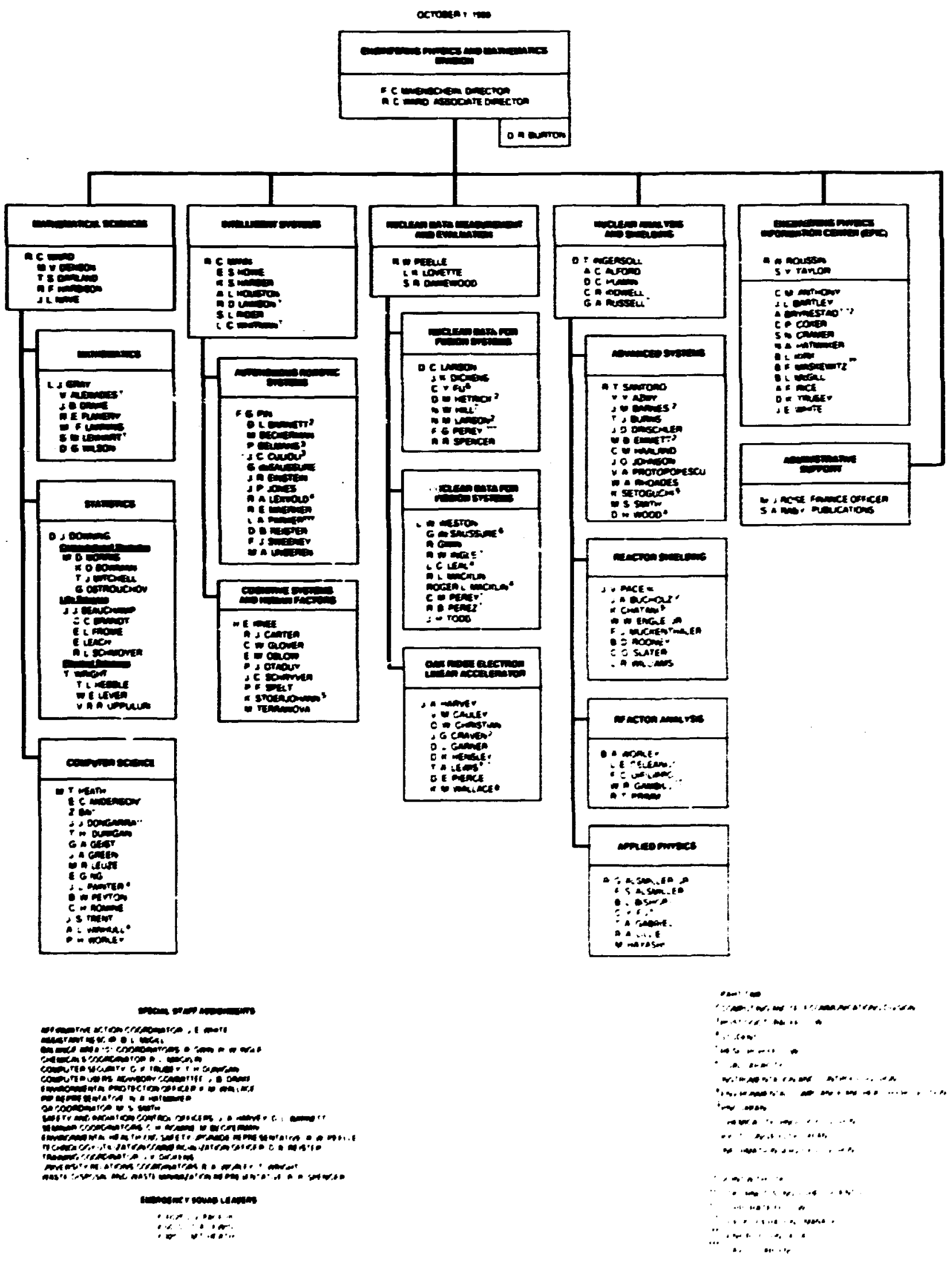




\section{AUTHOR INDEX}

Abo-Shehadeh, M. 4.15,4.16

Aldrich, T. E. 1.139

Alexander, C. W. 2.15

Alexiades, V. 1.54, 1.55,1.56,1.57,1.58

Alsmiller, F. S. 4.35,4.36,4.37,4.38,4.47

Alsmiller, Jr., R. G. $4.21,4.25,4.35,4.36,4.37,4.38$, 4.43,4.47,4.52,4.53

Arnold, S. F. 1.116

Aster, A. L. 1.80

Auerbach, S. 1. $3.100,3.101$

Axiz, M. J. 1.85

Azmy, Y. Y. 4.1,4.2,4.3,4.4,4.5,4.6,4.7,4.8,4.67,4.68, $4.69,4.70,4.81,5.3,5.4,5.5$

Barhen, J. 3.17,3.74

Barnes, J. M. 4.36,4.52,4.53,4.54,4.55,4.57,4.58,4.60, $4.63,4.78,4.80,4.85,4.86$

Barnett, D. L. 3.1,3.2,3.3,3.5,3.10

Bartell, S. 1.89

Beauchamp, J. J. 1.109,1.110,1.130,1 132,1.135

Beckerman, M. 3.4,3.5,3.6,3.7,3.21,3.32,3.60

Beer, H. 2.1,2.2

Belmans, P. F. B. 3.8

Bennett, C. A. 2.11

Herger, M. J. 5.16

Bhargave, T. N. 1.120

Bishop, B. L. $4.37,4.47,4.48,4.57$

Blencoe, J. G. 2.3

Bockhoff, K. H. 2.66

Boffi, V. C. 4.70

Bowman, K. O. 1.98,1.99,1.100,1.101,1.102,1.103, 1.104,1.121,1.122,1.123,1.131

Bowyer, K. W. 3.9,3.47

Brau, J. E. 4.39,4.40,4.48,4.50

Britton, C. 3.72,3.73,3.95

Brnadhead, B. L. 4.88

Broadway, J. 5.7

Bullock, J. 1.82,2.11

Burks, E. L. 3.10,3.11,3.43,3.44

Burns, T. J. 4.56,4.64

Burtis, L. S. 1.130

Buttemer, D. 4.92

Cacuci, D. G. $4.67,4.71,4.72,4.88$

Campbell, D. J. 4.91

Cao, Z. 2.19

Carlson, A. D. 2.66

Carlton, R. F. $2.4,2.25$

Carnes, S. 4.93

Carpenter, A. V. 1.124

Carreras, B. 1.73
Carter, R. J. 3.120,3.126,3.127,3.128,3.129, 3.130,3.131

Childs, R. L. $4.9,4.29,4.89,4.90$

Christensen, H. I. 3.48,3.49,3.50,

Christiansen, D. S. $3.97,3.98,3.107,3.108$

Chu, E. 1.18

Church, A., Jr. 1.87

Clapp, N. E., Jr. 3.116

Clinard, K. M. 3.58

Cohen, G. 3.62,3.63

Colyer, S. P. 1.134

Conde, H. 2.62

Connelly, E. M. 3.120

Conopast, J. V. 3.109

Coener, C. 4.73

Cox, R. L. 4.78

Cramer, S. N. 5.1,5.2,5.7,5.8

Crane, A. T. 3.100,3.101

Culioli, J.-C. $3.61,3.62,3.63,3.64,3.65,2.66,3.67$, $3.68,3.69,3.70,3.71,3.72,3.73$

Curlee, T. R. 3.99

Currin, C. 1.88

Dagenhart, W. K. 4.54

Das, R. K. 2.5

Davis, G. J. $1.23,1.27$

Davis, J. 3.116

de Sausoure, G. 2.16,2.22,2.26,2.27,2.28,2.29, 2.30,2.31,2.32,2.33,2.38,2.39,2.40,2.41,2.42,

2.44,2.60,3.1,3.2,3.12,3.13,3.33,3.35,3.37.

3.40,3.45

Derrien, H. $2.26,2.27,2.28,2.20$

D'hondt, P. 3.113,3.114

Dictens, J. K. 2.6,2.7,2.8,2.11,2.12,2.45,2.46, 2.67,2.68,2.69

Dickens, M. 3.51

Di Cirecio, A. 4.51

Difilippo, F. C. $2.22,4.7,4.10,4.11,4.12,4.13,4.14$, 4.15,4.16,4.32,4.75

Divadeenam, M. 4.41,4.42,4.49

Dockery, J. 4.78

Dodds, H. L. 3.123

Dolan, B. W. 1.125

Dowdy, L. W. 1.13

Downing, D. J. $1.89,1.126,1.127$

Drake, J. B. $1.57,1.58,1.60,1.68,1.69,1.72$, 1.73

Drischler, J. D. 4.55,4.57,4.58,4.63

DuFrain, R. J. 1.106

Lunford, C. L. 2.70,5.13

Dunigan, T. H. 1.1,1.2,1.3

Dycus, F. M. 1.81 
Sasterly, C. E. $1.139,3.130$

Ebeling-Koning, D. 3.94

Einstein, J. R. 3.5,3.21,3.43,3.44,3.45,3.51,3.60

Egan, B. Z. 1.130

Elliscin, B. C. 4.91

Emmett, M. B. 4.82

Engle, Jr., W. W. 4.17

Entriken, R. 1.4

Ericson, N. 3.72,3.73

Etheridge, Y. H. 1.105

Ezell, G. H. 1.130

Fabry, A. 3.113,3.114

Farkas, L. A. $3.4,3.6,3.21,3.60$

Farquharson, J. A. 4.91

Feezell, R. R. 3.43,3.44

Filippone, W. L. 4.43

Finlay, R. W. 2.5

Finley, M. 3.14

Finney, J. L. 3.116

Flanagan, G. F. $4.91,4.92,4.93,4.94,4.95$

Fianders, W. D. 1.124

Flanery, R. E. $1.68,1.69,1.70,1.71,1.74,4.30$

Fraize, W. E. $4.94,4.95$

Frome, E. L. 1.106,1.115,1.124,1.128,1.133,1.134

Fry. S. A. 1.124

Fryxell, R. C. 3.15

Fu, C. Y. $2.34,2.47,2.48,2.49,2.50,2.51,2.52,2.53$, $2.54,2.55,2.56,2.57,2.58,4.23,4.83,4.87$

Fulkerson, W. 3.100,3.101

Funderlic, R. E. 1.27

Furuta, K. 5.18

Gabriel, T. A. $2.21,4.24,4.36,4.37,4.39,4.40,4.41$, $4.42,4.43,4.44,4.45,4.46,4.47,4.48,4.49,4.50,4.51$, $4.52,4.53,4.55,4.57,4.58,4.61,4.63,4.65$

Gambill, W. R. 4.18

Geist, G. A. $1.5,1.6,1.19,1.20,1.21,1.22,1.23$, $1.24,1.25,1.26,1.27$

George, A. 1.18,1.34,1.35,1.36,1.37,1.38

Giles, G. E. $1.80,1.81,1.82,1.83$

Gillies, S. T. 1.75

Gilpin, H. 5.17

Glover, C. W. $3.6,3.21,3.51,3.52,3.53,3.60,3.64$

Goldstein, M. 3.74

Goodman, M. S. 4.51

Gonzalez, R. C. 3.22

Gray, L J. $1.72,1.76,1.77,1.78,1.79,1.80,1.81$, $1.82,1.83,1.84,1.85$

Greene, N. M. 4.27

Gröer, P. G. 1.125

Gulati, S. 3.17

Gung, $C, Y, 4.52$
Guthrie, P. G. 3.116

Guthrie, V. H. 4.91

Haaland, C. M. $4.84,4.85,4.86$

Hagan, C. W., Jr. 3.100

Haire, J. 3.132

Hamel, W. R. 3.16,3.20,3.42,3.46

Han, J. 3.21, 3.77,3.78,3.79

Hansen, P. C. $1.28,1.29$

Harvey, J. A. 2.4,2.5,2.9,2.10,2.20,2.22,2.66

Havener, C. C. 2.11

Heath, M. T. $1.6,1.7,1.8,1.9,1.10,1.11,1.12$, $1.30,1.31,1.34,1.39,1.40$

Heer, E. 3.45

Henderson, D. L. 4.19,4.20,4.21,4.22

Henkel, C. S. 1.31

Hermann, O. W. 4.36,4.37,4.38,4.47

Hershberger, R. L. 2.19

Hetrick, D. M. $2.52,2.54,2.55,2.56,2.57,2.58$

Hicks, H. R. 1.73

Hill, $N$. $W$. $2.4,2.9,2 \cdot 10,2.19,2.20,2.21$

Hopp, T. 1.123

Horwedel, J. E. $\quad 3.80,3.81,3.82,3.89,3.90$

Hubbell, J. H. 5.16

Humphrys, B. L. 3.132

Huncaker, C. T. 1.135

Hutchinson, D. P. 2.11

Ingersoll, D. T. $4.23,4.87,4.89,4.90$

Ingle, $R$. W. 2.16

Ingraffea, A. R. 1.84

Iyengar, S. S. $\quad 3.1,3.17,3.18,3.37,3.38,3.39$

Jammalamadaica, S. R. 1.129

Jimenez, B. D. 1.130

Johnson, C. H. $2.4,2.25$

Johnson, D. 4.92

Johneon, 3. O. $2.21,4.24,4.47,4.50,4.55,4.57,4.58$, $4.59,4.60,4.63,4.64,4.82$

Johnston, S. E. 3.4

Joiner, E. E. 1.134

Jones, D. T. 3.115

Jones, J. P. 3.4,3.5,3.6,3.10,3.21,3.47,3.50,

$3.54,3.55,3.56,3.57,3.58,3.59,3.60,3.64$

Jones, T. D. $1.137,1.138$

Kacker, R. 1.123

Kaizerman, S. 3.86

Kam, F. B. K. 3.110

Kaplan, כิ. 4.92

Kaplan, T. 1.85

Karnofsky, B. 3.131

Kartachak, T. 4.94 
Kash, D. E. $3.100,3.101$

Kastenbaum, M. A. 1.131

Kelly, J. M. 1.132

Killough, S. M. 3.10,3.12,3.33

Kimball, K. T. 1.139

Kirchner, J. R. 4.91

Kirk, B. L. $4 . \overline{8}, 5.3,5.4,5.5,5.7$

Kirkmam, J. Q. 4.91

Kisner, R. A. 3.92

Knez, H. E. $3121,3.123,3.133$

Koger, K. H. 3.132

Kondo, S. 5.18

Kosako, K. 4.52

Krishnaprasad, P. S. 3.41

Krois, P. A. 3.120

Kuban, D. P. 3.46

Lake, C. H. 3.47

Lam, H. K. 1.10,

Larson, D. C. $2.7,2.12,2.52,2.53,2.54,2.55,2.56$, $2.57,2.58,2.59,2.66,2.70,2.71$

Larson, N. M. $2.20,2.29,2.31,2.35,2.36,2.37,2.42$

Lawlins, W. F. 1.73,1.75,1.86

Lawson, M. A. 1.75

Lazereth, 0. 4.41,4.42,4.49

Leal, L. C. $2.10,2.29,2.30,2.31,2.38,2.39,2.40$, $2.41,2.42,2.44,2.60$

Lee, J. C. 3.42

Lee, N. E. 1.130

Lee, T. Y. 5.8

Leenders, L. 3.113,3.114

Lenhart, S. M. $1.59,4.73$

Leuze, M. R. 1.13,1,15

Lewis, J. G. 1.41

Liepins, G. R. 1.107

Lillie, R. A. 4.17,4.25,4.57,4.88

Littlefield, L. G. 1.133,1.134

Liv, J. 1.34,1.35

Lu, A. 1.24

Lundegard, R. 1.123

Lynch, V. 1.73

Lyness, E. $3.35,3.40$

Ma, C. H. 2.11

Macklin, R. L. 2.1,2.2,2.13,2.14,2.15,2.17, 2.18,2.19,2.20,2.21

Macklin, Roger 2.16,2.44

Maerker, R. E. $3.83,3.84,3.90,3.110,3.111$, $3.112,3.113,3.114$

Manges, W. W. 3.43,3.44

Mann, R. C. 3.4,3.6,3.19,3.20,3.21,3.22,3.23, $3.42,3.59,3.60,3.77,3.78,3.79$

Manneschmidt, J. F. 3.132
Marable, J. H. 2.32

Merch-i uba, C. $3.24,3.25,3.96,4.74$

Martl.a, L. F. 1.84

Martin, W. R. 3.42

Maskewitz, B. F. 5.9,5.10,5.11,5.14

Maynard, C. W. 4.22

MeCarthy, J. F. 1.130

McConnell, J. W. 2.8

McDaniel, T. W. 1.135

MeEllistrem, M. T. 2.19

McKenzie, P. 1.82

MeKnight, R. 5.13

McNeany, S. 4.65

Meacham, S. A. 3.46

Mihlmester, P. E. 3.109

Mitchell, T. J. $1.87,1.88,1.90,1.91,1.92,1.96$,

1.97,1.108,1.109,1.110

Mochizuki, T. 4.18

Moore, M. S. 2.31,2.42

Mori, T. 4.52

Morris, M. D. $1.88,1.90,1.91,1.92,1.93,1.94$, $1.95,1.96,1.111,1.128,1.136,1.137,1.138,1.139$

Moses, D. L. 4.96

Muir, D. W. 2.63

Munoz-Cobo, J. L. 4.75

Nadkarny, S. 4.33

Nakagawa, M. 4.52

Nakamura, T. 4.52

Nakai, R. 3.132,3.133

Naney, M. T. 2.3

Narang, H. N. 1.60

$\mathrm{Ng}_{\mathrm{g}}$ E. $1.25,1.34,1.35,1.36,1.37,1.38,1.42$, $1.43,1.44,1.45,1.46,1.47$

Noakes, M. W. 3.34

Oblow, E. M. 3.7,3.26,3.27,3.28,3.82,3.89,3.00

Oka, Y. 5.18

Ortega, J. M. 1.14

Ostrouchov, G. 1.112,1.113,1.114,1.115

Otaduy, P. J. $3.93,3.85,3.96$

Pace, III, J. V. 4.88

Park, K. H. 1.13

Parker, L. E. $3.29,3.30,3.31$

Paula, H. M. 4.91

Payne, G. B. 3.116

Peelle, R. W. 2.61,2.62,2.63

Perey, C. M. 2.20

Perey, F. G. $2.3,2.10,2.11,2.20,2.21,2.37,2.43,2.64$

Perez, R. B. 2.16,2 22,2.29,2.30,2.31,2.33,2.38,2.39,

$2.40,2.41,2.42,2.44,2.60,2.65,3.24,3.25,3.95,4.15$,

4. $16,4.74$ 
Perry, A. M. 3.100,3.101

Peyton, B. W. 1.6,1.41

Pettey, C. C. 1.15

Pin, F. G. $3.1,3.3,3.12,3.16,3.18,3.23,3.29,3.32$,

3.33,3.35,3.38,3.39,3.45,3.46,3.81,3.82,3.85,

$3.89,3.90$

Plemmons, R. J. 1.31

Poenits, W. P. 2.24

Pothen, A. 1.33,1.41

Powell, G. L. 2.11

Primm, III, R. T. 4.26,4.27,4.28,4.32

Protopopescu, V. 3.65,3.66,3.67,3.68,3.69,3.70, 3.71,3.72,3.73,4.67,4.68,4.69,4.70,4.71,4.72,4.73, 4.76,4.77,4.78,4.81

Ragland, W. A. 3.134

Rancoita, P. G. 4.40

Reister, D. B. $3.34,3.97,3.98,3.99,3.100,3.101,3.102$, 3.103,3.104,3.105,3.106,3.107,3.108,3.109

Renter, L. A. $1.61,1.62$

Rhoades, W. A. $4.9,4.29,4.30,4.82,4.89,4.90$

Richards, R. K. 2.11

Ritter, R. C. 1.75

Robinson, J. T. 3.32,3.91,3.92,3.93,3.94

Roe, C. 3.64

Romine, C. H. $1.14,1.16,1.17,1.26,1.32,1.33$

Rooney, B. D. 2.11,4.31

Roussin, R. W. 4.23,4.87,5.12,5.13,5.14,5.17

Rovere, L. A. $3.95,3.96$

Rusu, P. 4.79,4.80

Ryskanip, J. M. 4.32

Sabharwal, A. 3.1,3.37,3.38,3.39

Sacks, J. 1.97

Sintoro, R. T. 4.43,4.52,4.53,4.54,4.55,4.57,4.58, $4.59,4.60,4.61,4.63,4.65,4.66,4.78,4.80,4.85,4.86$

Sartory, W. K. 1.75

Sayer, A. M. 1.133,1.134

Sawhney, R. S. 3.122,3.123

Schmidt, D. M. 2.18

Schmoyer, R. L. 1.116

Schryver, J. C. $3.121,3.122,3.123,3.124,3.125$

Scollan, D. F. 4.81

Seamster, T. L. 3.115,3.119

Setoguchi, K. 3.132,3.133

Shenton, L. R. $1.98,1.99,1.100,1.101,1.102,1.103$, 1.104

Simpson, E. M. 3.59

Slater, C. O. 4.17,4.34

Smith, D. E. 3.116

Smith, M. S. 3.134,4.43,4.55,4.57,4.58,4.50,4,62, 4.63.4.64.4.65,4.66
Snyder, C. E. $3.115,3.116,3.117,3.119$

Spelt, P. F. 3.2,3.5,3.11,3.12,3.13,3.32,3.33,3.35, 3.40

Spencer, R. R. 2.11

Spergel, M. S. $4.41,4.42,4.49$

Sreenath, N. 3.36,3.41

Stallman, F. W. 3.110

Tantreraley, W. G. 1.124

Terranova, M. 3.115,3.116,3.117,3.118 3.119

Tesar, D. 3.22

Thompson, D. H. $\mathbf{3 . 4 3 , 3 . 4 4}$

Thompeon, S. 1.48,1.75

Todd, J. H. $2.21,2.23$

Todreas, N. E. 3.94

Toomarian, N. 3.74,3.75,3.86

Treitler, I. E. 3.119

Trubey, D. K. 5.6,5.14,5.15,5.16

Tulenko, J. S. $3.22,3.42$

Turnbull, B. W. 1.108

Tweed, G. L. 2.10

Uhrig, R. E. 3.129

Uppuluri, V. R. R. $1.107,1.120,1.125,1.129,1.140$

Voigt, R. G. 1.14

Wacholder, E. $3.21,3.76,3.77,3.78,3.79,3.86$

Wachspress, E. L. 1.24

Walker, W. J. 3.115

Wallace, S. A. 1.122

Ward, R. C. 1.27

Ward, T. E. $4.41,4.42,4.49$

Wasson, 0. A. 2.96

Watanabe, Y. 4.52

Webster, S. 3.116

Wehe, D. K. 3.22,3.42

Weisbin, C. R. 3.1,3.5,3.12,3.13,3.18,3.20,3.23, $3.30,3 .{ }^{2} 1,3.32,3.33,3.35,3.37,3.38,3.39,3.43,3.44$, $3.45,3.46$

Welch, W. J. 1.97

Wendel, M. W. 1.83

Wesolowski, D. J. 2.3

Weston, L. W. 2.22,2.23,2.24

White, J. E. 4.23,4.87,5.17

Williams, L. R. 4.9

Williams, M. A. 1.63

Williams, M. L. $3.110,4.33,4.34$

Wilson, D. G. $1.59,1.61,1.62,1.63,1.64,1.65,1.66$, $1.67,1.68,1.69,1.70,1.71$

Wilson, R. 4.51

Winters, R. R. $2,4,2.17,2.18,2,19,2,21,2.25,2,41$

Wood, R. T. 2.65 
Worley, B. A $\quad 3.81,3.82,3.84,3.87,3.88,3.89,3.90$

Worley, P. H. $1.6,1.12,1.49,1.50,1.51,1.52,1.53$

Wright, it. Q. 2.60,3.82,3.90,4.34

Wright, T. 1.105,1.117,1.118,1.119,1.141,1.142

Wynn, H. P. 1.97
Yesuda, H. 3.133

Ylvisaler, D. $1.88,1.92$

Young, P. G. 2.24,2.59,2.70,5.13

Yousoef, M. Z. 4.52

Yũcel, A. 4.33 


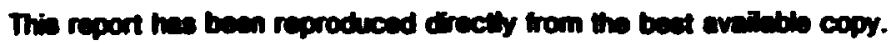

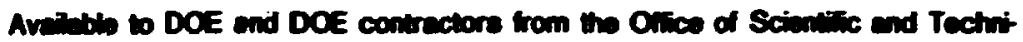

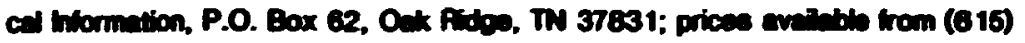
570-8101, FTS 628-8401.

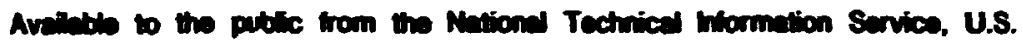
Depertinent of Commerce, 5285 Pon Royd Ad. Springliedd. VA 22161. NIIS price codere- Pristad Copy. Al6 Microliche $\mathrm{NO1}$

Thie repont was prepered es en sccount of work eponeored by en ecency of the Unived States Covernment. Nivither the Uhined Statise Covernment nor eny ecancy thereof, nor any of thai euployen, makes eny werrenty. expreses or

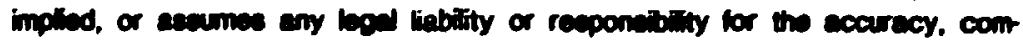

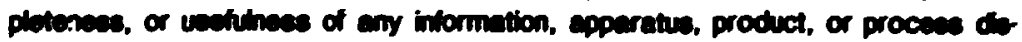

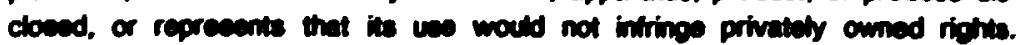
Peverence hercin to exy epecific commerciel product, procenes, or ecrvice by

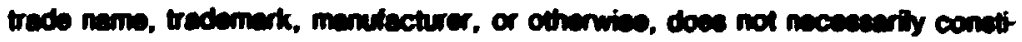

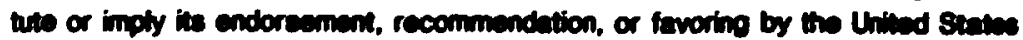
Covernment of any econcy thereof. The views and opinions of cuthors expreaend hersin do not necesearty etate or reflect those of the Uhiled States Covertunedt or any ecency thered. 